Supporting Information

\title{
3-Acetoxyquinuclidine as Catalyst in EDA Complex-Mediated Reactions triggered by Visible Light
}

\author{
Irene Bosque, ${ }^{*}$ Thorsten Bach \\ Department of Chemistry and Catalysis Research Center (CRC) \\ Technische Universität München, 85747 Garching, Germany \\ irene.bosque@ua.es
}

Table of contents:

1. General remarks

2. UV-visible spectra of the mixtures of compound $\mathbf{2 a}$ with different amines

S2

3. Optimization tables for products $\mathbf{3 b}, \mathbf{3 k}$ and $\mathbf{3 v}$

S3

4. Mechanistic insights

S3

5. Quantum yield calculation for the reaction of $\mathbf{2} \mathbf{b}^{6}$ to $3 \mathbf{b}^{6}$

S4

S7

6. Job Plot for the reaction of $\mathbf{2} \mathbf{b}^{\mathbf{6}}$ to $\mathbf{3} \mathbf{b}^{\mathbf{6}}$

$\mathrm{S} 11$

7. Cyclic voltammetry (CV) experiments of $1 \mathbf{a}$ and $\mathbf{2 a}$ to $\mathbf{2 y} \quad S 12$

8. UV-visible spectra of compounds $2 \mathbf{a}$ to $\mathbf{2 z}$ in the absence and in the presence of q-OAc $\quad$ S19

9. Synthesis of the electron donor (ED) catalyst 3-acetoxyquinuclidine (q-OAc) $\quad$ S26

$\begin{array}{ll}\text { 10. General synthetic procedures } & \text { S27 }\end{array}$

11. Guide of compounds $\quad$ S29

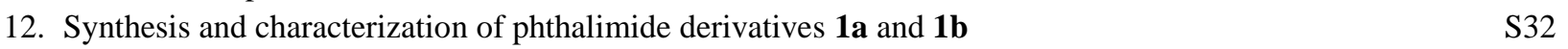

13. Synthesis and characterization of tetrachlorophthalimide derivatives $\mathbf{2 a}$ to $\mathbf{2 z} \quad$ S33

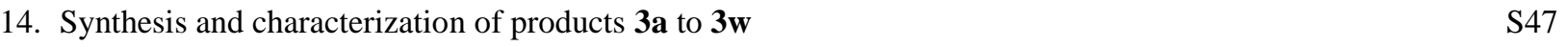

15. Synthesis and characterization of products $4 \mathbf{a}, \mathbf{4 k}, \mathbf{4 p}, \mathbf{4 r}, \mathbf{4 s}$ and $\mathbf{5 p} \quad$ S59

16. Synthesis and characterization of acids $\mathbf{S 1} \quad S 62$

17. Copy of the ${ }^{1} \mathrm{H}$ - and ${ }^{13} \mathrm{C}-\mathrm{NMR}$ spectra of the synthetized compounds and NOESY spectra of the $\quad$ S75 required compounds

18. References

S239 


\section{General remarks:}

Chemicals were purchased and used without further purification, unless otherwise noted. Tetrahydrofuran (THF), diethyl ether $\left(\mathrm{Et}_{2} \mathrm{O}\right)$ and dichloromethane (DCM) were purified using a MBSPS 800 MBraun solvent purification system. All other solvents were distilled prior to use.

Air-sensitive reactions were carried out in oven-dried glassware sealed either with a rubber septum or a screw cap under a positive pressure of dry Argon. Photocatalytic experiments were conducted in a photochemical reactor equipped with blue LEDs $(15 \mathrm{~W}, \lambda \max =455 \mathrm{~nm})$.

Analytical thin-layer chromatography (TLC) was performed on silica gel 60 (F254) glass plates. The TLC plates were visualized by either ultraviolet (UV) light $\left(\lambda=254 \mathrm{~nm}\right.$ ) or treatment with either $\mathrm{KMnO}_{4}, \mathrm{I}_{2}$ or Hanessian's stains followed by heating with a heat gun. Purification of products was performed using flash chromatography on silica gel 60 (230-400 mesh).

Melting points (mp) were measured in one replica using a Büchi M-565 apparatus.

Nuclear magnetic resonance (NMR) spectra in solution were recorded at room temperature on a Bruker AVHD300, AVHD-400, AVHD-500, or a Bruker AV-II-500 equipped with cryo probe head. Proton NMR ( $\left.{ }^{1} \mathrm{H}-\mathrm{NMR}\right)$ spectra were calibrated to the residual solvent signal of chloroform- $\mathrm{d}_{1}\left(\mathrm{CHCl}_{3} \delta=7.26 \mathrm{ppm}\right)$ and carbon NMR $\left({ }^{13} \mathrm{C}-\mathrm{NMR}\right)$ spectra to the ${ }^{13} \mathrm{C}$-D triplet of $\mathrm{CDCl}_{3}(\delta=77.16 \mathrm{ppm})$. Carbons corresponding to a $\mathrm{C}, \mathrm{CH}, \mathrm{CH}_{2}$ and $\mathrm{CH}_{3}$ have been assigned using phase sensitive 2-dimensional heteronuclear single quantum coherence (HSCQ), Heteronuclear Multiple Bond Correlation (HMBC) and Correlated Spectroscopy (COSY) experiments. Abbreviations used for single multiplicities: s-singlet, d-doublet, t-triplet, q-quartet, quin.-quintet, br-broad signal. Spectra showing a mixture of rotarmers or diastereomeric structures reflect partial integrations in signals separated for the two different components (e.g. for a mixture with 9:1 dr, separate signals are integrated as 0.1 $\mathrm{H}$ and $0.9 \mathrm{H})$.

Electronic spectra were measured on an Agilent Cary 60 UV-visible spectrophotometer using a $1 \mathrm{~cm}$ path length cuvette.

Electrospray ionization (ESI) or electronic impact (EI) mode was used in all cases to measure the highresolution mass spectrometry (HRMS) measured on a Thermo Scientific ${ }^{\mathrm{TM}}$ UltiMate $^{\mathrm{TM}} 3000$ HPLC System (loop mode) or a QTOF MS (Quadrupole time-of-flight mass spectrometry).

Electrochemical measurements (CV: cyclic voltammetry) were carried out with an $\mathrm{EmStat}^{3+}$ potentiostat using a three-electrode cell equipped with glassy carbon working and counter electrodes and a $\mathrm{Ag} / \mathrm{AgNO}_{3}$ reference electrode. Potentials are reported in $\mathrm{V}$ vs $\mathrm{SCE}\left(\mathrm{E}_{\mathrm{Ag} / \mathrm{AgNO} 3}=0.3 \mathrm{~V}\right.$ vs $\left.\mathrm{SCE}\right)$.

Infrared spectra (IR) were recorded on a PerkinElmer IR 4100 spectrometer directly measuring in solid or liquid phase via a total reflexion method (ATR). 
2. UV-visible spectra of compound $2 \mathrm{a}$ in the presence of different amines:

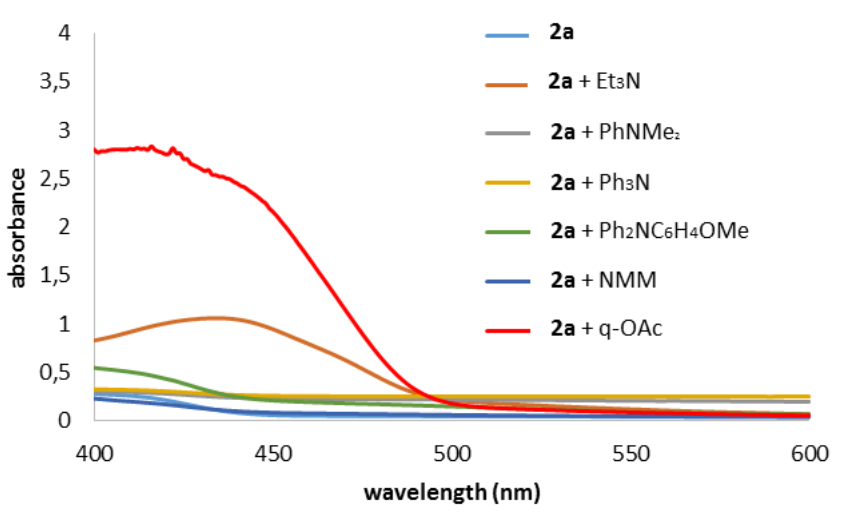

Scheme S1: UV-visible spectra of a 1:1 mixture of 2a with different amines in a $15 \mathrm{mM}$ solution in MeCN. Path length of $1 \mathrm{~cm}$. NMM: $N$-methylmorpholine. q-OAc: 3 -acetoxyquinuclidine.

\section{Optimization tables of the different reactions.}

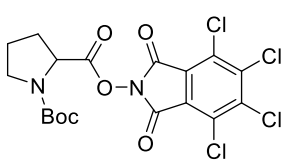

$\mathbf{2 b}$

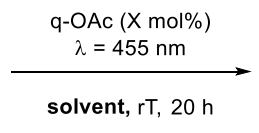

solvent, rT, $20 \mathrm{~h}$

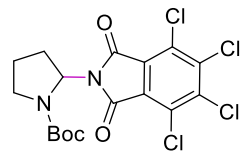

3b

\begin{tabular}{ccc} 
q-OAc $(X \mathrm{~mol} \%)$ & solvent & yield of 3b \\
\hline- & MeCN & $0 \%$ \\
$5 \mathrm{~mol} \%$ & $\mathrm{MeCN}$ & $58 \%$ \\
$15 \mathrm{~mol} \%$ & $\mathrm{MeCN}$ & $69 \%$ \\
$25 \mathrm{~mol} \%$ & $\mathrm{MeCN}$ & $69 \%$ \\
$30 \mathrm{~mol} \%$ & $\mathrm{MeCN}$ & $71 \%$
\end{tabular}

\begin{tabular}{ccc} 
q-OAc $(\mathrm{X} \mathrm{mol} \%)$ & solvent & yield of $\mathbf{3 b}$ \\
\hline- & DMF & $39 \%(4 \mathrm{~h})$ \\
$5 \mathrm{~mol} \%$ & DMF & $83 \%(6 \% \mathbf{2 b})$ \\
$\mathbf{1 0 ~ \mathrm { mol } \%}$ & DMF & $\mathbf{8 9 \%}$ \\
$15 \mathrm{~mol} \%$ & DMF & $90 \%$ \\
$25 \mathrm{~mol} \%$ & DMF & $91 \%$ \\
$30 \mathrm{~mol} \%$ & DMF & $91 \%$
\end{tabular}

Scheme S2: Catalyst loading and solvent optimization of the aminodecarboxylation reaction from $\mathbf{2 b}$ to $\mathbf{3 b}$.

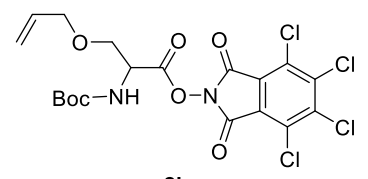

2k

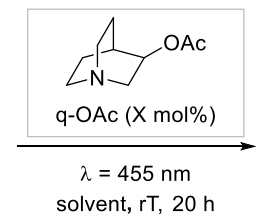

solvent, rT, $20 \mathrm{~h}$

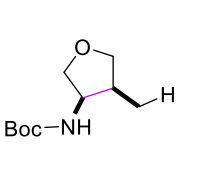

3k (dr 4:1)

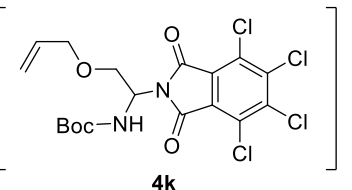

4k

\begin{tabular}{ccc} 
q-OAc $(\mathrm{X} \mathrm{mol} \%)$ & solvent & yield of $\mathbf{3 k}$ \\
\hline $25 \mathrm{~mol} \%$ & MeCN & $0 \%$ (degradation of $\mathbf{2 k})$ \\
- & DMF & $0 \%(98 \% \mathbf{2 k})$ \\
$5 \mathrm{~mol} \%$ & DMF & $31 \%(+10 \% \mathbf{4 k}, 18 \% \mathbf{2 k})$ \\
$15 \mathrm{~mol} \%$ & DMF & $46 \%(+7 \% \mathbf{4 k}, 3 \% \mathbf{2 k})$ \\
$25 \mathrm{~mol} \%$ & DMF & $59 \%(+8 \% \mathbf{4 k}$, no $\mathbf{2 k})$ \\
$30 \mathrm{~mol} \%$ & DMF & $58 \%(+8 \% \mathbf{4 k}$, no $\mathbf{2 k})$
\end{tabular}

Scheme S3: Catalyst loading and solvent optimization of the cyclization reaction from $2 \mathbf{k}$ to $3 \mathbf{k}$. 

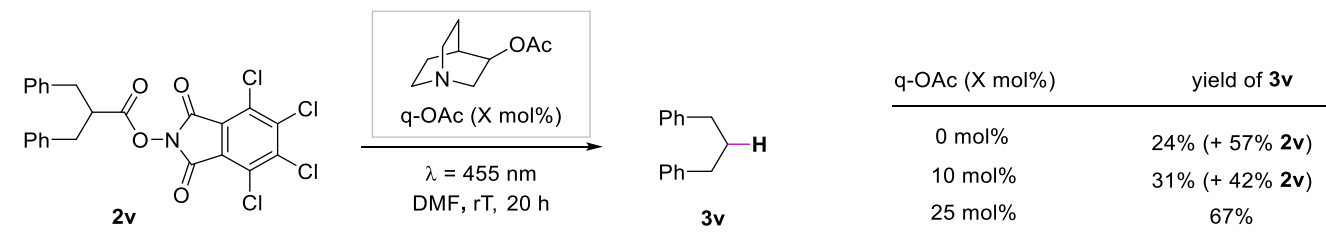

Scheme S4: Catalyst loading optimization of the hydrodecarboxylation reaction from $2 \mathbf{v}$ to $3 \mathbf{v}$.

\section{Mechanistic insights:}

\section{a. Use of TEMPO as radical scavenger:}

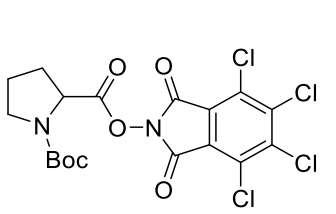

2b

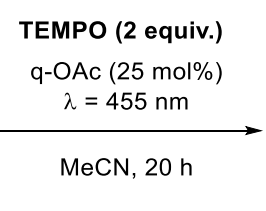

$66 \%$ recovered $2 b$

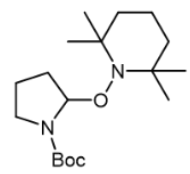

$[\mathrm{M}+\mathrm{H}]^{+}: 327$<smiles>O=C(O)N1CCCC1N1C(=O)c2c(Cl)c(Cl)c(Cl)c(Cl)c2C1=O</smiles>

3b

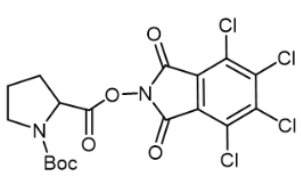

$[\mathrm{M}+2 \mathrm{H}-\mathrm{Boc}]^{+}: 397$

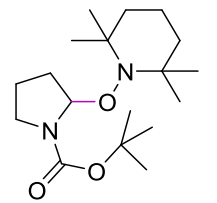

Exact Mass: 326,26

Observed $327(\mathrm{M}+\mathrm{H})$

MS detection

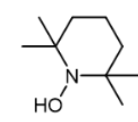

$[\mathrm{M}+\mathrm{H}]^{+}: 158$

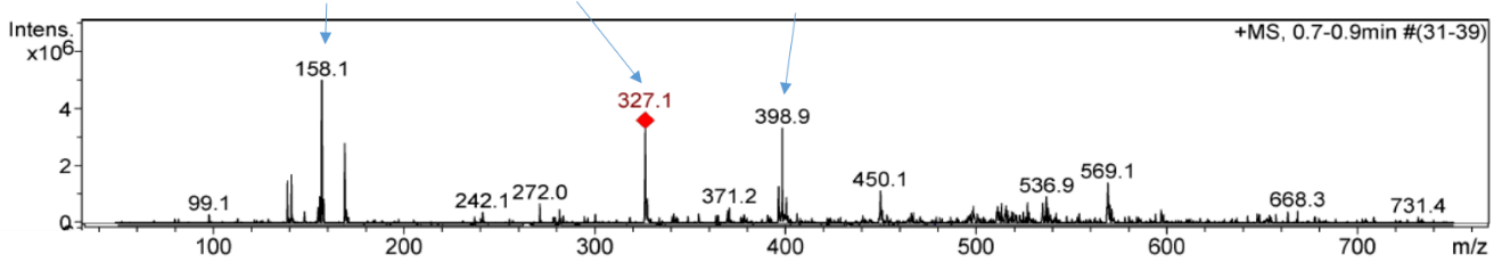

Scheme S5: Experiment under the initial reaction conditions in the presence of (2,2,6,6-Tetramethylpiperidin1-yl)oxyl (TEMPO) as radical scavenge (top). Mass spectroscopy (MS) analysis of the reaction mixture (bottom).

\section{b. Reaction using $\mathrm{Et}_{3} \mathrm{~N}$ as donor.}<smiles>[R]N1CCCC1C(=O)ON1C(=O)c2c(Cl)c(Cl)c(Cl)c(Cl)c2C1=O</smiles>

2

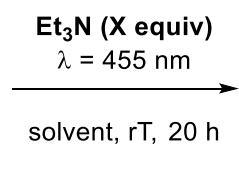

2b $(\mathrm{R}=\mathrm{Boc}) \quad \mathrm{MeCN}$ 2b' (R=Cbz) $\quad M e C N$ 2b' (R=Cbz) 2b' (R=Cbz)<smiles>[R]N1CCCC1N1C(=O)c2c(Cl)c(Cl)c(Cl)c(Cl)c2C1=O</smiles>

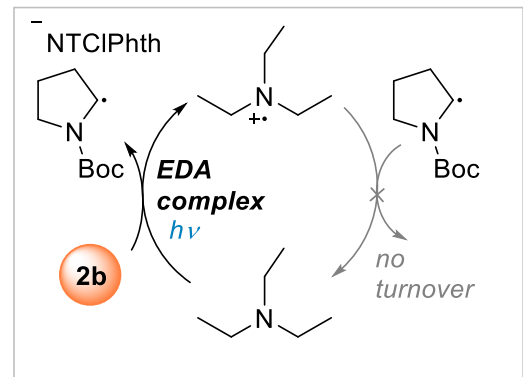

Scheme S6: Reaction using 1 or 0.1 equivalents of $\mathrm{Et}_{3} \mathrm{~N}$ as amine in different solvents. 


\section{c. Explanation of the role of DMF as possible HAT.}

Literature precedents show the use of DMF as HAT source in different reactions. ${ }^{[1-4]}$ Calculations show that the formyl and the methyl positions of the DMF have similar BDEs $\left[\mathrm{H}-\mathrm{CH}_{2} \mathrm{~N}(\mathrm{Me}) \mathrm{CHO}\right.$ or $\left.\mathrm{Me}_{2} \mathrm{NC}(\mathrm{O})-\mathrm{H}\right]=89-$ $90 \mathrm{kcal} / \mathrm{mol} .^{[2]}$ The unexpected weakness of these $\mathrm{C}-\mathrm{H}$ bonds derives from the fact that the radical formed has an unusual stability because of its planarity and its several resonance forms.

DMF has been reported to even serve as HAT source in radical hydrogenation of benzylic positions at high temperatures, even though the BDEs are very similar $\left(\mathrm{BDE}\left(\mathrm{C}_{6} \mathrm{H}_{5}-\mathrm{H}\right)=84-90 \mathrm{kcal} / \mathrm{mol}{ }^{[5]}\right)$. Labeling experiments were performed in the mentioned work.

In this work, we observed the crucial necessity of DMF in the reaction of $\mathbf{2 k}$ to give $3 \mathbf{k}$ since the reaction in $\mathrm{MeCN}$ gave no product formation and only starting material degradation (Scheme S3, vide supra). This result indicates two things: (1) the EDA complex is formed in $\mathrm{MeCN}$, and this implies decarboxylation, but since there is no HAT source, (2) the product is not formed and the radical is quenched via unproductive pathways, thus degrading the starting material.

On the other hand, the experiment with DMF- $\mathrm{d}_{7}$ gave traces of $\mathbf{3 k}$, with no deuterium incorporation, and partial degradation of the SM (Scheme S6a). The fact that there is no deuterium incorporation can be explained by the presence of available hydrogen atoms to abstract in the substrate itself and in the product (an ether functionality is present in both). This also reveals that the abstraction of the deuterium atom is beyond the kinetic barrier and that the absence of a great excess of abstractable hydrogen atoms in the reaction media results in the quenching of the formed radical via unproductive pathway giving no significant product formation and partial degradation of the starting material.

a)

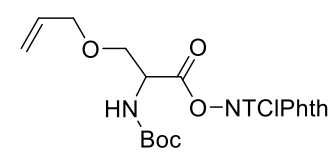

2k

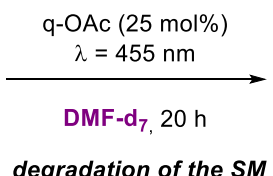

degradation of the SM

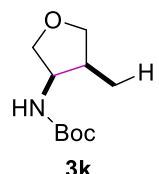

traces

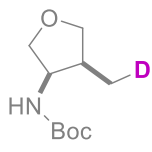

not observed b)

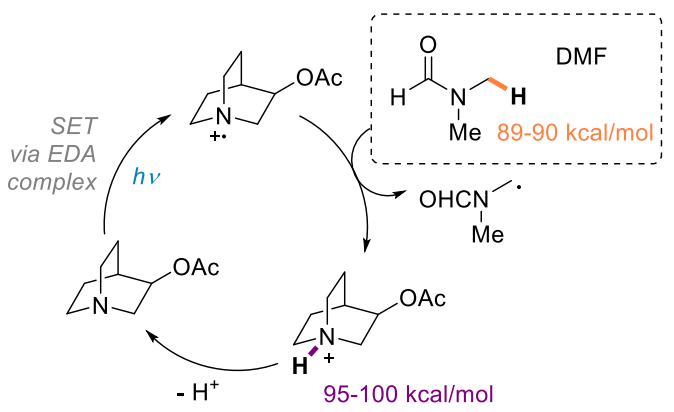

c)

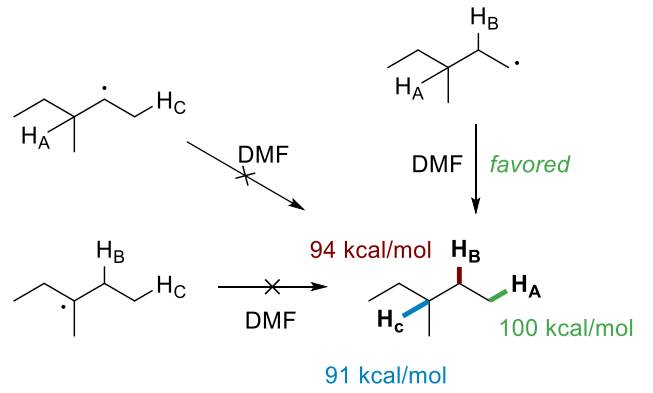

Scheme S7: Rationale for the proposed mechanism of radical cyclization. a) Experiment of $\mathbf{2 k}$ in the presence of DMF- $\mathrm{d}_{7}$. b) regeneration of the catalyst q-OAc using DMF as HAT source. c) difference in BDE of a primary, secondary and tertiary alkyl radicals and their tendency of abstracting a hydrogen atom from DMF. Bond dissociation energies (BDEs) are given for the different $\mathrm{X}-\mathrm{H}$ or $\mathrm{C}-\mathrm{H}$ bonds in $\mathrm{kcal} / \mathrm{mol} .{ }^{[2,6,7]}$ SET: single electron transfer. EDA: electron donor-acceptor. HAT: hydrogen atom transfer. 


\section{d. Selectivity of the hydrodecarboxylation reaction:}

This selectivity was observed when substrates $\mathbf{2 x}, \mathbf{2 u}$ or $\mathbf{2 y}$ where submitted to the optimized reaction conditions. For substrate $\mathbf{2 u}, \mathbf{3} \mathbf{u}$ was obtained in $62 \%$ yield. In the case of substrate $\mathbf{2 x}$, EDA complex formation in the UV-visible spectra is observed but the reaction did not take place, probably due to the instability of the primary radical, thus, decarboxylation might not be taking place in this case. However for substrate $\mathbf{2 y}$, no EDA complex formation is observed. We hypothesize that steric reasons might take an important role in this case, avoiding the approach of the q-OAc and, as consequence, the formation of the EDA complex.<smiles>O=C(CCCCc1ccccc1)ON1C(=O)c2c(Cl)c(Cl)c(Cl)c(Cl)c2C1=O</smiles>

$2 x$<smiles>CC(C)(C)N1CCC(C(=O)ON2C(=O)c3c(Cl)c(Cl)c(Cl)c(Cl)c3C2=O)CC1</smiles>

$2 \mathrm{u}$

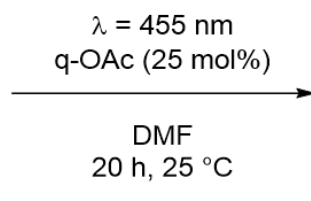<smiles>C=CCCCC1(C(=O)ON2C(=O)c3c(Cl)c(Cl)c(Cl)c(Cl)c3C2=O)CCN(S)CC1</smiles>

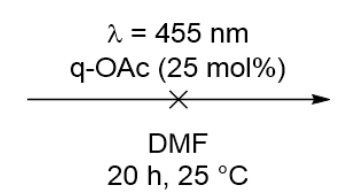

$2 y$
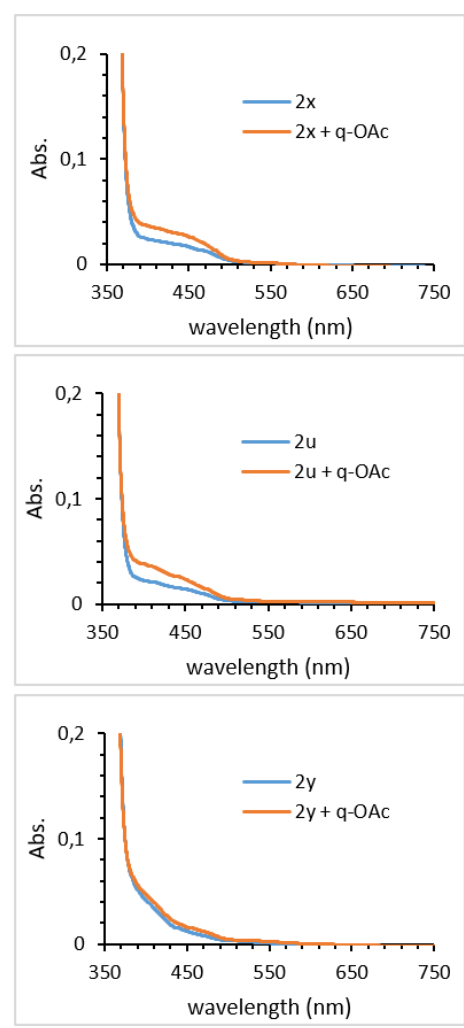

Scheme S8: Selective hydrodecarboxylation via catalytic EDA complex formation with q-OAc. Unreactive substrates $\mathbf{2 x}$ and $\mathbf{2 y}$, and reactive example $\mathbf{2 u}$. UV-visible spectra of substrates in DMF (9 $\mathrm{mM})$ in the absence and in the presence of q-OAc.

e. Comparison of the optimized reaction to the use of $\mathrm{Ru}(\mathrm{bpy})_{3} \mathrm{Cl}_{2}$ or Eosin $\mathrm{Y}$ as catalysts.<smiles>O=C(O)ON1C(=O)c2c(Cl)c(Cl)c(Cl)c(Cl)c2C1=O</smiles>

$2 b^{\prime}, 2 n$ or $2 u$

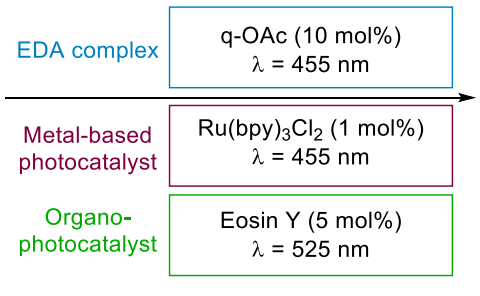

DMF, $17-20 \mathrm{~h}, 25^{\circ} \mathrm{C}$

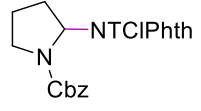

Yield of $\mathbf{3} \mathbf{b}^{\prime}$ and $\left(\mathbf{2} \mathbf{b}^{\prime}\right)$

$72 \%(0 \%)$

$0 \%(78 \%)$

$0 \%(71 \%)$

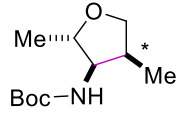

Yield of $3 n$ and $(2 n)$ $51 \%$, 9:1 dr (0\%)

$45 \%, 9: 1 \mathrm{dr}(0 \%)$ $0 \%(84 \%)$

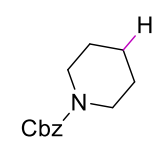

Yield of $3 u$ and $(2 u)$

$62 \%(0 \%)$

$0 \%(78 \%)$

$0 \%(77 \%)$

Scheme S9: Reaction of $\mathbf{2 b}, \mathbf{2 n}$ and $\mathbf{2} \mathbf{u}$ using q-OAc, $\mathrm{Ru}(\mathrm{bpy})_{3} \mathrm{Cl}_{2}$ or Eosin $\mathrm{Y}$ as catalyst. 


\section{f. UV-visible spectra of $2 \mathrm{~b}$ and $3 \mathrm{~b}$.}

$\mathrm{UV}$-visible spectra for starting material $\mathbf{2} \mathbf{b}$ and product $\mathbf{3} \mathbf{b}$ was recorded and for the mixture of both of them $(\mathbf{2 b}+\mathbf{3 b})$. No remarkable EDA complex band was observed when $\mathbf{2 b}$ and $\mathbf{3 b}$ were mixed. (Scheme S10, top). UV-visible spectra of $\mathbf{2 b}$ was recorded at increasing concentrations. From $13 \mathrm{mM}$ on, the solution became slightly turbid, which can explain the general increase of the absorption in the scanned range. The addition of q-OAc gave a completely yellow-clear solution (observed in the absorption decrease in the baseline at 650-750 $\mathrm{nm}$ range). No appreciable concentration dependency of the absorption spectrum of $\mathbf{2} \mathbf{b}$ is observed.

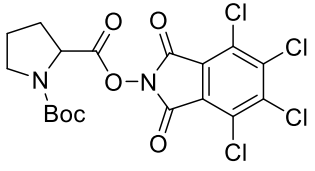

2b

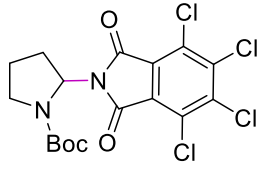

$3 b$

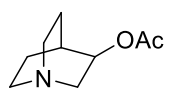

q-OAc
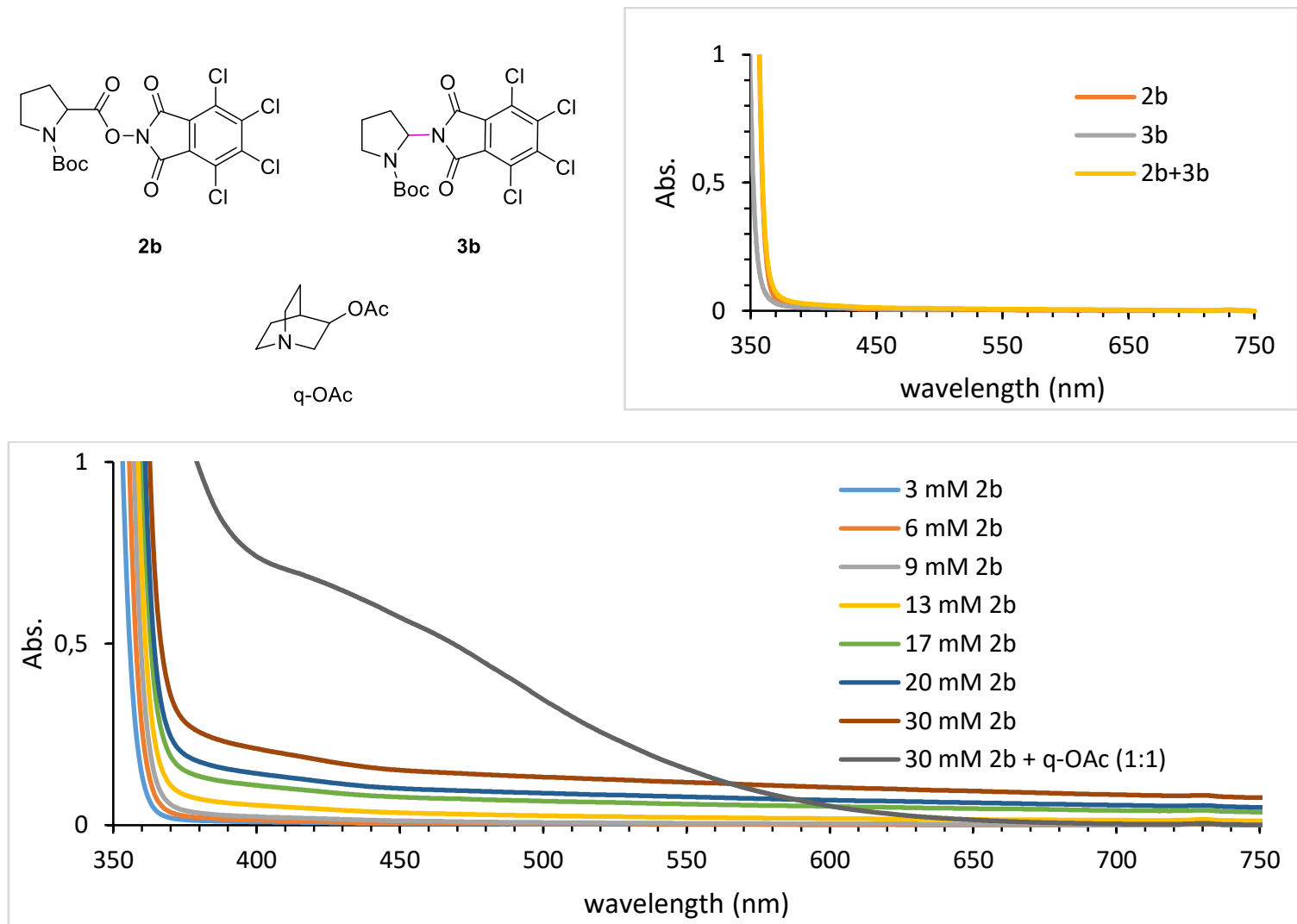

Scheme S10: Top: UV-visible spectra recorded for $9 \mathrm{mM}$ solutions in MeCN; Bottom: UV-visible spectra recorded for solutions ranging from 3 to $30 \mathrm{mM}$ in $\mathrm{MeCN}$. Cuvette of $1 \mathrm{~cm}$ path length. 


\section{Quantum yield experiment:}

The set up used in order to perform the quantum yield calculation was described in our previous publications. ${ }^{[8]}$ An LED of $455 \pm 10 \mathrm{~nm}$ was situated at $21 \mathrm{~cm}$ from the cuvette and focused using a set of focusing lenses ( $\mathrm{f}=$ $25, \mathrm{f}=150$ ), giving an incident area in the cuvette of about $9.0 \mathrm{~mm} \times 9.0 \mathrm{~mm}$. The cuvette holder was equipped with a magnetic stir bar driver ( 2 mag, cuvetteMixtdrive1) which was set to $400 \mathrm{rpm}$ in order to perform the reactions. This setup was built inside a black box equipped with a red lamp and a red-filtered window.

The QY is calculated according to the following equation:

$$
\Phi(1 \mathrm{~h})=\frac{\text { mols of } \boldsymbol{X}}{\text { photon flux } \times t \times f}
$$

were mols of $\mathbf{X}$ are the moles of product formed after a certain irradiation time ( $t$ in $s$ ), photon flux refers to the number of photons that reach the reaction mixture per second $\left(E \cdot \mathrm{s}^{-1}\right)$ and $\mathrm{f}$ refers to the fraction of light absorbed by the reaction mixture.

The photon flux after thermal equilibration of the LED ( $0.7 \mathrm{~V}$ of constant current), was calculated to be $90 \mathrm{~mW}$ according to the incident area mentioned above. These calculations were performed using a radiometrically calibrated setup (OceanOptics, USB-4000). The value obtained correspond to a photon flux of $3.402 \cdot 10^{-7} \mathrm{E} \cdot \mathrm{s}^{-1}$.

The quantum yield (QY) measurements were performed for substrate $\mathbf{2} \mathbf{b}$ ' as the control reaction in the absence of amine gave no conversion. The reaction solution was prepared inside a glove box under strict red-light conditions. In a cuvette equipped with a J Young valve and a magnetic stir bar, $2 \mathrm{mmol}$ (106 $\mathrm{mg}$ ) of $\mathbf{2} \mathbf{b}$ ' were added, followed by $2 \mathrm{~mL}$ of DMF and $1 \mathrm{~mL}$ of the stock solution of q-OAc $(10 \mathrm{~mol} \%)$ [The stock solution was prepared by dissolving $11.9 \mathrm{mg}$ of q-OAc in $3.5 \mathrm{~mL}$ of DMF]. The cuvette was sealed, covered with aluminum foil and taken out of the glove box.

The UV-visible spectra of the reaction mixture was recorded in order to calculate the fraction of photons absorbed by the reaction solution (Scheme S11).

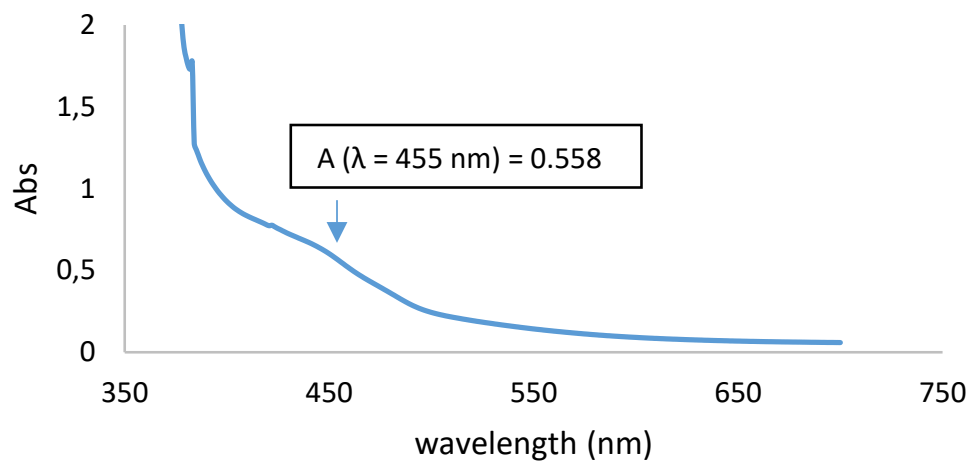

Scheme S11: UV-visible spectra recorded for the reaction mixture before irradiation.

The absorbance at $\lambda=455 \mathrm{~nm}$ was determined to be 0.558 . In consequence, the transmittance was calculated to be 27.7 , meaning that $72.3 \%$ of the light is being absorbed by the sample. In consequence, the fraction of light absorbed (f) is 0.723 .

The cuvette was then introduced into the black box and placed in the reaction holder with magnetic stirring. The reaction mixture was irradiated for $1 \mathrm{~h}$.

During the irradiation time, a stock solution of the internal standard was prepared. In this case, 1,2,4,5tetrabromobenzene $\left(\mathrm{Mw}=394\right.$, signal at $7.68 \mathrm{ppm}$ in $\left.\mathrm{CDCl}_{3}\right)$ was selected as internal standard (IS) to avoid overlapping with the signals of the starting material $\mathbf{2} \mathbf{b}^{\prime}$ ' and product $\mathbf{3} \mathbf{b}$ '. This stock solution was prepared by 
introducing $197 \mathrm{mg}$ of the IS into a $10 \mathrm{~mL}$ volumetric flask and diluting with $\mathrm{CDCl}_{3}$ to a final volume of 10 $\mathrm{mL}\left(0.05 \mathrm{mmol} \cdot \mathrm{mL}^{-1}\right)$. Note: a more concentrated solution gave no complete dissolution of the IS.

After $1 \mathrm{~h}$ of irradiation, the reaction mixture was diluted with EtOAc and water and extracted with EtOAc (x 3). The organic layers were washed with water (x 2) and brine, dried over $\mathrm{Na}_{2} \mathrm{SO}_{4}$, filtered and concentrated, all under red light conditions. After evaporation, $2 \mathrm{~mL}$ of the stock solution of IS $(0.1 \mathrm{mmol}$ IS, 0.5 equiv. vs 2b') were added to the crude, and an additional $3 \mathrm{~mL}$ of $\mathrm{CDCl}_{3}$ were added to ensure complete dissolution of the crude material. ${ }^{1} \mathrm{H}-\mathrm{NMR}$ analysis revealed the formation of product $\mathbf{3} \mathbf{b}$ ' in a $9 \%$ yield, equivalent to 0.018 mmol of product formed (Scheme S12).

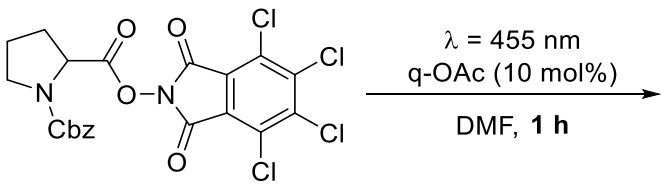

2b'

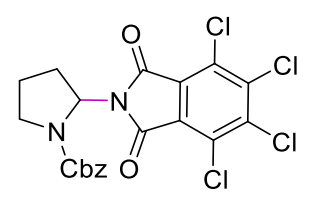

3b'

Scheme S12: Reaction used for the quantum yield calculation after $1 \mathrm{~h}$ of irradiation.

In consequence, knowing that $0.018 \mathrm{mmol}$ of $\mathrm{X}$ were formed after $\mathrm{t}=1 \mathrm{~h}$, in a solution with $\mathrm{f}=0.723$ and using an LED providing a photon flux of $3.402 \cdot 10^{-7} \mathrm{E} \cdot \mathrm{s}^{-1}$, the quantum yield was calculated to be 0.02 :

$\Phi(1 \mathrm{~h})=\frac{\text { mols of } \mathbf{3} \boldsymbol{b}^{\prime}}{\text { photon flux } \times t \times f}=\frac{1.8 \cdot 10^{-5}}{3.402 \cdot 10^{-7} \times 3600 \times 0.723}=0.02$ 
Characteristics of the LED used for the quantum yield calculation:

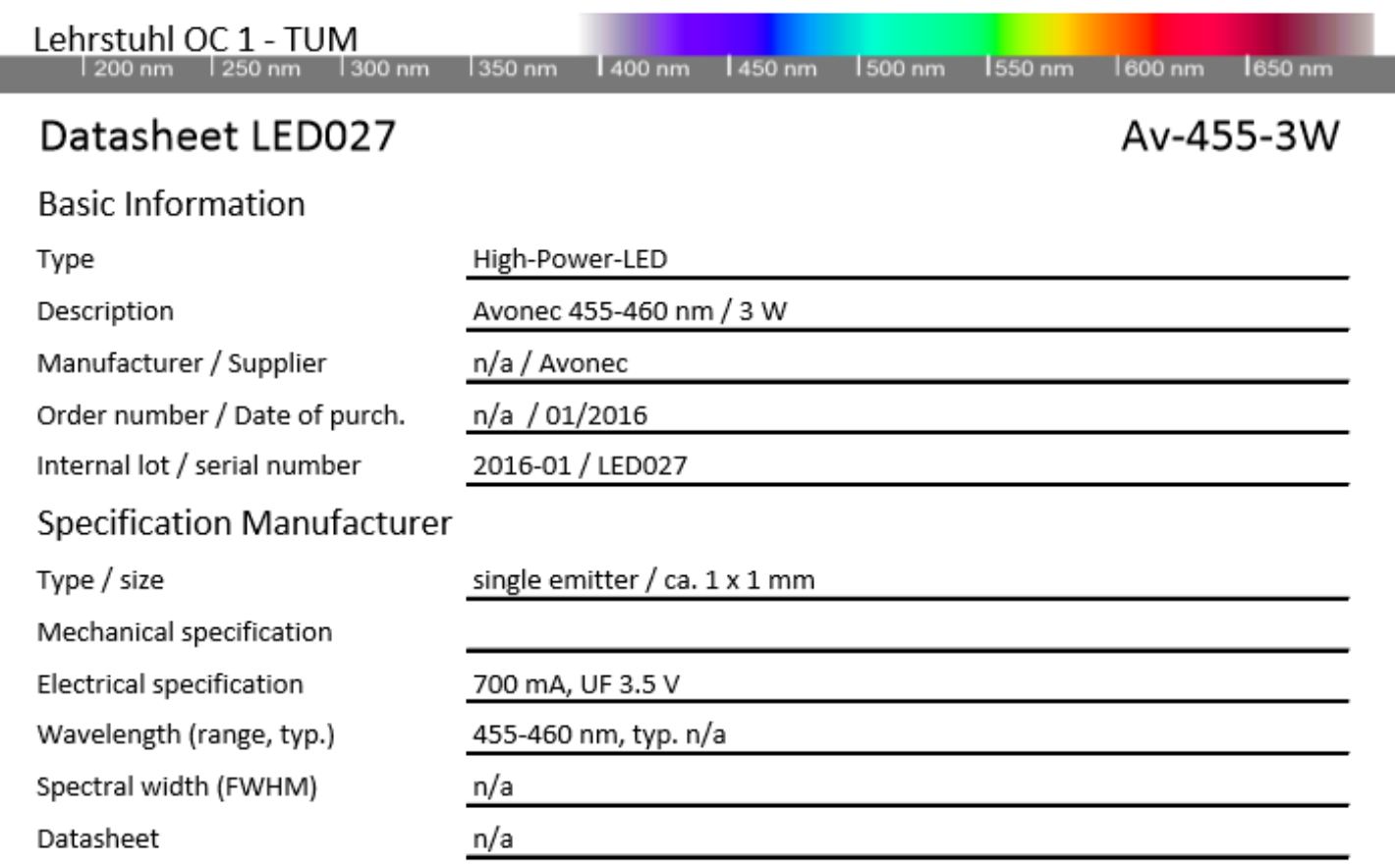

\section{Characterization}

Description of measurement

Measured with Ocean-optics USB4000 spectrometer using a

calibrated setup (cosine corrector/fibre).

The distance between the emitting surface and the surface of

the cosine corrector was $20 \mathrm{~mm}$. The LED was operated at

$700 \mathrm{~mA}$ on a passive heat-sink at approx. $20^{\circ} \mathrm{C}$

Measured wavelength $455 \mathrm{~nm}$

Measured spectral width

Integral Reference intensity

$22 \mathrm{~nm}$

$60400 \mu \mathrm{W} / \mathrm{cm}^{2}$ (400-550 nm @ 20 mm distance, 4 mmcosine corr.)

Spectrum

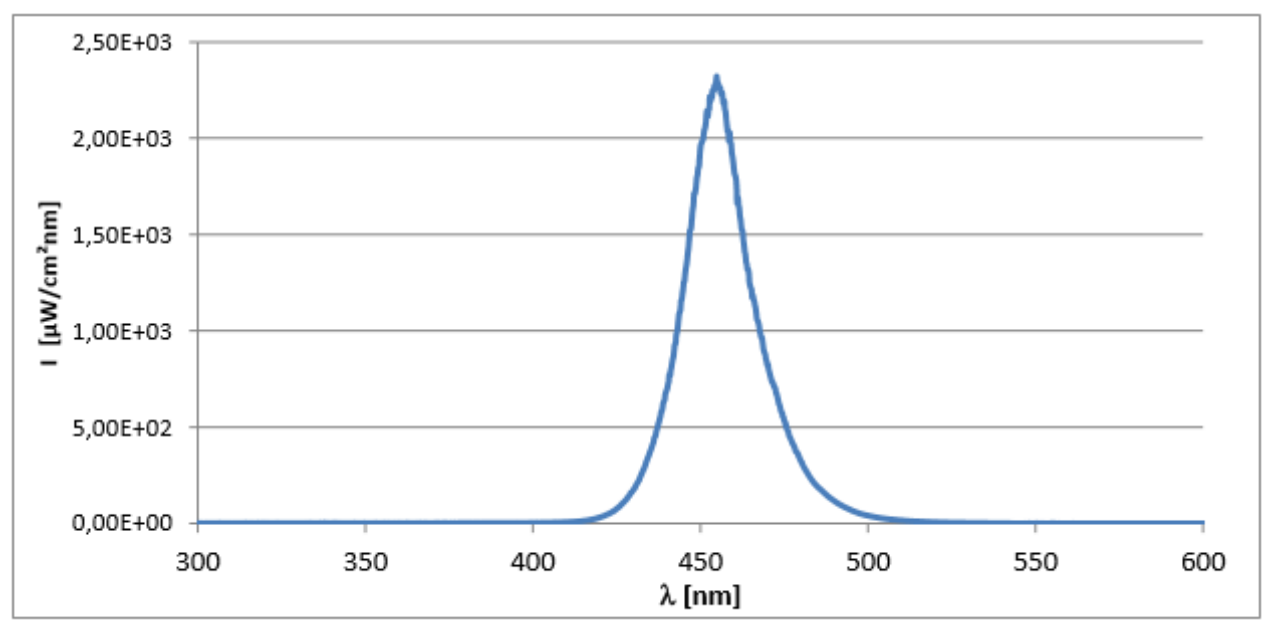




\section{Job plot:}

In order to determine the optimal ratio of starting material and q-OAc catalyst and calculate the extinction coefficient of the EDA complex, a Job Plot of compound 2b' was performed. A $1 \mathrm{~cm}$ x $1 \mathrm{~mm}$ cuvette was used (total volume of $250 \mu \mathrm{L}$, path length $1 \mathrm{~mm}$ ). Two stock solutions of compound $\mathbf{2} \mathbf{b}$ ' and q-OAc were prepared with a concentration of $100 \mathrm{mM}$. For this, $107 \mathrm{mg}$ of $\mathbf{2} \mathbf{b}$ ' were dissolved in $2 \mathrm{~mL}$ of MeCN and $32 \mathrm{mg}$ of qOAc were dissolved in $2 \mathrm{~mL}$ of $\mathrm{MeCN}$.

Seven measurements with ratios 2b':q-OAc of 0:100, 20:80, 35:65, 50:50, 65:35, 80:20, 100:0 were recorded, with a mixing of the corresponding stock solutions in the amounts shown in the table (Scheme S13a). The UVvisible measurements are shown (Scheme S13b). From each curve, the absorbance obtained at $455 \mathrm{~nm}$ was selected (Scheme S13a) and plotted in the Job Plot (Scheme S13c).

\begin{tabular}{|c|c|c|c|c|c|c|}
\hline & d) & $2 \mathbf{b}^{\prime}(\%)$ & $\mathrm{q}-\mathrm{OAc}(\%)$ & Solution of $\mathbf{2 d}(\mu \mathrm{L})$ & Solution of q-OAc $(\mu \mathrm{L})$ & Abs. \\
\hline & & 0 & 100 & 0 & 250 & 0,0396 \\
\hline $\mathrm{q}-\mathrm{OAc}$ & & 20 & 80 & 50 & 200 & 0,1447 \\
\hline & & 35 & 65 & 87 & 163 & 0,2073 \\
\hline & & 50 & 50 & 125 & 125 & 0,2427 \\
\hline & & 65 & 35 & 163 & 87 & 0,2469 \\
\hline & & 80 & 20 & 200 & 50 & 0,2072 \\
\hline & & 100 & 0 & 250 & 0 & 0,0469 \\
\hline
\end{tabular}

subs. (2b')

b)

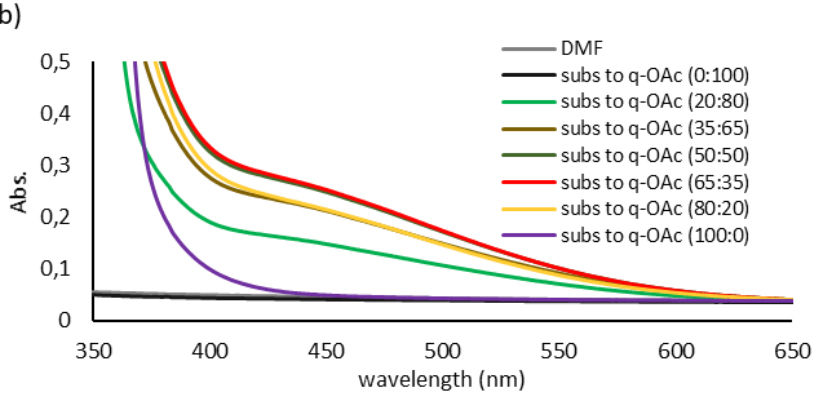

c)

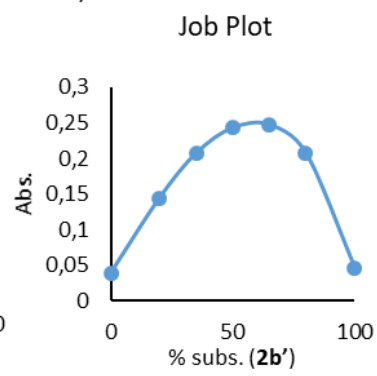

Scheme S13: a) Table with the amounts of stock solutions needed for each measurement and the absorbance at $455 \mathrm{~nm}$ observed in each case. b) UV-visible spectra of each mixture. c) Job plot at $455 \mathrm{~nm}$.

The extinction coefficient of the EDA complex in 1:1 ratio was calculated form the Beer-Lambert equation:

$$
A=\varepsilon \cdot l \cdot c
$$

Where A represents the Absorbance, $\varepsilon$ is the extinction coefficient $\left(\mathrm{L} \cdot \mathrm{mol}^{-1} \cdot \mathrm{cm}^{-1}\right), 1$ is the path length $(\mathrm{cm})$, and $\mathrm{c}$ is the concentration (M). As consequence, the extinction coefficient of the 1:1 EDA complex $\mathbf{2} \mathbf{b}^{\mathbf{2}}$ :q-OAc was found to be:

$$
\varepsilon=\frac{A}{l \cdot c}=\frac{0.2427}{0.1 \mathrm{~cm} \cdot 0.1 \mathrm{M}}=24.27 \mathrm{~L} \cdot \mathrm{mol}^{-1} \cdot \mathrm{cm}^{-1}
$$




\section{Cyclic voltammetry (CV) experiments of 1a and 2a to 2y:}

Cyclic voltammetry $(\mathrm{CV})$ experiments were measured in a three-electrode cell equipped with glassy carbon working and counter electrodes and a $\mathrm{Ag} / \mathrm{AgNO}_{3}(0.01 \mathrm{M}$ in $\mathrm{MeCN})$ reference electrode. A value of $300 \mathrm{mV}$ was added to the values given in order to obtain the corresponding potentials vs SCE. In the cell were added 5 $\mathrm{mL}$ of a $0.1 \mathrm{M}$ solution of recrystallized tetrabutylammoniumhexafluorophosphate $\left(\mathrm{TBAPF}_{6}\right)$ in $\mathrm{MeCN}$ and about $5 \mathrm{mg}$ of substrate. The CVs were measured at a scan rate of $200 \mathrm{mV} / \mathrm{s}$. The value of the potential at the inflexion point of each oxidation curve $\left(\mathrm{E}_{1 / 2}\right)$ was selected as the reduction potential of the corresponding substrate.

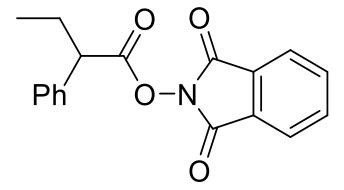

$\mathrm{E}_{1 / 2}(\mathbf{1 a})=-1.24 \mathrm{~V}$ vs SCE

$1 a$
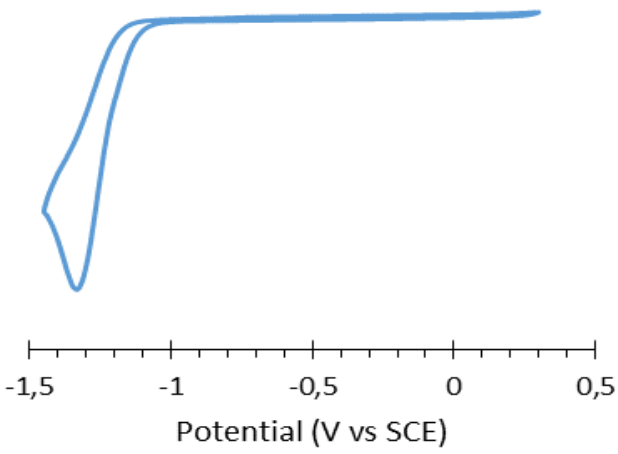<smiles>CCC(C(=O)ON1C(=O)c2c(Cl)c(Cl)c(Cl)c(Cl)c2C1=O)c1ccccc1</smiles>

$\mathrm{E}_{1 / 2}(\mathbf{2 a})=-0.64 \mathrm{~V}$ vs SCE

\section{$2 a$}

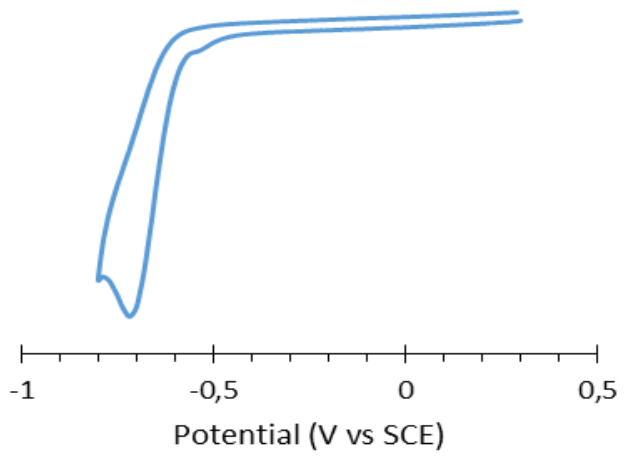<smiles>CC(C)(C)OC(=O)N1CCCC1C(=O)ON1C(=O)c2c(Cl)c(Cl)c(Cl)c(Cl)c2C1=O</smiles>

$\mathrm{E}_{1 / 2}(\mathbf{2 b})=-0.63 \mathrm{~V}$ vs SCE

2b

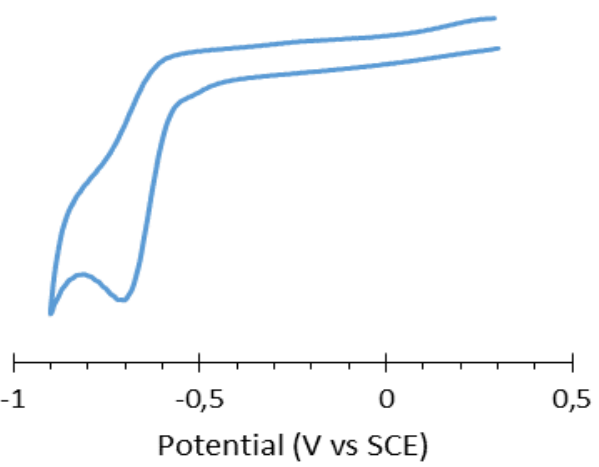<smiles>C=CCO[C@H]1CC(C(=O)ON2C(=O)c3c(Cl)c(Cl)c(Cl)c(Cl)c3C2=O)C(=O)N1C(=O)OC(C)(C)C</smiles>

$\mathrm{E}_{1 / 2}(\mathbf{2 c})=-0.70 \mathrm{~V}$ vs SCE

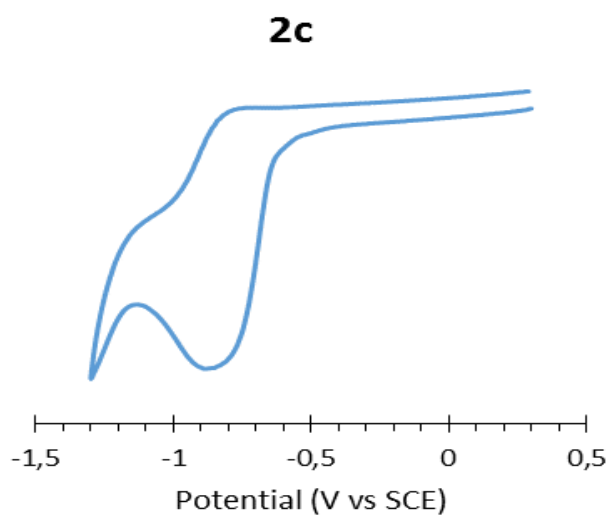


<smiles>O=C(ON1C(=O)c2c(Cl)c(Cl)c(Cl)c(Cl)c2C1=O)C1CCCN1C(=O)c1ccccc1</smiles>

$\mathrm{E}_{1 / 2}\left(\mathbf{2} \mathbf{b}^{\prime}\right)=-0.61 \mathrm{~V}$ vs SCE

2b'
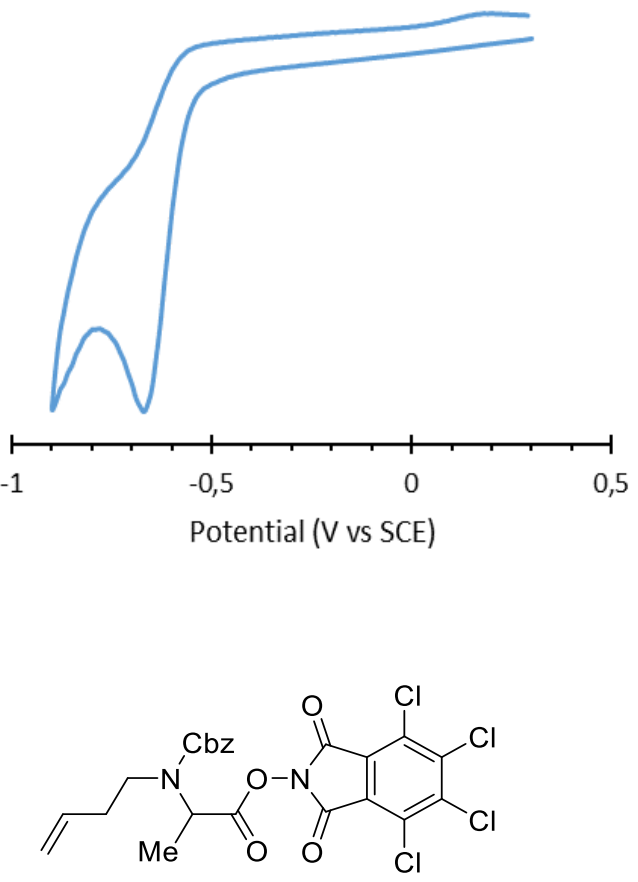

$\mathrm{E}_{1 / 2}(\mathbf{2 e})=-0.64 \mathrm{~V}$ vs SCE

$2 e$
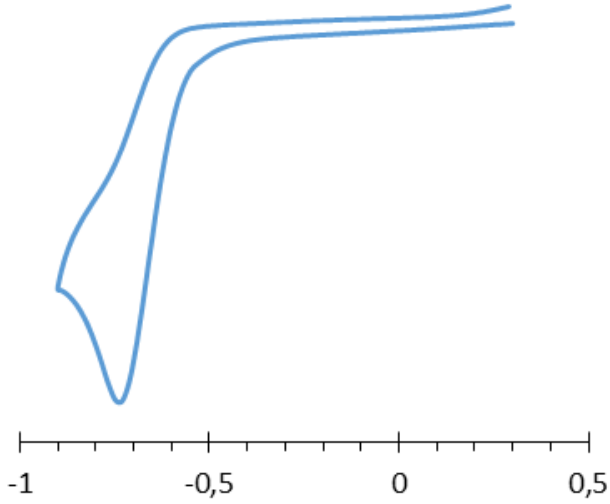

Potential (V vs SCE)

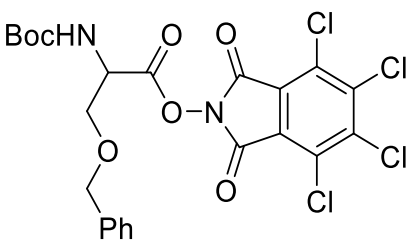

$\mathrm{E}_{1 / 2}(\mathbf{2 f})=-0.61 \mathrm{~V}$ vs SCE

$2 f$
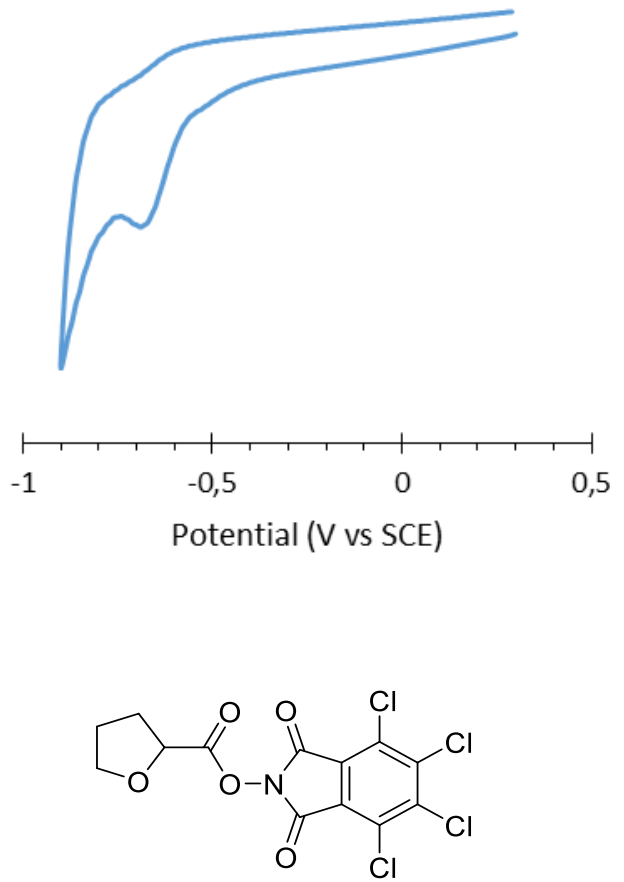

$\mathrm{E}_{1 / 2}(\mathbf{2 g})=-0.61 \mathrm{~V}$ vs SCE

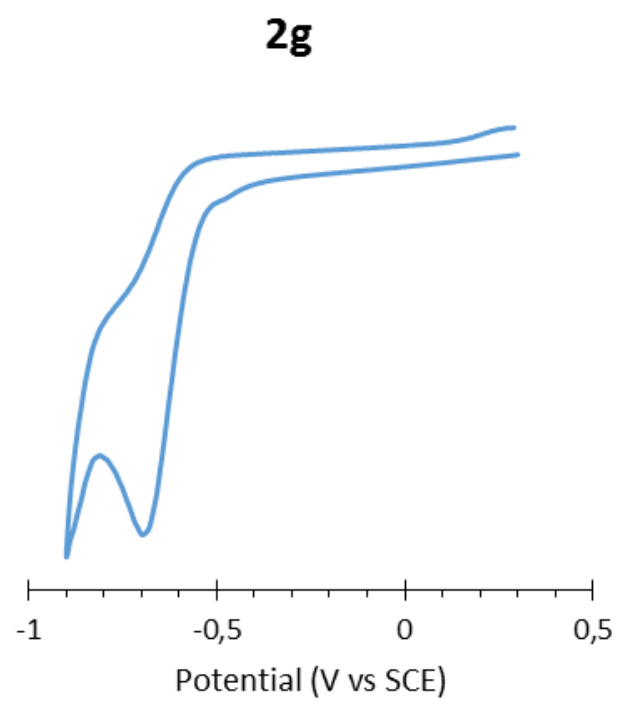




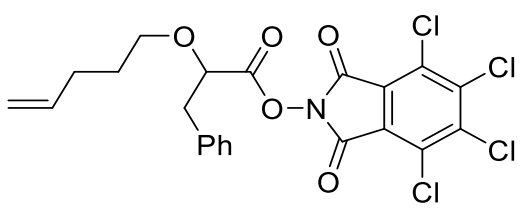

$\mathrm{E}_{1 / 2}(\mathbf{2 h})=-0.62 \mathrm{~V}$ vs SCE
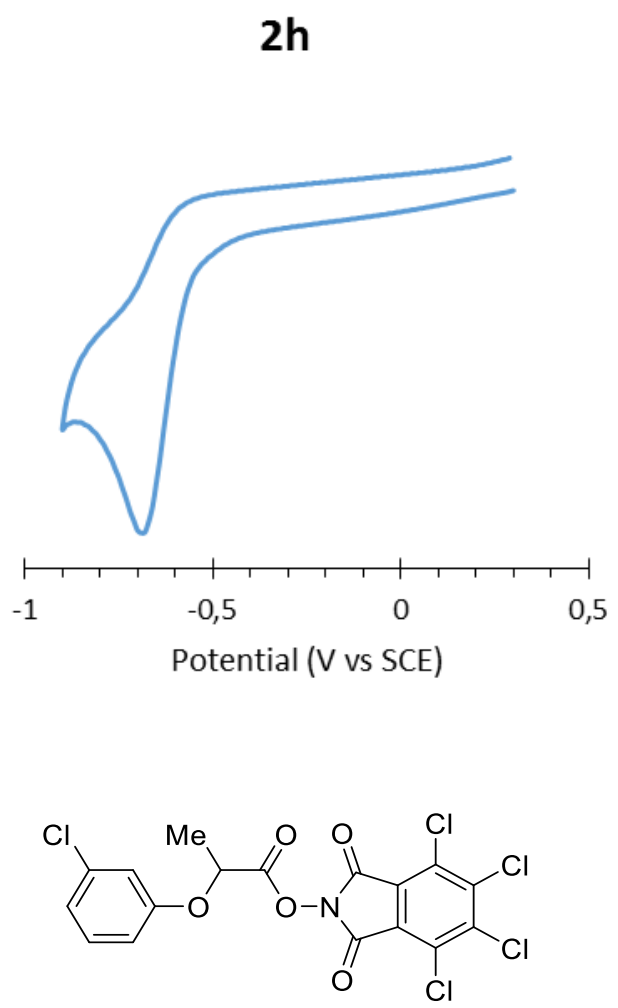

$\mathrm{E}_{1 / 2}(\mathbf{2 i})=-0.57 \mathrm{~V}$ vs SCE

2i
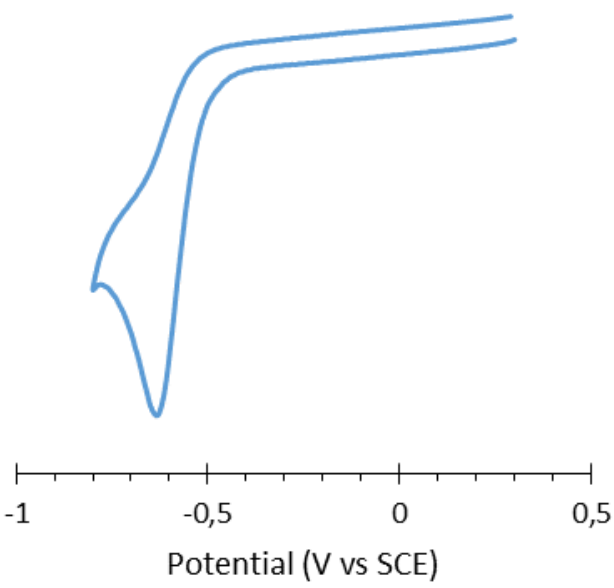

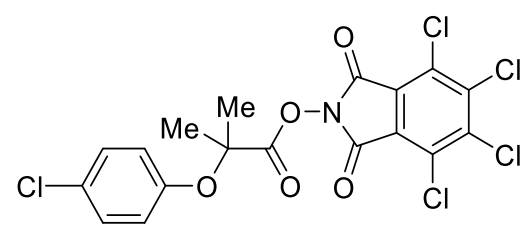

$E_{1 / 2}(\mathbf{2} \mathbf{j})=-0.54 \mathrm{~V}$ vs SCE

2j
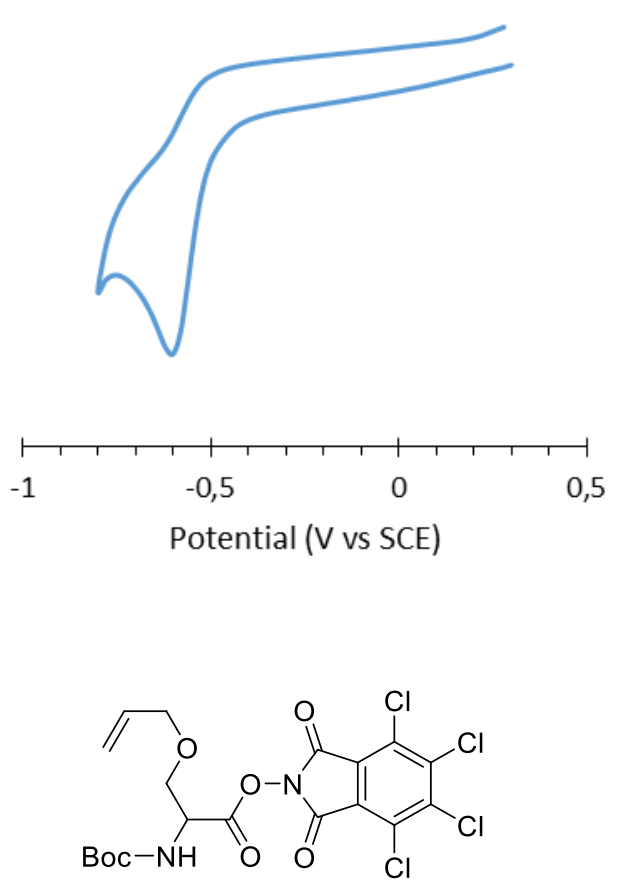

$\mathrm{E}_{1 / 2}(\mathbf{2 k})=-0.63 \mathrm{~V}$ vs SCE

\section{2k}
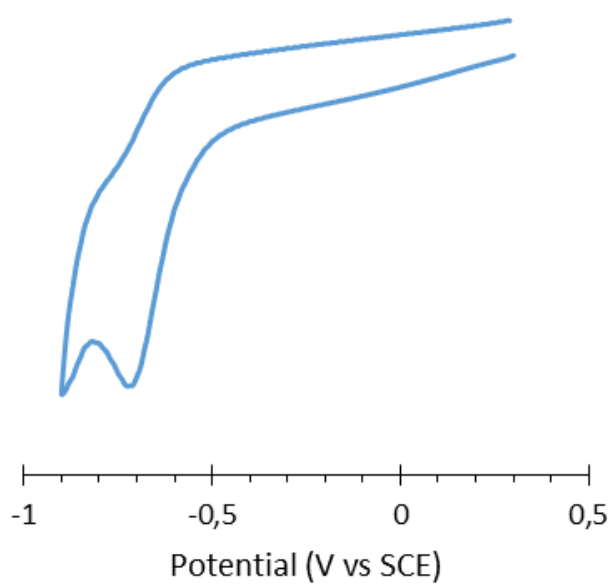


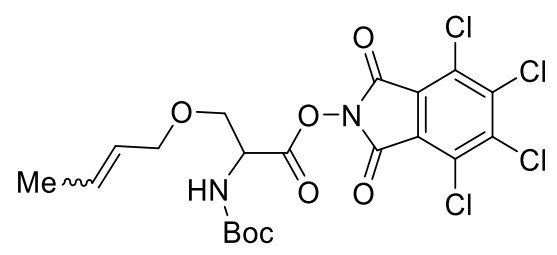

$\mathrm{E}_{1 / 2}(\mathbf{2 l})=-0.65 \mathrm{~V}$ vs SCE
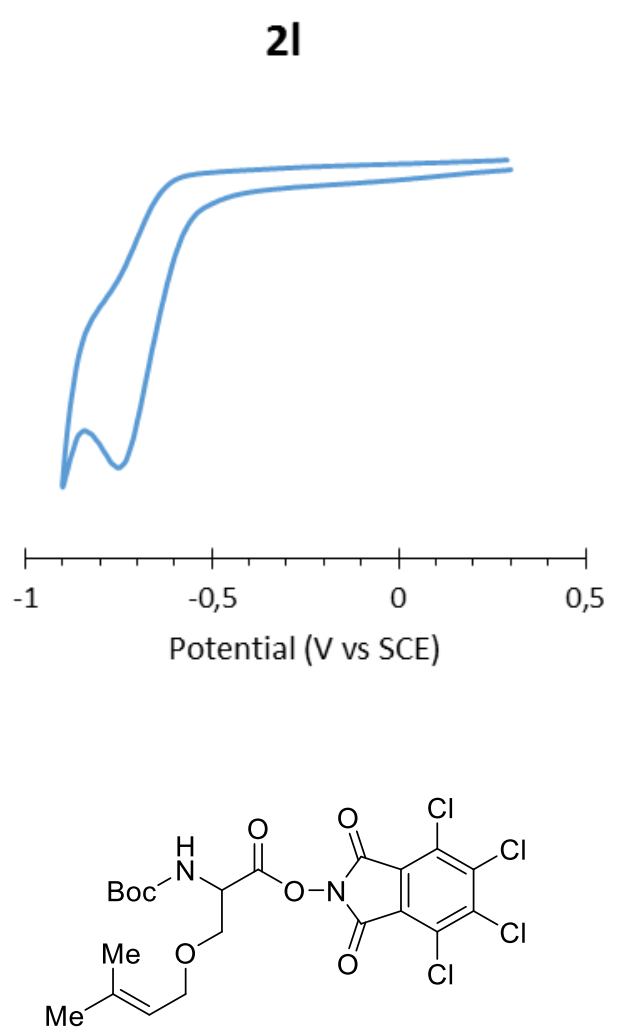

$\mathrm{E}_{1 / 2}(\mathbf{2 m})=-0.54 \mathrm{~V}$ vs SCE

$2 m$
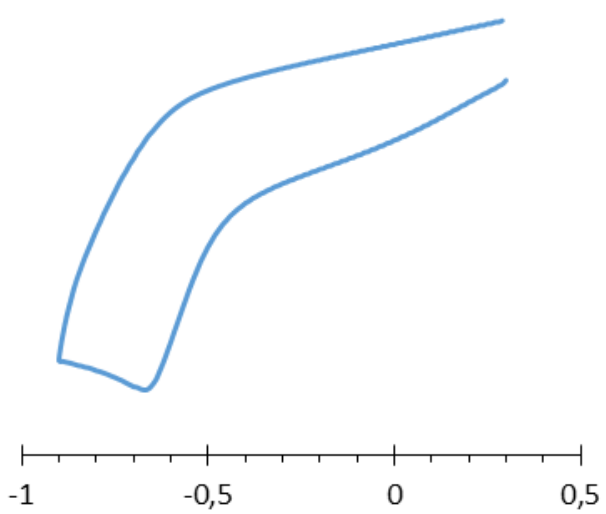

Potential (V vs SCE)

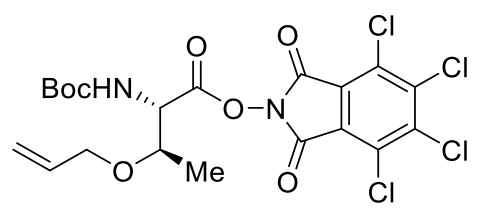

$\mathrm{E}_{1 / 2}(\mathbf{2 n})=-0.59 \mathrm{~V}$ vs SCE
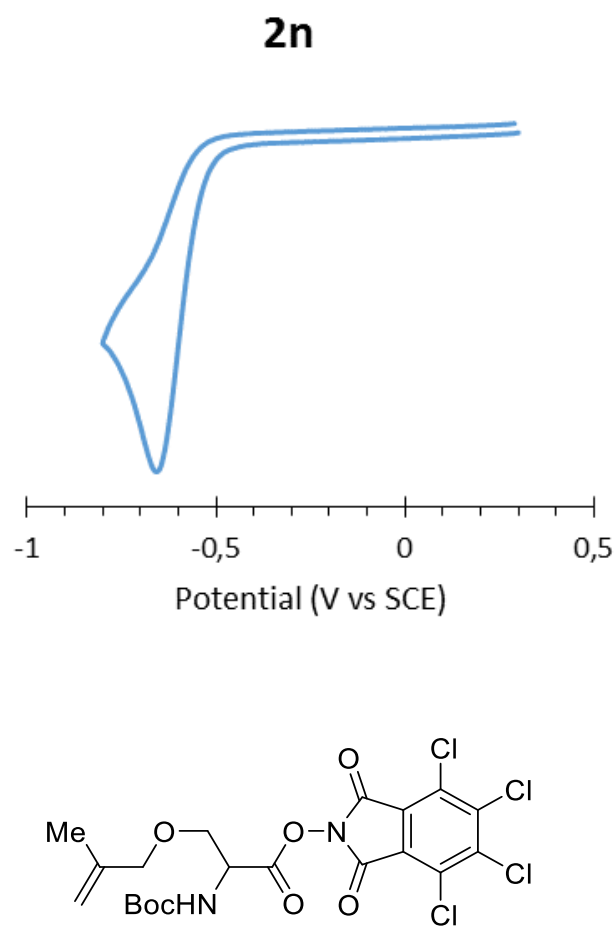

$\mathrm{E}_{1 / 2}(\mathbf{2 o})=-0.64 \mathrm{~V}$ vs SCE

20

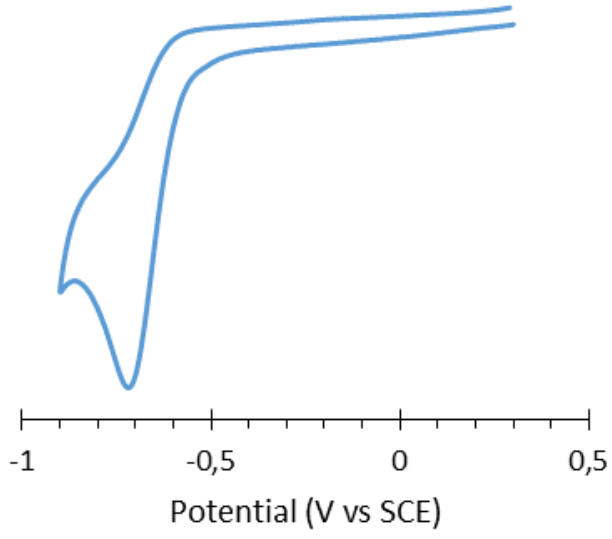




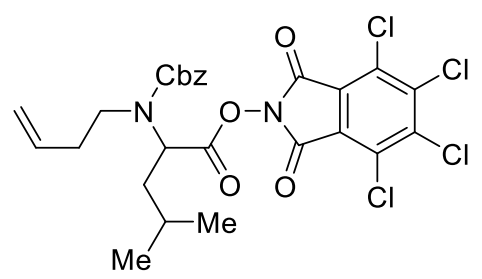

$\mathrm{E}_{1 / 2}(\mathbf{2 p})=-0.61 \mathrm{~V}$ vs SCE

$2 p$
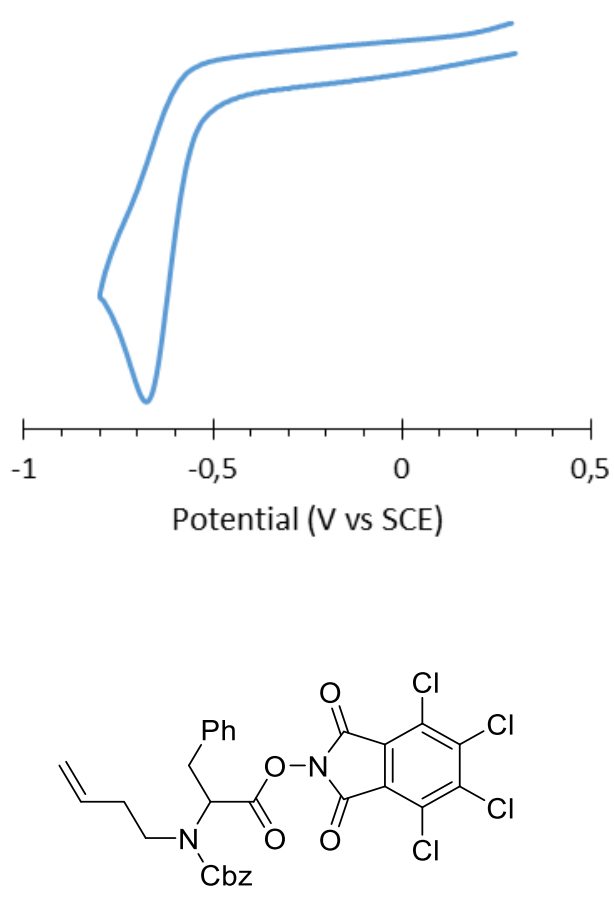

$\mathrm{E}_{1 / 2}(\mathbf{2 q})=-0.61 \mathrm{~V}$ vs SCE

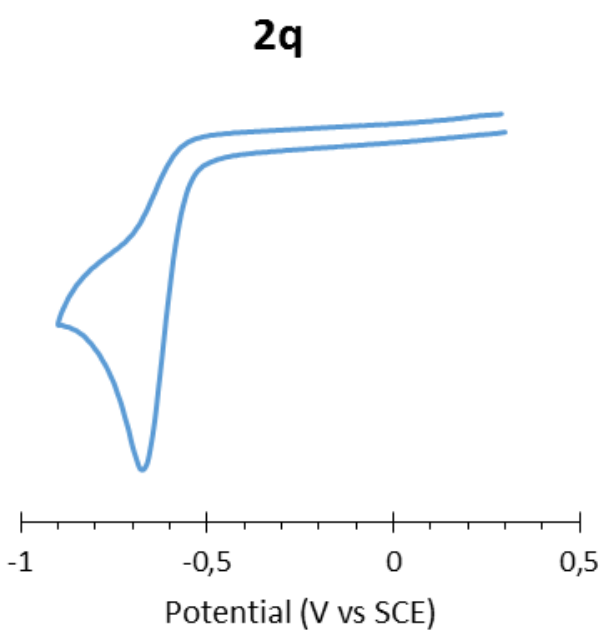

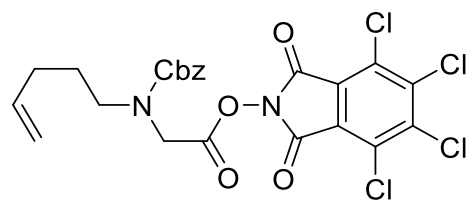

$\mathrm{E}_{1 / 2}(\mathbf{2 r})=-0.63 \mathrm{~V}$ vs SCE

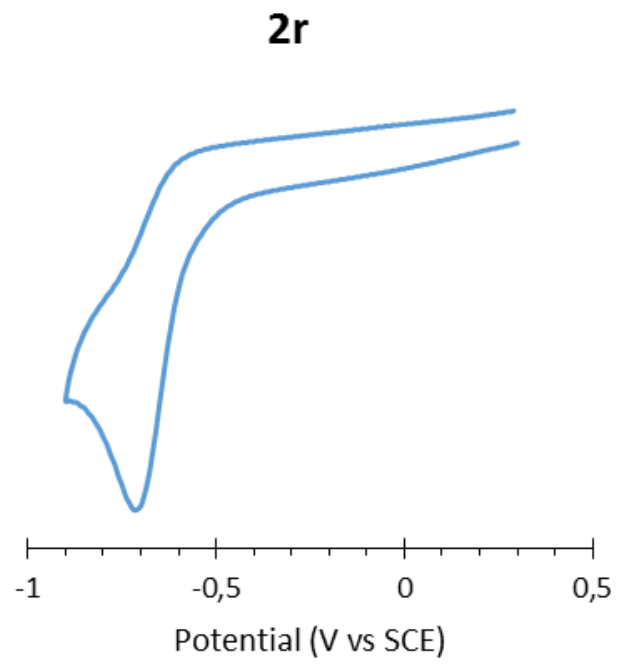

$\mathrm{E}_{1 / 2}(2 \mathrm{~s})=-0.56 \mathrm{~V}$ vs SCE

\section{$2 s$}

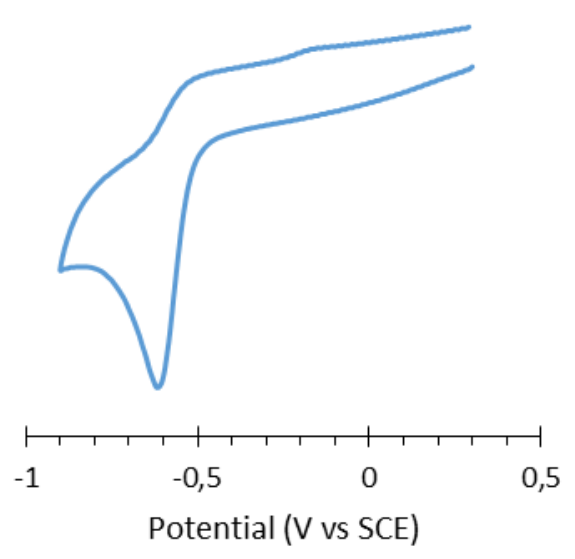




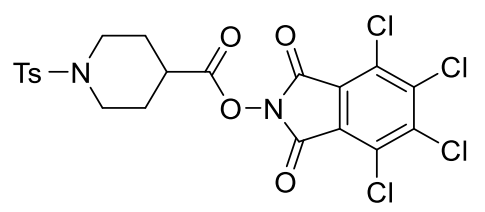

$\mathrm{E}_{1 / 2}(\mathbf{2 t})=-0.63 \mathrm{~V}$ vs SCE
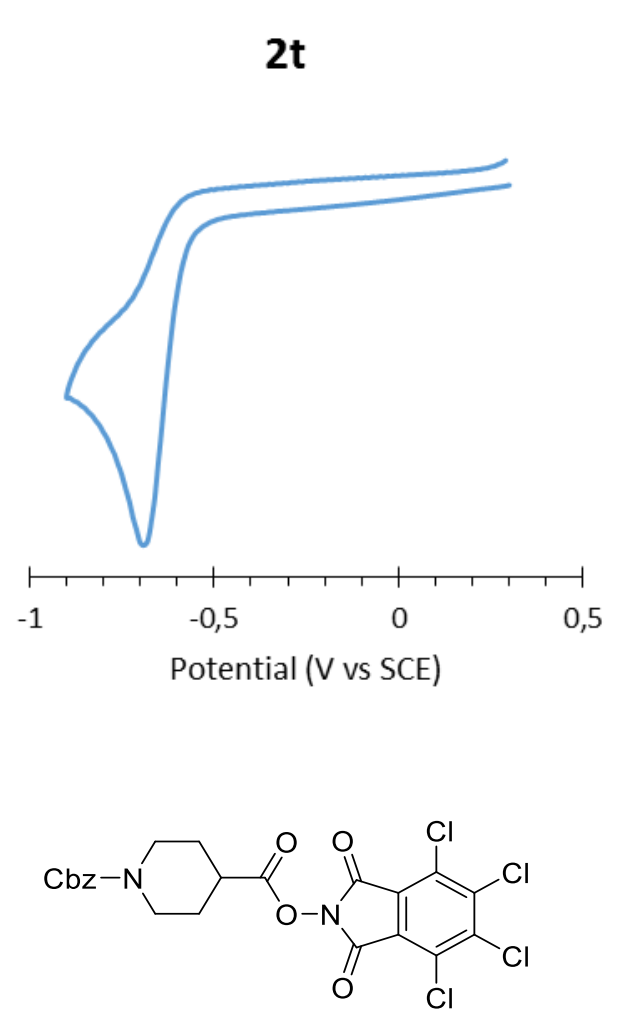

$\mathrm{E}_{1 / 2}(\mathbf{2 u})=-0.60 \mathrm{~V}$ vs SCE

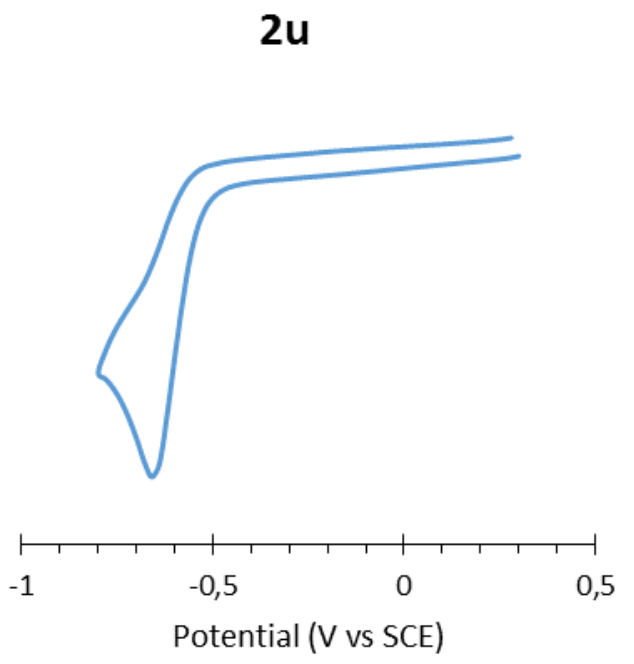

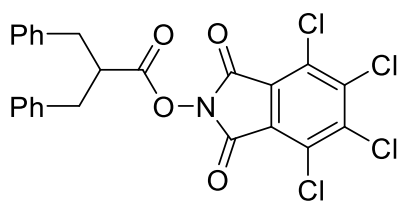

$\mathrm{E}_{1 / 2}(\mathbf{2 v})=-0.64 \mathrm{~V}$ vs SCE

2v
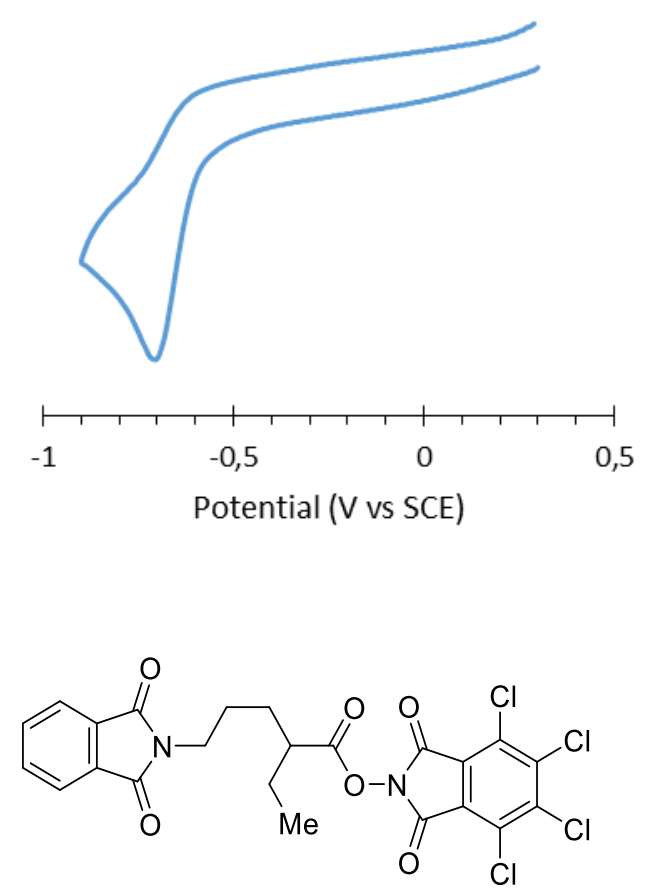

$\mathrm{E}_{1 / 2}(\mathbf{2} \mathbf{w})=-0.65 \mathrm{~V}$ vs SCE

$2 w$

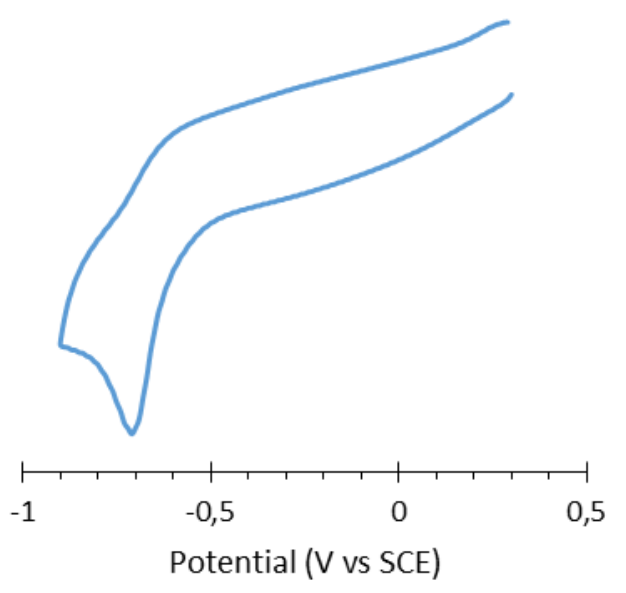




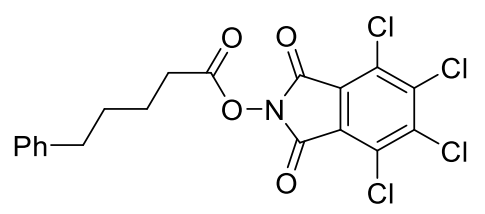

$\mathrm{E}_{1 / 2}(\mathbf{2 x})=-0.66 \mathrm{~V}$ vs SCE

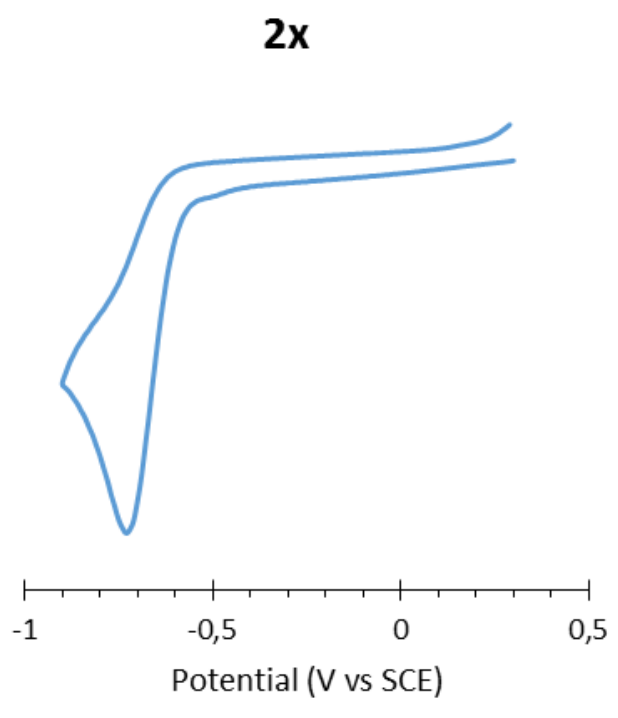

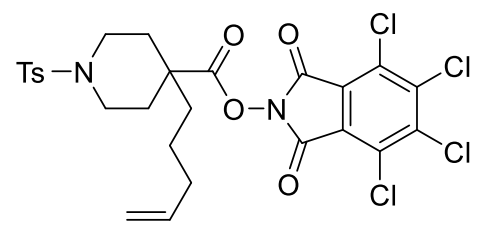

$\mathrm{E}_{1 / 2}(\mathbf{2 y})=-0.63 \mathrm{~V}$ vs SCE

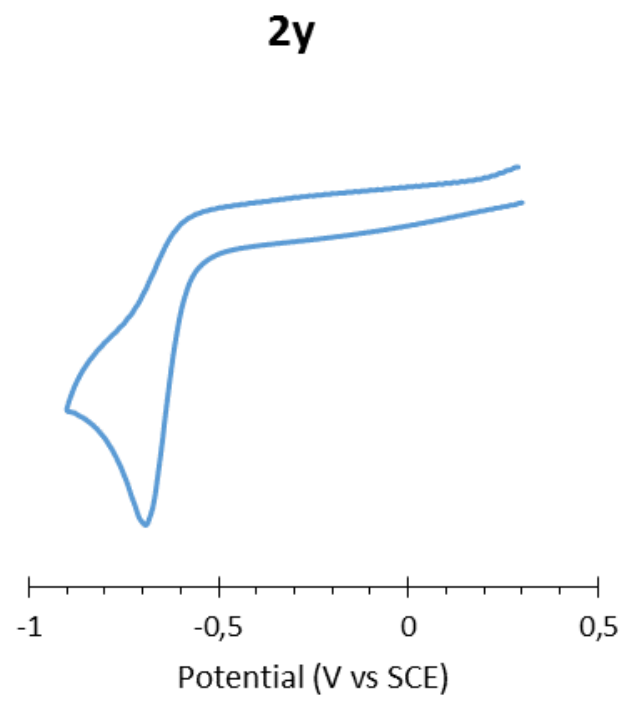




\section{UV-visible spectra of compounds $2 a$ to $2 y$ in the absence and in the presence of q-OAc:}

UV-visible spectra of a 1:1 mixture of each starting material (1a, 2a-2y) in the absence or in the presence of qOAc was measured in a $9 \mathrm{mM}$ solutions in DMF in a $1 \mathrm{~cm}$ of path length cuvette.
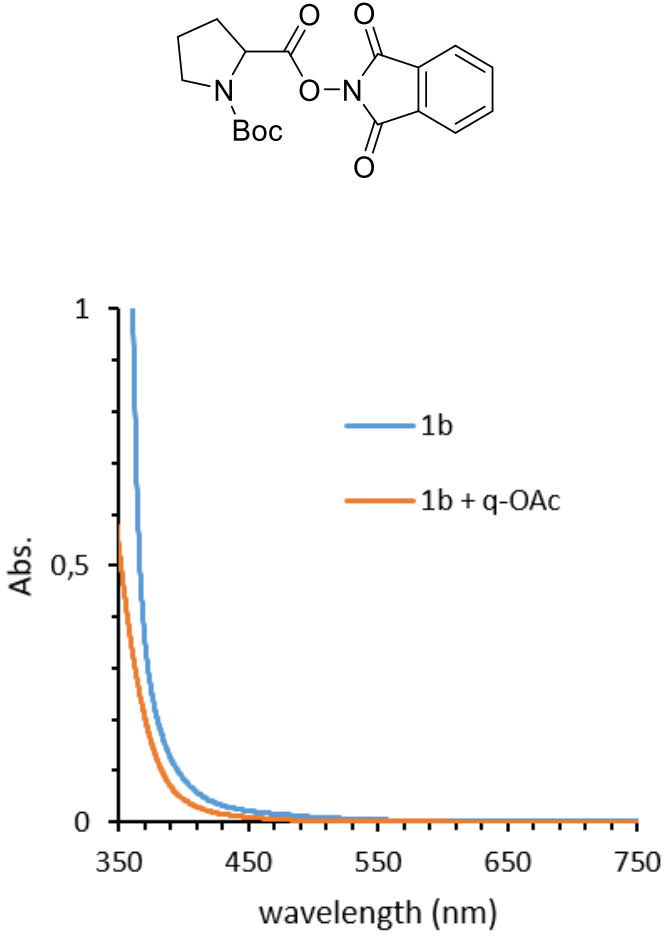<smiles>CCC(C(=O)ON1C(=O)c2c(Cl)c(Cl)c(Cl)c(Cl)c2C1=O)c1ccccc1</smiles>

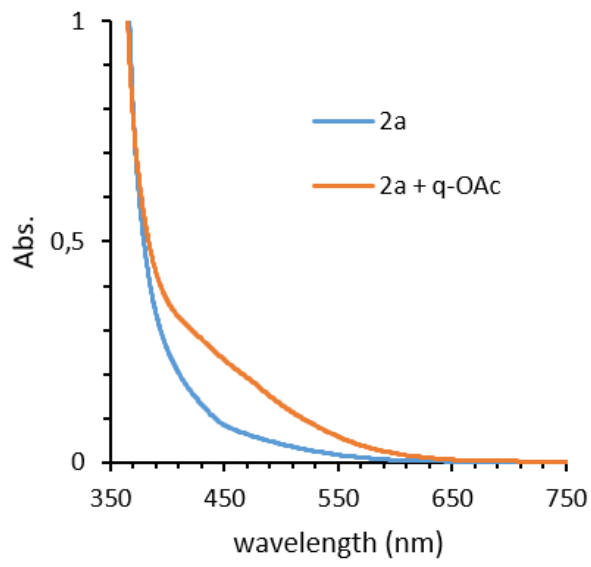<smiles>O=C(ON1C(=O)c2c(Cl)c(Cl)c(Cl)c(Cl)c2C1=O)C1CCCN1C(=O)c1ccccc1</smiles>

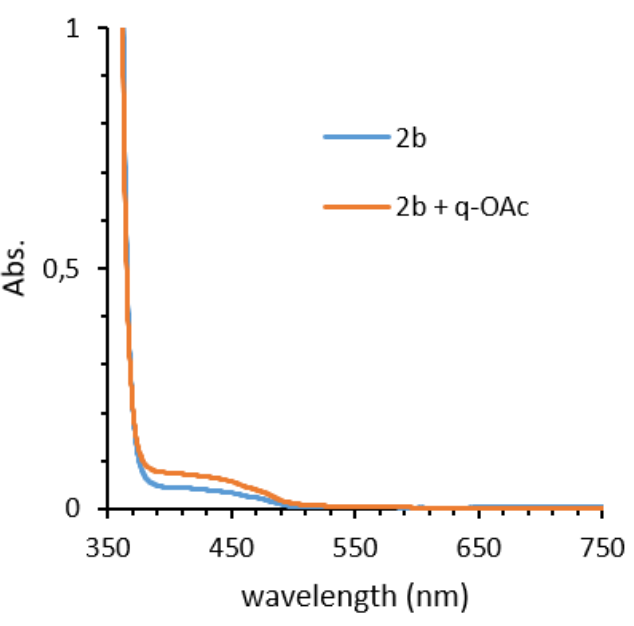<smiles></smiles>

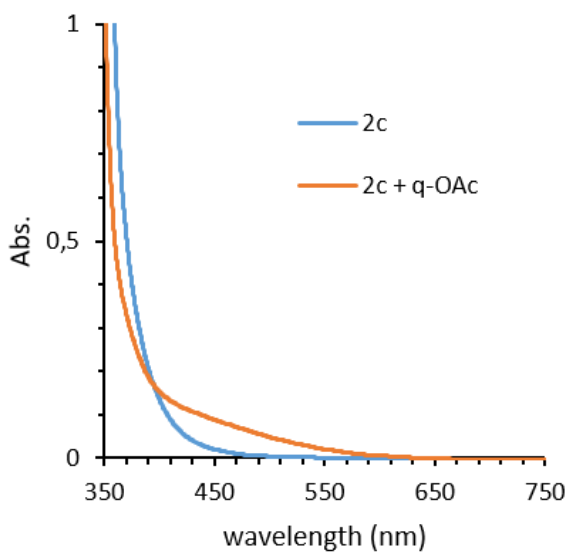


<smiles>O=C(ON1C(=O)c2c(Cl)c(Cl)c(Cl)c(Cl)c2C1=O)C1CCCN1C(=O)c1ccccc1</smiles>
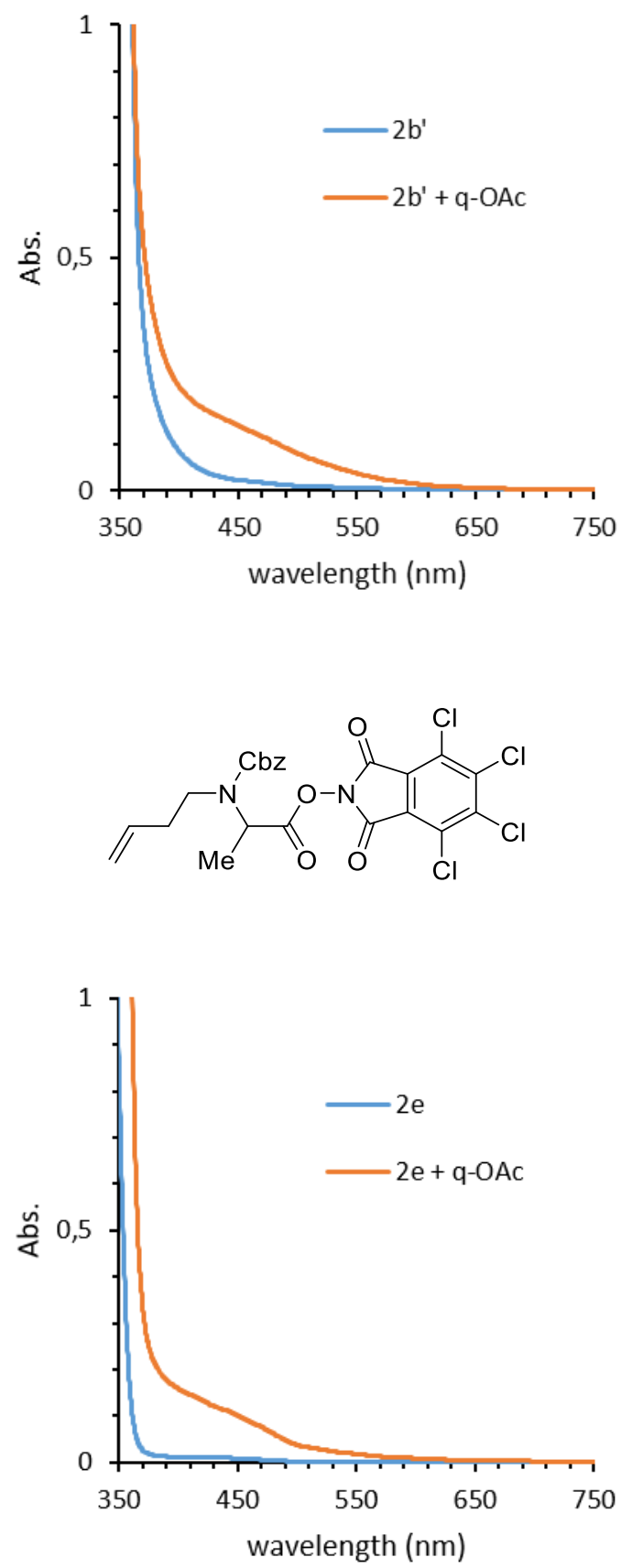<smiles>O=C(NC(COCc1ccccc1)C(=O)ON1C(=O)c2c(Cl)c(Cl)c(Cl)c(Cl)c2C1=O)c1ccccc1</smiles>
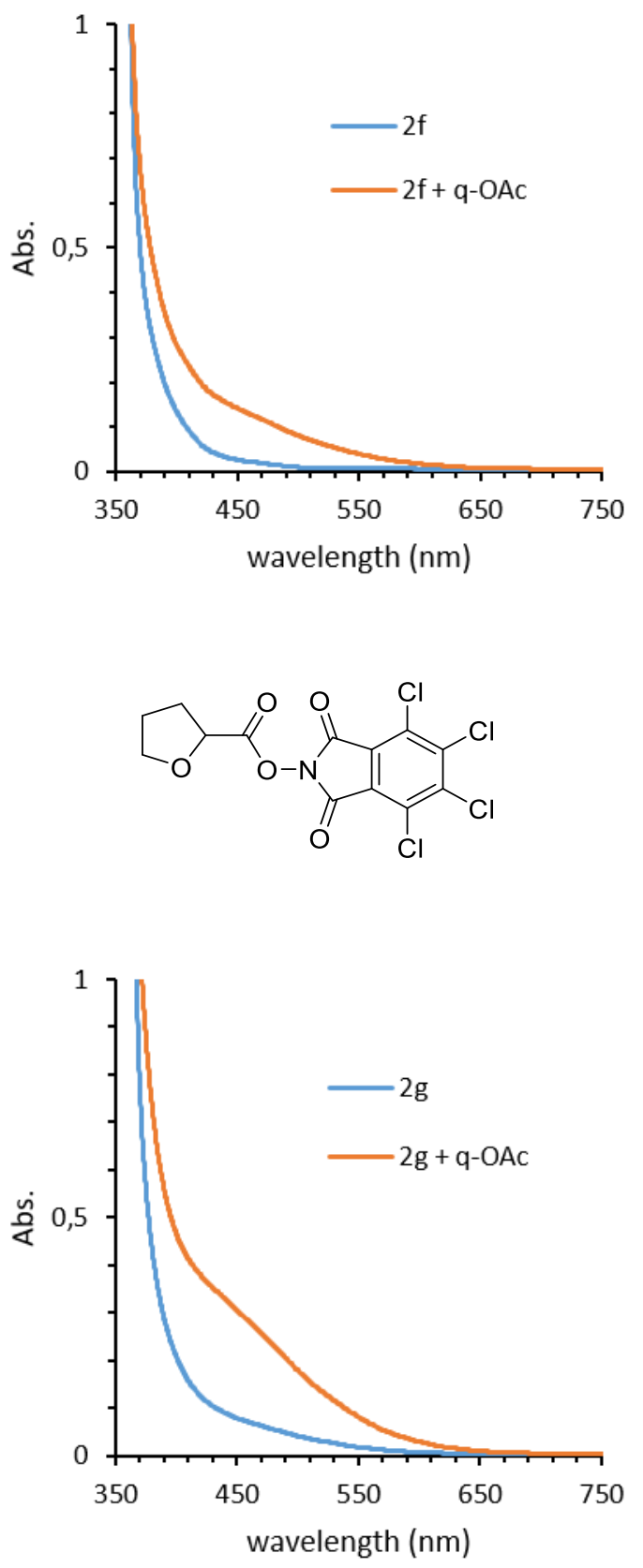

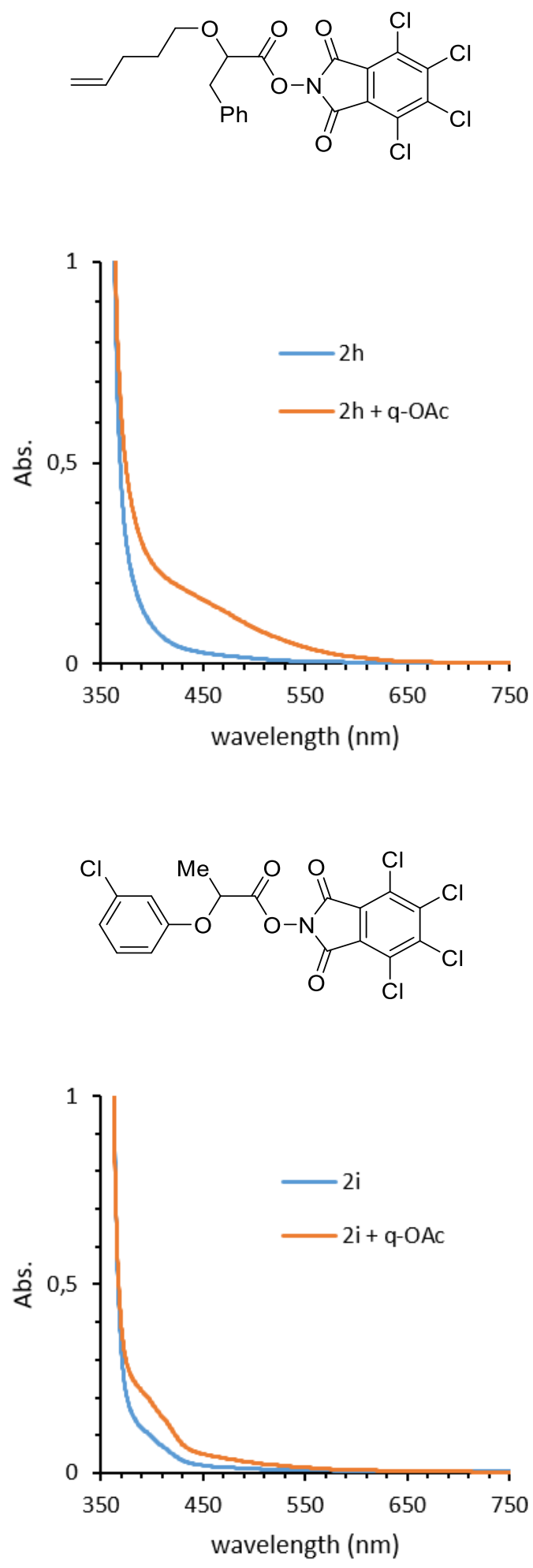
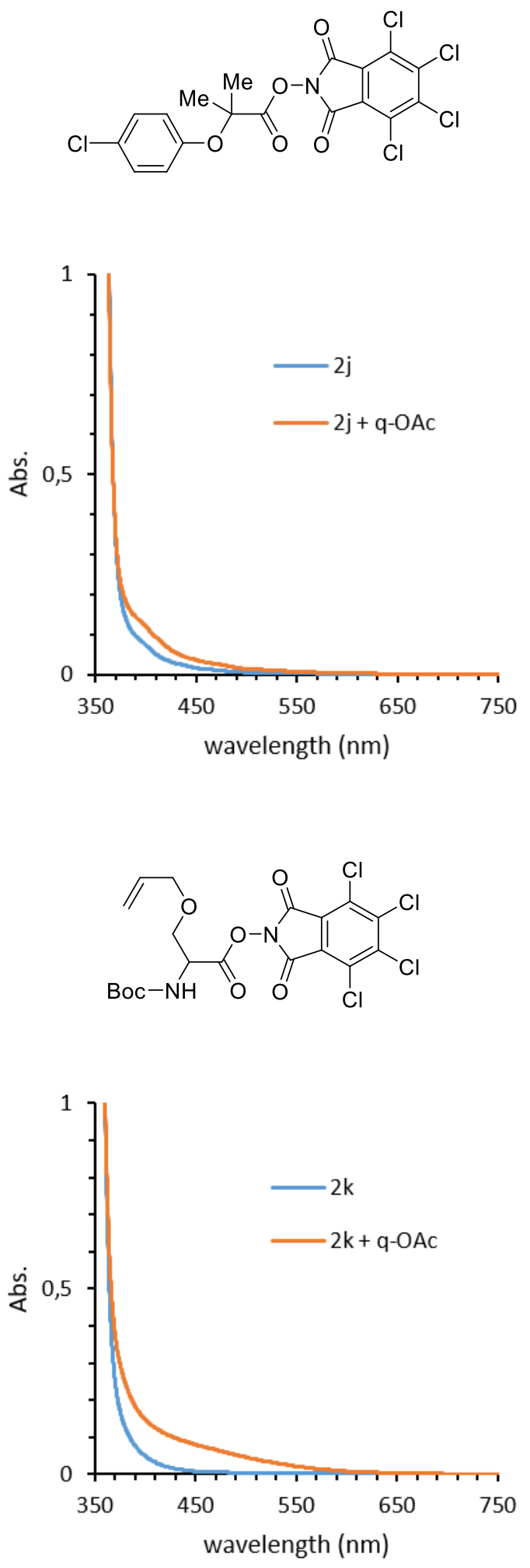

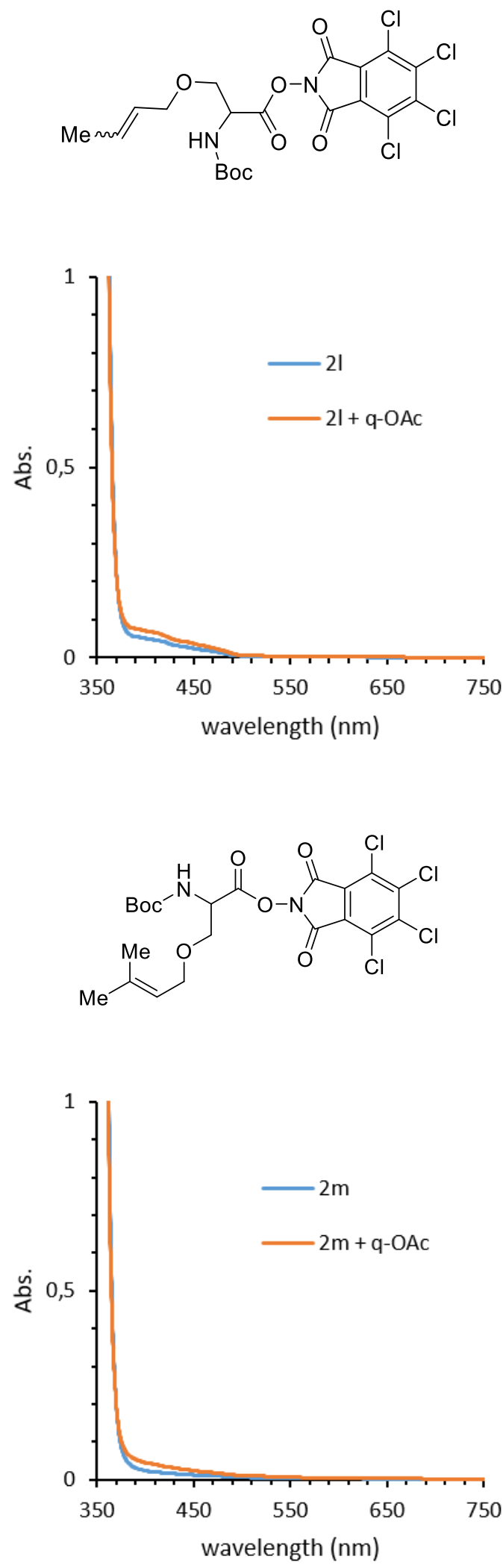

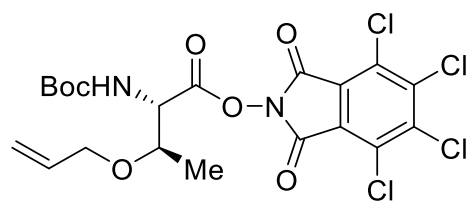

$E_{1 / 2}(\mathbf{2 n})=-0.59 \mathrm{~V}$ vs SCE

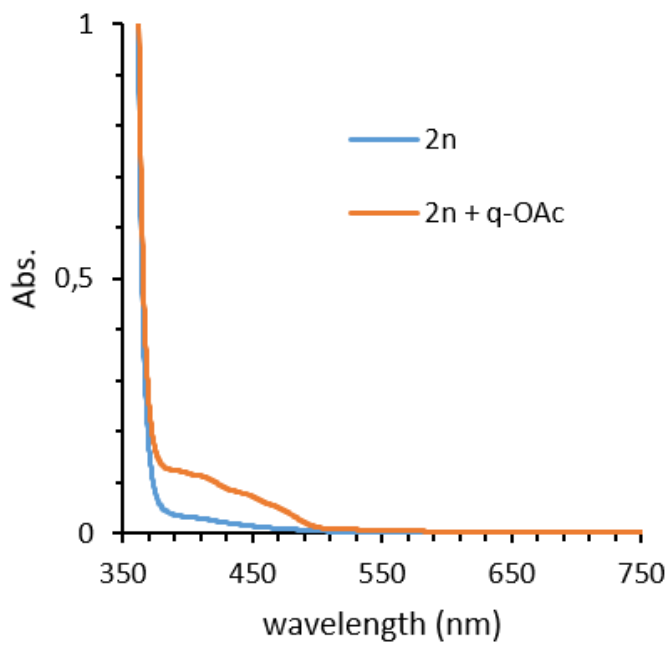<smiles>C=C(C)COCC(NC(=O)OC(=O)c1ccccc1)C(=O)ON1C(=O)c2c(Cl)c(Cl)c(Cl)c(Cl)c2C1=O</smiles>

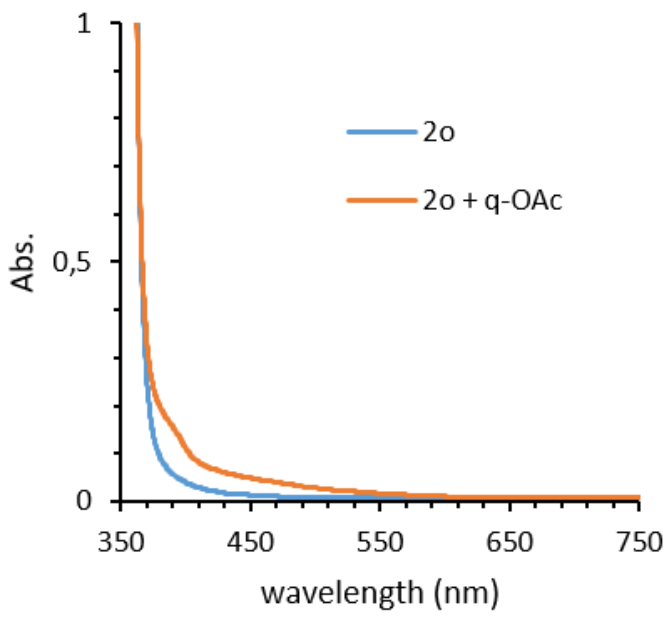



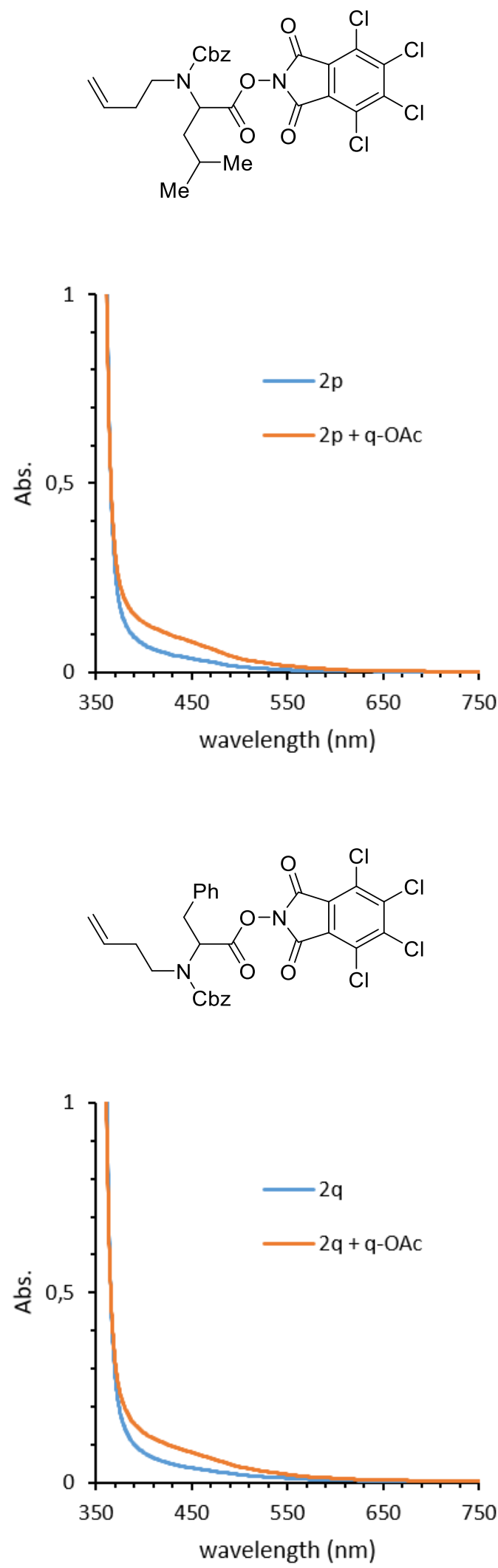
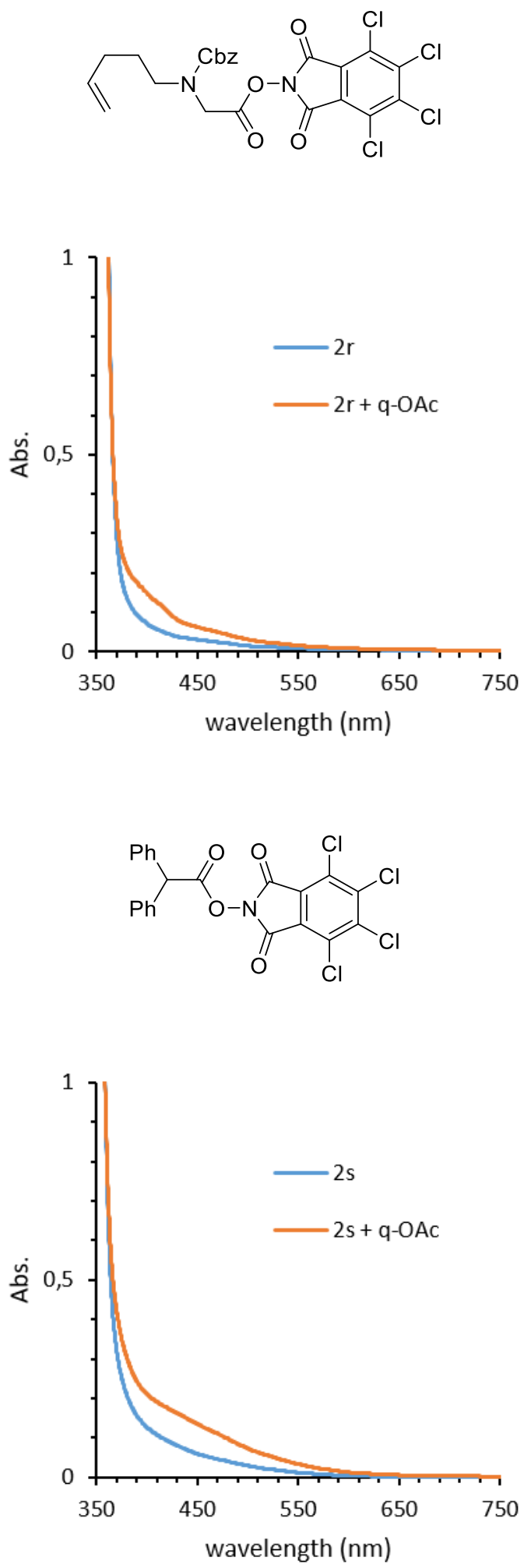

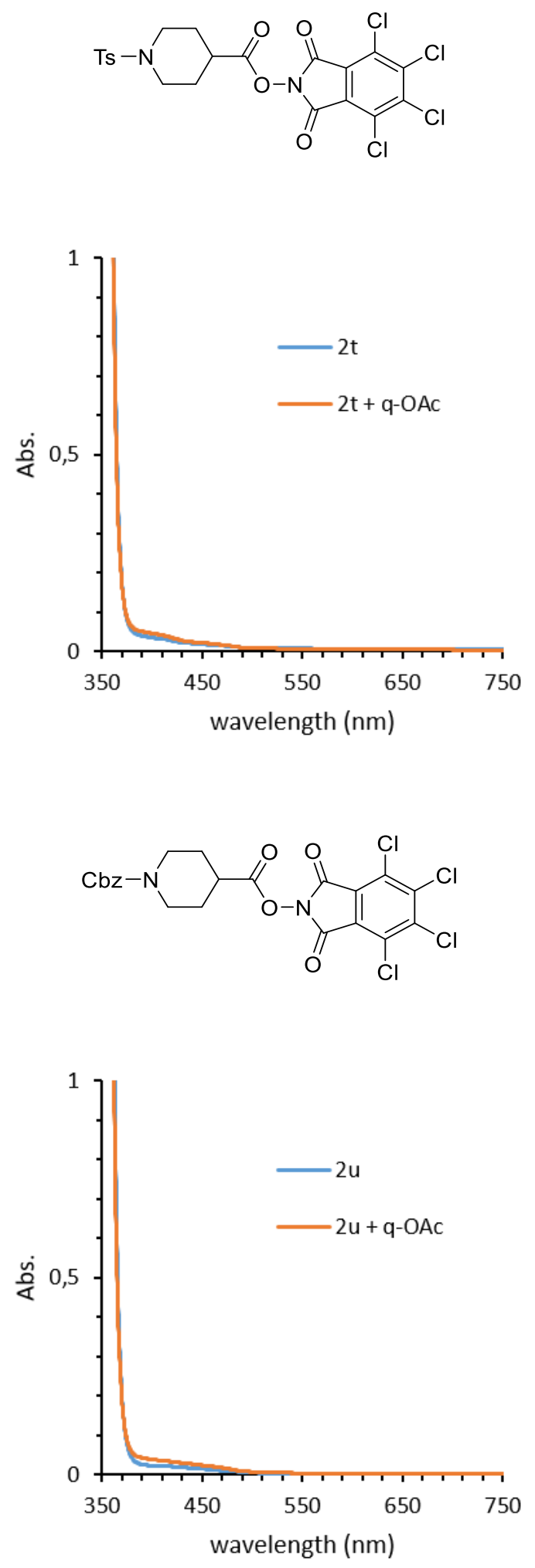
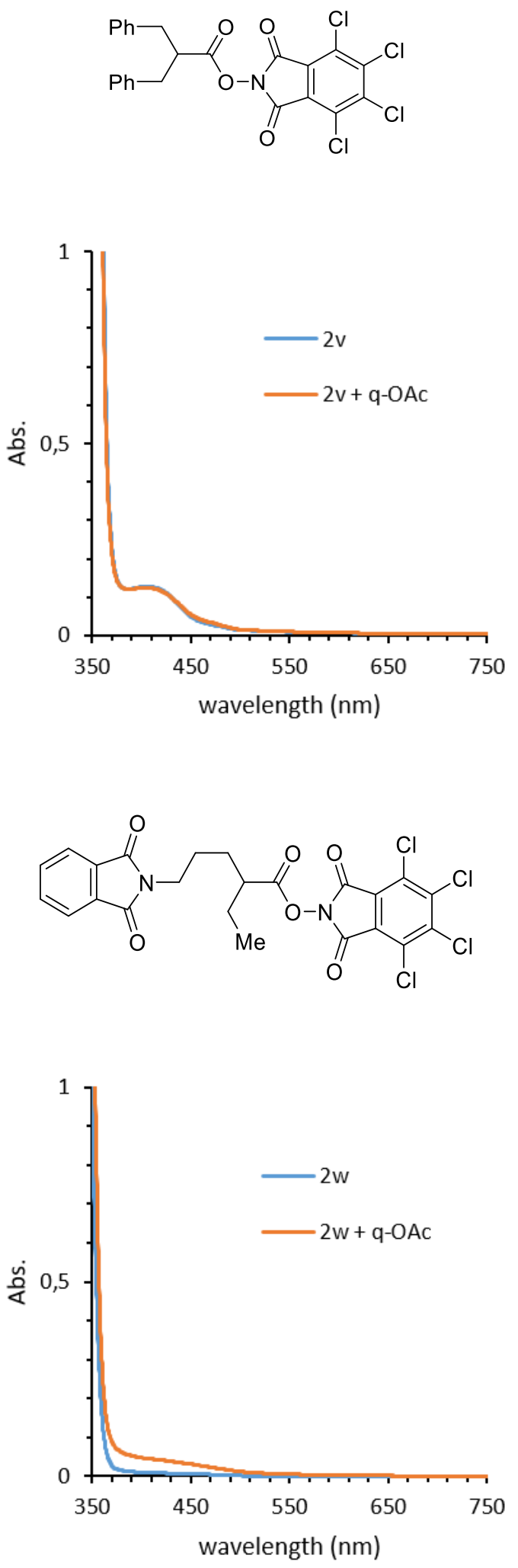

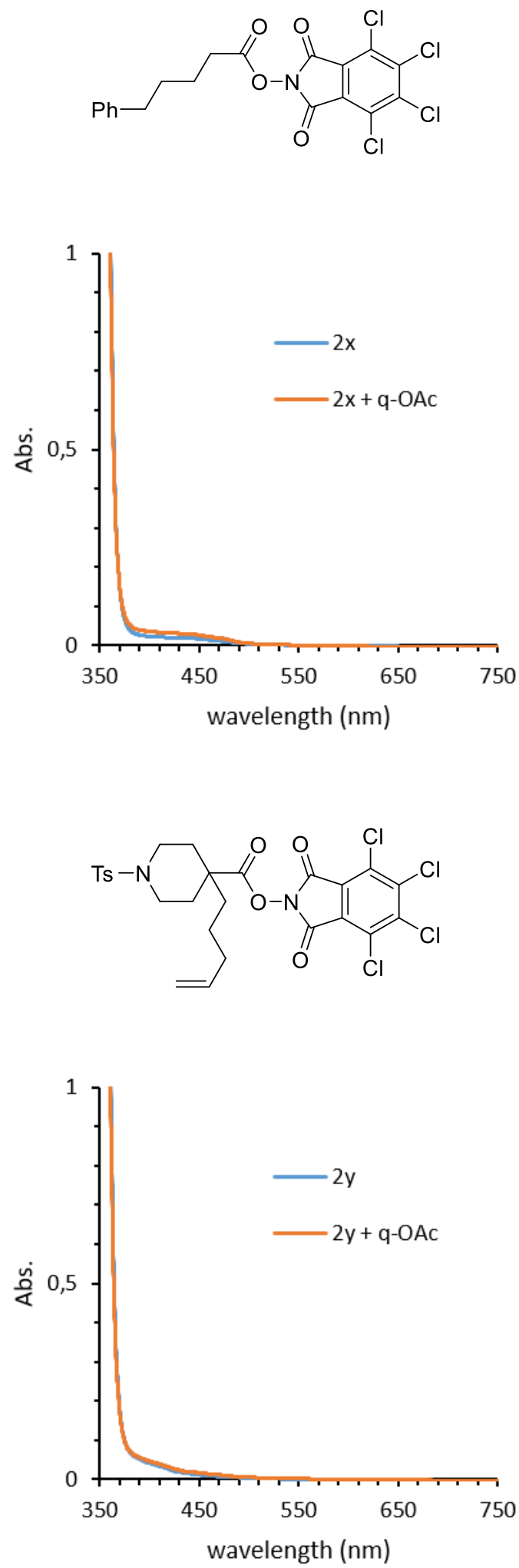
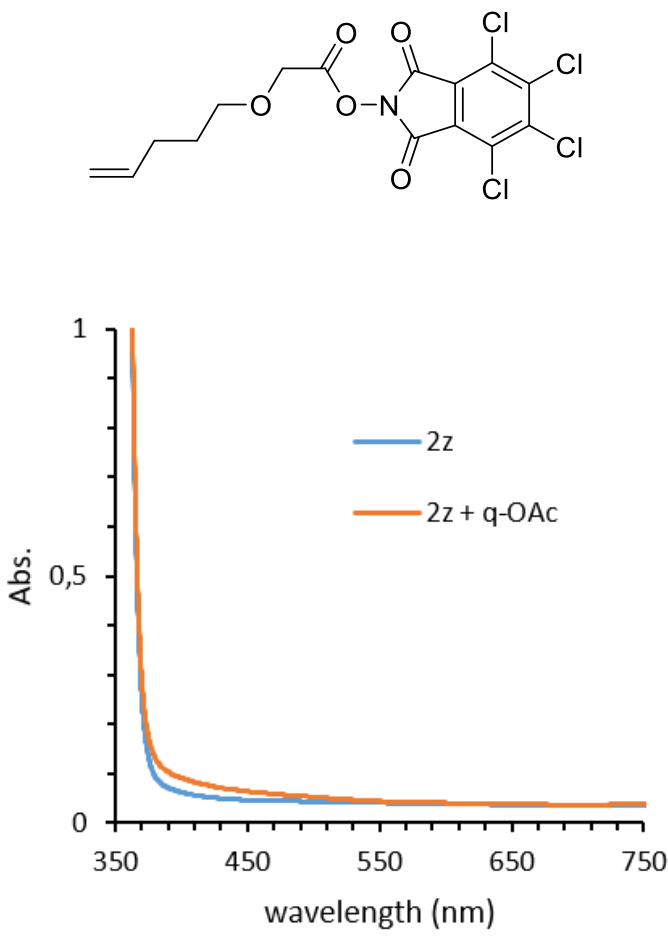


\section{Synthesis of the ED catalyst 3-acetoxyquinuclidine (q-OAc)}

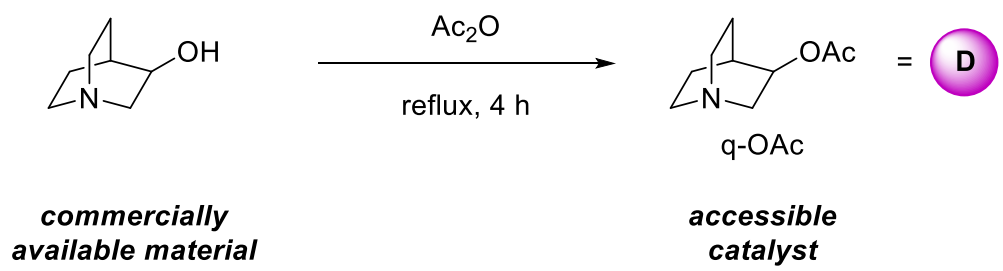

A modification of a procedure found in the literature was used: ${ }^{[9]}$

3-quinuclidinol (1.0 g, $7.87 \mathrm{mmol})$ was added in a flame dried round bottom flask under Argon atmosphere. Acetic anhydride $(33 \mathrm{~mL})$ was added and the flask was connected to a reflux condenser under Argon atmosphere, and refluxed for $4 \mathrm{~h}$. After this time, the reaction was allowed to reach room temperature. The condenser was substituted by a micro-distillation apparatus and the solvent was distilled. The residue was carefully dissolved in $20 \mathrm{~mL}$ of $\mathrm{NaHCO}_{3}$ sat. aq. solution and $15 \mathrm{~mL}$ of chloroform. The heterogeneous mixture was vigorously stirred for $30 \mathrm{~min}$. The organic phase was separated and kept, and the aqueous phase was extracted with chloroform $(5 \mathrm{x} 10 \mathrm{~mL})$. Organic phases were combined, dried over $\mathrm{MgSO}_{4}$, filtered and concentrated. The brownish residue was distilled under vacuum $\left(110^{\circ} \mathrm{C}, 2 \mathrm{mbar}\right)$. The product was obtained as a colorless wax (689 mg, 52\% yield) that was stored in the fridge under Argon atmosphere for further use.

${ }^{1}$ H-NMR: (400 MHz, Chloroform- $d$ ) $\delta 4.76-4.64(\mathrm{~m}, 1 \mathrm{H}), 3.17$ (ddd, $\left.J=14.6,8.4,2.1 \mathrm{~Hz}, 1 \mathrm{H}\right), 2.88-2.64$ $(\mathrm{m}, 4 \mathrm{H}), 2.61(\mathrm{dt}, J=14.8,2.7 \mathrm{~Hz}, 1 \mathrm{H}), 2.00(\mathrm{~s}, 3 \mathrm{H}), 1.92(\mathrm{q}, J=3.3 \mathrm{~Hz}, 1 \mathrm{H}), 1.77$ (tdd, $J=9.9,5.2,2.5 \mathrm{~Hz}$, $1 \mathrm{H}), 1.67-1.56(\mathrm{~m}, 1 \mathrm{H}), 1.54-1.43(\mathrm{~m}, 1 \mathrm{H}), 1.39-1.26(\mathrm{~m}, 1 \mathrm{H})$.

${ }^{13}$ C-NMR: (101 MHz, $\left.\mathrm{CDCl}_{3}\right) \delta 170.9(\mathrm{C}), 71.4(\mathrm{CH}), 55.5\left(\mathrm{CH}_{2}\right), 47.4\left(\mathrm{CH}_{2}\right), 46.5\left(\mathrm{CH}_{2}\right), 25.2(\mathrm{CH}), 24.6$ $\left(\mathrm{CH}_{2}\right), 21.2\left(\mathrm{CH}_{3}\right), 19.5\left(\mathrm{CH}_{2}\right)$.

IR (ATR): $\tilde{v}\left(\mathrm{~cm}^{-1}\right)$ 2951, 2916, 2875, 1718, 1380, 1362, 1289, 1249, 1021, 778.

HRMS: calculated for $\mathrm{C}_{9} \mathrm{H}_{16} \mathrm{NO}_{2}\left(\mathrm{M}+\mathrm{H}^{+}\right)$170.1176, found 170.1169 . 


\section{General synthetic procedures:}

\section{General procedure A for the synthesis of the tetrachlorophthalimide derivatives 1 and 2:}

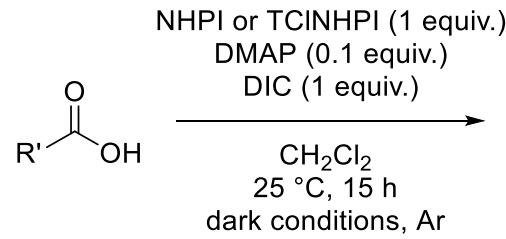<smiles>[R]C(=O)ON1C(=O)c2c([R])c([R])c([R])c([R])c2C1=O</smiles>

$1(\mathrm{R}=\mathrm{H})$

$2(\mathrm{R}=\mathrm{Cl})$
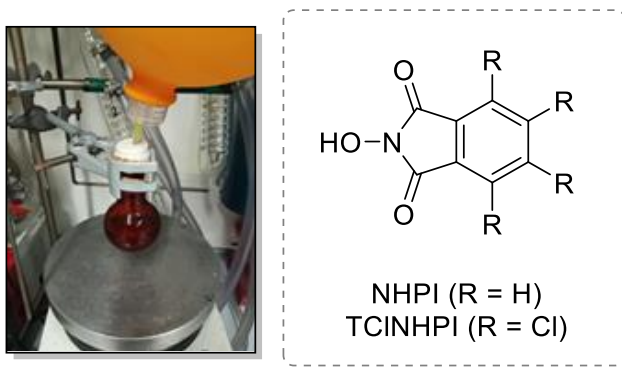

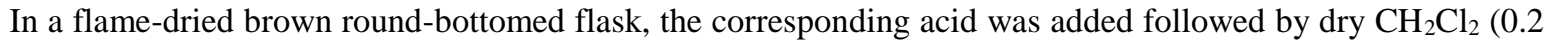
M). Then, $N$-hydroxyphthalimide (NHPI) or tetrachloro- $N$-hydroxyphthalimide (TCINHPI) (1 equiv.) and DMAP (0.1 equiv.) were added. DIC was added dropwise and the reaction mixture was stirred at room temperature for $15 \mathrm{~h}$. After this time, the reaction mixture was filtered using $\mathrm{CH}_{2} \mathrm{Cl}_{2}$ to remove the insoluble urea, and the filtrate was collected in a brown round-bottomed flask. The mixture was concentrated immediately before purification by column chromatography to give the corresponding products $\mathbf{1}$ or $\mathbf{2}$. Note: All products $\mathbf{2}$, while in the reaction mixture, proved to be unstable under light irradiation from the lamps of the lab due to the necessity of an amine (DMAP) to perform the reaction. For this reason, brown round-bottomed flasks were always used to carry out this reaction. After column, products were found stable; even so, they were kept under dark conditions in order to avoid decomposition over time. For this reason, purification of every product was performed immediately after filtration and concentration.

\section{General procedure B for the photocatalyzed reaction using q-OAc as ED catalyst.}<smiles>[R]C(=O)ON1C(=O)c2c(Cl)c(Cl)c(Cl)c(Cl)c2C1=O</smiles>

2
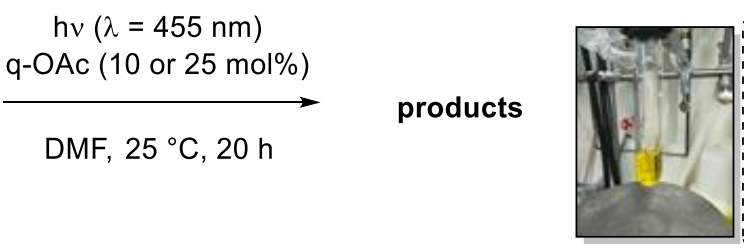

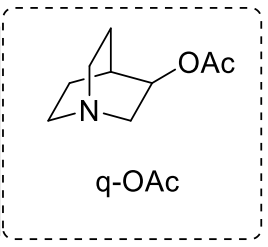

A $25 \mathrm{~mL}$ screw cap Schlenk tube equipped with a stir bar was flame dried and placed under Argon atmosphere. $0.1 \mathrm{mmol}$ of ester 2 were added, followed by $1 \mathrm{~mL}$ of dry DMF (exclusion of water was crucial for reproducible results) and 0.01 or $0.025 \mathrm{mmol}$ of 3-acetoxyquinuclidine (q-OAc). The tube was sealed and freeze-pump-thaw was performed three times to avoid any presence of oxygen during the reaction. The reaction vial was irradiated at $455 \mathrm{~nm}$ for $17-20 \mathrm{~h}$ at room temperature. After this time, the reaction mixture was diluted in EtOAc and water. The aqueous layer was extracted three times with EtOAc. The organic layers were washed with water and brine, dried over $\mathrm{Na}_{2} \mathrm{SO}_{4}$, filtered and concentrated. The crude was purified by column chromatography. Control reactions in the absence of q-OAc were run using $0.05 \mathrm{mmol}$ of ester 2 and $0.5 \mathrm{~mL}$ of DMF. 


\section{General procedure C:}

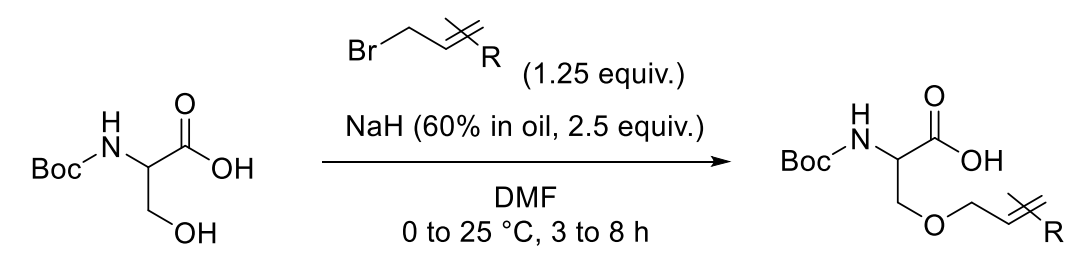

$\mathrm{N}$-Boc-serine ( $3.0 \mathrm{~g}, 14.6 \mathrm{mmol})$ was dissolved in $48 \mathrm{~mL}$ of dry DMF under Argon atmosphere. In a separate flask, $\mathrm{NaH}$ (60\% in oil) (2.5 equiv.) was suspended in $12 \mathrm{~mL}$ of DMF and this suspension was cooled down to $0{ }^{\circ} \mathrm{C}$. The solution of $\mathrm{N}$-Boc-serine was added via cannula to this suspension. After complete addition, the reaction mixture was allowed to reach room temperature and stir for $1 \mathrm{~h}$. Then, the corresponding allylic bromide was added and the reaction was stirred at room temperature for 3 to $8 \mathrm{~h}$. Water $(3 \mathrm{~mL})$ was then carefully added to quench the excess of $\mathrm{NaH}$ and the mixture was concentrated. The crude was dissolved in water $(20 \mathrm{~mL})$ and acidified to $\mathrm{pH} 2$ using $2 \mathrm{M} \mathrm{HCl}$ or solid citric acid. This aqueous phase was extracted with EtOAc (3 times), dried over $\mathrm{Na}_{2} \mathrm{SO}_{4}$, filtered and concentrated to give the corresponding acid. This acid was used in the next step (general procedure $\mathbf{A}$ ) without further purification.

\section{General procedure D:}

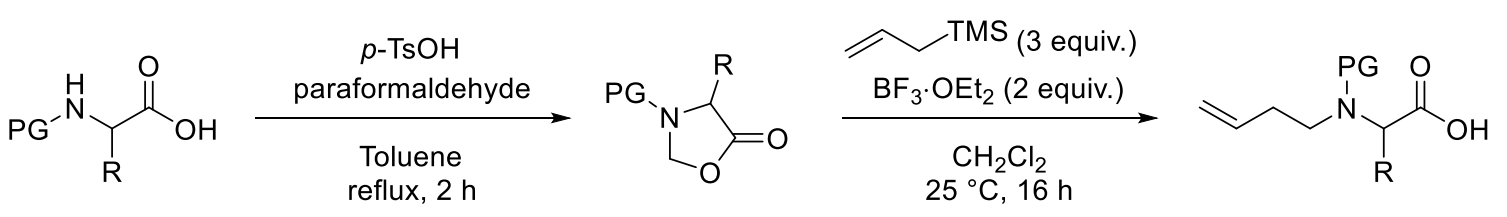

Paraformaldehyde $(1.0 \mathrm{~g})$ and p-TsOH $(100 \mathrm{mg})$ were added to a solution of the corresponding $\mathrm{N}$-protected aminoacid $(5 \mathrm{mmol})$ in toluene $(100 \mathrm{~mL})$. The reaction mixture was refluxed under Dean-Stark conditions for $2 \mathrm{~h}$. Then, the reaction mixture was allowed to reach room temperature and washed twice with $\mathrm{NaHCO}_{3}$ saturated aqueous solution. The organic layer (toluene) was dried over $\mathrm{Na}_{2} \mathrm{SO}_{4}$, filtered and concentrated giving the corresponding oxazolidinone. Column chromatography was performed to give pure product.

A solution of the corresponding oxazolidinone $(2 \mathrm{mmol})$ in dry $\mathrm{CH}_{2} \mathrm{Cl}_{2}(20 \mathrm{~mL})$ under Argon atmosphere was cooled down to $0{ }^{\circ} \mathrm{C} . \mathrm{BF}_{3} \cdot \mathrm{OEt}_{2}(502 \mu \mathrm{L}, 4 \mathrm{mmol}, 2$ equiv.) was added dropwise followed by allyltrimethylsilane ( $950 \mu \mathrm{L}, 6 \mathrm{mmol}, 3$ equiv.). The reaction was stirred for $16 \mathrm{~h}$. $\mathrm{Et}_{2} \mathrm{O}$ and $\mathrm{NaHCO}_{3}$ saturated aqueous solution were added and the aqueous phase was separated. The organic phase was concentrated. This crude was redissolved in $\mathrm{Et}_{2} \mathrm{O}$ and extracted 3-5 times with $\mathrm{NaHCO}_{3}$ saturated aqueous solution. All the collected aqueous phases were combined and the $\mathrm{pH}$ was adjusted to $\mathrm{pH} 2-3$ with $\mathrm{HCl}$ (1 $\mathrm{M}$ aqueous solution) or solid citric acid. The acidified aqueous phase was extracted three times with EtOAc. The organic phases were combined, dried over $\mathrm{Na}_{2} \mathrm{SO}_{4}$, filtered and concentrated to give the corresponding acid that was used without further purification. 
11. Guide of compounds:

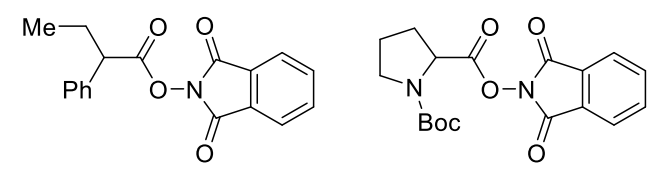

$1 \mathrm{a}$

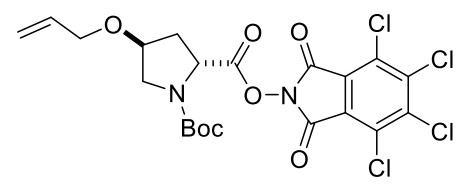

2c

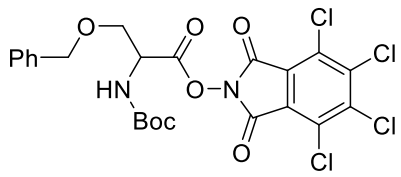

$2 f$<smiles>CC(Oc1cccc(Cl)c1)C(=O)ON1C(=O)c2c(Cl)c(Cl)c(Cl)c(Cl)c2C1=O</smiles>

$2 \mathbf{i}$
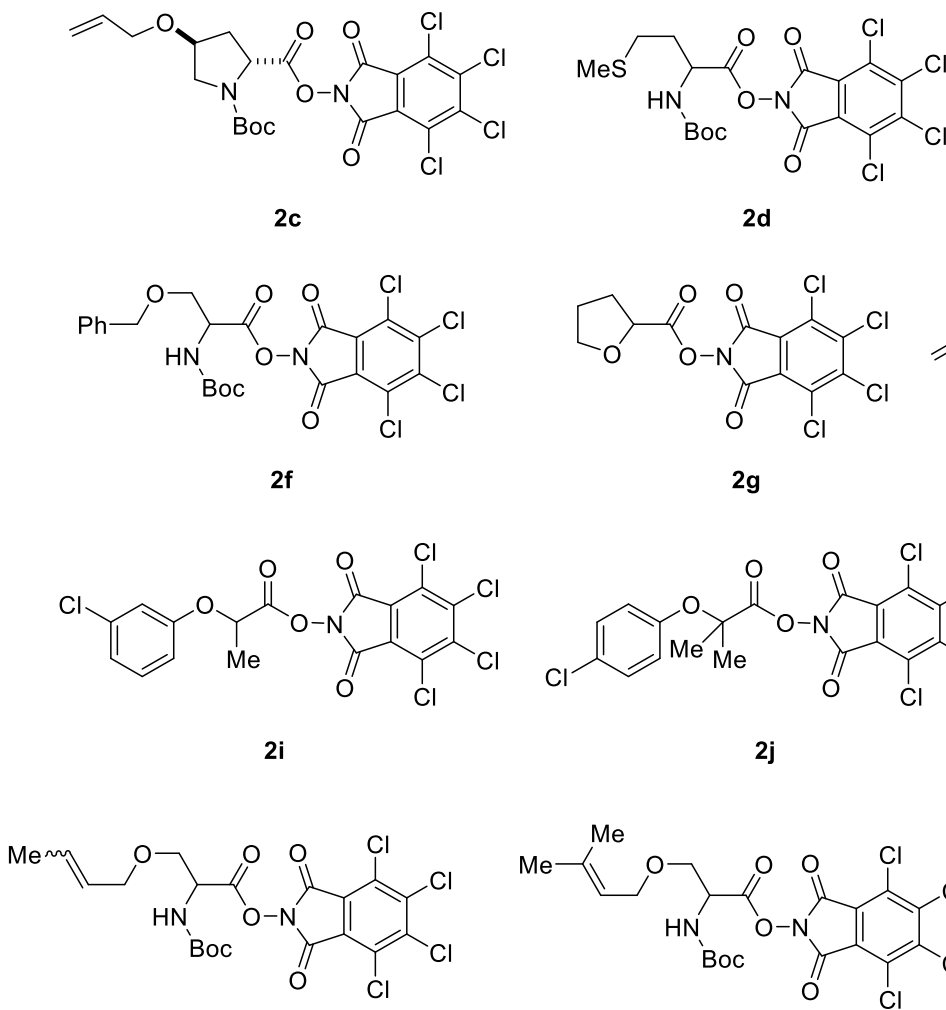

2d

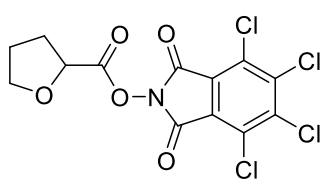

$2 g$<smiles>CCC(C(=O)ON1C(=O)c2c(Cl)c(Cl)c(Cl)c(Cl)c2C1=O)c1ccccc1</smiles>

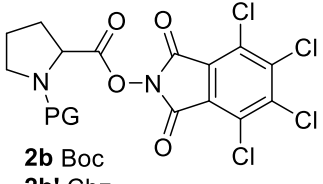

2b' Cbz

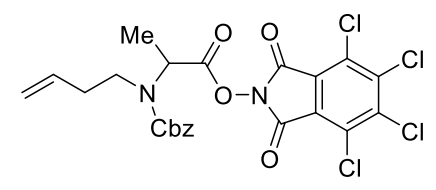

$2 e$

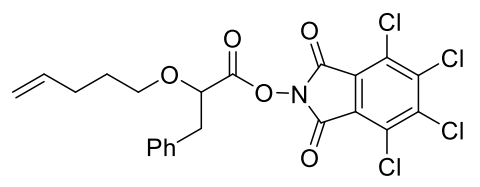

$2 h$

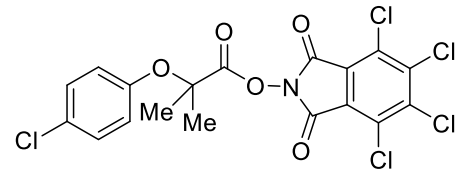

2j

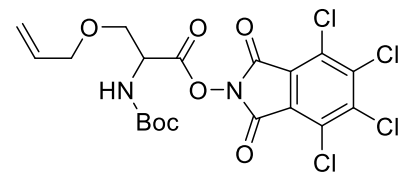

2k
21

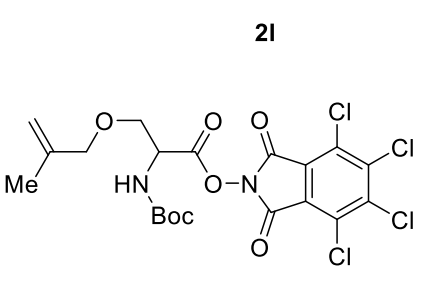

20

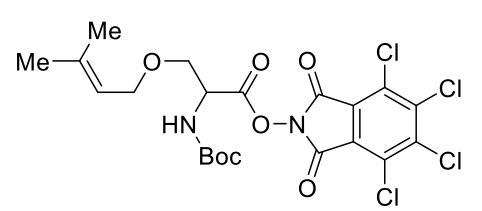

$2 \mathrm{~m}$

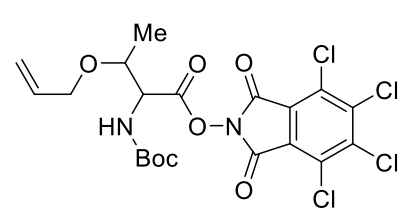

$2 n$

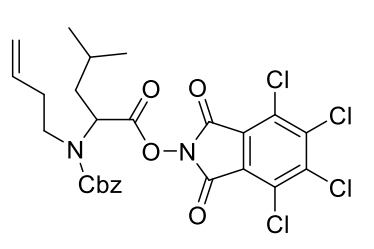

$2 p$

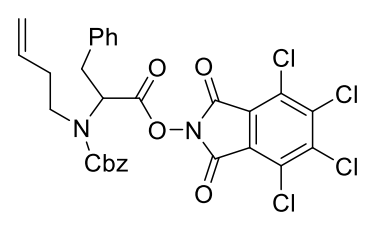

$2 q$

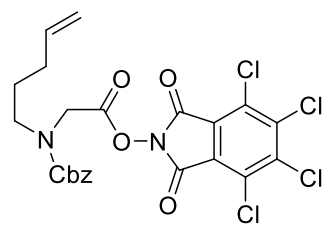

$2 r$

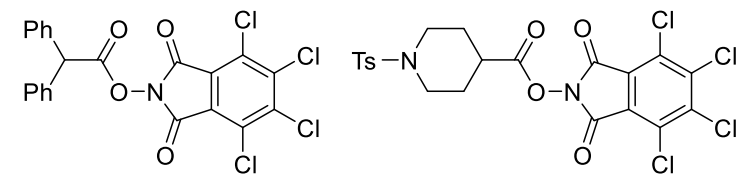

2s

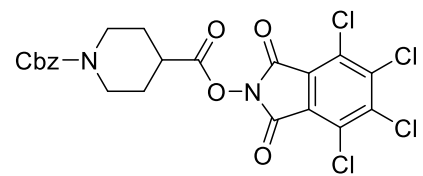

$2 u$

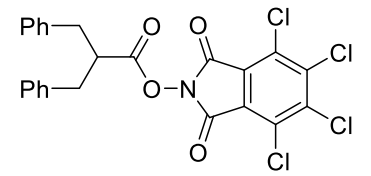

2v
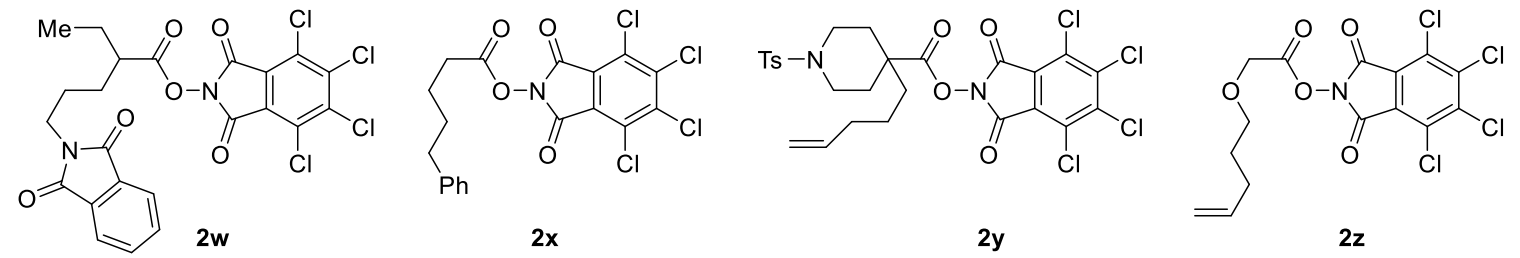
<smiles>CCC(c1ccccc1)C(CC)c1ccccc1</smiles>

3a<smiles>O=C1c2c(Cl)c(Cl)c(Cl)c(Cl)c2C(=O)N1C1CCCN1P(=O)(O)c1ccccc1</smiles>

3b Boc<smiles>C=CCOC1CC(N2C(=O)c3c(Cl)c(Cl)c(Cl)c(Cl)c3C2=O)N(C(=O)OC(C)(C)C)C1</smiles>

3c-cis<smiles>C=CCOC1C[C@@H](N2C(=O)c3c(Cl)c(Cl)c(Cl)c(Cl)c3C2=O)N(C(=O)OC(C)(C)C)C1</smiles>

3c-trans<smiles>CSCCC(NC(=O)OC(C)(C)C)N1C(=O)c2c(Cl)c(C)c(Cl)c(Cl)c2C1=O</smiles>

3d<smiles>O=C1c2c(Cl)c(Cl)c(Cl)c(Cl)c2C(=O)N1C1CCCO1</smiles>

$3 g$<smiles>[M]C(N(C)CCC=C)N1C(=O)c2c(Cl)c(Cl)c(Cl)c(Cl)c2C1=O</smiles>

$3 e$<smiles>C=CCCCOC(Cc1ccccc1)N1C(=O)c2c(Cl)c(Cl)c(Cl)c(Cl)c2C1=O</smiles>

3h

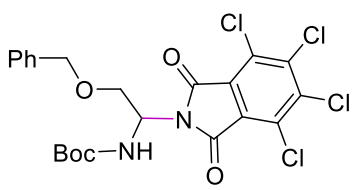

$3 f$<smiles>CC(Oc1cccc(Cl)c1)N1C(=O)c2c(Cl)c(Cl)c(Cl)c(Cl)c2C1=O</smiles>

$3 \mathbf{i}$<smiles>CC(C)(Oc1ccc(Cl)cc1)N1C(=O)c2c(Cl)c(Cl)c(Cl)c(Cl)c2C1=O</smiles>

3j<smiles>CC(C)(C)OC(=O)NC1COCC1[N+](=O)[O-]</smiles>
3k (4:1 dr)

Boc-NH<smiles>CCC1COCC1NC(=O)OC(C)(C)C</smiles>

3I (2:1 dr)<smiles>[Y]C(C)C1COCC1NC(=O)OC(C)(C)C</smiles>

$3 m$<smiles>[M]C1COC([M])C1NC(C)(C)C</smiles>
3n $(9: 1 \mathrm{dr})$

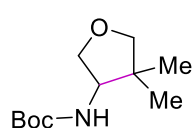

30<smiles>CC(C)CC1C(C)CCN1C(=O)OCc1ccccc1</smiles>

$3 p$

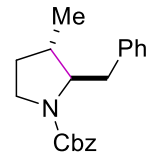

$3 q(>9: 1 d r)$<smiles>CC1CCCN(C(=O)OCc2ccccc2)C1</smiles>

$3 r$<smiles>c1ccc(C(c2ccccc2)c2ccccc2)cc1</smiles>

3s<smiles></smiles>

$3 t$<smiles>O=C(c1ccccc1)N1CCCCC1</smiles>

3u
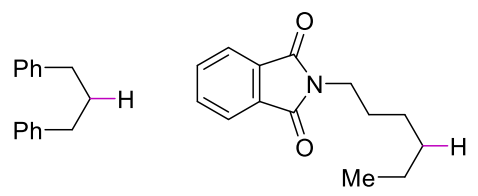

$3 v$<smiles>C=C(C)COCC(NC(=O)OC(C)(C)C)N1C(=O)c2c(Cl)c(Cl)c(Cl)c(Cl)c2C1=O</smiles>

$4 k$<smiles>C=CCCN(CC(C)C)C(CC(C)C)N1C(=O)c2c(Cl)c(Cl)c(Cl)c(Cl)c2C1=O</smiles>

4s
$5 p$ 


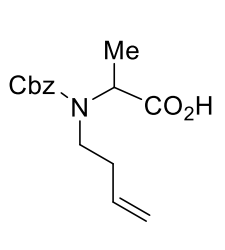

s1e<smiles>C=CCCCOC(Cc1ccccc1)C(=O)O</smiles>

s1h<smiles>C=CCCN(C(=O)O)C(CC(C)(C)C)C(=O)O</smiles>

S1p<smiles>C=CCCN(C(=O)O)C(Cc1ccccc1)C(=O)O</smiles>

s1q<smiles>C=CCCCN(C)CC(=O)O</smiles>

S1r<smiles>O=C(O)C1CCN([12F])CC1</smiles>

s1t<smiles>O=C(O)C1CCN(C(=O)OCc2ccccc2)CC1</smiles>

S1u<smiles>O=C(O)C(Cc1ccccc1)Cc1ccccc1</smiles>

S1v

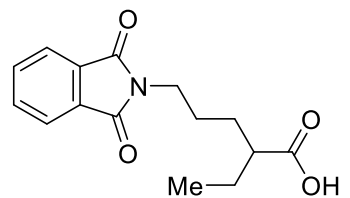

S1w<smiles>C=CCCCC1(C(=O)O)CCN([As])CC1</smiles>

S1y<smiles>C=CCCCOCC(=O)OCCCOC(Cc1ccccc1)C(=O)OC</smiles><smiles>C=CCCCNCC(=O)OCC</smiles><smiles>O=C(O)C(C(=O)O)(c1ccccc1)c1ccccc1</smiles>

S2r

S2v<smiles>CCC(CCCN1C(=O)c2ccccc2C1=O)(C(=O)O)C(=O)O</smiles>

S2w<smiles>CCOC(=O)C(C(=O)OCC)(c1ccccc1)c1ccccc1</smiles>

S3v<smiles>CCOC(=O)C(CC)(CCCN1C(=O)c2ccccc2C1=O)C(=O)OCC</smiles>

S3w

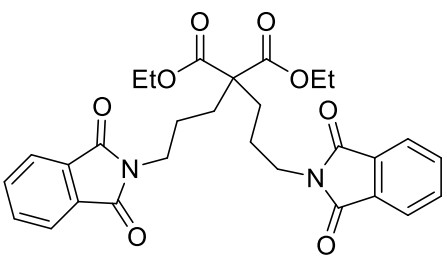

S5w 


\section{Characterization of phthalimide derivatives $1 \mathrm{a}$ and $1 \mathrm{~b}$ :}

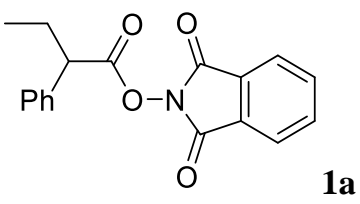

Obtained following general procedure A, from $10 \mathrm{mmol}$ of 2-phenylbutyric acid. Column chromatography (9:1 pentane:EtOAc) afforded the product in $87 \%$ yield $(2.68 \mathrm{~g})$ as a white solid ( $\left.\mathbf{m p}: 60.2^{\circ} \mathrm{C}\right)$.

${ }^{1}$ H-NMR: (500 MHz, Chloroform- $d$ ) $\delta 7.91-7.85(\mathrm{~m}, 2 \mathrm{H}), 7.83-7.76(\mathrm{~m}, 2 \mathrm{H}), 7.44-7.39(\mathrm{~m}, 4 \mathrm{H}), 7.39-$ $7.32(\mathrm{~m}, 1 \mathrm{H}), 3.88(\mathrm{t}, J=7.6 \mathrm{~Hz}, 1 \mathrm{H}), 2.27(\mathrm{dt}, J=13.6,7.3 \mathrm{~Hz}, 1 \mathrm{H}), 1.99(\mathrm{dt}, J=13.7,7.3 \mathrm{~Hz}, 1 \mathrm{H}), 1.06(\mathrm{t}, J$ $=7.4 \mathrm{~Hz}, 3 \mathrm{H})$.

${ }^{13}$ C-NMR: (126 MHz, $\left.\mathrm{CDCl}_{3}\right) \delta 170.3(2 \mathrm{xC}), 161.9(\mathrm{C}), 136.8(\mathrm{C}), 134.7(2 \mathrm{xCH}), 128.9(2 \mathrm{xC}), 128.9(2 \mathrm{xCH})$, $128.1(\mathrm{CH}), 127.9(2 \times \mathrm{CH}), 123.9(2 \times \mathrm{CH}), 50.4(\mathrm{CH}), 27.2\left(\mathrm{CH}_{2}\right), 11.9\left(\mathrm{CH}_{3}\right)$.

$\mathrm{E}_{1 / 2}(\mathbf{1 a})=-1.24 \mathrm{~V}$ vs SCE.

HRMS: calculated for $\mathrm{C}_{18} \mathrm{H}_{16} \mathrm{NO}_{4}\left[\mathrm{M}+\mathrm{H}^{+}\right] 310.1074$, found 310.1070 .

Rf: 0.48 (8:2 pentane:EtOAc).

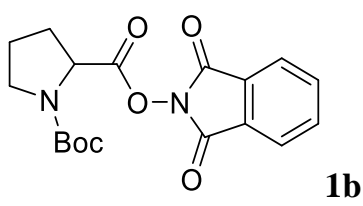

Obtained following general procedure A, from $6.5 \mathrm{mmol}$ of $N$-Boc-proline. Column chromatography (9:1 to $7: 3$ pentane:EtOAc) afforded the product in $93 \%$ yield $(2.17 \mathrm{~g})$.

${ }^{1}$ H-NMR: (300 MHz, Chloroform- $d$ ) $\delta 7.91(\mathrm{dd}, J=5.5,3.1 \mathrm{~Hz}, 2 \mathrm{H}), 7.81(\mathrm{dd}, J=5.5,3.1 \mathrm{~Hz}, 2 \mathrm{H}), 4.73(\mathrm{t}, J$ $=6.2 \mathrm{~Hz}, 0.2 \mathrm{H}), 4.63(\mathrm{dd}, J=8.6,3.9 \mathrm{~Hz}, 0.8 \mathrm{H}), 3.73-3.58(\mathrm{~m}, 1 \mathrm{H}), 3.59-3.39(\mathrm{~m}, 1 \mathrm{H}), 2.49-2.35(\mathrm{~m}, 2 \mathrm{H})$, $2.17-1.94(\mathrm{~m}, 2 \mathrm{H}), 1.54(\mathrm{~s}, 7.3 \mathrm{H}), 1.51(\mathrm{~s}, 1.8 \mathrm{H})$.

${ }^{13}$ C-NMR: $\left(75 \mathrm{MHz}, \mathrm{CDCl}_{3}\right) \delta 169.6(2 \mathrm{xC}), 161.7(\mathrm{C}), 153.5(\mathrm{C}), 134.8(2 \mathrm{xCH}), 128.9(2 \mathrm{xC}), 124.0(2 \mathrm{xCH})$, 81.1 (C), 57.2 (CH), $46.3\left(\mathrm{CH}_{2}\right), 31.4\left(\mathrm{CH}_{2}\right), 28.4 / 28.1\left(3 \times \mathrm{CH}_{3}\right), 23.5\left(\mathrm{CH}_{2}\right)$.

HRMS: calculated for $\mathrm{C}_{18} \mathrm{H}_{21} \mathrm{~N}_{2} \mathrm{O}_{6}\left[\mathrm{M}+\mathrm{H}^{+}\right]$361.1394, found 361.1391 .

Rf: 0.45 (8:2 pentane:EtOAc). 


\section{Characterization of tetrachlorophthalimide derivatives 2a to 2z:}<smiles>CCC(ON1C(=O)c2c(Cl)c(Cl)c(Cl)c(Cl)c2C1=O)c1ccccc1</smiles>

Obtained following general procedure $\mathbf{A}$, from $6.7 \mathrm{mmol}$ of 2-phenylbutyric acid. Column chromatography (95:5 to 8:2 pentane:EtOAc) afforded the product in $94 \%$ yield $(2.82 \mathrm{~g})$ as a white-off solid (mp: $\left.126.8{ }^{\circ} \mathrm{C}\right)$.

${ }^{1}$ H-NMR: (500 MHz, Chloroform- $d$ ) $\delta 7.45-7.36(\mathrm{~m}, 4 \mathrm{H}), 7.35(\mathrm{td}, J=6.5,6.1,2.5 \mathrm{~Hz}, 1 \mathrm{H}), 3.88(\mathrm{t}, J=7.6$ $\mathrm{Hz}, 1 \mathrm{H}), 2.26$ (dt, $J=13.6,7.4 \mathrm{~Hz}, 1 \mathrm{H}), 1.99(\mathrm{dp}, J=14.6,7.4 \mathrm{~Hz}, 1 \mathrm{H}), 1.07$ (t, $J=7.4 \mathrm{~Hz}, 3 \mathrm{H})$.

${ }^{13}$ C-NMR: $\left(126 \mathrm{MHz}, \mathrm{CDCl}_{3}\right) \delta 169.8(2 \mathrm{xC}), 157.5(\mathrm{C}) 141.0(2 \mathrm{xC}), 136.4(\mathrm{C}), 130.4(2 \mathrm{xC}), 129.0(2 \mathrm{xCH})$, $128.0(\mathrm{CH}), 128.0(2 \times \mathrm{CH}), 124.7(2 \times \mathrm{C}), 77.3,50.4(\mathrm{CH}), 27.1\left(\mathrm{CH}_{2}\right), 11.9\left(\mathrm{CH}_{3}\right)$.

$\mathrm{E}_{1 / 2}(\mathbf{2 a})=-0.64 \mathrm{~V}$ vs SCE.

IR (ATR): $\tilde{v}\left(\mathrm{~cm}^{-1}\right)$ 3064, 2969, 1816, 1791, 1746, 1704, 1377, 1366, 1198, 1053, 1036, 731, 697.

HRMS: calculated for $\mathrm{C}_{18} \mathrm{H}_{12} \mathrm{Cl}_{4} \mathrm{NO}_{4}\left[\mathrm{M}+\mathrm{H}^{+}\right] 445.9515$, found 445.9522 .

Rf: 0.30 (95:5 pentane:EtOAc).

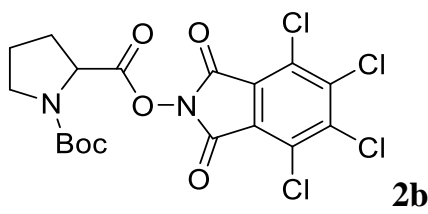

Obtained following general procedure $\mathbf{A}$, from $6.7 \mathrm{mmol}$ of $N$-Boc-proline. Column chromatography (9:1 to 7:3 pentane:EtOAc) afforded the product in $61 \%$ yield $(2.01 \mathrm{~g})$ as a white solid (mp: $\left.196.1{ }^{\circ} \mathrm{C}\right)$.

${ }^{1}$ H-NMR: (500 MHz, Chloroform- $d$ ) $\delta 4.73$ (dd, $\left.J=8.4,3.8 \mathrm{~Hz}, 0.2 \mathrm{H}\right), 4.64(\mathrm{dd}, J=8.9,3.6 \mathrm{~Hz}, 0.8 \mathrm{H}), 3.65$ (ddd, $J=10.4,7.9,4.4 \mathrm{~Hz}, 0.8 \mathrm{H}), 3.58(\mathrm{ddd}, J=11.9,7.9,4.3 \mathrm{~Hz}, 0.2 \mathrm{H}), 3.51(\mathrm{dt}, J=10.4,7.5 \mathrm{~Hz}, 0.8 \mathrm{H}), 3.45$ $(\mathrm{dt}, J=10.0,7.5 \mathrm{~Hz}, 0.2 \mathrm{H}), 2.52-2.40(\mathrm{~m}, 0.8 \mathrm{H}), 2.41-2.33(\mathrm{~m}, 1.2 \mathrm{H}), 2.15-2.06(\mathrm{~m}, 1 \mathrm{H}), 2.07-1.96(\mathrm{~m}$, $1 \mathrm{H}), 1.53(\mathrm{~s}, 7.2 \mathrm{H}), 1.50(\mathrm{~s}, 1.8 \mathrm{H})$.

${ }^{13}$ C-NMR: $\left(126 \mathrm{MHz}, \mathrm{CDCl}_{3}\right) \delta$ 169.3/168.9 (C), 157.3 (C), 154.1/153.4 (C), 141.1/141.0 (C), 130.5 (C), 124.7/124.6 (C), 81.3/80.6 (C), 57.2/57.1 (CH), 46.5/46.3 ( $\left.\mathrm{CH}_{2}\right), 31.4 / 30.2\left(\mathrm{CH}_{2}\right), 28.4 / 28.2\left(3 \mathrm{xCH}_{3}\right), 24.5 / 23.5$ $\left(\mathrm{CH}_{2}\right)$.

$\mathrm{E}_{1 / 2}(\mathbf{2 b})=-0.63 \mathrm{~V}$ vs SCE.

IR (ATR): $\tilde{v}\left(\mathrm{~cm}^{-1}\right)$ 2981, 2873, 1827, 1795, 1746, 1703, 1378, 1367, 1056, 726.

HRMS: calculated for $\mathrm{C}_{18} \mathrm{H}_{16} \mathrm{Cl}_{4} \mathrm{~N}_{2} \mathrm{O}_{6} \mathrm{Na}\left(\mathrm{M}+\mathrm{Na}^{+}\right)$520.9631; found 520.9623

Rf: 0.47 (8:2 pentane:EtOAc). 
<smiles>O=C(ON1C(=O)c2c(Cl)c(Cl)c(Cl)c(Cl)c2C1=O)C1CCCN1C(=O)O</smiles>

Obtained following general procedure A from $2.00 \mathrm{mmol}$ of $\mathrm{N}$-Cbz-proline. Column chromatography (7:3 pentane:EtOAc) afforded the product in $60 \%$ yield $(629 \mathrm{mg})$ as a white solid $\left(\mathbf{m p :} 80.1{ }^{\circ} \mathrm{C}\right)$.

${ }^{1}$ H-NMR: (400 MHz, Chloroform- $d$ ) mixture of rotamers $\delta 7.46-7.29(\mathrm{~m}, 5 \mathrm{H}), 5.31(\mathrm{~d}, J=12.3 \mathrm{~Hz}, 0.6 \mathrm{H})$, $5.25(\mathrm{~d}, J=12.4 \mathrm{~Hz}, 0.4 \mathrm{H}), 5.15(\mathrm{~d}, J=12.3 \mathrm{~Hz}, 1 \mathrm{H}), 4.80(\mathrm{dd}, J=8.3,4.0 \mathrm{~Hz}, 0.4 \mathrm{H}), 4.73(\mathrm{dd}, J=8.6,3.8$ $\mathrm{Hz}, 0.6 \mathrm{H}), 3.76-3.61(\mathrm{~m}, 1 \mathrm{H}), 3.63-3.48(\mathrm{~m}, 1 \mathrm{H}), 2.52-2.32(\mathrm{~m}, 2 \mathrm{H}), 2.18-1.97(\mathrm{~m}, 2 \mathrm{H})$.

${ }^{13}$ C-NMR: $\left(101 \mathrm{MHz}, \mathrm{CDCl}_{3}\right) \delta$ mixture of rotamers 168.9/168.7 (2xC), 157.2/157.1 (C), 154.7/153.9 (C), 141.1/141.0 (2xC), 136.4/136.3 (C), 130.5/130.4 (2xC), 128.5/128.4 (2xCH), 128.3/128.1 (2xCH), 128.1/128.0 $(\mathrm{CH})$, 124.7/124.7 (2xC), 67.6/67.4 $\left(\mathrm{CH}_{2}\right)$, 57.4/57.0 (CH), 46.9/46.4 $\left(\mathrm{CH}_{2}\right)$, 31.4/30.3 $\left(\mathrm{CH}_{2}\right), 24.4 / 23.5\left(\mathrm{CH}_{2}\right)$.

$\mathrm{E}_{1 / 2}(\mathbf{2 d})=-0.61 \mathrm{~V}$ vs SCE.

IR (ATR): $\tilde{v}\left(\mathrm{~cm}^{-1}\right):$ 2931, 1795, 1736, 1671, 1431, 1361, 1302, 1127, 1039, 770, 731.

HRMS: calculated for $\mathrm{C}_{21} \mathrm{H}_{15} \mathrm{Cl}_{4} \mathrm{~N}_{2} \mathrm{O}_{6}\left[\mathrm{M}+\mathrm{H}^{+}\right]$532.9655, found 532.9655 .

Rf: 0.22 (8:2 pentane:EtOAc).<smiles>C=CCO[C@H]1C[C@@H](C(=O)ON2C(=O)c3c(Cl)c(Cl)c(Cl)c(Cl)c3C2=O)C(=O)O1</smiles>

Obtained following general procedure $\mathbf{B}$ followed by general procedure $\mathbf{A}$, from $0.96 \mathrm{mmol}$ of $N$-Boc-trans-4hydroxyproline and $1.20 \mathrm{mmol}$ of prenyl bromide. Column chromatography (9:1 pentane:EtOAc) afforded the product in $41 \%$ yield over two steps $(217 \mathrm{mg})$ as a white foam $\left(\mathbf{m p}: 61.6^{\circ} \mathrm{C}\right)$.

${ }^{1} \mathrm{H}$-NMR: (400 MHz, Chloroform- $d$ ) Mixture of rotamers is observed $\delta 5.91$ (ddt, $J=17.2,10.8,5.6 \mathrm{~Hz}, 1 \mathrm{H}$ ), $5.31(\mathrm{dq}, J=17.2,1.6 \mathrm{~Hz}, 1 \mathrm{H}), 5.23(\mathrm{dq}, J=10.4,1.4 \mathrm{~Hz}, 1 \mathrm{H}), 4.83-4.74(\mathrm{t}, J=7.8 \mathrm{~Hz}, 0.2 \mathrm{H}), 4.73(\mathrm{t}, J=$ $7.8 \mathrm{~Hz}, 0.8 \mathrm{H}), 4.28-4.16(\mathrm{~m}, 1 \mathrm{H}), 4.08-3.96(\mathrm{~m}, 2 \mathrm{H}), 3.77-3.68(\mathrm{~m}, 0.8 \mathrm{H}), 3.67-3.55(\mathrm{~m}, 1.2 \mathrm{H}), 2.61$ (dddd, $J=13.6,8.4,3.5,1.5 \mathrm{~Hz}, 0.8 \mathrm{H}$ ), 2.53 (dt, $J=8.2,4.5 \mathrm{~Hz}, 0.2 \mathrm{H}$ ), 2.43 (ddd, $J=13.2,7.4,5.0 \mathrm{~Hz}, 1 \mathrm{H}$ ), $1.51(\mathrm{~s}, 7.2 \mathrm{H}), 1.50(\mathrm{~s}, 1.8 \mathrm{H})$.

${ }^{13}$ C-NMR: $\left(101 \mathrm{MHz}, \mathrm{CDCl}_{3}\right) \delta$ 169.3/168.7 (C), 157.2/157.1 (C), 154.1/153.4 (C), 141.1/141.0 (C), 134.1(CH), $130.5(\mathrm{C}), 124.7 / 124.6(\mathrm{C}), 117.6 / 117.5\left(\mathrm{CH}_{2}\right), 81.6 / 80.9(\mathrm{C}), 75.7(\mathrm{CH}), 70.4 / 70.3\left(\mathrm{CH}_{2}\right)$, 56.2/56.0 (CH), 51.8/51.3 $\left(\mathrm{CH}_{2}\right), 37.3 / 35.8\left(\mathrm{CH}_{2}\right), 28.3 / 28.1\left(3 \mathrm{xCH}_{3}\right)$.

$\mathrm{E}_{1 / 2}(\mathbf{2 c})=-0.70 \mathrm{~V}$ vs SCE.

IR: (ATR): $\tilde{v}\left(\mathrm{~cm}^{-1}\right): 3082,1934,1978,1796,1736,1656,1699,1395,1370,1157,1090,923,792,731,691$.

HRMS: calculated for $\mathrm{C}_{21} \mathrm{H}_{21} \mathrm{Cl}_{4} \mathrm{~N}_{2} \mathrm{O}_{7}[\mathrm{M}+\mathrm{H}]^{+}, 555.0068$; found, 555.0066.

Rf: 0.54 ( $8: 2$ pentane:EtOAc). 


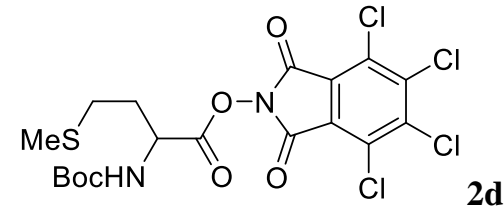

Obtained following general procedure A from $2.00 \mathrm{mmol}$ of $N$-Boc-Methionine. Column chromatography (7:3 pentane:EtOAc) afforded the product in $58 \%$ yield $(613 \mathrm{mg})$ as a white solid $\left(\mathbf{m p}: 150.3{ }^{\circ} \mathrm{C}\right)$.

${ }^{1} \mathbf{H}-\mathbf{N M R}$ : mixture of rotamers is observed $\left(400 \mathrm{MHz}, \mathrm{CDCl}_{3}\right) \delta 5.25(\mathrm{~d}, J=8.0 \mathrm{~Hz}, 1 \mathrm{H}), 4.90(\mathrm{br} \mathrm{s}, 0.75 \mathrm{H})$, 4.68 (br s, 0.25H), $2.80-2.53(\mathrm{~m}, 2 \mathrm{H}), 2.38-2.23(\mathrm{~m}, 1 \mathrm{H}), 2.23-2.09(\mathrm{~m}, 1 \mathrm{H}), 2.17(\mathrm{~s}, 3 \mathrm{H}), 1.48(\mathrm{~s}, 9 \mathrm{H})$.

${ }^{13}$ C-NMR: (101 MHz, $\left.\mathrm{CDCl}_{3}\right) \delta 168.7(2 \mathrm{xC}), 157.1(\mathrm{C}), 154.8(\mathrm{C}), 141.1(2 \mathrm{xC}), 130.5(2 \mathrm{xC}), 124.6(2 \mathrm{xC})$, $80.9(\mathrm{C}), 51.3(\mathrm{CH}), 32.1\left(\mathrm{CH}_{2}\right), 29.6\left(\mathrm{CH}_{2}\right), 28.2\left(3 \mathrm{xCH}_{3}\right), 15.4\left(\mathrm{CH}_{3}\right)$.

IR (ATR): $\tilde{v}\left(\mathrm{~cm}^{-1}\right):$ 2975, 2929, 1821, 1794, 1748, 1702, 1378, 1366, 1160, 1032, 730.

HRMS: calculated for $\mathrm{C}_{13} \mathrm{H}_{10} \mathrm{Cl}_{4} \mathrm{~N}_{2} \mathrm{O}_{4} \mathrm{~S}\left(\mathrm{M}+\mathrm{H}^{+}-\mathrm{C}_{5} \mathrm{H}_{9} \mathrm{O}_{2}\right) 431.9080$, found 431.9082 .

Rf: 0.31 (8:2 hexane:EtOAc)<smiles>C=CCCN(C(=O)OCc1ccccc1)C(C)C(=O)ON1C(=O)c2c(Cl)c(Cl)c(Cl)c(Cl)c2C1=O</smiles>

Obtained following general procedure A from $1.0 \mathrm{mmol}$ of acid S1e. Column chromatography (9:1 pentane:EtOAc) afforded the product in $67 \%$ yield $(373 \mathrm{mg})$ as a white solid (mp: $\left.56^{\circ} \mathrm{C}\right)$.

${ }^{1}$ H-NMR: (300 MHz, Chloroform-d) $\delta 7.45-7.32$ (m, 5H), 5.79 (br s, 1H), $5.42-5.12$ (m, $\left.2 \mathrm{H}\right), 5.16-4.97$ (m, 2.5H), 4.71 (br s, 0.5H), 3.56 (br s, 1H), $3.47-3.18$ (m, 1H), 2.43 (br s, 2H), 1.65 (br s, $3 \mathrm{H})$.

${ }^{13}$ C-NMR: $\left(126 \mathrm{MHz} \mathrm{CDCl}_{3}\right) \delta 168.1(2 \times C), 157.2(\mathrm{C}), 155.9 / 155.1(\mathrm{C}), 141.1(2 \times C), 136.2(\mathrm{C}), 134.8 / 134.7$ $(\mathrm{CH}), 130.5(2 \times C), 128.5 / 128.5(2 \times C H), 128.4 / 128.1(2 \times C H), 128.0(\mathrm{CH}), 124.6(2 \times C), 117.1\left(\mathrm{CH}_{2}\right), 67.9$ $\left(\mathrm{CH}_{2}\right)$, 54.1/53.8 $(\mathrm{CH}), 47.0 / 45.7\left(\mathrm{CH}_{2}\right), 34.2 / 33.4\left(\mathrm{CH}_{2}\right), 16.2 / 15.7\left(\mathrm{CH}_{3}\right)$.

$\mathrm{E}_{1 / 2}(\mathbf{2 e})=-0.64 \mathrm{~V}$ vs SCE.

IR (ATR): $\tilde{v}\left(\mathrm{~cm}^{-1}\right): 3034,3068,2947,1820,1794,1747,1702,1471,1454,1416,1377,1367,1293,1198$, $1040,912,793,729,696$.

HRMS: calculated for $\mathrm{C}_{23} \mathrm{H}_{19} \mathrm{Cl}_{4} \mathrm{~N}_{2} \mathrm{O}_{6}\left(\mathrm{M}+\mathrm{H}^{+}\right)$560.9962, found 560.9961 .

Rf: 0.53 (8:2 pentane:EtOAc). 


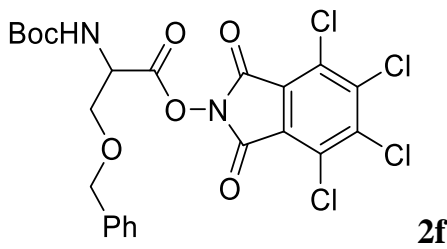

Obtained following general procedure $\mathbf{C}$ and general procedure $\mathbf{A}$, from $1.73 \mathrm{mmol}$ of $N$-Boc-serine and 2.17 mmol of benzyl bromide. Column chromatography (9:1 to 8:2 pentane:EtOAc) afforded the product in $39 \%$ yield over two steps $(391 \mathrm{mg})$ as a yellow-off solid ( $\left.\mathbf{m p}: 146.2{ }^{\circ} \mathrm{C}\right)$.

${ }^{1}$ H-NMR: (400 MHz, Chloroform- $d$ ) $\delta 7.51-7.31(\mathrm{~m}, 5 \mathrm{H}), 5.49(\mathrm{~d}, J=9.1 \mathrm{~Hz}, 0.8 \mathrm{H}), 5.30(\mathrm{t}, J=4.6 \mathrm{~Hz}$, $0.2 \mathrm{H}), 4.92(\mathrm{dt}, J=9.3,3.3 \mathrm{~Hz}, 0.8 \mathrm{H}), 4.72(\mathrm{~d}, J=11.9 \mathrm{~Hz}, 1 \mathrm{H}), 4.64(\mathrm{~s}, 0.2 \mathrm{H}), 4.61(\mathrm{~d}, J=11.9 \mathrm{~Hz}, 1 \mathrm{H}), 4.06$ $(\mathrm{dd}, J=9.9,3.2 \mathrm{~Hz}, 0.8 \mathrm{H}), 4.05-3.98(\mathrm{~m}, 0.2 \mathrm{H}), 3.87(\mathrm{dd}, J=9.8,3.5 \mathrm{~Hz}, 1 \mathrm{H}), 1.52(\mathrm{~s}, 1.8 \mathrm{H}), 1.51(\mathrm{~s}, 7.2 \mathrm{H})$.

${ }^{13}$ C-NMR: $\left(101 \mathrm{MHz}, \mathrm{CDCl}_{3}\right) \delta 167.3(2 \mathrm{xC}), 157.0(\mathrm{C}), 154.9(\mathrm{C}), 141.1(2 \mathrm{xC}), 137.1(\mathrm{C}), 130.5(2 \times C), 128.5$ (3xCH), $128.0(2 \times C H), 124.7(2 \times C), 80.7(\mathrm{C}), 73.8\left(\mathrm{CH}_{2}\right), 69.6\left(\mathrm{CH}_{2}\right), 53.0(\mathrm{CH}), 28.3\left(3 \times \mathrm{CH}_{3}\right)$.

$\mathrm{E}_{1 / 2}(\mathbf{2 f})=-0.61 \mathrm{~V}$ vs SCE.

IR (ATR): $\tilde{v}\left(\mathrm{~cm}^{-1}\right):$ 2977, 2931, 2873, 1827, 1796, 1749, 1712, 1509, 1496, 1366, 1161, $1038,731$.

HRMS: calculated for $\mathrm{C}_{23} \mathrm{H}_{21} \mathrm{Cl}_{4} \mathrm{~N}_{2} \mathrm{O}_{7}\left(\mathrm{M}+\mathrm{H}^{+}\right)$579.0068, found 579.0067.

Rf: 0.35 (8:2 pentane:EtOAc).

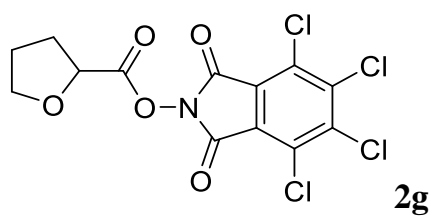

Obtained following general general procedure A from $6.5 \mathrm{mmol}$ of 2-tetrahydrofurancarboxylic acid. Column chromatography (8:2 to 7:3 pentane:EtOAc) afforded the product in $27 \%$ yield $(688 \mathrm{mg})$ as a white solid (mp: $\left.138.4^{\circ} \mathrm{C}\right)$.

${ }^{1}$ H-NMR: (500 MHz, Chloroform- $\left.d\right) \delta 4.90(\mathrm{dd}, J=8.6,4.8 \mathrm{~Hz}, 1 \mathrm{H}), 4.11(\mathrm{dd}, J=14.8,7.3 \mathrm{~Hz}, 1 \mathrm{H}), 4.08-$ $3.99(\mathrm{~m}, 1 \mathrm{H}), 2.52-2.43(\mathrm{~m}, 1 \mathrm{H}), 2.44-2.33(\mathrm{~m}, 1 \mathrm{H}), 2.17-2.08(\mathrm{~m}, 1 \mathrm{H}), 2.09-2.00(\mathrm{~m}, 1 \mathrm{H})$.

${ }^{13}$ C-NMR: (126 MHz, $\left.\mathrm{CDCl}_{3}\right) \delta 169.4(2 \times C), 157.4(\mathrm{C}), 141.1$ (2xC), 130.5 (2xC), $124.6(2 \times C), 74.9(\mathrm{CH})$, $70.0\left(\mathrm{CH}_{2}\right), 30.9\left(\mathrm{CH}_{2}\right), 25.1\left(\mathrm{CH}_{2}\right)$.

$\mathrm{E}_{1 / 2}(\mathbf{2 g})=-0.61 \mathrm{~V}$ vs SCE

IR: (ATR): $\tilde{v}\left(\mathrm{~cm}^{-1}\right):$ 2984, 2884, 1821, 1795, 1747, 1449, 1378, 1367, 1301, 1198, 1059, 842, $794,732$.

HRMS: calculated for $\mathrm{C}_{13} \mathrm{H}_{8} \mathrm{Cl}_{4} \mathrm{NO}_{5}\left[\mathrm{M}+\mathrm{H}^{+}\right]$399.9122, found 399.9120 .

Rf: 0.43 (8:2 pentane:EtOAc). 


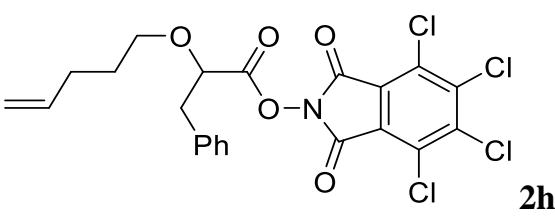

Obtained following general procedure A from $0.81 \mathrm{mmol}$ of acid S1h. Column chromatography (19:1 pentane:EtOAc) afforded the product in $56 \%$ yield $(233 \mathrm{mg})$ as a white solid $\left(\mathbf{m p}: 74.2{ }^{\circ} \mathrm{C}\right)$.

${ }^{1}$ H-NMR: (500 MHz, Chloroform- $d$ ) $\delta 7.39-7.30(\mathrm{~m}, 5 \mathrm{H}), 5.83-5.67(\mathrm{~m}, 1 \mathrm{H}), 4.96(\mathrm{t}, J=1.4 \mathrm{~Hz}, 1 \mathrm{H}), 4.94$ $-4.91(\mathrm{~m}, 1 \mathrm{H}), 4.39(\mathrm{dd}, J=8.4,4.7 \mathrm{~Hz}, 1 \mathrm{H}), 3.76(\mathrm{dt}, J=9.1,6.1 \mathrm{~Hz}, 1 \mathrm{H}), 3.42(\mathrm{ddd}, J=9.0,6.9,5.8 \mathrm{~Hz}$, $1 \mathrm{H}), 3.27$ (dd, $J=14.1,4.7 \mathrm{~Hz}, 1 \mathrm{H}), 3.21(\mathrm{dd}, J=14.1,8.4 \mathrm{~Hz}, 1 \mathrm{H}), 2.07$ (dddt, $J=8.8,7.3,3.5,1.8 \mathrm{~Hz}, 2 \mathrm{H})$, $1.79-1.60(\mathrm{~m}, 2 \mathrm{H})$.

${ }^{13}$ C-NMR: (126 MHz, $\left.\mathrm{CDCl}_{3}\right) \delta 168.4(2 \mathrm{xC}), 157.3(\mathrm{C}), 141.2(2 \mathrm{xC}), 137.9(\mathrm{CH}), 135.9(\mathrm{C}), 130.5(2 \times \mathrm{C}), 129.6$ $(2 \times C H), 128.5(2 x C H), 127.1(\mathrm{CH}), 124.7(2 \times C), 115.0\left(\mathrm{CH}_{2}\right), 78.8(\mathrm{CH}), 70.7\left(\mathrm{CH}_{2}\right), 39.5\left(\mathrm{CH}_{2}\right), 29.9\left(\mathrm{CH}_{2}\right)$, $28.6\left(\mathrm{CH}_{2}\right)$.

$\mathrm{E}_{1 / 2}(\mathbf{2 h})=-0.62 \mathrm{~V}$ vs SCE.

IR (ATR): $\tilde{v}\left(\mathrm{~cm}^{-1}\right): 3031,2933,1818,1793,1748,1640,1496,1377,1365,1300,1152,1065,1035,918,793$, $731,699$.

HRMS: calculated for $\mathrm{C}_{20} \mathrm{H}_{15} \mathrm{Cl}_{4} \mathrm{NO}_{5}\left[\mathrm{M}-\mathrm{C}_{2} \mathrm{H}_{2}+\mathrm{H}^{+}\right]$488.9699, found 488.9704 .

Rf: 0.67 (9:1 pentane:EtOAc).

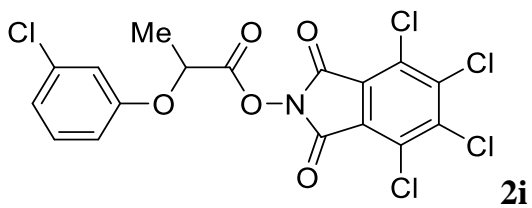

Obtained following general procedure A from $3.35 \mathrm{mmol}$ of 2-(3-chlorophenoxy)propanoic acid. Column chromatography (8:2 to $1: 1$ pentane:EtOAc) afforded the product in $61 \%$ yield $(974 \mathrm{mg})$ as a white solid (mp: $\left.155.3^{\circ} \mathrm{C}\right)$.

${ }^{1}$ H-NMR: (500 MHz, Chloroform- $\left.d\right) \delta 7.31(\mathrm{~d}, J=8.2 \mathrm{~Hz}, 1 \mathrm{H}), 7.06(\mathrm{dd}, J=8.2,2.2 \mathrm{~Hz}, 1 \mathrm{H}), 7.04(\mathrm{t}, J=2.2$ $\mathrm{Hz}, 1 \mathrm{H}), 6.88(\mathrm{dd}, J=8.2,2.2 \mathrm{~Hz}, 1 \mathrm{H}), 5.12(\mathrm{q}, J=6.9 \mathrm{~Hz}, 1 \mathrm{H}), 1.89(\mathrm{~d}, J=6.9 \mathrm{~Hz}, 3 \mathrm{H})$.

${ }^{13}$ C-NMR: (126 MHz, $\left.\mathrm{CDCl}_{3}\right) \delta 167.9(2 \times C), 157.5(\mathrm{C}), 157.2(\mathrm{C}), 141.3(2 \times C), 135.2(\mathrm{C}), 130.6$ (C), 130.6 (2xC), $124.6(\mathrm{CH}), 122.8(2 \times \mathrm{C}), 116.4(\mathrm{CH}), 112.9(\mathrm{CH}), 71.0(\mathrm{CH}), 18.8(\mathrm{CH})$.

$\mathrm{E}_{1 / 2}(\mathbf{2 i})=-0.57 \mathrm{~V}$ vs SCE.

IR (ATR): $\tilde{v}\left(\mathrm{~cm}^{-1}\right):$ 2928, 1830, 1796, 1749, 1592, 1476, 1388, 1197, 1071, 1040, 910, 860, $793,732$.

HRMS: calculated for $\mathrm{C}_{17} \mathrm{H}_{6} \mathrm{Cl}_{5} \mathrm{NNaO}_{5}\left(\mathrm{M}+\mathrm{Na}^{+}-2 \mathrm{H}^{+}\right)$501.8586, found 501.8590.

Rf: 0.49 (8:2 pentane:EtOAc). 
<smiles>CC(C)(Oc1ccc(Cl)cc1)C(=O)ON1C(=O)c2c(Cl)c(Cl)c(Cl)c(Cl)c2C1=O</smiles>

Obtained following general procedure A from $3.35 \mathrm{mmol}$ of 2-(4-chlorophenoxy)-2-methylpropanoic acid. Column chromatography (9:1 to 8:2 pentane:EtOAc) afforded the product in $50 \%$ yield $(833 \mathrm{mg})$ as a white solid (mp: $\left.163.8^{\circ} \mathrm{C}\right)$.

${ }^{1}$ H-NMR: (500 MHz, Chloroform- $d$ ) $\delta 7.33$ (d, $\left.J=8.8 \mathrm{~Hz}, 2 \mathrm{H}\right), 7.04(\mathrm{~d}, J=8.8 \mathrm{~Hz}, 2 \mathrm{H}), 1.79(\mathrm{~s}, 6 \mathrm{H})$.

${ }^{13}$ C-NMR: $\left(126 \mathrm{MHz}, \mathrm{CDCl}_{3}\right) \delta 170.3(2 \mathrm{xC}), 157.4(\mathrm{C}), 153.0(\mathrm{C}), 141.3(2 \mathrm{xC}), 130.6(2 \mathrm{xC}), 129.5(2 \mathrm{xCH})$, $128.6(2 \times \mathrm{C}), 124.6(\mathrm{C}), 121.5(2 \times \mathrm{CH}), 79.0(\mathrm{C}), 25.6\left(2 \mathrm{xCH}_{3}\right)$.

$\mathrm{E}_{1 / 2}(\mathbf{2 j})=-0.54 \mathrm{~V}$ vs SCE .

IR (ATR): $\tilde{v}\left(\mathrm{~cm}^{-1}\right):$ 3098, 2993, 2939, 1815, 1790, 1746, 1487, 1976, 1197, 1052, 907, 793, 727.

HRMS: calculated for $\mathrm{C}_{18} \mathrm{H}_{10} \mathrm{Cl}_{5} \mathrm{NaNO}_{5}\left(\mathrm{M}+\mathrm{Na}^{+}\right)$519.8864, found 519.8863 .

Rf: 0.68 (8:2 pentane:EtOAc).

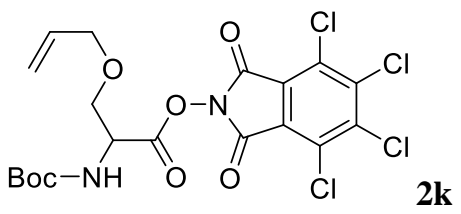

Obtained following general procedure $\mathbf{C}$ and general procedure $\mathbf{A}$, from $2.07 \mathrm{mmol}$ of $\mathrm{N}$-Boc-serine and 2.59 mmol of allylbromide. Column chromatography (9:1 pentane:EtOAc) afforded the product in $43 \%$ yield over two steps $(469 \mathrm{mg})$ as a white solid ( $\left.\mathbf{m p}: 133.7^{\circ} \mathrm{C}\right)$. Rotameric structures are observed.

${ }^{1}$ H-NMR: (500 MHz, Chloroform- $d$ ) 5.97 (ddt, $\left.J=16.4,10.9,5.7 \mathrm{~Hz}, 1 \mathrm{H}\right), 5.46(\mathrm{~d}, J=9.0 \mathrm{~Hz}, 1 \mathrm{H}), 5.34$ (dq, $J=17.3,1.6 \mathrm{~Hz}, 1 \mathrm{H}), 5.27(\mathrm{dq}, J=10.5,1.4 \mathrm{~Hz}, 1 \mathrm{H}), 4.91(\mathrm{~d}, J=9.0 \mathrm{~Hz}, 1 \mathrm{H}), 4.13(\mathrm{dq}, J=6.0,1.3 \mathrm{~Hz}, 2 \mathrm{H})$, $4.02(\mathrm{dd}, J=9.8,3.2 \mathrm{~Hz}, 1 \mathrm{H}), 3.84(\mathrm{dd}, J=9.7,3.5 \mathrm{~Hz}, 1 \mathrm{H}), 1.49$ (s, 9H).

${ }^{13}$ C-NMR: (126 MHz, $\left.\mathrm{CDCl}_{3}\right) \delta$ 167.3/167.2 (2xC), 157.0/156.6 (C), 154.9/154.2 (C), 141.3/141.1 (2xC), 133.9/133.7 (CH), 130.7/130.5 (2xC), 124.6/124.6 (2xC), 118.3/118.1 $\left(\mathrm{CH}_{2}\right)$, 81.7/80.8 (C), 72.7/72.4 $\left(\mathrm{CH}_{2}\right)$, 69.4/68.5 $\left(\mathrm{CH}_{2}\right), 54.0 / 52.7(\mathrm{CH}), 28.3 / 28.1\left(3 \mathrm{xCH}_{3}\right)$.

$\mathrm{E}_{1 / 2}(\mathbf{2 k})=-0.63 \mathrm{~V}$ vs SCE.

IR (ATR): $\tilde{v}\left(\mathrm{~cm}^{-1}\right):$ 2979, 2932, 2872, 1829, 1797, 1751, 1717, 1502, 1368, 1251, 1197, 1161, 1039, 929, 731.

HRMS: calculated for $\mathrm{C}_{19} \mathrm{H}_{18} \mathrm{Cl}_{4} \mathrm{~N}_{2} \mathrm{NaO}_{7}\left(\mathrm{M}^{+}+\mathrm{Na}\right), 550.9736$; found 550.9730 .

Rf: 0.35 (4:1 Pentane: EtOAc). 
<smiles>CC=CCOCC(NC(=O)OC(C)(C)C)C(=O)ON1C(=O)c2c(Cl)c(Cl)c(Cl)c(Cl)c2C1=O</smiles>

Obtained following general procedure $\mathbf{C}$ and general procedure $\mathbf{A}$, from $2.67 \mathrm{mmol}$ of $\mathrm{N}$-Boc-serine and 3.33 mmol of crotyl bromide. Column chromatography (9:1 pentane:EtOAc) afforded the product in $27 \%$ yield over two steps (388 $\mathrm{mg})$ as a white-off solid ( $\left.\mathbf{m p}: 123.3^{\circ} \mathrm{C}\right)$.

${ }^{1}$ H-NMR: (400 MHz, Chloroform- $d$ ) Mixture of rotamers and diastereoisomers $\delta 5.78$ (ddt, $J=14.5,7.7,5.7$ $\mathrm{Hz}, 1 \mathrm{H}), 5.68-5.56(\mathrm{~m}, 1 \mathrm{H}), 5.46(\mathrm{~d}, J=9.2 \mathrm{~Hz}, 0.7 \mathrm{H}), 5.25-5.17(\mathrm{~m}, 0.2 \mathrm{H}), 4.93-4.83(\mathrm{~m}, 0.7 \mathrm{H}), 4.60(\mathrm{~d}$, $J=10.5 \mathrm{~Hz}, 0.2 \mathrm{H}), 4.24-3.89(\mathrm{~m}, 3 \mathrm{H}), 3.81(\mathrm{dd}, J=9.9,3.5 \mathrm{~Hz}, 1 \mathrm{H}), 1.75(\mathrm{dd}, J=6.5,1.5 \mathrm{~Hz}, 2.1 \mathrm{H}), 1.71$ (dd, $J=7.0,1.8 \mathrm{~Hz}, 0.9 \mathrm{H}), 1.52(\mathrm{~s}, 2.7 \mathrm{H}), 1.48(\mathrm{~s}, 6.3 \mathrm{H})$.

${ }^{13}$ C-NMR: (101 MHz, $\left.\mathrm{CDCl}_{3}\right) \delta 167.3(2 \mathrm{xC}), 156.9(\mathrm{C}), 155.0(\mathrm{C}), 141.0(2 \mathrm{xC}), 130.5(2 \mathrm{xC}), 130.5(2 \mathrm{xC})$, $126.8(\mathrm{CH}), 124.7(\mathrm{CH}), 80.7(\mathrm{C}), 72.4\left(\mathrm{CH}_{2}\right), 69.0\left(\mathrm{CH}_{2}\right), 52.7(\mathrm{CH}), 29.7 / 28.3\left(3 \mathrm{xCH}_{3}\right), 17.8 / 13.2\left(\mathrm{CH}_{3}\right)$.

$\mathrm{E}_{1 / 2}(\mathbf{2 l})=-0.65 \mathrm{~V}$ vs SCE.

IR (ATR): $\tilde{v}\left(\mathrm{~cm}^{-1}\right): 3320,2978,2934,1828,1797,1751,1719,1510,1367,1197,1164,1037,866,792,732$.

HRMS: calculated for $\mathrm{C}_{20} \mathrm{H}_{21} \mathrm{Cl}_{4} \mathrm{~N}_{2} \mathrm{O}_{7}[\mathrm{M}+\mathrm{H}]^{+}$, 543.0078; found, 543.0067.

Rf: 0.46 (8:2 pentane:EtOAc).<smiles>CC(C)=CCOCC(NC(=O)OCc1ccccc1)C(=O)ON1C(=O)c2c(Cl)c(Cl)c(Cl)c(Cl)c2C1=O</smiles>

Obtained following general procedure $\mathbf{C}$ and general procedure $\mathbf{A}$, from $0.98 \mathrm{mmol}$ of $\mathrm{N}$-Boc-serine and 1.23 mmol of prenyl bromide. Column chromatography (9:1 pentane:EtOAc) afforded the product in $18 \%$ yield over two steps $(98 \mathrm{mg})$ as a white foam ( $\left.\mathbf{m p}: 110.1^{\circ} \mathrm{C}\right)$.

${ }^{1}$ H-NMR: (500 MHz, Chloroform- $d$ ) $\delta 5.47(\mathrm{~d}, J=9.2 \mathrm{~Hz}, 0.8 \mathrm{H}), 5.39(\mathrm{t}, J=5.7 \mathrm{~Hz}, 1 \mathrm{H}), 5.17$ (br s, 0.2H), $4.87(\mathrm{~d}, J=8.7 \mathrm{~Hz}, 0.8 \mathrm{H}), 4.62(\mathrm{br} \mathrm{s}, 0.2 \mathrm{H}), 4.12(\mathrm{t}, J=7.5 \mathrm{~Hz}, 2 \mathrm{H}), 3.98(\mathrm{~d}, J=7.1 \mathrm{~Hz}, 1 \mathrm{H}), 3.83(\mathrm{~d}, J=9.6$ $\mathrm{Hz}, 1 \mathrm{H}), 1.79$ (s, 3H), $1.71(\mathrm{~s}, 3 \mathrm{H}), 1.52(\mathrm{~s}, 2 \mathrm{H}), 1.49(\mathrm{~s}, 7 \mathrm{H})$.

${ }^{13}$ C-NMR: $\left(126 \mathrm{MHz}, \mathrm{CDCl}_{3}\right) \delta 167.4(2 \mathrm{xC})$ 157.0/(C), $155.0(\mathrm{C}), 141.0(2 \mathrm{xC}), 138.0(\mathrm{C}), 130.5(2 \mathrm{xC}), 124.7$ $(2 \times C), 120.3(\mathrm{CH}), 80.7(\mathrm{C}), 69.0\left(\mathrm{CH}_{2}\right), 68.0\left(\mathrm{CH}_{2}\right), 54.0 / 52.7(\mathrm{CH}), 28.3 / 28.2\left(3 \mathrm{xCH}_{3}\right), 25.8\left(\mathrm{CH}_{3}\right), 18.1$ $\left(\mathrm{CH}_{3}\right)$.

$\mathrm{E}_{1 / 2}(\mathbf{2 m})=-0.54 \mathrm{~V}$ vs SCE.

IR (ATR): $\tilde{v}\left(\mathrm{~cm}^{-1}\right): 3302,2977,2931,1828,1797,1750,1735,1505,1452,1367,1197,1161,1038,911,859$, 731.

HRMS: calculated for $\mathrm{C}_{21} \mathrm{H}_{23} \mathrm{Cl}_{4} \mathrm{~N}_{2} \mathrm{O}_{7}\left[\mathrm{M}+\mathrm{H}^{+}\right]$, 557.0224; found, 557.0224

Rf: 0.42 (4:1 pentane:acetone). 
<smiles>C=CCOC([C@H](NC(=O)OC)C(=O)O)[C@@H](O)C(=O)ON1C(=O)c2c(Cl)c(Cl)c(Cl)c(Cl)c2C1=O</smiles>

Obtained following general procedure $\mathbf{C}$ and general procedure $\mathbf{A}$, from $1.93 \mathrm{mmol}$ of $\mathrm{N}$-Boc-methionine and $2.41 \mathrm{mmol}$ of allyl bromide. Column chromatography (9:1 to 8:2 pentane:EtOAc) afforded the product in $46 \%$ yield over two steps $(479 \mathrm{mg})$ as a white solid ( $\left.\mathbf{m p}: 114.7^{\circ} \mathrm{C}\right)$.

${ }^{1}$ H-NMR: (400 MHz, Chloroform- $d$ ) $\delta 5.98(\mathrm{ddt}, J=16.5,9.9,5.9 \mathrm{~Hz}, 1 \mathrm{H}), 5.37(\mathrm{~d}, J=9.7 \mathrm{~Hz}, 0.8 \mathrm{H}), 5.32$ $(\mathrm{dq}, J=17.2,1.4 \mathrm{~Hz}, 1 \mathrm{H}), 5.21(\mathrm{dq}, J=10.2,1.4 \mathrm{~Hz}, 1 \mathrm{H}), 5.13(\mathrm{~d}, J=8.8 \mathrm{~Hz}, 0.2 \mathrm{H}), 4.74(\mathrm{dd}, J=9.7,2.3 \mathrm{~Hz}$, $0.8 \mathrm{H}), 4.43(\mathrm{~d}, J=8.8 \mathrm{~Hz}, 0.2 \mathrm{H}), 4.30-4.18(\mathrm{~m}, 1 \mathrm{H}), 4.19-4.03(\mathrm{~m}, 2 \mathrm{H}), 1.52(\mathrm{~s}, 1.8 \mathrm{H}), 1.48(\mathrm{~s}, 7.2 \mathrm{H}), 1.37$ $(\mathrm{d}, J=6.8 \mathrm{~Hz}, 0.6 \mathrm{H}), 1.32(\mathrm{~d}, J=6.2 \mathrm{~Hz}, 2.4 \mathrm{H})$.

${ }^{13}$ C-NMR: (101 MHz, $\left.\mathrm{CDCl}_{3}\right) \delta$ 167.7/167.5 (2xC), 157.1/157.0 (C), $155.5(\mathrm{C}), 141.0(2 \mathrm{xC}), 134.5 / 134.3(\mathrm{CH})$, $130.5(2 \mathrm{xC}), 124.7(2 \mathrm{xC}), 117.6\left(\mathrm{CH}_{2}\right), 81.5 / 80.5(\mathrm{C}), 74.4 / 73.6(\mathrm{CH}), 70.7\left(\mathrm{CH}_{2}\right), 58.5 / 57.0(\mathrm{CH}), 28.3 / 28.1$ $\left(3 \mathrm{xCH}_{3}\right), 17.2 / 16.5\left(\mathrm{CH}_{3}\right)$.

$\mathrm{E}_{1 / 2}(\mathbf{2 n})=-0.59 \mathrm{~V}$ vs SCE.

IR (ATR): $\tilde{v}\left(\mathrm{~cm}^{-1}\right): 3443,2980,1825,1796,1748,1716,1499,1366,1300,1161,1054,907,729$.

HRMS: calculated for $\mathrm{C}_{20} \mathrm{H}_{21} \mathrm{Cl}_{4} \mathrm{~N}_{2} \mathrm{O}_{7}\left(\mathrm{M}+\mathrm{H}^{+}\right)$543.0068, found 543.0066.

Rf: 0.51 (4:1 pentane:EtOAc).<smiles>C=C(C)COCC(NC(=O)OCc1ccccc1)C(=O)ON1C(=O)c2c(Cl)c(Cl)c(Cl)c(Cl)c2C1=O</smiles>

Obtained following general procedure $\mathbf{C}$ and general procedure $\mathbf{A}$, from $1.46 \mathrm{mmol}$ of $\mathrm{N}$-Boc-serine and 1.82 mmol of methallyl bromide. Column chromatography (9:1 pentane:EtOAc) afforded the product in $48 \%$ yield over two steps $(378 \mathrm{mg})$ as a white solid ( $\left.\mathbf{m p}: 120.8^{\circ} \mathrm{C}\right)$.

${ }^{1}$ H-NMR: (400 MHz, Chloroform- $d$ ) $\delta 5.47(\mathrm{~d}, J=9.1 \mathrm{~Hz}, 0.75 \mathrm{H}), 5.20(\mathrm{br} \mathrm{s}, 0.25 \mathrm{H}), 5.01(\mathrm{~s}, 1 \mathrm{H}), 4.96(\mathrm{~s}$, $1 \mathrm{H}), 4.96-4.85$ (m, 0.75H), 4.64 (br s, $0.25 \mathrm{H}), 4.07-4.03(\mathrm{~m}, 2 \mathrm{H}), 4.01-3.95(\mathrm{~m}, 1 \mathrm{H}), 3.81$ (dd, $J=9.8,3.6$ $\mathrm{Hz}, 1 \mathrm{H}), 1.78(\mathrm{~s}, 3 \mathrm{H}), 1.53(\mathrm{~s}, 2.25 \mathrm{H}), 1.49$ (s, 6.75H).

${ }^{13}$ C-NMR: (126 MHz, $\left.\mathrm{CDCl}_{3}\right) \delta 167.3(2 \mathrm{xC}), 156.9$ (C), 154.5 (C), $141.4(\mathrm{C}), 141.1$ (2xC), 130.5 (2xC), 124.6 $(2 \times C), 113.4 / 113.3\left(\mathrm{CH}_{2}\right)$, 81.7/80.7 (C), $75.8\left(\mathrm{CH}_{2}\right), 69.3 / 68.5\left(\mathrm{CH}_{2}\right), 53.9 / 52.6(\mathrm{CH}), 28.3 / 28.1\left(3 \mathrm{xCH}_{3}\right), 19.4$ $\left(\mathrm{CH}_{3}\right)$.

$\mathrm{E}_{1 / 2}(\mathbf{2 o})=-0.64 \mathrm{~V}$ vs SCE.

IR (ATR): $\tilde{v}\left(\mathrm{~cm}^{-1}\right): 3439,2978,1932,1827,1797,1751,1721,1502,1452,1368,1300,1252,1163,1112$, $1038,906,868,792,732$.

HRMS: calculated for $\mathrm{C}_{20} \mathrm{H}_{21} \mathrm{Cl}_{4} \mathrm{~N}_{2} \mathrm{O}_{7}\left(\mathrm{M}+\mathrm{H}^{+}\right)$543.0068, found 543.0068.

Rf: 0.56 (8:2 pentane:EtOAc). 
<smiles>C=CCCN(Cc1ccccc1)C(CC(C)C)C(=O)ON1C(=O)c2c(Cl)c(Cl)c(Cl)c(Cl)c2C1=O</smiles>

Obtained following general procedure A from $1.0 \mathrm{mmol}$ of acid S1p. Column chromatography (9:1 pentane:EtOAc) afforded the product in $62 \%$ yield $(374 \mathrm{mg})$ as a colourless wax.

${ }^{1}$ H-NMR: (500 MHz, Chloroform- $d$ ) $\delta 7.40-7.28(\mathrm{~m}, 5 \mathrm{H}), 5.90-5.65(\mathrm{~m}, 1 \mathrm{H}), 5.42-5.14(\mathrm{~m}, 3 \mathrm{H}), 5.06(\mathrm{~d}$, $J=12.1 \mathrm{~Hz}, 1.6 \mathrm{H}), 4.83(\mathrm{~s}, 0.4 \mathrm{H}), 3.58(\mathrm{br} \mathrm{d}, J=49.1 \mathrm{~Hz}, 1 \mathrm{H}), 3.20(\mathrm{br} \mathrm{s}, 1 \mathrm{H}), 2.54-2.31(\mathrm{~m}, 2 \mathrm{H}), 1.88(\mathrm{~s}$, 2H), $1.72(\mathrm{~s}, 1 \mathrm{H}), 0.99(\mathrm{~d}, J=43.7 \mathrm{~Hz}, 6 \mathrm{H})$.

${ }^{13}$ C-NMR: $\left(126 \mathrm{MHz}, \mathrm{CDCl}_{3}\right) \delta 168.1(2 \mathrm{xC}), 157.2(\mathrm{C}), 156.4 / 155.4(\mathrm{C}), 141.1$ (2xC), $136.2(\mathrm{C}), 134.8(\mathrm{C})$, $130.5(2 \times C), 128.5(2 \times C H), 128.2 / 128.1(2 \times C H), 127.9(\mathrm{CH}), 124.6(2 \times C), 117.0\left(\mathrm{CH}_{2}\right), 67.9\left(\mathrm{CH}_{2}\right), 56.4 / 55.9$ $(\mathrm{CH}), 46.9 / 45.3\left(\mathrm{CH}_{2}\right), 39.0 / 38.4\left(\mathrm{CH}_{2}\right), 34.1 / 33.1\left(\mathrm{CH}_{2}\right), 24.7 / 24.6(\mathrm{CH}), 22.9\left(\mathrm{CH}_{3}\right), 21.9 / 21.7\left(\mathrm{CH}_{3}\right)$.

$\mathrm{E}_{1 / 2}(\mathbf{2} \mathbf{p})=-0.61 \mathrm{~V}$ vs SCE.

IR (ATR): $\tilde{v}\left(\mathrm{~cm}^{-1}\right): 3069,2959,2872,1819,1794,1749,1705,1468,1414,1377,1368,1299,1224,1156$, 961, 916, 877, 794, 731, 698.

HRMS: calculated for $\mathrm{C}_{22} \mathrm{H}_{25} \mathrm{Cl}_{4} \mathrm{~N}_{2} \mathrm{O}_{6}\left[\mathrm{M}+\mathrm{H}^{+}\right]$603.0432, found 603.0429.

Rf: 0.48 (9:1 pentane:EtOAc).<smiles>C=CCCN(Cc1ccccc1)C(Cc1ccccc1)C(=O)ON1C(=O)c2c(Cl)c(Cl)c(Cl)c(Cl)c2C1=O</smiles>

Obtained following general procedure A from $1.1 \mathrm{mmol}$ of acid S1q. Column chromatography (95:5 to 9:1 pentane:EtOAc) afforded the product in $53 \%$ yield $(370 \mathrm{mg})$ as a white solid $\left(\mathbf{m p}: 56.1^{\circ} \mathrm{C}\right)$.

${ }^{1}$ H-NMR: (500 MHz, Chloroform- $d$ ) 1:1 mixture of rotamers $\delta 7.46(\mathrm{~d}, J=7.5 \mathrm{~Hz}, 1 \mathrm{H}), 7.39(\mathrm{~d}, J=4.4 \mathrm{~Hz}$, 3H), $7.39-7.28(\mathrm{~m}, 2 \mathrm{H}), 7.27$ (s, 2H), $7.20(\mathrm{~d}, J=7.2 \mathrm{~Hz}, 1 \mathrm{H}), 7.05(\mathrm{~d}, J=6.9 \mathrm{~Hz}, 1 \mathrm{H}), 5.75-5.57(\mathrm{~m}, 1 \mathrm{H})$, $5.50(\mathrm{~d}, J=12.1 \mathrm{~Hz}, 0.5 \mathrm{H}), 5.30(\mathrm{~d}, J=12.4 \mathrm{~Hz}, 0.5 \mathrm{H}), 5.23(\mathrm{~d}, J=12.3 \mathrm{~Hz}, 0.5 \mathrm{H}), 5.15(\mathrm{~d}, J=12.1 \mathrm{~Hz}, 0.5 \mathrm{H})$, $5.06-4.92(\mathrm{~m}, 2 \mathrm{H}), 4.76$ (t, $J=7.5 \mathrm{~Hz}, 0.5 \mathrm{H}), 4.56$ (br s, $0.5 \mathrm{H}), 3.50-3.22(\mathrm{~m}, 3 \mathrm{H}), 2.84$ (ddd, $J=15.1,9.4$, $6.3 \mathrm{~Hz}, 0.5 \mathrm{H}), 2.74(\mathrm{dq}, J=14.6,6.9 \mathrm{~Hz}, 0.5 \mathrm{H}), 2.24-2.09(\mathrm{~m}, 2 \mathrm{H})$.

${ }^{13}$ C-NMR: $\left(126 \mathrm{MHz}, \mathrm{CDCl}_{3}\right)$ mixture of rotamers $\delta$ 167.1/166.9 (2xC), 157.2 (C), 155.7/154.9 (C), 141.2/141.0 (2xC), 136.5 (C), 136.3 (C), 136.2/136.1 (C), 134.8/134.7 (=CH), 130.6/130.5 (2xC), 129.2 $(2 \mathrm{XCH}), 128.8 / 128.8(2 \mathrm{xCH}), 128.7 / 128.0(2 \mathrm{xCH}), 128.6 / 128.5(2 \mathrm{xCH}), 128.3 / 128.1(\mathrm{CH}), 127.2 / 127.1(\mathrm{CH})$, 124.7/124.6 (2xC), $116.9\left(=\mathrm{CH}_{2}\right), 68.1 / 67.8\left(\mathrm{CH}_{2}\right), 60.9 / 60.8(\mathrm{CH}), 49.3 / 48.2\left(\mathrm{CH}_{2}\right), 36.3 / 35.3\left(\mathrm{CH}_{2}\right), 33.2 / 32.4$ $\left(\mathrm{CH}_{2}\right)$.

$\mathrm{E}_{1 / 2}(\mathbf{2 q})=-0.61 \mathrm{~V}$ vs SCE.

IR (ATR): $\tilde{v}\left(\mathrm{~cm}^{-1}\right)$ 3066, 3031, 2943, 1822, 1739, 1746, 1702, 1469, 1455, 1376, 1365, 1299, 1036, 767, 729, 698.

HRMS: calculated for $\mathrm{C}_{29} \mathrm{H}_{23} \mathrm{Cl}_{4} \mathrm{~N}_{2} \mathrm{O}_{6}\left(\mathrm{M}+\mathrm{H}^{+}\right)$637.0275, found 637.0267.

Rf: 0.52 (8:2 pentane:EtOAc). 
<smiles>C=CCCN(CCCCC)CC(=O)ON1C(=O)c2c(Cl)c(Cl)c(Cl)c(Cl)c2C1=O</smiles>

Obtained following general procedure A from $2.89 \mathrm{mmol}$ of acid S1r. Column chromatography (8:2 pentane:EtOAc) afforded the product in $59 \%$ yield $(952 \mathrm{mg})$ as a white solid $\left(\mathbf{m p}: 144.8^{\circ} \mathrm{C}\right)$.

${ }^{1}$ H-NMR: (400 MHz, Chloroform- $d$ ) $\delta 7.52-7.30(\mathrm{~m}, 5 \mathrm{H}), 5.95-5.67(\mathrm{~m}, 1 \mathrm{H}), 5.21(\mathrm{~s}, 2 \mathrm{H}), 5.12-4.87(\mathrm{~m}$, 2H), $4.43(\mathrm{~d}, J=32.2 \mathrm{~Hz}, 2 \mathrm{H}), 3.43(\mathrm{dt}, J=11.2,7.5 \mathrm{~Hz}, 2 \mathrm{H}), 2.25-1.95(\mathrm{~m}, 2 \mathrm{H}), 1.71(\mathrm{~h}, J=7.3 \mathrm{~Hz}, 2 \mathrm{H})$.

${ }^{13}$ C-NMR: (101 MHz, $\left.\mathrm{CDCl}_{3}\right) \delta$ 166.1/166.0 (2xC), 157.1/157.0 (C), 156.3/155.3 (C), 141.3/141.2 (2xC), 141.1/141.1 $\left(\mathrm{CH}_{2}\right), 137.5 / 137.4(\mathrm{C}), 136.2 / 136.1(2 \mathrm{xC}), 130.6(2 \mathrm{xCH}), 128.5 / 128.2(2 \mathrm{xCH}), 128.1 / 127.9(\mathrm{CH})$, 124.6 (2xC), 115.4/115.3 (CH), 68.0/67.9 $\left(\mathrm{CH}_{2}\right), 48.5 / 47.8\left(\mathrm{CH}_{2}\right), 47.0 / 46.8\left(\mathrm{CH}_{2}\right), 30.7 / 30.6\left(\mathrm{CH}_{2}\right), 27.4 / 27.1$ $\left(\mathrm{CH}_{2}\right)$.

$\mathrm{E}_{1 / 2}(\mathbf{2 r})=-0.63 \mathrm{~V}$ vs SCE.

IR (ATR): $\tilde{v}\left(\mathrm{~cm}^{-1}\right):$ 2939, 1833, 1797, 1750, 1718, 1401, 1378, 1322, 1199, 1076, 915, 879, 793, 732.

HRMS: calculated for $\mathrm{C}_{23} \mathrm{H}_{19} \mathrm{Cl}_{4} \mathrm{~N}_{2} \mathrm{O}_{6}\left(\mathrm{M}+\mathrm{H}^{+}\right)$560.9962, found 560.9960 .

Rf: 0.29 (9:1 pentane:EtOAc).<smiles>O=C(ON1C(=O)c2c(Cl)c(Cl)c(Cl)c(Cl)c2C1=O)c1ccccc1</smiles>

Obtained following general procedure A from $1.17 \mathrm{mmol}$ of diphenylacetic acid. Column chromatography (8:2 to 0:1 pentane:acetone) afforded the product in $42 \%$ yield $(241 \mathrm{mg})$ as a pale yellow solid ( $\left.\mathrm{mp}: 159.8^{\circ} \mathrm{C}\right)$.

${ }^{1}$ H-NMR: (500 MHz, Chloroform- $d$ ) $\delta 7.45-7.39$ (m, 8H), $7.38-7.32(\mathrm{~m}, 2 \mathrm{H}), 5.44(\mathrm{~s}, 1 \mathrm{H})$.

${ }^{13}$ C-NMR: (126 MHz, $\left.\mathrm{CDCl}_{3}\right) \delta 168.6(2 \mathrm{xC}), 157.4(\mathrm{C}), 141.1$ (2xC), $136.4(2 \mathrm{xC}), 130.5(2 \mathrm{xC}), 129.0(4 \mathrm{xCH})$, $128.7(4 \mathrm{xCH}), 128.0(2 \times \mathrm{CH}), 124.7(2 \times \mathrm{C}), 54.0(\mathrm{CH})$.

$\mathrm{E}_{1 / 2}(2 \mathrm{~s})=-0.56 \mathrm{~V}$ vs SCE.

IR (ATR): $\tilde{v}\left(\mathrm{~cm}^{-1}\right)$ 3031, 3064, 1816, 1792, 1747, 1377, 1365, 1199, 1061, 1035, 731, 698.

HRMS: calculated for $\mathrm{C}_{22} \mathrm{H}_{12} \mathrm{Cl}_{4} \mathrm{NO}_{4}\left[\mathrm{M}+\mathrm{H}^{+}\right]$495.9485, found 495.9488 .

Rf: 0.48 (9:1 pentane:EtOAc). 


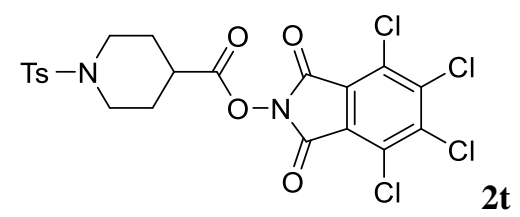

Obtained following general procedure A from $3.02 \mathrm{mmol}$ of 1-tosylpiperidine-4-carboxylic acid (S1t). Column chromatography (8:2 to 1:1 pentane:EtOAc) afforded the product in $93 \%$ yield $(1.58 \mathrm{~g})$ as a white foam (mp: $214.4^{\circ} \mathrm{C}$ ).

1H-NMR: (400 MHz, Chloroform- $d$ ) $\delta 7.67(\mathrm{~d}, J=8.4 \mathrm{~Hz}, 2 \mathrm{H}), 7.36(\mathrm{~d}, J=8.5 \mathrm{~Hz}, 2 \mathrm{H}), 3.66(\mathrm{t}, J=4.6 \mathrm{~Hz}$, $1 \mathrm{H}), 3.63(\mathrm{t}, J=4.4 \mathrm{~Hz}, 1 \mathrm{H}), 2.76(\mathrm{tt}, J=9.9,4.2 \mathrm{~Hz}, 1 \mathrm{H}), 2.74-2.62(\mathrm{~m}, 2 \mathrm{H}), 2.46(\mathrm{~s}, 3 \mathrm{H}), 2.20-2.11(\mathrm{~m}$, $2 \mathrm{H}), 2.09-1.98(\mathrm{~m}, 2 \mathrm{H})$.

${ }^{13}$ C-NMR: (101 MHz, $\left.\mathrm{CDCl}_{3}\right) \delta$ 169.7, (2xC) $157.4(\mathrm{C}), 143.8(\mathrm{C}), 141.2(2 \mathrm{xC}), 133.0(\mathrm{C}), 130.5$ (2xC), 129.8 $(2 \mathrm{xCH}), 127.7(2 \mathrm{xCH}), 124.6(2 \mathrm{xC}), 44.8\left(2 \mathrm{xCH}_{2}\right), 37.5(\mathrm{CH}), 27.2\left(2 \mathrm{xCH}_{2}\right), 21.6\left(\mathrm{CH}_{3}\right)$.

$\mathrm{E}_{1 / 2}(\mathbf{2 t})=-0.63 \mathrm{~V}$ vs SCE .

IR (ATR): $\tilde{v}\left(\mathrm{~cm}^{-1}\right)$ 2981, 1818, 1794, 1747, 1495, 1451, 1379, 1367, 1161, 1038, 815, 730.

HRMS: calculated for $\mathrm{C}_{21} \mathrm{H}_{17} \mathrm{Cl}_{4} \mathrm{~N}_{2} \mathrm{O}_{6} \mathrm{~S}\left(\mathrm{M}^{+}+\mathrm{H}\right)$ 566.9526; found 566.9517.

Rf: 0.21 (4:1 pentane:EtOAc)

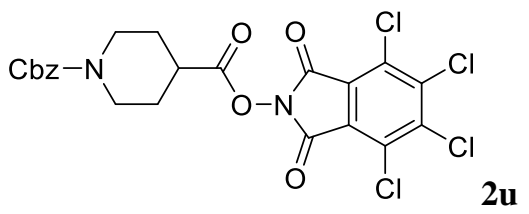

Obtained following general procedure A from $2.13 \mathrm{mmol}$ of 1-benzylosycarbonylpiperidine-4-carboxylic acid (S1u). Column chromatography (8:2 to 7:3 pentane:EtOAc) afforded the product in $47 \%$ yield (549 $\mathrm{mg})$ as a white solid (mp: $\left.164.7^{\circ} \mathrm{C}\right)$.

${ }^{1}$ H-NMR: (500 MHz, Chloroform- $\left.d\right) \delta 7.45-7.32(\mathrm{~m}, 5 \mathrm{H}), 5.16(\mathrm{~s}, 2 \mathrm{H}), 4.11(\mathrm{br} \mathrm{s}, 2 \mathrm{H}), 3.16(\mathrm{t}, J=10.1 \mathrm{~Hz}$, 2H), $3.05-2.86(\mathrm{~m}, 1 \mathrm{H}), 2.08$ (br s, $2 \mathrm{H}), 1.90(\mathrm{~d}, J=10.0 \mathrm{~Hz}, 2 \mathrm{H})$.

${ }^{13}$ C-NMR: (126 MHz, $\left.\mathrm{CDCl}_{3}\right) \delta 170.1(2 \times C), 157.5$ (C), 155.1 (C), 141.1 (C), 136.6 (C), 130.5 (C), 128.5 (2xCH), $128.1(\mathrm{CH}), 127.9(2 \times \mathrm{CH}), 124.6(\mathrm{C}), 67.3\left(\mathrm{CH}_{2}\right), 42.7\left(2 \mathrm{xCH}_{2}\right), 38.2(\mathrm{CH}), 27.5\left(2 \mathrm{xCH}_{2}\right)$.

$\mathrm{E}_{1 / 2}(\mathbf{2 u})=-0.60 \mathrm{~V}$ vs SCE.

IR (ATR): $\tilde{v}\left(\mathrm{~cm}^{-1}\right)$ 2956, 2929, 2860, 1816, 1791, 14745, 1694, 1470, 1449, 1378, 1365, 1228, $1037,729$.

HRMS: calculated for $\left(\mathrm{M}+\mathrm{H}^{+}\right)$, found.

Rf: 0.20 (8:2 pentane:EtOAc). 


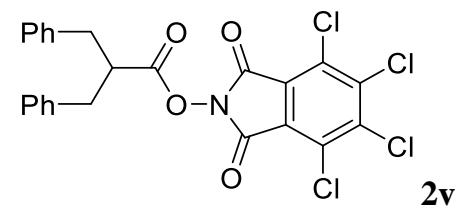

Obtained following general procedure A from $1.50 \mathrm{mmol}$ of 2-benzyl-3-phenylpropanoic acid. Column chromatography (95:5 pentane:EtOAc) afforded the product in 51\% yield (398 $\mathrm{mg}$ ) as a white solid (mp: 148.7 $\left.{ }^{\circ} \mathrm{C}\right)$.

${ }^{1}$ H-NMR: (400 MHz, Chloroform- $d$ ) $\delta 7.29-7.27(\mathrm{~m}, 4 \mathrm{H}), 7.22-7.11(\mathrm{~m}, 6 \mathrm{H}), 3.25(\mathrm{p}, J=6.7 \mathrm{~Hz}, 1 \mathrm{H}), 3.10$ (dd, $J=14.0,7.7 \mathrm{~Hz}, 2 \mathrm{H}), 2.86(\mathrm{dd}, J=14.0,6.5 \mathrm{~Hz}, 2 \mathrm{H})$.

${ }^{13}$ C-NMR: (101 MHz, $\left.\mathrm{CDCl}_{3}\right) \delta 170.8(2 \mathrm{xC}), 157.3(\mathrm{C}), 140.9$ (2xC), $137.6(2 \mathrm{xC}), 130.4(2 \mathrm{xC}), 129.0(4 \mathrm{xCH})$, $128.7(4 \mathrm{xCH}), 126.9(2 \times \mathrm{CH}), 124.7(2 \mathrm{xC}), 46.8(\mathrm{CH}), 37.4\left(2 \mathrm{xCH}_{2}\right)$.

$\mathrm{E}_{1 / 2}(\mathbf{2 v})=-0.64 \mathrm{~V}$ vs SCE.

IR (ATR): $\tilde{v}\left(\mathrm{~cm}^{-1}\right)$ 3029, 2927, 1815, 1791, 1746, 1603, 1496, 1454, 1377, 1366, 1301, 1197, 1155, 1039, 977 , $910,823,730,700$.

HRMS: calculated for $\mathrm{C}_{24} \mathrm{H}_{16} \mathrm{Cl}_{4} \mathrm{NO}_{4}\left(\mathrm{M}+\mathrm{H}^{+}\right)$523.9798, found 523.9798.

Rf: 0.33 (95:5 pentane:EtOAc).

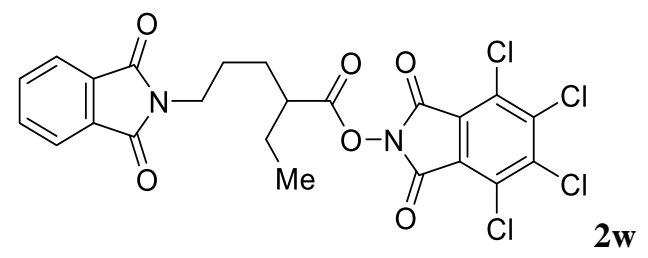

Obtained following general procedure A from $1.16 \mathrm{mmol}$ of $\mathbf{S 1 w}$. Column chromatography (8:2 to 1:1 pentane:EtOAc) afforded the product in $63 \%$ yield $(408 \mathrm{mg})$ as a white solid $\left(\mathbf{m p}: 166.3{ }^{\circ} \mathrm{C}\right)$.

${ }^{1}$ H-NMR: (400 MHz, Chloroform- $d$ ) $\delta 7.79(\mathrm{dd}, J=5.4,3.1 \mathrm{~Hz}, 2 \mathrm{H}), 7.65(\mathrm{dd}, J=5.4,3.1 \mathrm{~Hz}, 2 \mathrm{H}), 3.70(\mathrm{q}, J$ $=6.6 \mathrm{~Hz}, 2 \mathrm{H}), 2.69(\mathrm{td}, J=8.7,4.5 \mathrm{~Hz}, 1 \mathrm{H}), 1.97-1.55(\mathrm{~m}, 6 \mathrm{H}), 0.99(\mathrm{~d}, J=14.9 \mathrm{~Hz}, 3 \mathrm{H})$.

${ }^{13}$ C-NMR: $\left(101 \mathrm{MHz}, \mathrm{CDCl}_{3}\right) \delta 171.5(2 \mathrm{xC}), 168.4(2 \mathrm{xC}), 157.6(\mathrm{C}), 141.0(2 \mathrm{xC}), 133.9(2 \times \mathrm{CH}), 132.1(2 \times C)$, $130.4(2 \times C), 124.8(2 \times C), 123.3(2 \times C H), 44.2(\mathrm{CH}), 37.4\left(\mathrm{CH}_{2}\right), 29.1\left(\mathrm{CH}_{2}\right), 26.1\left(\mathrm{CH}_{2}\right), 25.6\left(\mathrm{CH}_{2}\right), 11.5$ $\left(\mathrm{CH}_{3}\right)$.

$\mathrm{E}_{1 / 2}(\mathbf{2 w})=-0.65 \mathrm{~V}$ vs SCE.

IR (ATR): $\tilde{v}\left(\mathrm{~cm}^{-1}\right)$ 2967, 2938, 1790, 1747, 1708, 1396, 1377, 1366, 1197, 1045, 720.

HRMS: calculated for $\mathrm{C}_{23} \mathrm{H}_{17} \mathrm{Cl}_{4} \mathrm{~N}_{2} \mathrm{O}_{6}\left(\mathrm{M}+\mathrm{H}^{+}\right)$558.9806, found 558.9807.

Rf: 0.27 (8:2 pentane:EtOAc). 


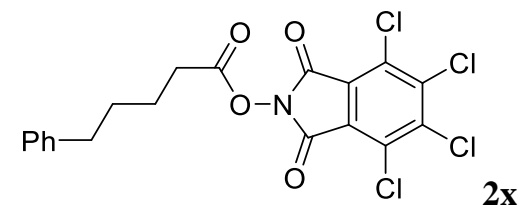

Obtained following general procedure A from 2 mmol of 5-phenylvaleric acid. Column chromatography (95:5 to $8: 2$ pentane:EtOAc) afforded the product in $51 \%$ yield $(464 \mathrm{mg})$ as a white solid $\left(\mathbf{m p}: 76.5{ }^{\circ} \mathrm{C}\right)$.

${ }^{1}$ H-NMR: (500 MHz, Chloroform- $d$ ) $\delta 7.34-7.28(\mathrm{~m}, 2 \mathrm{H}), 7.26-7.17(\mathrm{~d}, J=6.6 \mathrm{~Hz}, 3 \mathrm{H}), 2.76-2.55(\mathrm{~m}$, $4 \mathrm{H}), 2.00-1.75(\mathrm{~m}, 4 \mathrm{H})$.

${ }^{13}$ C-NMR: (101 MHz, $\left.\mathrm{CDCl}_{3}\right) \delta$ 169. (2xC), $157.6(\mathrm{C}), 141.6(\mathrm{C}), 141.0(2 \times C), 130.5(2 \times C), 128.4(4 \times C H)$, $125.9(\mathrm{CH}), 124.7(2 \times \mathrm{C}), 35.3\left(\mathrm{CH}_{2}\right), 30.8\left(\mathrm{CH}_{2}\right), 30.3\left(\mathrm{CH}_{2}\right), 24.2\left(\mathrm{CH}_{2}\right)$.

$\mathrm{E}_{1 / 2}(\mathbf{2 x})=-0.66 \mathrm{~V}$ vs SCE.

IR (ATR): $\tilde{v}\left(\mathrm{~cm}^{-1}\right): 2937,1819,1793,1746,1378,1198,1157,1043,731$.

HRMS: calculated for C19H12CL4NO4 $\left(\mathrm{M}-\mathrm{H}^{+}\right)$459.9496, found 459.9496.

Rf: 0.39 (9:1 pentane:EtOAc).

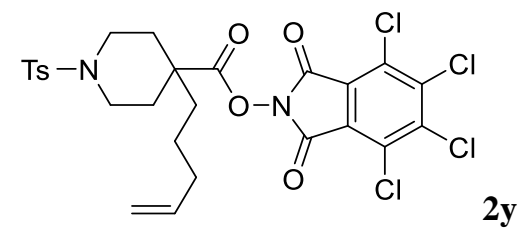

Obtained following general procedure A from $0.80 \mathrm{mmol}$ of $\mathbf{S 1 y}$. Column chromatography (8:2 pentane:EtOAc) afforded the product in $55 \%$ yield $(278 \mathrm{mg})$ as a white solid $\left(\mathbf{m p}: 196.5^{\circ} \mathrm{C}\right)$.

${ }^{1} \mathbf{H}$-NMR: (500 MHz, Chloroform- $d$ ) $\delta$ mixture of rotamers is observed $7.67(\mathrm{~d}, J=7.9 \mathrm{~Hz}, 1.7 \mathrm{H}), 7.59(\mathrm{~d}, J=$ $7.8 \mathrm{~Hz}, 0.3 \mathrm{H}), 7.36(\mathrm{~d}, J=7.9 \mathrm{~Hz}, 1.7 \mathrm{H}), 7.32(\mathrm{~d}, J=8.0 \mathrm{~Hz}, 0.3 \mathrm{H}), 5.81(\mathrm{ddt}, J=16.9,10.3,6.7 \mathrm{~Hz}, 0.85 \mathrm{H})$, 5.67 (ddt, $J=17.0,10.4,6.8 \mathrm{~Hz}, 0.15 \mathrm{H}), 5.10-4.85(\mathrm{~m}, 2 \mathrm{H}), 3.74(\mathrm{~d}, J=12.3 \mathrm{~Hz}, 1.7 \mathrm{H}), 3.58(\mathrm{~d}, J=12.0 \mathrm{~Hz}$, $0.3 \mathrm{zH}), 2.57(\mathrm{t}, J=12.3 \mathrm{~Hz}, 1.7 \mathrm{H}), 2.46(\mathrm{~s}, 3 \mathrm{H}), 2.40(\mathrm{~d}, J=12.8 \mathrm{~Hz}, 0.3 \mathrm{H}), 2.35(\mathrm{~d}, J=13.8 \mathrm{~Hz}, 1.7 \mathrm{H}), 2.12$ $(\mathrm{q}, J=7.0 \mathrm{~Hz}, 1.7 \mathrm{H}), 2.06(\mathrm{~d}, J=14.0 \mathrm{~Hz}, 0.3 \mathrm{H}), 1.97(\mathrm{q}, J=7.0 \mathrm{~Hz}, 0.3 \mathrm{H}), 1.76-1.66(\mathrm{~m}, 3 \mathrm{H}), 1.60-1.44$ $(\mathrm{m}, 3 \mathrm{H})$.

${ }^{13}$ C-NMR: $\left(101 \mathrm{MHz}, \mathrm{CDCl}_{3}\right) \delta$ 171.1/170.8 (2xC), $157.3(\mathrm{C}), 143.8 / 143.5(\mathrm{C}), 141.1(2 \times C), 137.7 / 137.4(\mathrm{CH})$, 133.1/132.9 (C), 130.4/129.7 (2xCH), $127.6(2 \times C H), 127.5(2 \times C), 124.6(2 \times C), 115.6 / 115.4\left(\mathrm{CH}_{2}\right), 46.4 / 45.2$ (C), 43.6/43.4 ( $\left(\mathrm{CH}_{2}\right)$, 39.7/38.9 $\left(\mathrm{CH}_{2}\right), 33.6 / 33.5\left(\mathrm{CH}_{2}\right), 33.2 / 32.7\left(\mathrm{CH}_{2}\right), 22.9 / 22.7\left(\mathrm{CH}_{2}\right), 21.6 / 21.6\left(\mathrm{CH}_{3}\right)$.

$\mathrm{E}_{1 / 2}(\mathbf{2 y})=-0.63 \mathrm{~V}$ vs SCE.

IR (ATR): $\tilde{v}\left(\mathrm{~cm}^{-1}\right)$ 2931, 2861, 1809, 1785, 1748, 1378, 1346, 1163, 1051, 932, 725.

HRMS: calculated for $\mathrm{C}_{26} \mathrm{H}_{25} \mathrm{Cl}_{4} \mathrm{~N}_{2} \mathrm{O}_{6} \mathrm{~S}\left[\mathrm{M}+\mathrm{H}^{+}\right]$635.0152, found 635.0149.

Rf: 0.334 (8:2 pentane:EtOAc). 


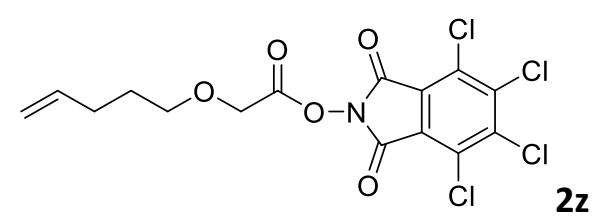

Obtained following general general procedure $\mathbf{A}$ from $0.74 \mathrm{mmol}$ of acid S1z. Column chromatography (9:1 to 8:2 hexane:EtOAc) afforded the product in $49 \%$ yield $(155 \mathrm{mg})$ as a white solid $\left(\mathbf{m p :} 112.7^{\circ} \mathrm{C}\right)$.

${ }^{1} \mathbf{H}-\mathbf{N M R}:\left(400 \mathrm{MHz}, \mathrm{CDCl}_{3}\right) \delta 5.84(\mathrm{ddt}, J=16.9,10.2,6.7 \mathrm{~Hz}, 1 \mathrm{H}), 5.06(\mathrm{ddd}, J=17.1,3.4,1.6 \mathrm{~Hz}, 1 \mathrm{H})$, $5.00(\mathrm{ddd}, J=10.2,2.0,1.1 \mathrm{~Hz}, 1 \mathrm{H}), 4.50(\mathrm{~s}, 2 \mathrm{H}), 3.67(\mathrm{t}, J=6.4 \mathrm{~Hz}, 2 \mathrm{H}), 2.23-2.13(\mathrm{~m}, 2 \mathrm{H}), 1.81-1.71(\mathrm{~m}$, $2 \mathrm{H})$.

${ }^{13}$ C-NMR: $\left(101 \mathrm{MHz}, \mathrm{CDCl}_{3}\right) \delta 166.6(2 \mathrm{xC}), 157.2(\mathrm{C}), 141.1(2 \mathrm{xC}), 137.8\left(\mathrm{CH}_{2}\right), 130.6(2 \mathrm{xC}), 124.6(2 \mathrm{xC})$, $115.1(\mathrm{CH}), 71.8\left(\mathrm{CH}_{2}\right), 66.1\left(\mathrm{CH}_{2}\right), 30.0\left(\mathrm{CH}_{2}\right), 28.6\left(\mathrm{CH}_{2}\right)$.

IR (ATR): $\tilde{v}\left(\mathrm{~cm}^{-1}\right):$ 2924, 1823, 1794, 1724, 1380, 1153, 1067, 730, 718.

Rf: 0.33 (9:1 Hexane:EtOAc) 


\section{Characterization of products 3a to $3 w$ :}

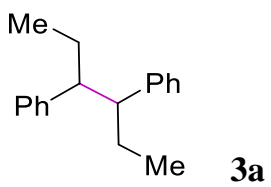

Obtained following general procedure $\mathbf{B}$ from $45 \mathrm{mg}(0.1 \mathrm{mmol})$ of $\mathbf{2 a}$ and $4 \mathrm{mg}$ of q-OAc $(25 \mathrm{~mol} \%)$. Column chromatography (9:1 to 7:3 pentane:EtOAc) afforded major product 3a in 67\% yield (13 $\mathrm{mg})$ as a white solid (mp: $93.1^{\circ} \mathrm{C}$ ). The control reaction in the absence of q-OAc gave no product formation.

${ }^{1}$ H-NMR: (500 MHz, Chloroform- $d$ ) $\delta 7.35(\mathrm{t}, J=7.5 \mathrm{~Hz}, 2 \mathrm{H}), 7.25(\mathrm{~d}, J=7.3 \mathrm{~Hz}, 1 \mathrm{H}), 7.24-7.20(\mathrm{~m}, 2 \mathrm{H})$, $7.15(\mathrm{dd}, J=8.1,6.5 \mathrm{~Hz}, 2 \mathrm{H}), 7.12-7.07(\mathrm{~m}, 1 \mathrm{H}), 6.91-6.87(\mathrm{~m}, 2 \mathrm{H}), 2.79-2.71(\mathrm{~m}, 1 \mathrm{H}), 2.62-2.58(\mathrm{~m}$, $1 \mathrm{H}), 1.91$ (dddd, $J=14.5,10.9,9.2,5.5 \mathrm{~Hz}, 1 \mathrm{H}), 1.62(\mathrm{dtd}, J=13.7,7.4,3.6 \mathrm{~Hz}, 1 \mathrm{H}), 1.45-1.25(\mathrm{~m}, 3 \mathrm{H}), 0.76$ $(\mathrm{t}, J=7.3 \mathrm{~Hz}, 3 \mathrm{H}), 0.55(\mathrm{t}, J=7.4 \mathrm{~Hz}, 3 \mathrm{H})$.

${ }^{13}$ C-NMR: (126 MHz, $\left.\mathrm{CDCl}_{3}\right) \delta$ 144.6/143.0 (C), 129.1/128.4 (2xCH), 128.2/127.4 (2xCH), 126.0/125.6 (CH), 54.2/53.2 (CH), 27.5/25.8 $\left(\mathrm{CH}_{2}\right), 12.4 / 12.2\left(\mathrm{CH}_{3}\right)$.

IR (ATR): $\tilde{v}\left(\mathrm{~cm}^{-1}\right)$ 3082, 3061, 3024, 2966, 2923, 2874, 1494, 1451, 761, 699.

HRMS: calculated for $\mathrm{C}_{18} \mathrm{H}_{23}\left[\mathrm{M}+\mathrm{H}^{+}\right] 239.1794$, found 239.1788 .

Rf: 0.82 (9:1 pentane:EtOAc).<smiles>O=C(O)N1CCCC1N1C(=O)c2c(Cl)c(Cl)c(Cl)c(Cl)c2C1=O</smiles>

Obtained following general procedure $\mathbf{B}$ from $49.5 \mathrm{mg}(0.1 \mathrm{mmol})$ of $\mathbf{2 b}$ and $1.7 \mathrm{mg}$ of q-OAc $(10 \mathrm{~mol} \%)$ after $4 \mathrm{~h}$ of irradiation. Column chromatography (9:1 pentane:EtOAc) afforded the product in $89 \%$ yield $(40 \mathrm{mg})$ as a white solid ( $\mathbf{m p :} 245^{\circ} \mathrm{C}$ ). The control reaction in the absence of q-OAc gave only $39 \%$ of product. The product was found to be highly insoluble in $\mathrm{CDCl}_{3}$, but very soluble in acetone- $\mathrm{d}_{6}$.

${ }^{1}$ H-NMR: (500 MHz, Acetone- $d_{6}$ ) rotamers observed $\delta 5.97(\mathrm{dd}, J=8.2,3.3 \mathrm{~Hz}, 1 \mathrm{H}), 3.64(\mathrm{dd}, J=11.4,5.5$ $\mathrm{Hz}, 1 \mathrm{H}), 3.56(\mathrm{dd}, J=11.6,6.1 \mathrm{~Hz}, 1 \mathrm{H}), 2.60-2.33(\mathrm{~m}, 1 \mathrm{H}), 2.32-2.17(\mathrm{~m}, 2 \mathrm{H}), 2.04-1.77(\mathrm{~m}, 1 \mathrm{H}), 1.39$ (s, 4H), 1.27 (s, 5H).

${ }^{13}$ C-NMR: (126 MHz, Acetone- $\left.d_{6}\right) \delta$ 162.8/162.5 (2xC), 153.2/152.5 (C), 139.2/139.1 (2xC), 129.0/128.9 $(2 \times C), 128.0(2 \times C), 79.5 / 79.3(\mathrm{C}), 65.1 / 64.4(\mathrm{CH}), 47.1 / 46.8\left(\mathrm{CH}_{2}\right), 31.3 / 30.8\left(\mathrm{CH}_{2}\right), 27.6 / 27.5\left(3 \times \mathrm{xCH}_{3}\right)$, 23.6/23.1 $\left(\mathrm{CH}_{2}\right)$.

IR: (ATR): $\tilde{v}\left(\mathrm{~cm}^{-1}\right)$ 2977, 2933, 1780, 1721, 1702, 1394, 1368, 1338, 1323, 1143, 752, 739.

HRMS: calculated for $\mathrm{C}_{17} \mathrm{H}_{17} \mathrm{Cl}_{4} \mathrm{~N}_{2} \mathrm{O}_{6}(\mathrm{M}+\mathrm{H}), 454,9913$; found, 454,9913.

Rf: 0.63 (7:3 pentane:EtOAc). 
<smiles>O=C([O-])N1CCCC1N1C(=O)c2c(Cl)c(Cl)c(Cl)c(Cl)c2C1=O</smiles>

Obtained following general procedure $\mathbf{B}$ from $53 \mathrm{mg}(0.1 \mathrm{mmol})$ of $\mathbf{2} \mathbf{b}$ ' and $1.7 \mathrm{mg}$ of q-OAc (10 mol\%). Column chromatography $(9: 1$ pentane:EtOAc) afforded the product in $72 \%$ yield $(35 \mathrm{mg})$ as a white foam (mp: $140.8^{\circ} \mathrm{C}$ ). The control reaction in the absence of q-OAc gave no product formation.

${ }^{1}$ H-NMR: (400 MHz, Chloroform- $d$ ) $\delta 7.49-7.31(\mathrm{~m}, 2 \mathrm{H}), 7.15-6.97(\mathrm{~m}, 3 \mathrm{H}), 6.08(\mathrm{dd}, J=7.7,4.4 \mathrm{~Hz}$, $0.4 \mathrm{H}), 5.96(\mathrm{dd}, J=7.8,4.6 \mathrm{~Hz}, 0.6 \mathrm{H}), 5.46(\mathrm{~d}, J=11.7 \mathrm{~Hz}, 0.6 \mathrm{H}), 5.15(\mathrm{~d}, J=12.5 \mathrm{~Hz}, 0.4 \mathrm{H}), 5.01(\mathrm{~d}, J=$ $12.4 \mathrm{~Hz}, 0.4 \mathrm{H}), 4.60(\mathrm{~d}, J=11.7 \mathrm{~Hz}, 0.6 \mathrm{H}), 3.90-3.74(\mathrm{~m}, 1 \mathrm{H}), 3.75-3.56(\mathrm{~m}, 1 \mathrm{H}), 2.47-2.19(\mathrm{~m}, 3 \mathrm{H}), 2.02$ $-1.88(\mathrm{~m}, 1 \mathrm{H})$.

${ }^{13}$ C-NMR: $\left(126 \mathrm{MHz}, \mathrm{CDCl}_{3}\right) \delta$ 162.8/162.42 (2xC), 154.2/153.3 (C), 140.2/139.9 (2xC), 136.2 (C), 129.8/129.5 (2xC), 128.5/128.4 (2xCH), 128.1/128.0 (2xCH), 127.8/127.7 (CH), 127.3/127.0 (2xC), 67.1/66.8 $\left(\mathrm{CH}_{2}\right), 65.0 / 64.0(\mathrm{CH}), 47.4 / 47.1\left(\mathrm{CH}_{2}\right), 31.6 / 30.8\left(\mathrm{CH}_{2}\right), 24.0 / 23.4\left(\mathrm{CH}_{2}\right)$.

IR (ATR): v ( $\left.\mathrm{cm}^{-1}\right)$ 2961, 2893, 1779, 1721, 1497, 1404, 1369, 10321, 1195, 1144, 944, 914, 751, 739.

HRMS: calculated for $\mathrm{C}_{20} \mathrm{H}_{15} \mathrm{Cl}_{4} \mathrm{~N}_{2} \mathrm{O}_{4}\left(\mathrm{M}+\mathrm{H}^{+}\right) 488.9751$, found 488.9751 .

Rf: 0.33 (8:2 pentane:EtOAc).

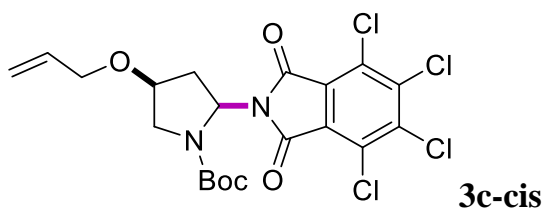

Obtained following general procedure $\mathbf{B}$ from $55.4 \mathrm{mg}(0.1 \mathrm{mmol})$ of $\mathbf{2 c}$ and $1.7 \mathrm{mg}$ of q-OAc $(10 \mathrm{~mol} \%)$. Column chromatography (9:1 to 8:2 pentane:EtOAc) afforded 3c-cis as the major product in $42 \%$ yield (21 $\mathrm{mg}$ ). The control reaction in the absence of q-OAc gave only $31 \%$ of combined major and minor products.

${ }^{1}$ H-NMR: (400 MHz, Chloroform- $d$ ) $\delta 6.03-5.83(\mathrm{~m}, 2 \mathrm{H}), 5.30(\mathrm{dd}, J=17.2,6.4 \mathrm{~Hz}, 1 \mathrm{H}), 5.25-5.15(\mathrm{~m}$, $1 \mathrm{H}), 4.18-3.83(\mathrm{~m}, 4 \mathrm{H}), 3.62$ (ddd, $J=21.3,10.0,7.4 \mathrm{~Hz}, 1 \mathrm{H}), 2.62$ (ddd, $J=20.2,10.5,6.5 \mathrm{~Hz}, 1 \mathrm{H}), 2.42$ (ddt, $J=37.3,12.8,7.6 \mathrm{~Hz}, 1 \mathrm{H}), 1.43(\mathrm{~s}, 4 \mathrm{H}), 1.31(\mathrm{~s}, 5 \mathrm{H})$.

${ }^{13}$ C-NMR: $\left(126 \mathrm{MHz}, \mathrm{CDCl}_{3}\right) \delta$ 162.6/162.3 (2xC), 153.4/152.9 (C), 140.4/140.0 (2xC), $134.31(\mathrm{CH})$, 129.8/129.7 (2xC), 127.4/127.2 (2xC), 117.7/117.4 $\left(\mathrm{CH}_{2}\right)$, 81.1/80.8 (C), 75.0/74.4 (CH), 71.0/70.7 $\left(\mathrm{CH}_{2}\right)$, 63.4/62.8 (CH), 51.0/50.2 $\left(\mathrm{CH}_{2}\right), 36.3 / 36.0\left(\mathrm{CH}_{2}\right), 28.4 / 28.3\left(3 \mathrm{xCH}_{3}\right)$.

IR: (ATR): $\tilde{v}\left(\mathrm{~cm}^{-1}\right): 2977,2929,1780,1723,1476,1455,1397,1368,1329,1142,738$.

HRMS: calculated for $\mathrm{C}_{20} \mathrm{H}_{21} \mathrm{Cl}_{4} \mathrm{~N}_{2} \mathrm{O}_{5}\left(\mathrm{M}+\mathrm{H}^{+}\right)$511.0170, found 511.0169.

Rf: 0.56 (8:2 pentane: EtOAc). 
<smiles>C=CCO[C@H]1C[C@@H](N2C(=O)c3c(Cl)c(Cl)c(Cl)c(Cl)c3C2=O)N(C(=O)[O-])C1</smiles>

Obtained following general procedure $\mathbf{B}$ from $55.4 \mathrm{mg}(0.1 \mathrm{mmol})$ of $\mathbf{2 c}$ and $1.7 \mathrm{mg}$ of q-OAc $(10 \mathrm{~mol} \%)$. Column chromatography (9:1 to 8:2 pentane:EtOAc) afforded 3c-trans as minor product in $24 \%$ yield (12 mg) as minor product. The control reaction in the absence of q-OAc gave only $31 \%$ of combined major and minor products.

${ }^{1}$ H-NMR: (500 MHz, Chloroform- $d$ ) mixture of rotamers $\delta 6.16$ (ddd, $J=22.2,8.1,5.8 \mathrm{~Hz}, 1 \mathrm{H}$ ), 5.93 (ddt, $J$ $=16.3,10.7,5.5 \mathrm{~Hz}, 1 \mathrm{H}), 5.32(\mathrm{dd}, J=17.2,1.8 \mathrm{~Hz}, 1 \mathrm{H}), 5.23(\mathrm{dd}, J=10.5,1.7 \mathrm{~Hz}, 1 \mathrm{H}), 4.37$ (br s, $1 \mathrm{H}), 4.09$ $-3.95(\mathrm{~m}, 2 \mathrm{H}), 3.90-3.60(\mathrm{~m}, 2 \mathrm{H}), 2.60-2.35(\mathrm{~m}, 2 \mathrm{H}), 1.42(\mathrm{~s}, 4 \mathrm{H}), 1.33(\mathrm{~s}, 5 \mathrm{H})$.

${ }^{13}$ C-NMR: $\left(101 \mathrm{MHz}, \mathrm{CDCl}_{3}\right) \delta$ 162.7/162.4 (C), 153.7/153.2 (C), 140.4/140.2 (C), $134.3(\mathrm{CH}), 129.8(\mathrm{C})$, 127.4/127.2 (C), 117.3/117.2 $\left(\mathrm{CH}_{2}\right)$, 81.0/80.6 (C), 76.2 (CH), $70.0\left(\mathrm{CH}_{2}\right)$, 63.4/63.3 (CH), 52.6/51.9 $\left(\mathrm{CH}_{2}\right)$, 37.6/36.8 $\left(\mathrm{CH}_{2}\right), 28.4 / 28.3\left(3 \mathrm{xCH}_{3}\right)$.

IR (ATR): $\tilde{v}\left(\mathrm{~cm}^{-1}\right):$ 2931, 2977, 1781, 1723, 1457, 1396, 1368, 1342, 1166, 1141, 739.

HRMS: calculated for $\mathrm{C}_{20} \mathrm{H}_{21} \mathrm{Cl}_{4} \mathrm{~N}_{2} \mathrm{O}_{5}\left(\mathrm{M}+\mathrm{H}^{+}\right)$511.0170, found 511.0169.

Rf: 0.53 (8:2 pentane:EtOAc).<smiles>CC(=O)OCCC(NC(CCSC(C)(C)C)C(=O)O)N1C(=O)c2c(Cl)c(Cl)c(Cl)c(Cl)c2C1=O</smiles>

Obtained following general procedure $\mathbf{B}$ from $53 \mathrm{mg}(0.1 \mathrm{mmol})$ of $\mathbf{2 d}$ and $1.7 \mathrm{mg}$ of q-OAc (10 mol\%). Column chromatography (97:3 to 8:2 hexane:EtOAc) afforded the product in $76 \%$ yield $(36 \mathrm{mg})$ as a white solid ( $\mathbf{m p}$ $\left.148.1{ }^{\circ} \mathrm{C}\right)$. The control reaction in the absence of q-OAc gave no product formation.

${ }^{1}$ H-NMR: $\left(300 \mathrm{MHz}, \mathrm{CDCl}_{3}\right) \delta 6.04(\mathrm{dt}, J=9.7,7.6 \mathrm{~Hz}, 1 \mathrm{H}), 5.69(\mathrm{br} \mathrm{s}, 1 \mathrm{H}), 2.67-2.42(\mathrm{~m}, 2 \mathrm{H}), 2.41-2.21$ (m, 2H), $2.10(\mathrm{~s}, 3 \mathrm{H}), 1.43(\mathrm{~s}, 9 \mathrm{H})$.

${ }^{13}$ C-NMR: $\left(101 \mathrm{MHz}, \mathrm{CDCl}_{3}\right) \delta 162.8(2 \mathrm{xC}), 154.0(\mathrm{C}), 140.4(2 \times \mathrm{x}), 129.9(2 \mathrm{xC}), 127.3(2 \mathrm{xC}), 80.9(\mathrm{C}), 58.7$ $(\mathrm{CH}), 32.5\left(\mathrm{CH}_{2}\right), 29.9\left(\mathrm{CH}_{2}\right), 28.2\left(3 \times \mathrm{xCH}_{3}\right), 15.6\left(\mathrm{CH}_{3}\right)$.

IR (ATR): $\tilde{v}\left(\mathrm{~cm}^{-1}\right)$ : 3387, 2976, 2922, 1780, 1717, 1498, 1321, 1160, 737.

HRMS: calculated for $\mathrm{C}_{17} \mathrm{H}_{19} \mathrm{Cl}_{4} \mathrm{~N}_{2} \mathrm{O}_{4} \mathrm{~S}\left(\mathrm{M}+\mathrm{H}^{+}\right)$489.9814, found 489.9808 .

R:: 0.35 (8:2 hexane:EtOAc) 
<smiles>C=CCCN(C(=O)O)C(N1C(=O)c2c(Cl)c(Cl)c(Cl)c(Cl)c2C1=O)[N+]([O-])([O-])O</smiles>

Obtained following general procedure B from $56 \mathrm{mg}(0.1 \mathrm{mmol})$ of $2 \mathbf{e}$ and $4 \mathrm{mg}$ of q-OAc $(25 \mathrm{~mol} \%)$. Column chromatography (97:3 to 9:1 pentane:EtOAc) afforded the product in 53\% yield ( $27 \mathrm{mg})$. The control reaction in the absence of q-OAc gave no product formation.

${ }^{1}$ H-NMR: (500 MHz, Chloroform- $d$ ) $\delta 7.41-7.27$ (m, 3H), $7.25-7.18$ (m, 2H), 6.45 (br s, 0.5H), 6.29 (br s, $0.5 \mathrm{H}), 5.76$ (br s, 1H), 5.33 (br s, 0.5H), 5.25 (br s, 0.5H), $5.11-4.96(\mathrm{~m}, 3 \mathrm{H}), 3.66(\mathrm{~s}, 2 \mathrm{H}), 2.39$ (br s, $1 \mathrm{H})$, 2.29 (br s, $1 \mathrm{H}), 1.77$ (s, 3H).

${ }^{13}$ C-NMR: $\left(126 \mathrm{MHz}, \mathrm{CDCl}_{3}\right) \delta$ 162.9/162.8 (2xC), 155.9/155.1 (C), $140.2(2 \mathrm{xC}), 136.2(\mathrm{C}), 135.0(\mathrm{CH}), 129.8$ (2xC), $128.4(2 \times C H), 128.0(2 \times C H), 127.8(\mathrm{CH}), 127.1(\mathrm{C}), 116.9\left(\mathrm{CH}_{2}\right), 67.5\left(\mathrm{CH}_{2}\right), 60.3(\mathrm{CH}), 44.2 / 43.6$ $\left(\mathrm{CH}_{2}\right), 34.7 / 33.9\left(\mathrm{CH}_{2}\right), 17.3 / 16.9\left(\mathrm{CH}_{3}\right)$.

IR (ATR): $\tilde{v}\left(\mathrm{~cm}^{-1}\right): 3068,2978,2951,1778,1721,1497,1476,1454,1402,1371,1343,1320,1204,1001$, 966, 739 .

HRMS: calculated for $\mathrm{C}_{22} \mathrm{H}_{19} \mathrm{Cl}_{4} \mathrm{~N}_{2} \mathrm{O}_{4}\left(\mathrm{M}+\mathrm{H}^{+}\right)$517.0064, found 517.0064.

Rf: 0.40 (8:2 pentane:EtOAc).<smiles>O=C1c2c(Cl)c(Cl)c(Cl)c(Cl)c2C(=O)N1C(COCc1ccccc1)NCc1ccccc1</smiles>

Obtained following general procedure $\mathbf{B}$ from $58 \mathrm{mg}(0.1 \mathrm{mmol})$ of $\mathbf{2 f}$ and $1.7 \mathrm{mg}$ of q-OAc (10 mol\%). Column chromatography (9:1 pentane:EtOAc) afforded the product in $89 \%$ yield $(48 \mathrm{mg})$. The control reaction in the absence of q-OAc gave no product formation.

${ }^{1}$ H-NMR: (400 MHz, Chloroform- $d$ ) $\delta 7.49-7.18$ (m, 5H), 6.21 (br s, 1H), 5.71 (br s, 1H), 4.60 (d, $J=12.0$ $\mathrm{Hz}, 1 \mathrm{H}), 4.49(\mathrm{~d}, J=12.0 \mathrm{~Hz}, 1 \mathrm{H}), 3.91(\mathrm{dd}, J=10.3,8.9 \mathrm{~Hz}, 1 \mathrm{H}), 3.77(\mathrm{dd}, J=10.3,5.5 \mathrm{~Hz}, 1 \mathrm{H}), 1.44(\mathrm{~s}$, 9H).

${ }^{13}$ C-NMR: (101 MHz, $\left.\mathrm{CDCl}_{3}\right) \delta 162.9(2 \times \mathrm{C}), 154.0(\mathrm{C}), 140.2(2 \times \mathrm{x}), 137.2(\mathrm{CH}), 129.9(2 \mathrm{xC}), 128.4(2 \times \mathrm{xH})$, 127.8 (CH), $127.8(2 \times C H), 127.3(2 \times C), 81.0(\mathrm{C}), 73.1\left(\mathrm{CH}_{2}\right), 68.1\left(\mathrm{CH}_{2}\right), 57.8(\mathrm{CH}), 28.2\left(3 \mathrm{xCH}_{3}\right)$.

IR (ATR): $\tilde{v}\left(\mathrm{~cm}^{-1}\right)$ 2931, 2872, 1780, 1723, 1496, 1454, 1368, 1162, 1047, 739.

HRMS: calculated for $\mathrm{C}_{22} \mathrm{H}_{21} \mathrm{Cl}_{4} \mathrm{~N}_{2} \mathrm{O}_{4}\left[\mathrm{M}+\mathrm{H}^{+}\right]$535.0170, found 535.0170.

Rf: 0.45 (8:2 pentane:EtOAc). 
<smiles>O=C1c2c(Cl)c(Cl)c(Cl)c(Cl)c2C(=O)N1C1CCCO1</smiles>

Obtained following general procedure $\mathbf{B}$ from $39.6 \mathrm{mg}(0.1 \mathrm{mmol})$ of $\mathbf{2 g}$ and $1.7 \mathrm{mg}$ of q-OAc (10 mol\%). Column chromatography (9:1 pentane:EtOAc) afforded the product in $30 \%$ yield $(11 \mathrm{mg})$ as a thick colorless wax. The control reaction in the absence of q-OAc gave no product formation.

${ }^{1}$ H-NMR: (300 MHz, Chloroform- $d$ ) $\delta 6.06(\mathrm{dd}, J=7.8,4.4 \mathrm{~Hz}, 1 \mathrm{H}), 4.24(\mathrm{q}, J=7.6 \mathrm{~Hz}, 1 \mathrm{H}), 3.99$ (td, $J=$ $7.7,4.3 \mathrm{~Hz}, 1 \mathrm{H}), 2.66-2.24(\mathrm{~m}, 3 \mathrm{H}), 2.15-1.95(\mathrm{~m}, 1 \mathrm{H})$.

${ }^{13}$ C-NMR: $\left(75 \mathrm{MHz}, \mathrm{CDCl}_{3}\right) \delta 163.1(2 \mathrm{xC}), 140.3(2 \mathrm{xC}), 129.8(2 \mathrm{xC}), 127.4(2 \mathrm{xC}), 81.7(\mathrm{CH}), 70.2\left(\mathrm{CH}_{2}\right)$, $29.3\left(\mathrm{CH}_{2}\right), 25.9\left(\mathrm{CH}_{2}\right)$.

IR (ATR): $\tilde{v}\left(\mathrm{~cm}^{-1}\right)$ 2982, 2880, 1825, 1453, 1377, 1308, 1201, 794, 733.

Rf: 0.49 (8:2 pentane:EtOAc).<smiles>C=CCCCOC(Cc1ccccc1)N1C(=O)c2c(Cl)c(Cl)c(Cl)c(Cl)c2C1=O</smiles>

Obtained following general procedure $\mathbf{X}$ from $52 \mathrm{mg}(0.1 \mathrm{mmol})$ of $\mathbf{X}$ and $1.7 \mathrm{mg}$ of q-OAc (10 mol\%). Column chromatography (9:1 pentane:EtOAc) afforded the product in $66 \%$ yield $(31 \mathrm{mg})$ as a white solid $\left(\mathbf{m p}: 82{ }^{\circ} \mathrm{C}\right)$. The control reaction in the absence of q-OAc gave no product formation.

${ }^{1}$ H-NMR: (500 MHz, Chloroform- $d$ ) $\delta 7.30-7.26(\mathrm{~m}, 2 \mathrm{H}), 7.25-7.19(\mathrm{~m}, 3 \mathrm{H}), 5.72(\mathrm{ddt}, J=16.9,10.2,6.7$ $\mathrm{Hz}, 1 \mathrm{H}), 5.61(\mathrm{t}, J=7.3 \mathrm{~Hz}, 1 \mathrm{H}), 5.01-4.86(\mathrm{~m}, 2 \mathrm{H}), 3.60(\mathrm{dd}, J=14.0,7.0 \mathrm{~Hz}, 1 \mathrm{H}), 3.54-3.41(\mathrm{~m}, 3 \mathrm{H})$, $2.12-2.02(\mathrm{~m}, 2 \mathrm{H}), 1.78-1.61(\mathrm{~m}, 2 \mathrm{H})$.

${ }^{13}$ C-NMR: $\left(101 \mathrm{MHz}, \mathrm{CDCl}_{3}\right) \delta 163.2(2 \mathrm{xC}), 140.4(2 \mathrm{xC}), 137.8(\mathrm{CH}), 136.0(\mathrm{C}), 129.9(2 \mathrm{xC}), 129.2(2 \mathrm{xCH})$, $128.6(2 \mathrm{xCH}), 127.0(\mathrm{CH}), 126.9(2 \times \mathrm{C}), 115.0\left(\mathrm{CH}_{2}\right), 83.5(\mathrm{CH}), 68.7\left(\mathrm{CH}_{2}\right), 38.7\left(\mathrm{CH}_{2}\right), 29.9\left(\mathrm{CH}_{2}\right), 28.3$ $\left(\mathrm{CH}_{2}\right)$.

IR (ATR): $\tilde{v}\left(\mathrm{~cm}^{-1}\right)$ 3029, 3065, 2929, 1779, 1720, 1496, 1370, 1336, 1320, 1116, 955, 884, 739.

HRMS: calculated for $\mathrm{C}_{21} \mathrm{H}_{18} \mathrm{Cl}_{4} \mathrm{O}_{3}\left[\mathrm{M}+\mathrm{H}^{+}\right]$474.0006, found 474.0001 .

Rf: 0.72 (9:1 pentane:EtOAc). 


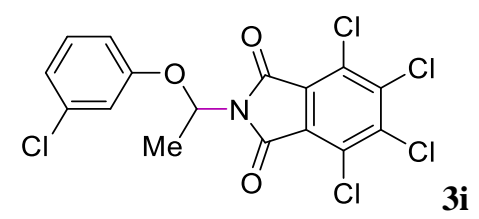

Obtained following general procedure $\mathbf{B}$ from $48 \mathrm{mg}(0.1 \mathrm{mmol})$ of $\mathbf{2 i}$ and $1.7 \mathrm{mg}$ of q-OAc (10 mol\%). Column chromatography (9:1 pentane:EtOAc) afforded the product in $78 \%$ yield $(34 \mathrm{mg})$. The control reaction in the absence of q-OAc gave only $38 \%$ of product formation.

${ }^{1}$ H-NMR: (500 MHz, Chloroform- $d$ ) $\delta 7.20(\mathrm{t}, J=8.1 \mathrm{~Hz}, 1 \mathrm{H}), 7.05(\mathrm{t}, J=2.1 \mathrm{~Hz}, 1 \mathrm{H}), 6.99(\mathrm{dd}, J=8.0,1.9$ $\mathrm{Hz}, 1 \mathrm{H}), 6.93(\mathrm{dd}, J=8.4,2.4 \mathrm{~Hz}, 1 \mathrm{H}), 6.35(\mathrm{q}, J=6.2 \mathrm{~Hz}, 1 \mathrm{H}), 1.97(\mathrm{~d}, J=6.2 \mathrm{~Hz}, 3 \mathrm{H})$.

${ }^{13}$ C-NMR: (126 MHz, $\left.\mathrm{CDCl}_{3}\right) \delta 162.4(2 \mathrm{xC}), 156.2(\mathrm{C}), 140.7$ (2xC), $135.2(\mathrm{C}), 130.6(\mathrm{CH}), 130.1(2 \times C)$, $126.9(2 \times C), 122.9(\mathrm{CH}), 117.4(\mathrm{CH}), 114.0(\mathrm{CH}), 76.4(\mathrm{CH}), 19.1\left(\mathrm{CH}_{3}\right)$.

IR (ATR): $\tilde{v}\left(\mathrm{~cm}^{-1}\right):$ 2931, 1777, 1727, 1592, 1475, 1442, 1391, 1328, 1279, 1220, 978, 944, 740.

HRMS: calculated for $\mathrm{C}_{16} \mathrm{H}_{9} \mathrm{Cl}_{5} \mathrm{NO}_{3}\left(\mathrm{M}+\mathrm{H}^{+}\right) 441.8961$, found 441.8960 .

Rf: 0.76 (8:2 pentane:EtOAc).

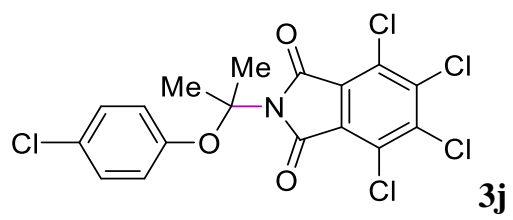

Obtained following general procedure B from $49.4 \mathrm{mg}(0.1 \mathrm{mmol})$ of $\mathbf{2 j}$ and $1.7 \mathrm{mg}$ of q-OAc $(10 \mathrm{~mol} \%)$. Column chromatography (9:1 pentane:EtOAc) afforded the product in $70 \%$ yield (32 $\mathrm{mg})$. The control reaction in the absence of q-OAc gave only $19 \%$ of product formation.

${ }^{1}$ H-NMR: (400 MHz, Chloroform- $d$ ) $\delta 7.22(\mathrm{~d}, J=7.5 \mathrm{~Hz}, 2 \mathrm{H}), 6.90(\mathrm{~d}, J=7.7 \mathrm{~Hz}, 2 \mathrm{H}), 2.07$ (s, 4H).

${ }^{13}$ C-NMR: $\left(126 \mathrm{MHz} \mathrm{CDCl}_{3}\right) \delta 163.4(2 \mathrm{xC}), 152.4(\mathrm{C}), 140.6(2 \mathrm{xC}), 129.9(2 \mathrm{xC}), 129.5(2 \mathrm{xCH}), 129.4(2 \mathrm{xC})$, $126.7(\mathrm{C}), 123.0(2 \mathrm{xCH}), 92.5(\mathrm{C}), 27.5\left(2 \mathrm{xCH}_{3}\right)$.

IR (ATR): $\tilde{v}\left(\mathrm{~cm}^{-1}\right):$ 3005, 1780, 1718, 1487, 1370, 1319, 1147, 736.

HRMS: calculated for $\mathrm{C}_{17} \mathrm{H}_{11} \mathrm{Cl}_{5} \mathrm{NO}_{3}\left(\mathrm{M}+\mathrm{H}^{+}\right)$451.9176, found 451.9174 .

Rf: 0.56 (9:1 pentane:EtOAc). 
$\sum_{B o c-N H}^{O} d r 4: 1$

Obtained following general procedure $\mathbf{B}$ from $52.5 \mathrm{mg}(0.1 \mathrm{mmol})$ of $\mathbf{2 k}$ and $4 \mathrm{mg}$ of q-OAc $(25 \mathrm{~mol} \%)$. Column chromatography (9:1 to 7:3 pentane:EtOAc) afforded major product $3 \mathbf{k}$ in 59\% yield (12 $\mathrm{mg}$ ) as a 4:1 mixture of diastereomers. The control reaction in the absence of q-OAc gave no product formation.

${ }^{1}$ H-NMR: ${ }^{1} \mathrm{H}$ NMR major: $(400 \mathrm{MHz}$, Chloroform- $d$ ) $\delta 4.54(\mathrm{~d}, J=9.6 \mathrm{~Hz}, 1 \mathrm{H}), 4.16(\mathrm{dq}, J=9.5,4.7 \mathrm{~Hz}$, $1 \mathrm{H}), 3.92-3.84(\mathrm{~m}, 2 \mathrm{H}), 3.54(\mathrm{dd}, J=9.3,3.1 \mathrm{~Hz}, 1 \mathrm{H}), 3.28(\mathrm{t}, J=8.5 \mathrm{~Hz}, 1 \mathrm{H}), 2.36(\mathrm{p}, J=7.9,7.4 \mathrm{~Hz}, 1 \mathrm{H})$, $1.38(\mathrm{~s}, 9 \mathrm{H}), 0.93(\mathrm{~d}, J=6.9 \mathrm{~Hz}, 3 \mathrm{H})$; minor: (400 MHz, Chloroform- $d$ ) $\delta 4.33$ (br s, $1 \mathrm{H}), 4.00-3.92(\mathrm{~m}, 2 \mathrm{H})$, 3.74 (br s, 1H), $3.51-3.47(\mathrm{~m}, 1 \mathrm{H}), 3.29(\mathrm{t}, J=8.4 \mathrm{~Hz}, 1 \mathrm{H}), 2.09-1.95(\mathrm{~m}, 1 \mathrm{H}), 1.38(\mathrm{~s}, 9 \mathrm{H}), 1.03(\mathrm{~d}, J=6.9$ $\mathrm{Hz}, 3 \mathrm{H})$.

${ }^{13}$ C-NMR: $\left(101 \mathrm{MHz}, \mathrm{CDCl}_{3}\right) \delta$ 155.6/155.5 (C), $79.5(\mathrm{C}), 74.0 / 73.5\left(\mathrm{CH}_{2}\right), 73.3\left(\mathrm{CH}_{2}\right)$ 63.1/53.9 (CH), 41.1/36.9 (CH), 28.4/28.3 $\left(3 \mathrm{xCH}_{3}\right), 16.6 / 11.4\left(\mathrm{CH}_{3}\right)$.

IR (ATR): $\tilde{v}\left(\mathrm{~cm}^{-1}\right)$ 3320, 2972, 2932, 2875, 1685, 1525, 1365, 1248, 1165, 1090, 1049, 922, 698.

HRMS: calculated for $\mathrm{C}_{10} \mathrm{H}_{20} \mathrm{NO}_{3}(\mathrm{M}+\mathrm{H})$ 202.1438; found, 202.1438

Rf: 0.29 (4:1 pentane:EtOAc).

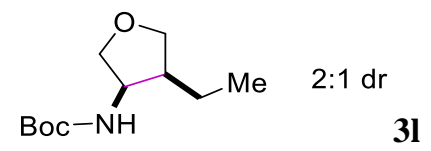

Obtained following general procedure $\mathbf{B}$ from $54 \mathrm{mg}(0.1 \mathrm{mmol})$ of $\mathbf{2 l}$ and $4 \mathrm{mg}$ of q-OAc $(25 \mathrm{~mol} \%)$. Column chromatography (9:1 to 8:2 pentane:EtOAc) afforded the product in $49 \%$ yield (11 $\mathrm{mg}$ ) of a colorless wax as a 2:1 mixture of diastereomers. The product was isolated as an inseparable mixture with an impurity, assumed to be the corresponding aminodecarboxylated product. The control reaction in the absence of q-OAc gave no product formation.

${ }^{1}$ H-NMR: 2:1 mixture of diastereomers $(500 \mathrm{MHz}$, Chloroform- $d$ ) $\delta 4.67$ (br s, $1 \mathrm{H}), 4.29$ (br s, $\left.1 \mathrm{H}\right), 4.06(\mathrm{t}, J$ $=8.1 \mathrm{~Hz}, 0.35 \mathrm{H}), 4.01(\mathrm{t}, J=8.4 \mathrm{~Hz}, 0.65 \mathrm{H}), 3.98-3.89(\mathrm{~m}, 1 \mathrm{H}), 3.69(\mathrm{dd}, J=9.3,2.2 \mathrm{~Hz}, 0.65 \mathrm{H}), 3.60(\mathrm{br} \mathrm{s}$, $0.35 \mathrm{H}), 3.44(\mathrm{dd}, J=8.9,6.6 \mathrm{~Hz}, 0.35 \mathrm{H}), 3.42(\mathrm{t}, J=9.0 \mathrm{~Hz}, 0.65 \mathrm{H}), 2.25(\mathrm{~h}, J=7.7 \mathrm{~Hz}, 0.65 \mathrm{H}), 1.91(\mathrm{pd}, J=$ 6.9, $4.3 \mathrm{~Hz}, 0.35 \mathrm{H}), 1.47$ (s, 9H), $1.41-1.23(\mathrm{~m}, 2 \mathrm{H}), 0.97(\mathrm{t}, J=7.4 \mathrm{~Hz}, 3 \mathrm{H})$.

${ }^{13}$ C-NMR: 2:1 mixture of diastereomers $\left(126 \mathrm{MHz}, \mathrm{CDCl}_{3}\right) \delta$ 155.5/155.4 (C), $79.5(\mathrm{C}), 74.3 / 73.24\left(\mathrm{CH}_{2}\right)$, 72.5/71.7 $\left(\mathrm{CH}_{2}\right), 56.7 / 52.9(\mathrm{CH}), 48.5 / 44.6(\mathrm{CH}), 28.4 / 28.3\left(3 \mathrm{xCH}_{3}\right), 25.1 / 20.1\left(\mathrm{CH}_{2}\right), 12.8 / 12.4\left(\mathrm{CH}_{3}\right)$.

IR (ATR): $\tilde{v}\left(\mathrm{~cm}^{-1}\right)$ 3323, 2970, 2933, 2876, 1713, 1696, 1528, 1391, 1366, 1276, 1171, 1056, 899, 781.

HRMS: calculated for $\mathrm{C}_{13} \mathrm{H}_{25} \mathrm{~N}_{2} \mathrm{O}_{3}\left[\mathrm{M}+\mathrm{MeCN}+\mathrm{H}^{+}\right]$257.1859, found 257.1850.

Rf: 0.3 ( $8: 2$ pentane:EtOAc). 


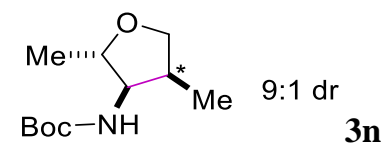

Obtained following general procedure $\mathbf{B}$ from $54 \mathrm{mg}(0.1 \mathrm{mmol})$ of $\mathbf{2 n}$ and $4 \mathrm{mg}$ of q-OAc (25 mol\%). Column chromatography (9:1 to 8:2 pentane:EtOAc) afforded the product in $51 \%$ yield $(11 \mathrm{mg})$ as a 9:1 mixture of diastereomers. The control reaction in the absence of q-OAc gave no product formation.

${ }^{1}$ H-NMR: as a 9:1 mixture of diastereomers (400 MHz, Chloroform- $d$ ) $\delta 4.43$ (br d, $J=10.0 \mathrm{~Hz}, 0.9 \mathrm{H}$ ), 4.31 (br s, 0.1H), 3.97 (dd, $J=8.8,7.3 \mathrm{~Hz}, 0.9 \mathrm{H}), 3.91-3.85(\mathrm{~m}, 0.1 \mathrm{H}), 3.70(\mathrm{q}, J=7.5 \mathrm{~Hz}, 0.9 \mathrm{H}), 3.57$ (p, $J=6.1$ $\mathrm{Hz}, 0.9 \mathrm{H}), 3.48(\mathrm{t}, J=6.7 \mathrm{~Hz}, 0.1 \mathrm{H}), 3.41(\mathrm{t}, J=8.2 \mathrm{~Hz}, 0.1 \mathrm{H}), 3.25(\mathrm{t}, J=8.2 \mathrm{~Hz}, 0.9 \mathrm{H}), 3.14(\mathrm{dd}, J=8.9,7.3$ $\mathrm{Hz}, 0.1 \mathrm{H}), 2.39(\mathrm{dt}, J=14.4,7.1 \mathrm{~Hz}, 0.9 \mathrm{H}), 2.03-1.91(\mathrm{~m}, 0.1 \mathrm{H}), 1.35(\mathrm{~s}, 9 \mathrm{H}), 1.20(\mathrm{~d}, J=6.4 \mathrm{~Hz}, 0.3 \mathrm{H}), 1.17$ $(\mathrm{d}, J=6.3 \mathrm{~Hz}, 2.7 \mathrm{H}), 1.00(\mathrm{~d}, J=6.8 \mathrm{~Hz}, 0.3 \mathrm{H}), 0.85(\mathrm{~d}, J=7.0 \mathrm{~Hz}, 2.7 \mathrm{H})$.

${ }^{13}$ C-NMR: $\left(101 \mathrm{MHz}, \mathrm{CDCl}_{3}\right) \delta 155.7(\mathrm{C}), 79.6(\mathrm{CH}), 79.5(\mathrm{C}), 73.6\left(\mathrm{CH}_{2}\right), 59.2(\mathrm{CH}), 35.4(\mathrm{CH}), 28.3$ $\left(3 \mathrm{xCH}_{3}\right), 19.6\left(\mathrm{CH}_{3}\right), 11.9\left(\mathrm{CH}_{3}\right)$.

IR (ATR): $\tilde{v}\left(\mathrm{~cm}^{-1}\right)$ 3324, 2972, 2932, 2876, 1689, 1526, 1455, 1366, 1247, 1167, 1043, 1013, 932, 863, 782, 673.

HRMS: calculated for $\mathrm{C}_{13} \mathrm{H}_{25} \mathrm{~N}_{2} \mathrm{O}_{3}\left[\mathrm{M}+\mathrm{MeCN}+\mathrm{H}^{+}\right]$257.1859; found 257.1860.

Rf: 0.31 (4:1 pentane:EtOAc).

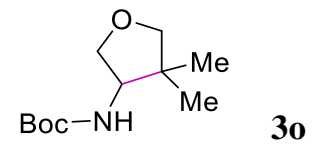

Obtained following general procedure B from $54 \mathrm{mg}(0.1 \mathrm{mmol})$ of $\mathbf{2 0}$ and $4 \mathrm{mg}$ of q-OAc (25 mol\%). Column chromatography (9:1 to 8:2 pentane:EtOAc) afforded the major product 30 in $42 \%$ yield (9 mg) as a colorless wax. The control reaction in the absence of q-OAc gave no product formation.

${ }^{1}$ H-NMR: (400 MHz, Chloroform-d) $\delta 4.57$ (br s, 1H), 4.17 (dd, J = 9.2, 7.0 Hz, 1H), 4.01 (br s, $1 \mathrm{H}$ ), 3.60 $3.47(\mathrm{~m}, 3 \mathrm{H}), 1.46(\mathrm{~s}, 9 \mathrm{H}), 1.13(\mathrm{~s}, 3 \mathrm{H}), 1.03(\mathrm{~s}, 3 \mathrm{H})$.

${ }^{13}$ C-NMR: $\left(101 \mathrm{MHz}, \mathrm{CDCl}_{3}\right) \delta 155.6(\mathrm{C}), 79.6\left(\mathrm{CH}_{2}\right), 79.5(\mathrm{C}), 72.4\left(\mathrm{CH}_{2}\right), 59.2(\mathrm{CH}), 41.6(\mathrm{C}), 28.3\left(3 \times \mathrm{XH}_{3}\right)$, $25.3(\mathrm{CH} 3), 19.9\left(\mathrm{CH}_{3}\right)$.

IR (ATR): $\tilde{v}\left(\mathrm{~cm}^{-1}\right): 3324,2968,2932,2873,1685,1529,1391,1366,1249,1169,1065,940,912,887,856$, 696.

HRMS: calculated for $\mathrm{C}_{11} \mathrm{H}_{22} \mathrm{NO}_{3}\left[\mathrm{M}+\mathrm{H}^{+}\right] 216.1594$, found 216.1595

Rf: 0.27 (8:2 pentane:EtOAc). 


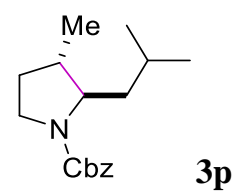

Obtained following general procedure $\mathbf{X}$ from $60 \mathrm{mg}(0.1 \mathrm{mmol})$ of $\mathbf{X}$ and $4 \mathrm{mg}$ of q-OAc (25 mol\%). Column chromatography (97:3 to 9:1 pentane:EtOAc) afforded the minor product $\mathbf{3 p}$ in $15 \%$ yield (4 mg) as a colorless wax and as a single diastereoisomer. The control reaction in the absence of q-OAc gave no product formation.

${ }^{1}$ H-NMR: (500 MHz, Chloroform- $\left.d\right) \delta 7.43-7.30(\mathrm{~m}, 5 \mathrm{H}), 5.24-5.09(\mathrm{~m}, 2 \mathrm{H}), 3.64-3.49(\mathrm{~m}, 2 \mathrm{H}), 3.40(\mathrm{t}$, $J=8.9 \mathrm{~Hz}, 1 \mathrm{H}), 2.11-2.02(\mathrm{~m}, 2 \mathrm{H}), 1.70-1.62(\mathrm{~m}, 1 \mathrm{H}), 1.56-1.43(\mathrm{~m}, 2 \mathrm{H}), 1.35-1.17(\mathrm{~m}, 2 \mathrm{H}), 1.03(\mathrm{~s}$, $3 \mathrm{H}), 1.00-0.94(\mathrm{t}, J=6.6 \mathrm{~Hz}, 3 \mathrm{H}), 0.86(\mathrm{t}, J=6.7 \mathrm{~Hz}, 3 \mathrm{H})$.

${ }^{13} \mathrm{C}$-NMR: mixture of rotamers is observed $\left(126 \mathrm{MHz}, \mathrm{CDCl}_{3}\right) \delta 155.2 / 155.0(\mathrm{C}), 137.2 / 137.0(\mathrm{C}), 128.4 / 128.3$ (2xCH), 128.0/127.8 (2xCH), 127.8/127.7 (CH), 66.7/66.4 $\left(\mathrm{CH}_{2}\right), 63.5 / 63.1(\mathrm{CH}), 44.6 / 44.3\left(\mathrm{CH}_{2}\right), 44.0 / 43.3$ $\left(\mathrm{CH}_{2}\right), 37.7 / 36.9(\mathrm{CH}), 30.8 / 29.9\left(\mathrm{CH}_{2}\right), 25.7(\mathrm{CH}), 23.7\left(\mathrm{CH}_{3}\right), 22.0 / 21.9\left(\mathrm{CH}_{3}\right), 19.8 / 19.7\left(\mathrm{CH}_{3}\right)$.

IR (ATR): $\tilde{v}\left(\mathrm{~cm}^{-1}\right)$ 2957, 1702, 1455, 1411, 1349, 1199, 1102, 1029, 912, 768, 740, 697.

HRMS: calculated for $\mathrm{C}_{17} \mathrm{H}_{26} \mathrm{NO}_{2}\left(\mathrm{M}+\mathrm{H}^{+}\right)$276.1958, found 276.1959.

Rf: 0.18 (95:5 pentane:EtOAc).

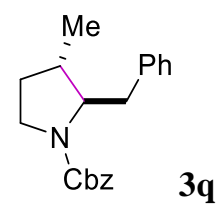

Obtained following general procedure B from $63 \mathrm{mg}(0.1 \mathrm{mmol})$ of $\mathbf{2 q}$ and $4 \mathrm{mg}$ of q-OAc (25 mol\%). Column chromatography (9:1 pentane:EtOAc) afforded the product in $26 \%$ yield $(8 \mathrm{mg})$ as a single diastereoisomer. The control reaction in the absence of q-OAc gave no product formation.

${ }^{1}$ H-NMR: (500 MHz, Chloroform- $d$ ) rotamers are observed $\delta 7.48-7.31(\mathrm{~m}, 5 \mathrm{H}), 7.26-7.04(\mathrm{~m}, 5 \mathrm{H}), 5.21$ (s, 2H), $3.70(\mathrm{dd}, J=16.9,8.7 \mathrm{~Hz}, 1 \mathrm{H}), 3.58$ (d, $J=6.8 \mathrm{~Hz}, 1 \mathrm{H}), 3.29$ (br s, 1H), $3.17-2.99$ (m, $1 \mathrm{H}), 2.86-$ 2.68 (m, 1H), 2.10 (br s, 1H), 1.89 (d, J=7.0 Hz, 1H), 1.44 (br s, 1H), 0.89 (d, J = 6.9 Hz, 3H).

${ }^{13}$ C-NMR: $\left(126 \mathrm{MHz} \mathrm{CDCl}_{3}\right) \delta$ 155.1/154.9 (C), $138.6(\mathrm{C}), 137.2 / 136.8(\mathrm{C}), 129.7 / 129.4(2 \times C H), 129.2(\mathrm{CH})$, $128.5(2 \times C H), 128.4 / 128.3(2 x C H), 128.0 / 127.8(2 x C H), 126.3 / 126.2(\mathrm{CH}), 67.0 / 66.5\left(\mathrm{CH}_{2}\right), 66.0 / 65.8(\mathrm{CH})$, 45.4/45.2 $\left(\mathrm{CH}_{2}\right)$, 40.2/38.7 $\left(\mathrm{CH}_{2}\right), 36.8 / 35.8(\mathrm{CH}), 31.1 / 30.2\left(\mathrm{CH}_{2}\right), 19.4 / 19.2\left(\mathrm{CH}_{3}\right)$.

IR (ATR): $\tilde{v}\left(\mathrm{~cm}^{-1}\right)$ 3029, 2958, 1697, 1495, 1453, 1412, 1345, 1094, 768, 698.

HRMS: calculated for $\mathrm{C}_{20} \mathrm{H}_{24} \mathrm{NO}_{2}\left(\mathrm{M}+\mathrm{H}^{+}\right) 310.1802$, found 310.1802.

Rf: 0.44 (8:2 pentane:EtOAc). 


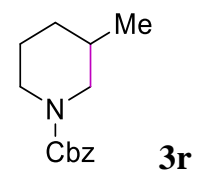

Obtained following general procedure B from $56 \mathrm{mg}(0.1 \mathrm{mmol})$ of $\mathbf{2 r}$ and $4 \mathrm{mg}$ of q-OAc $(25 \mathrm{~mol} \%)$. Column chromatography (95:5 to 9:1 pentane:EtOAc) afforded the major product $3 \mathbf{r}$ in $41 \%$ yield (9.6 mg) as a colorless oil. The control reaction in the absence of q-OAc gave no product formation.

${ }^{1}$ H-NMR: (400 MHz, Chloroform- $d$ ) $\delta 7.40-7.35(\mathrm{~m}, 4 \mathrm{H}), 7.35-7.29(\mathrm{~m}, 1 \mathrm{H}), 5.22(\mathrm{~s}, 2 \mathrm{H}), 4.07(\mathrm{~d}, J=11.5$ $\mathrm{Hz}, 1 \mathrm{H}), 4.00$ (br s, 1H), 2.78 (ddd, $J=13.1,11.7,3.1 \mathrm{~Hz}, 1 \mathrm{H}), 2.43$ (br s, 1H), 1.81 (ddd, $J=11.6,3.9,1.9$ $\mathrm{Hz}, 2 \mathrm{H}), 1.70-1.51(\mathrm{~m}, 2 \mathrm{H}), 1.08(\mathrm{qd}, J=12.2,3.9 \mathrm{~Hz}, 1 \mathrm{H}), 0.90(\mathrm{~d}, J=6.6 \mathrm{~Hz}, 3 \mathrm{H})$.

${ }^{13}$ C-NMR: (126 MHz, $\left.\mathrm{CDCl}_{3}\right) \delta 155.0(\mathrm{C}), 137.0(\mathrm{C}), 128.5(2 \times \mathrm{CH}), 127.9(\mathrm{CH}), 127.8(2 \times \mathrm{CH}), 66.9\left(\mathrm{CH}_{2}\right)$, 51.3 $\left(\mathrm{CH}_{2}\right), 44.4\left(\mathrm{CH}_{2}\right), 32.9\left(\mathrm{CH}_{2}\right), 30.9(\mathrm{CH}), 25.3 / 24.9\left(\mathrm{CH}_{2}\right), 18.9\left(\mathrm{CH}_{3}\right)$.

IR (ATR): $\tilde{v}\left(\mathrm{~cm}^{-1}\right)$ 2929, 2853, 1698, 1497, 1467, 1428, 1257, 1232, 1154, 1096, 912, 765, 697.

HRMS: calculated for $\mathrm{C}_{14} \mathrm{H}_{20} \mathrm{NO}_{2}\left(\mathrm{M}+\mathrm{H}^{+}\right)$234.1489, found 234.1489.

Rf: 0.66 ( 7:3 pentane:EtOAc).<smiles>c1ccc(C(c2ccccc2)P(c2ccccc2)c2ccccc2)cc1</smiles>

Obtained following general procedure B from $49 \mathrm{mg}(0.1 \mathrm{mmol})$ of $\mathbf{2 s}$ and $4 \mathrm{mg}$ of q-OAc $(25 \mathrm{~mol} \%)$. Column chromatography (95:5 to 9:1 pentane:EtOAc) afforded the major product $3 \mathbf{s}$ in $24 \%$ yield (3 mg) as colorless needles ( $\mathbf{m p :} 202.2^{\circ} \mathrm{C}$ ). The control reaction in the absence of q-OAc gave only $10 \%$ of product formation.

${ }^{1}$ H-NMR: (500 MHz, Chloroform- $\left.d\right) \delta 7.22-7.17(\mathrm{~m}, 8 \mathrm{H}), 7.13(\mathrm{t}, J=7.6 \mathrm{~Hz}, 8 \mathrm{H}), 7.08-7.00(\mathrm{~m}, 4 \mathrm{H}), 4.80$ $(\mathrm{s}, 2 \mathrm{H})$.

${ }^{13}$ C-NMR: (126 MHz, $\left.\mathrm{CDCl}_{3}\right) \delta 143.4(4 \mathrm{xC}), 128.5(8 \mathrm{xCH}), 128.1(8 \mathrm{xCH}), 125.8(4 \mathrm{xCH}), 56.3(2 \mathrm{xCH})$.

IR (ATR): $\tilde{v}\left(\mathrm{~cm}^{-1}\right)$ 3025, 2924, 1598, 1493, 1449, 1072, 744, 695.

HRMS: calculated for $\mathrm{C}_{16} \mathrm{H}_{21}\left(\mathrm{M}+\mathrm{H}^{+}\right) 333.1649$, found 333.1640 .

Rf: 0.54 (8:2 pentane:EtOAc).

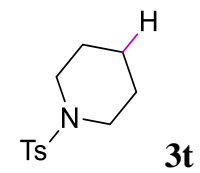

Obtained following general procedure B from $56 \mathrm{mg}(0.1 \mathrm{mmol})$ of $\mathbf{2 t}$ and $4 \mathrm{mg}$ of q-OAc $(25 \mathrm{~mol} \%)$. Column chromatography (8:2 pentane:EtOAc) afforded the product in $77 \%$ yield $(18 \mathrm{mg})$ as a white solid (mp: 71.2 ${ }^{\circ} \mathrm{C}$ ). The control reaction in the absence of q-OAc gave only $14 \%$ of product formation. 
${ }^{1}$ H-NMR: (500 MHz, Chloroform- $d$ ) $\delta 7.66(\mathrm{~d}, J=8.2 \mathrm{~Hz}, 2 \mathrm{H}), 7.34(\mathrm{~d}, J=8.0 \mathrm{~Hz}, 2 \mathrm{H}), 3.26-2.88(\mathrm{~m}, 4 \mathrm{H})$, $2.46(\mathrm{~s}, 3 \mathrm{H}), 1.66(\mathrm{p}, J=5.8 \mathrm{~Hz}, 4 \mathrm{H}), 1.48-1.34(\mathrm{~m}, 2 \mathrm{H})$.

${ }^{13}$ C-NMR: (126 MHz, $\left.\mathrm{CDCl}_{3}\right) \delta 143.3(\mathrm{C}), 133.2(\mathrm{C}), 129.5(2 \mathrm{xCH}), 127.7(2 \mathrm{xCH}), 46.9\left(2 \mathrm{xCH}_{2}\right), 25.2$ $\left(2 \mathrm{xCH}_{2}\right), 23.5\left(\mathrm{CH}_{2}\right), 21.6\left(\mathrm{CH}_{3}\right)$.

IR (ATR): $\tilde{v}\left(\mathrm{~cm}^{-1}\right): 3212,2925,2854,1771,1704,1516,1454,1365,1318,1164,1084,926,752,713$.

HRMS: calculated for $\mathrm{C}_{12} \mathrm{H}_{18} \mathrm{NO}_{2} \mathrm{~S}\left[\mathrm{M}+\mathrm{H}^{+}\right] 240.1053$, found 240.1049 .

Rf: 0.34 ( $8: 2$ pentane:EtOAc)

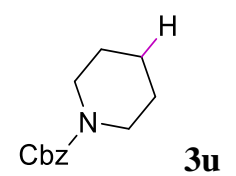

Obtained following general procedure B from $54 \mathrm{mg}(0.1 \mathrm{mmol})$ of $\mathbf{2 u}$ and $4 \mathrm{mg}$ of q-OAc $(25 \mathrm{~mol} \%)$. Column chromatography (9:1 pentane:EtOAc) afforded the product in $62 \%$ yield $(13.4 \mathrm{mg})$ as a colorless wax. The control reaction in the absence of q-OAc gave no product formation.

${ }^{1}$ H-NMR: (500 MHz, Chloroform- $d$ ) $\delta 7.38(\mathrm{~d}, J=4.3 \mathrm{~Hz}, 4 \mathrm{H}), 7.38-7.29(\mathrm{~m}, 1 \mathrm{H}), 5.15(\mathrm{~s}, 2 \mathrm{H}), 3.62-3.40$ (m, 4H), 1.61 (dd, $J=8.4,4.2 \mathrm{~Hz}, 2 \mathrm{H}), 1.56$ (br s, 4H).

${ }^{13}$ C-NMR: $\left(101 \mathrm{MHz}, \mathrm{CDCl}_{3}\right) \delta 155.3(\mathrm{C}), 137.1(\mathrm{C}), 128.4(2 \mathrm{xCH}), 127.9(\mathrm{CH}), 127.8(2 \mathrm{xCH}), 66.9\left(\mathrm{CH}_{2}\right)$, $44.9\left(2 \mathrm{xCH}_{2}\right), 25.7\left(2 \mathrm{xCH}_{2}\right), 24.4\left(\mathrm{CH}_{2}\right)$.

IR (ATR): $\tilde{v}\left(\mathrm{~cm}^{-1}\right)$ 2937, 2855, 1697, 1497, 1469, 1428, 1262, 1233, 1147, 1025, 697.

HRMS: calculated for $\mathrm{C}_{13} \mathrm{H}_{18} \mathrm{NO}_{2}\left(\mathrm{M}+\mathrm{H}^{+}\right) 220.1332$, found 220.1332 .

Rf: 0.38 (8:2 pentane: EtOAc).

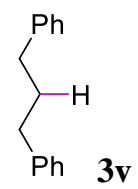

Obtained following general procedure B from $52 \mathrm{mg}(0.1 \mathrm{mmol})$ of $2 \mathrm{v}$ and $4 \mathrm{mg}$ of q-OAc $(25 \mathrm{~mol} \%)$. Column chromatography ( $97: 3$ pentane:EtOAc) afforded the product in $67 \%$ yield $(13 \mathrm{mg}$ ) as a colorless oil. The control reaction in the absence of q-OAc gave only $24 \%$ of product formation.

${ }^{1}$ H-NMR: (500 MHz, Chloroform- $d$ ) $\delta 7.33-7.29(\mathrm{~m}, 4 \mathrm{H}), 7.23-7.19(\mathrm{~m}, 6 \mathrm{H}), 2.83-2.62(\mathrm{~m}, 4 \mathrm{H}), 2.00$ (tt, $J=9.2,7.0 \mathrm{~Hz}, 2 \mathrm{H})$.

${ }^{13}$ C-NMR: (126 MHz, $\left.\mathrm{CDCl}_{3}\right) \delta 142.3(2 \mathrm{xC}), 128.5(4 \mathrm{xCH}), 128.3(4 \mathrm{xCH}), 125.7(2 \mathrm{xCH}), 35.5\left(2 \mathrm{xCH}_{2}\right), 33.0$ $\left(\mathrm{CH}_{2}\right)$.

IR (ATR): $\tilde{v}\left(\mathrm{~cm}^{-1}\right):$ 3062, 3026, 2926, 2856, 1603, 1496, 1453, 743, 697. 
HRMS: calculated for $\mathrm{C}_{15} \mathrm{H}_{15}\left[\mathrm{M}-\mathrm{H}^{+}\right]$195.1179, found 195.1179 .

Rf: 0.77 (95:5 Pentane:EtOAc).

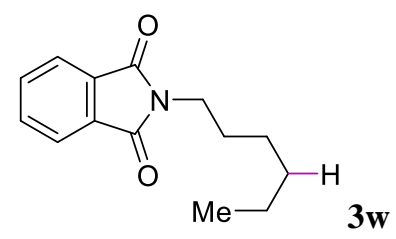

Obtained following general procedure $\mathbf{B}$ from $28 \mathrm{mg}(0.05 \mathrm{mmol})$ of $\mathbf{2 w}$ and $2 \mathrm{mg}$ of q-OAc $(25 \mathrm{~mol} \%)$ in 0.5 $\mathrm{mL}$ of DMF. Column chromatography (95:5 pentane:EtOAc) afforded the product in $62 \%$ yield $(7.2 \mathrm{mg}$ ) as a colorless wax. The control reaction in the absence of q-OAc gave no product formation.

${ }^{1}$ H-NMR: (500 MHz, Chloroform- $d$ ) $\delta 7.86(\mathrm{dd}, J=5.4,3.0 \mathrm{~Hz}, 2 \mathrm{H}), 7.73(\mathrm{dd}, J=5.4,3.0 \mathrm{~Hz}, 2 \mathrm{H}), 3.70(\mathrm{t}, J$ $=7.3 \mathrm{~Hz}, 2 \mathrm{H}), 1.69(\mathrm{p}, J=6.9 \mathrm{~Hz}, 2 \mathrm{H}), 1.45-1.20(\mathrm{~m}, 6 \mathrm{H}), 0.90(\mathrm{t}, J=6.9 \mathrm{~Hz}, 3 \mathrm{H})$.

${ }^{13}$ C-NMR: $\left(75 \mathrm{MHz}, \mathrm{CDCl}_{3}\right) \delta 168.5(2 \mathrm{xC}), 133.8(2 \mathrm{xCH}), 132.2(2 \mathrm{xC}), 123.1(2 \mathrm{xCH}), 38.1\left(\mathrm{CH}_{2}\right), 31.4\left(\mathrm{CH}_{2}\right)$, $28.6\left(\mathrm{CH}_{2}\right), 26.5\left(\mathrm{CH}_{2}\right), 22.5\left(\mathrm{CH}_{2}\right), 14.0\left(\mathrm{CH}_{3}\right)$.

IR (ATR): $\tilde{v}\left(\mathrm{~cm}^{-1}\right)$ 2931, 2859, 1773, 1711, 1467, 1437, 1396, 1367, 719.

HRMS: calculated for $\mathrm{C}_{14} \mathrm{H}_{18} \mathrm{NO}_{2}\left(\mathrm{M}+\mathrm{H}^{+}\right)$232.1332, found 232.1333.

Rf: 0.50 (pentane:EtOAc). 


\section{Characterization of products $4 a, 4 k, 4 p, 4 r, 4 s$ and $5 p$ :}<smiles>CCC(c1ccccc1)N1C(=O)c2c(Cl)c(Cl)c(Cl)c(Cl)c2C1=O</smiles>

Obtained following general procedure B from $45 \mathrm{mg}(0.1 \mathrm{mmol})$ of $\mathbf{2 a}$ and $4 \mathrm{mg}$ of q-OAc $(25 \mathrm{~mol} \%)$. Column chromatography (9:1 to 7:3 pentane:EtOAc) afforded minor product $4 \mathbf{a}$ in $3 \%$ yield ( $2 \mathrm{mg}$ ) as a colorless wax. The control reaction in the absence of q-OAc gave no product formation.

${ }^{1}$ H-NMR: (500 MHz, Chloroform- $d$ ) $\delta 7.46(\mathrm{dd}, J=7.7,1.6 \mathrm{~Hz}, 2 \mathrm{H}), 7.42-7.30(\mathrm{~m}, 3 \mathrm{H}), 5.27(\mathrm{t}, J=7.0 \mathrm{~Hz}$, $1 \mathrm{H}), 2.22(\mathrm{dp}, J=14.3,7.5 \mathrm{~Hz}, 1 \mathrm{H}), 1.98(\mathrm{dp}, J=14.3,7.5 \mathrm{~Hz}, 1 \mathrm{H}), 1.01(\mathrm{t}, J=7.5 \mathrm{~Hz}, 1 \mathrm{H})$.

${ }^{13}$ C-NMR: (126 MHz, $\left.\mathrm{CDCl}_{3}\right) \delta 159.3(2 \mathrm{xC}), 140.5(2 \mathrm{xC}), 137.5(\mathrm{C}), 129.8(2 \mathrm{xC}), 129.2(\mathrm{CH}), 128.4(2 \mathrm{xCH})$, $128.0(2 \times C H), 124.5(2 \times C), 91.2(\mathrm{CH}), 28.0\left(\mathrm{CH}_{2}\right), 10.1\left(\mathrm{CH}_{3}\right)$.

IR (ATR): $\tilde{v}\left(\mathrm{~cm}^{-1}\right)$ 2925, 1765, 1736, 1455, 1366, 1197, 1031, 730.

HRMS: calculated for $\mathrm{C}_{17} \mathrm{H}_{12} \mathrm{Cl}_{4} \mathrm{NO}_{2}\left(\mathrm{M}+\mathrm{H}^{+}\right)$401.9617, found 401.9625 .

Rf: 0.62 (9:1 pentane:EtOAc).<smiles>C=CCOCC(NC(=O)c1ccccc1)N1C(=O)c2c(Cl)c(Cl)c(Cl)c(Cl)c2C1=O</smiles>

Obtained following general procedure $\mathbf{B}$ from $52.5 \mathrm{mg}(0.1 \mathrm{mmol})$ of $\mathbf{2 k}$ and $4 \mathrm{mg}$ of q-OAc ( $25 \mathrm{~mol} \%)$. Column chromatography (9:1 to 7:3 pentane:EtOAc) afforded minor product $\mathbf{4 k}$ in $8 \%$ yield (4 $\mathrm{mg}$ ) as a colorless wax.

${ }^{1}$ H-NMR: (500 MHz, Chloroform- $d$ ) $\delta 6.17(\mathrm{~s}, 1 \mathrm{H}), 5.83$ (ddt, $\left.J=16.3,10.9,5.6 \mathrm{~Hz}, 1 \mathrm{H}\right), 5.71(\mathrm{~s}, 1 \mathrm{H}), 5.25$ (dd, $J=17.2,1.8 \mathrm{~Hz}, 1 \mathrm{H}), 5.22-5.16(\mathrm{~m}, 1 \mathrm{H}), 4.03(\mathrm{qd}, J=12.8,5.5 \mathrm{~Hz}, 2 \mathrm{H}), 3.88(\mathrm{dd}, J=10.3,8.8 \mathrm{~Hz}, 1 \mathrm{H})$, $3.73(\mathrm{dd}, J=10.3,5.7 \mathrm{~Hz}, 1 \mathrm{H}), 1.45(\mathrm{~s}, 9 \mathrm{H})$.

${ }^{13}$ C-NMR: (126 MHz, $\left.\mathrm{CDCl}_{3}\right) \delta 163.0(2 \mathrm{xC}), 154.2(\mathrm{C}), 140.3(2 \mathrm{xC}), 133.8(\mathrm{CH}), 129.9(2 \mathrm{xC}), 127.3(2 \mathrm{xC})$, 117.9 $\left(\mathrm{CH}_{2}\right), 81.0(\mathrm{C}), 72.0\left(\mathrm{CH}_{2}\right), 67.9\left(\mathrm{CH}_{2}\right), 57.7(\mathrm{CH}), 28.2\left(3 \mathrm{xCH}_{3}\right)$.

IR (ATR): $\tilde{v}\left(\mathrm{~cm}^{-1}\right): 3251,2977,1707,1368,1319,1163,1084,753,714$.

HRMS: calculated for $\mathrm{C}_{18} \mathrm{H}_{19} \mathrm{Cl}_{4} \mathrm{~N}_{2} \mathrm{O}_{5}\left[\mathrm{M}+\mathrm{H}^{+}\right] 483.0043$, found 483.0038 .

$\mathbf{R}_{\mathbf{f}}$ 0.75 (8:2 pentane:EtOAc). 


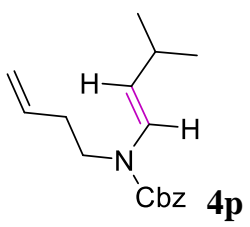

Obtained following general procedure $\mathbf{X}$ from $60 \mathrm{mg}(0.1 \mathrm{mmol})$ of $\mathbf{X}$ and $4 \mathrm{mg}$ of q-OAc $(25 \mathrm{~mol} \%)$. Column chromatography (97:3 to 9:1 pentane:EtOAc) afforded major product $\mathbf{4 p}$ in $35 \%$ yield (9.5 $\mathrm{mg}$ ) as a colorless wax. The control reaction in the absence of q-OAc gave no product formation. This enamine was found to be highly unstable over time. Signals of the hydrolyzed product can be observed in the ${ }^{13} \mathrm{C}-\mathrm{NMR}$ spectra. Only the signals of the enamine product are described.

${ }^{1}$ H-NMR: mixture of rotamers is observed (500 MHz, Chloroform- $d$ ) $\delta 7.46-7.31(\mathrm{~m}, 5 \mathrm{H}), 6.83(\mathrm{dd}, J=59.2$, $14.1 \mathrm{~Hz}, 1 \mathrm{H}), 5.78(\mathrm{dq}, J=21.7,6.3,5.2 \mathrm{~Hz}, 1 \mathrm{H}), 5.21(\mathrm{~s}, 2 \mathrm{H}), 5.14-5.00(\mathrm{~m}, 2 \mathrm{H}), 4.93-4.82(\mathrm{~m}, 1 \mathrm{H}), 3.74$ $-3.35(\mathrm{~m}, 2 \mathrm{H}), 2.41-2.18(\mathrm{~m}, 3 \mathrm{H}), 1.05(\mathrm{~s}, 3 \mathrm{H}), 1.03(\mathrm{~s}, 3 \mathrm{H})$.

${ }^{13} \mathrm{C}$-NMR: mixture of rotamers is observed $\left(126 \mathrm{MHz}, \mathrm{CDCl}_{3}\right) \delta 154.3 / 153.8(\mathrm{C}), 136.6 / 136.4(\mathrm{CH})$, 135.1/135.0 (C), $128.5(2 \times C H), 128.1(\mathrm{CH}), 128.0(2 \times C H), 124.8 / 124.1(\mathrm{CH}), 117.4 / 117.2(\mathrm{CH}), 116.7 / 116.6$ $\left(\mathrm{CH}_{2}\right), 67.5\left(\mathrm{CH}_{2}\right), 43.5 / 43.2\left(\mathrm{CH}_{2}\right), 31.7 / 31.1\left(\mathrm{CH}_{2}\right), 29.7 / 29.6(\mathrm{CH}), 23.5\left(\mathrm{CH}_{3}\right), 23.4 / 22.6\left(\mathrm{CH}_{3}\right)$.

IR (ATR): $\tilde{v}\left(\mathrm{~cm}^{-1}\right)$ 3068, 3033, 2957, 2867, 1708, 1661, 1642, 1456, 1409, 1355, 1280, 1253, 1204, 1149, 915, $792,697$.

HRMS: calculated for $\mathrm{C}_{17} \mathrm{H}_{24} \mathrm{NO}_{2}\left(\mathrm{M}+\mathrm{H}^{+}\right)$274.1807, found 274.1802.

Rf: 0.47 (95:5 pentane:EtOAc).

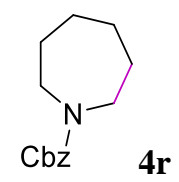

Obtained following general procedure B from $56 \mathrm{mg}(0.1 \mathrm{mmol})$ of $\mathbf{2 r}$ and $4 \mathrm{mg}$ of q-OAc $(25 \mathrm{~mol} \%)$. Column chromatography (95:5 to 9:1 pentane:EtOAc) afforded minor product $4 \mathbf{r}$ in $23 \%$ yield (5.4 $\mathrm{mg}$ ) as a colorless oil. The control reaction in the absence of q-OAc gave no product formation.

${ }^{1}$ H-NMR: (500 MHz, Chloroform- $\left.d\right) \delta 7.38(\mathrm{~d}, J=4.3 \mathrm{~Hz}, 4 \mathrm{H}), 7.36-7.29(\mathrm{~m}, 1 \mathrm{H}), 5.16(\mathrm{~s}, 2 \mathrm{H}), 3.47(\mathrm{dt}, J$ $=20.2,6.0 \mathrm{~Hz}, 4 \mathrm{H}), 1.83-1.62(\mathrm{~m}, 4 \mathrm{H}), 1.63-1.49(\mathrm{~m}, 4 \mathrm{H})$.

${ }^{13}$ C-NMR: $\left(126 \mathrm{MHz} \mathrm{CDCl}_{3}\right) \delta 156.2(\mathrm{C}), 137.2(\mathrm{C}), 128.4(2 \times \mathrm{CH}), 127.8(\mathrm{CH}), 127.7(2 \times \mathrm{CH}), 66.8\left(\mathrm{CH}_{2}\right)$, 47.1/46.6 $\left(2 \mathrm{xCH}_{2}\right), 28.6 / 28.3\left(2 \mathrm{xCH}_{2}\right), 27.4 / 27.0\left(2 \mathrm{xCH}_{2}\right)$.

IR (ATR): $\tilde{v}\left(\mathrm{~cm}^{-1}\right)$ 2931, 1668, 1422, 1351, 1260, 1202, 1076, 996, 757, 597.

HRMS: calculated for $\mathrm{C}_{14} \mathrm{H}_{20} \mathrm{NO}_{2}\left(\mathrm{M}+\mathrm{H}^{+}\right)$234.1489, found 234.1489.

Rf: 0.61 ( $7: 3$ pentane:EtOAc). 
<smiles>c1ccc([PbH](c2ccccc2)c2ccccc2)cc1</smiles>

Obtained following general procedure $\mathbf{B}$ from $49 \mathrm{mg}(0.1 \mathrm{mmol})$ of $2 \mathrm{~s}$ and $4 \mathrm{mg}$ of q-OAc $(25 \mathrm{~mol} \%)$. Column chromatography (95:5 to 9:1 pentane:EtOAc) afforded the minor product $4 \mathrm{~s}$ in $18 \%$ yield (2 $\mathrm{mg}$ ) as yelloworange needles ( $\mathbf{m p :} 222.3{ }^{\circ} \mathrm{C}$ ). The control reaction in the absence of q-OAc gave no product formation.

${ }^{1}$ H-NMR: (500 MHz, Chloroform- $d$ ) $\delta 7.16-7.07$ (m, 12H), $7.09-7.02(\mathrm{~m}, 8 \mathrm{H})$.

${ }^{13}$ C-NMR: (126 MHz, $\left.\mathrm{CDCl}_{3}\right) \delta 143.7$ (2xC), $140.9(4 \mathrm{xC}), 131.3(8 \mathrm{xCH}), 127.6(8 \mathrm{xCH}), 126.4(4 \mathrm{xCH})$.

IR (ATR): $\tilde{v}\left(\mathrm{~cm}^{-1}\right)$ 3051, 2922, 2852, 1596, 1490, 1443, 1029, 746, 760, 698.

HRMS: calculated for $\mathrm{C}_{16} \mathrm{H}_{20}\left(\mathrm{M}^{+}\right) 332.1565$, found 332.1559 .

Rf: 0.80 (8:2 pentane:EtOAc).

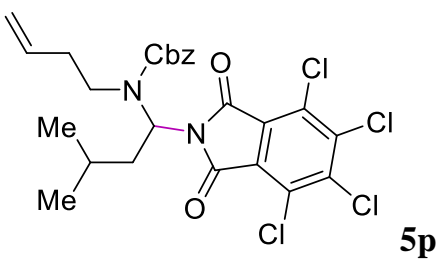

Obtained following general procedure $\mathbf{X}$ from $60 \mathrm{mg}(0.1 \mathrm{mmol})$ of $\mathbf{X}$ and $4 \mathrm{mg}$ of q-OAc $(25 \mathrm{~mol} \%)$. Column chromatography (97:3 to 9:1 pentane:EtOAc) afforded minor product $\mathbf{5 p}$ in $14 \%$ yield $(8 \mathrm{mg})$ as a colorless wax. The control reaction in the absence of q-OAc gave no product formation.

${ }^{1}$ H-NMR: (500 MHz, Chloroform- $d$ ) $\delta 7.36(\mathrm{~d}, J=7.1 \mathrm{~Hz}, 4 \mathrm{H}), 7.35-7.29(\mathrm{~m}, 1 \mathrm{H}), 6.40(\mathrm{~d}, J=56.9 \mathrm{~Hz}, 1 \mathrm{H})$, $5.73(\mathrm{br} \mathrm{s}, 1 \mathrm{H}), 5.27$ (br s, 1H), $5.13(\mathrm{dd}, J=23.3,10.5 \mathrm{~Hz}, 1 \mathrm{H}), 5.03(\mathrm{~d}, J=14.4 \mathrm{~Hz}, 1 \mathrm{H}), 4.97$ (d, $J=10.1$ $\mathrm{Hz}, 1 \mathrm{H}), 3.63-3.54(\mathrm{~m}, 2 \mathrm{H}), 2.38-2.23(\mathrm{~m}, 3 \mathrm{H}), 1.88$ (ddd, $J=14.0,8.3,6.0 \mathrm{~Hz}, 1 \mathrm{H}), 1.47$ (br s, $1 \mathrm{H}), 1.05$ $-0.93(\mathrm{~m}, 6 \mathrm{H})$.

${ }^{13} \mathrm{C}$-NMR: mixture of rotamers is observed $\left(126 \mathrm{MHz}, \mathrm{CDCl}_{3}\right) \delta 163.3(2 \times \mathrm{x}), 155.8 / 155.3(\mathrm{C}), 140.3(2 \times \mathrm{C})$, $136.2(\mathrm{C}), 135.0(\mathrm{CH}), 129.9(2 \times \mathrm{C}), 128.4(2 \mathrm{xCH}), 128.0(2 \mathrm{xCH}), 127.8(2 \mathrm{xC}), 127.1(\mathrm{CH}), 116.9\left(\mathrm{CH}_{2}\right), 67.6$ $\left(\mathrm{CH}_{2}\right), 62.7(\mathrm{CH}), 44.3 / 43.8\left(\mathrm{CH}_{2}\right), 39.0 / 38.7\left(\mathrm{CH}_{2}\right), 34.6 / 33.7\left(\mathrm{CH}_{2}\right), 24.9(\mathrm{CH}), 23.0 / 21.9\left(\mathrm{CH}_{3}\right)$.

IR (ATR): $\tilde{v}\left(\mathrm{~cm}^{-1}\right)$ 3067, 2959, 2871, 1780, 1724, 1455, 1402, 1370, 1339, 1319, 1261, 1173, 1093, 995, 855, 739.

HRMS: calculated for $\mathrm{C}_{25} \mathrm{H}_{25} \mathrm{Cl}_{4} \mathrm{~N}_{2} \mathrm{O}_{4}\left(\mathrm{M}+\mathrm{H}^{+}\right)$559.0533, found 559.0534 .

Rf: 0.27 (95:5 pentane:EtOAc). 


\section{Synthesis and characterization of acids S1:}

\section{Synthesis of acid S1e:}

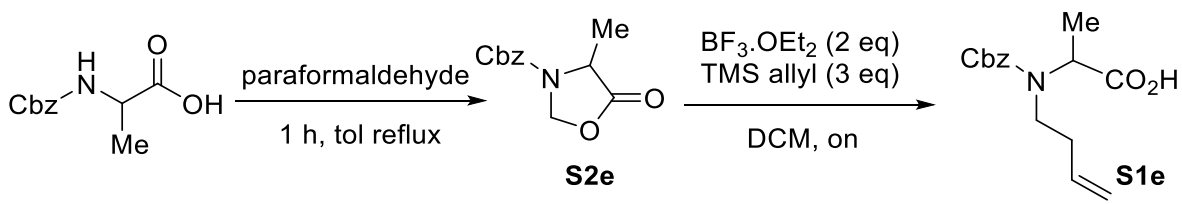<smiles>CC(C)N1COC(=O)C1[N+]([O-])([O-])[O-]</smiles>

Obtained following general procedure B from $5 \mathrm{mmol}$ of $\mathrm{N}$-Cbz-alanine. Column chromatography (8:2 pentane:EtOAc) afforded the product in $85 \%$ yield $(1.01 \mathrm{~g})$ as a white solid $\left(\mathbf{m p}: 62.9^{\circ} \mathrm{C}\right)$.

${ }^{1}$ H-NMR: (500 MHz, Chloroform- $d$ ) $\delta 7.44-7.35$ (m, 5H), 5.50 (br s, 1H), 5.31 (d, $\left.J=4.4 \mathrm{~Hz}, 1 \mathrm{H}\right), 5.27-$ $5.15(\mathrm{~m}, 2 \mathrm{H}), 4.33$ (br s, 1H), 1.56 (br s, 3H).

${ }^{13}$ C-NMR: $\left(126 \mathrm{MHz}, \mathrm{CDCl}_{3}\right) \delta 172.9(\mathrm{C}), 152.8 / 152.3(\mathrm{C}), 135.4(\mathrm{C}), 128.7(2 \mathrm{xCH}), 128.6(2 \mathrm{xCH}), 128.3$ $(\mathrm{CH}), 77.5\left(\mathrm{CH}_{2}\right), 67.9\left(\mathrm{CH}_{2}\right), 50.7(\mathrm{CH}), 17.0 / 16.3\left(\mathrm{CH}_{3}\right)$.

IR (ATR): $\tilde{v}\left(\mathrm{~cm}^{-1}\right): 3066,3034,1796,1709,1499,1453,1411,1355,1247,1170,1129,1043,914,750,765$, 698.

HRMS: calculated for $\mathrm{C}_{29} \mathrm{H}_{23} \mathrm{Cl}_{4} \mathrm{~N}_{2} \mathrm{O}_{6}\left(\mathrm{M}+\mathrm{H}^{+}\right)$637.0275, found 637.0267.

R: 0.26 (8:2 pentane:EtOAc).

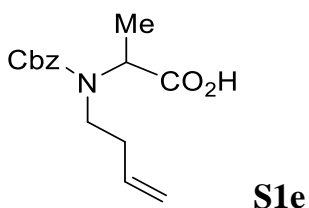

Obtained following general procedure B from $2 \mathrm{mmol}$ of oxazolidinone S2e as a colorless oil in 68\% yield (379 $\mathrm{mg})$. No further purification was performed.

${ }^{1}$ H-NMR: (500 MHz, Chloroform- $d$ ) 6:4 mixture of rotamers $\delta 7.42-7.32(\mathrm{~m}, 5 \mathrm{H}), 5.90-5.69(\mathrm{~m}, 1 \mathrm{H}), 5.23$ $-5.00(\mathrm{~m}, 4 \mathrm{H}), 4.49(\mathrm{q}, J=7.3 \mathrm{~Hz}, 0.6 \mathrm{H}), 4.34(\mathrm{q}, J=7.3,6.9 \mathrm{~Hz}, 0.4 \mathrm{H}), 3.50(\mathrm{tdd}, J=15.0,8.8,4.7 \mathrm{~Hz}, 1 \mathrm{H})$, 3.25 (dddd, $J=20.6,15.0,9.4,6.0 \mathrm{~Hz}, 1 \mathrm{H}$ ), 2.41 (ddt, $J=22.6,15.2,7.8 \mathrm{~Hz}, 1 \mathrm{H}$ ), 2.31 (dt, $J=14.4,7.2 \mathrm{~Hz}$, $1 \mathrm{H}), 1.53(\mathrm{~d}, J=7.3 \mathrm{~Hz}, 1.8 \mathrm{H}), 1.50(\mathrm{~d}, J=7.3 \mathrm{~Hz}, 1.2 \mathrm{H})$.

${ }^{13}$ C-NMR: $\left(126 \mathrm{MHz}, \mathrm{CDCl}_{3}\right) \delta$ 177.7/177.1 (C), 156.4/155.6 (C), 136.3/136.2 (C), 135.2/134.9 (CH), 128.5/128.4 (2xCH), 128.1/128.0 (CH), 127.9/127.8 (2xCH), 116.9/116.8 $\left(\mathrm{CH}_{2}\right), 67.6\left(\mathrm{CH}_{2}\right)$, 56.0/55.4 $(\mathrm{CH})$, 47.0/46.2 $\left(\mathrm{CH}_{2}\right), 34.1 / 33.4\left(\mathrm{CH}_{2}\right), 15.9 / 15.2\left(\mathrm{CH}_{3}\right)$.

IR (ATR): $\tilde{v}\left(\mathrm{~cm}^{-1}\right): 3069,3034,2983,2945,1743,1701,1476,1454,1422,1255,1203,1156,1066,1016$, 915, 786, 698 .

HRMS: calculated for $\mathrm{C}_{15} \mathrm{H}_{18} \mathrm{NO}_{4}\left(\mathrm{M}-\mathrm{H}^{+}\right)$276.1241, found 276.1243 . 


\section{Synthesis of acid S1h:}<smiles>COC(=O)C(O)Cc1ccccc1</smiles>

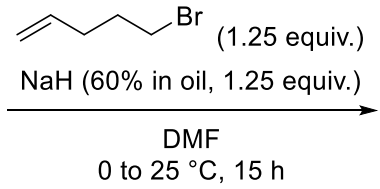

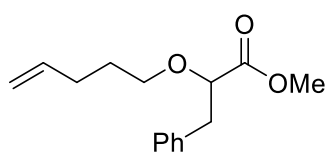

S2h

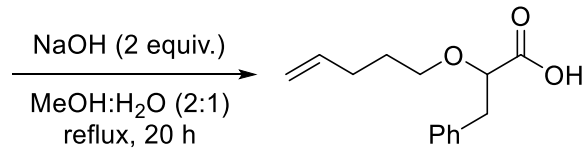

S1h

Methyl 3-phenyl lactate (721 mg, $4.0 \mathrm{mmol}$ ) was dissolved in $13.2 \mathrm{~mL}$ of dry DMF under Argon atmosphere. This solution was added to a cold $\left(0{ }^{\circ} \mathrm{C}\right)$ suspension of $\mathrm{NaH}(60 \%$ in oil $)(200 \mathrm{mg}, 5 \mathrm{mmol})$ in $3.3 \mathrm{~mL}$ of DMF. After complete addition, the reaction mixture was allowed to reach room temperature and stir for $3 \mathrm{~h}$. Then, 1bromo-4-pentene $(592 \mu \mathrm{L}, 5 \mathrm{mmol})$ was added and the reaction was stirred at room temperature for $15 \mathrm{~h}$. Water $(3 \mathrm{~mL})$ was then carefully added to quench the excess of $\mathrm{NaH}$. Extraction with EtOAc (x3) was followed by wash of the organic layers with thiosulphate (10\% aq. solution), water and brine. The organic layer was dried over $\mathrm{Na}_{2} \mathrm{SO}_{4}$, filtered and concentrated. The crude acid was purified using column chromatography (pentane:EtOAc 9:1 to 8:2) to give pure ester S2h as a colorless oil (329 $\mathrm{mg}, 33 \%$ ).

Substrate S2h (300 mg, $1.21 \mathrm{mmol})$ were dissolved in a 2:1 MeOH:H $2 \mathrm{O}$ mixture (10 mL). NaOH (96 mg, 2.42 mmol) were added and the solution was heated at $85{ }^{\circ} \mathrm{C}$ for $15 \mathrm{~h}$. After this time, the reaction was allowed to reach room temperature and the $\mathrm{MeOH}$ was eliminated under reduced vaccuo. The residual aqueous solution was washed once with EtOAc and acidified to $\mathrm{pH} 2$ using $1 \mathrm{M} \mathrm{HCl}$ aq. solution. Extraction with EtOAc (x3) was followed by wash of the organic layers with brine. The organic layer was dried over $\mathrm{Na}_{2} \mathrm{SO}_{4}$, filtered and concentrated to give acid $\mathbf{S 1 h}(236 \mathrm{mg}, 83 \%)$. This product was used in the next reaction without further purification.

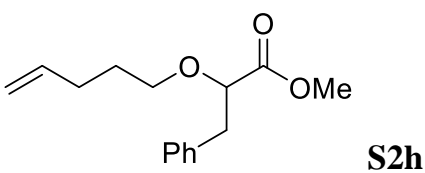

${ }^{1}$ H-NMR: (500 MHz, Chloroform- $d$ ) $\delta 7.37-7.28(\mathrm{~m}, 3 \mathrm{H}), 7.27-7.23(\mathrm{~m}, 2 \mathrm{H}), 5.74(\mathrm{ddt}, J=18.3,9.4,6.7$ $\mathrm{Hz}, 1 \mathrm{H}), 4.94(\mathrm{~s}, 1 \mathrm{H}), 4.91(\mathrm{dd}, J=6.0,1.7 \mathrm{~Hz}, 1 \mathrm{H}), 4.04(\mathrm{dd}, J=8.4,4.9 \mathrm{~Hz}, 1 \mathrm{H}), 3.74(\mathrm{~s}, 3 \mathrm{H}), 3.60(\mathrm{dt}, J=$ 9.2, $6.2 \mathrm{~Hz}, 1 \mathrm{H}), 3.27(\mathrm{dt}, J=9.1,6.6 \mathrm{~Hz}, 1 \mathrm{H}), 3.06(\mathrm{dd}, J=13.9,4.9 \mathrm{~Hz}, 1 \mathrm{H}), 3.01(\mathrm{dd}, J=13.9,8.4 \mathrm{~Hz}, 1 \mathrm{H})$, $2.03(\mathrm{pt}, J=7.8,6.4 \mathrm{~Hz}, 2 \mathrm{H}), 1.74-1.51(\mathrm{~m}, 2 \mathrm{H})$.

${ }^{13}$ C-NMR: (101 MHz, $\left.\mathrm{CDCl}_{3}\right) \delta 172.9(\mathrm{C}), 138.1(\mathrm{CH}), 137.2(\mathrm{C}), 129.4(2 \times \mathrm{xH}), 128.3(2 \times \mathrm{xH}), 126.6(\mathrm{CH})$, $114.8\left(\mathrm{CH}_{2}\right), 80.4(\mathrm{CH}), 70.1\left(\mathrm{CH}_{2}\right), 51.8\left(\mathrm{CH}_{3}\right), 39.4\left(\mathrm{CH}_{2}\right), 30.0\left(\mathrm{CH}_{2}\right), 28.7\left(\mathrm{CH}_{2}\right)$.

IR (ATR): $\tilde{v}\left(\mathrm{~cm}^{-1}\right)$ : 3065, 6060, 2950, 2869, 1752, 1640, 1496, 1454, 1436, 1175, 1196, 1121, 1017, 913, 699.

HRMS: calculated for $\mathrm{C}_{15} \mathrm{H}_{21} \mathrm{O}_{3}\left(\mathrm{M}+\mathrm{H}^{+}\right)$249.1485, found 249.1485 .

Rf: 0.68 (8:2 pentane:EtOAc). 


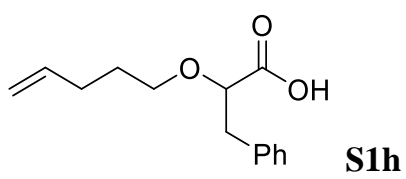

${ }^{1}$ H-NMR: (400 MHz, Chloroform- $d$ ) $\delta 7.36-7.30(\mathrm{~m}, 3 \mathrm{H}), 7.25-7.24(\mathrm{~m}, 2 \mathrm{H}), 5.74(\mathrm{ddt}, J=16.5,9.6,4.7$ $\mathrm{Hz}, 1 \mathrm{H}), 4.99-4.96(\mathrm{~m}, 1 \mathrm{H}), 4.96-4.92(\mathrm{~m}, 1 \mathrm{H}), 4.10(\mathrm{dd}, J=8.1,4.1 \mathrm{~Hz}, 1 \mathrm{H}), 3.59(\mathrm{dt}, J=9.1,6.3 \mathrm{~Hz}, 1 \mathrm{H})$, $3.38(\mathrm{dt}, J=9.2,6.5 \mathrm{~Hz}, 1 \mathrm{H}), 3.17(\mathrm{dd}, J=14.1,4.0 \mathrm{~Hz}, 1 \mathrm{H}), 3.02(\mathrm{dd}, J=14.1,8.1 \mathrm{~Hz}, 1 \mathrm{H}), 2.05(\mathrm{tt}, J=14.8$, $7.1 \mathrm{~Hz}, 2 \mathrm{H}), 1.66(\mathrm{pd}, J=6.7,2.5 \mathrm{~Hz}, 2 \mathrm{H}) .{ }^{13} \mathrm{C}-\mathbf{N M R}:\left(101 \mathrm{MHz}, \mathrm{CDCl}_{3}\right) \delta 137.8(\mathrm{CH}), 136.5(\mathrm{C}), 129.5$ (2xCH), $128.4(2 \times C H), 126.9(\mathrm{CH}), 115.1\left(\mathrm{CH}_{2}\right), 79.9(\mathrm{CH}), 70.8\left(\mathrm{CH}_{2}\right), 38.7\left(\mathrm{CH}_{2}\right), 30.0\left(\mathrm{CH}_{2}\right), 28.6\left(\mathrm{CH}_{2}\right)$. IR (ATR): $\tilde{v}\left(\mathrm{~cm}^{-1}\right): 3031,2939,1720,1641,1455,1203,1150,913,754,599$. HRMS: calculated for $\mathrm{C}_{14} \mathrm{H}_{17} \mathrm{O}_{3}$ $\left(\mathrm{M}-\mathrm{H}^{+}\right)$233.1178, found 233.1184 .

\section{Synthesis of acid S1p:}

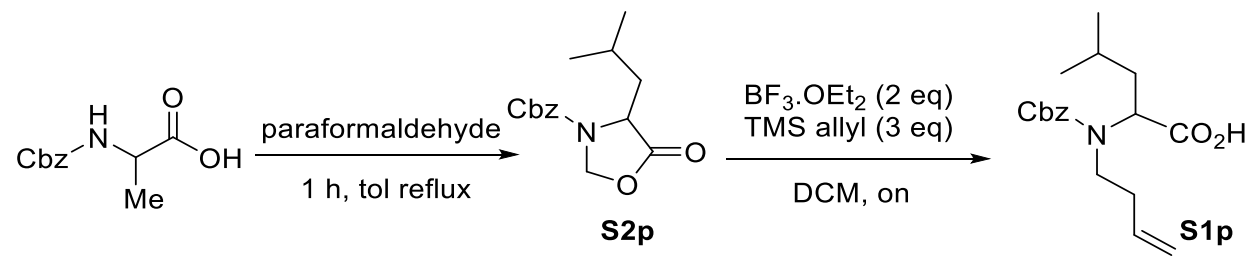<smiles>CC(C)CC1C(=O)OCN1C(=O)O</smiles>

Obtained following general procedure $\mathbf{B}$ from $5 \mathrm{mmol}$ of $\mathrm{N}$-Cbz-leucine. Column chromatography (9:1 to 8:2 pentane:EtOAc) afforded the product in $86 \%$ yield $(1.19 \mathrm{~g})$ as a white solid $\left(\mathbf{m p :} 62.0{ }^{\circ} \mathrm{C}\right)$.

1H-NMR: (500 MHz, Chloroform- $d$ ) $\delta 7.45$ - 7.34 (m, 5H), 5.62 (br s, 1H), 5.25 (d, J = $7.3 \mathrm{~Hz}, 1 \mathrm{H}), 5.23$ (s, 1H), $5.18(\mathrm{~d}, J=12.1 \mathrm{~Hz}, 1 \mathrm{H}), 4.37$ (br s, 1H), $2.09-1.67$ (m, 3H), 0.95 (br s, 3H).

${ }^{13}$ C-NMR: (126 MHz, $\left.\mathrm{CDCl}_{3}\right) \delta 172.8(\mathrm{C}), 153.2(\mathrm{C}), 135.3(\mathrm{C}), 128.7(2 \mathrm{xCH}), 128.7(2 \mathrm{xCH}), 128.4(\mathrm{CH})$, $77.6\left(\mathrm{CH}_{2}\right), 68.1\left(\mathrm{CH}_{2}\right), 53.6(\mathrm{CH}), 39.5\left(\mathrm{CH}_{2}\right), 24.4(\mathrm{CH}), 22.5\left(\mathrm{CH}_{3}\right), 22.4\left(\mathrm{CH}_{3}\right)$.

IR (ATR): $\tilde{v}\left(\mathrm{~cm}^{-1}\right): 3034,2959,2872,1801,1715,1499,1455,1413,1356,1216,1133,962,823,765,751$, 698.

HRMS: calculated for $\mathrm{C}_{15} \mathrm{H}_{20} \mathrm{NO}_{4}\left(\mathrm{M}+\mathrm{H}^{+}\right)$278.1387, found 278.1378.

Rf: 0.39 (8:2 pentane:EtOAc). 


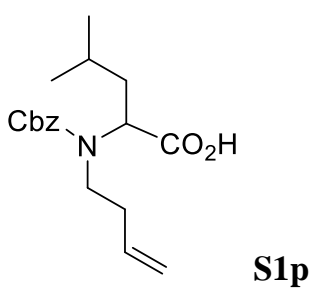

Obtained following general procedure B from $2 \mathrm{mmol}$ of oxazolidinone S2p in $71 \%$ yield (452 mg). The product was used in the next step without further purification.

${ }^{1}$ H-NMR: (500 MHz, Chloroform- $d$ ) mixture of rotamers $\delta 7.43-7.29(\mathrm{~m}, 5 \mathrm{H}), 5.81(\mathrm{dd}, J=17.0,10.2 \mathrm{~Hz}$, $0.4 \mathrm{H}), 5.73(\mathrm{ddt}, J=17.1,10.4,7.0 \mathrm{~Hz}, 0.6 \mathrm{H}), 5.21-5.02(\mathrm{~m}, 2 \mathrm{H}), 5.14-4.99(\mathrm{~m}, 2 \mathrm{H}), 4.56(\mathrm{dd}, J=9.6,5.4$ $\mathrm{Hz}, 0.6 \mathrm{H}), 4.51-4.43(\mathrm{~m}, 0.4 \mathrm{H}), 3.55-3.47(\mathrm{~m}, 1 \mathrm{H}), 3.15-3.09(\mathrm{~m}, 1 \mathrm{H}), 2.54-2.38(\mathrm{~m}, 1 \mathrm{H}), 2.35-2.28$ $(\mathrm{m}, 1 \mathrm{H}), 1.87(\mathrm{ddd}, J=14.3,9.1,5.7 \mathrm{~Hz}, 0.6 \mathrm{H}), 1.82-1.76(\mathrm{~m}, 1.4 \mathrm{H}), 1.69-1.64(\mathrm{~m}, 1 \mathrm{H}) 1.00-0.99(\mathrm{~m}$, $2 \mathrm{H}), 0.98(\mathrm{~d}, J=2.5 \mathrm{~Hz}, 2 \mathrm{H}), 0.94(\mathrm{~d}, J=6.5 \mathrm{~Hz}, 1 \mathrm{H}), 0.91(\mathrm{~d}, J=6.2 \mathrm{~Hz}, 1 \mathrm{H})$.

${ }^{13} \mathrm{C}-\mathrm{NMR}$ : $\left(126 \mathrm{MHz}, \mathrm{CDCl}_{3}\right)$ mixture of rotamers $\delta$ 177.6/176.8 (C), 157.1/155.9 (C), 136.2 (C), 135.2/134.9 $(\mathrm{CH}), 128.5 / 128.4(2 \times C H), 128.1 / 128.0(\mathrm{CH}), 127.9 / 127.8(2 \mathrm{xCH}), 116.9 / 116.7\left(\mathrm{CH}_{2}\right), 67.7 / 67.5\left(\mathrm{CH}_{2}\right)$, 58.9/57.9 (CH), 47.0/46.6 $\left(\mathrm{CH}_{2}\right), 38.7 / 38.1\left(\mathrm{CH}_{2}\right), 33.9 / 33.0\left(\mathrm{CH}_{2}\right), 24.8 / 24.7(\mathrm{CH}), 23.0 / 22.9\left(\mathrm{CH}_{3}\right), 21.8 / 21.6$ $\left(\mathrm{CH}_{3}\right)$.

IR (ATR): $\tilde{v}\left(\mathrm{~cm}^{-1}\right): 3069,3034,2958,1741,1704,1470,1368,1296,1191,1154,1047,1029,966,826,770$, 698.

HRMS: calculated for $\mathrm{C}_{18} \mathrm{H}_{24} \mathrm{NO}_{4}\left(\mathrm{M}-\mathrm{H}^{+}\right) 318.1711$, found 318.1713 .

\section{Synthesis of acid S1q:}

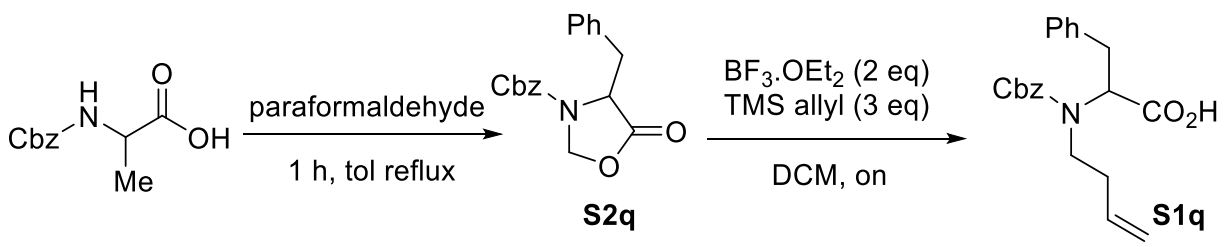<smiles>O=C1OCN(C(=O)OCc2ccccc2)C1Cc1ccccc1</smiles>

Obtained following general procedure B from $2.5 \mathrm{mmol}$ of $\mathrm{N}$-Cbz-phenylalanine. Column chromatography (8:2 pentane:EtOAc) afforded the product in $93 \%$ yield $(720 \mathrm{mg})$ as a white solid $\left(\mathbf{m p}: 81.8^{\circ} \mathrm{C}\right)$.

${ }^{1}$ H-NMR: (500 MHz, Chloroform-d) $\delta 7.51-7.32(\mathrm{~m}, 5 \mathrm{H}), 7.28-7.20(\mathrm{~m}, 3 \mathrm{H}), 7.16-6.96(\mathrm{~m}, 2 \mathrm{H}), 5.45-$ 5.12 (m, 3H), 4.60 (br s, 1H), 4.27 (br s, 1H), 3.37 (br d, $J=106.7 \mathrm{~Hz}, 1 \mathrm{H}), 3.21$ (br s, 1H).

${ }^{13}$ C-NMR: $\left(126 \mathrm{MHz} \mathrm{CDCl}_{3}\right) \delta$ 172.0/171.8 (C), 152.6/152.1 (C), 135.6 (C), 134.6/134.4 (C), 129.6 (4xCH), $128.8(4 x \mathrm{xH}), 128.4(\mathrm{CH}), 127.6(\mathrm{CH}), 78.2 / 77.9\left(\mathrm{CH}_{2}\right)$, 68.1/67.8 $\left(\mathrm{CH}_{2}\right)$, 56.5/56.4 $(\mathrm{CH}), 36.3 / 35.1\left(\mathrm{CH}_{2}\right)$.

IR (ATR): $\tilde{v}\left(\mathrm{~cm}^{-1}\right):$ 3064, 3032, 2917, 1800, 1714, 1497, 1454, 1418, 1359, 1162, 1051, 759, 700.

HRMS: calculated for $\mathrm{C}_{18} \mathrm{H}_{18} \mathrm{NO}_{4}\left(\mathrm{M}+\mathrm{H}^{+}\right)$312.1230, found 312.1236.

Rf: 0.37 (8:2 pentane:EtOAc) 


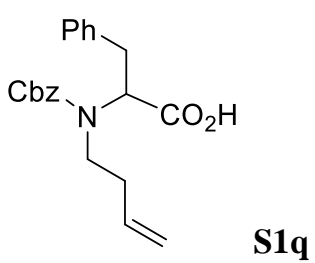

Obtained following general procedure B from $1 \mathrm{mmol}$ of oxazolidinone S2q in $80 \%$ yield (283 mg). The product was used in the next step without further purification.

${ }^{1}$ H-NMR: (500 MHz, Chloroform- $d$ ) mixture of rotamers $\delta 7.47-7.35(\mathrm{~m}, 5 \mathrm{H}), 7.29-7.20(\mathrm{~m}, 3 \mathrm{H}), 7.20-$ $7.05(\mathrm{~m}, 2 \mathrm{H}), 5.69(\mathrm{ddt}, J=17.2,10.5,6.9 \mathrm{~Hz}, 0.3 \mathrm{H}), 5.59(\mathrm{ddt}, J=17.3,10.6,6.9 \mathrm{~Hz}, 0.7 \mathrm{H}), 5.29-5.10(\mathrm{~m}$, $2 \mathrm{H}), 5.03-4.91(\mathrm{~m}, 2 \mathrm{H}), 4.24(\mathrm{dd}, J=10.3,4.9 \mathrm{~Hz}, 0.3 \mathrm{H}), 4.17(\mathrm{dd}, J=10.1,5.6 \mathrm{~Hz}, 0.7 \mathrm{H}), 3.47-3.25(\mathrm{~m}$, 2.7H), $3.15(\mathrm{dd}, J=14.2,10.4 \mathrm{~Hz}, 0.3 \mathrm{H}), 2.70(\mathrm{ddd}, J=14.7,8.9,5.6 \mathrm{~Hz}, 1 \mathrm{H}), 2.16(\mathrm{q}, J=7.9 \mathrm{~Hz}, 0.6 \mathrm{H}), 2.05$ $(\mathrm{dp}, J=22.6,7.5 \mathrm{~Hz}, 1.4 \mathrm{H})$.

${ }^{13}$ C-NMR: $\left(126 \mathrm{MHz}, \mathrm{CDCl}_{3}\right) \delta$ 175.5/174.4 (C), 156.6/155.4 (C), 137.4/137.3 (C), 136.2/136.1 (C), 135.2/134.7 (CH), $129.2(2 \times C H), 128.6(2 \times C H), 128.5(2 \times C H), 128.3 / 128.2(\mathrm{CH}), 127.9(2 \times C H), 126.8(\mathrm{CH})$, 116.9/116.6 $\left(\mathrm{CH}_{2}\right), 67.7\left(\mathrm{CH}_{2}\right)$, 64.4/62.4 (CH), 49.3/49.2 $\left(\mathrm{CH}_{2}\right), 36.0 / 34.9\left(\mathrm{CH}_{2}\right)$, 32.9/32.4 $\left(\mathrm{CH}_{2}\right)$.

IR (ATR): $\tilde{v}\left(\mathrm{~cm}^{-1}\right): 3065,3031,2942,1803,1703,1497,1473,1454,1421,1291,1219,1179,1140,1080$, 1048, 997, 915, 822, 751, 699.

HRMS: calculated for $\mathrm{C}_{21} \mathrm{H}_{24} \mathrm{NO}_{4}\left(\mathrm{M}+\mathrm{H}^{+}\right)$354.1700, found 354.1699.

\section{Synthesis of acid $\mathrm{S} 1 \mathrm{r}^{[10]}$ :}

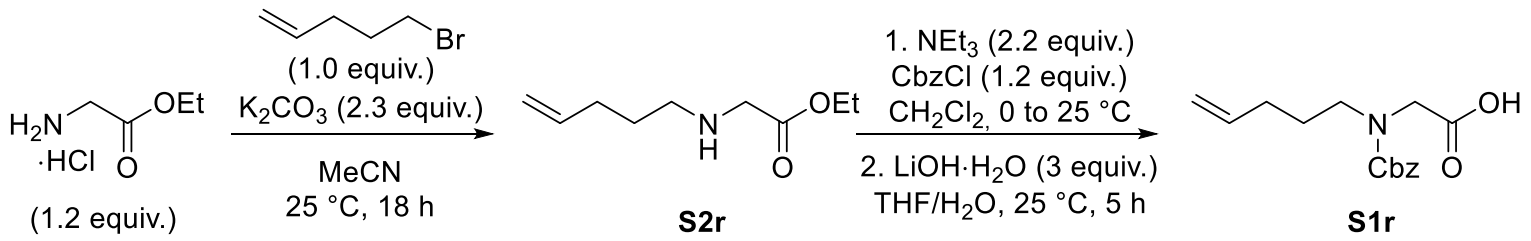

$\mathrm{K}_{2} \mathrm{CO}_{3}$ (2.68 g, $\left.19.4 \mathrm{mmol}\right)$ was added to a solution of ethyl glycinate hydrochloric acid (1.44 $\left.\mathrm{g}, 10 \mathrm{mmol}\right) \mathrm{in}$ $\mathrm{MeCN}$ (44 mL). The reaction mixture was stirred for $1 \mathrm{~h}$ before the addition of the 5-bromo-1-pentene (1.0 mL, $8.4 \mathrm{mmol}$ ). The reaction mixture was stirred for $18 \mathrm{~h}$ at room temperature. After this time, the reaction mixture was filtrated to remove inorganic salts and the filtrate was concentrated under vacuo. Water (44 mL) was added and the crude was extracted with $\mathrm{Et}_{2} \mathrm{O}$ five times to ensure complete extraction of product $\mathbf{S 2 r}$. The organic layers were washed with brine twice, dried, filtrated over $\mathrm{Na}_{2} \mathrm{SO}_{4}$ and concentrated to give product $\mathbf{S 2 r}$ as an ethereal solution due to the volatility of the product ( $73 \%$ in $\mathrm{Et}_{2} \mathrm{O}$ according to ${ }^{1} \mathrm{H}-\mathrm{NMR}$ spectra) in a $66 \%$ yield (941 mg of calculated pure product).

Product S2r (941 mg of calculated pure product, $73 \%$ solution in $\mathrm{Et}_{2} \mathrm{O}$ ), was dissolved in $\mathrm{CH}_{2} \mathrm{Cl}_{2}(14 \mathrm{~mL})$ and this solution was cooled down to $0{ }^{\circ} \mathrm{C}$. Et3N $(1.70 \mathrm{~mL}, 12.2 \mathrm{mmol})$ was added followed by $\mathrm{CbzCl}(915 \mu \mathrm{L}$, $6.41 \mathrm{mmol}$ ) that was added dropwise. The reaction was allowed to reach room temperature and stirred over 2 days. Volatiles were removed under reduced pressure and the crude was dissolved in a 4:1 THF/ $\mathrm{H}_{2} \mathrm{O}$ mixture $(15 \mathrm{~mL})$ followed by the addition of $\mathrm{LiOH} \cdot \mathrm{H} 2 \mathrm{O}(696 \mathrm{mg}, 16.6 \mathrm{mmol})$ and the reaction was stirred for $5 \mathrm{~h}$ at room temperature. The reaction mixture was then diluted with water $(10 \mathrm{~mL})$ and washed twice with $\mathrm{Et}_{2} \mathrm{O}$. The aqueous layer was acidified with concentrated $6 \mathrm{M} \mathrm{HCl}$ aqueous solution until $\mathrm{pH} 2$ at $0{ }^{\circ} \mathrm{C}$ followed by extraction with EtOAc (5 times). The organic layer was dried over $\mathrm{Na}_{2} \mathrm{SO}_{4}$, filtered and concentrated to give compound S1r as a clear colourless oil in a $52 \%$ yield $(803 \mathrm{mg})$. 


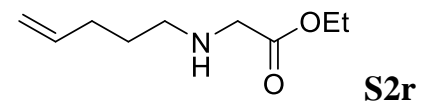

${ }^{1}$ H-NMR: $\left(500 \mathrm{MHz}, \mathrm{CDCl}_{3}\right) \delta 5.89-5.77(\mathrm{~m}, 1 \mathrm{H}), 5.03(\mathrm{dd}, J=17.1,7.7 \mathrm{~Hz}, 1 \mathrm{H}), 5.00-4.94(\mathrm{~m}, 1 \mathrm{H}), 4.31$ $-4.15(\mathrm{~m}, 2 \mathrm{H}), 3.37$ (dd, $J=39.4,1.6 \mathrm{~Hz}, 2 \mathrm{H}), 2.75-2.55(\mathrm{~m}, 2 \mathrm{H}), 2.10$ (dt, J = 22.3, $7.0 \mathrm{~Hz}, 2 \mathrm{H}), 1.59$ (ddd, $J=26.2,10.6,4.3 \mathrm{~Hz}, 2 \mathrm{H}), 1.34-1.26(\mathrm{~m}, 3 \mathrm{H})$.

${ }^{13}$ C-NMR: (101 MHz, $\left.\mathrm{CDCl}_{3}\right)(101 \mathrm{MHz}, \mathrm{CDCl} 3) \delta$ 172.5/171.6 (C), 138.6/138.3 (CH) , 114.7/114.5 ( $\left.\mathrm{CH}_{2}\right)$, 60.7/60.2 $\left(\mathrm{CH}_{2}\right)$, 55.14/53.8 $\left(\mathrm{CH}_{2}\right)$, 51.0/49.0 $\left(\mathrm{CH}_{2}\right)$, 31.4/31.4 $\left(\mathrm{CH}_{2}\right), 29.2 / 26.8\left(\mathrm{CH}_{2}\right), 14.3 / 14.2\left(\mathrm{CH}_{3}\right)$.

IR (ATR): $\tilde{v}\left(\mathrm{~cm}^{-1}\right):$ 3343, 3077, 2979, 2934, 2855, 1739, 1641, 1370, 1186, 1029, 911, 862, 745.

HRMS: calculated for $\mathrm{C}_{9} \mathrm{H}_{18} \mathrm{NO}_{2}\left(\mathrm{M}+\mathrm{H}^{+}\right)$172.1332, found 172.1332.

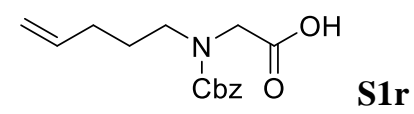

${ }^{1}$ H-NMR: (400 MHz, Chloroform- $d$ ) $\delta 9.01$ (br s, 1H), $7.52-7.10$ (m, 5H), $5.88-5.53$ (m, 1), $5.08(\mathrm{~d}, J=$ $15.9 \mathrm{~Hz}, 2 \mathrm{H}), 5.01-4.80(\mathrm{~m}, 2 \mathrm{H}), 3.96(\mathrm{~d}, J=19.5 \mathrm{~Hz}, 2 \mathrm{H}), 3.28(\mathrm{q}, J=8.3 \mathrm{~Hz}, 2 \mathrm{H}), 1.98(\mathrm{dq}, J=16.3,6.7$ $\mathrm{Hz}, 2 \mathrm{H}), 1.57(\mathrm{~h}, J=7.2 \mathrm{~Hz}, 2 \mathrm{H})$.

${ }^{13}$ C-NMR: $\left(101 \mathrm{MHz}, \mathrm{CDCl}_{3}\right) \delta$ 175.2/174.8 (C), 156.8/155.9 (C), 137.7/137.5 (CH), $136.3(\mathrm{C}), 128.5 / 128.4$ $(2 \mathrm{xCH}), 128.1 / 128.0(\mathrm{CH}), 127.8 / 127.8(2 \mathrm{xCH}), 115.3 / 115.2\left(\mathrm{CH}_{2}\right), 67.7 / 67.5\left(\mathrm{CH}_{2}\right), 49.1 / 48.6\left(\mathrm{CH}_{2}\right)$, 48.9/47.9 $\left(\mathrm{CH}_{2}\right), 30.8 / 30.7\left(\mathrm{CH}_{2}\right), 27.5 / 27.1\left(\mathrm{CH}_{2}\right)$.

IR (ATR): $\tilde{v}\left(\mathrm{~cm}^{-1}\right): 3067,2938,1700,1672,1454,1476,1430,1218,1180,912,770,697$.

HRMS: calculated for $\mathrm{C}_{15} \mathrm{H}_{18} \mathrm{NO}_{4}\left(\mathrm{M}-\mathrm{H}^{+}\right) 276.1241$, found 276.1244. 


\section{Synthesis of acid S1t:}

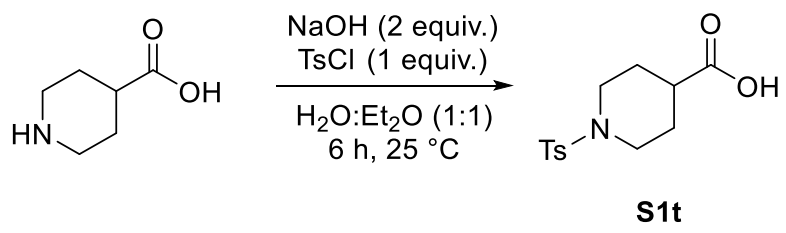

Piperidine-4-carboxylic acid (3.2 g, $25 \mathrm{mmol})$ was dissolved in $25 \mathrm{~mL}$ of $\mathrm{Et}_{2} \mathrm{O}$ and $25 \mathrm{~mL}$ of $2 \mathrm{M}$ aqueous solution of $\mathrm{NaOH}$ were carefully added at $0{ }^{\circ} \mathrm{C}$. Then $\mathrm{TsCl}(4.75 \mathrm{~g}, 25 \mathrm{mmol})$ was carefully added and the mixture was stirred at room temperature for $6 \mathrm{~h}$. A precipitate was formed over the reaction time. After this time the reaction mixture was further diluted with water until the precipitate was completely dissolved and the layers were separated. The aqueous layer was acidified to $\mathrm{pH} 3$ by addition of $4 \mathrm{M} \mathrm{HCl}$ aqueous solution and extracted with EtOAc three times. The organic layer was dried over $\mathrm{Na}_{2} \mathrm{SO}_{4}$, filtered and concentrated to give compound S1t (4.98 g, $70 \%$ yield) as a white foam (mp: $\left.169.8^{\circ} \mathrm{C}\right)$.

${ }^{1}$ H-NMR: (500 MHz, Chloroform- $d$ ) $\delta 7.66(\mathrm{~d}, J=8.2 \mathrm{~Hz}, 2 \mathrm{H}), 7.35(\mathrm{~d}, J=8.0 \mathrm{~Hz}, 2 \mathrm{H}), 3.66(\mathrm{dt}, J=11.9,3.9$ $\mathrm{Hz}, 2 \mathrm{H}), 2.49$ (td, $J=11.2,2.9 \mathrm{~Hz}, 2 \mathrm{H}), 2.46(\mathrm{~s}, 3 \mathrm{H}), 2.32$ (tt, $J=10.7,4.0 \mathrm{~Hz}, 1 \mathrm{H}), 2.07-1.96(\mathrm{~m}, 2 \mathrm{H}), 1.85$ (dtd, $J=14.8,10.8,4.0 \mathrm{~Hz}, 2 \mathrm{H})$.

${ }^{13}$ C-NMR: $\left(126 \mathrm{MHz}, \mathrm{CDCl}_{3}\right) \delta 179.7(\mathrm{C}), 143.7(\mathrm{C}), 132.9(\mathrm{C}), 129.7(2 \mathrm{xCH}), 127.7(2 \mathrm{xCH}), 45.4\left(2 \mathrm{xCH}_{2}\right)$, $39.8(\mathrm{CH}), 27.2\left(2 \mathrm{xCH}_{2}\right), 21.6\left(\mathrm{CH}_{3}\right)$.

IR: 2989, 2934, 2855, 1697, 1427, 1451, 1352, 1334, 1159, 919, 810, 725.

HRMS: calculated for $\mathrm{C}_{13} \mathrm{H}_{18} \mathrm{NO}_{4} \mathrm{~S}\left(\mathrm{M}+\mathrm{H}^{+}\right)$284.0951, found 284.0951 .

\section{Synthesis of acid S1u:}

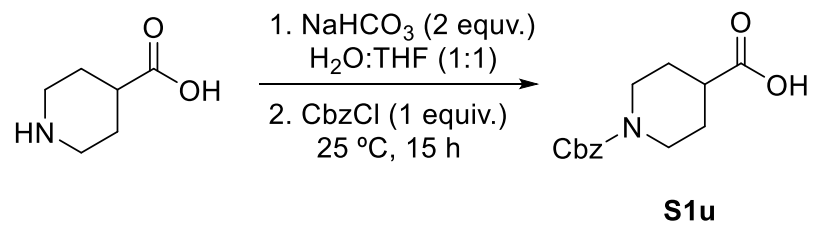

Piperidine-4-carboxylic acid ( $1.3 \mathrm{~g}, 10 \mathrm{mmol})$ was dissolved in $8 \mathrm{~mL}$ of a 1:1 mixture of $\mathrm{H}_{2} \mathrm{O}$ :THF. NaHCO3 $(1.68 \mathrm{~g}, 20 \mathrm{mmol})$ were carefully added at $0{ }^{\circ} \mathrm{C}$ and the solution was stirred for $1 \mathrm{~h}$. Then $\mathrm{CbzCl}(1.4 \mathrm{~mL}, 10$ $\mathrm{mmol}$ ) was added dropwise and the mixture was stirred at room temperature for $15 \mathrm{~h}$. Then, the organic solvent was eliminated and $5 \mathrm{~mL}$ of water were added. The aqueous phase was washed with EtOAc (x2) and acidified to $\mathrm{pH} 3$ by addition of $1 \mathrm{M} \mathrm{HCl}$ aqueous solution. The aqueous layer was extracted with EtOAc (x3), dried over $\mathrm{Na}_{2} \mathrm{SO}_{4}$, filtered and concentrated. Column chromatography (6:4 pentane:EtOAc) was perfomed to obtain compound S1u (1.06 g, 40\% yield).

${ }^{1}$ H-NMR: (500 MHz, Chloroform- $d$ ) $\delta 7.47-7.31$ (m, 5H), 5.16 (s, 2H), 4.12 (br s, 2H), 2.98 (br s, 2H), 2.55 (tt, $J=10.8,3.9 \mathrm{~Hz}, 1 \mathrm{H}), 1.95(\mathrm{~s}, 2 \mathrm{H}), 1.70(\mathrm{~d}, J=10.4 \mathrm{~Hz}, 2 \mathrm{H})$.

${ }^{13}$ C-NMR: $\left(126 \mathrm{MHz}, \mathrm{CDCl}_{3}\right) \delta 179.9(\mathrm{C}), 155.2(\mathrm{C}), 136.6(\mathrm{C}), 128.5(2 \times \mathrm{xH}), 128.1(\mathrm{CH}), 127.9(2 \times \mathrm{CH})$, $67.2\left(\mathrm{CH}_{2}\right), 43.2\left(2 \mathrm{xCH}_{2}\right), 40.6(\mathrm{CH}), 27.6\left(2 \mathrm{xCH}_{2}\right)$. 
IR (ATR): $\tilde{v}\left(\mathrm{~cm}^{-1}\right)$ 3033, 2955, 2863, 1731, 1699, 1434, 1219, 1030, 697.

HRMS: calculated for $\mathrm{C}_{14} \mathrm{H}_{16} \mathrm{NO}_{4}\left(\mathrm{M}-\mathrm{H}^{+}\right) 262.1085$, found 262.1087 .

Rf: 0.11 (4:6 pentane:EtOAc).

\section{Synthesis of acid S1v:}

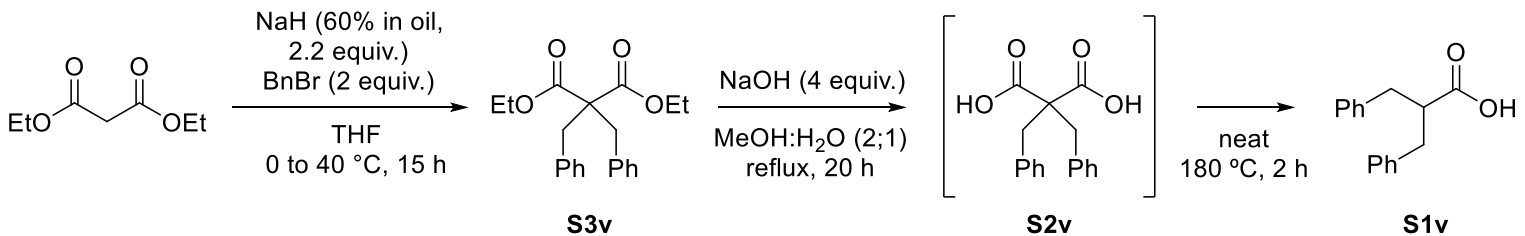

Diethyl malonate $(1.75 \mathrm{~mL}, 11.5 \mathrm{mmol})$ was dissolved in $30 \mathrm{~mL}$ of dry THF under Argon atmosphere. This solution was cooled to $0{ }^{\circ} \mathrm{C}$ and $\mathrm{NaH}(60 \%$ in oil) $(1.01 \mathrm{~g}, 25.3 \mathrm{mmol})$ was carefully added in portions under a stream of Argon. After complete addition, the reaction mixture was allowed to reach room temperature and stir for $3 \mathrm{~h}$. Then, benzyl bromide $(2.73 \mathrm{~mL}, 23 \mathrm{mmol})$ was added and the reaction was stirred at $40{ }^{\circ} \mathrm{C}$ for $15 \mathrm{~h}$. Water $(10 \mathrm{~mL})$ was then carefully added to quench the excess of $\mathrm{NaH}$. The organic solvent was eliminated and extraction with $\mathrm{Et}_{2} \mathrm{O}$ (x3) was followed by wash of the organic layers with water and brine. The organic layer was dried over $\mathrm{Na}_{2} \mathrm{SO}_{4}$, filtered and concentrated. The crude acid was purified using column chromatography (pentane:EtOAc 9:1 to 8:2) to give pure product $\mathbf{S 3 v}$ as a colorless oil $(3.46 \mathrm{~g}, 88 \%$ ).

Product S3v (3.0 g, $8.8 \mathrm{mmol}$ ) were dissolved in a 2:1 MeOH: $\mathrm{H}_{2} \mathrm{O}$ mixture $(66 \mathrm{~mL})$ and pulverized $\mathrm{NaOH}$ was added. The suspension was heated to reflux for $20 \mathrm{~h}$. After this time, the reaction was allowed to reach room temperature and the $\mathrm{MeOH}$ was eliminated under reduced vaccuo. The residual aqueous solution was washed once with EtOAc and acidified to $\mathrm{pH} 2$ using $1 \mathrm{M} \mathrm{HCl}$ aq. solution. Extraction with EtOAc (x3) was followed by wash of the organic layers with brine. The organic layer was dried over $\mathrm{Na}_{2} \mathrm{SO}_{4}$, filtered and concentrated to give diacid S2v (860 mg, 34\%). $420 \mathrm{mg}$ of crude product $\mathbf{S 2 v}$ were heated to $180{ }^{\circ} \mathrm{C}$ under neat conditions, to afford decarboxylated acid S1v (350 mg, quantitative) that was used in the next reaction without further purification.<smiles>CCOC(=O)C(Cc1ccccc1)(C(=O)OCC)c1ccccc1</smiles>

${ }^{1}$ H-NMR: (500 MHz, Chloroform- $d$ ) $\delta 7.28(\mathrm{p}, J=6.9 \mathrm{~Hz}, 6 \mathrm{H}), 7.20(\mathrm{~d}, J=7.2 \mathrm{~Hz}, 4 \mathrm{H}), 4.13(\mathrm{q}, J=7.1 \mathrm{~Hz}$, 4H), $3.26(\mathrm{~s}, 4 \mathrm{H}), 1.18(\mathrm{t}, J=7.1 \mathrm{~Hz}, 6 \mathrm{H})$.

${ }^{13}$ C-NMR: (126 MHz, $\left.\mathrm{CDCl}_{3}\right) \delta 171.0(2 \mathrm{xC}), 136.4(2 \mathrm{xC}), 130.1$ (4xCH), $128.2(4 \mathrm{xCH}), 126.9(2 \mathrm{xCH}), 61.2$ $\left(2 \mathrm{xCH}_{2}\right), 60.2(\mathrm{C}), 39.1\left(2 \mathrm{xCH}_{2}\right), 13.9\left(2 \mathrm{xCH}_{3}\right)$.

IR (ATR): $\tilde{v}\left(\mathrm{~cm}^{-1}\right): 3063,3088,3031,2938,1727,1495,1454,1269,1248,1197,1180,1040,742,700$.

HRMS: calculated for $\mathrm{C}_{21} \mathrm{H}_{25} \mathrm{O}_{4}\left(\mathrm{M}+\mathrm{H}^{+}\right) 341.1747$, found 341.1747. 
R $\mathbf{R}_{\mathbf{f}} 0.6$ (8:2 pentane:EtOAc).<smiles>O=C(O)C(Cc1ccccc1)Cc1ccccc1</smiles>

${ }^{1}$ H-NMR: (400 MHz, Chloroform- $d$ ) $\delta 7.34-7.27$ (m, 3H), $7.27-7.17$ (m, 7H), $3.09-2.96(\mathrm{~m}, 3 \mathrm{H}), 2.89-$ $2.77(\mathrm{~m}, 2 \mathrm{H})$.

${ }^{13}$ C-NMR: (101 MHz, $\left.\mathrm{CDCl}_{3}\right) \delta 179.0(\mathrm{C}), 138.7(2 \mathrm{xC}), 128.9(4 \mathrm{xCH}), 128.5(4 \mathrm{xCH}), 126.5(2 \mathrm{xCH}), 49.0$ $(\mathrm{CH}), 37.8\left(2 \mathrm{xCH}_{2}\right)$.

IR (ATR): ̃ ( $\left(\mathrm{cm}^{-1}\right):$ 3029, 2927, 1706, 1496, 1454, 1241, 1081, 744, 698.

HRMS: calculated for $\mathrm{C}_{16} \mathrm{H}_{15} \mathrm{O}_{2}\left(\mathrm{M}-\mathrm{H}^{+}\right)$239.1078, found 239.1077.

\section{Synthesis of acid S1w:}

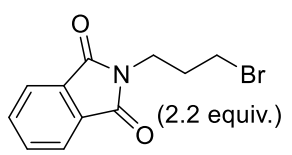

$\mathrm{NaH}(60 \%$ in oil, 1.32 equiv.)

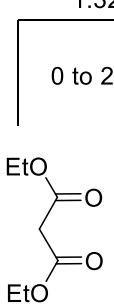
to $25^{\circ} \mathrm{C}, 15 \mathrm{~h}$<smiles>CCOC(=O)C(CCCN1C(=O)c2ccccc2C1=O)(CCCN1C(=O)c2ccccc2C1=O)C(=O)OCC</smiles>

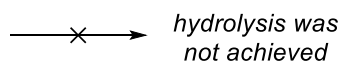

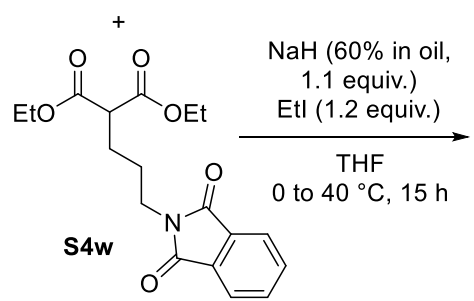

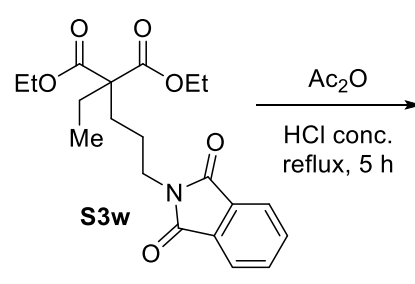

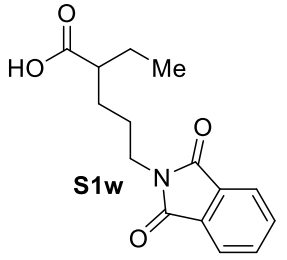

neat $160^{\circ} \mathrm{C}, 2 \mathrm{~h}$

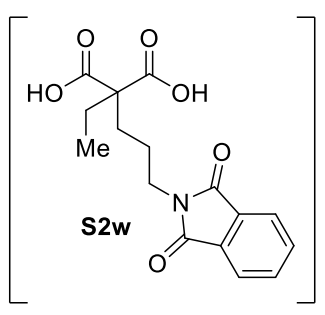

Diethyl malonate $(1.53 \mathrm{~mL}, 10 \mathrm{mmol})$ was dissolved in $30 \mathrm{~mL}$ of dry THF under Argon atmosphere. This solution was cooled to $0{ }^{\circ} \mathrm{C}$ and $\mathrm{NaH}(60 \%$ in oil) $(527 \mathrm{mg}, 13.2 \mathrm{mmol})$ was carefully added in portions under a stream of Argon. After complete addition, the reaction mixture was allowed to reach room temperature and stir for $1 \mathrm{~h}$. Then, 3-phthalimidepropyl bromide $(5.9 \mathrm{~g}, 22 \mathrm{mmol})$ was added and the reaction was stirred at room temperature for $15 \mathrm{~h}$. Water $(10 \mathrm{~mL})$ was then carefully added to quench the excess of $\mathrm{NaH}$. The organic solvent was eliminated and extraction with $\mathrm{Et}_{2} \mathrm{O}(\mathrm{x} 3)$ was followed by wash of the organic layers with water and brine. The organic layer was dried over $\mathrm{Na}_{2} \mathrm{SO}_{4}$, filtered and concentrated. Column chromatography (pentane:EtOAc 9:1 to 1:1) afforded monoalkylated product $\mathbf{S 4 w}(1.49 \mathrm{~g}, 43 \%)$ as a white solid (mp: $\left.44.8{ }^{\circ} \mathrm{C}\right)$ and dialkylated product S5w (1.03 g, 19\%) as a white solid (mp: $\left.158.5^{\circ} \mathrm{C}\right)$.

Monoalkylated product $\mathbf{S 4 w}$ (394 $\mathrm{mg}, 2 \mathrm{mmol}$ ) was dissolved in $3 \mathrm{~mL}$ of dry THF under Argon atmosphere. This solution was cooled to $0{ }^{\circ} \mathrm{C}$ and $\mathrm{NaH}(60 \%$ in oil) $(88 \mathrm{mg}, 2.2 \mathrm{mmol})$ was carefully added in portions under a stream of Argon. After complete addition, the reaction mixture was allowed to reach room temperature 
and stir for $1 \mathrm{~h}$. Then ethyl iodide $(177 \mu \mathrm{L}, 2.2 \mathrm{mmol})$ was added and the reaction was stirred at room temperature for 2 additional hours. Saturated $\mathrm{NH}_{4} \mathrm{Cl}$ aq. solution $(5 \mathrm{~mL})$ was then carefully added to quench the excess of $\mathrm{NaH}$. The organic solvent was eliminated and extraction with EtOAc (x3) was followed by wash of the organic layers with water and brine. The organic layer was dried over $\mathrm{Na}_{2} \mathrm{SO}_{4}$, filtered and concentrated. Column chromatography (pentane:EtOAc 8:2) afforded product S3w (462 mg, 62\%).

In order to avoid the loose of the phthalimide group under alkaline media, literature conditions were used in this case ${ }^{[11]}$ : product $\mathbf{S 3} \mathbf{w}(450 \mathrm{mg}, 1.2 \mathrm{mmol})$ was dissolved in concentrated aq. $\mathrm{HCl}$ solution $(3.8 \mathrm{~mL})$ and acetic anhydride $(2.5 \mathrm{~mL})$ was added. The suspension was heated to reflux for $5 \mathrm{~h}$. After this time, the solvent was distilled out of the solution (using the necessary safety precautions) and the residue was triturated in EtOH and filtered to give diacid S2w. The solid was then heated to $160{ }^{\circ} \mathrm{C}$ under neat conditions, to afford decarboxylated product S1w (323 mg, < 98\%) in a crude form containing a small amount of the corresponding diester S3w. Further purification was not possible and the crude was directly used in the next reaction.

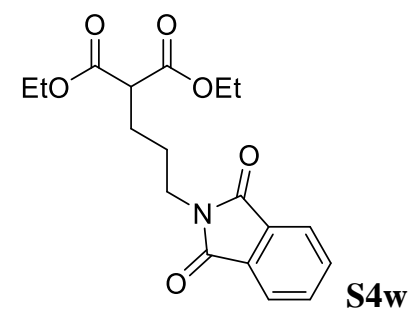

${ }^{1}$ H-NMR: (400 MHz, Chloroform- $d$ ) $\delta 7.77(\mathrm{dd}, J=5.4,3.1 \mathrm{~Hz}, 2 \mathrm{H}), 7.64(\mathrm{dd}, J=5.4,3.1 \mathrm{~Hz}, 2 \mathrm{H}), 4.11(\mathrm{qd}$, $J=7.1,2.0 \mathrm{~Hz}, 4 \mathrm{H}), 3.65(\mathrm{t}, J=7.0 \mathrm{~Hz}, 2 \mathrm{H}), 3.31(\mathrm{t}, J=7.5 \mathrm{~Hz}, 1 \mathrm{H}), 1.95-1.83(\mathrm{~m}, 2 \mathrm{H}), 1.74-1.61(\mathrm{~m}, 2 \mathrm{H})$, $1.18(\mathrm{t}, J=7.1 \mathrm{~Hz}, 6 \mathrm{H})$.

${ }^{13}$ C-NMR: (101 MHz, $\left.\mathrm{CDCl}_{3}\right) \delta 169.1(2 \mathrm{xC}), 168.3(2 \mathrm{xC}), 133.9(2 \mathrm{xCH}), 132.1(2 \mathrm{xC}), 123.2(2 \mathrm{xCH}), 61.4$ $\left(2 \mathrm{xCH}_{2}\right), 51.4(\mathrm{CH}), 37.4\left(\mathrm{CH}_{2}\right), 26.3\left(\mathrm{CH}_{2}\right), 25.9\left(\mathrm{CH}_{2}\right), 14.1\left(2 \mathrm{xCH}_{3}\right)$.

IR (ATR): $\tilde{v}\left(\mathrm{~cm}^{-1}\right):$ 2982, 1773, 1711, 1439, 1467, 1397, 1369, 1339, 1262, 1235, 1187, 1126, 1040, 720.

HRMS: calculated for $\mathrm{C}_{18} \mathrm{H}_{22} \mathrm{NO}_{6}\left(\mathrm{M}+\mathrm{H}^{+}\right) 348.1442$, found 348.1449 .

Rf: 0.14 (9:1 pentane:EtOAc) 


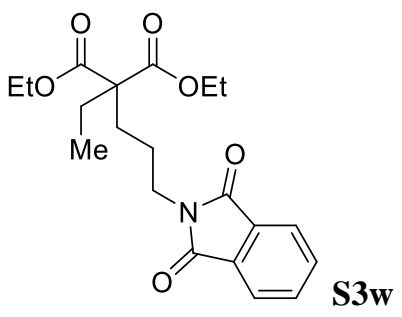

${ }^{1}$ H-NMR: (500 MHz, Chloroform- $d$ ) $\delta 7.86(\mathrm{dd}, J=5.4,3.1 \mathrm{~Hz}, 2 \mathrm{H}), 7.73(\mathrm{dd}, J=5.4,3.1 \mathrm{~Hz}, 2 \mathrm{H}), 4.18(\mathrm{q}, J$ $=7.1 \mathrm{~Hz}, 4 \mathrm{H}), 3.70(\mathrm{t}, J=7.3 \mathrm{~Hz}, 2 \mathrm{H}), 2.12-1.87(\mathrm{~m}, 4 \mathrm{H}), 1.73-1.47(\mathrm{~m}, 2 \mathrm{H}), 1.24(\mathrm{t}, J=7.1 \mathrm{~Hz}, 6 \mathrm{H}), 0.84$ $(\mathrm{t}, J=7.5 \mathrm{~Hz}, 3 \mathrm{H})$.

${ }^{13}$ C-NMR: $\left(126 \mathrm{MHz}, \mathrm{CDCl}_{3}\right) \delta 171.4(2 \mathrm{xC}), 168.3(2 \mathrm{xC}), 133.9(2 \mathrm{xCH}), 132.1(2 \mathrm{xC}), 123.2(2 \mathrm{xCH}), 61.2$ $\left(2 \mathrm{xCH}_{2}\right), 57.6(\mathrm{C}), 38.0\left(\mathrm{CH}_{2}\right), 28.9\left(\mathrm{CH}_{2}\right), 25.4\left(\mathrm{CH}_{2}\right), 23.3\left(\mathrm{CH}_{2}\right), 14.1\left(2 \mathrm{xCH}_{3}\right), 8.5\left(\mathrm{CH}_{3}\right)$.

IR (ATR): $\tilde{v}\left(\mathrm{~cm}^{-1}\right):$ 2978, 1773, 1712, 1466, 1396, 1204, 1034, 720.

HRMS: calculated for $\mathrm{C}_{20} \mathrm{H}_{26} \mathrm{NO}_{6}\left(\mathrm{M}+\mathrm{H}^{+}\right) 376.1755$, found 376.1747 .

Rf: 0.21 (8:2 pentane:EtOAc).

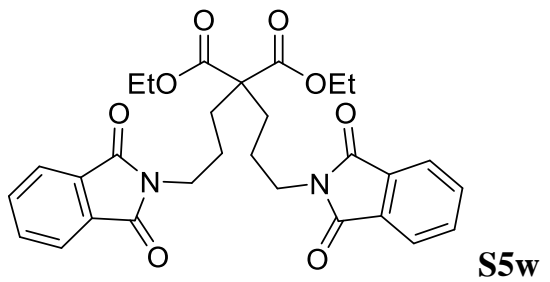

${ }^{1}$ H-NMR: (400 MHz, Chloroform- $d$ ) $\delta 7.76(\mathrm{dd}, J=5.4,3.1 \mathrm{~Hz}, 4 \mathrm{H}), 7.63(\mathrm{dd}, J=5.4,3.0 \mathrm{~Hz}, 4 \mathrm{H}), 4.07$ (q, $J$ $=7.1 \mathrm{~Hz}, 4 \mathrm{H}), 3.60(\mathrm{t}, J=7.1 \mathrm{~Hz}, 4 \mathrm{H}), 1.88-1.78(\mathrm{~m}, 4 \mathrm{H}), 1.60-1.51(\mathrm{~m}, 4 \mathrm{H}), 1.12(\mathrm{t}, J=7.1 \mathrm{~Hz}, 6 \mathrm{H})$.

${ }^{13}$ C-NMR: $\left(101 \mathrm{MHz}, \mathrm{CDCl}_{3}\right) \delta 171.0(2 \mathrm{xC}), 168.2(4 \mathrm{xC}), 133.9(2 \mathrm{xCH}), 132.1(2 \mathrm{xC}), 123.2(2 \mathrm{xCH}), 61.3$ $\left(2 \mathrm{xCH}_{2}\right), 57.0(\mathrm{C}), 37.9\left(2 \mathrm{xCH}_{2}\right), 30.1\left(2 \mathrm{xCH}_{2}\right), 23.5\left(2 \mathrm{xCH}_{2}\right), 14.0\left(2 \mathrm{xCH}_{3}\right)$.

IR (ATR): $\tilde{v}\left(\mathrm{~cm}^{-1}\right): 2940,1773,1710,1438,1397,1363,1219,1052,720$.

HRMS: calculated for $\mathrm{C}_{29} \mathrm{H}_{31} \mathrm{~N}_{2} \mathrm{O}_{8}\left(\mathrm{M}+\mathrm{H}^{+}\right)$535.2075, found 535.2075.

Rf: 0.05 (9:1 pentane:EtOAc). 


\section{Synthesis of acid S1y:}

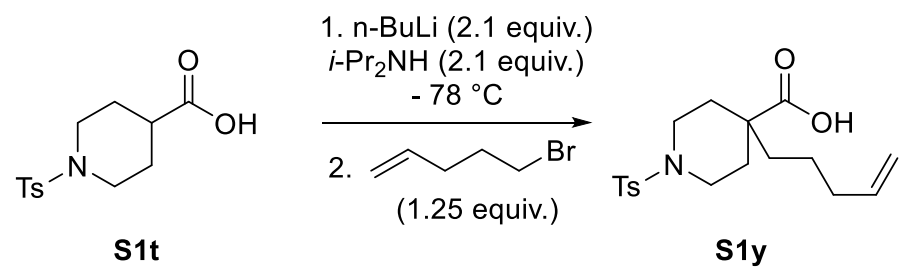

A solution of $n$-BuLi $(2.5 \mathrm{M}$ in hexane, $3.36 \mathrm{~mL}, 8.4 \mathrm{mmol})$ was added over a solution of $i$ - $\mathrm{Pr}_{2} \mathrm{NH}(1.18 \mathrm{~mL}$, $8.4 \mathrm{mmol})$ in dry THF $(40 \mathrm{~mL})$ at $-78{ }^{\circ} \mathrm{C}$ and the solution was stirred for $30 \mathrm{~min}$. Acid S1t $(4 \mathrm{mmol}, 1.13 \mathrm{~g})$ was dissolved in dry THF $(5 \mathrm{~mL})$ and added into the freshly prepared LDA solution at $-78^{\circ} \mathrm{C}$. The mixture was allowed to reach room temperature and stirred for $1 \mathrm{~h}$. Then, the reaction mixture was cooled to $-78{ }^{\circ} \mathrm{C}$ and 1-bromo-5-pentene $(591 \mu \mathrm{L}, 5 \mathrm{mmol})$ was added. The mixture was allowed to stir at room temperature for $20 \mathrm{~h}$. Then, the reaction mixture was poured into a mixture of $30 \mathrm{~g}$ of ice and $30 \mathrm{~mL}$ of pentane. The organic phase was separated and extracted with $\mathrm{NaHCO}_{3}$ saturated aqueous solution. The combined aqueous layers were acidified to $\mathrm{pH} 2$ with $1 \mathrm{M} \mathrm{HCl}$ aqueous solution and extracted three times with EtOAc. The combined organic layers were washed with water and brine, dried over $\mathrm{Na}_{2} \mathrm{SO}_{4}$, filtered and concentrated. Column chromatography (60:40:1 pentane: EtOAc: $\mathrm{AcOH})$ gave compound $\mathbf{S 1 y}$ in $45 \%$ yield $(640 \mathrm{mg})$ as a white solid (mp: $138.1^{\circ} \mathrm{C}$ ).

${ }^{1}$ H-NMR: (400 MHz, Chloroform- $d$ ) $\delta 7.64(\mathrm{~d}, J=8.2 \mathrm{~Hz}, 2 \mathrm{H}), 7.32(\mathrm{~d}, J=8.0 \mathrm{~Hz}, 2 \mathrm{H}), 5.72$ (ddt, $J=16.9$, $10.2,6.6 \mathrm{~Hz}, 1 \mathrm{H}), 5.14-4.85(\mathrm{~m}, 2 \mathrm{H}), 3.57$ (d, $J=12.4 \mathrm{~Hz}, 2 \mathrm{H}), 2.52(\mathrm{td}, J=11.9,2.6 \mathrm{~Hz}, 2 \mathrm{H}), 2.45(\mathrm{~s}, 3 \mathrm{H})$, $2.26-2.11(\mathrm{~m}, 2 \mathrm{H}), 2.00(\mathrm{q}, J=7.0 \mathrm{~Hz}, 2 \mathrm{H}), 1.62-1.50(\mathrm{~m}, 4 \mathrm{H}), 1.30(\mathrm{dtd}, J=14.8,7.4,4.3 \mathrm{~Hz}, 2 \mathrm{H})$.

${ }^{13}$ C-NMR: (101 MHz, $\left.\mathrm{CDCl}_{3}\right) \delta 180.5(\mathrm{C}), 143.5(\mathrm{C}), 137.8(\mathrm{CH}), 133.7(\mathrm{C}), 129.7(\mathrm{CH}), 127.6(\mathrm{CH}), 115.1$ $\left(\mathrm{CH}_{2}\right), 44.8(\mathrm{C}), 43.6\left(2 \mathrm{xCH}_{2}\right), 39.3\left(\mathrm{CH}_{2}\right), 33.6\left(\mathrm{CH}_{2}\right), 32.6\left(2 \mathrm{xCH}_{2}\right), 23.0\left(\mathrm{CH}_{2}\right), 21.5\left(\mathrm{CH}_{3}\right)$.

IR (ATR): $\tilde{v}\left(\mathrm{~cm}^{-1}\right)$ 3073, 2928, 1730, 1697, 1341, 1325, 1161, 911, 720.

HRMS: calculated for $\mathrm{C}_{18} \mathrm{H}_{24} \mathrm{NO}_{4} \mathrm{~S}\left(\mathrm{M}-\mathrm{H}^{+}\right) 350.1426$, found 350.1431 .

Rf: 0.36 (6:4:0.1 pentane:EtOAc:AcOH) 


\section{Synthesis of acid S1z:}

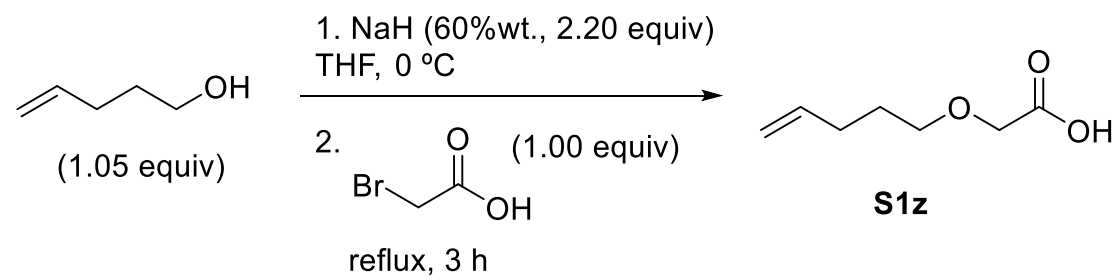

4-Penten-1-ol $(541 \mu \mathrm{L}, 5.25 \mathrm{mmol})$ was added dropwise to a stirring suspension of $\mathrm{NaH}(60 \% \mathrm{wt}, 264 \mathrm{mg}, 11$ $\mathrm{mmol})$ in $\mathrm{THF}(3.5 \mathrm{~mL})$ at $0{ }^{\circ} \mathrm{C}$. The mixture was stirred for $15 \mathrm{~min}$ at $0{ }^{\circ} \mathrm{C}$ and $15 \mathrm{~min}$ at room temperature. Then, the reaction mixture was cooled to $0{ }^{\circ} \mathrm{C}$ and a solution of 2 -Bromoacetic acid ( $\left.690 \mathrm{mg}, 5 \mathrm{mmol}\right) \mathrm{in} \mathrm{THF}$ $(3.5 \mathrm{~mL})$ was added dropwise. After complete addition, the reaction mixture was refluxed for $3 \mathrm{~h}$. Then, the solution was allowed to reach room temperature and cooled with an ice-bath and the remaining $\mathrm{NaH}$ was quenched by addition of water $(1 \mathrm{~mL})$. The heterogeneous solution was concentrated to evaporate the THF, and the residue was dilute in additional water $(2 \mathrm{~mL})$. A wash with $\mathrm{Et}_{2} \mathrm{O}$ was performed to eliminate the excess of alcohol and the aqueous layer was acidified with $\mathrm{HCl}(2 \mathrm{M})$ to $\mathrm{pH}$ 3-4. The product was extracted with EtOAc (x3). The organic layers were washed with brine, dried over $\mathrm{MgSO}_{4}$, filtrate and concentrate. The product was purified by column chromatography (8:2 to 7:3 Hexane:(3:1:0.1 EtOAc:EtOH:AcOH)) to give the final product as a pale yellow oil (320 mg, $44 \%$ yield).

${ }^{1}$ H-NMR: $\left(400 \mathrm{MHz}, \mathrm{CDCl}_{3}\right) \delta 5.83(\mathrm{ddt}, J=16.9,10.2,6.7 \mathrm{~Hz}, 1 \mathrm{H}), 5.06(\mathrm{ddd}, J=17.1,3.4,1.6 \mathrm{~Hz}, 1 \mathrm{H})$, $5.03-4.99(\mathrm{~m}, 1 \mathrm{H}), 4.13(\mathrm{~s}, 2 \mathrm{H}), 3.64-3.54(\mathrm{~m}, 2 \mathrm{H}), 2.21-2.13(\mathrm{~m}, 2 \mathrm{H}), 1.75(\mathrm{dd}, J=14.2,7.2 \mathrm{~Hz}, 2 \mathrm{H})$.

${ }^{13}$ C-NMR: $\left(101 \mathrm{MHz}, \mathrm{CDCl}_{3}\right) \delta 175.2(\mathrm{C}), 137.8(\mathrm{CH}), 115.1\left(\mathrm{CH}_{2}\right), 71.4\left(\mathrm{CH}_{2}\right), 67.7\left(\mathrm{CH}_{2}\right), 30.0\left(\mathrm{CH}_{2}\right), 28.6$ $\left(\mathrm{CH}_{2}\right)$.

IR (ATR): $\tilde{v}\left(\mathrm{~cm}^{-1}\right)$ 3111, 3048, 2940, 1731, 1640, 1210, 1129, 911.

HRMS: calculated for $\mathrm{C}_{7} \mathrm{H}_{12} \mathrm{O}_{3}\left(\mathrm{M}^{+}\right)$144.0781, found 144.0779.

Rf: 0.42 (7:3 Hexane:(3:1:0.1 EtOAc:EtOH:AcOH)) 
17. Copy of the ${ }^{1} \mathrm{H}$ - and ${ }^{13} \mathrm{C}$-NMR spectra of the synthetized compounds and NOESY of the required compounds. ${ }^{1} \mathrm{H}-\mathrm{NMR}$ of q-OAc

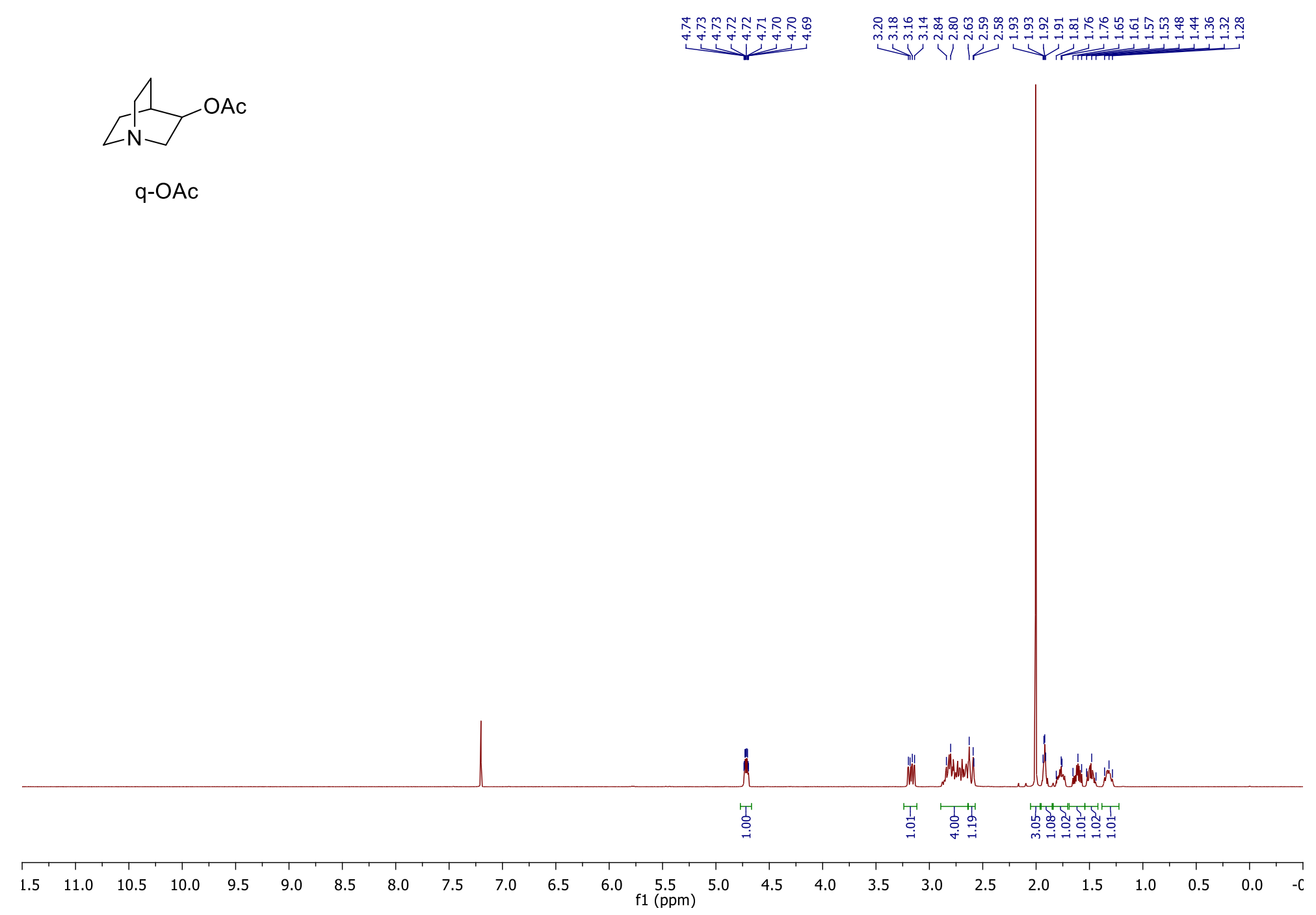


${ }^{13} \mathrm{C}-\mathrm{NMR}$ of q-OAc

要
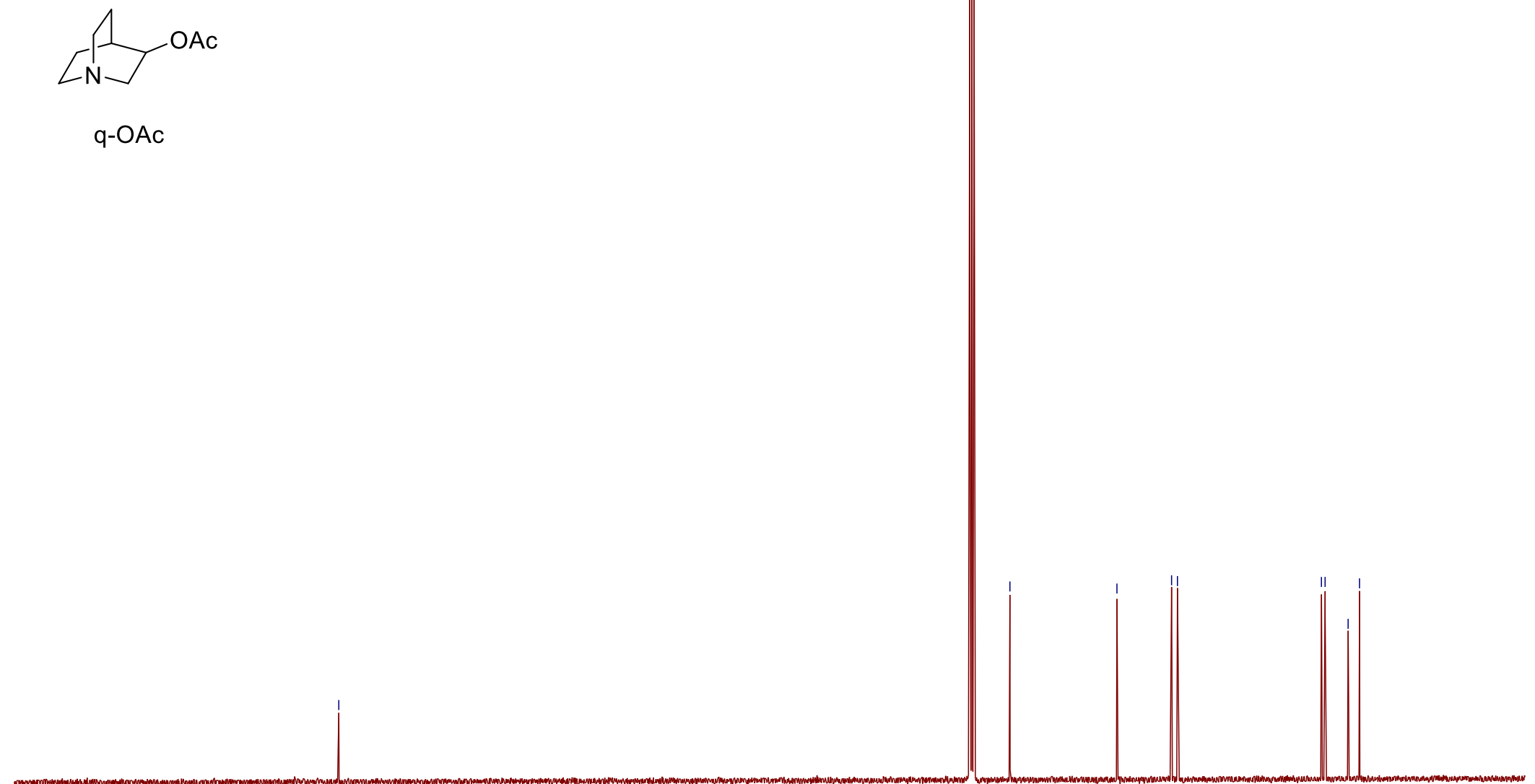

$\begin{array}{llllllllllllllllllllll}210 & 200 & 190 & 180 & 170 & 160 & 150 & 140 & 130 & 120 & \begin{array}{l}110 \\ \mathrm{f} 1(\mathrm{ppm})\end{array} & 100 & 90 & 80 & 70 & 60 & 50 & 40 & 30 & 20 & 10 & 0\end{array}$ 
${ }^{1} \mathrm{H}-\mathrm{NMR}$ of compound $\mathbf{1 a}$
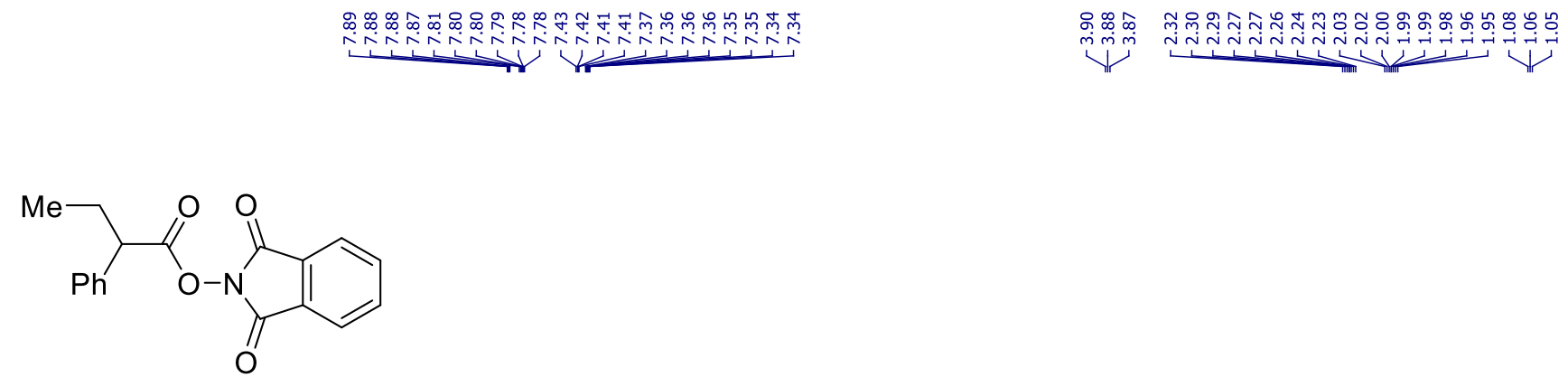

$1 \mathrm{a}$

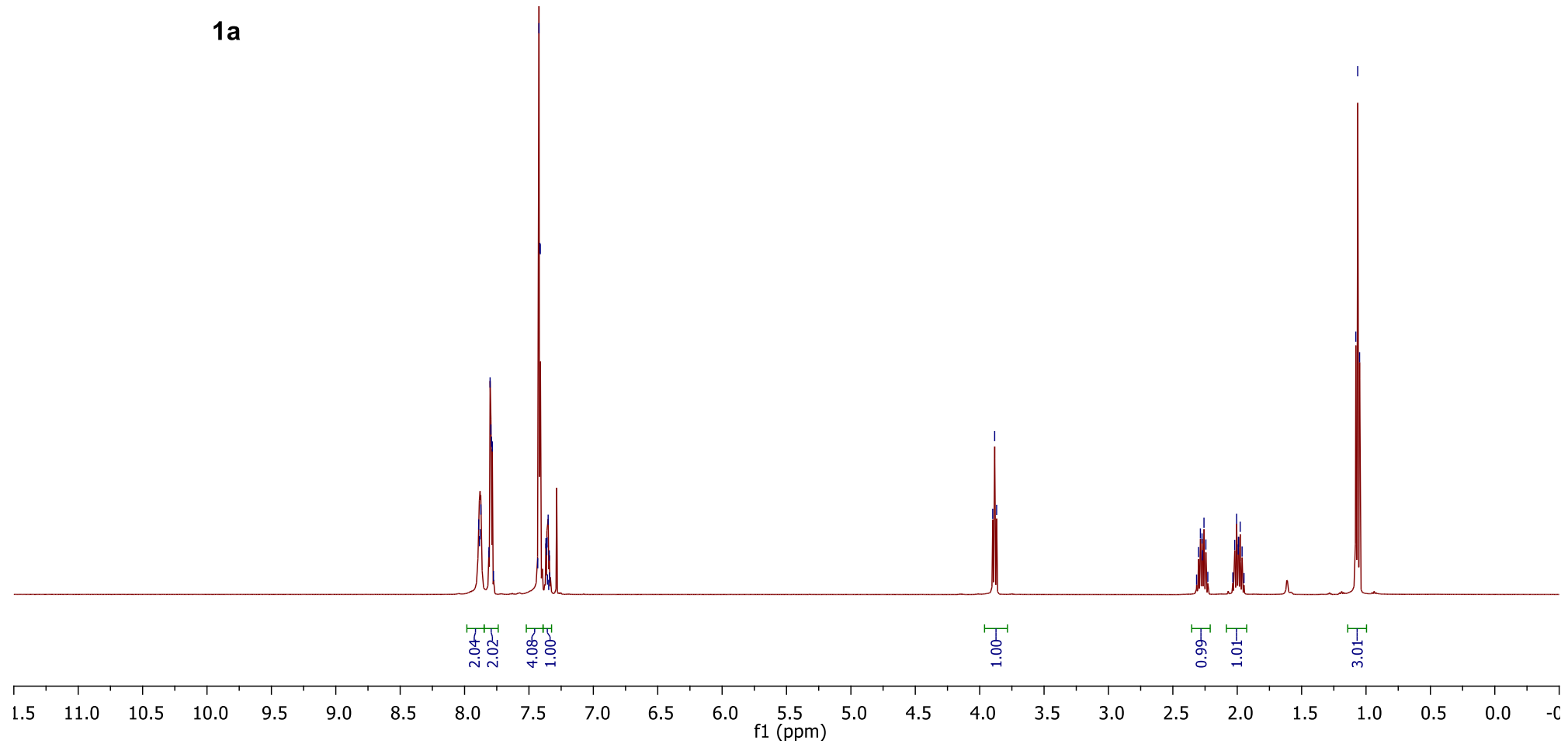


${ }^{13} \mathrm{C}$-NMR of compound 1a

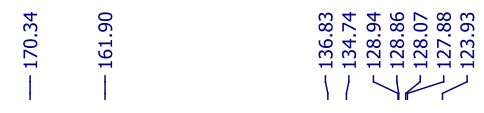

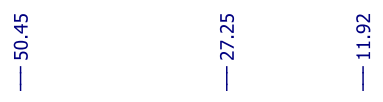

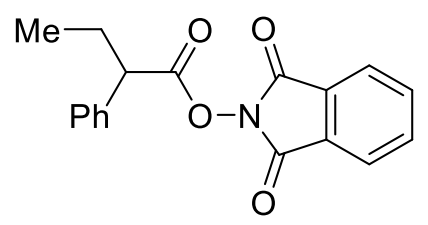

1a

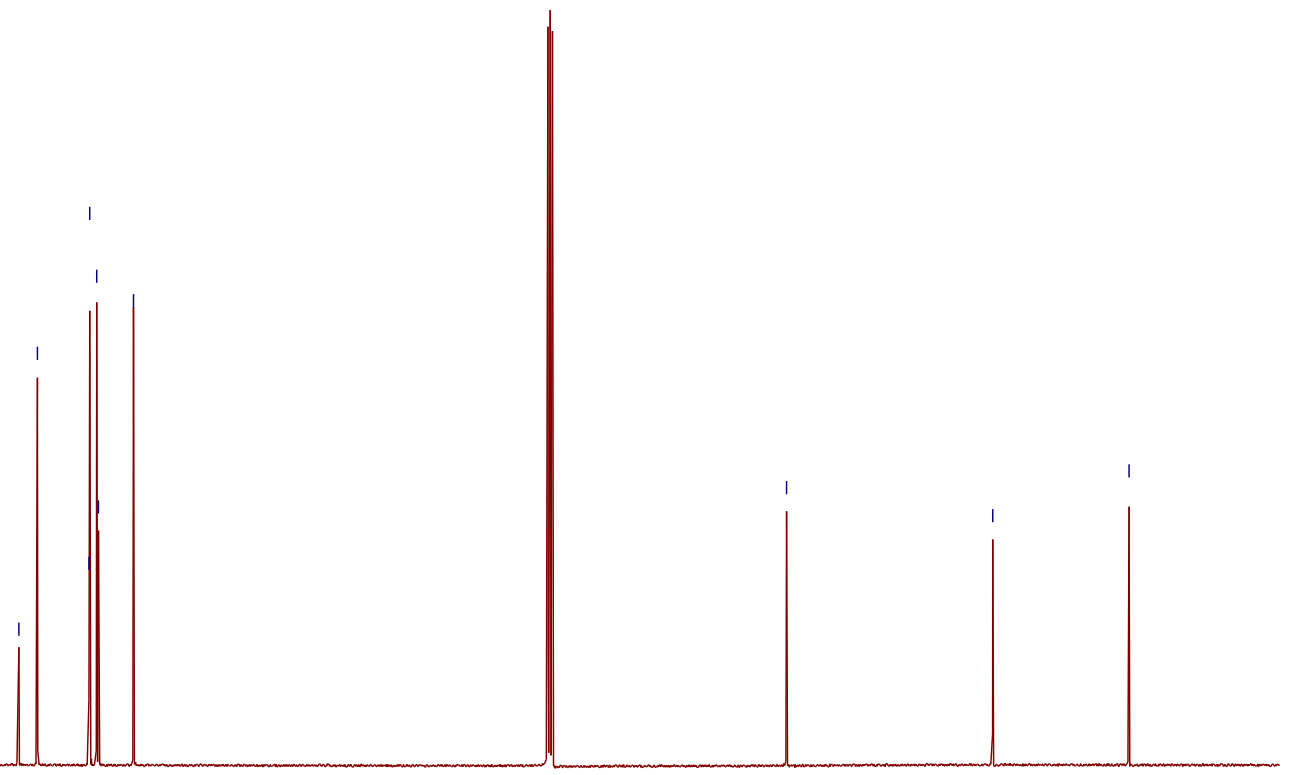

$210 \quad 200 \quad 190$

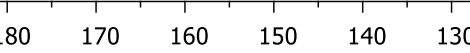

120

110100

$90 \quad 80$

$\begin{array}{lll}70 & 60 & 50\end{array}$

40

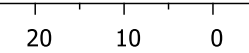


${ }^{1} \mathrm{H}-\mathrm{NMR}$ of compound $\mathbf{1 b}$

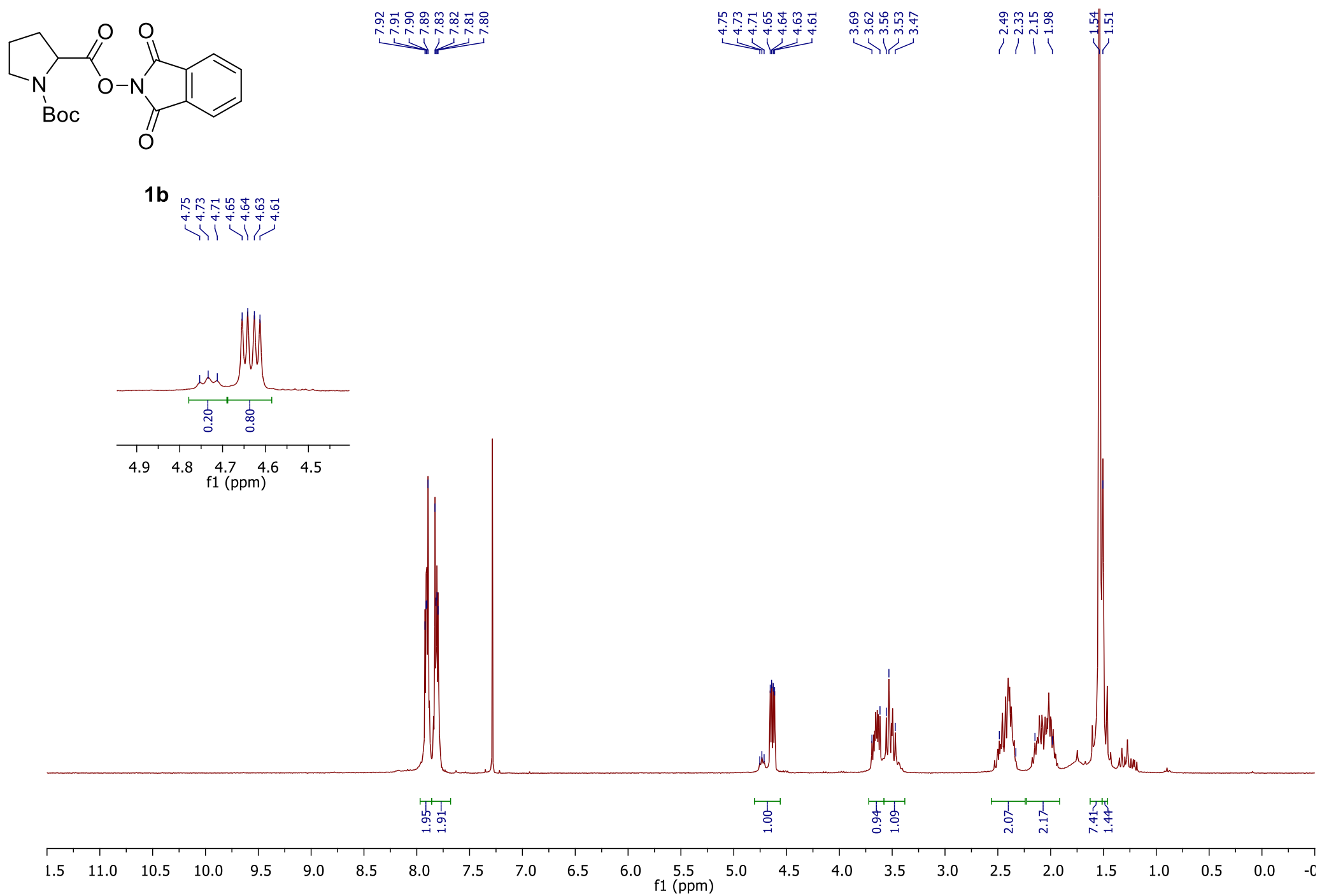


${ }^{13} \mathrm{C}-\mathrm{NMR}$ of compound $\mathbf{1 b}$

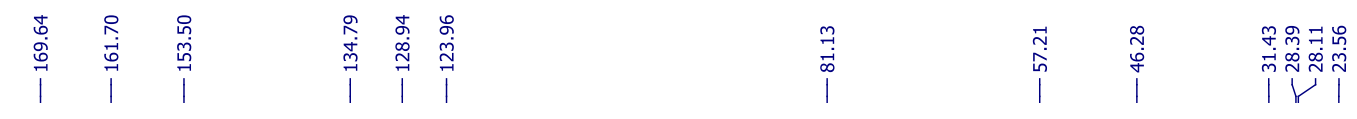

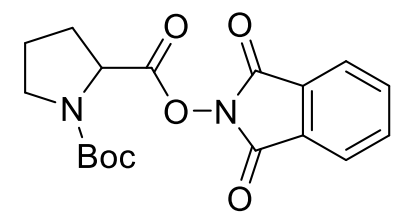

1b

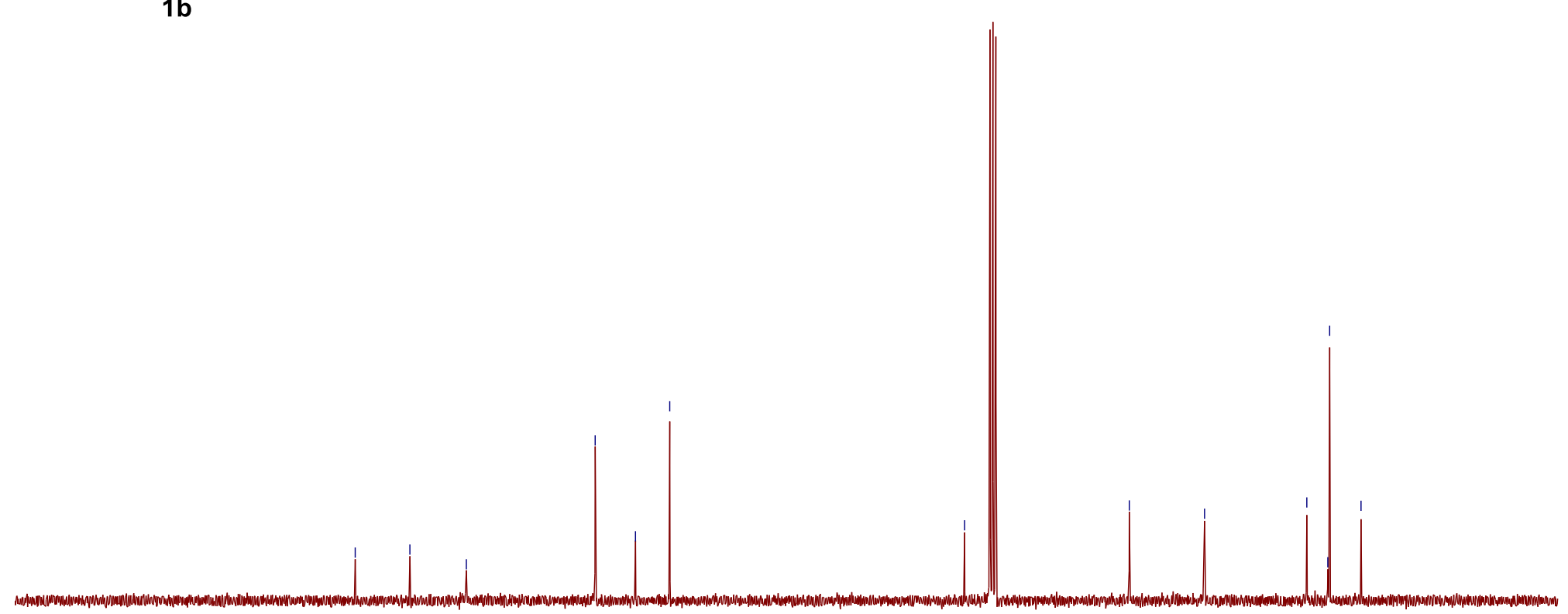


${ }^{1} \mathrm{H}-\mathrm{NMR}$ of compound 2a
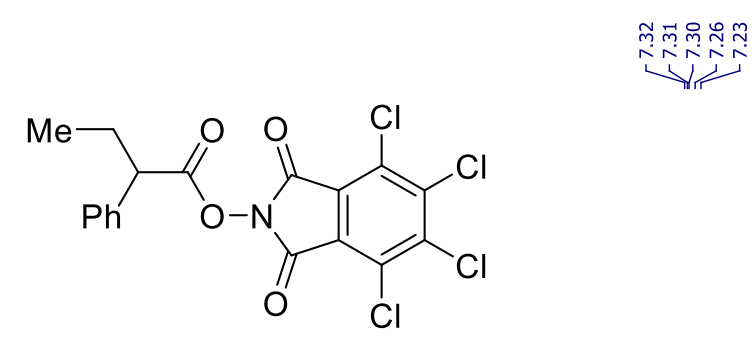

$2 a$

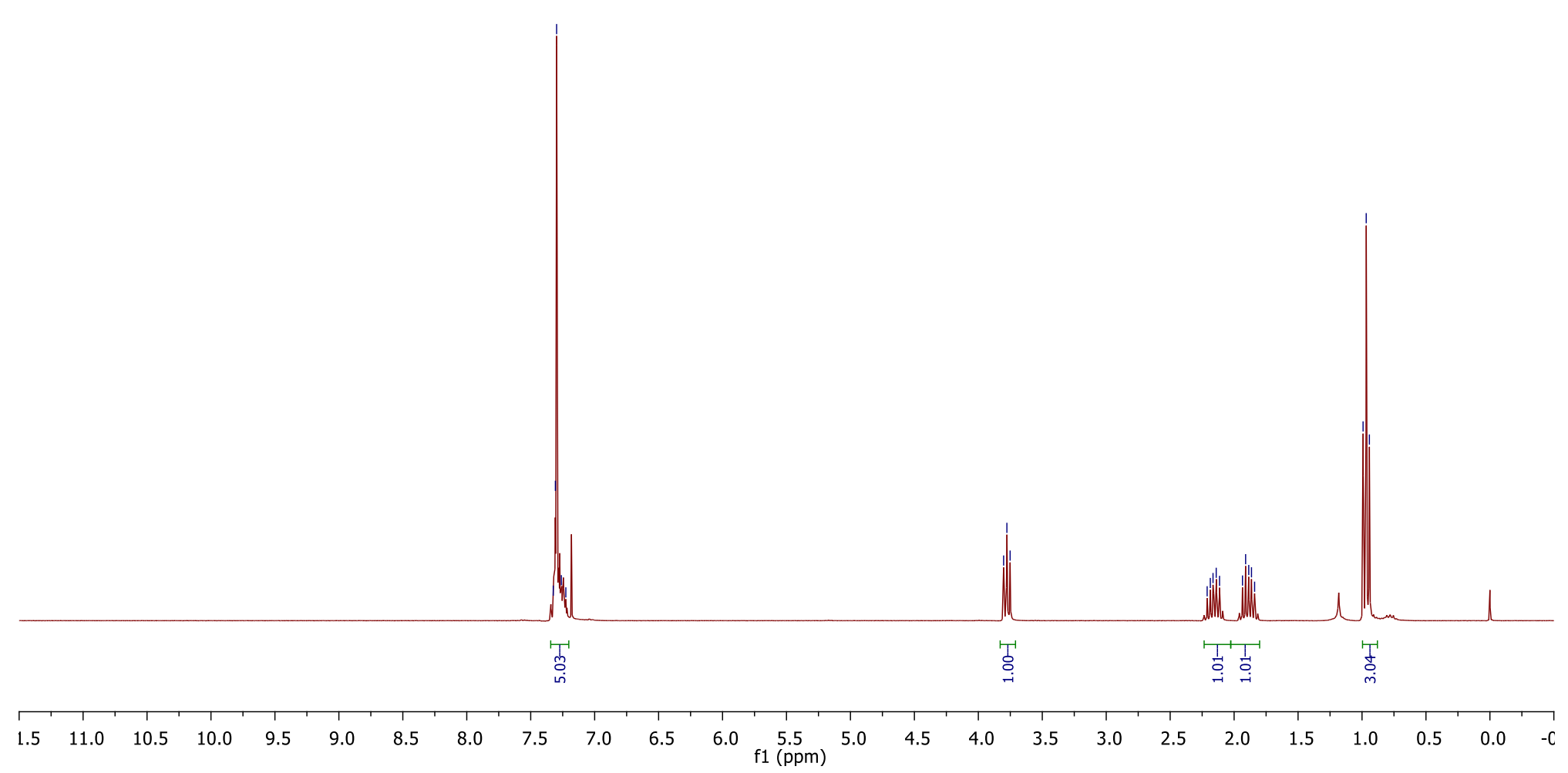


${ }^{13} \mathrm{C}-\mathrm{NMR}$ of compound $\mathbf{2 a}$

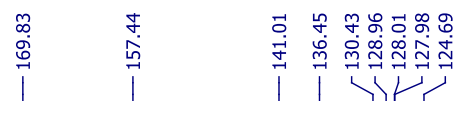

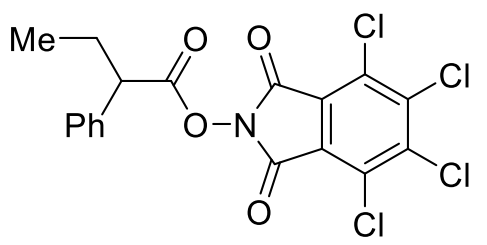

$2 a$

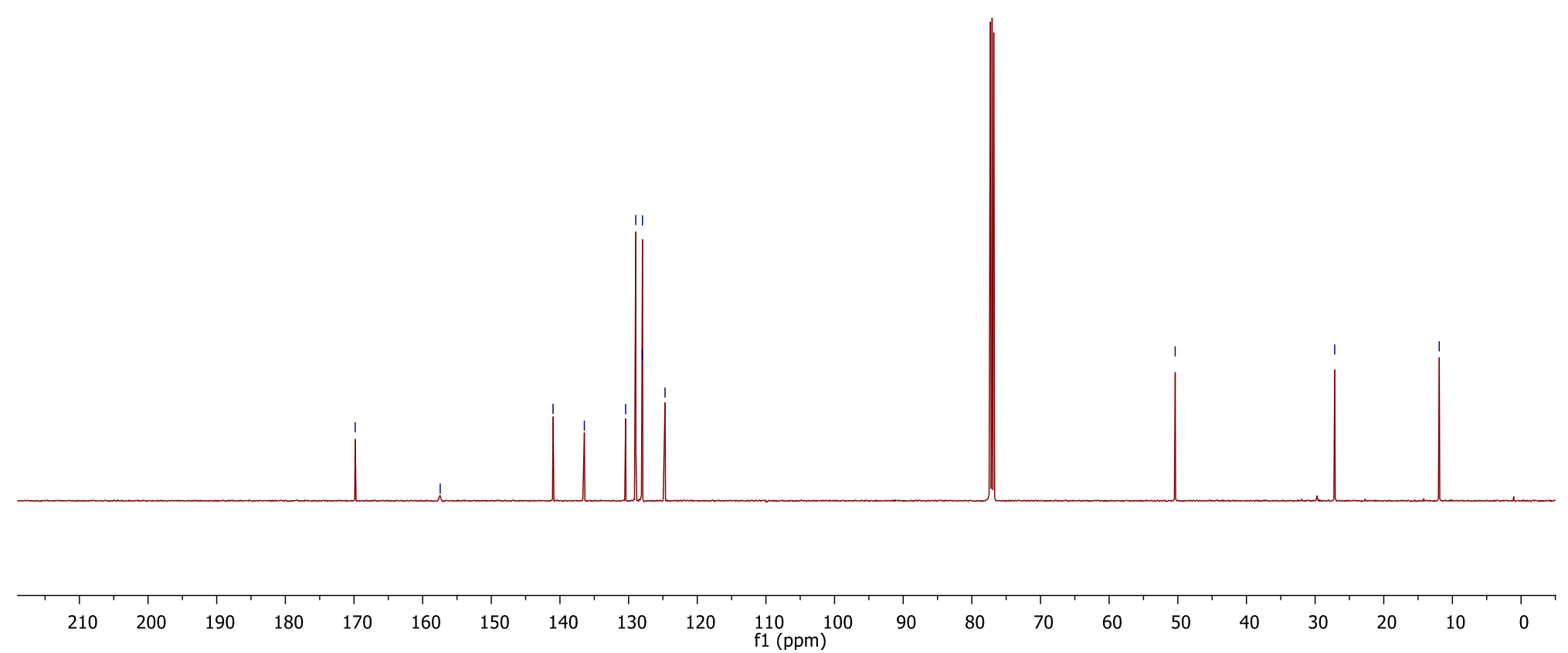


${ }^{1} \mathrm{H}-\mathrm{NMR}$ of compound $\mathbf{2 b}$

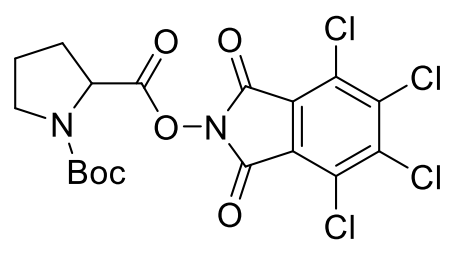

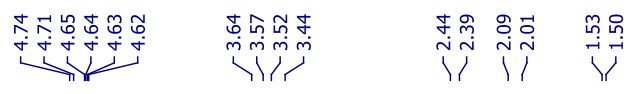

2b

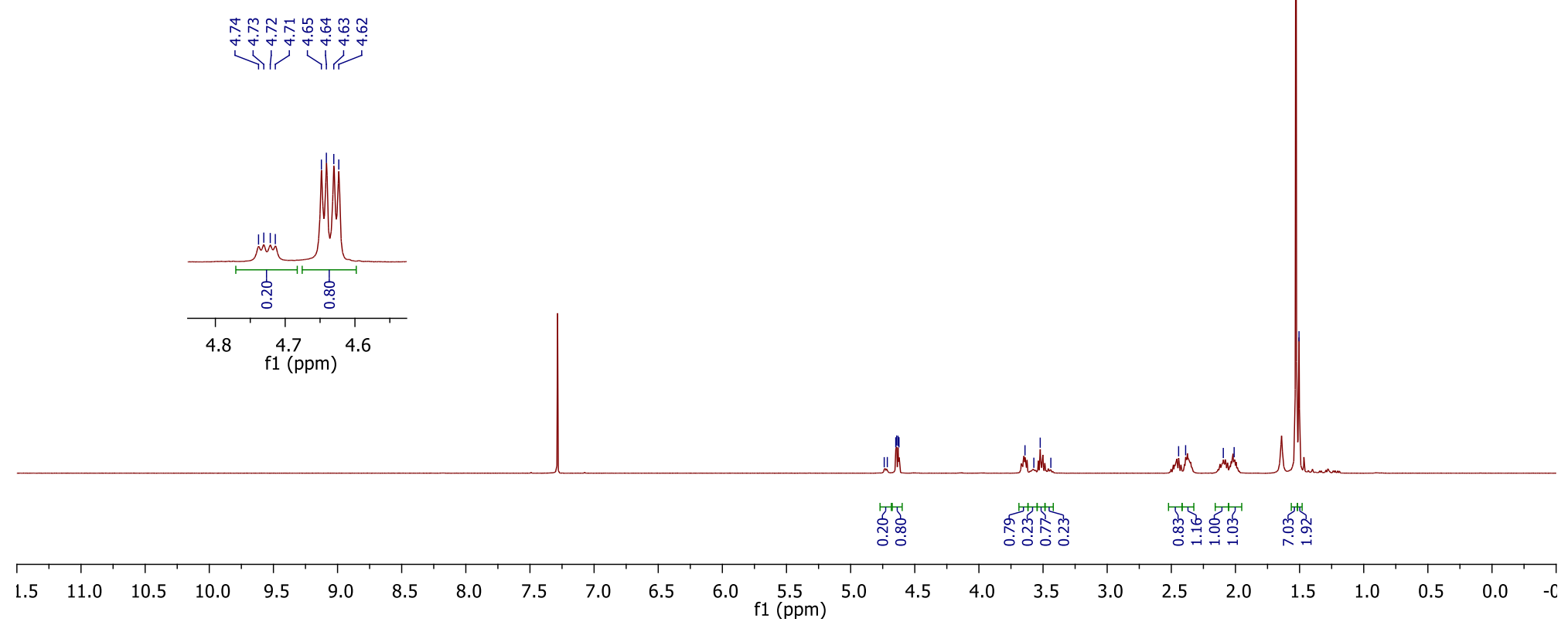


${ }^{13} \mathrm{C}-\mathrm{NMR}$ of compound $\mathbf{2 b}$

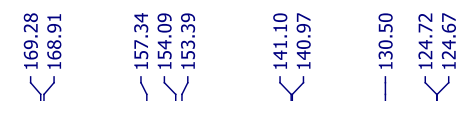

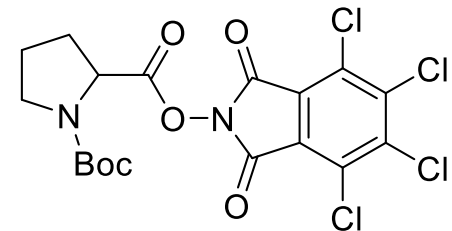

2b
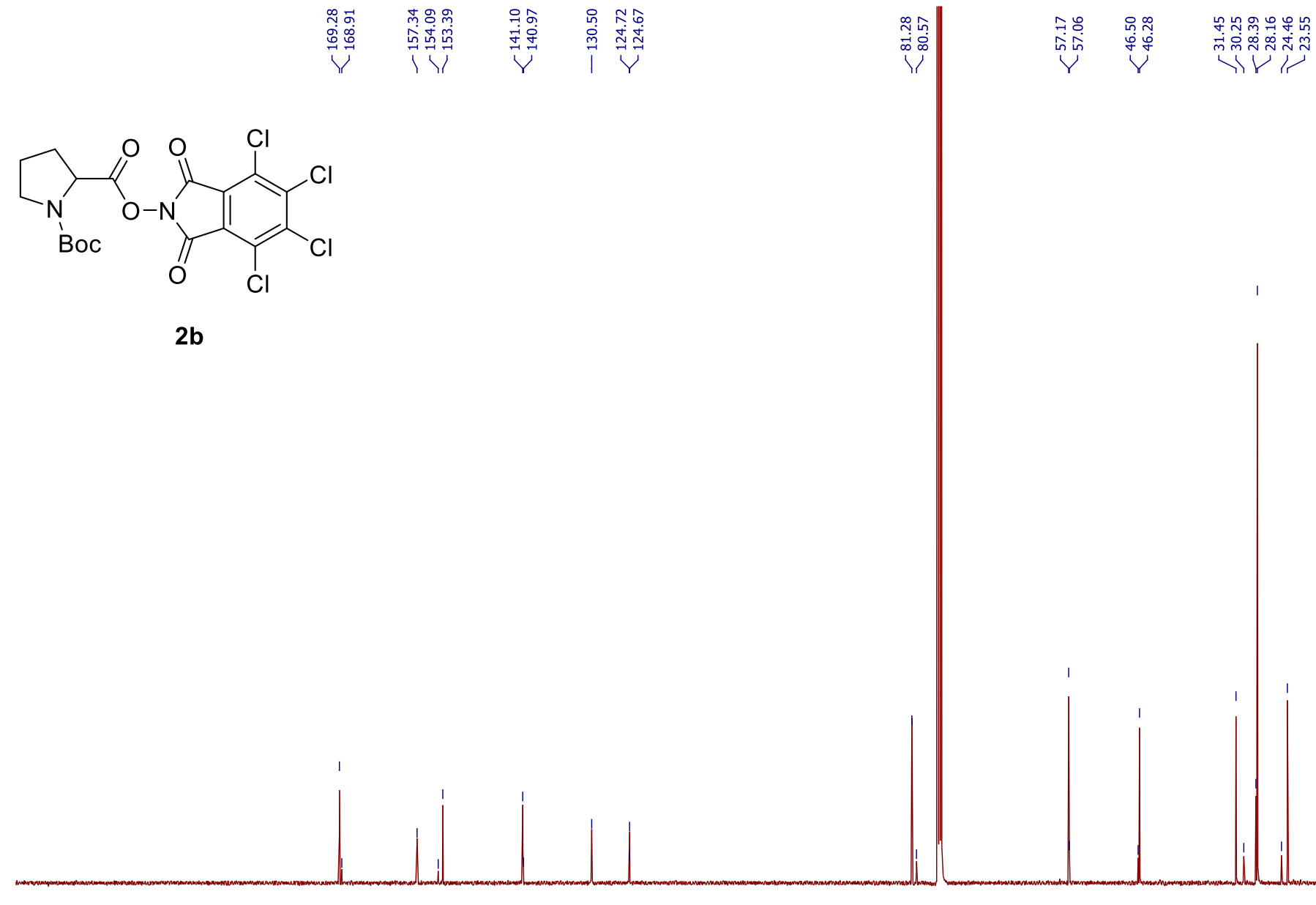

$210 \quad 200 \quad 190$ 
${ }^{1} \mathrm{H}-\mathrm{NMR}$ of compound $\mathbf{2 b}$,
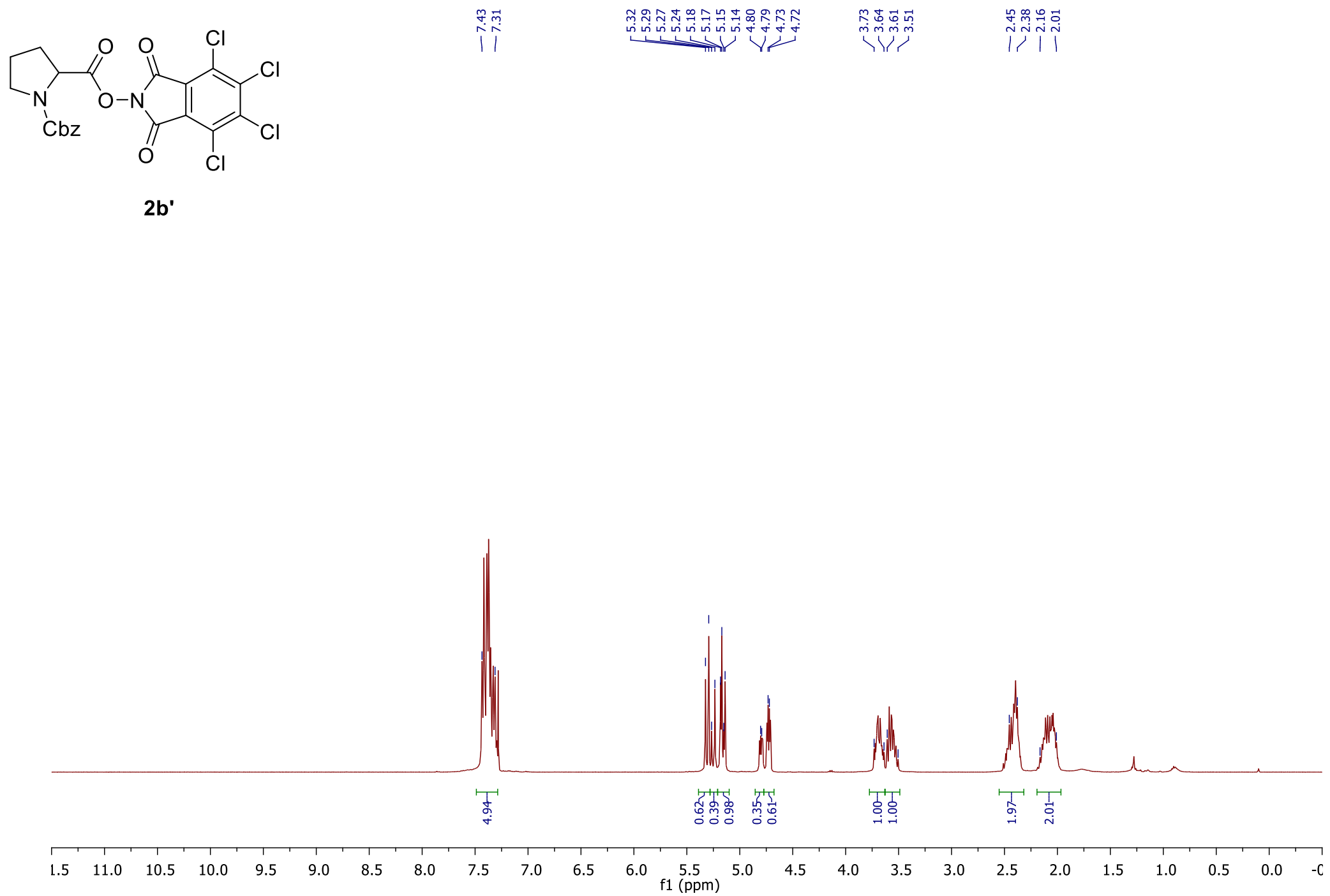
${ }^{13} \mathrm{C}-\mathrm{NMR}$ of compound $\mathbf{2 b}$ '

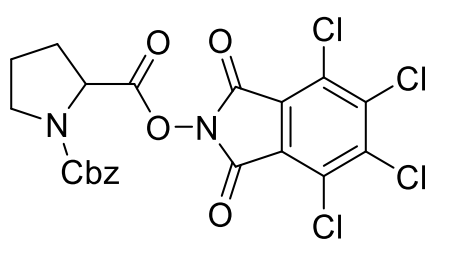

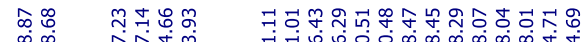

$\checkmark \quad Y r^{\prime}$

ن

$2 b^{\prime}$

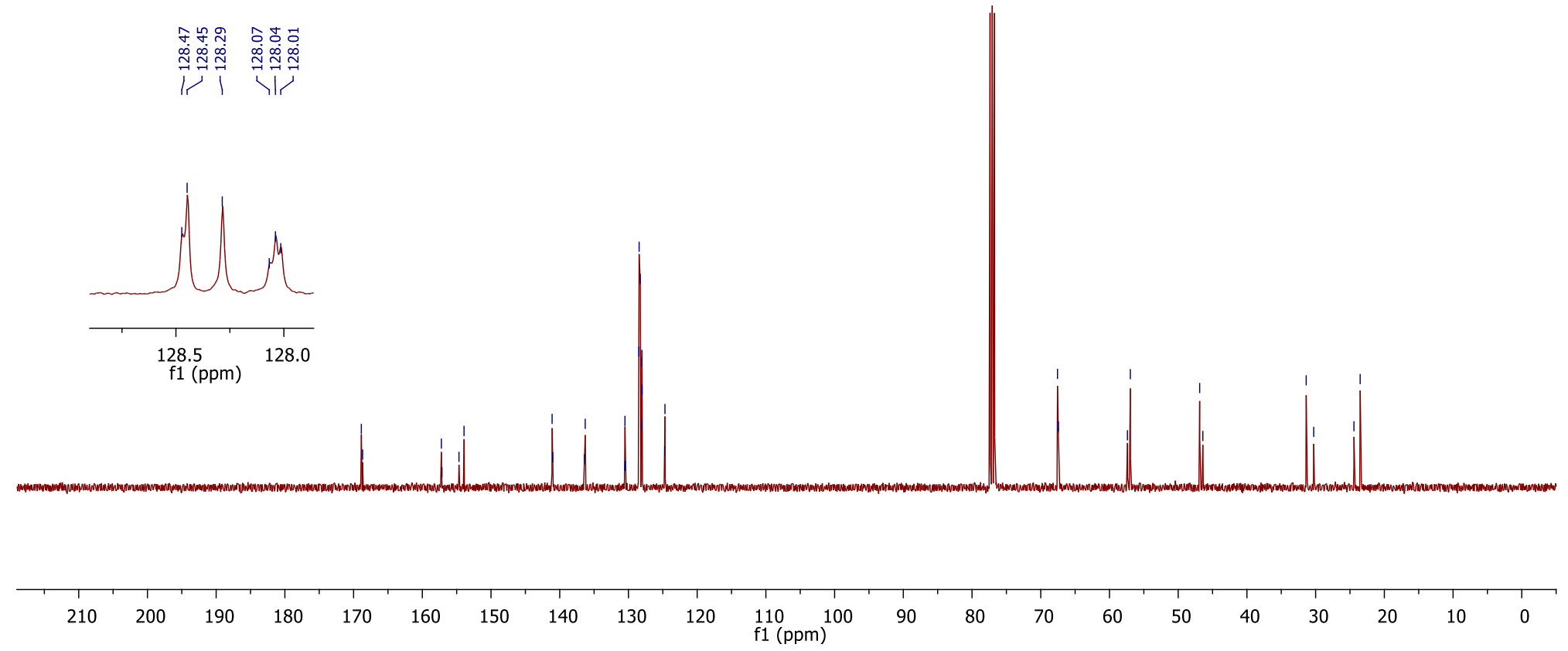


${ }^{1} \mathrm{H}-\mathrm{NMR}$ of compound $\mathbf{2 c}$

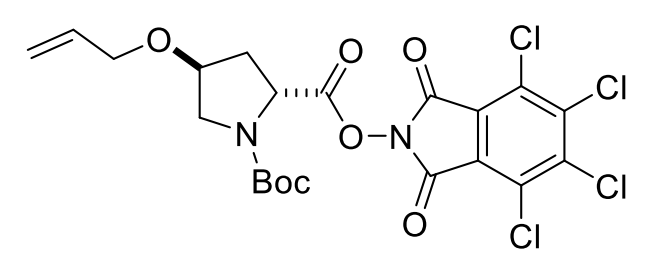

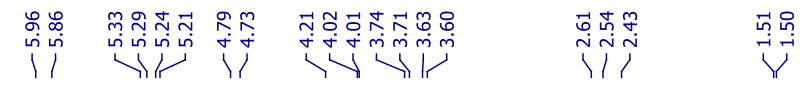

2c

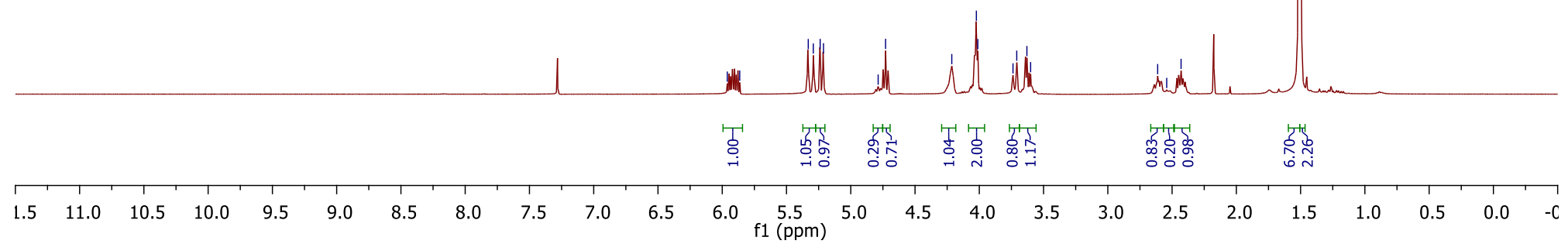


${ }^{13} \mathrm{C}$-NMR of compound $2 \mathrm{c}$
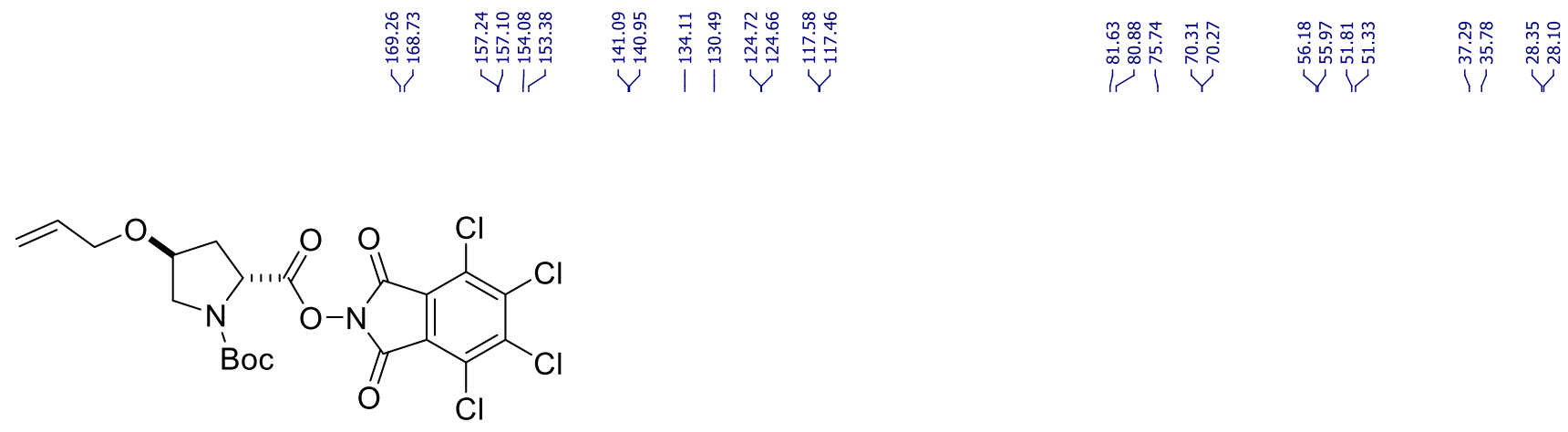

2c

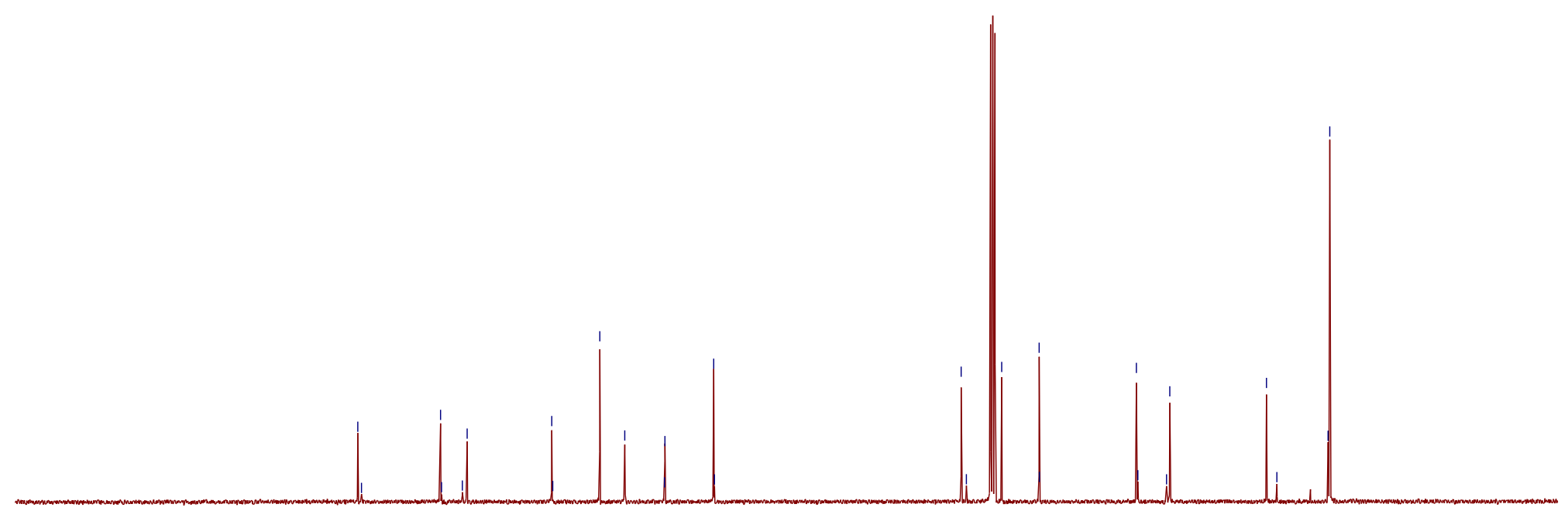


${ }^{1} \mathrm{H}$-NMR of compound $\mathbf{2 d}$

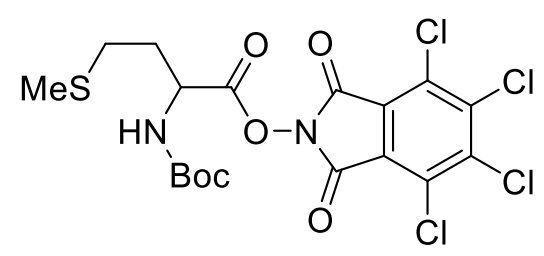

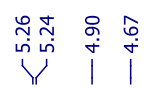

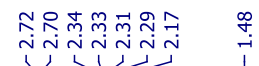

2d

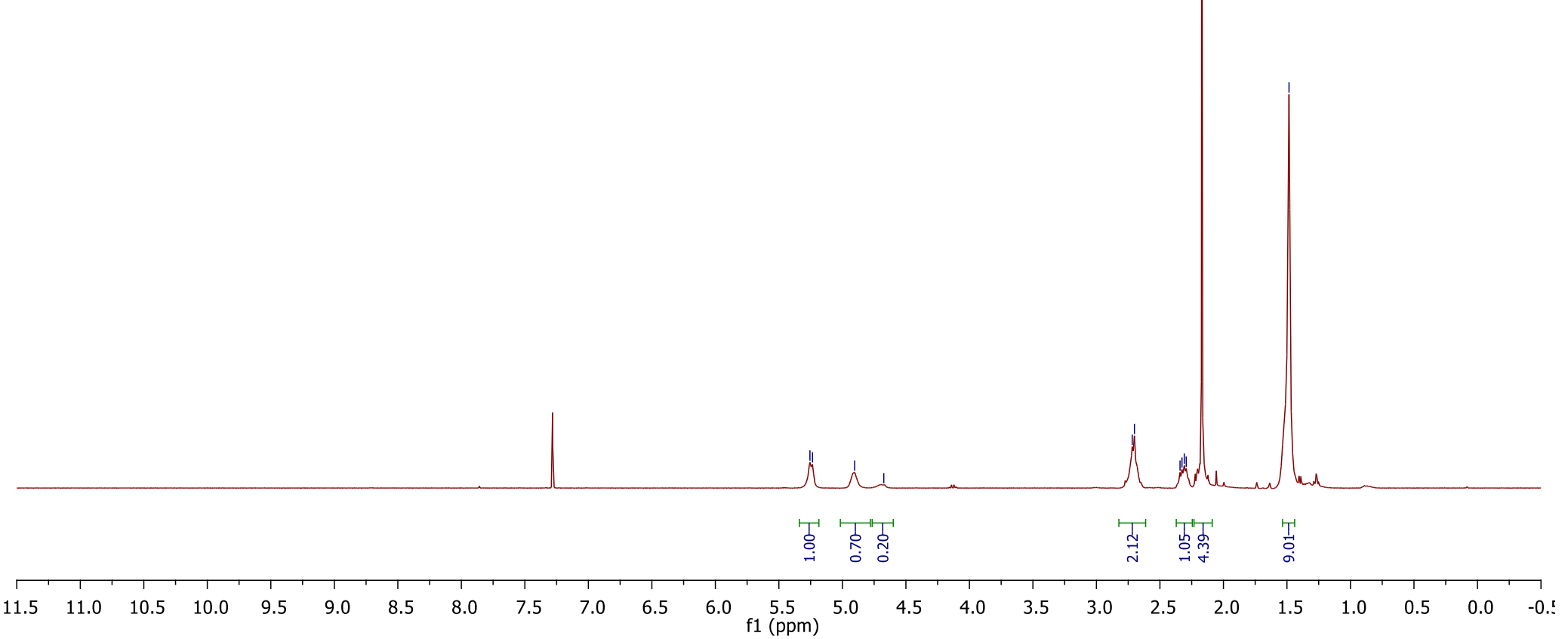


${ }^{13} \mathrm{C}$-NMR of compound $\mathbf{2 d}$

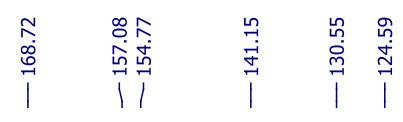

l

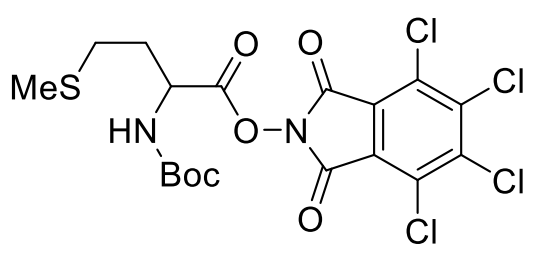

2d

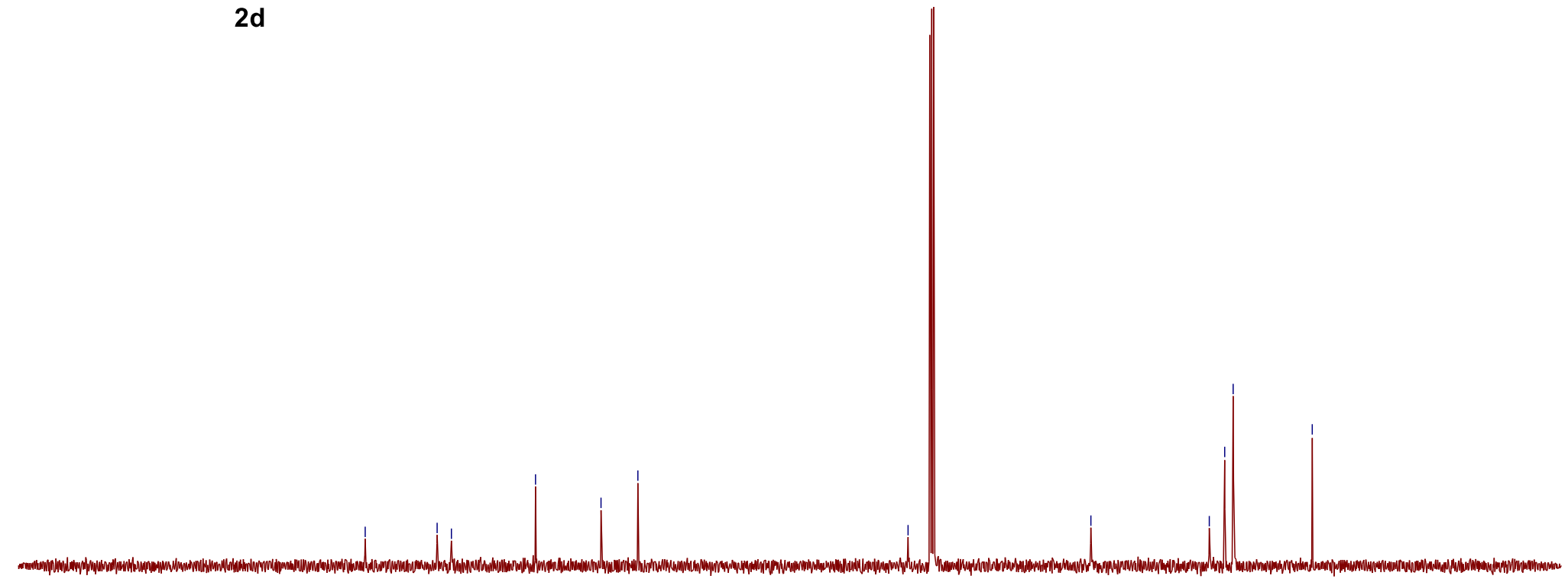

$\begin{array}{llllllllllll}220 & 210 & 200 & 190 & 180 & 170 & 160 & 150 & 140 & 130 & 120 & 110 \\ \mathrm{f} 1(\mathrm{ppm}) & 90\end{array}$ 
${ }^{1} \mathrm{H}$-NMR of compound $\mathbf{2 e}$

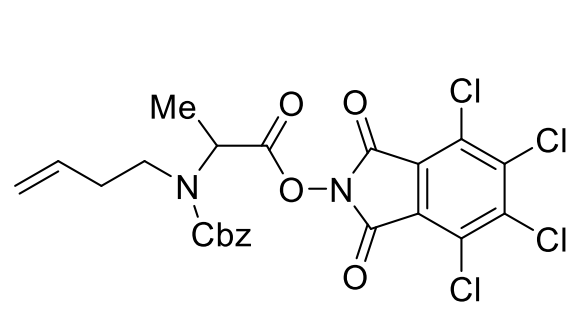

i

$2 e$

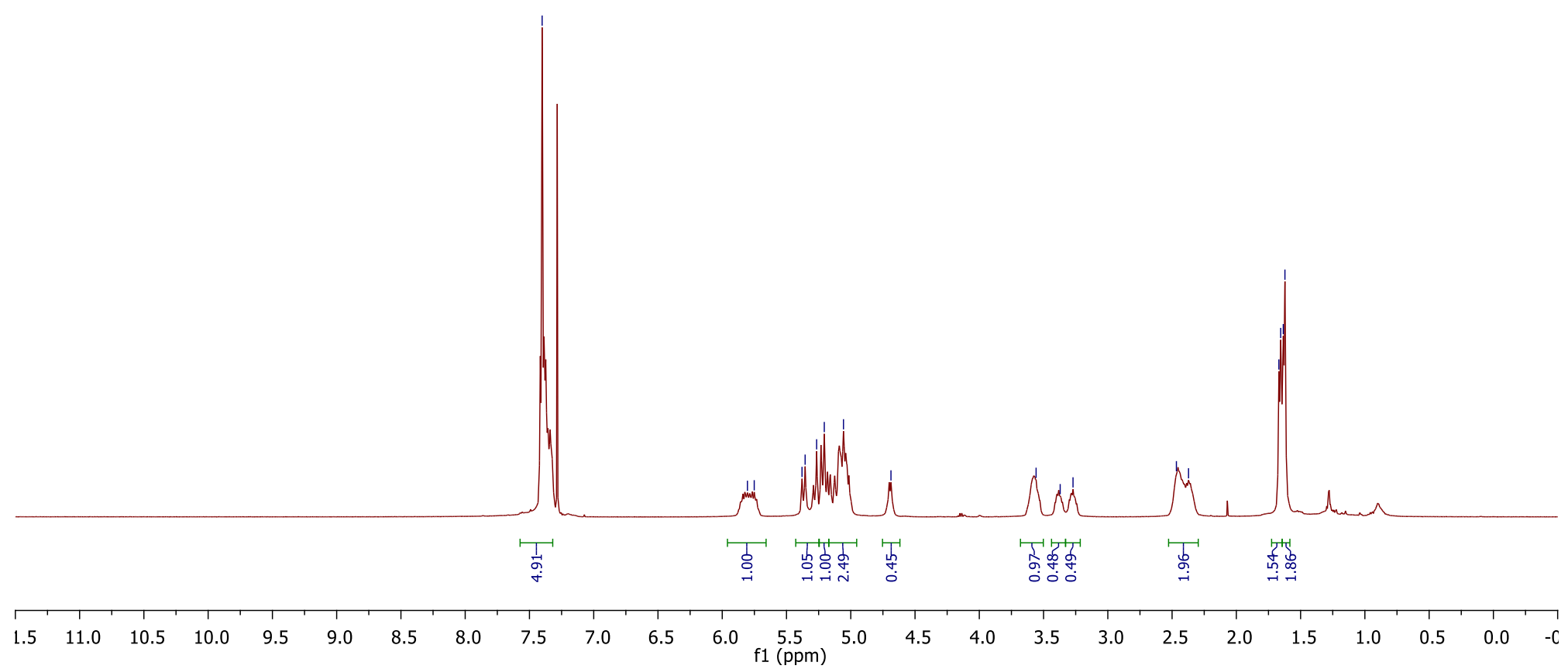


${ }^{13} \mathrm{C}-\mathrm{NMR}$ of compound $2 \mathrm{e}$

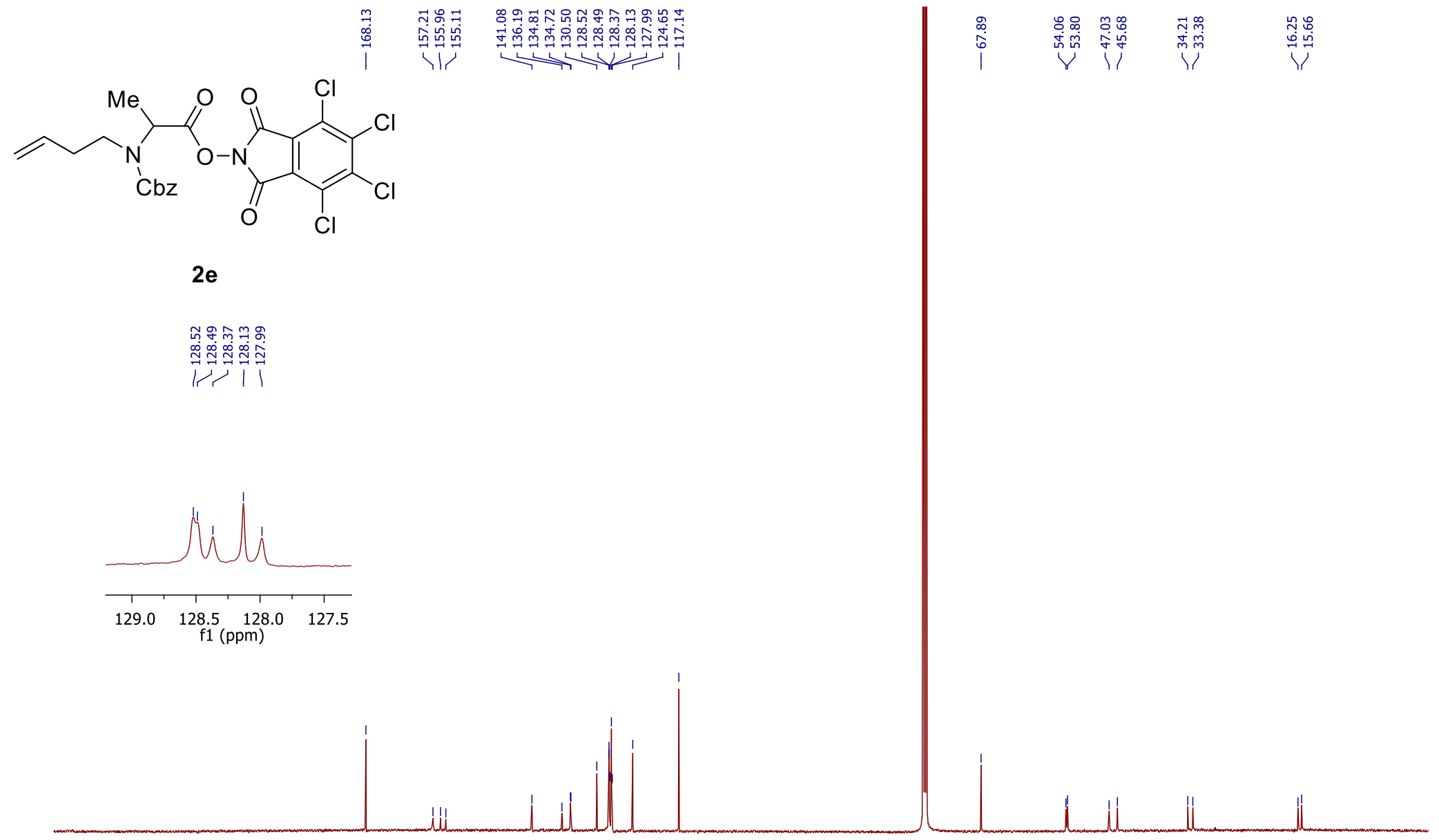

$\begin{array}{llllllllllllllllllllllllll}210 & 200 & 190 & 180 & 170 & 160 & 150 & 140 & 130 & 120 & 110 & 100 & 90 & 80 & 70 & 60 & 50 & 40 & 30 & 20 & 10 & 0\end{array}$ 
${ }^{1}$ H-NMR of compound $\mathbf{2 f}$

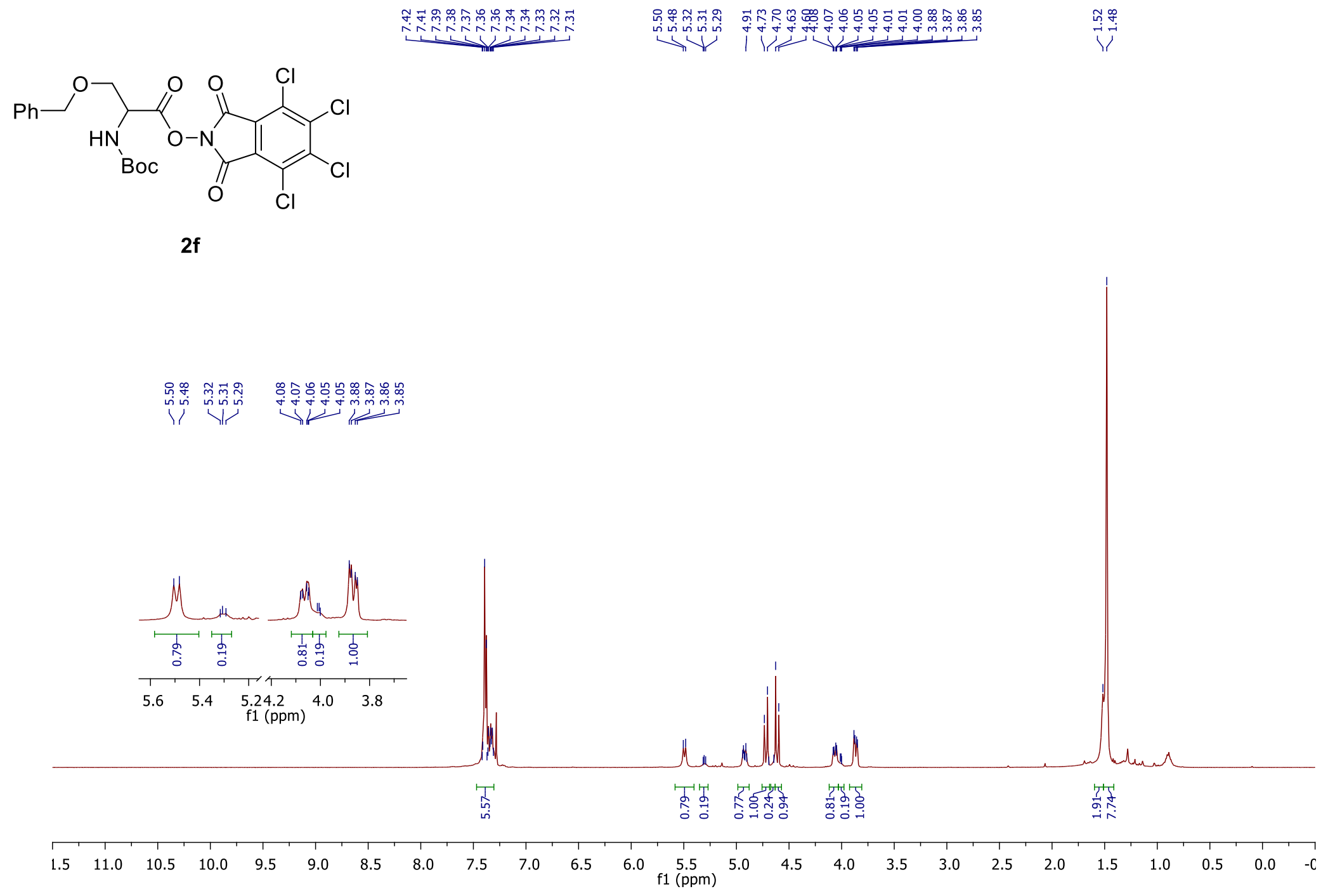


${ }^{13} \mathrm{C}$-NMR of compound $\mathbf{2 f}$

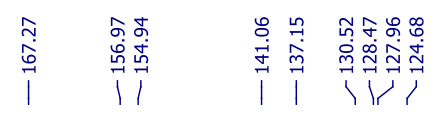

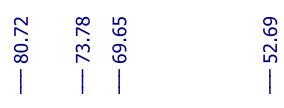

$\stackrel{\substack{\infty \\ \stackrel{1}{1}}}{i}$

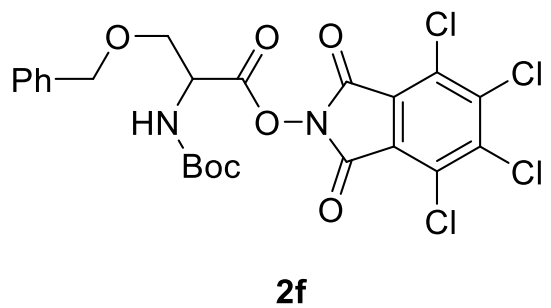

$2 f$

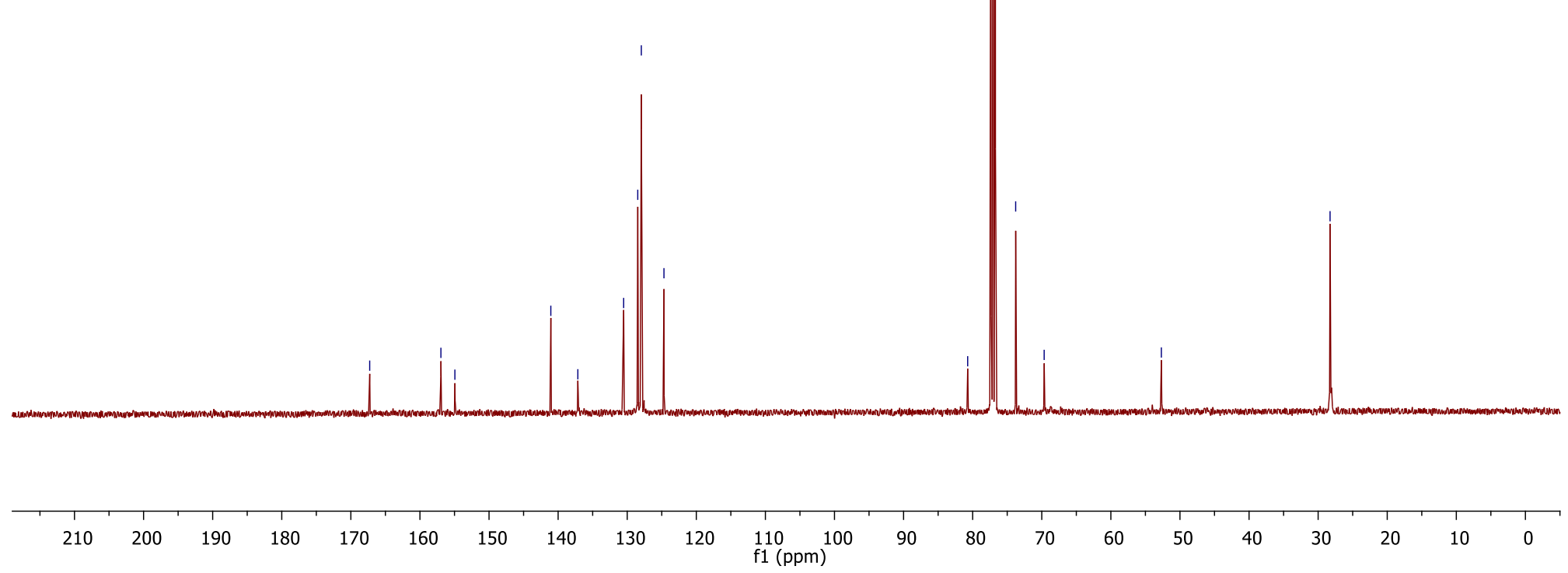


${ }^{1} \mathrm{H}$-NMR of compound $\mathbf{2 g}$

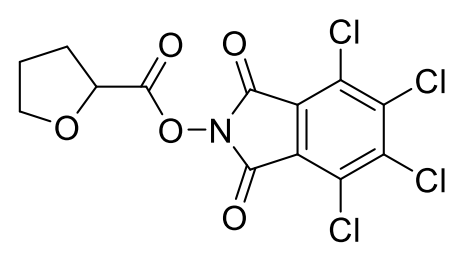

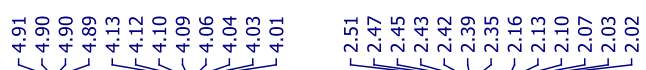

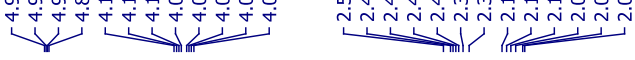

2g

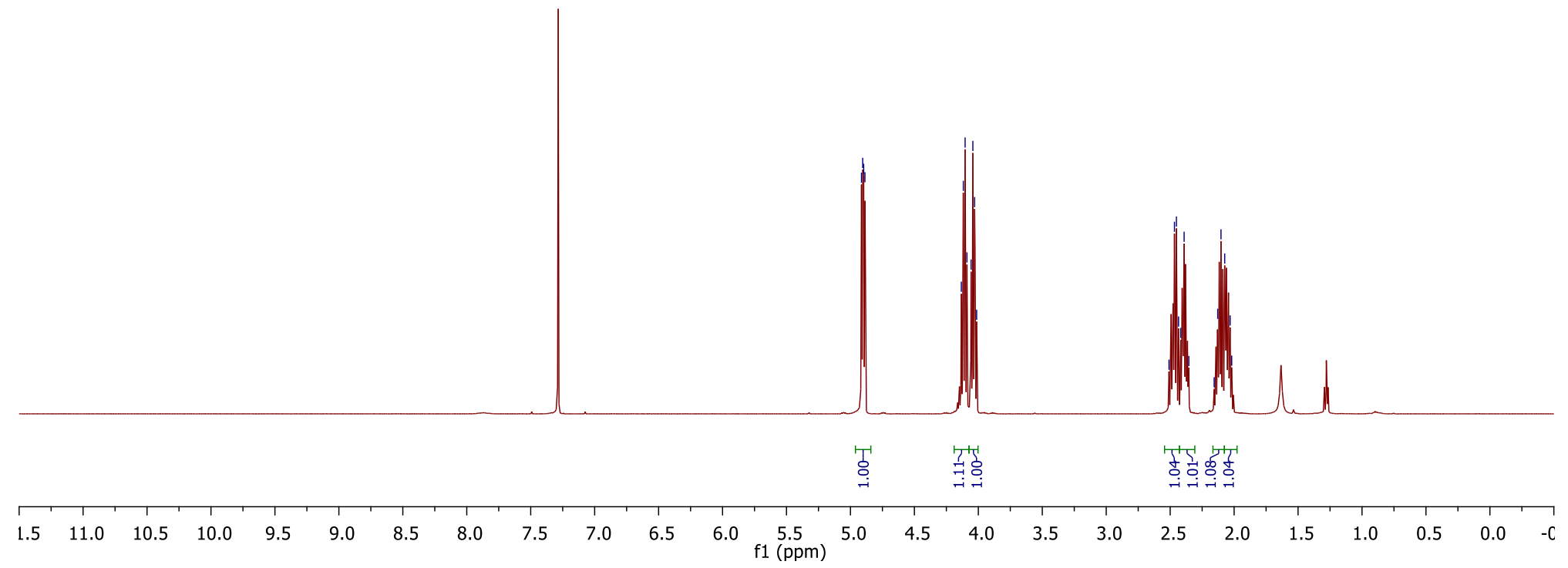


${ }^{13} \mathrm{C}-\mathrm{NMR}$ of compound $\mathbf{2 g}$

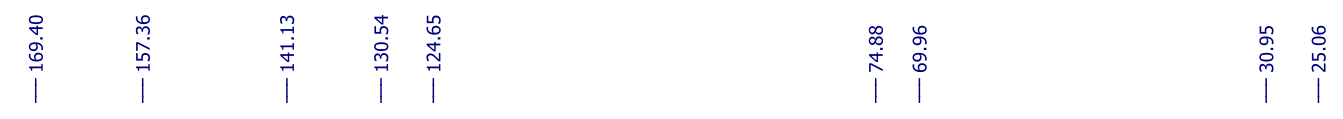

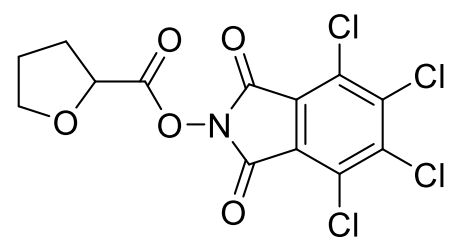

$2 g$

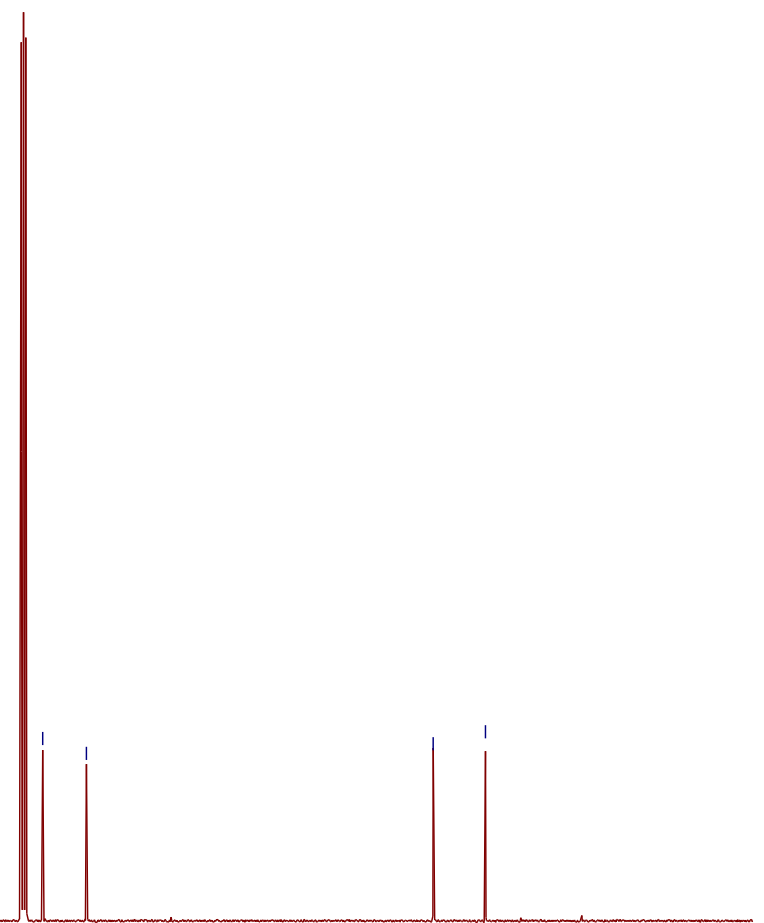

$210 \quad 200 \quad 190$

180
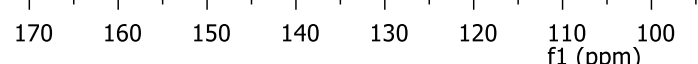

$90 \quad 80$

$\begin{array}{lll}70 & 60 & 50\end{array}$ 
${ }^{1}$ H-NMR of compound $\mathbf{2 h}$

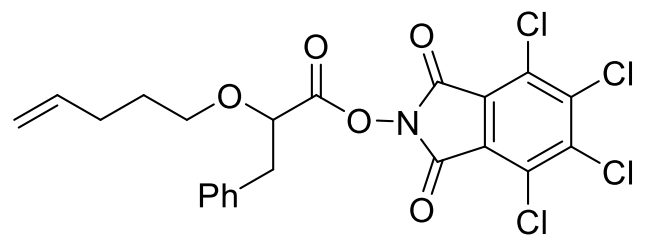

$2 \mathrm{~h}$

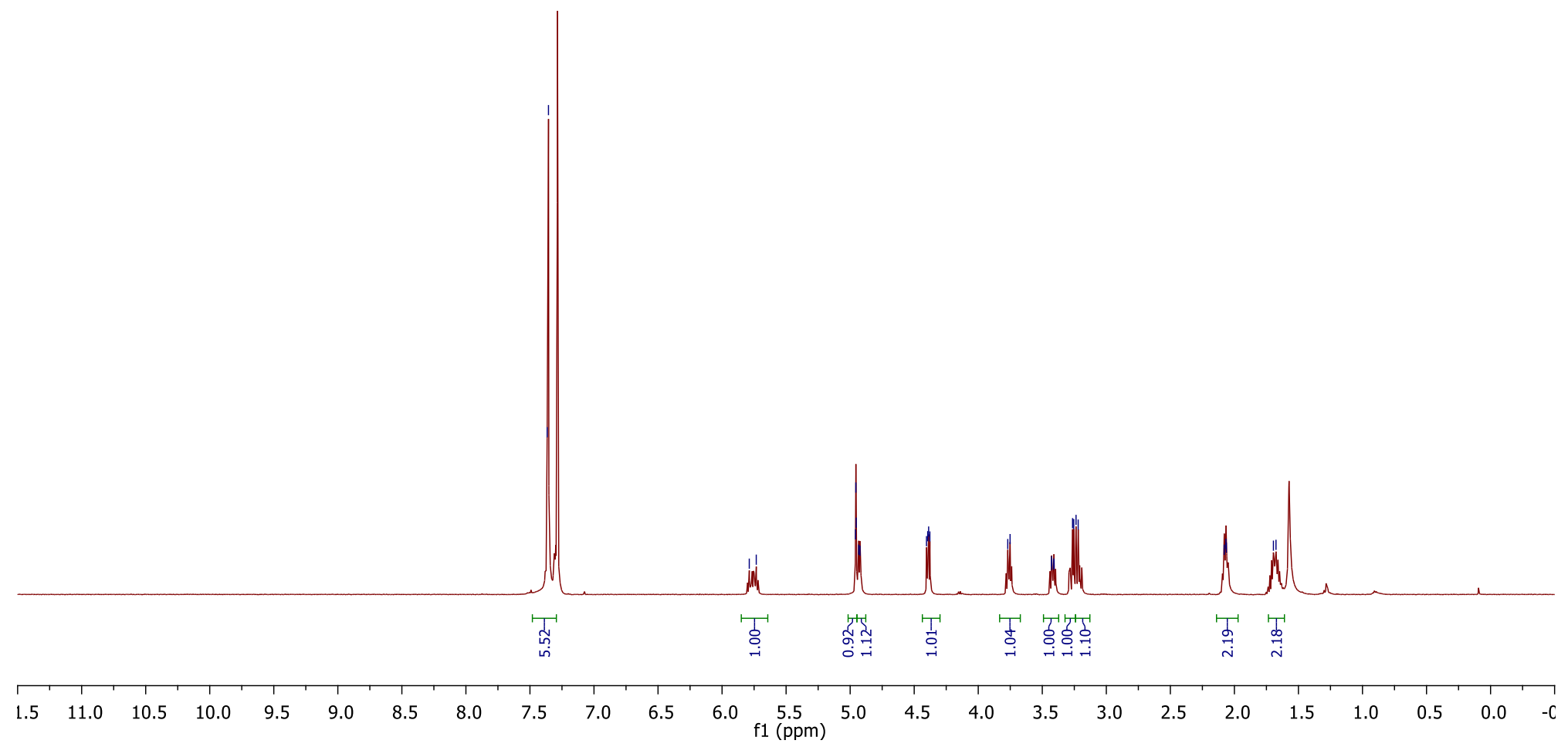


${ }^{13} \mathrm{C}-\mathrm{NMR}$ of compound $\mathbf{2 h}$

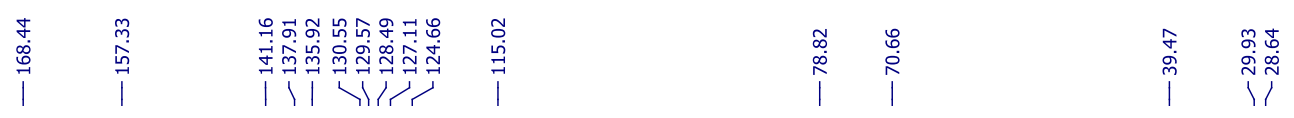

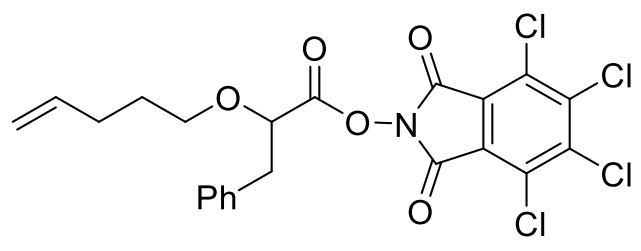

2h
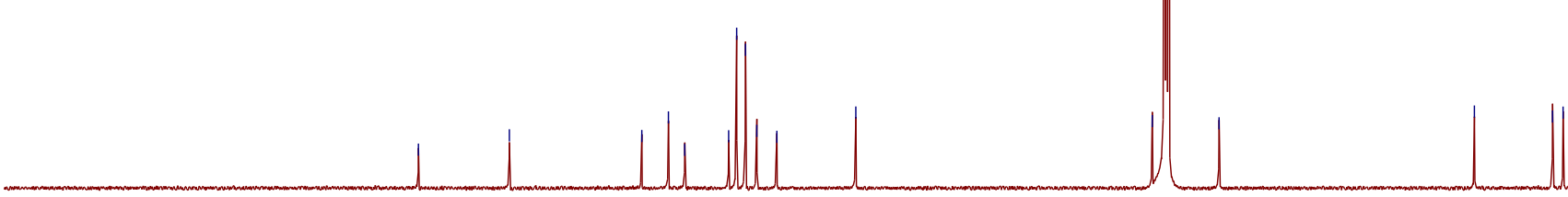

$\begin{array}{lll}210 & 200 \quad 190\end{array}$ 
${ }^{1} \mathrm{H}-\mathrm{NMR}$ of compound $\mathbf{2 i}$

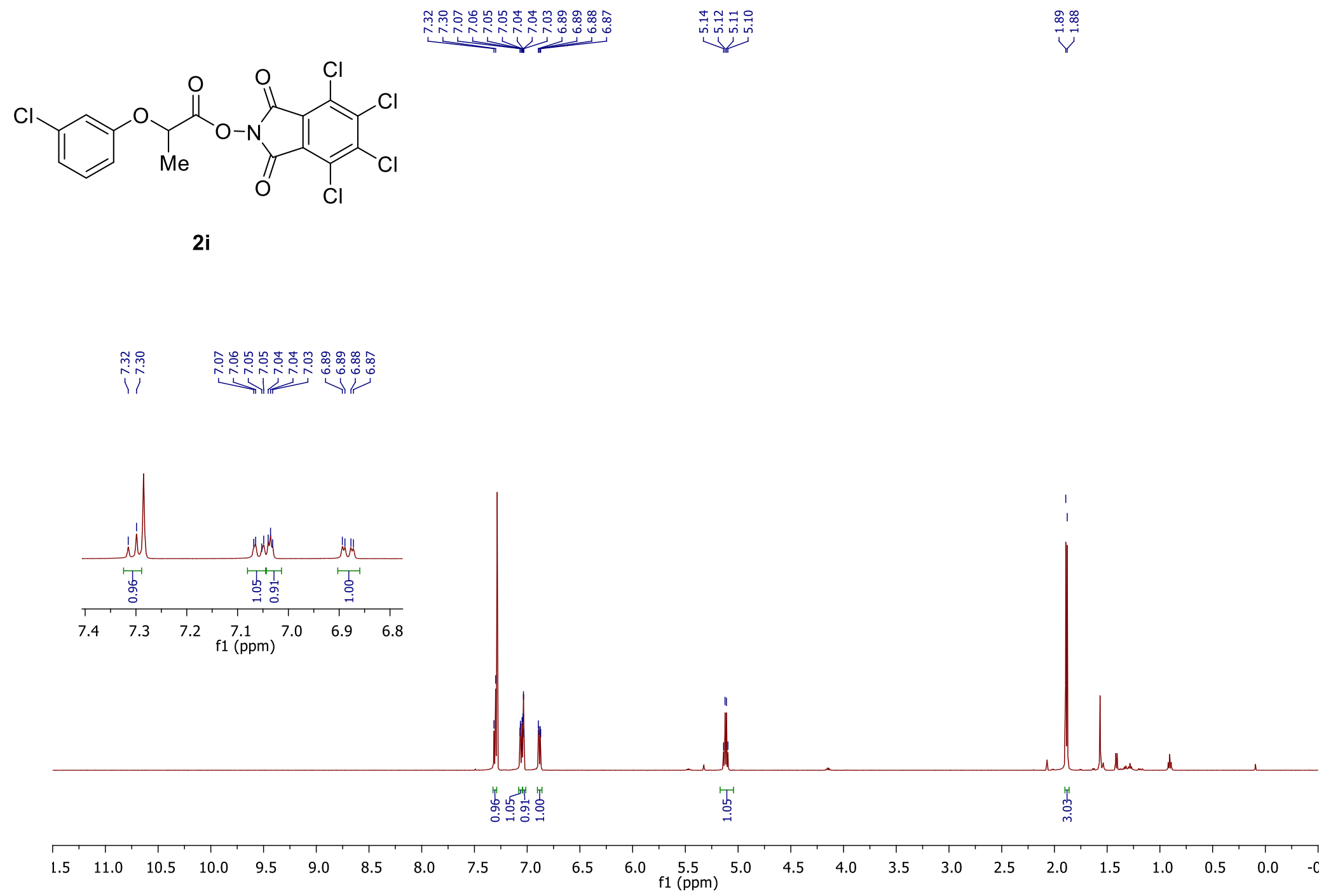


${ }^{13} \mathrm{C}-\mathrm{NMR}$ of compound $\mathbf{2 i}$
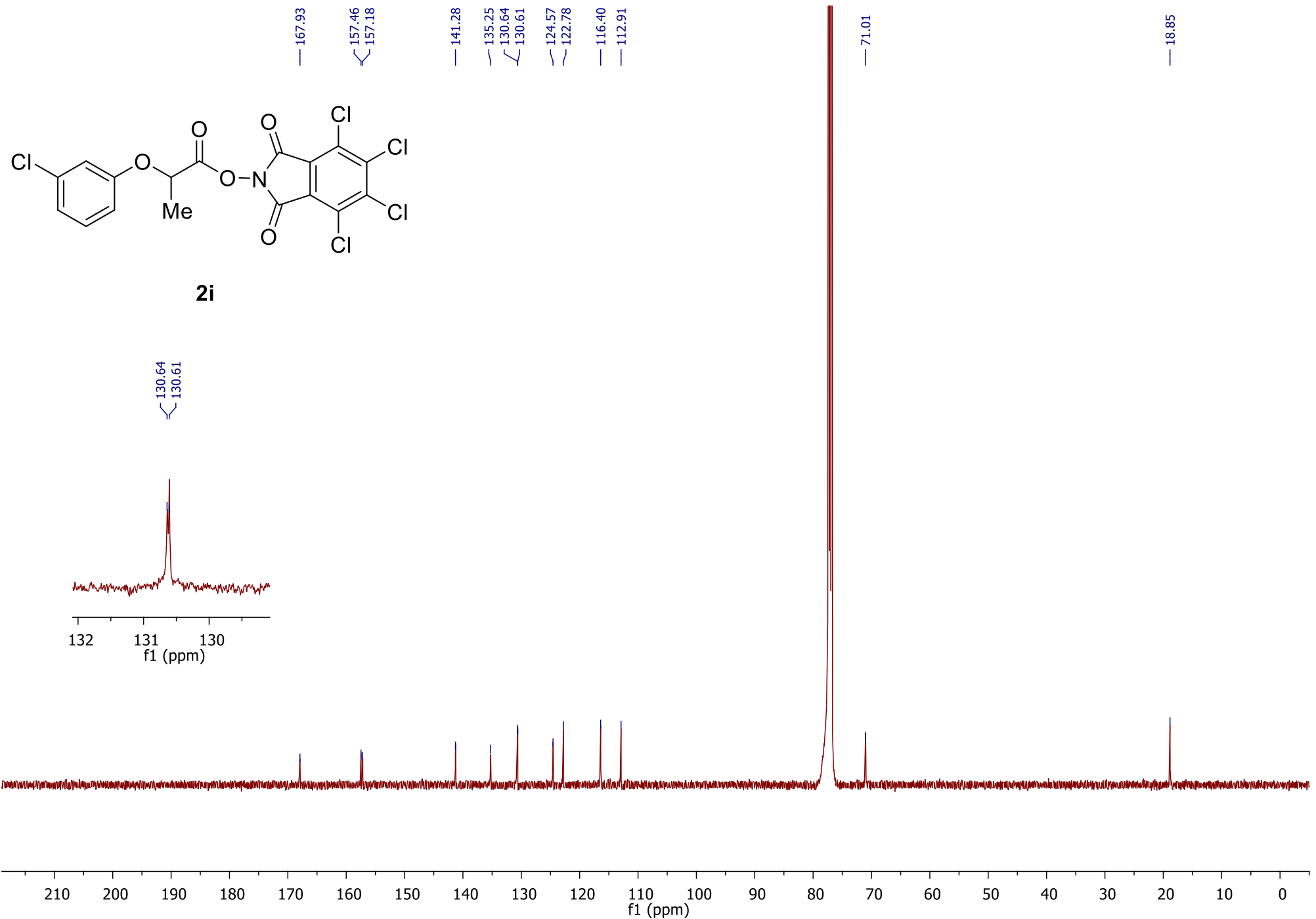
${ }^{1} \mathrm{H}$-NMR of compound $\mathbf{2 j}$

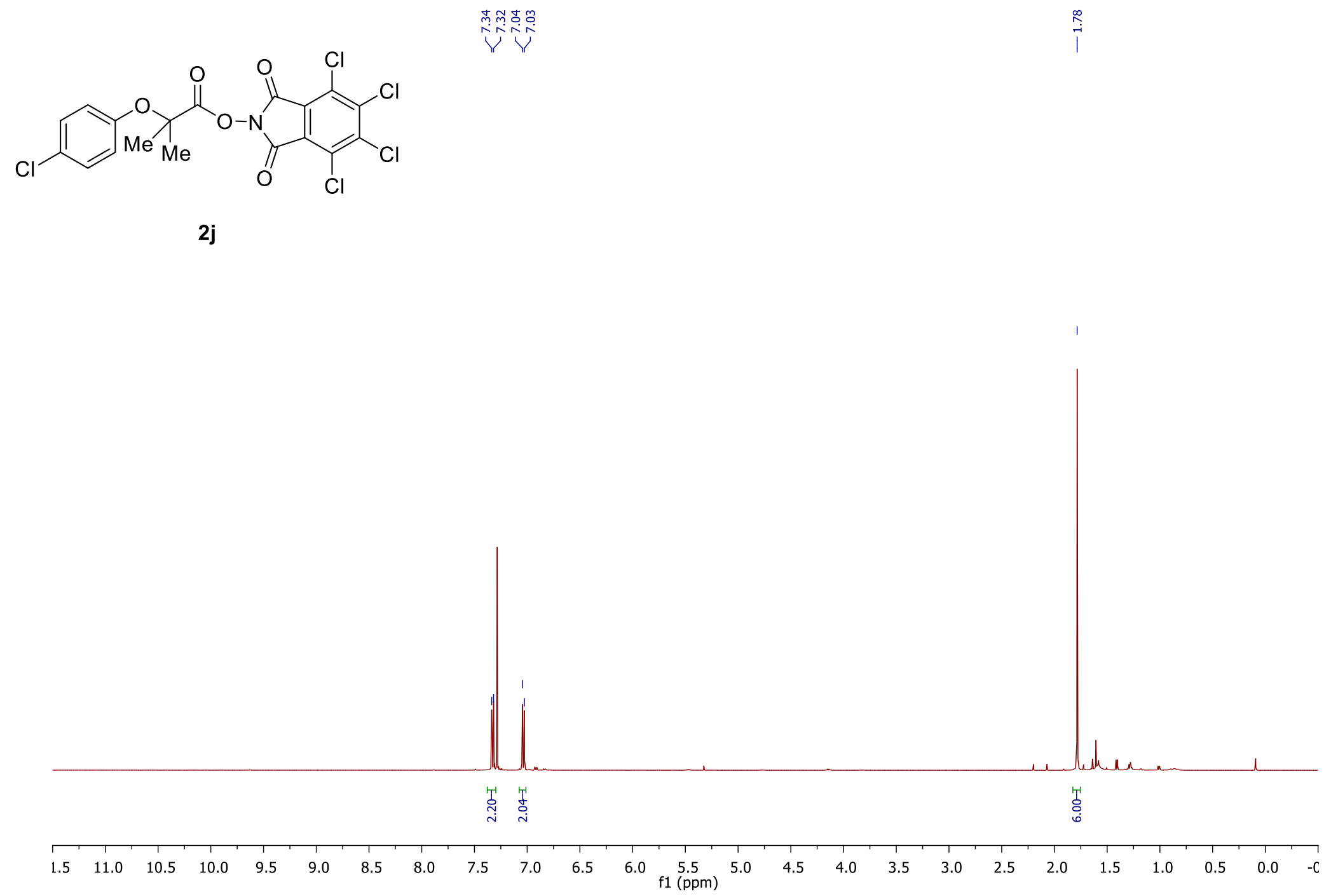


${ }^{13} \mathrm{C}$-NMR of compound $\mathbf{2} \mathbf{j}$

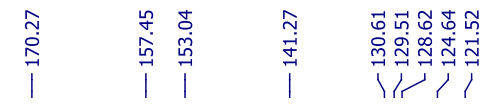

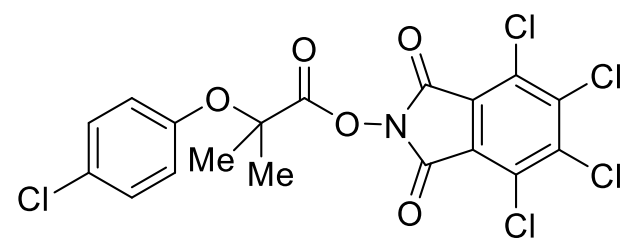

2j
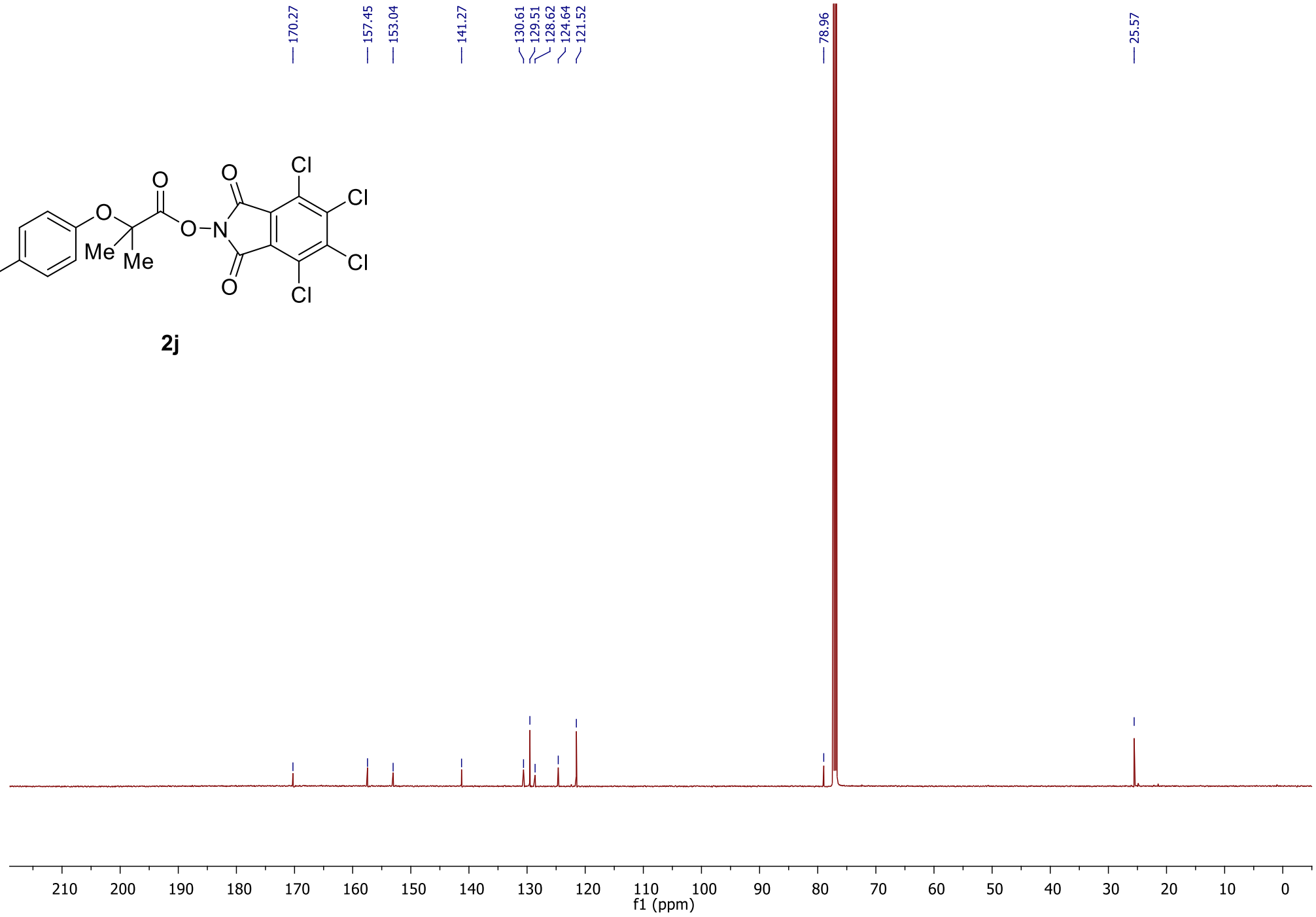
${ }^{1} \mathrm{H}$-NMR of compound $\mathbf{2 k}$

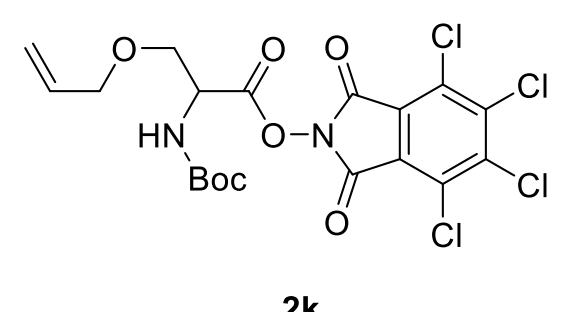

| \}

$2 k$

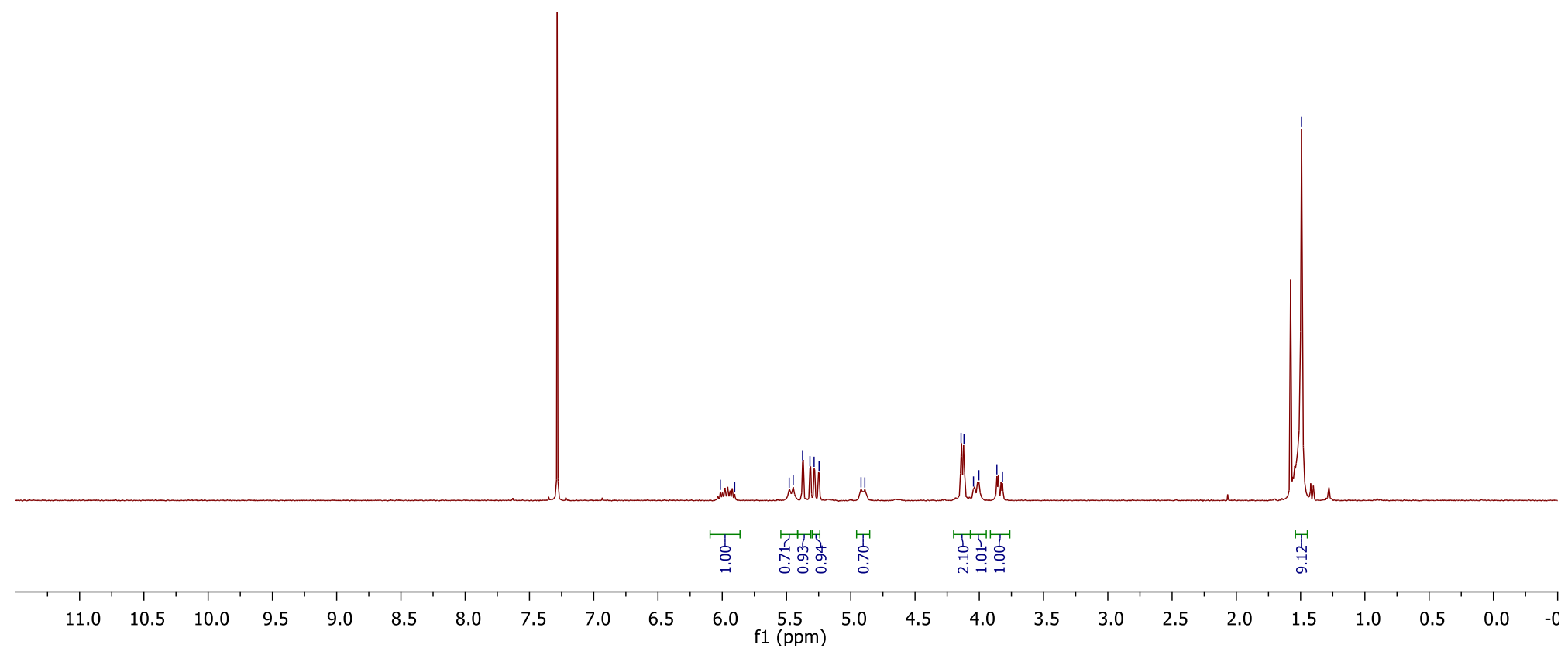


${ }^{13} \mathrm{C}-\mathrm{NMR}$ of compound $\mathbf{2 k}$
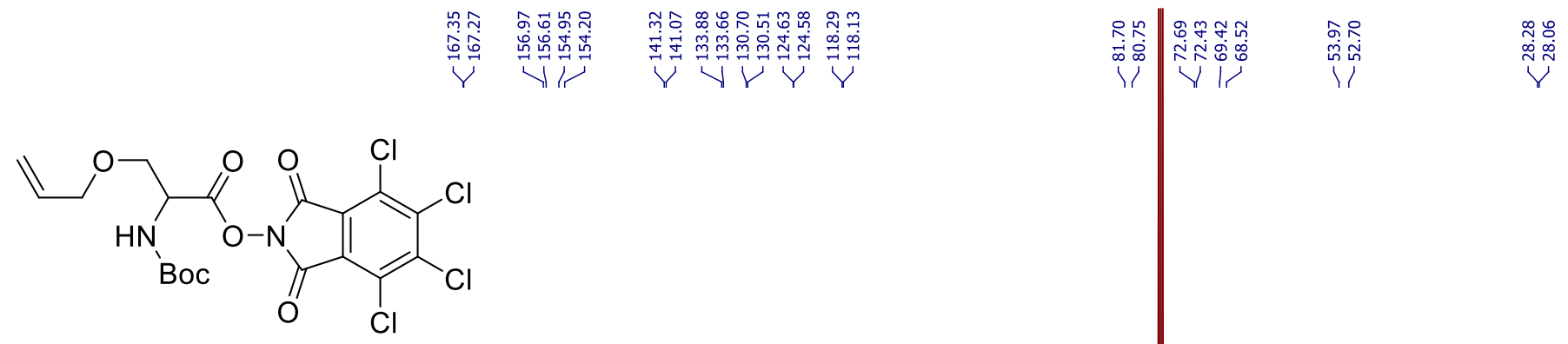

$2 k$

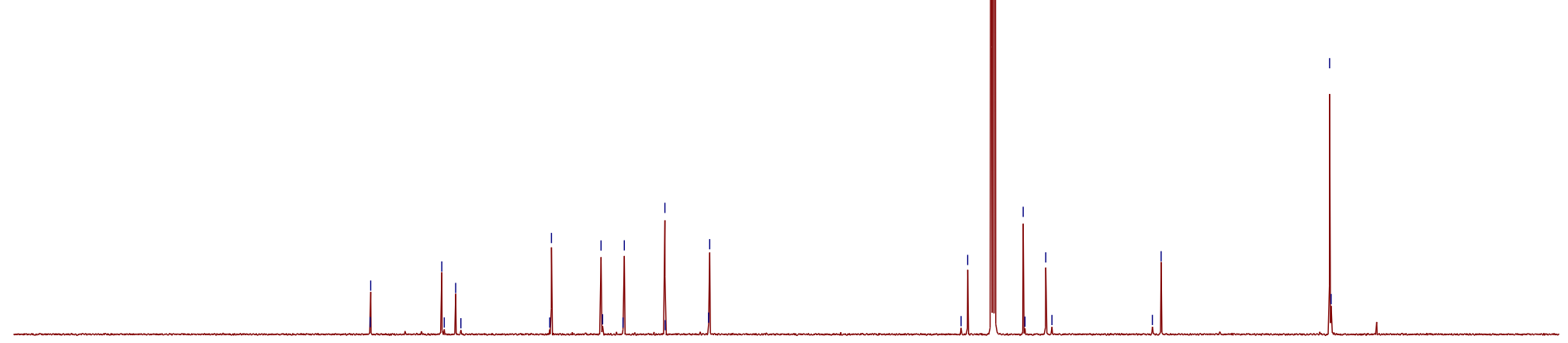


${ }^{1} \mathrm{H}-\mathrm{NMR}$ of compound $\mathbf{2 l}$

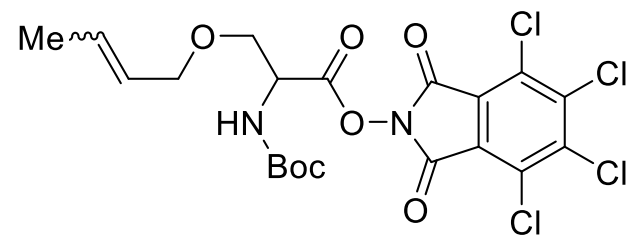

2 I

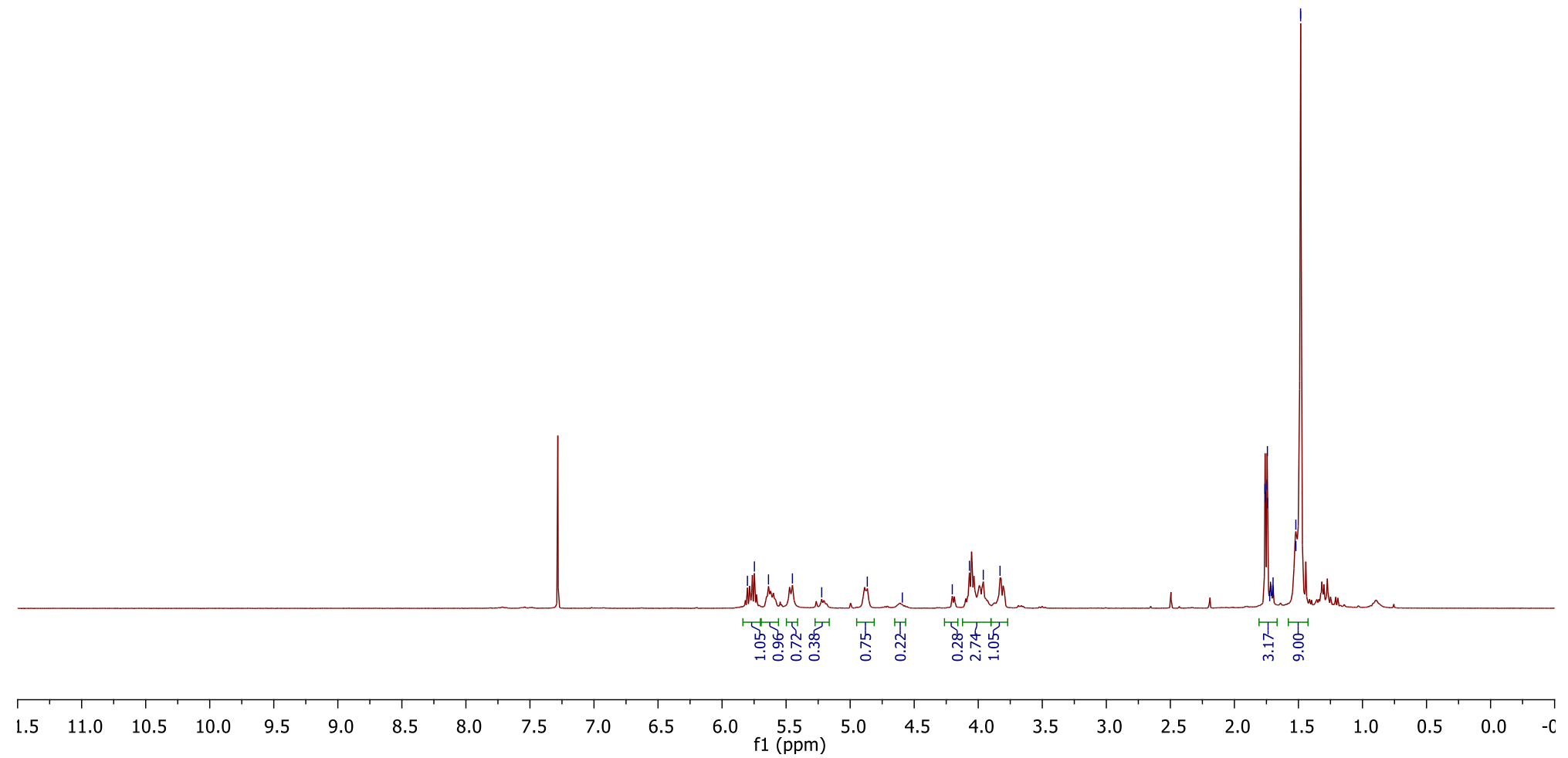


${ }^{13} \mathrm{C}-\mathrm{NMR}$ of compound $2 \mathrm{I}$

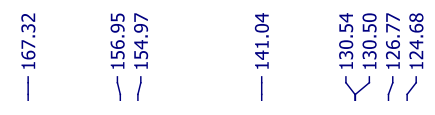

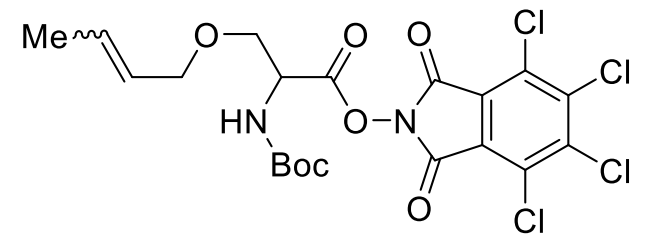

2I

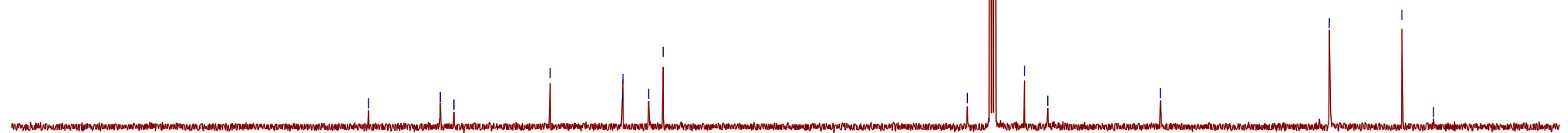


${ }^{1} \mathrm{H}$-NMR of compound $\mathbf{2 m}$

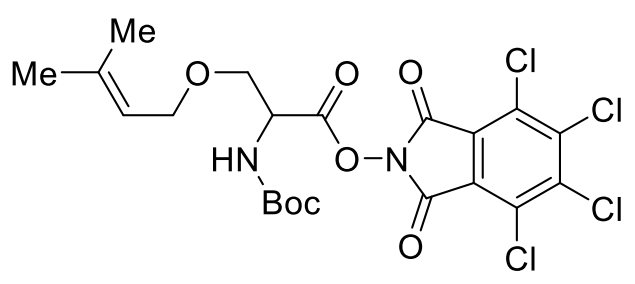

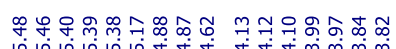

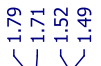

$2 m$

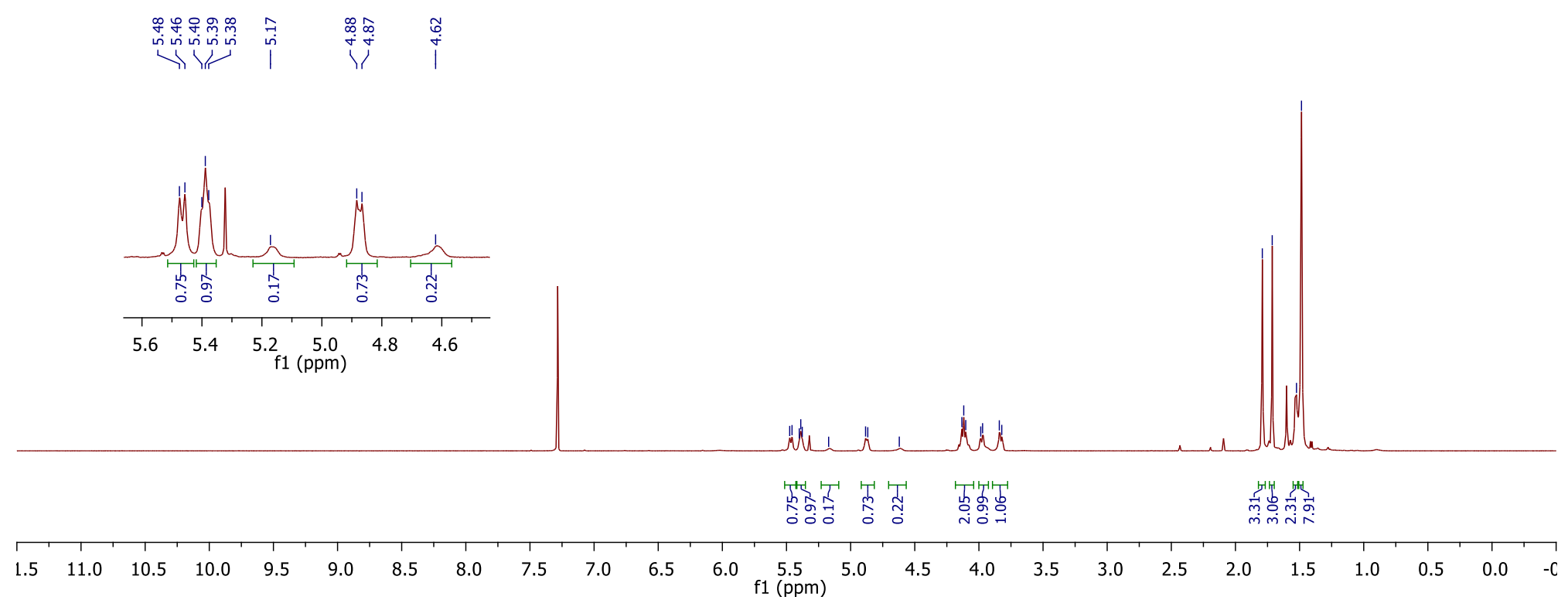


${ }^{13} \mathrm{C}-\mathrm{NMR}$ of compound $\mathbf{2 m}$

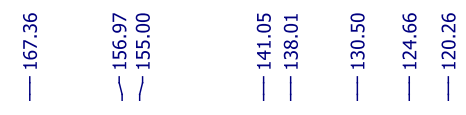

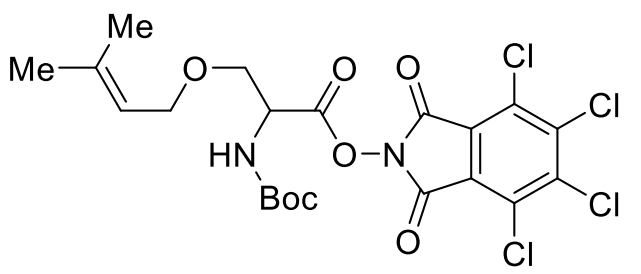

$2 m$

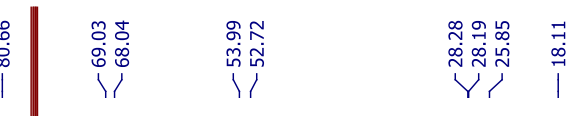


${ }^{1}$ H-NMR of compound 2 n

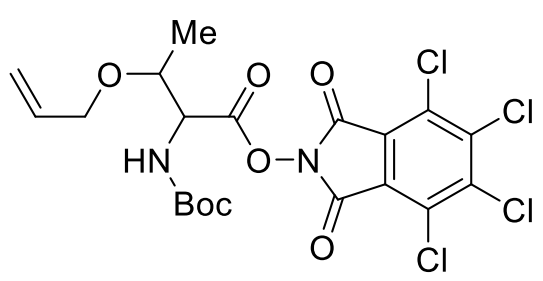

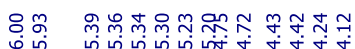

114

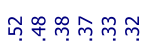
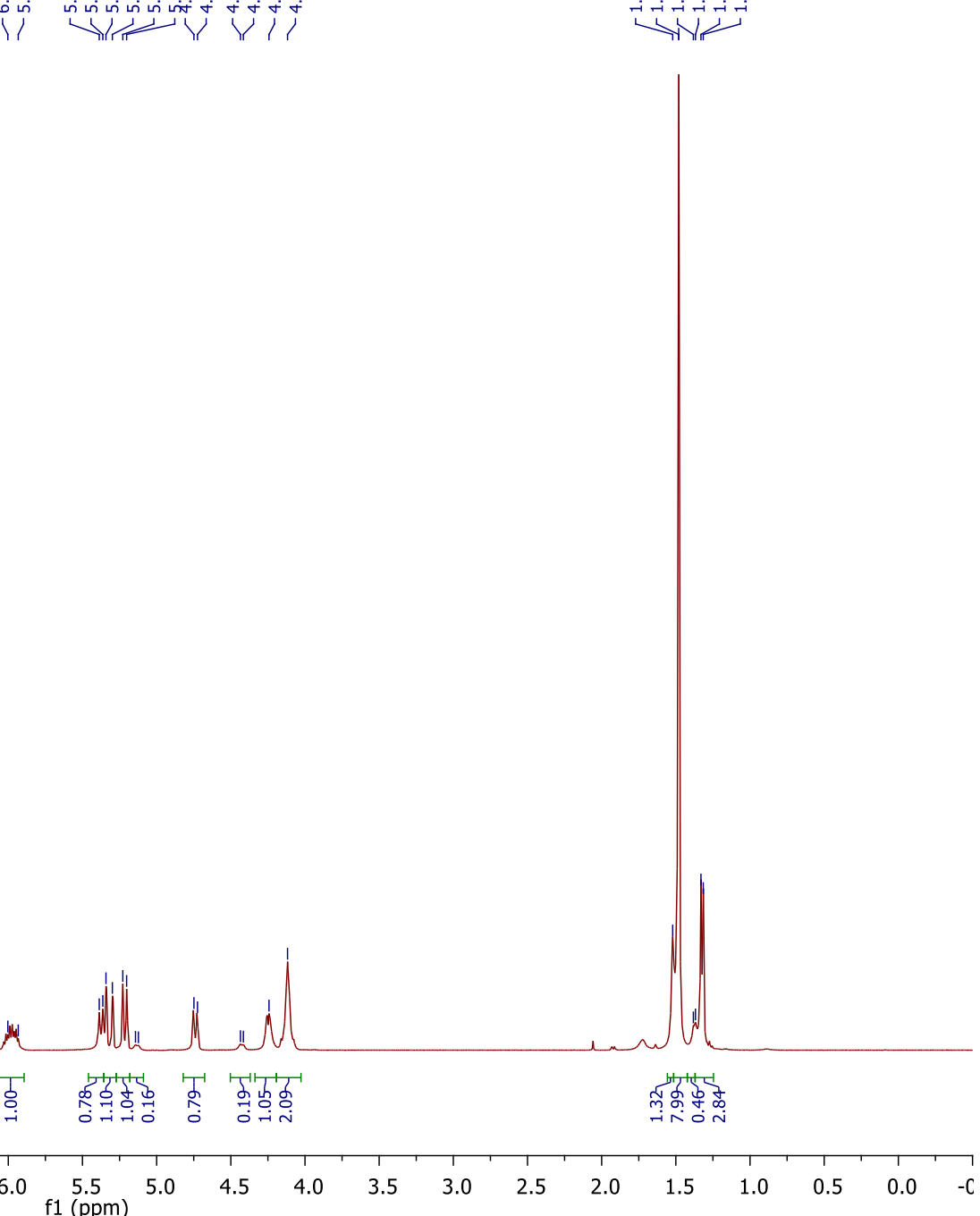
${ }^{13} \mathrm{C}-\mathrm{NMR}$ of compound $\mathbf{2 n}$

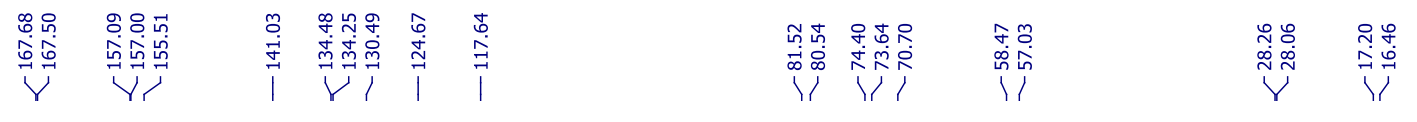

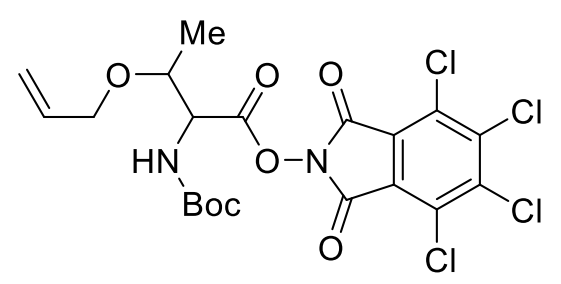

$2 n$

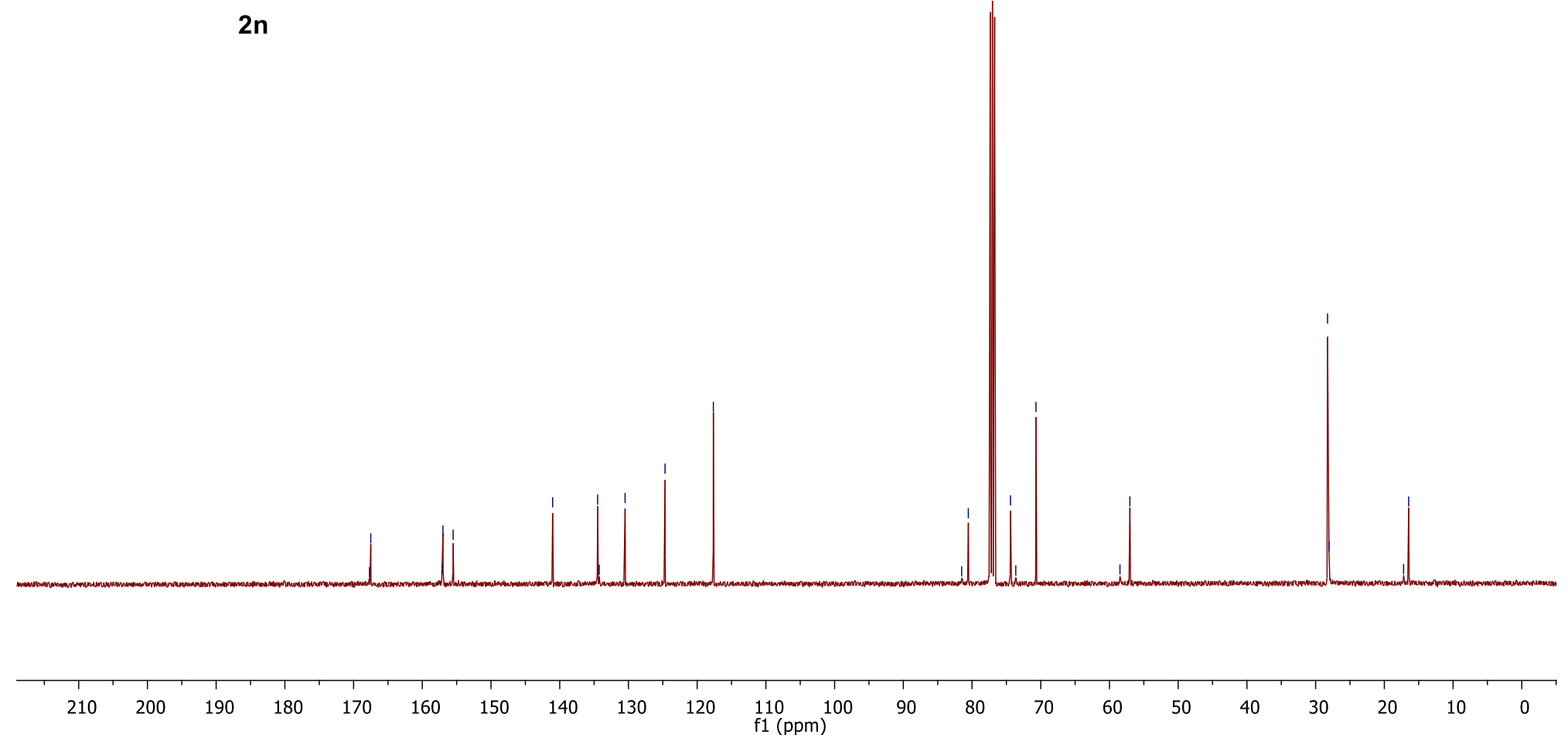


${ }^{1} \mathrm{H}-\mathrm{NMR}$ of compound $2 \mathrm{o}$

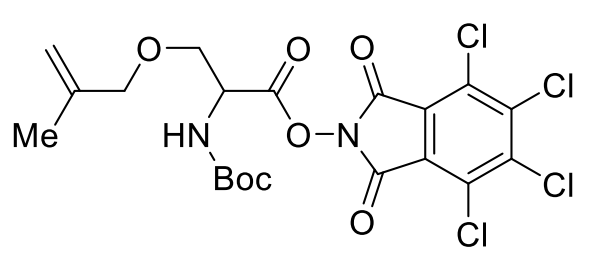

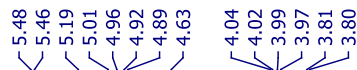

象饮

20

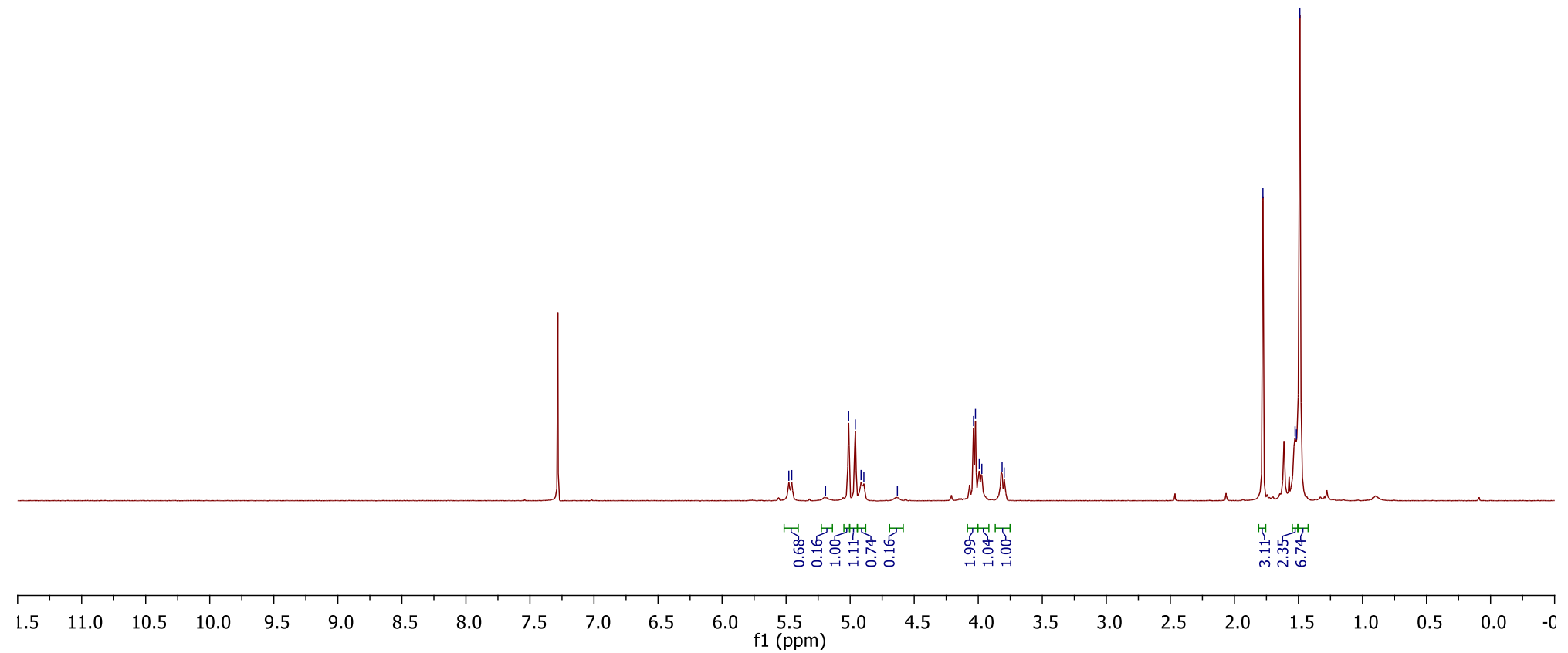


${ }^{13} \mathrm{C}-\mathrm{NMR}$ of compound $2 \mathrm{o}$

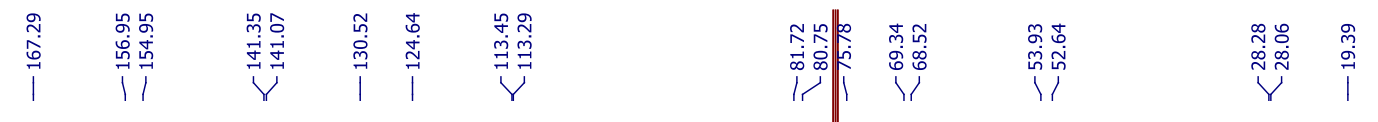

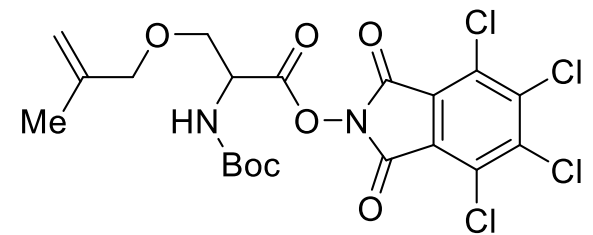

20

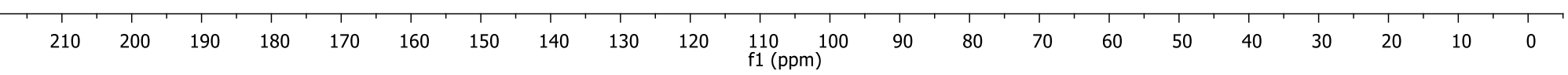


${ }^{1} \mathrm{H}$-NMR of compound $\mathbf{2 p}$

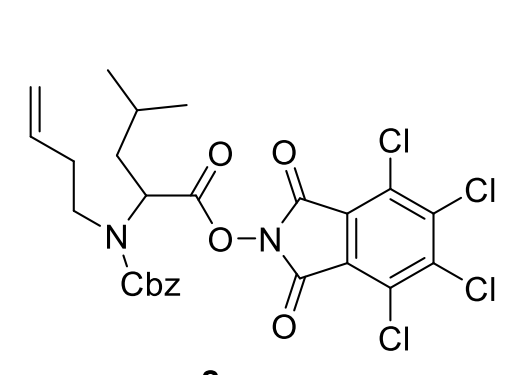

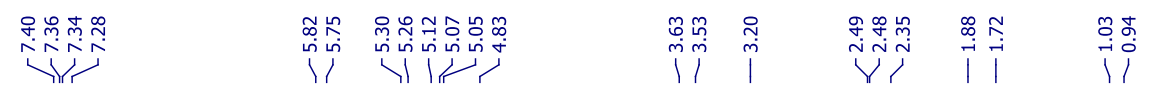

$2 p$

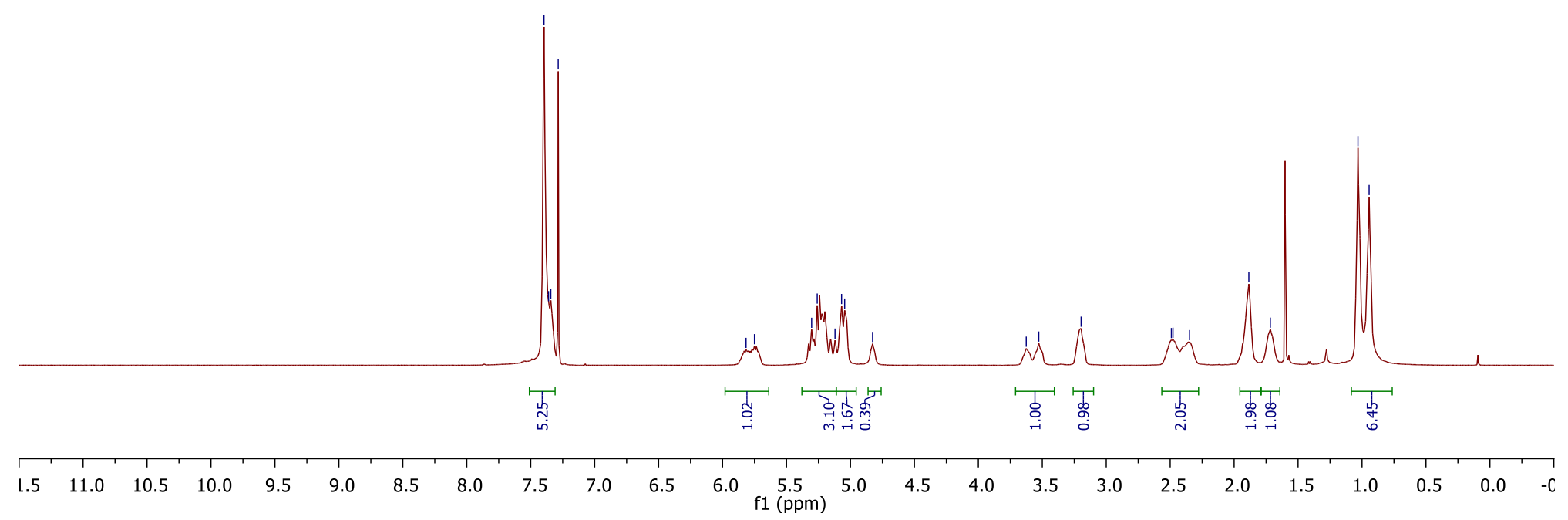


${ }^{13} \mathrm{C}-\mathrm{NMR}$ of compound $\mathbf{2 p}$

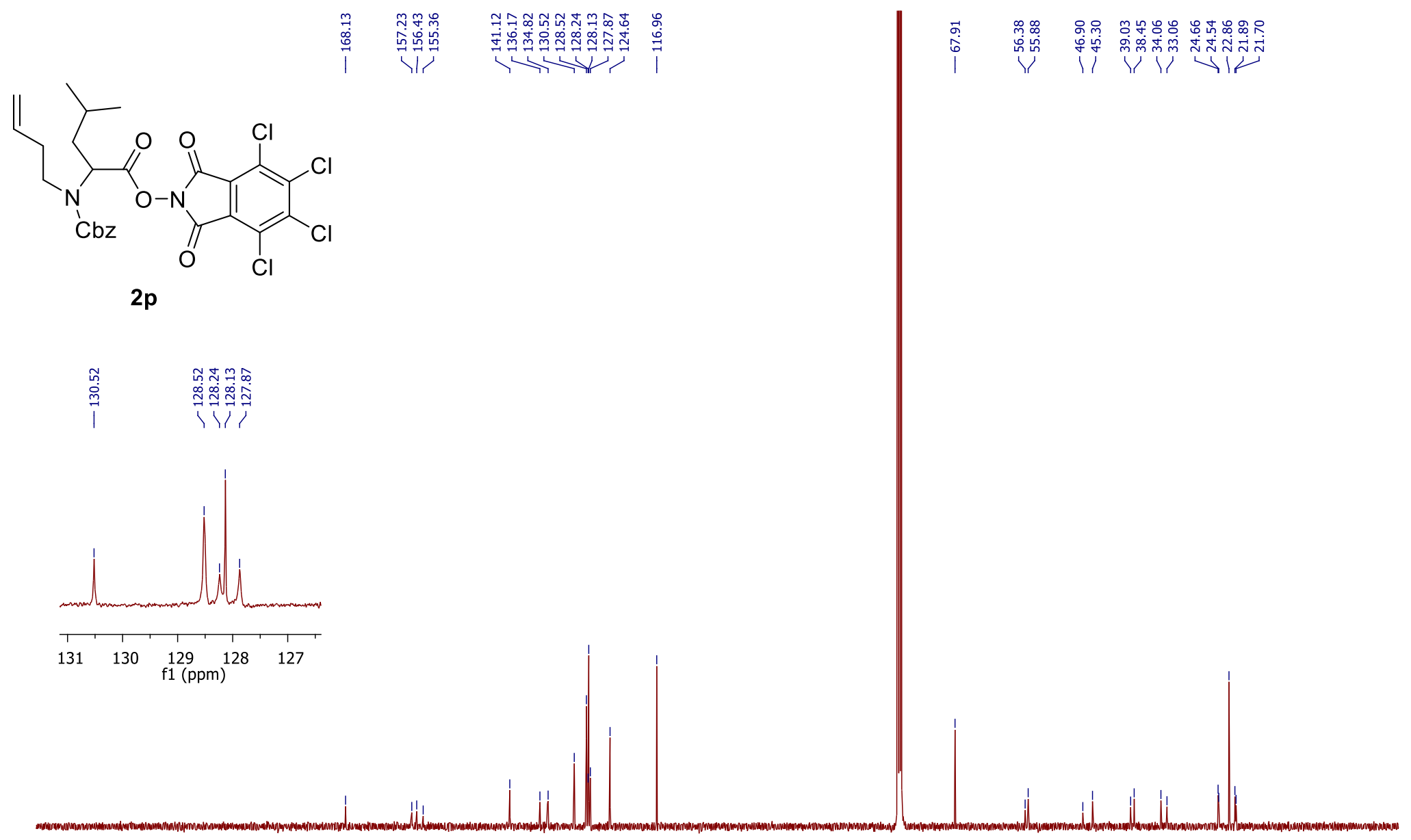

$\begin{array}{llllllll}210 & 200 & 190 & 180 & 170 & 160 & 150 & 140\end{array}$

$120 \quad 110 \quad 100$

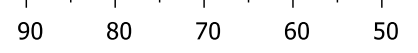


${ }^{1} \mathrm{H}-\mathrm{NMR}$ of compound $\mathbf{2 q}$

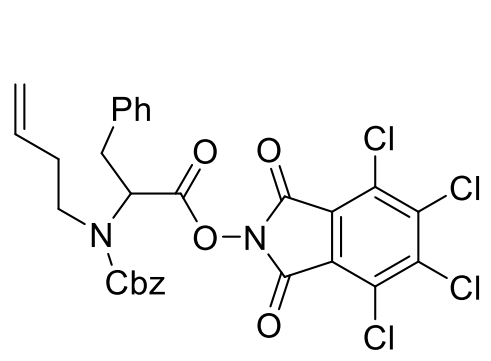

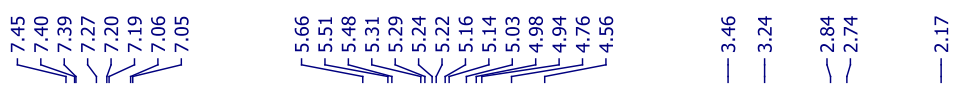

$2 q$

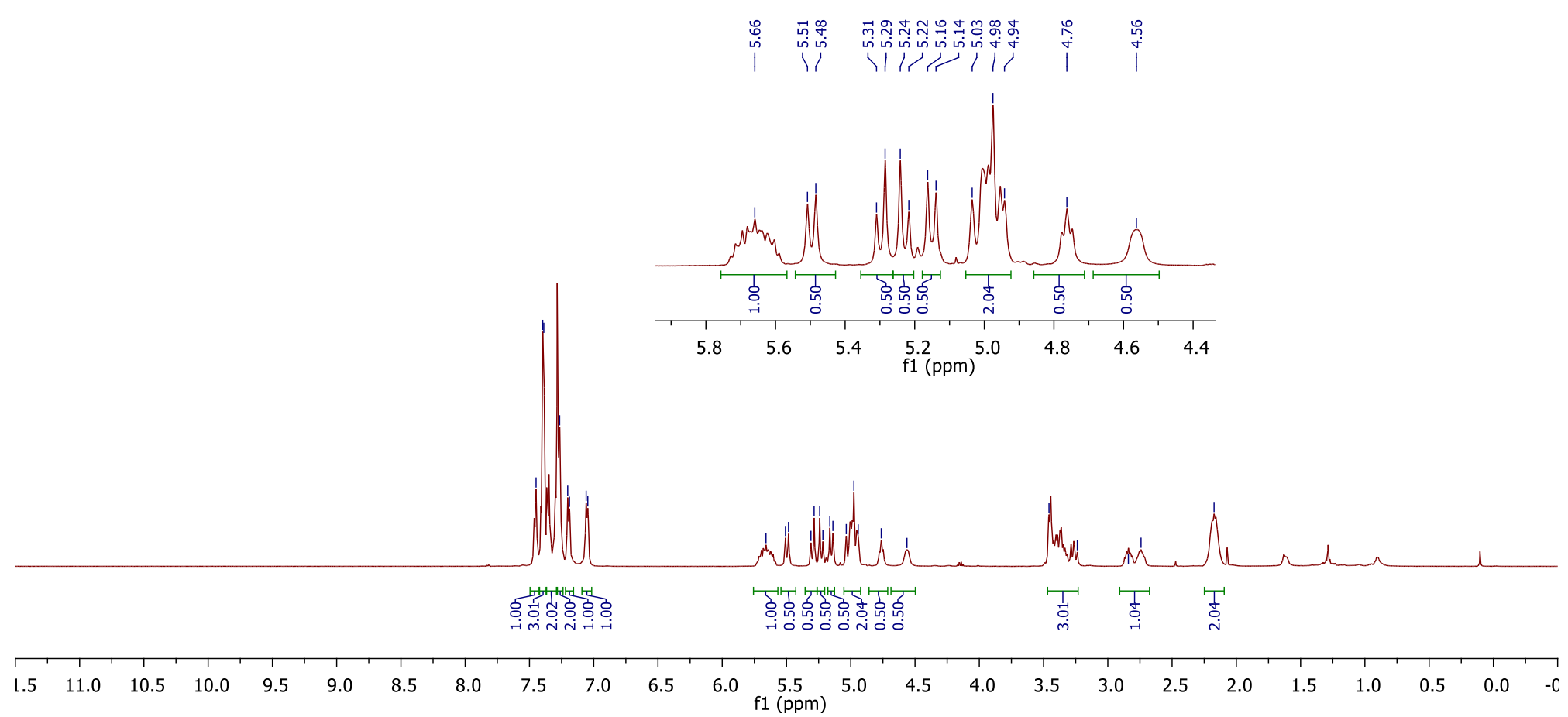


${ }^{13} \mathrm{C}-\mathrm{NMR}$ of compound $\mathbf{2 q}$

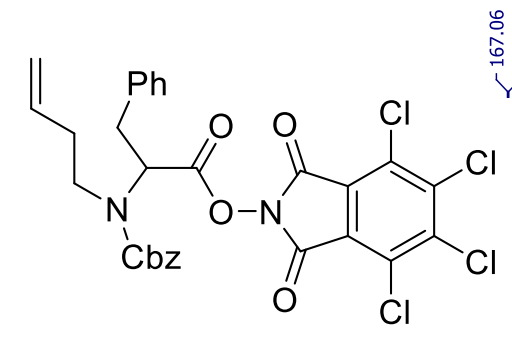

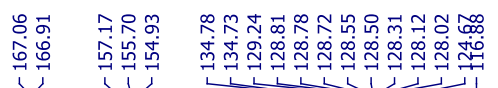

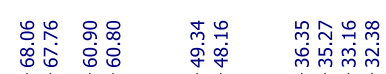

(1)

$2 q$
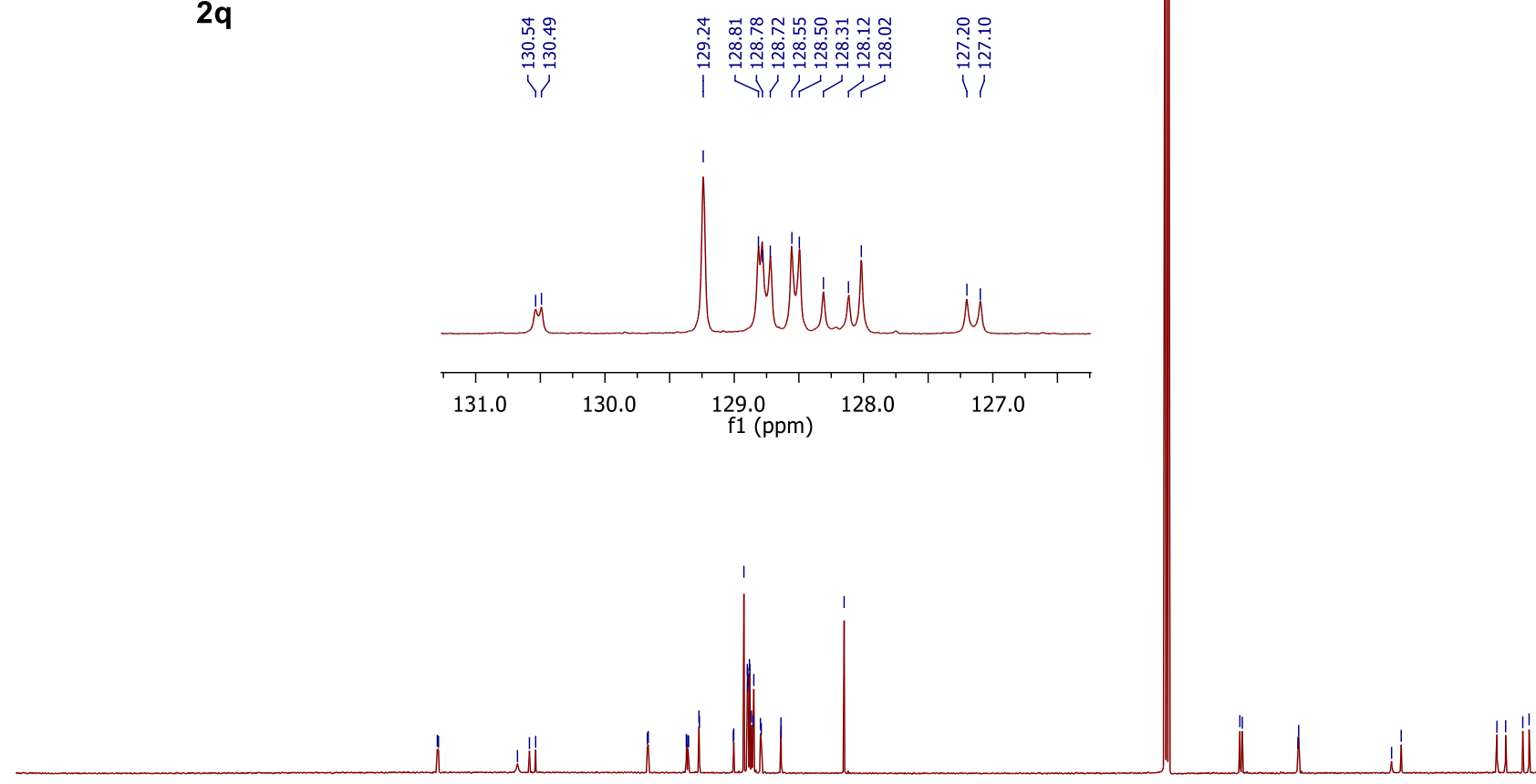

$210 \quad 200 \quad 190$ 
${ }^{1} \mathrm{H}$-NMR of compound $\mathbf{2 r}$

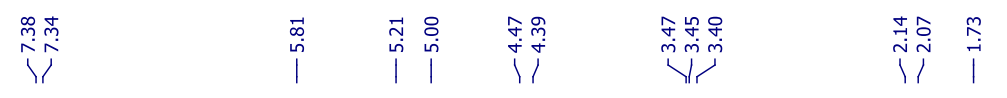
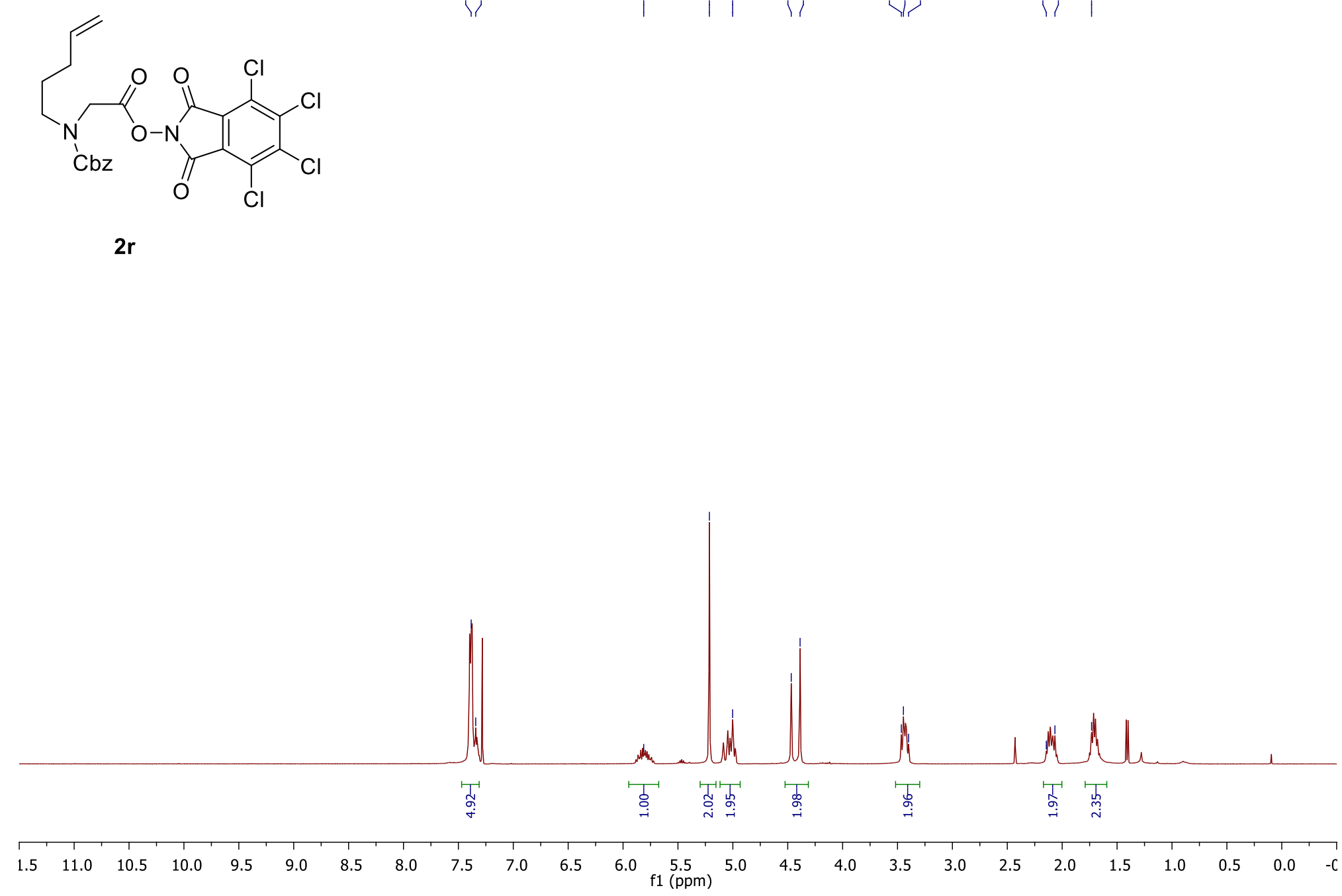
${ }^{13} \mathrm{C}$-NMR of compound $\mathbf{2 r}$

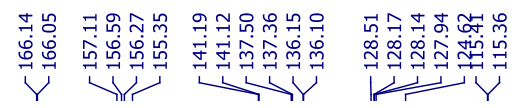

证
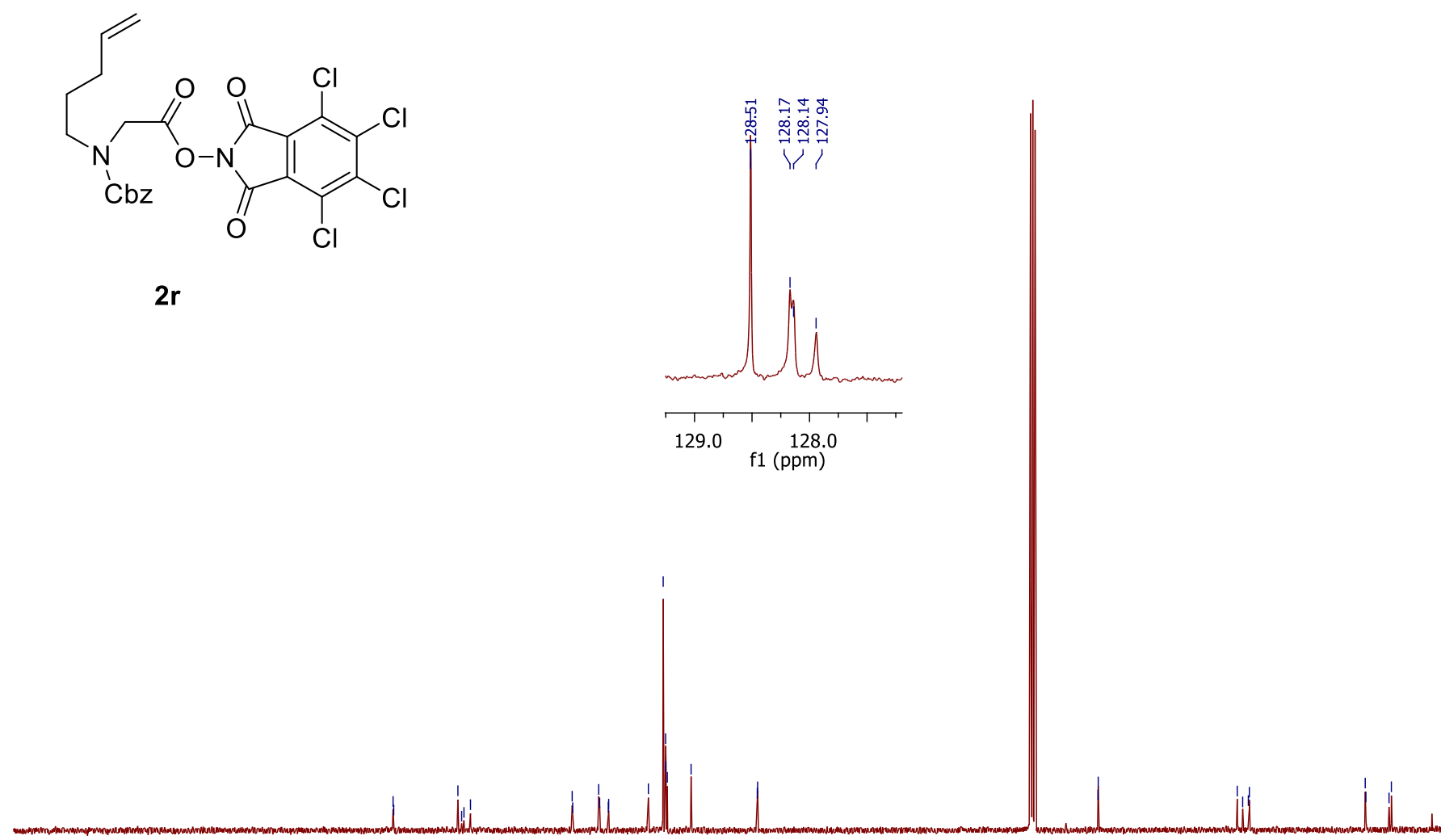

$\begin{array}{lllllllllll}210 & 200 & 190 & 180 & 170 & 160 & 150 & 140 & 130 & 120 & 110 \\ 100\end{array}$ 
${ }^{1} \mathrm{H}$-NMR of compound $2 \mathrm{~s}$
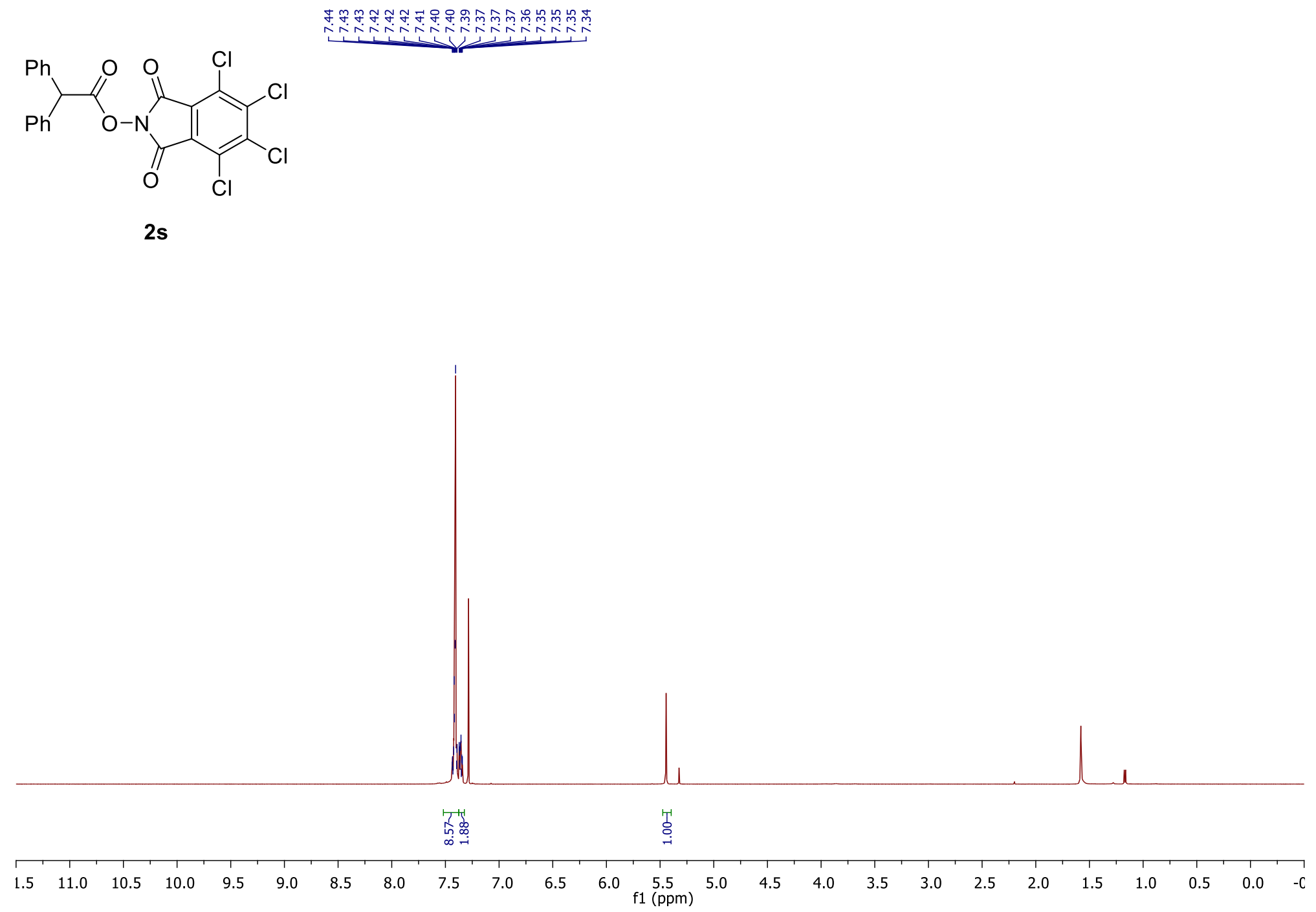
${ }^{13} \mathrm{C}-\mathrm{NMR}$ of compound $2 \mathrm{~s}$

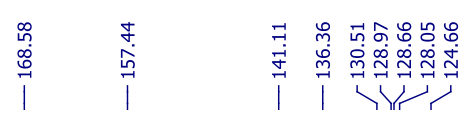

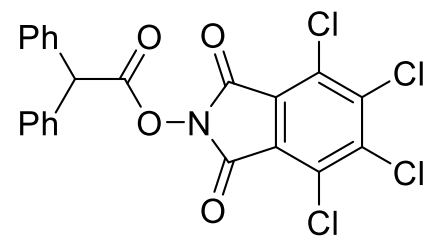

$2 s$
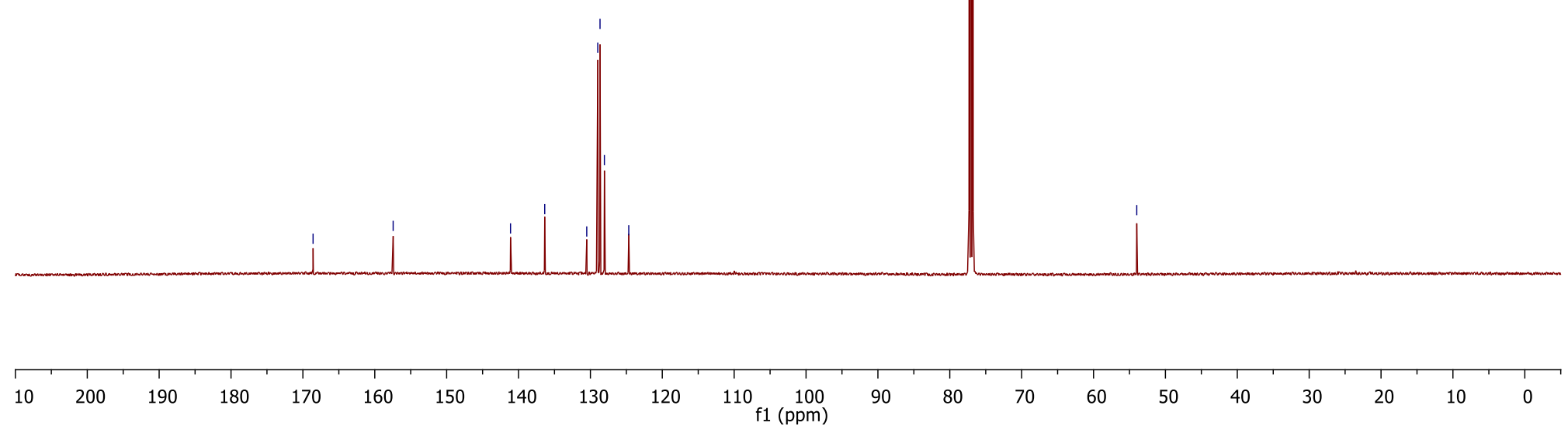

S120 
${ }^{1} \mathrm{H}$-NMR of compound $\mathbf{2 t}$
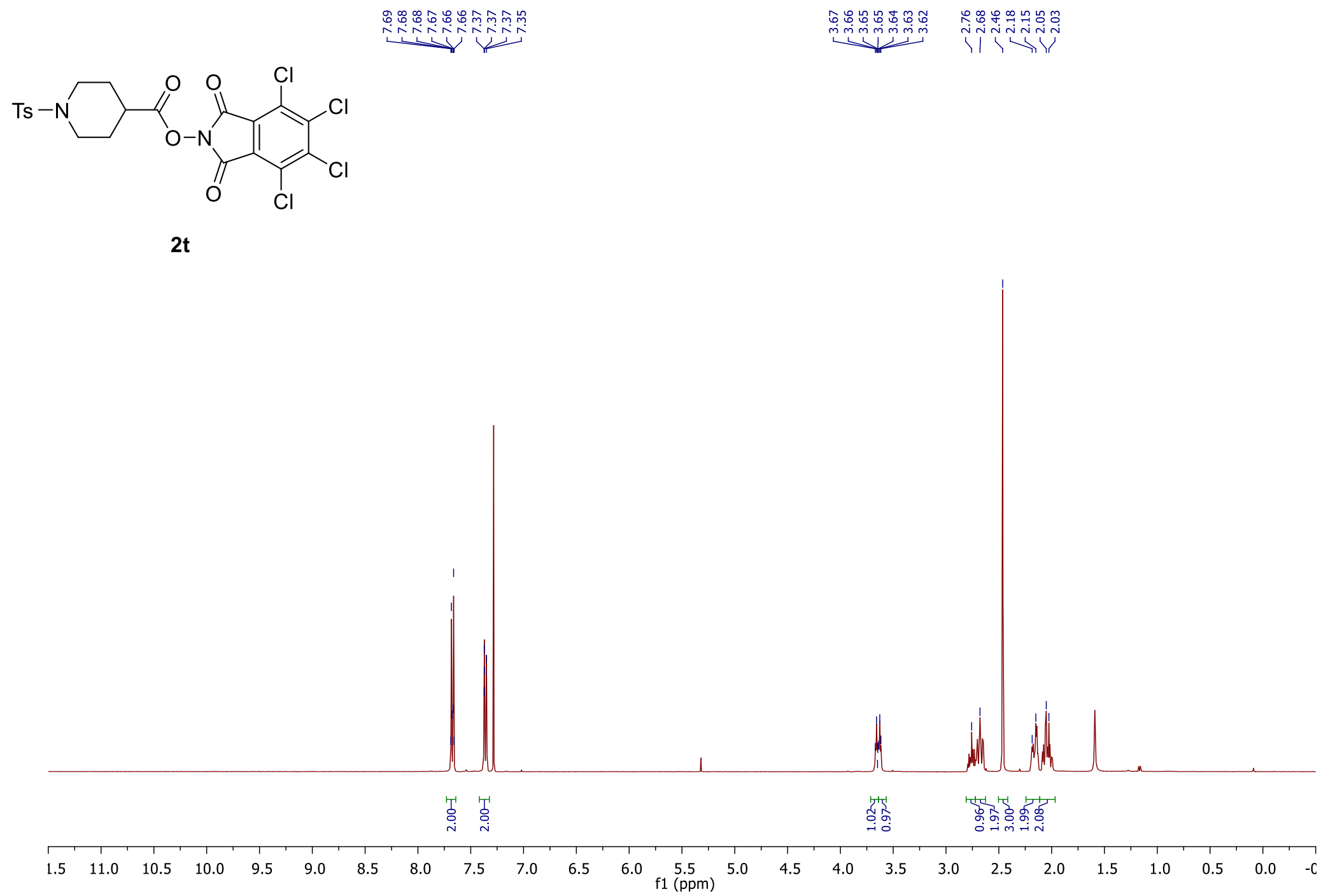
${ }^{13} \mathrm{C}$-NMR of compound $\mathbf{2 t}$

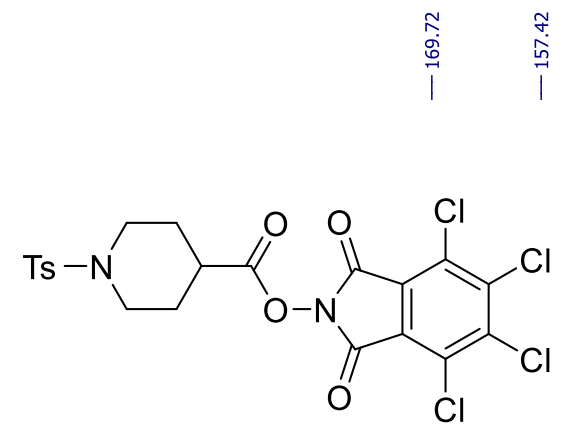

$2 t$

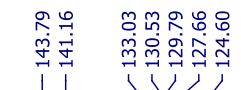

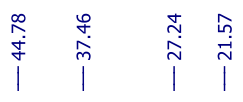

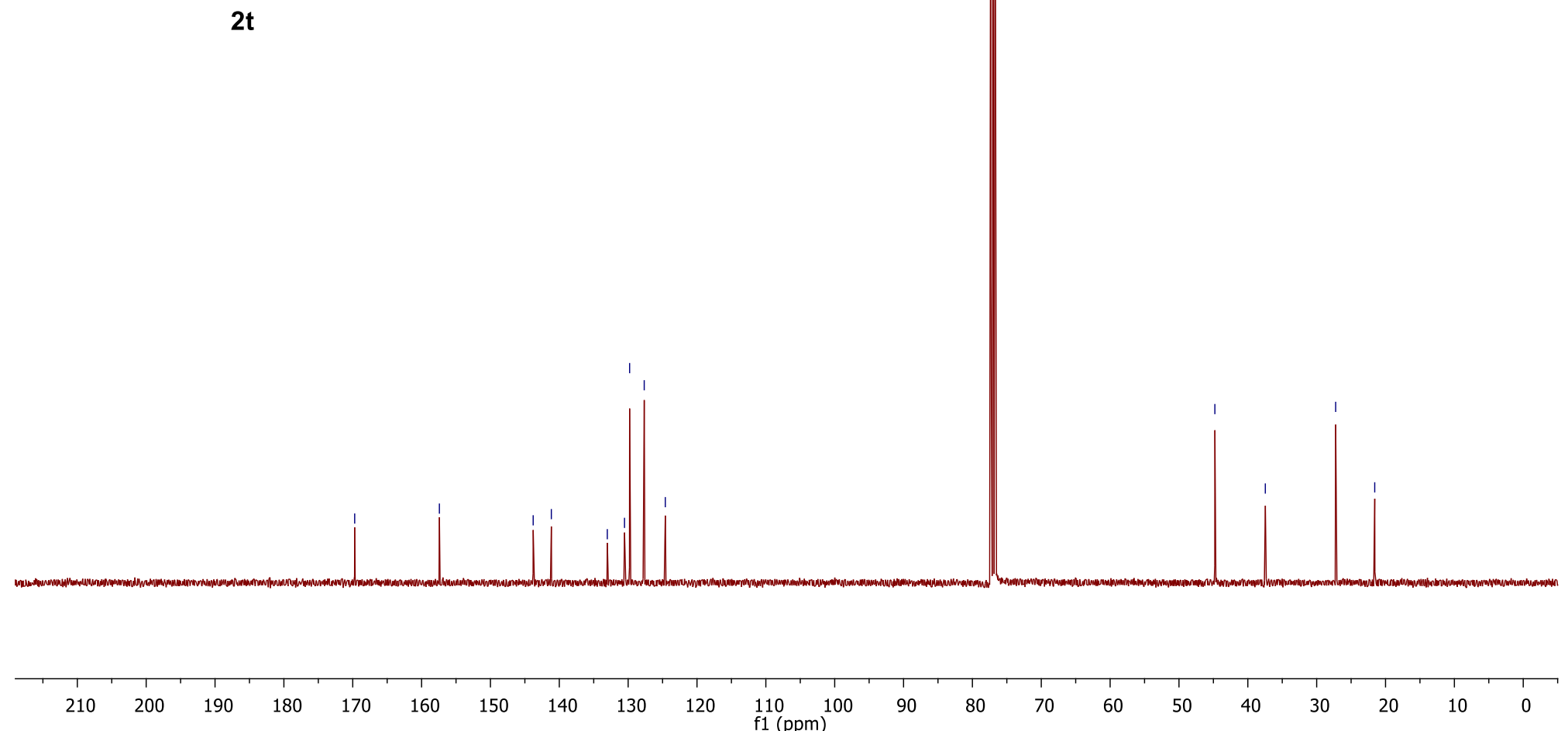


${ }^{1}$ H-NMR of compound $\mathbf{2 u}$
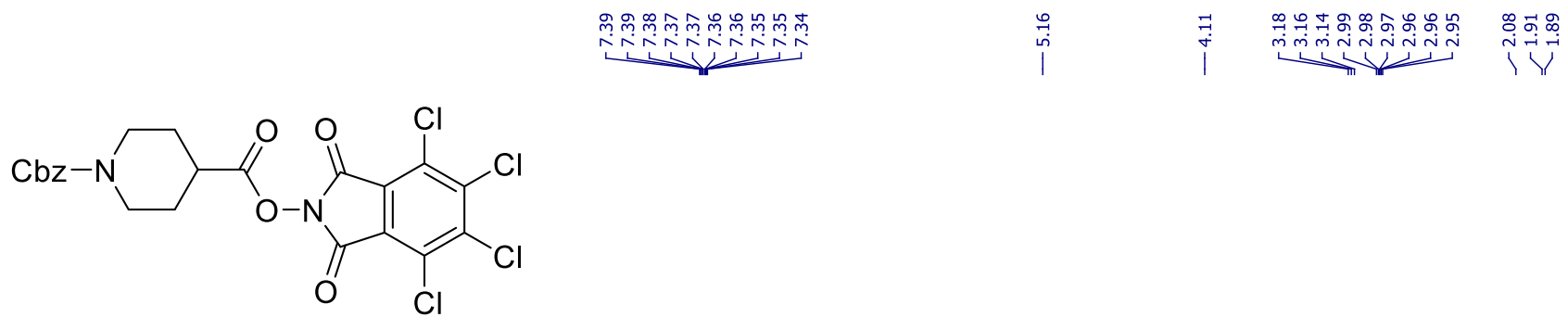

$2 u$

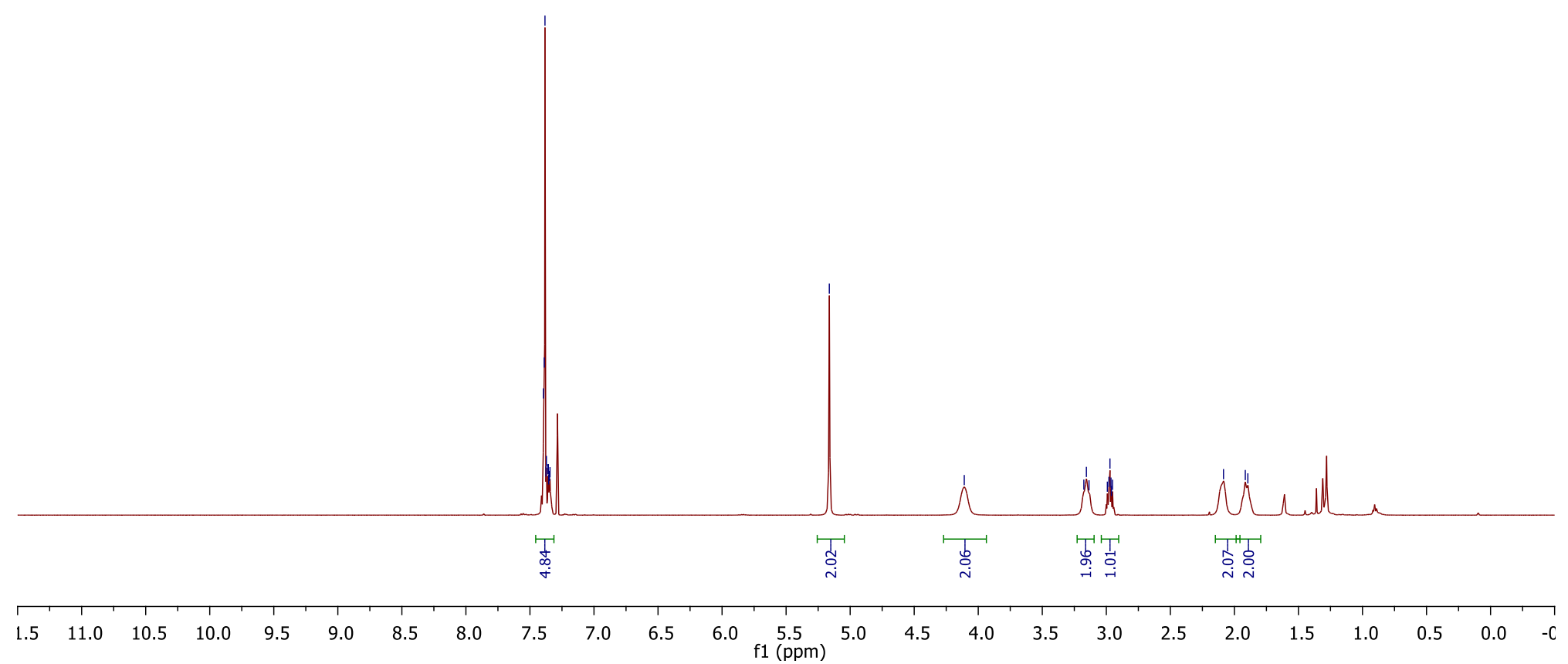


${ }^{13} \mathrm{C}-\mathrm{NMR}$ of compound $\mathbf{2 u}$

| |

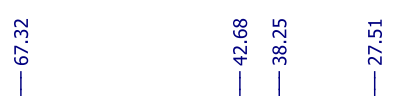

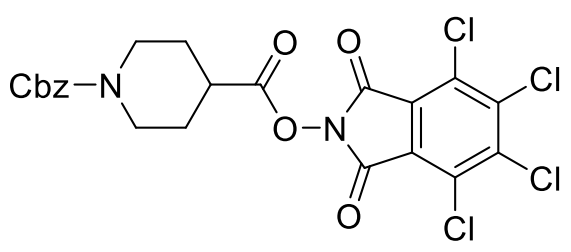

$2 u$

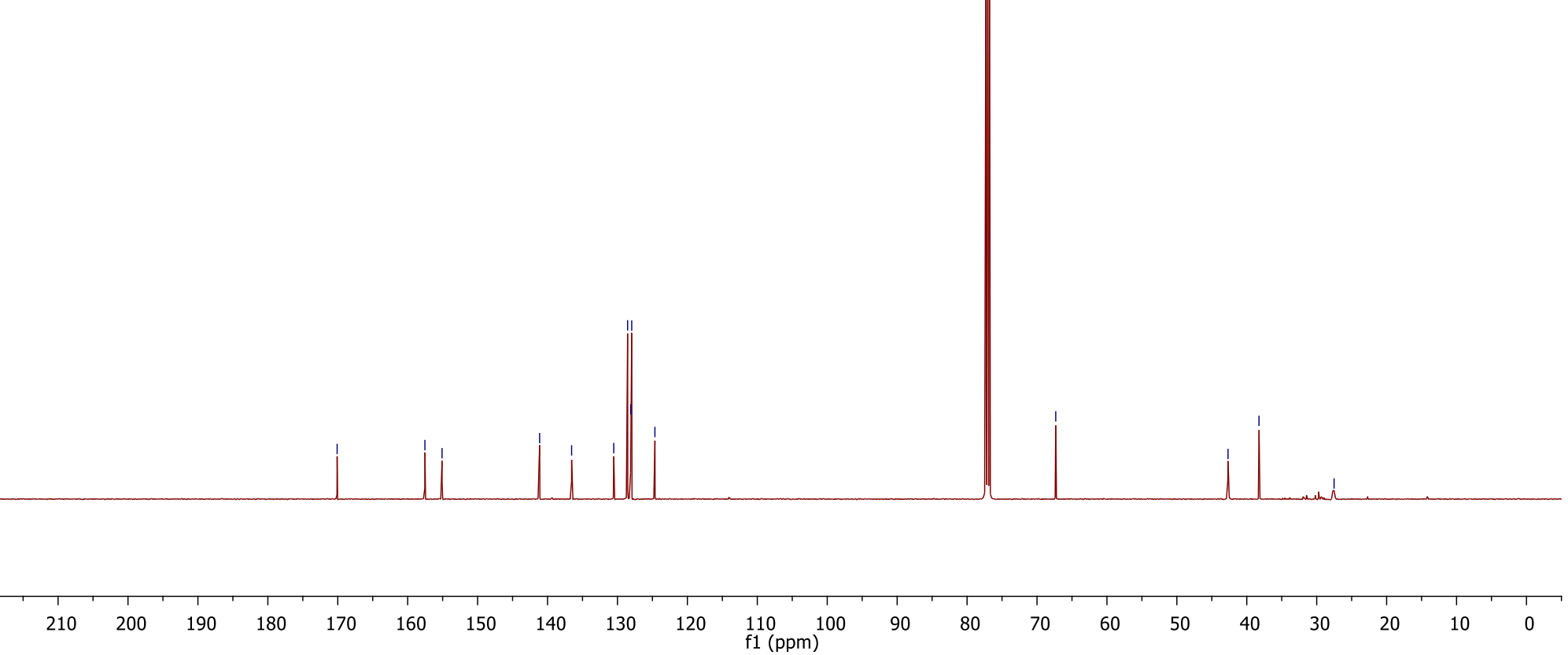


${ }^{1}$ H-NMR of compound $\mathbf{2 v}$

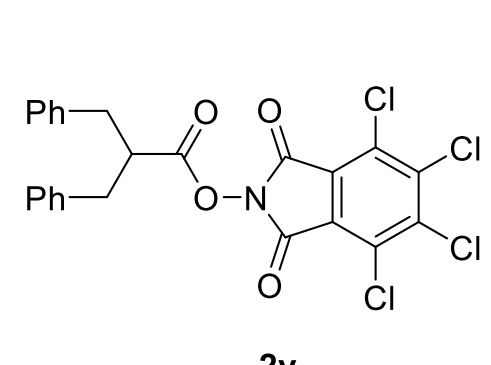

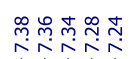

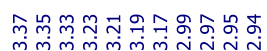

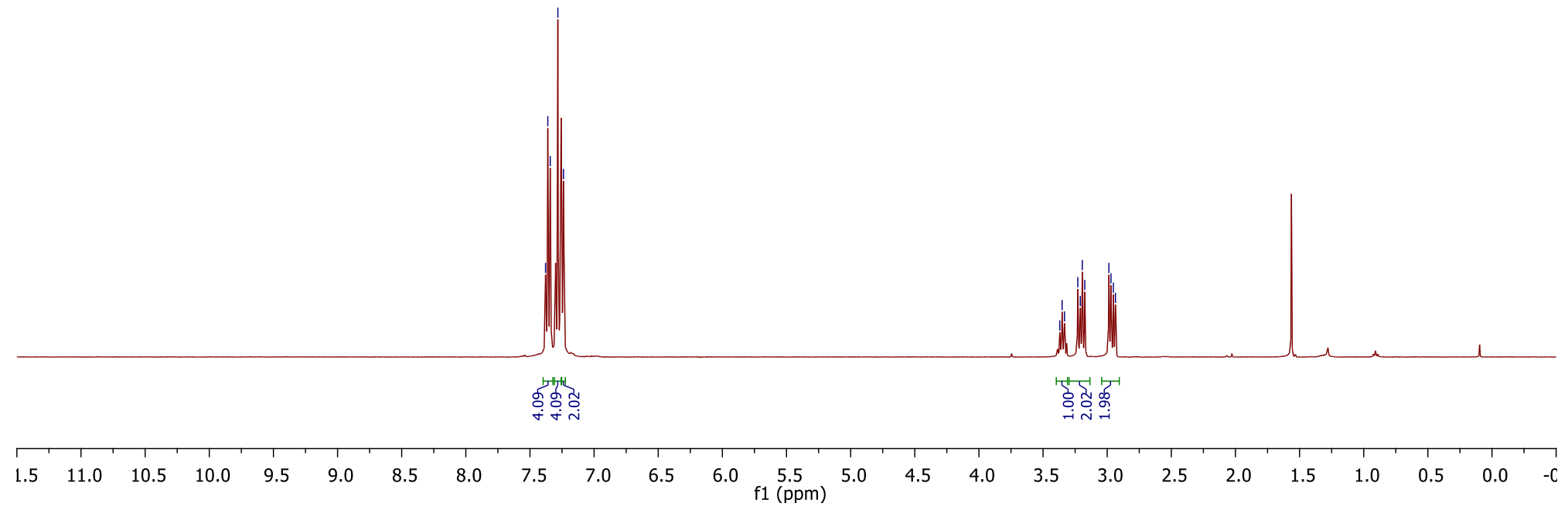


${ }^{13} \mathrm{C}$-NMR of compound $\mathbf{2 v}$

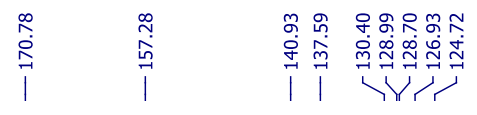

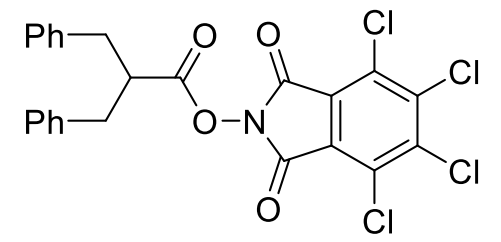

2v

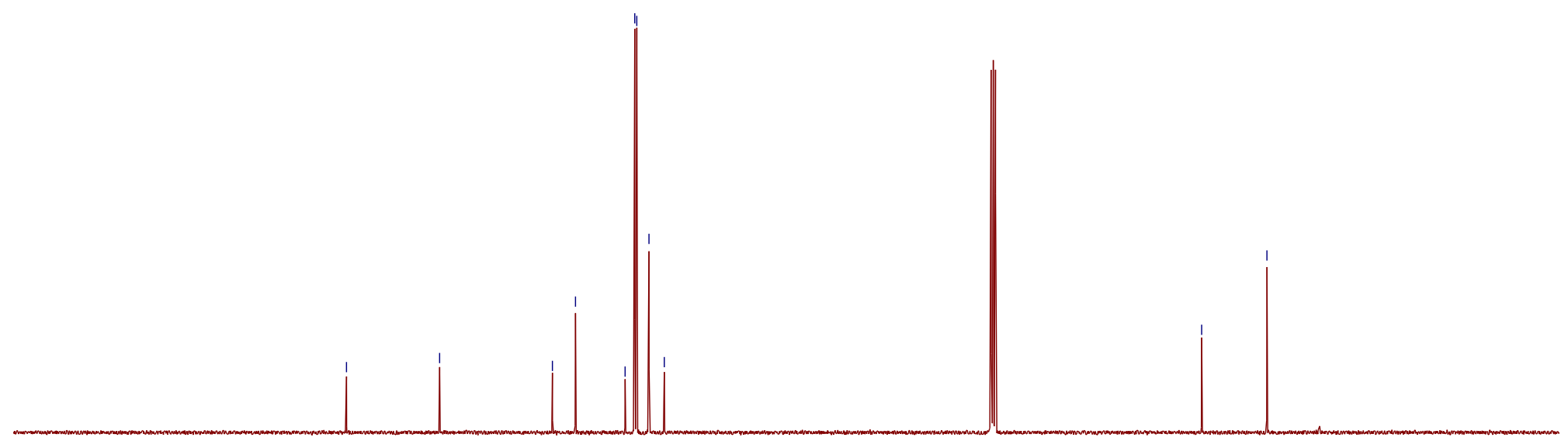


${ }^{1} \mathrm{H}$-NMR of compound $\mathbf{2 w}$

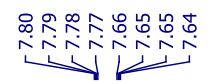

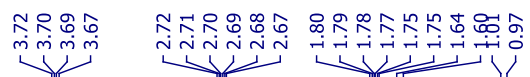

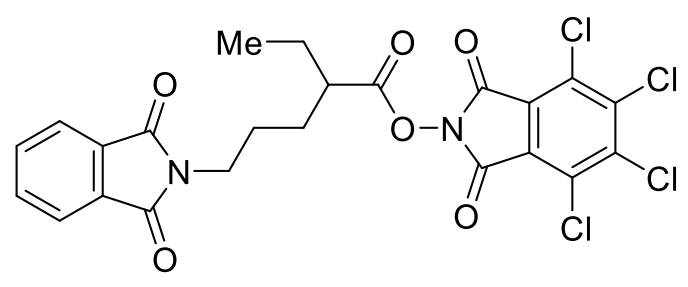

$2 w$

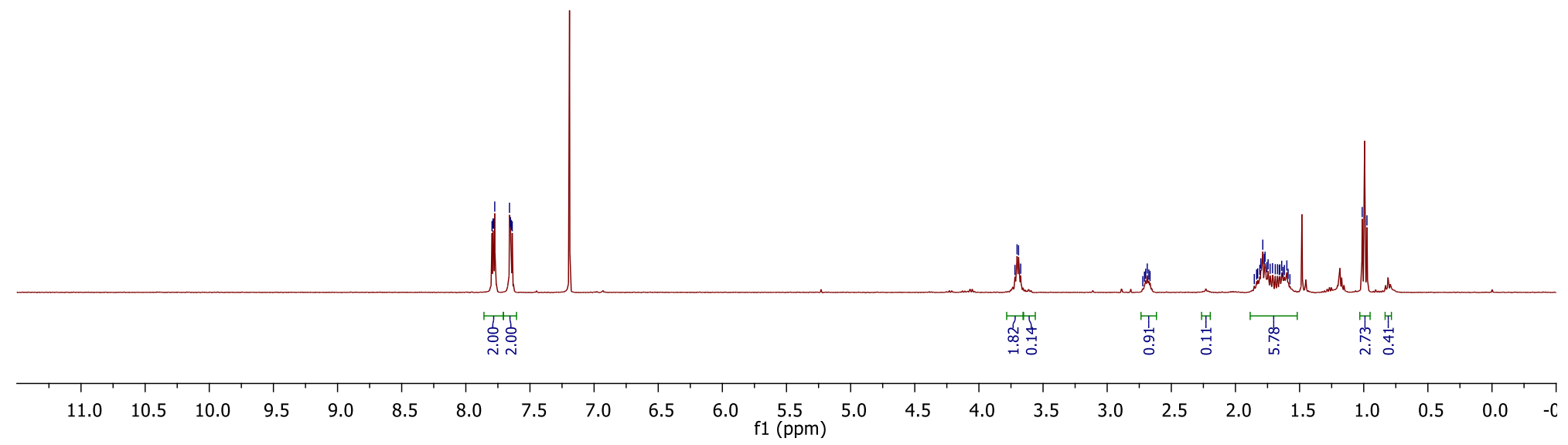


${ }^{13} \mathrm{C}-\mathrm{NMR}$ of compound $\mathbf{2 w}$

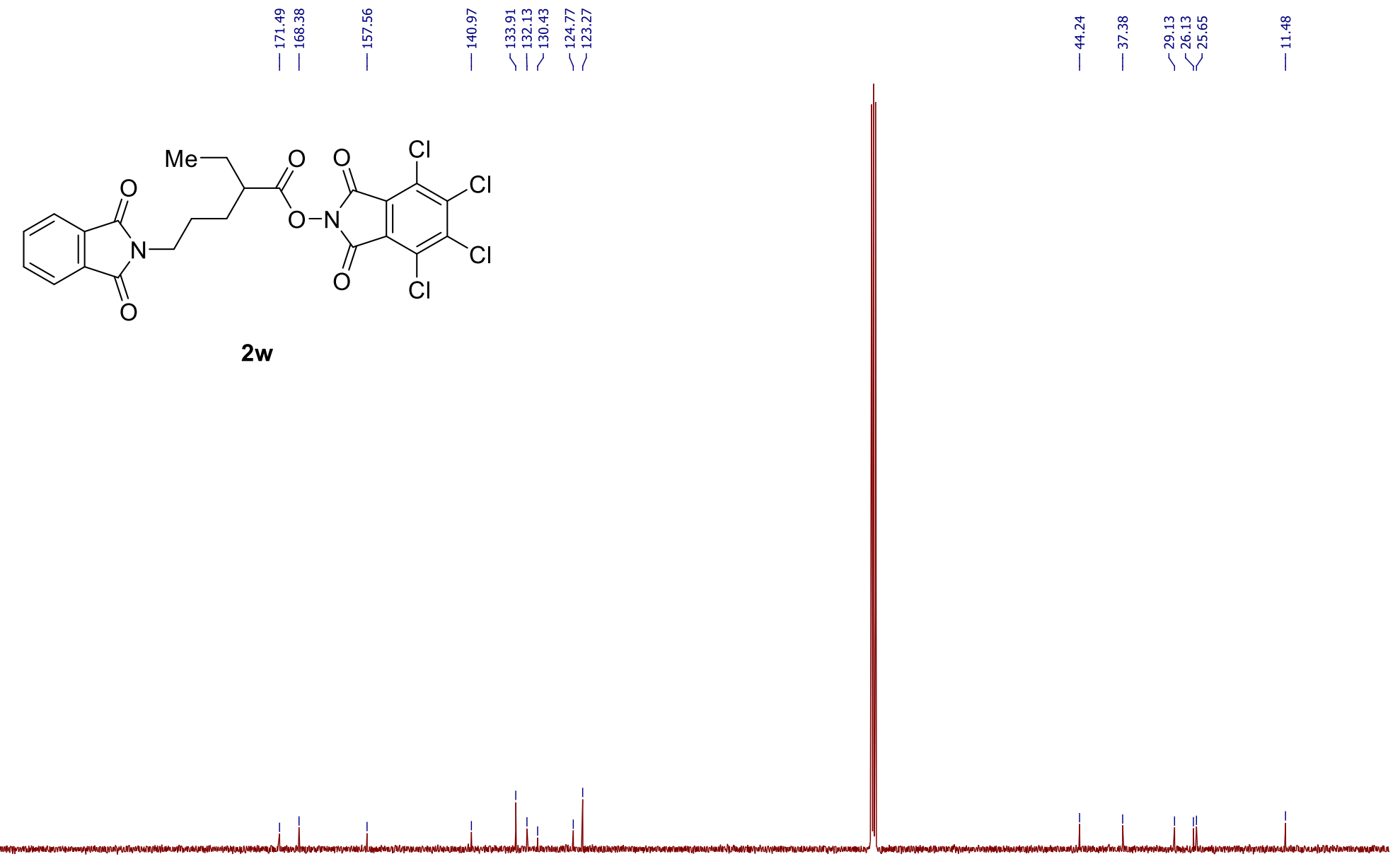

$\begin{array}{llllllllllllllllllllllllll}210 & 200 & 190 & 180 & 170 & 160 & 150 & 140 & 130 & 120 & 110 & 100 & 90 & 80 & 70 & 60 & 50 & 40 & 30 & 20 & 10 & 0\end{array}$ 
${ }^{1} \mathrm{H}$-NMR of compound $\mathbf{2 x}$

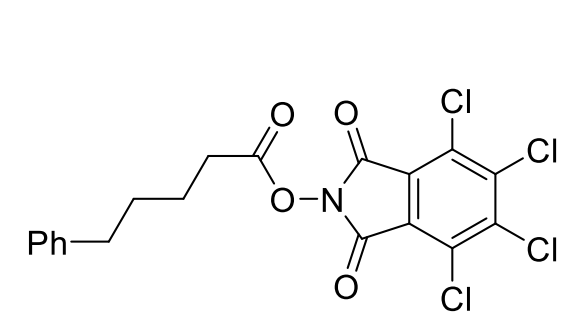

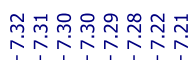

$\underbrace{2}$

2x

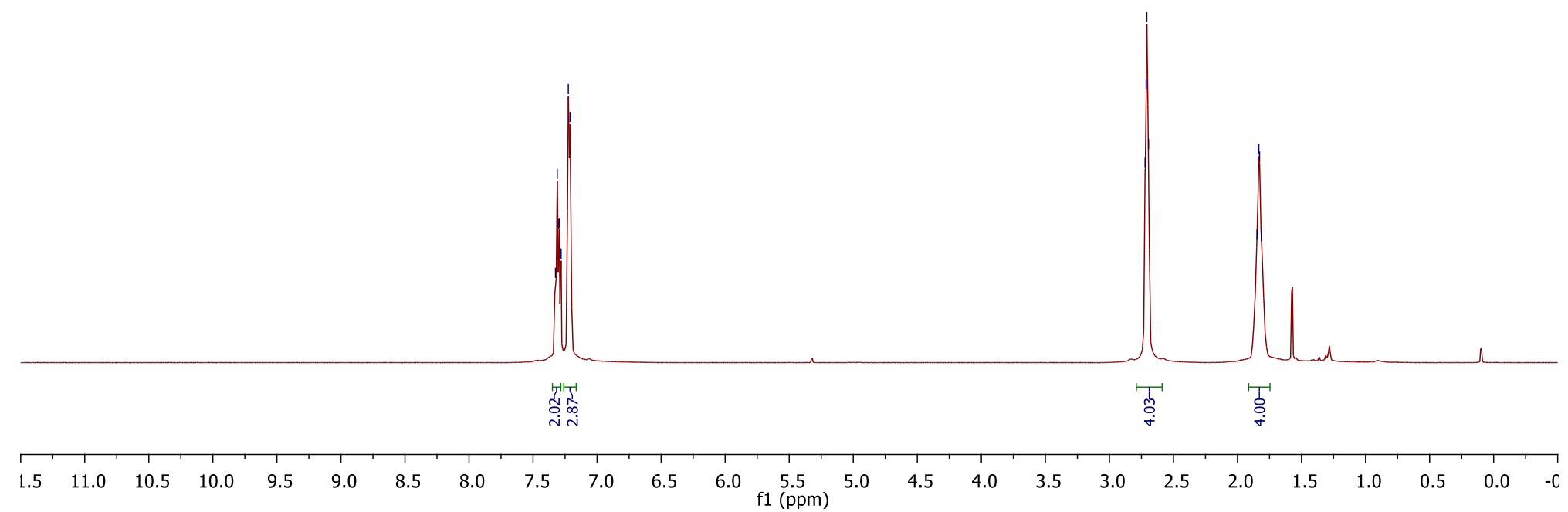


${ }^{13} \mathrm{C}$-NMR of compound $\mathbf{2 x}$

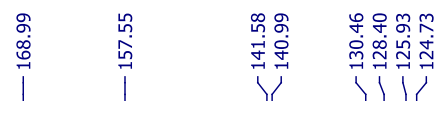

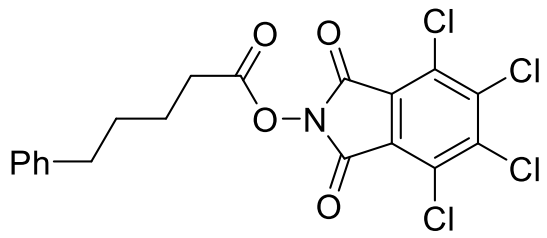

$2 \mathrm{x}$

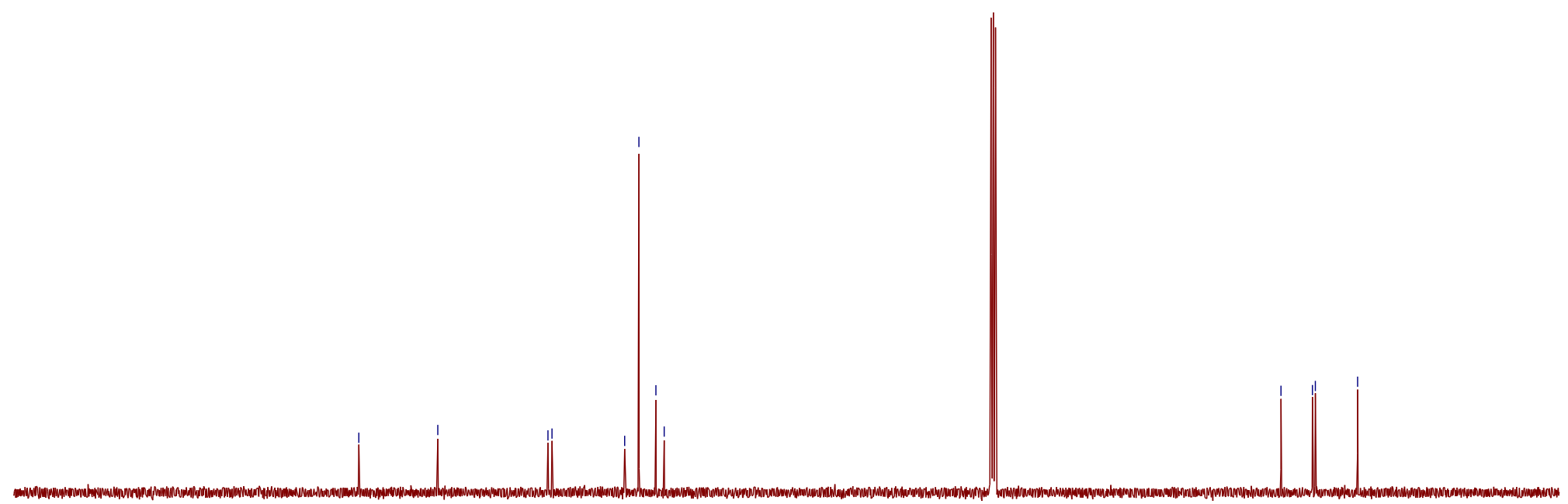


${ }^{1} \mathrm{H}-\mathrm{NMR}$ of compound $\mathbf{2 y}$

$$
\text { | }
$$

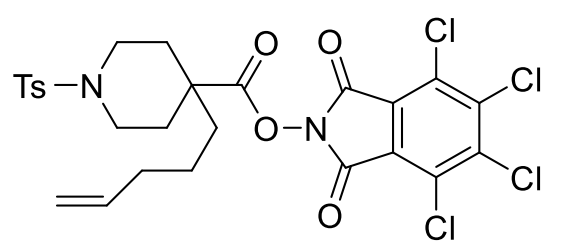

$2 y$

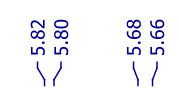
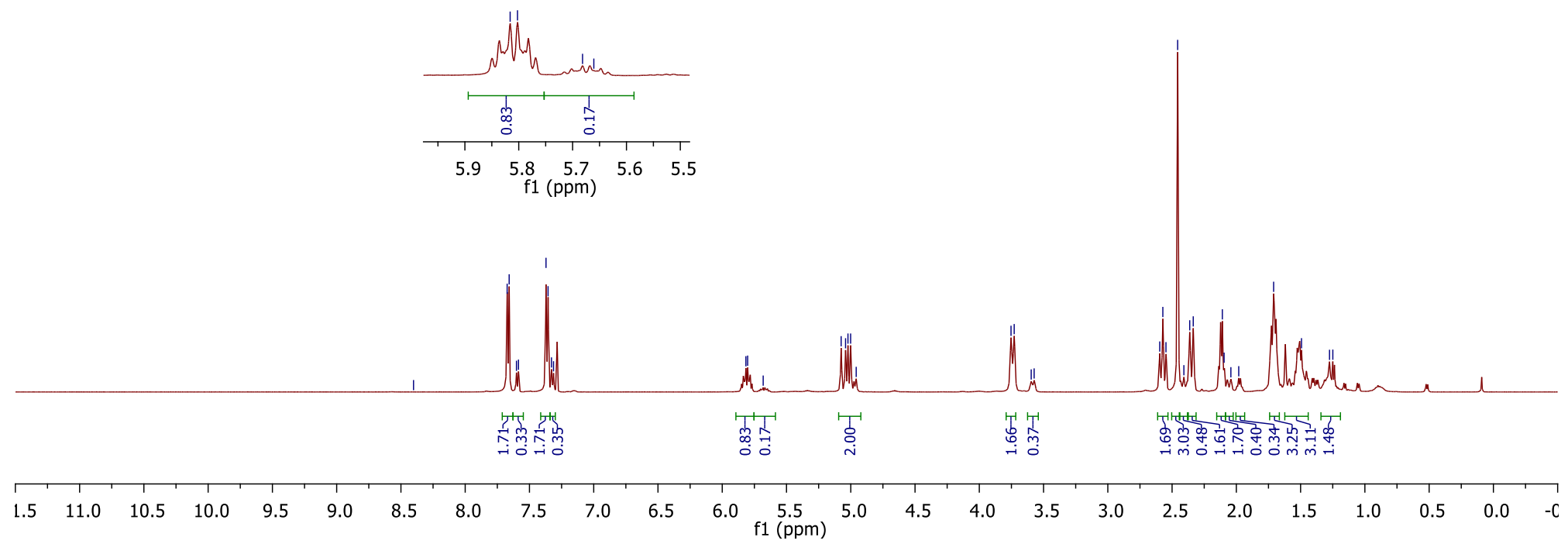
${ }^{13} \mathrm{C}$-NMR of compound $\mathbf{2 y}$

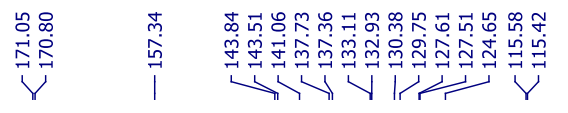

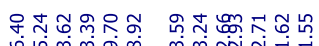

子于

पो

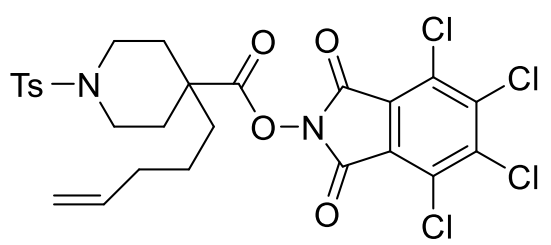

$2 y$

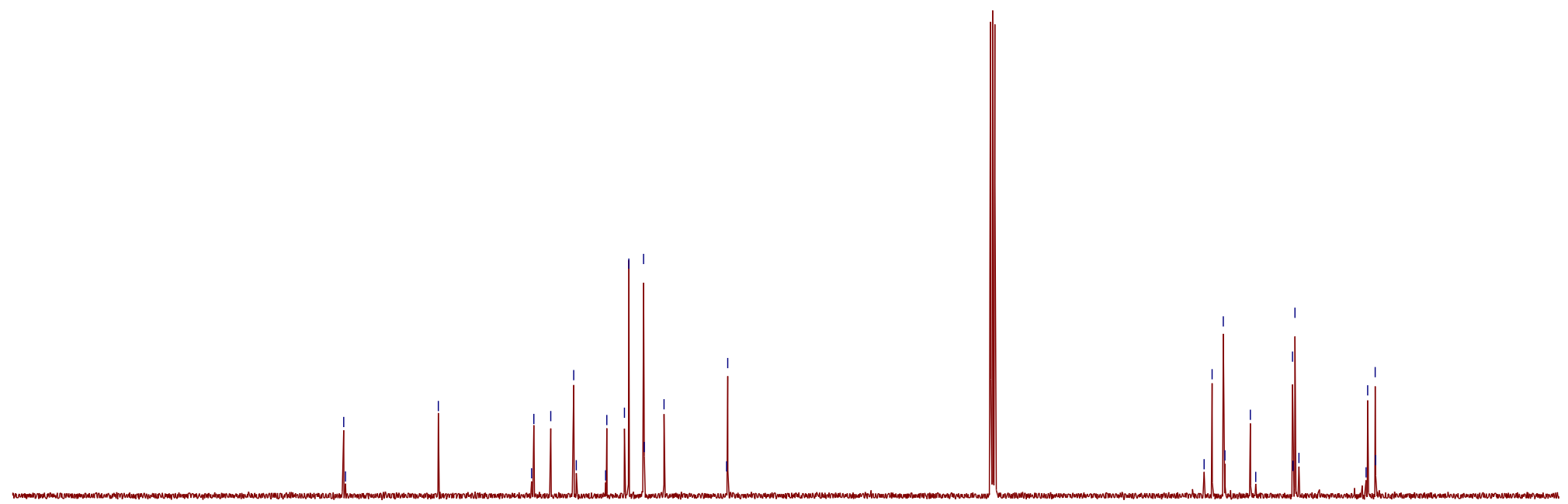


${ }^{1}$ H-NMR of compound $\mathbf{2 z}$
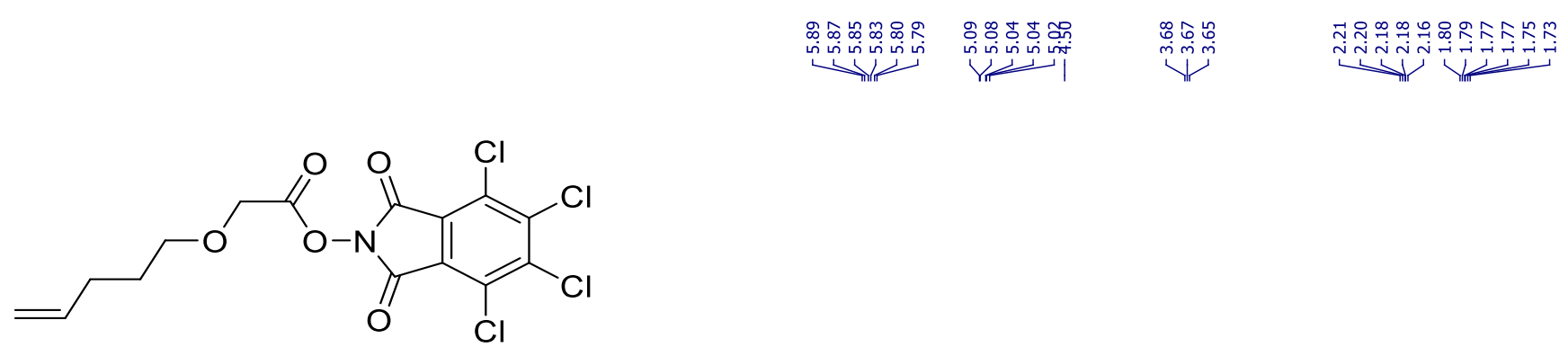

$2 z$

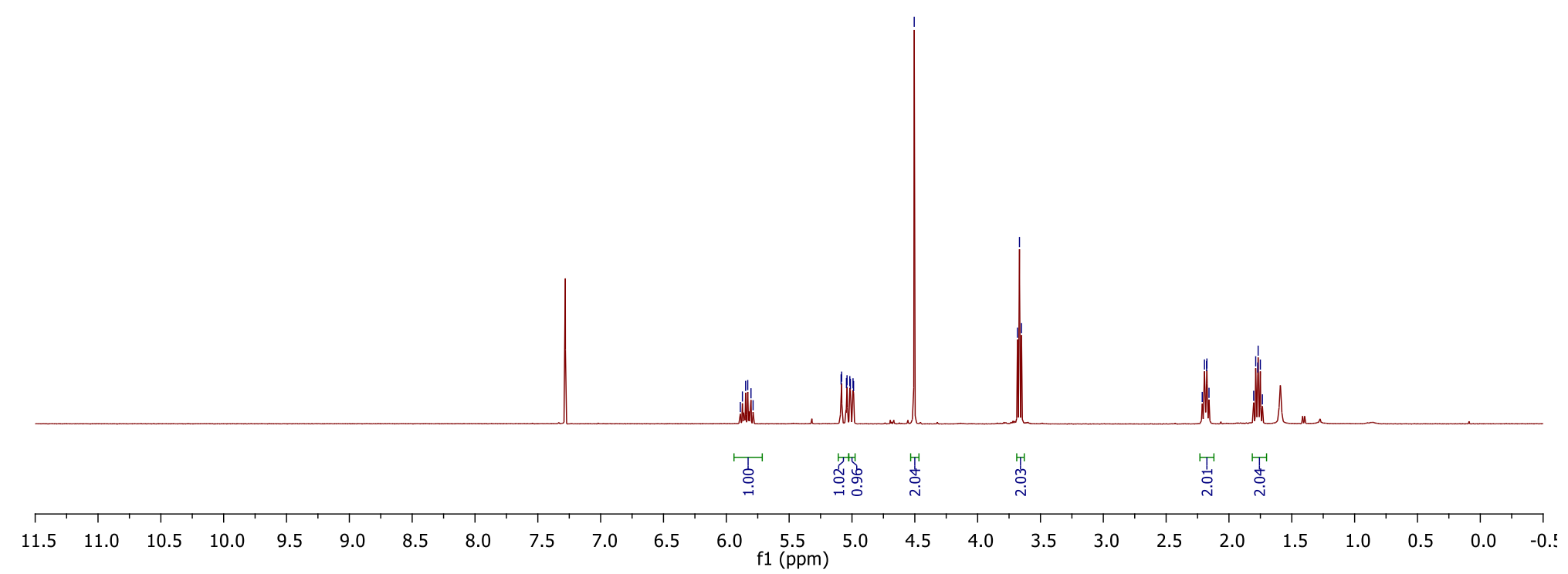


${ }^{13} \mathrm{C}$-NMR of compound $\mathbf{2 z}$

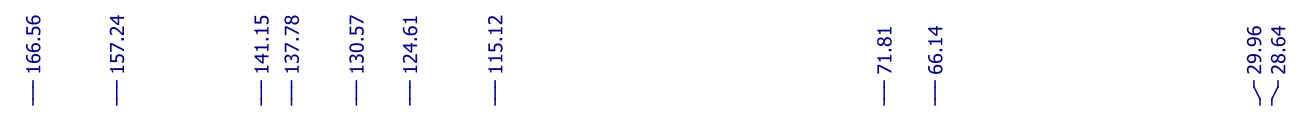

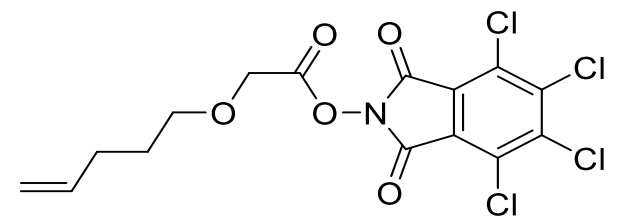

$2 z$

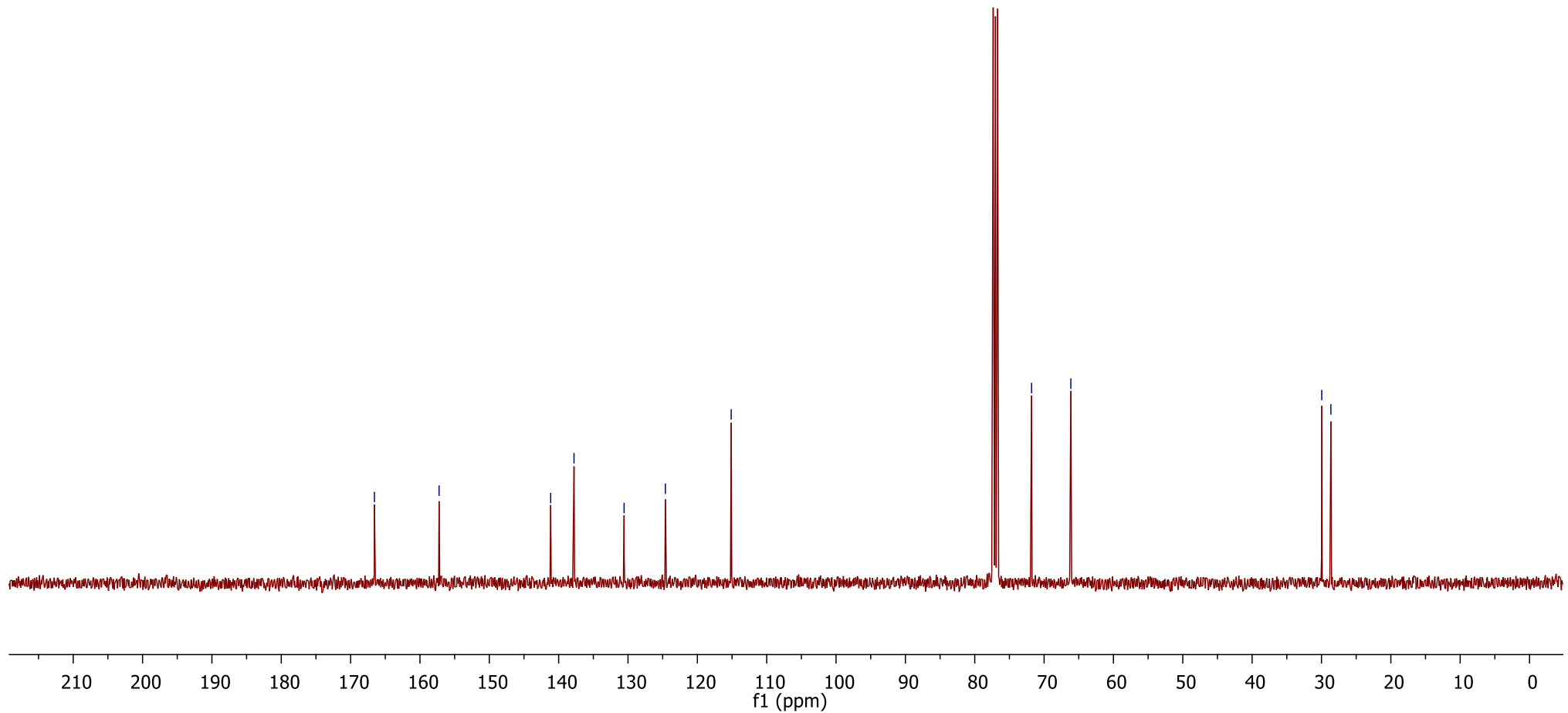


${ }^{1} \mathrm{H}-\mathrm{NMR}$ of compound 3a

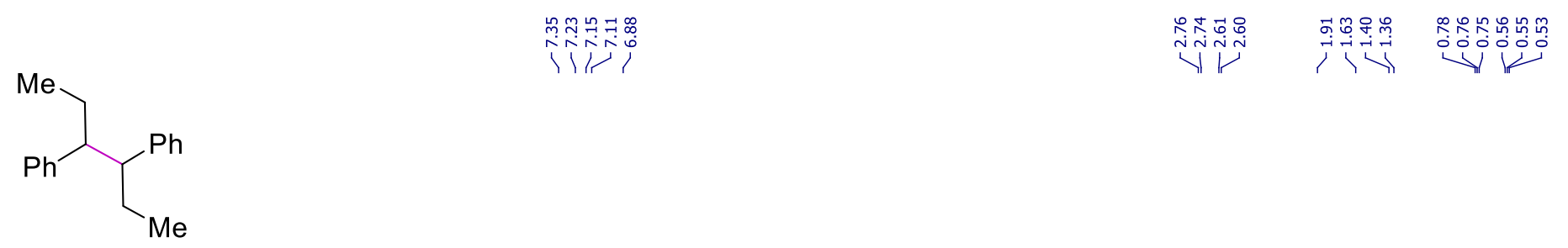

$3 a$

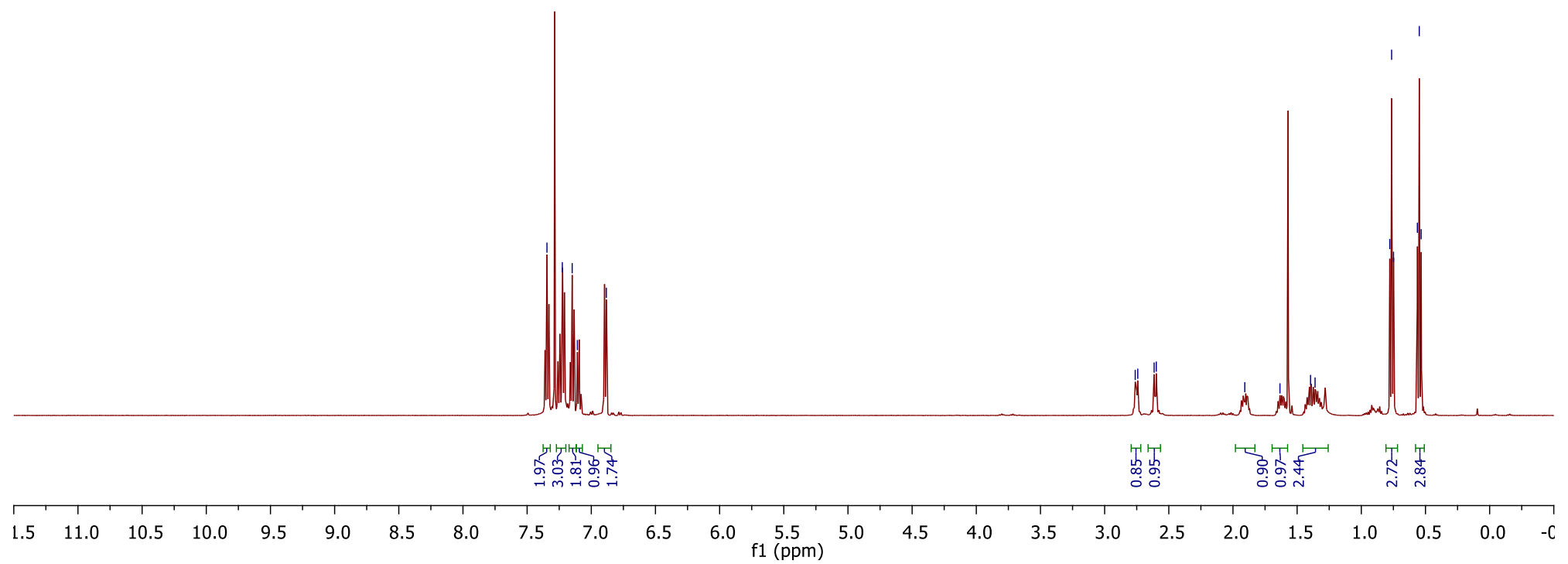


${ }^{13} \mathrm{C}-\mathrm{NMR}$ of compound $\mathbf{3 a}$

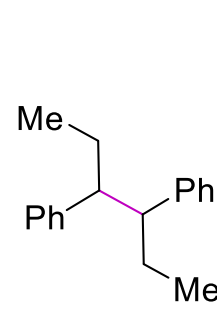

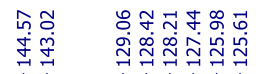

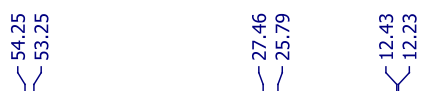

3a

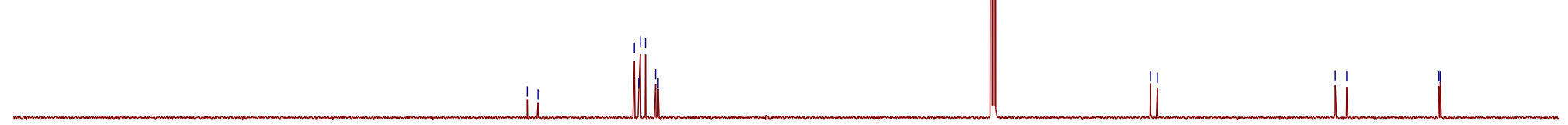

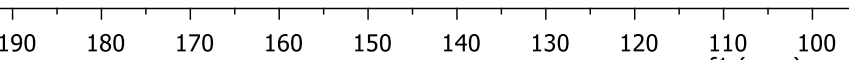

110
$f 1(p p m)$

$\begin{array}{lllll}90 & 80 & 70 & 60 & 50\end{array}$ 
${ }^{1} \mathrm{H}-\mathrm{NMR}$ of compound $\mathbf{3 b}$ (in acetone-d6)

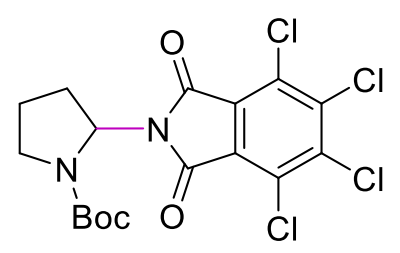

$3 b$

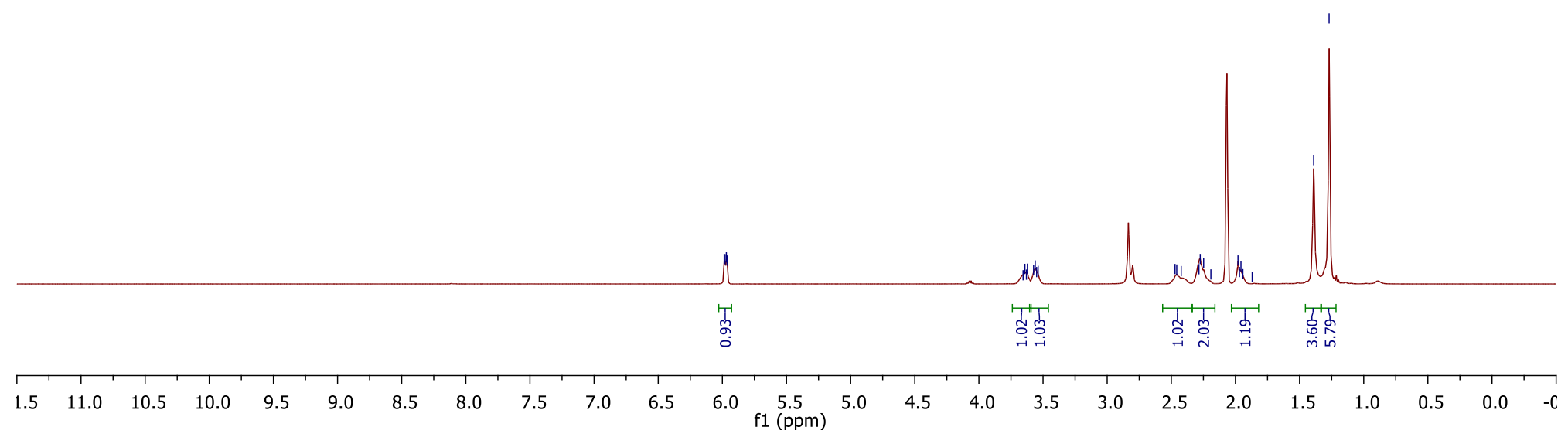


${ }^{13} \mathrm{C}-\mathrm{NMR}$ of compound $\mathbf{3 b}$ (in acetone-d6)

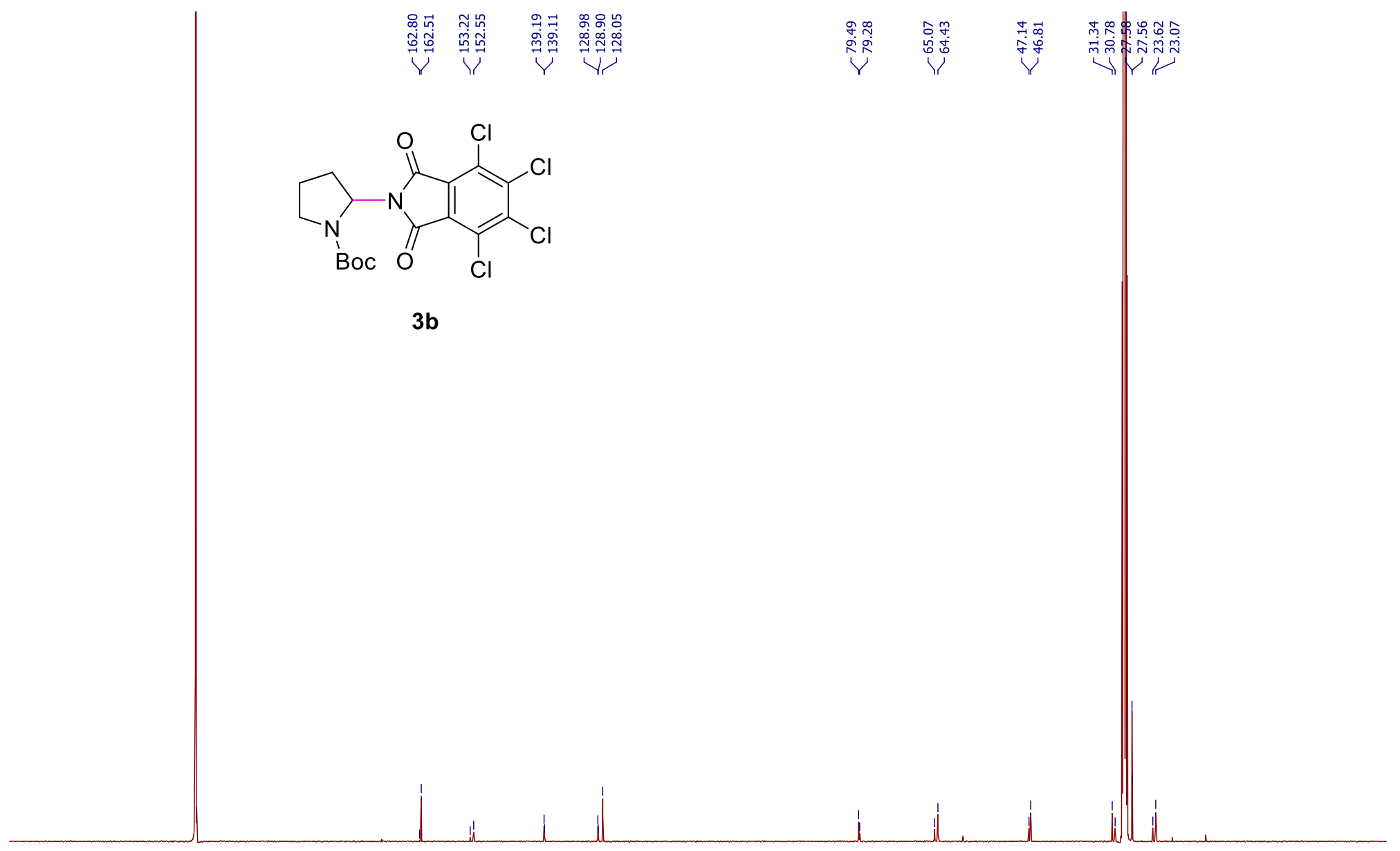

$\begin{array}{lllllllllllllllllllllllllllllllllllllllll}40 & 230 & 220 & 210 & 200 & 190 & 180 & 170 & 160 & 150 & 140 & 130 & 120 & 110 & 100 & 90 & 80 & 70 & 60 & 50 & 40 & 30 & 20 & 10 & 0 & -10 & -2\end{array}$ 
${ }^{1} \mathrm{H}-\mathrm{NMR}$ of compound $\mathbf{3 b}$ '

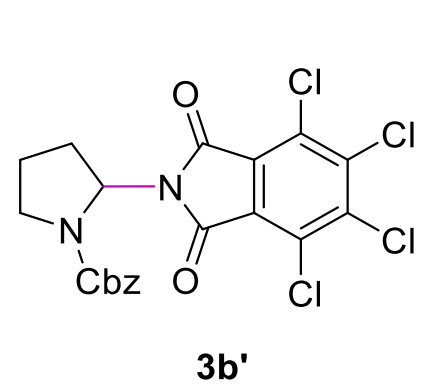

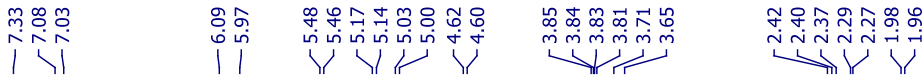

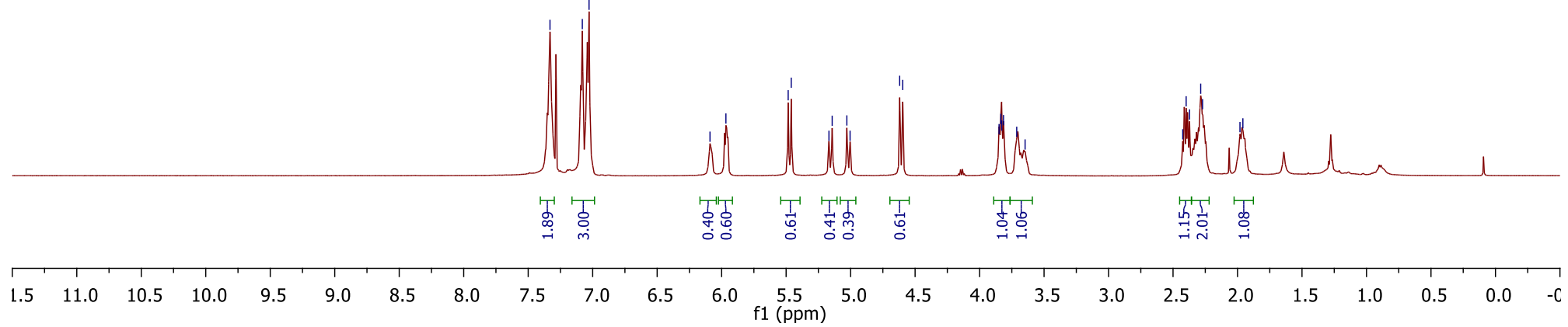


${ }^{13} \mathrm{C}-\mathrm{NMR}$ of compound $\mathbf{3 b}$ '
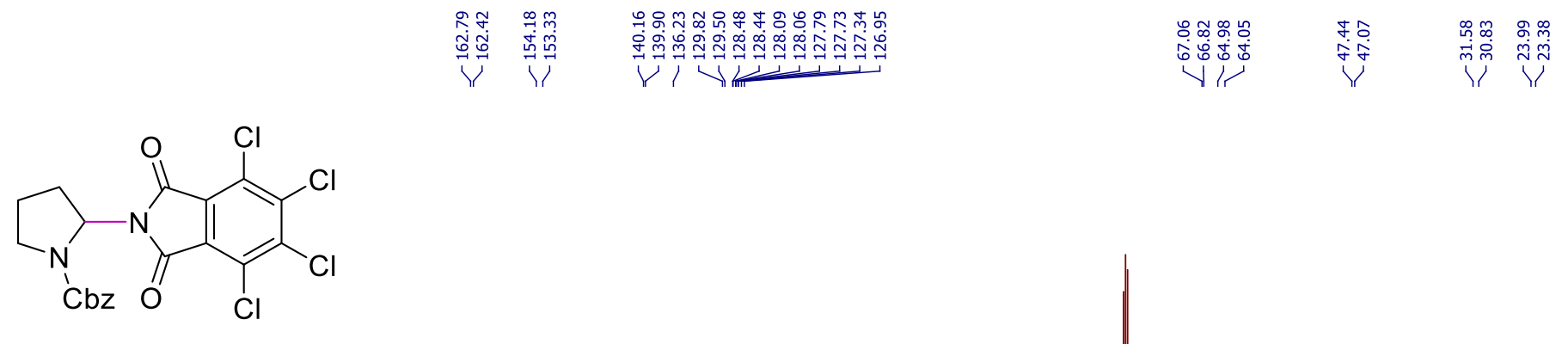

3b'

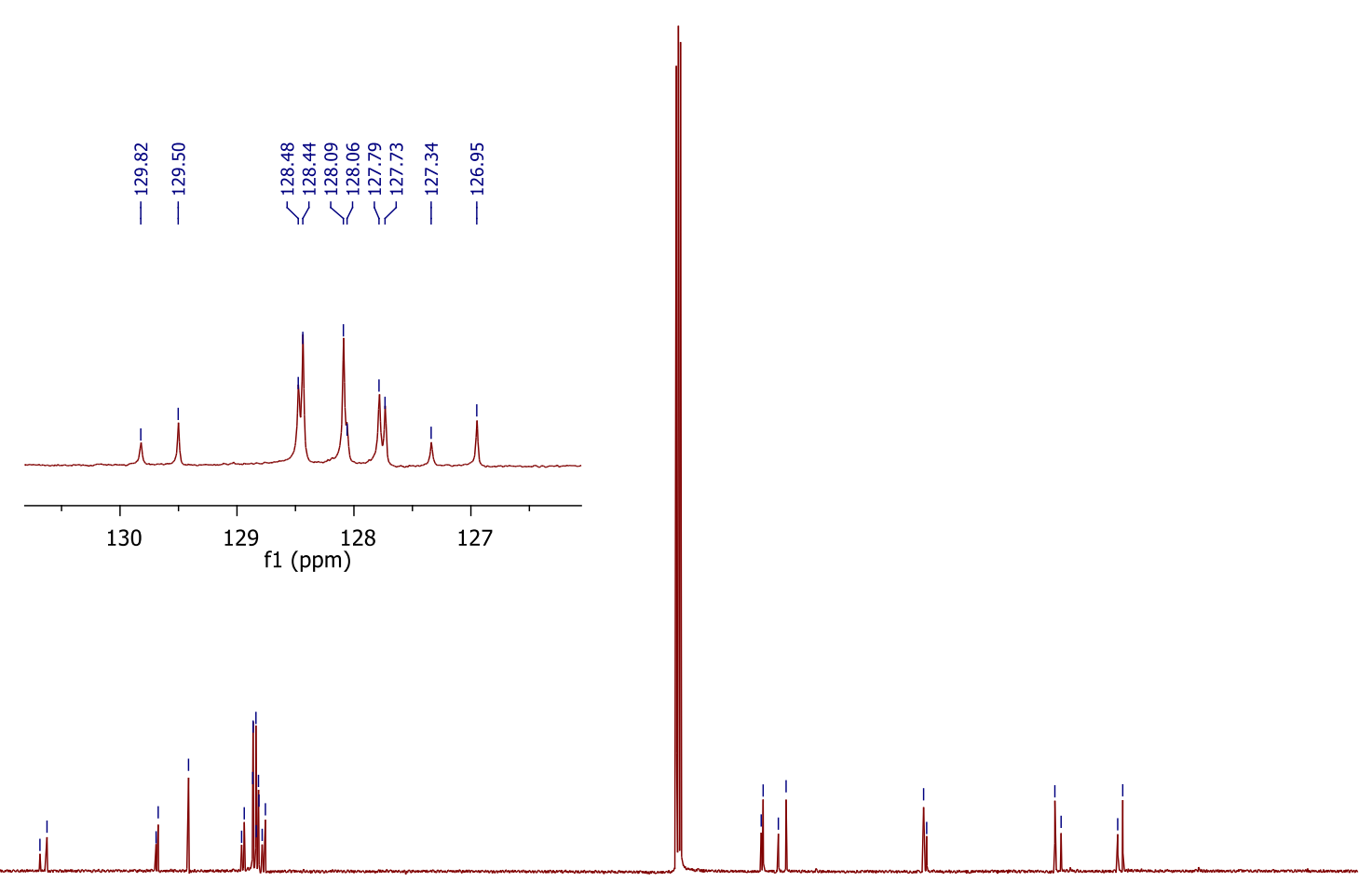

$210 \quad 200 \quad 190$

$180 \quad 170$

160

140

$30 \quad 120$

$110 \quad 100$

90

80

60

50 
${ }^{1} \mathrm{H}-\mathrm{NMR}$ of compound $\mathbf{3 c}$-cis

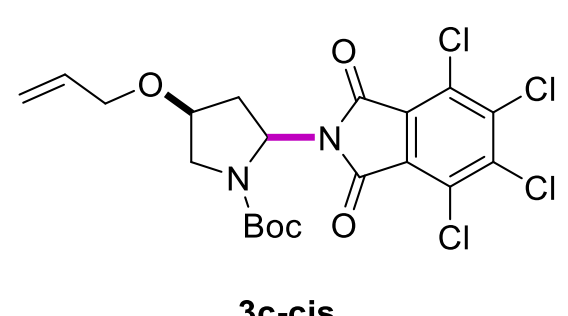

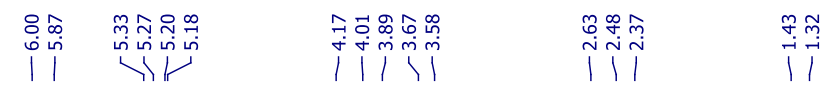

3c-cis

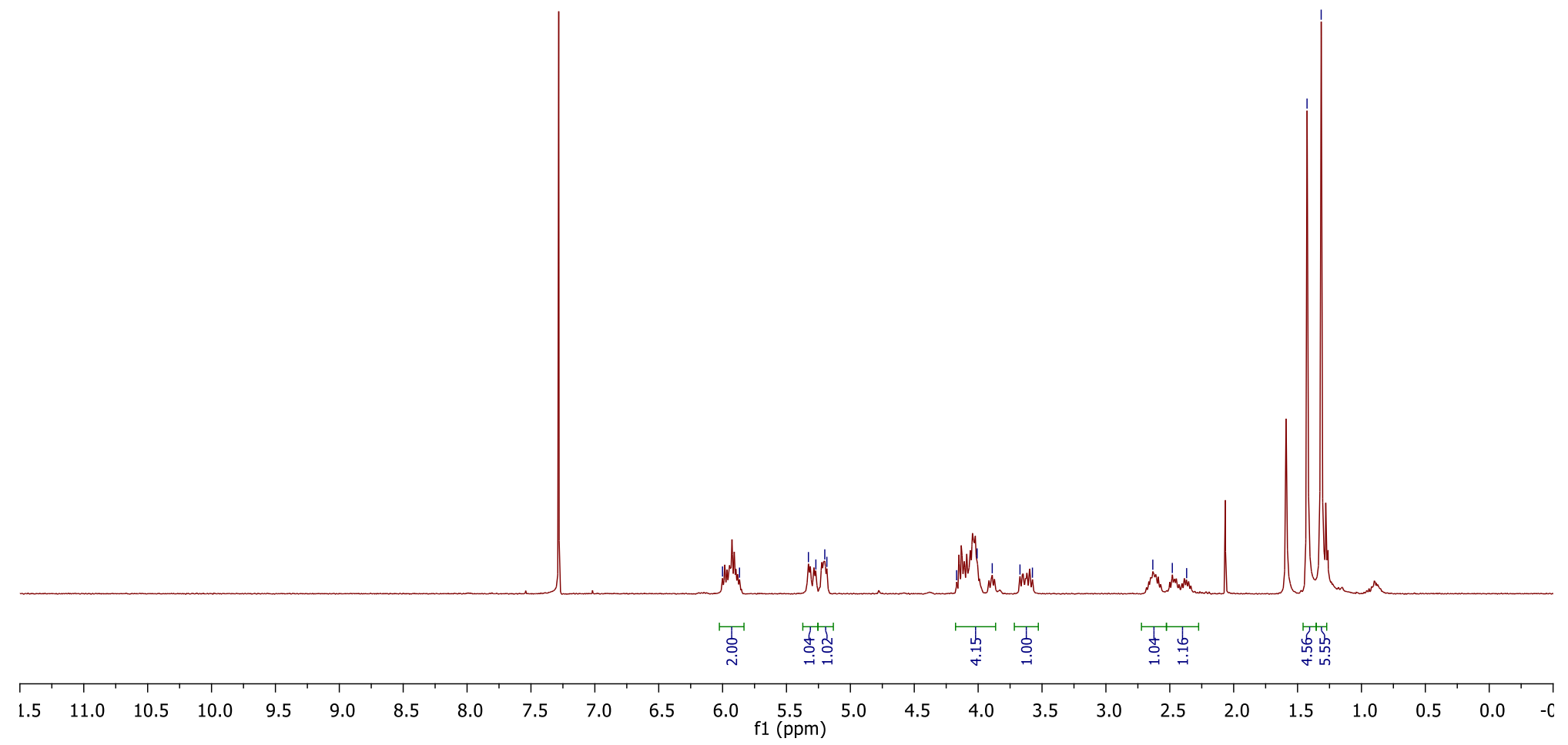


${ }^{13} \mathrm{C}$-NMR of compound 3c-cis

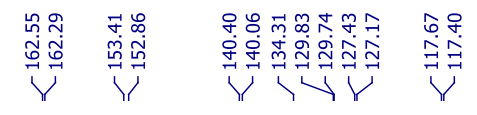

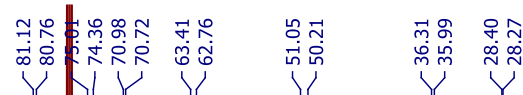

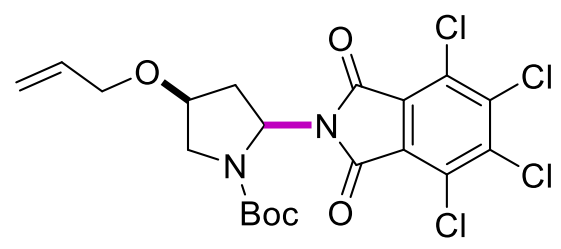

3c-cis

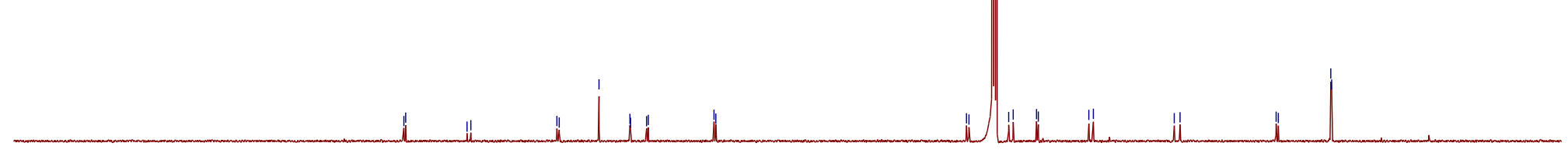


${ }^{1} \mathrm{H}-\mathrm{NMR}$ of compound $3 \mathrm{c}$-trans

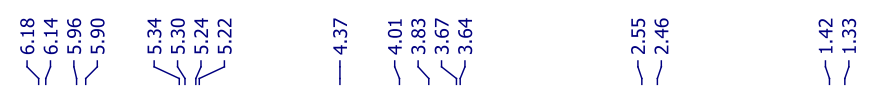

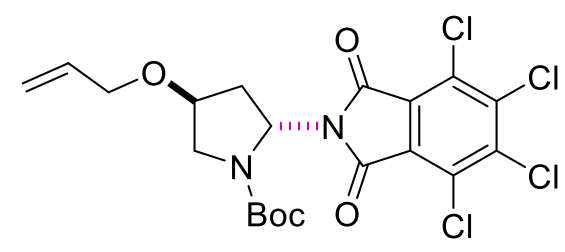

3c-trans

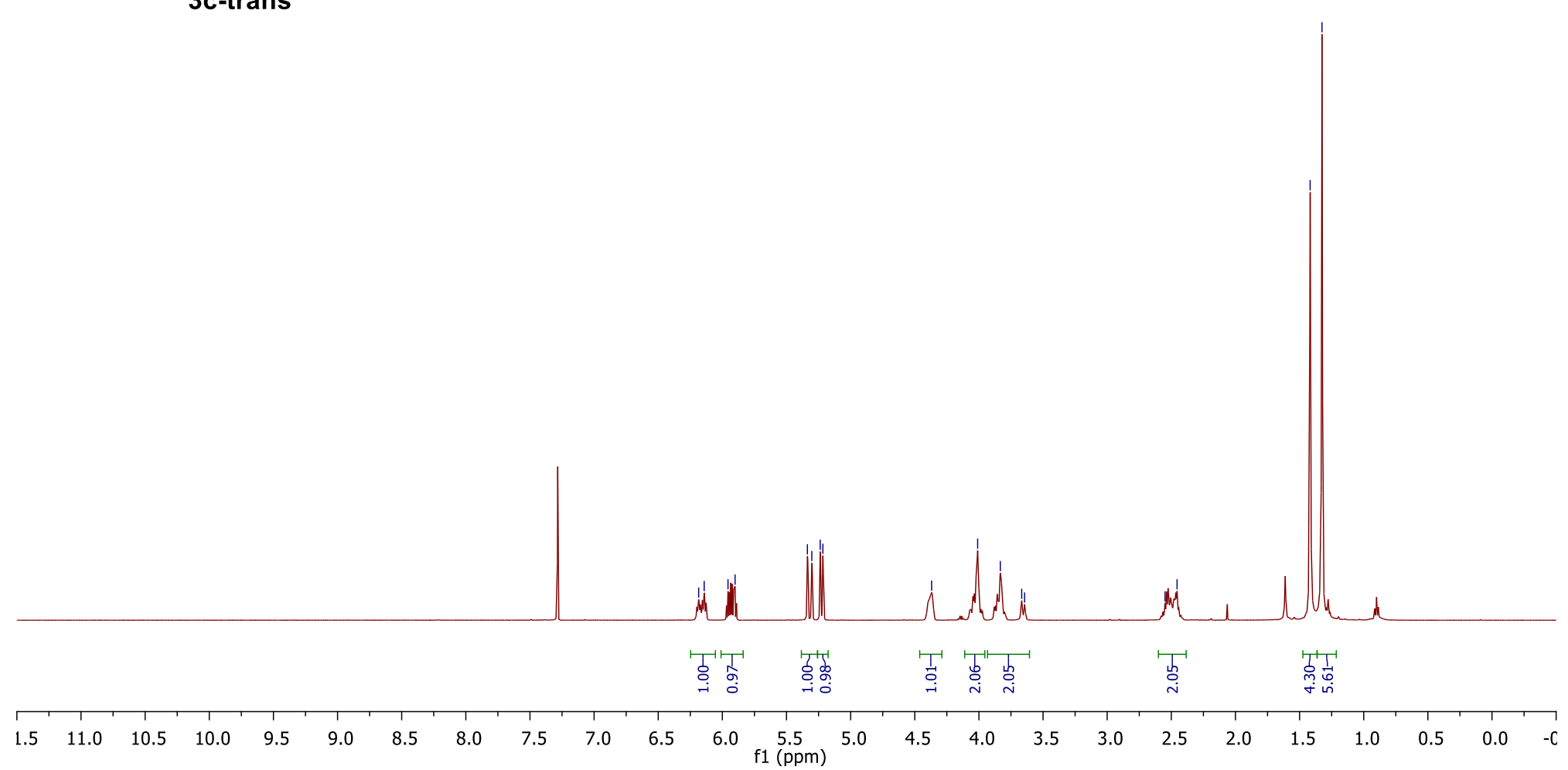


${ }^{13} \mathrm{C}-\mathrm{NMR}$ of compound $\mathbf{3 c}$-trans

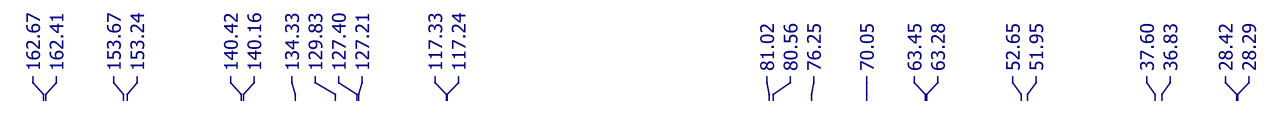

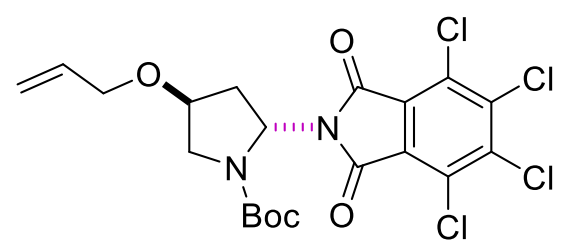

3c-trans

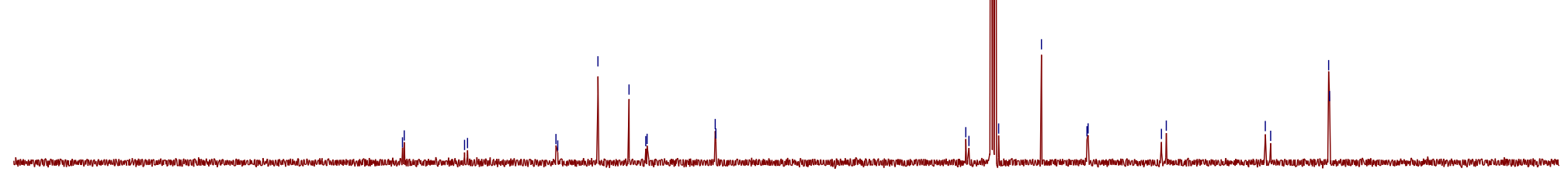


${ }^{1} \mathrm{H}-\mathrm{NMR}$ of compound $\mathbf{3 d}$
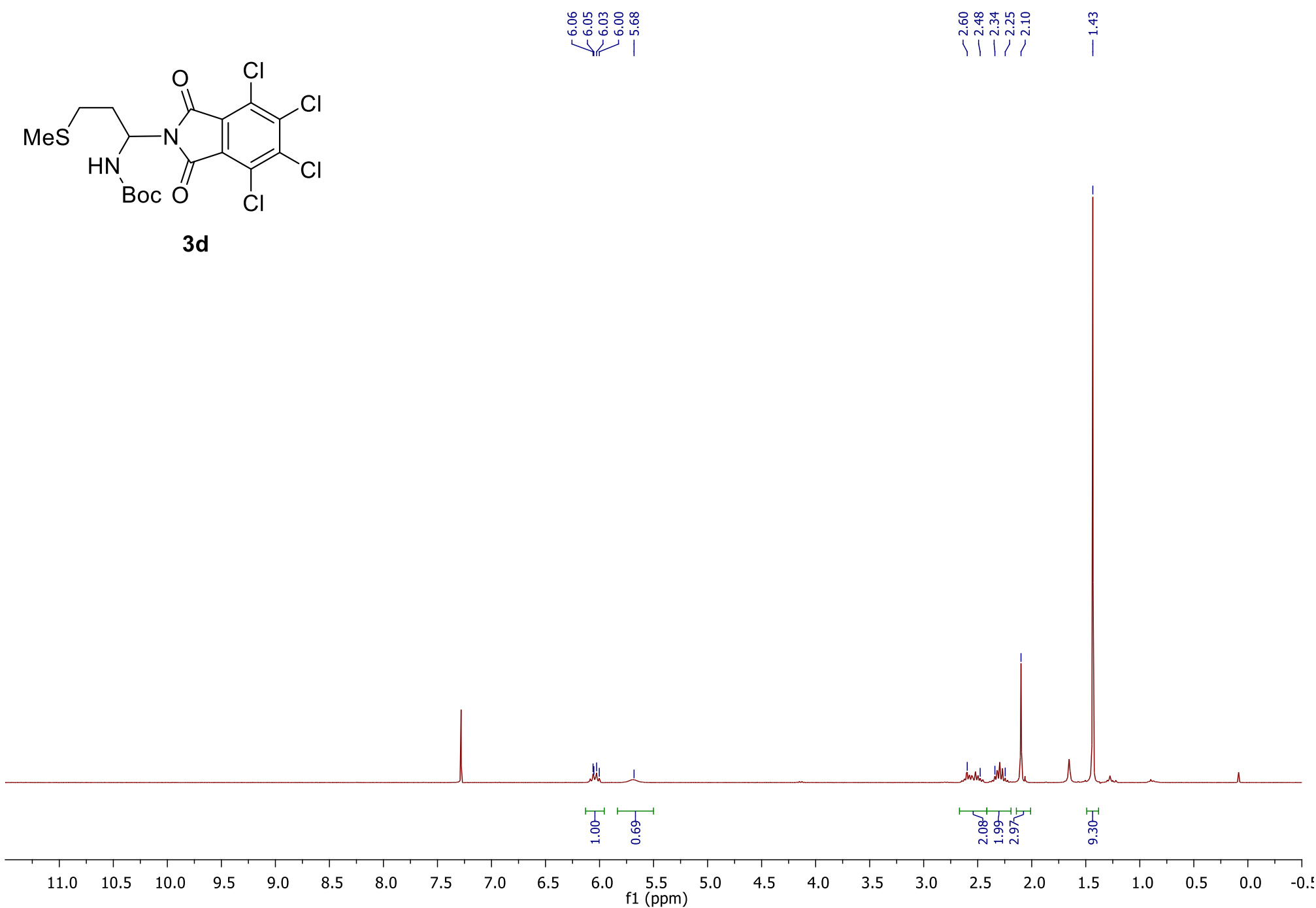
${ }^{13} \mathrm{C}-\mathrm{NMR}$ of compound $\mathbf{3 d}$

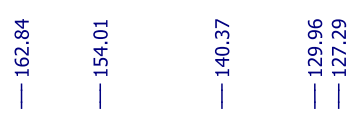

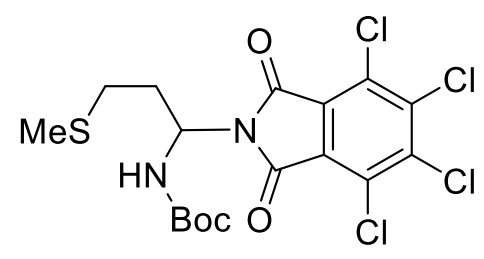

3d

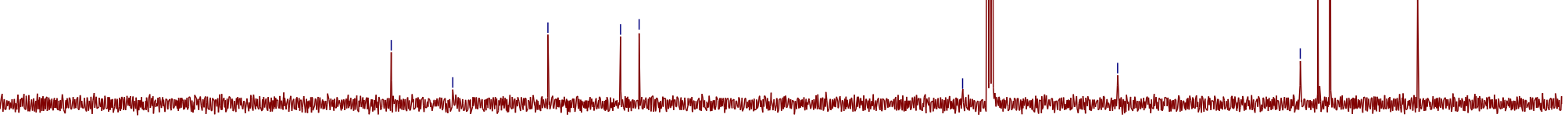


${ }^{1} \mathrm{H}-\mathrm{NMR}$ of compound $\mathbf{3 e}$

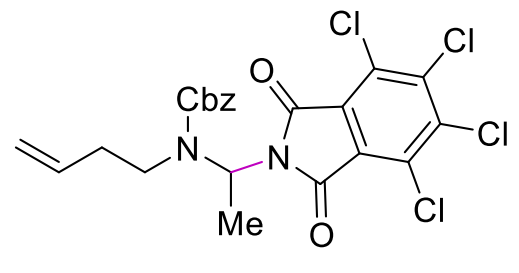

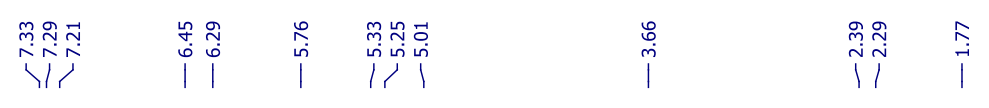

$3 e$

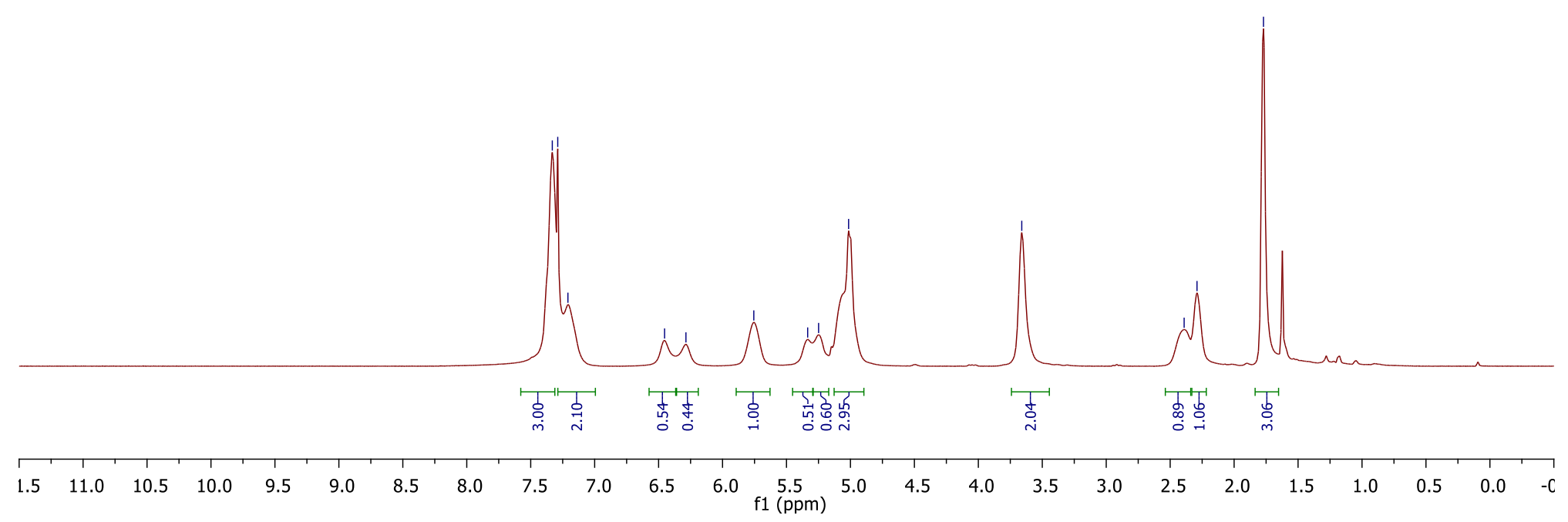


${ }^{13} \mathrm{C}-\mathrm{NMR}$ of compound $\mathbf{3 e}$

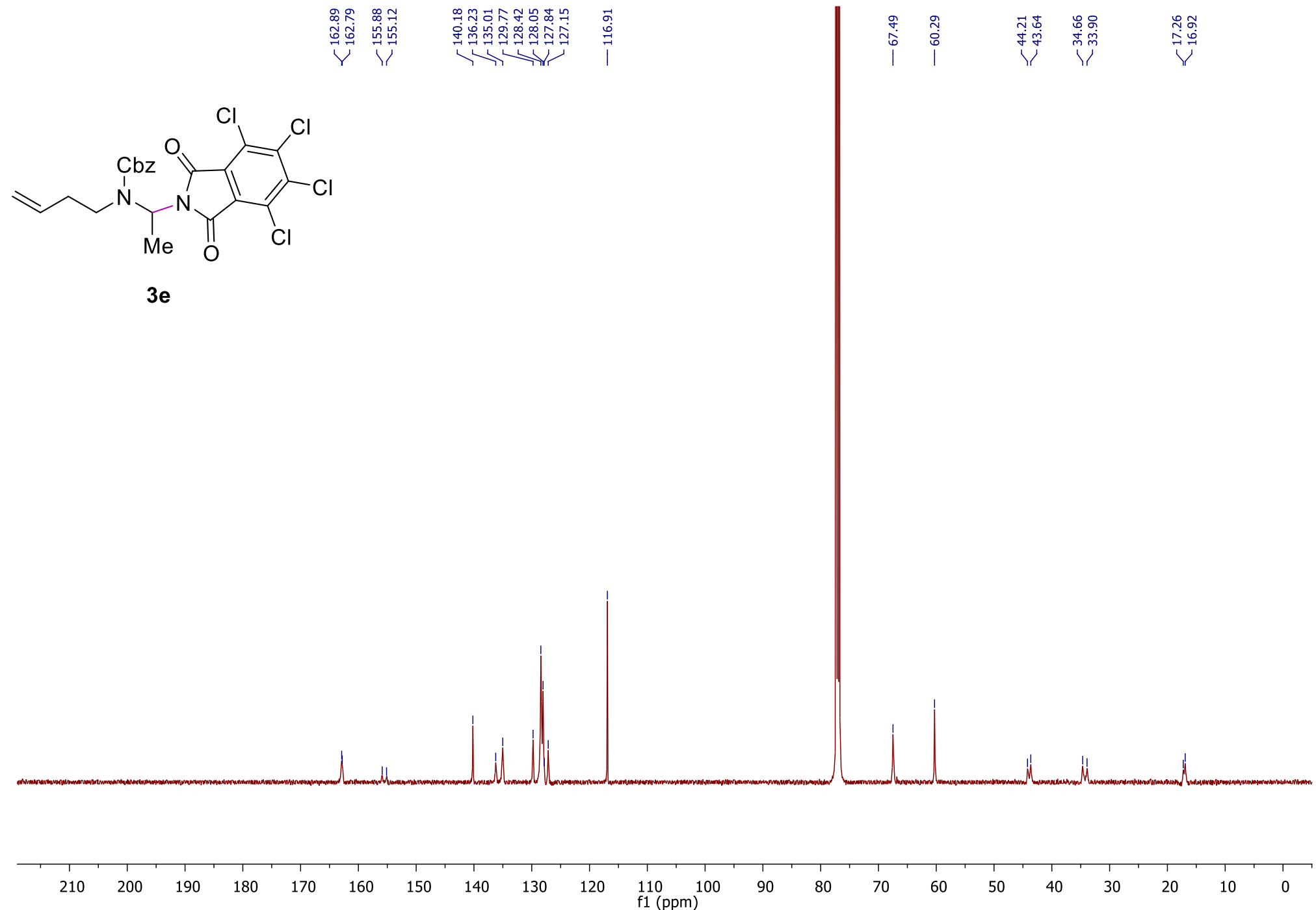


${ }^{1} \mathrm{H}-\mathrm{NMR}$ of compound $\mathbf{3 f}$

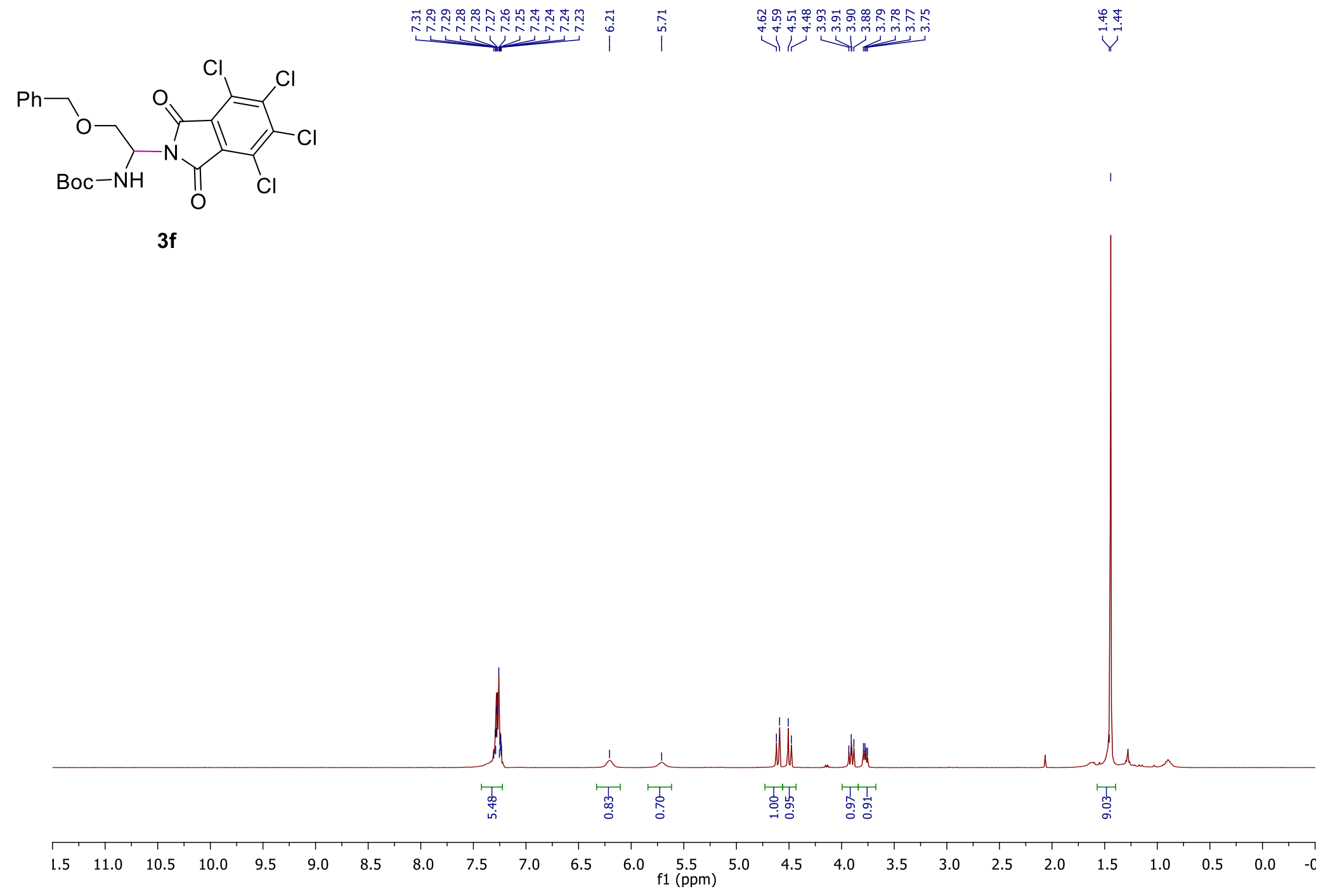


${ }^{13} \mathrm{C}$-NMR of compound $\mathbf{3 f}$

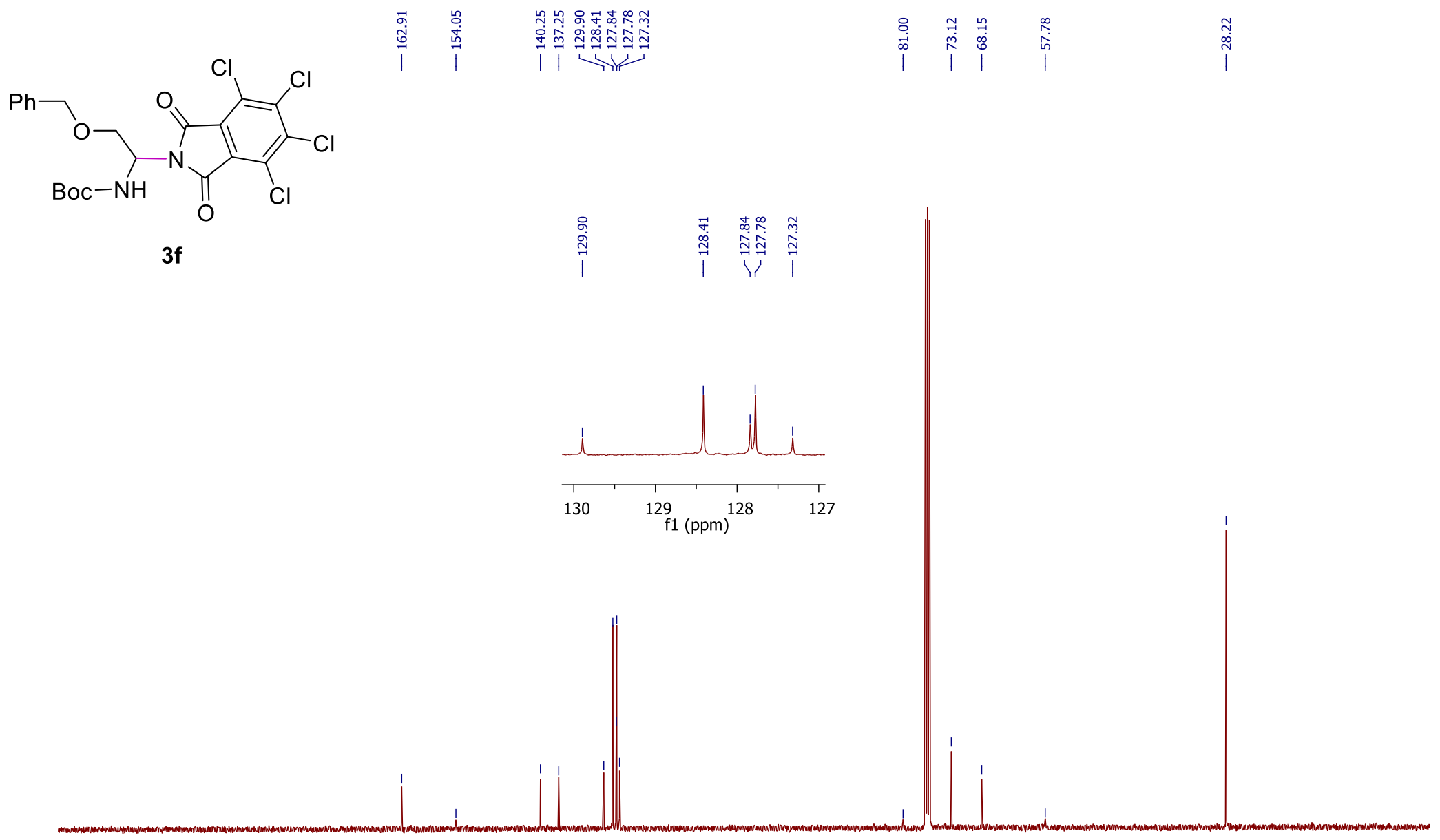

$\begin{array}{llllllllllllllllllllllllll}210 & 200 & 190 & 180 & 170 & 160 & 150 & 140 & 130 & 120 & \underset{\mathrm{f} 1}{110}(\mathrm{ppm}) & 100 & 90 & 80 & 70 & 60 & 50 & 40 & 30 & 20 & 10 & 0\end{array}$ 
${ }^{1} \mathrm{H}$-NMR of compound $\mathbf{3 g}$
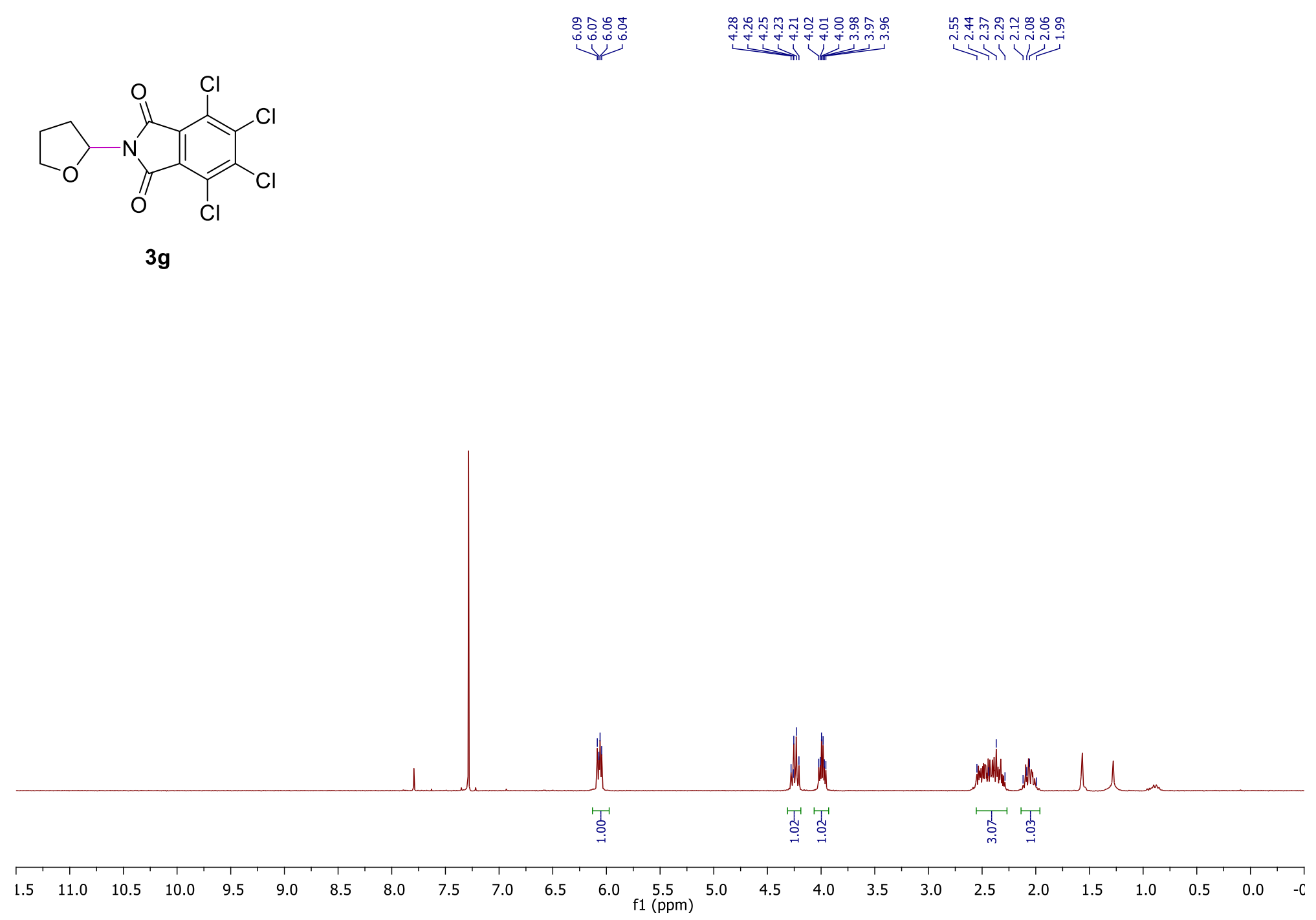
${ }^{13} \mathrm{C}-\mathrm{NMR}$ of compound $\mathbf{3 g}$

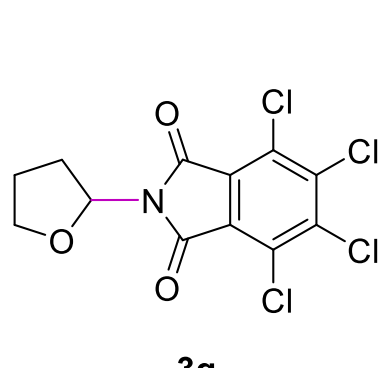

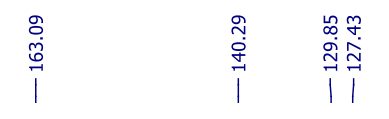

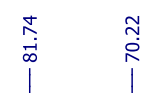

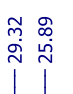

$3 \mathbf{g}$

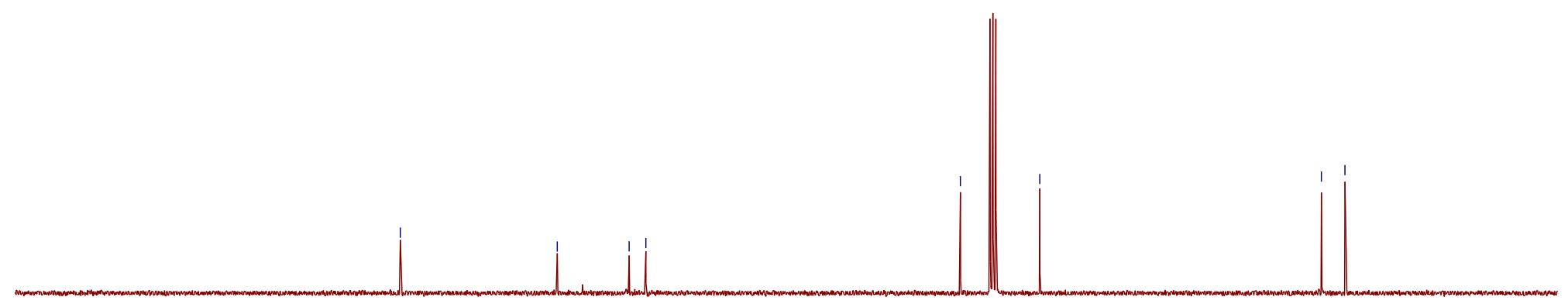


${ }^{1} \mathrm{H}-\mathrm{NMR}$ of compound $\mathbf{3 h}$
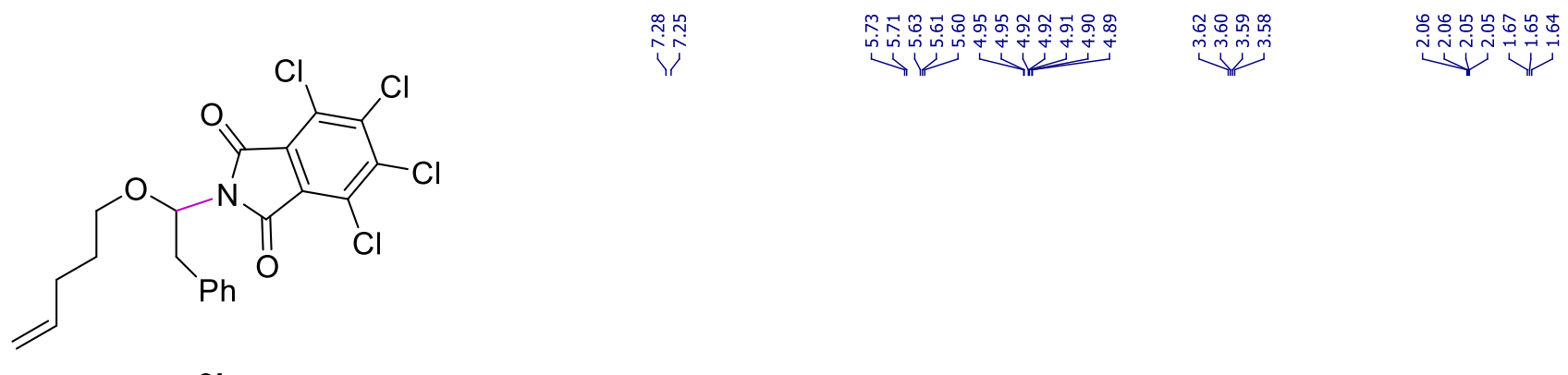

$3 h$

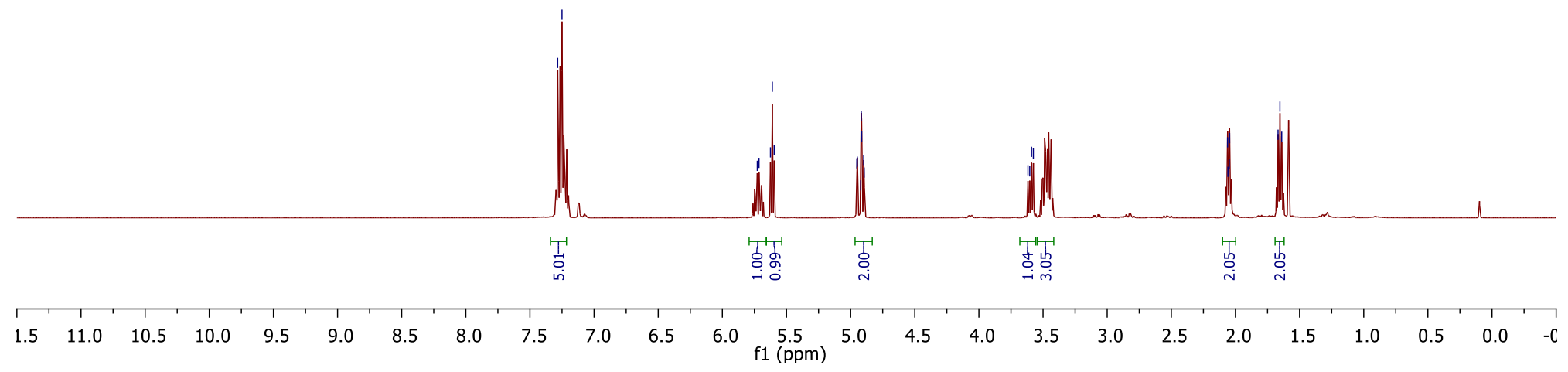


${ }^{13} \mathrm{C}-\mathrm{NMR}$ of compound $\mathbf{3 h}$

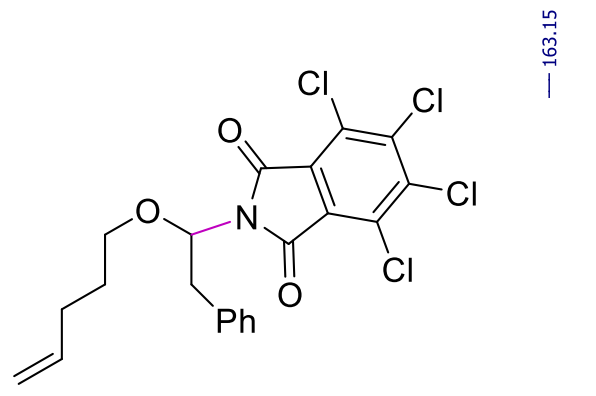

$3 h$

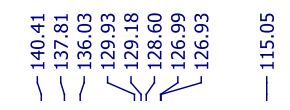

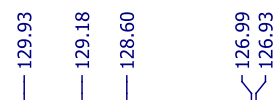

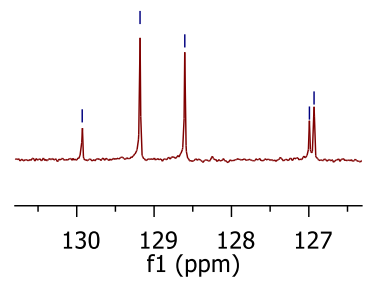

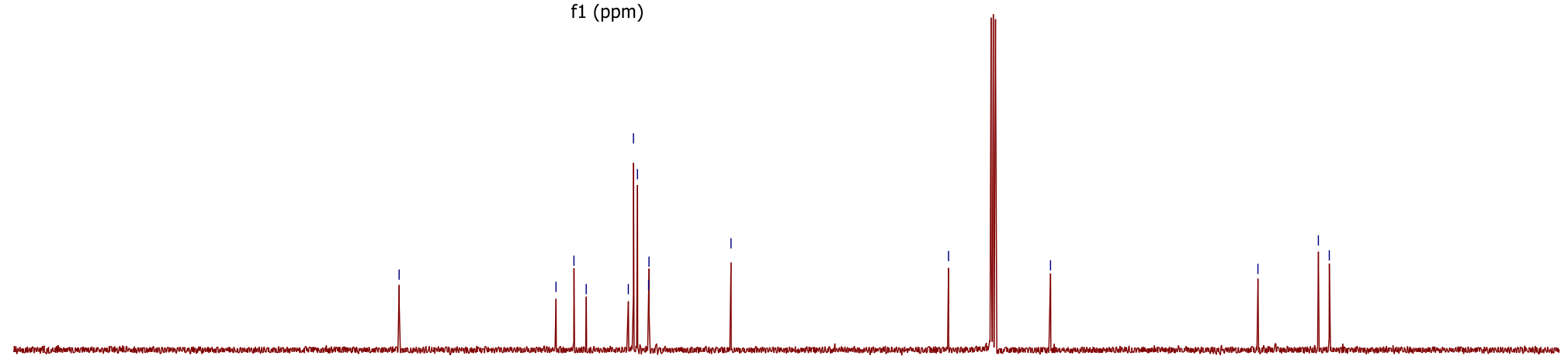

$210 \quad 200 \quad 190$ 
${ }^{1} \mathrm{H}-\mathrm{NMR}$ of compound $\mathbf{3 i}$

象

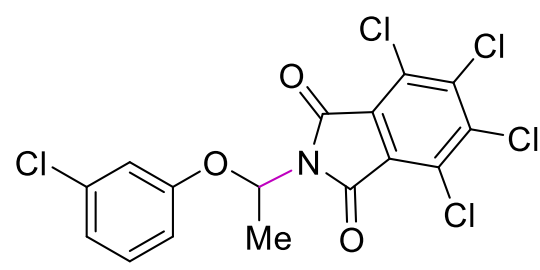

$3 \mathbf{i}$

\section{줏구 숫}
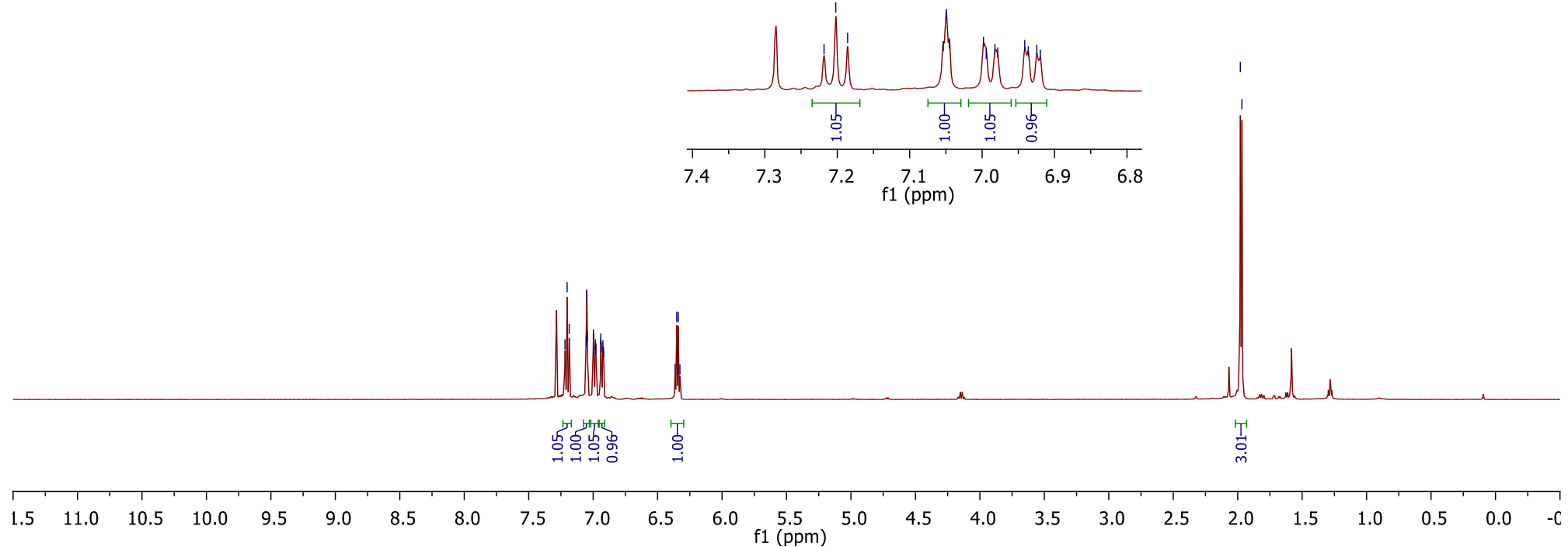
${ }^{13} \mathrm{C}-\mathrm{NMR}$ of compound $\mathbf{3 i}$

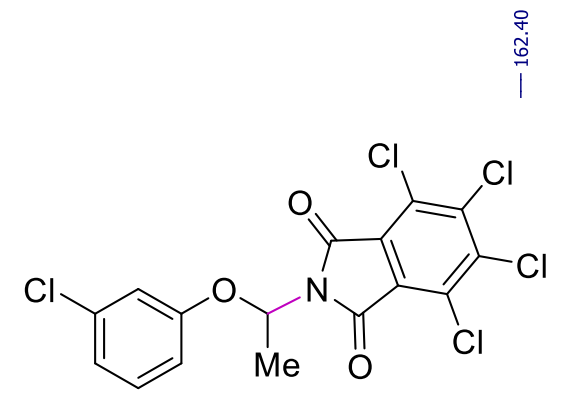

3i

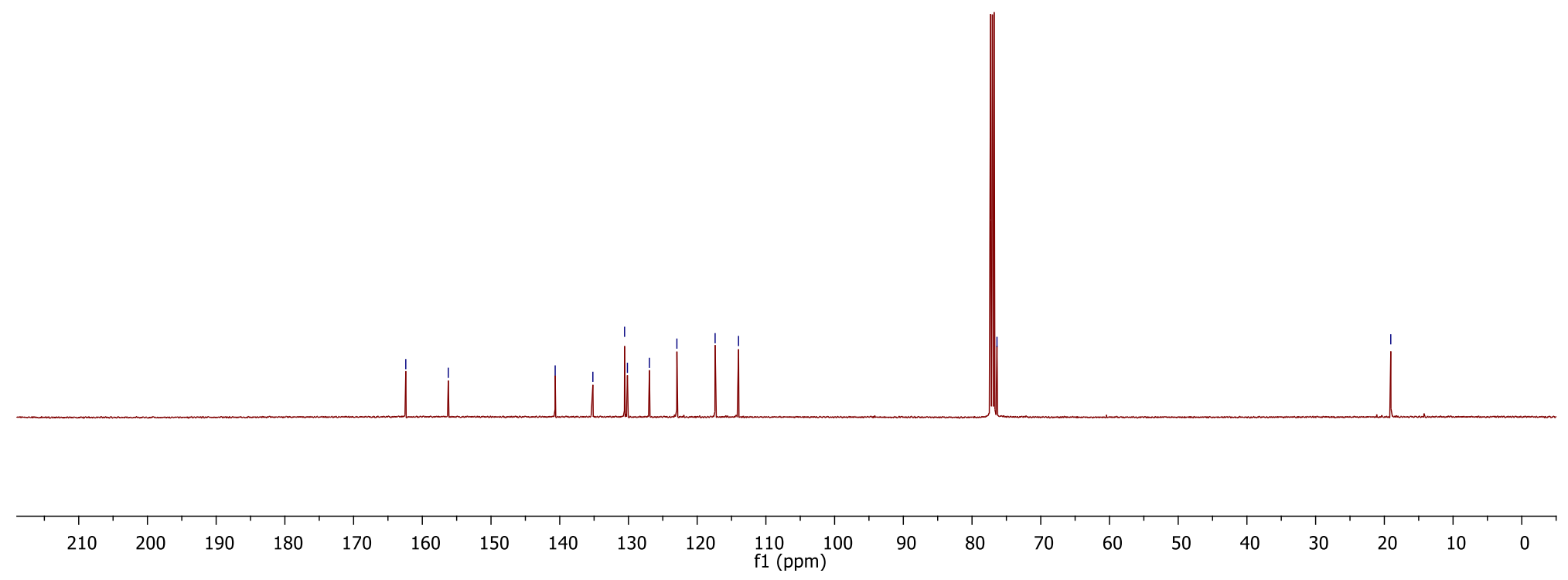


${ }^{1} \mathrm{H}$-NMR of compound $\mathbf{3 j}$

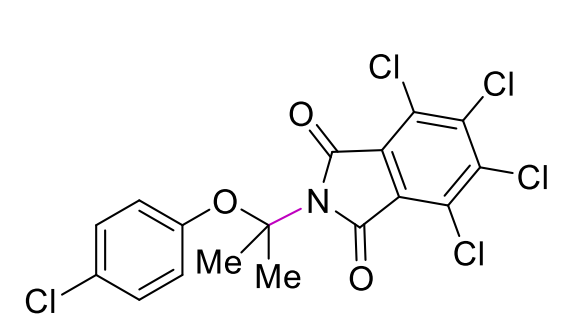

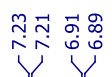

3j

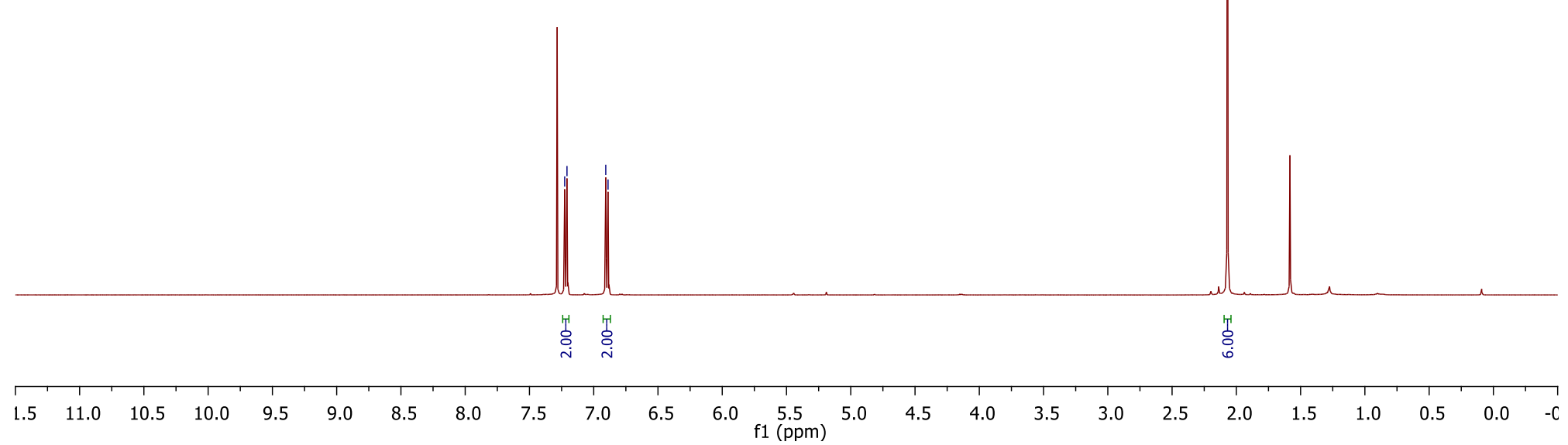


${ }^{13} \mathrm{C}-\mathrm{NMR}$ of compound $\mathbf{3 j}$

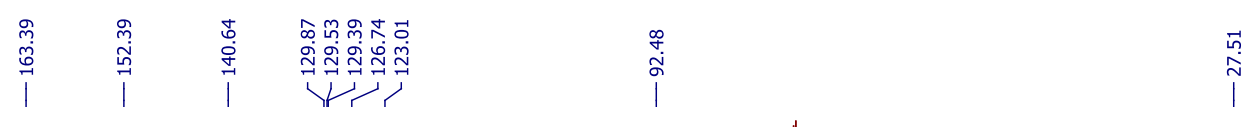

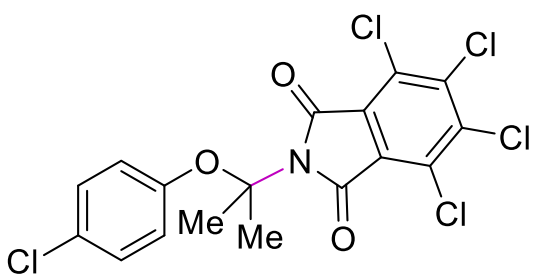

3j

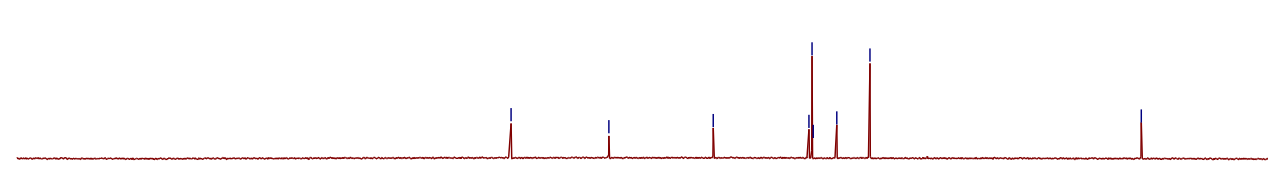


${ }^{1} \mathrm{H}-\mathrm{NMR}$ of compound $\mathbf{3 k}$

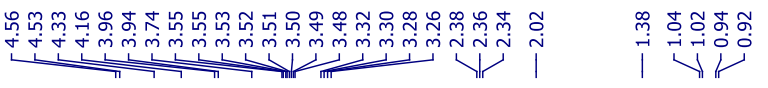

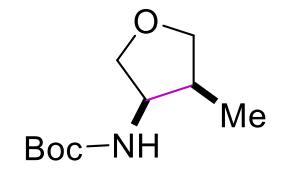

3k (4:1 dr)
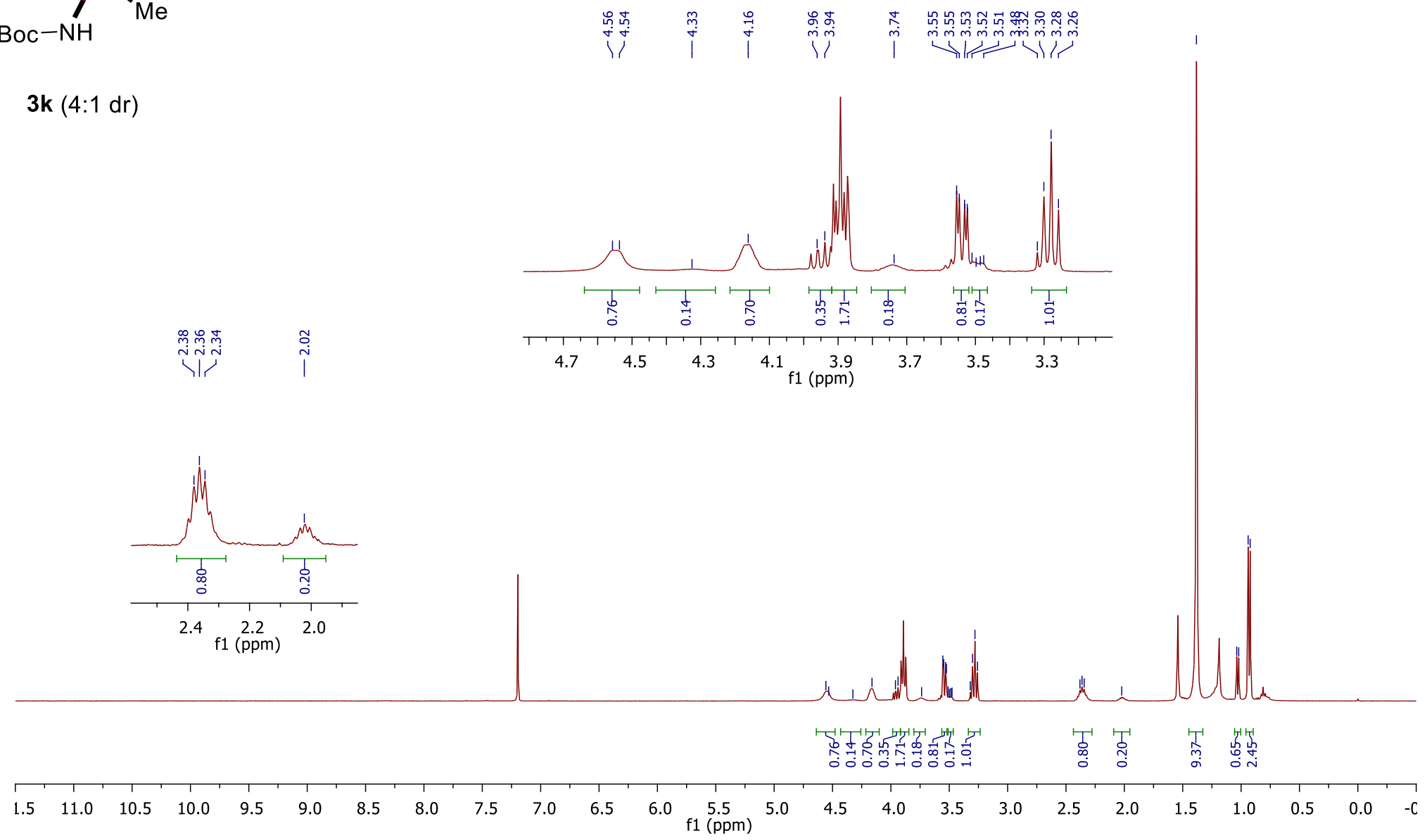
${ }^{13} \mathrm{C}-\mathrm{NMR}$ of compound $\mathbf{3 k}$

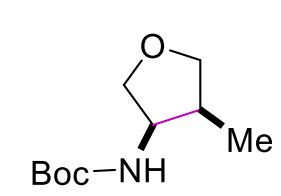

3k $(4: 1 \mathrm{dr})$ 
NOESY of compound $\mathbf{3 k}$

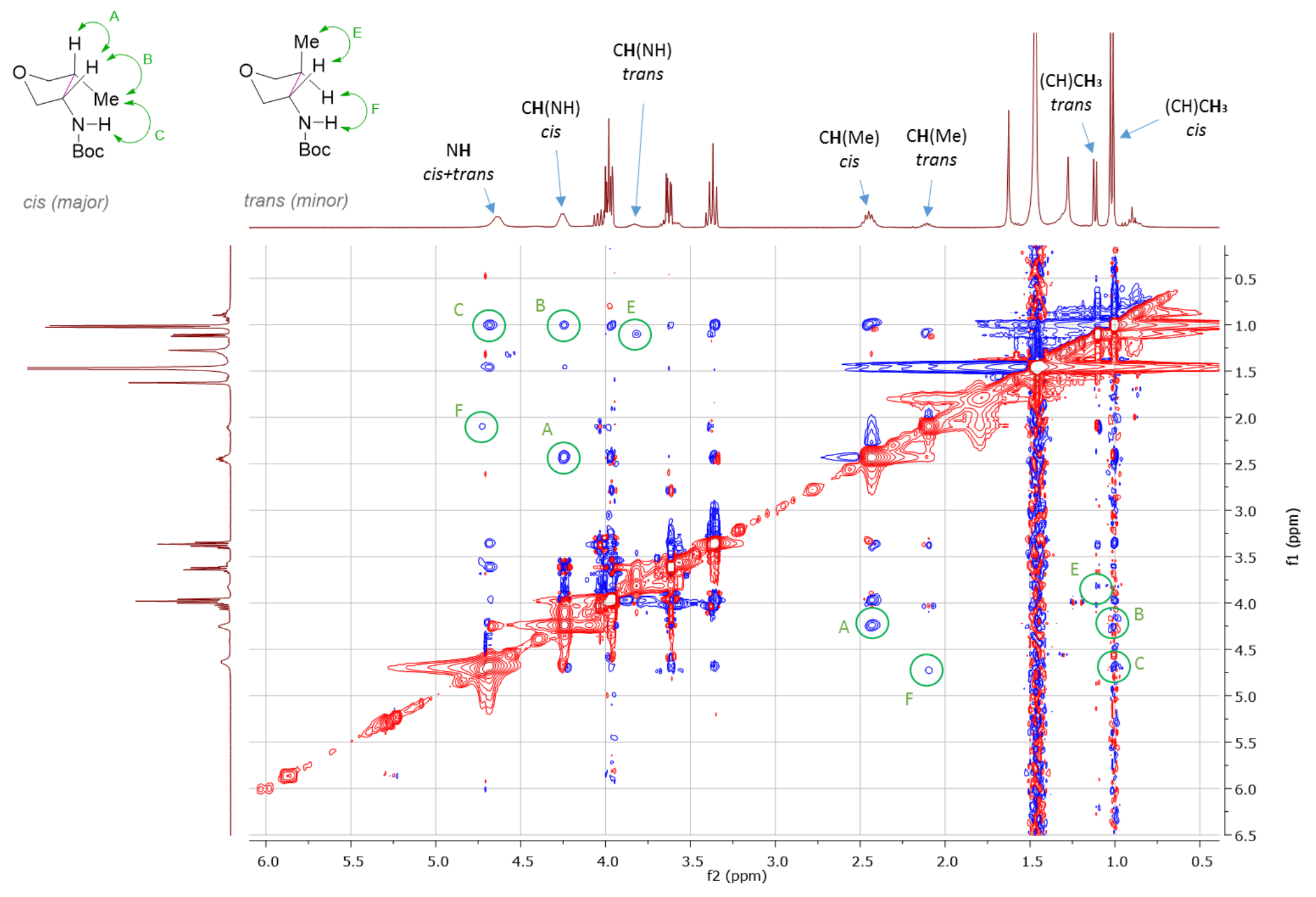


${ }^{1} \mathrm{H}-\mathrm{NMR}$ of compound 3l (contains impurities of an inseparable compound)

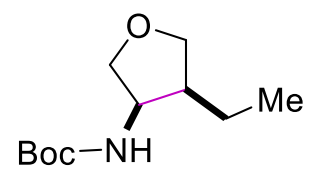

3I (2:1 dr)

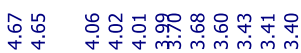

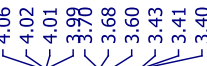

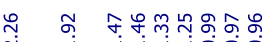

i i iritiogo

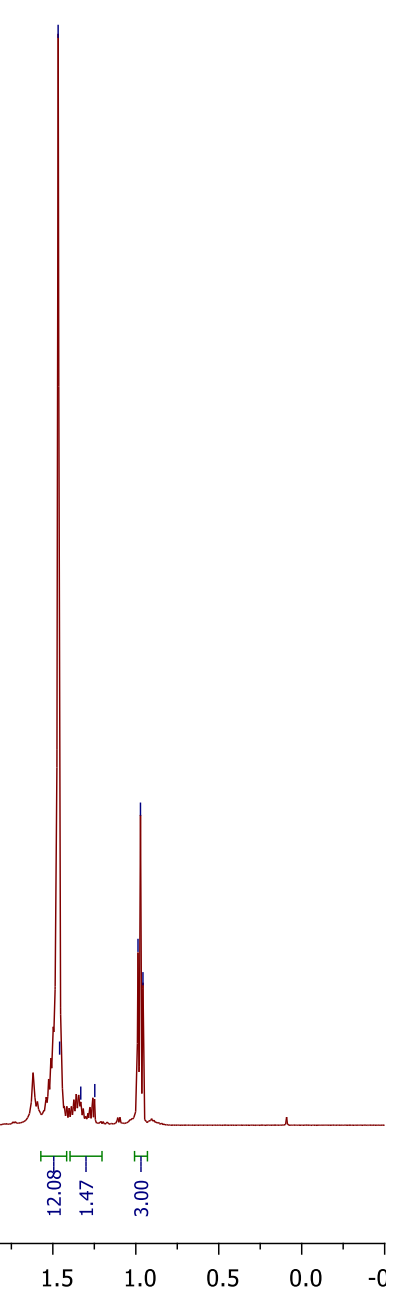


${ }^{13} \mathrm{C}-\mathrm{NMR}$ of compound $3 \mathrm{I}$

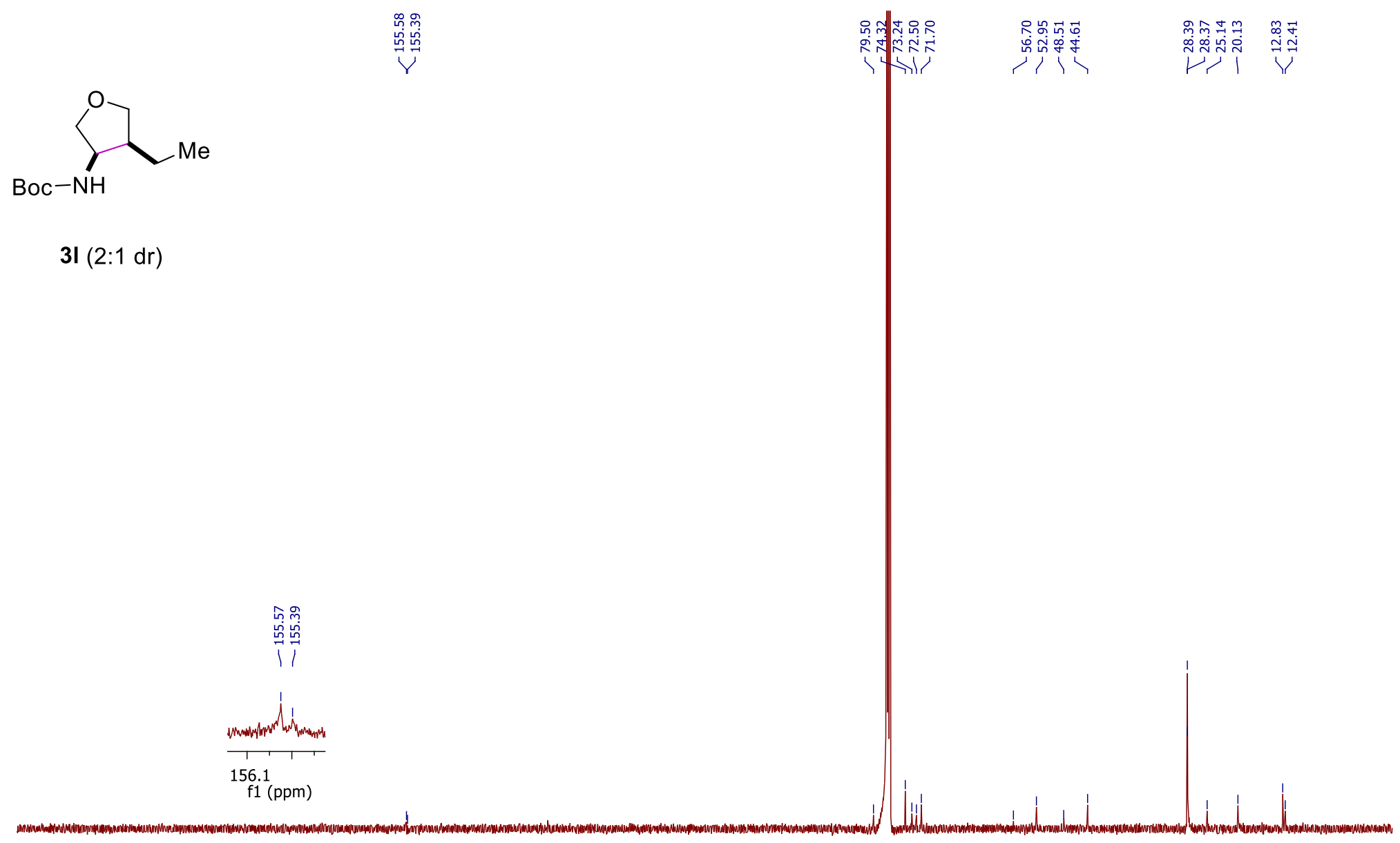


${ }^{1}$ H-NMR of compound $\mathbf{3 n}$
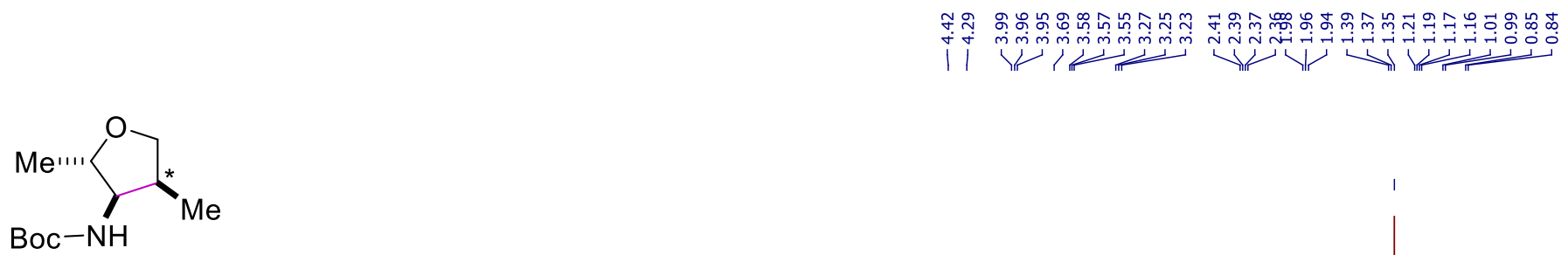

3n (9:1 dr)

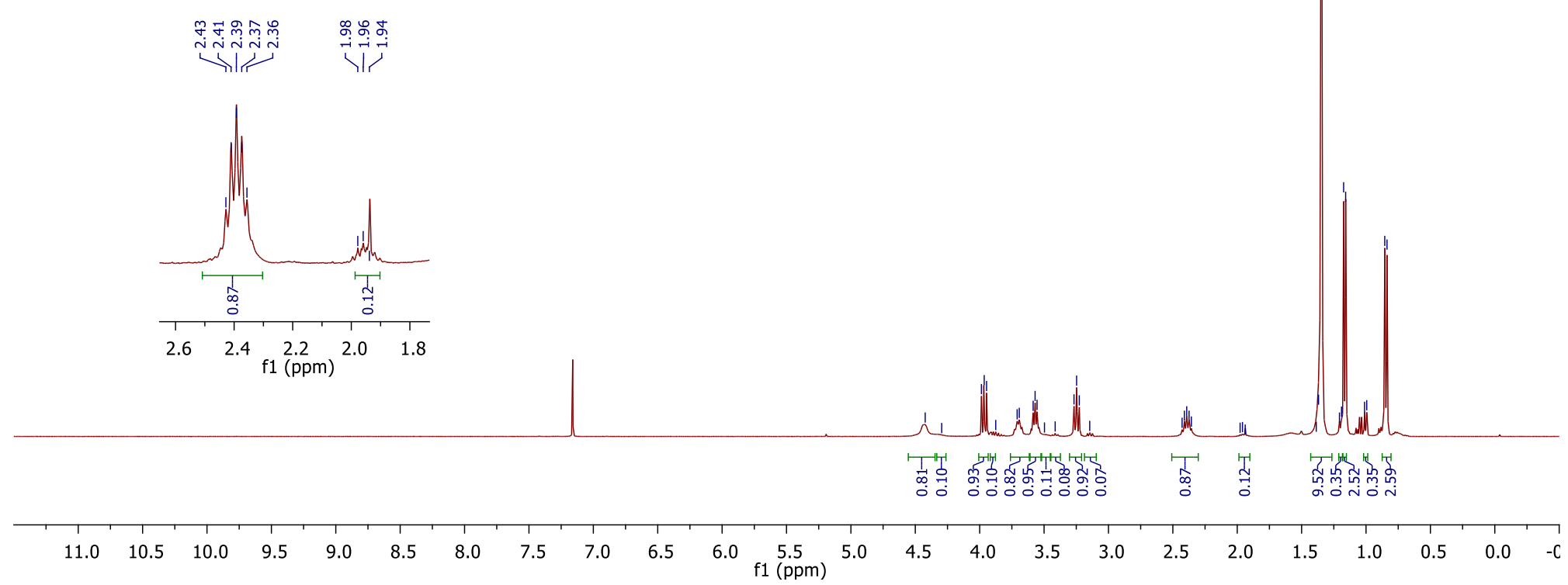


${ }^{13} \mathrm{C}-\mathrm{NMR}$ of compound $\mathbf{3 n}$

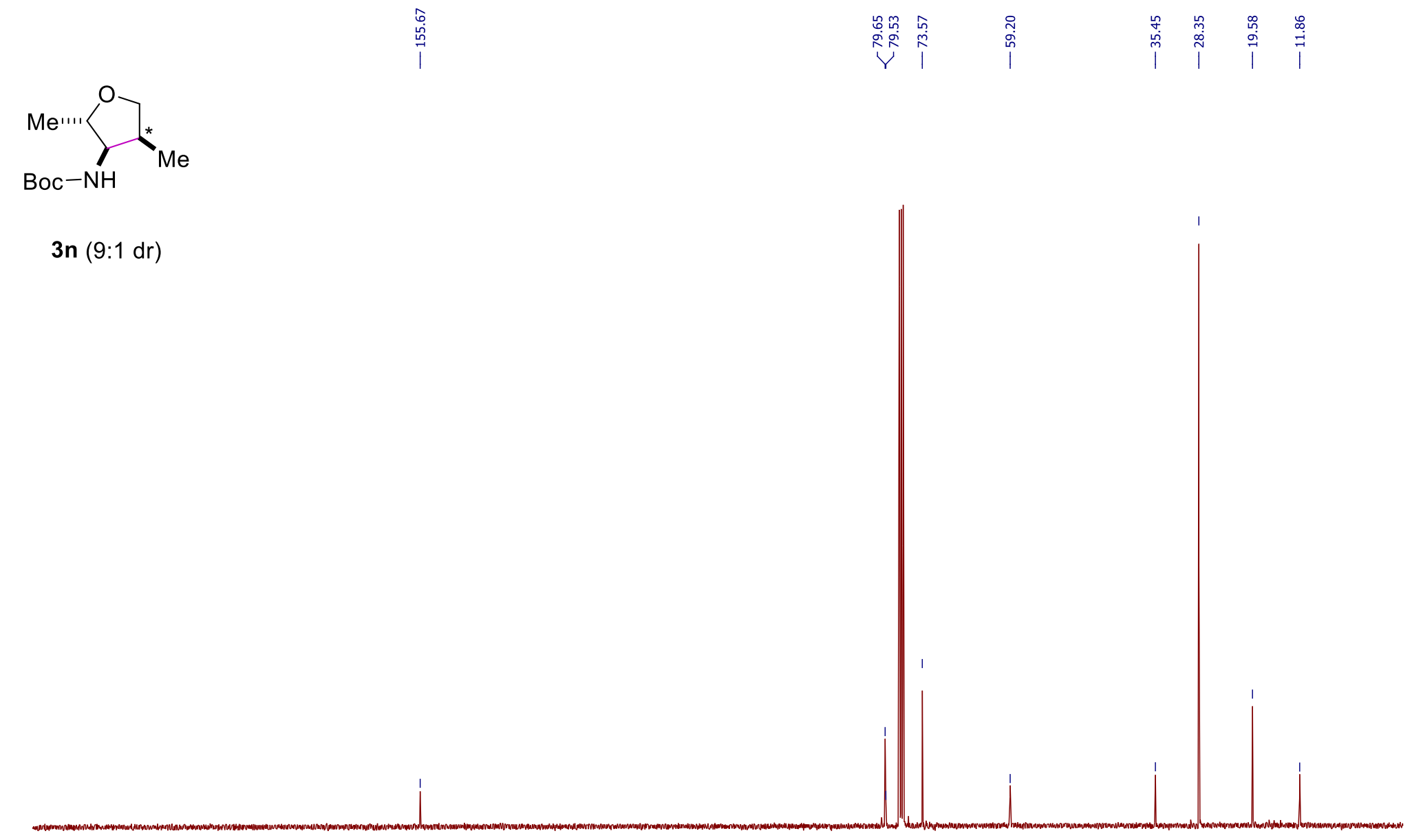

$\begin{array}{lllllllllllllllllllllll}210 & 200 & 190 & 180 & 170 & 160 & 150 & 140 & 130 & 120 & \underset{\mathrm{f} 1}{110}(\mathrm{ppm}) & 100 & 90 & 80 & 70 & 60 & 50 & 40 & 30 & 20 & 10 & 0\end{array}$ 
NOESY of compound $\mathbf{3 n}$

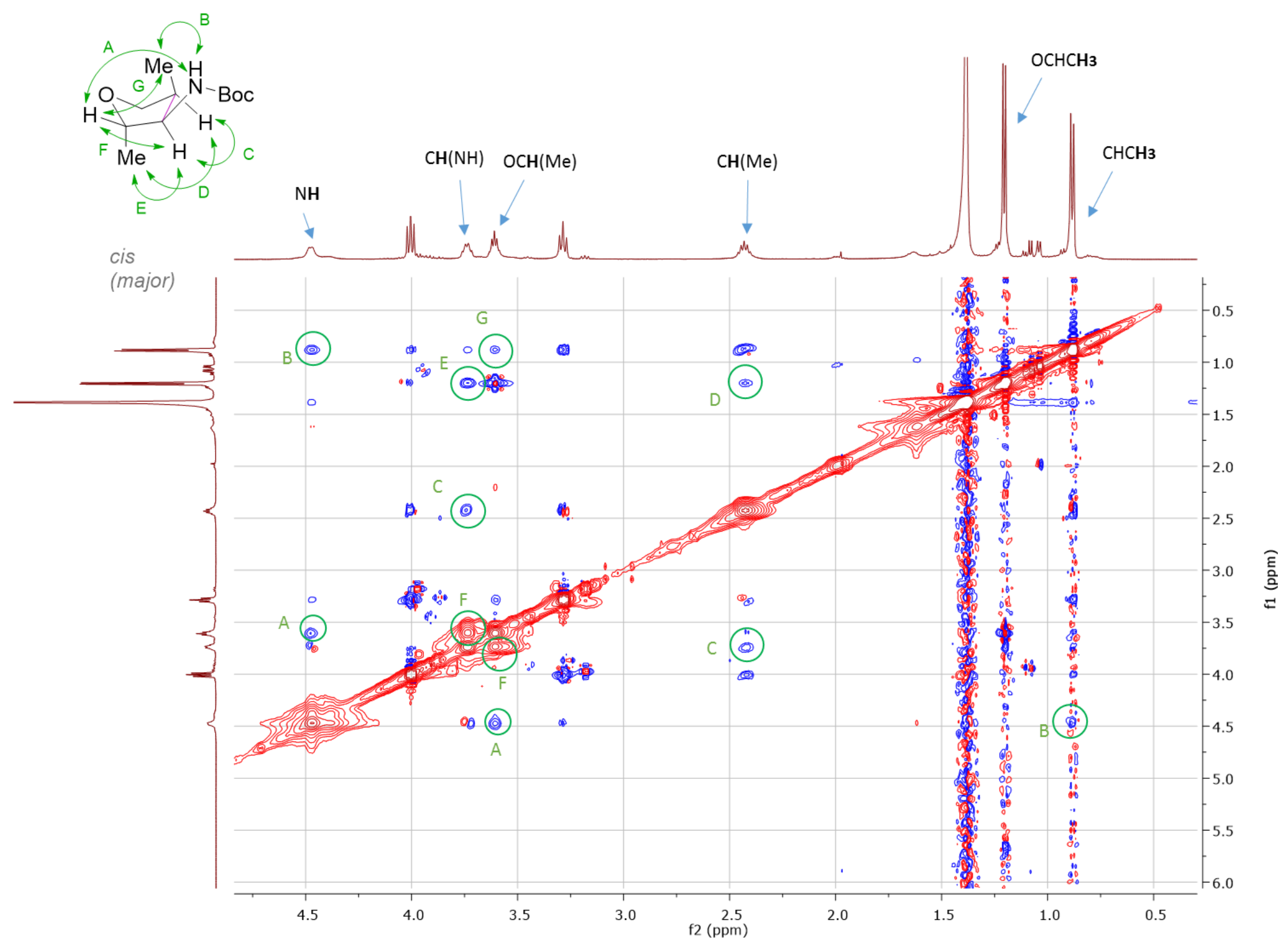


${ }^{1} \mathrm{H}-\mathrm{NMR}$ of compound $3 \mathrm{o}$
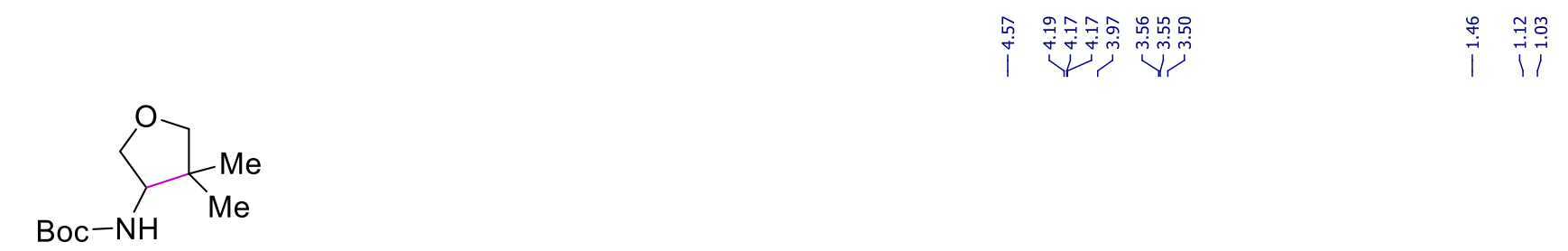

30

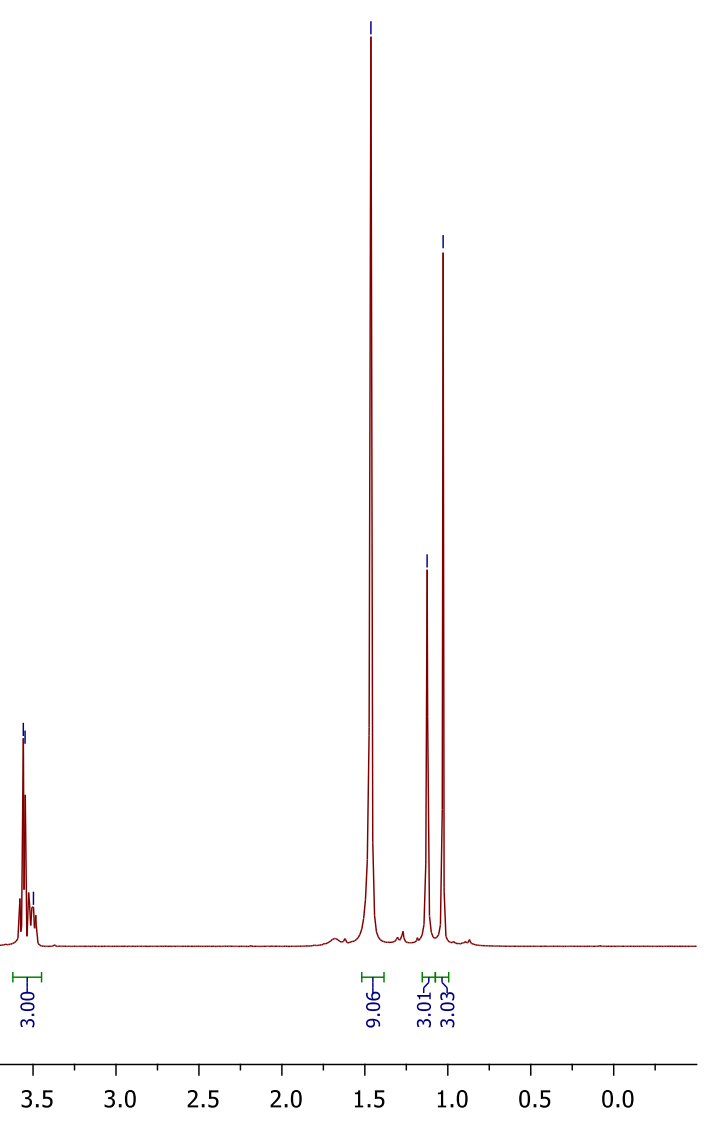


${ }^{13} \mathrm{C}-\mathrm{NMR}$ of compound $\mathbf{3 o}$

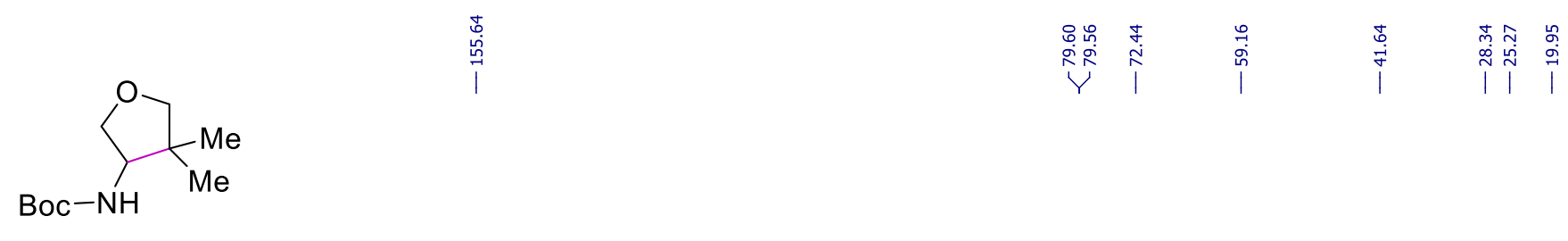

30

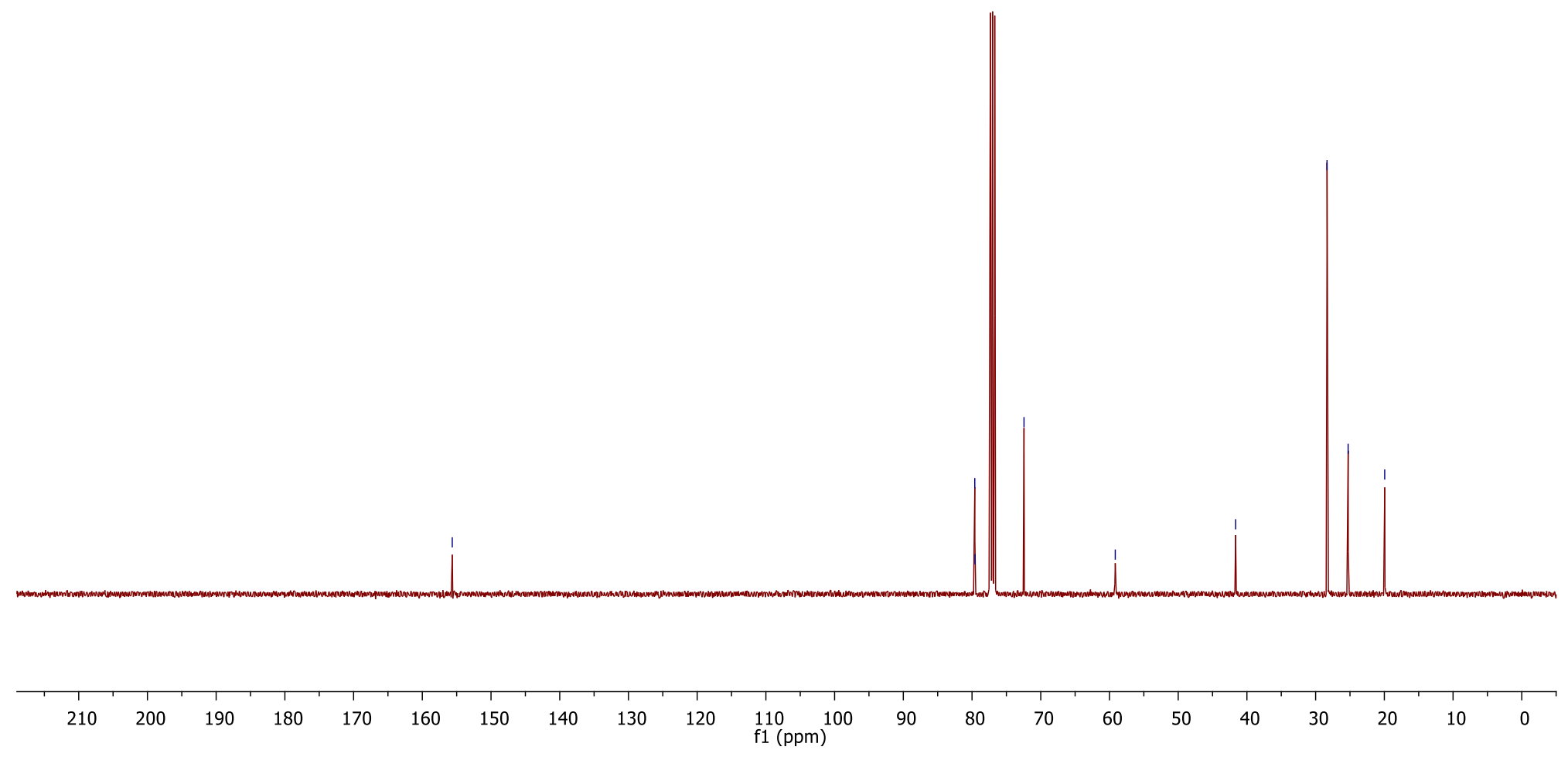


${ }^{1} \mathrm{H}$-NMR of compound $\mathbf{3 p}$
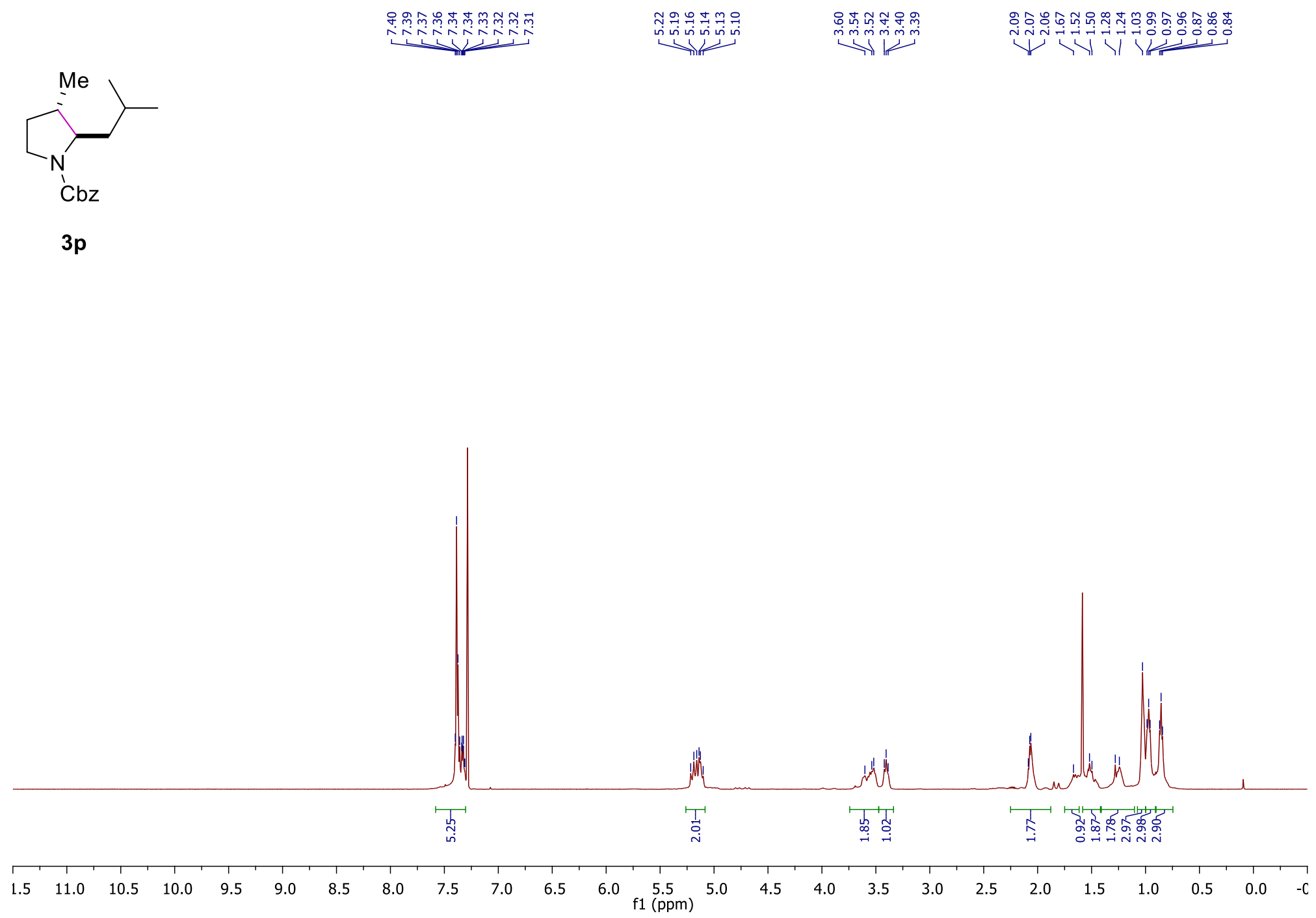
${ }^{13} \mathrm{C}-\mathrm{NMR}$ of compound $\mathbf{3 p}$

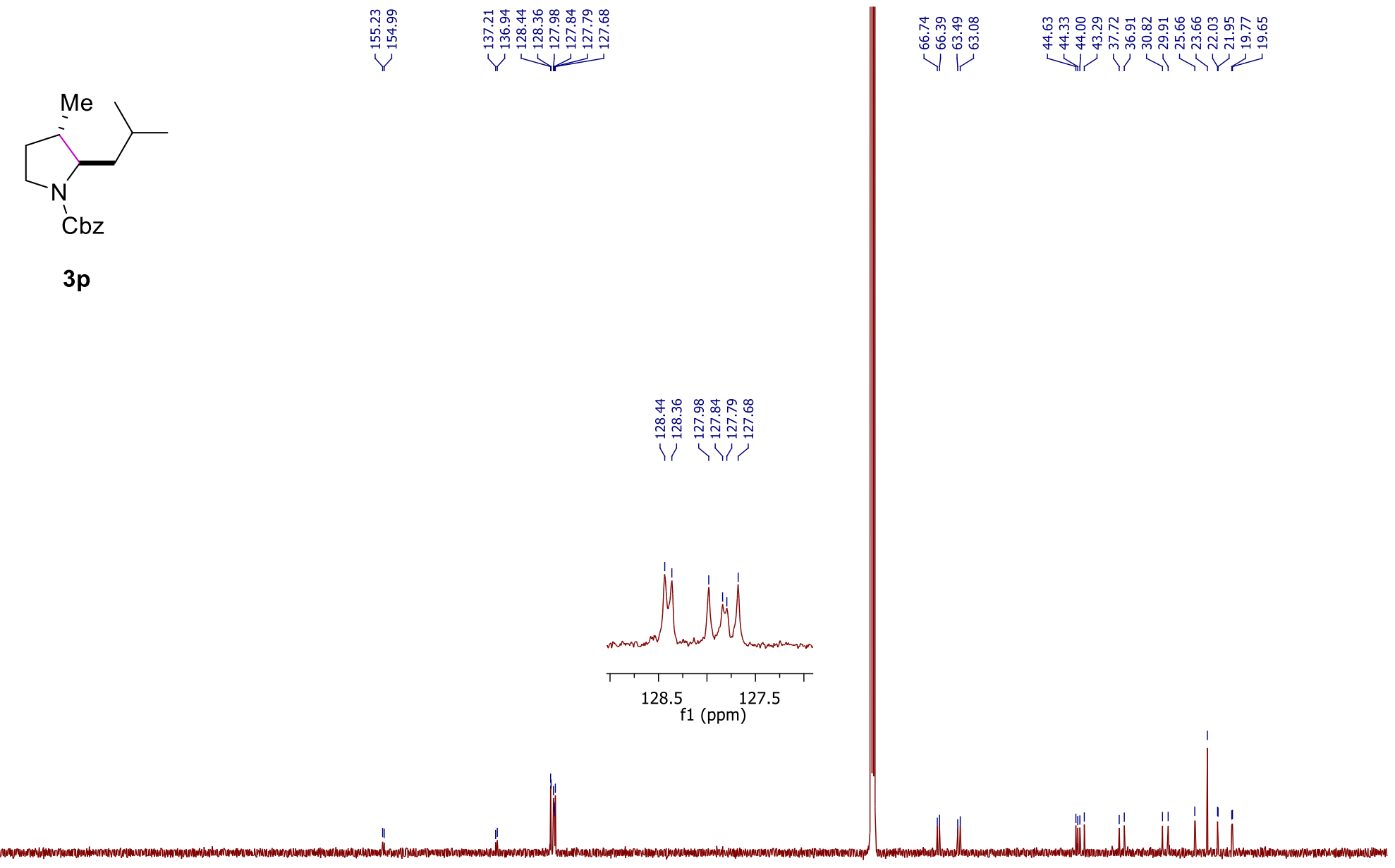

$\begin{array}{llllllll}210 & 200 & 190 & 180 & 170 & 160 & 150 & 140\end{array}$

120

110100

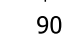

$\begin{array}{llll}80 & 70 & 60 & 50\end{array}$

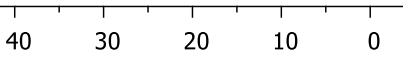


NOESY of compound $\mathbf{3 p}$

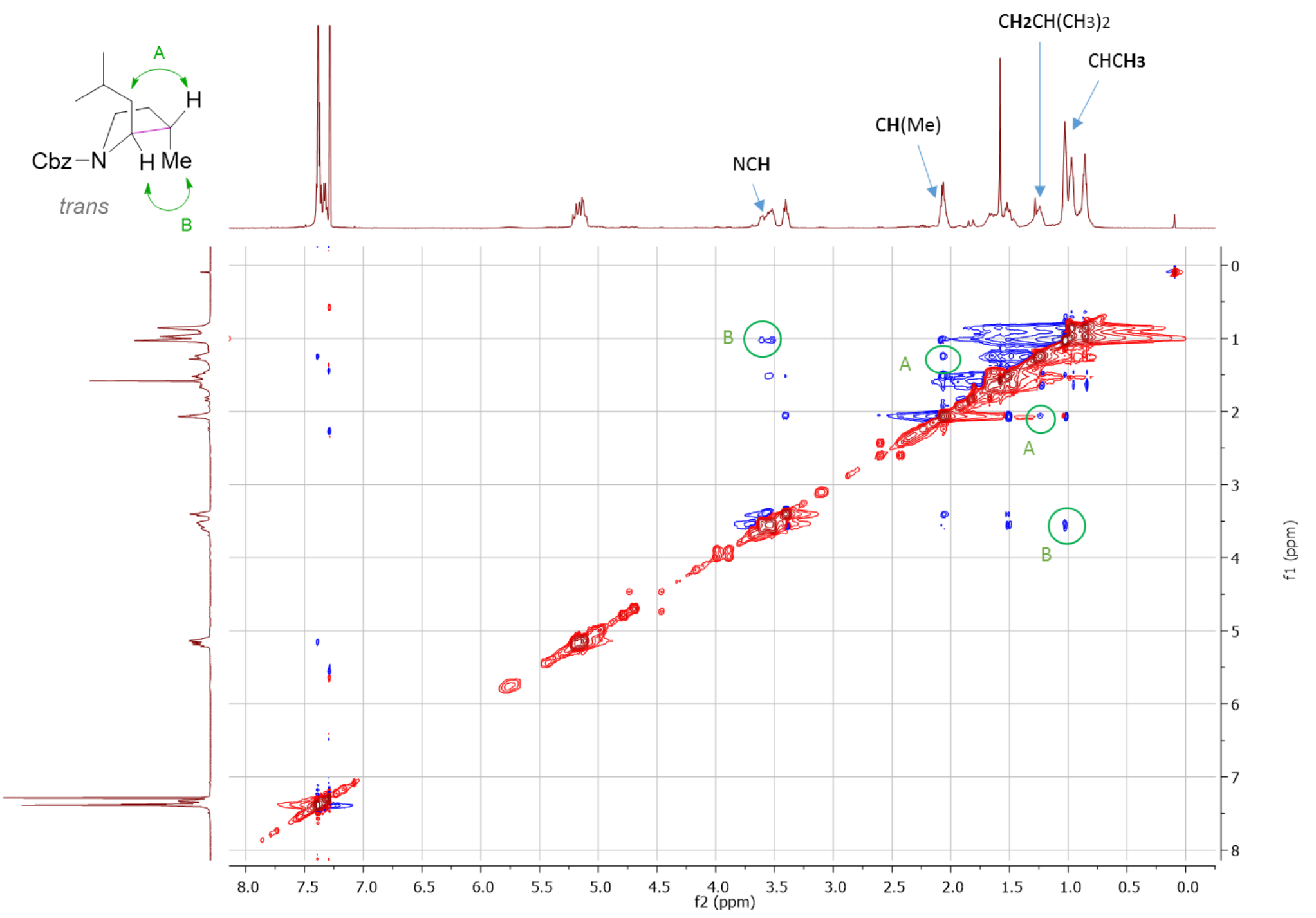


${ }^{1} \mathrm{H}-\mathrm{NMR}$ of compound $\mathbf{3 q}$
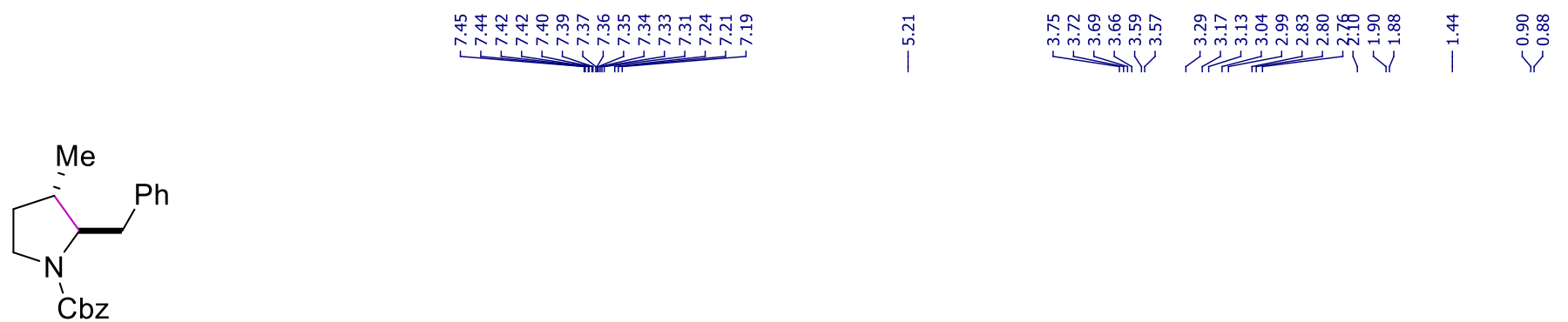

$3 q$

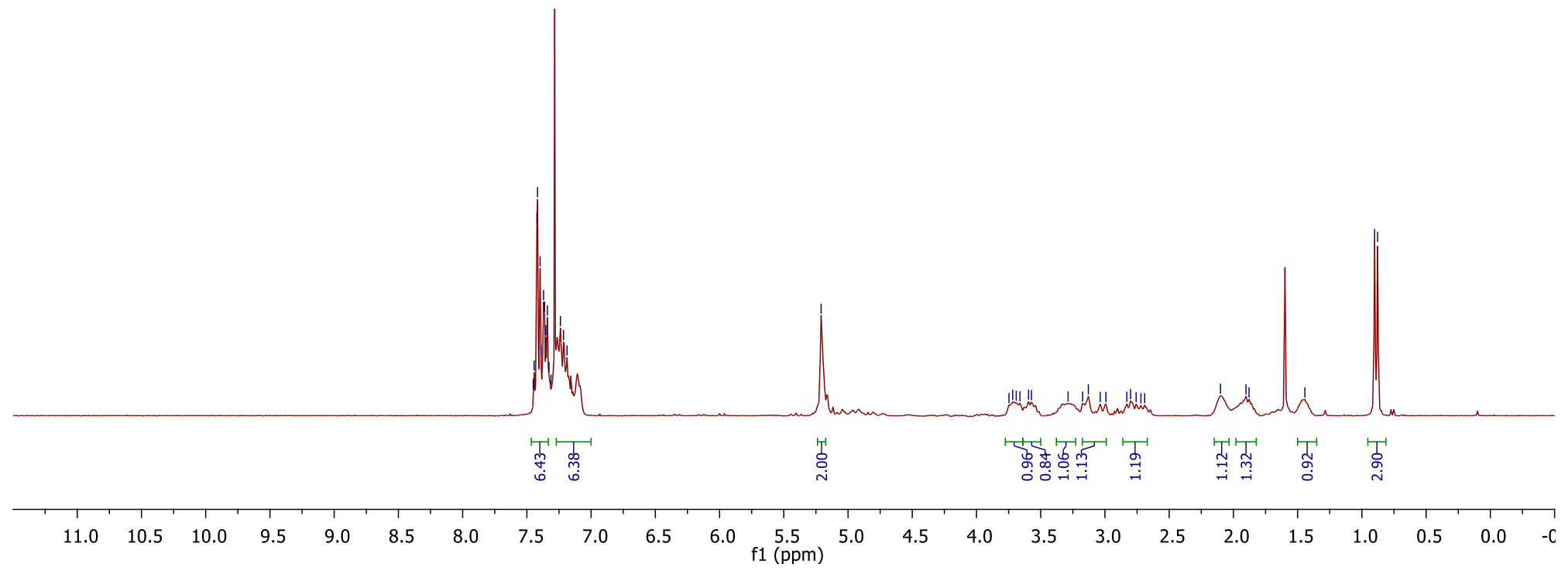


${ }^{13} \mathrm{C}-\mathrm{NMR}$ of compound $\mathbf{3 q}$

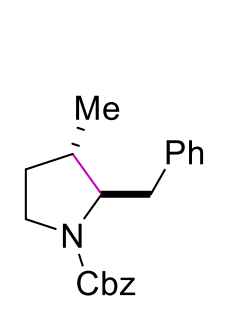

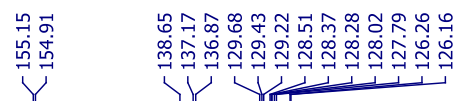

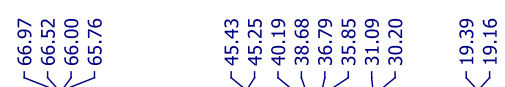

Vरी।

$3 q$

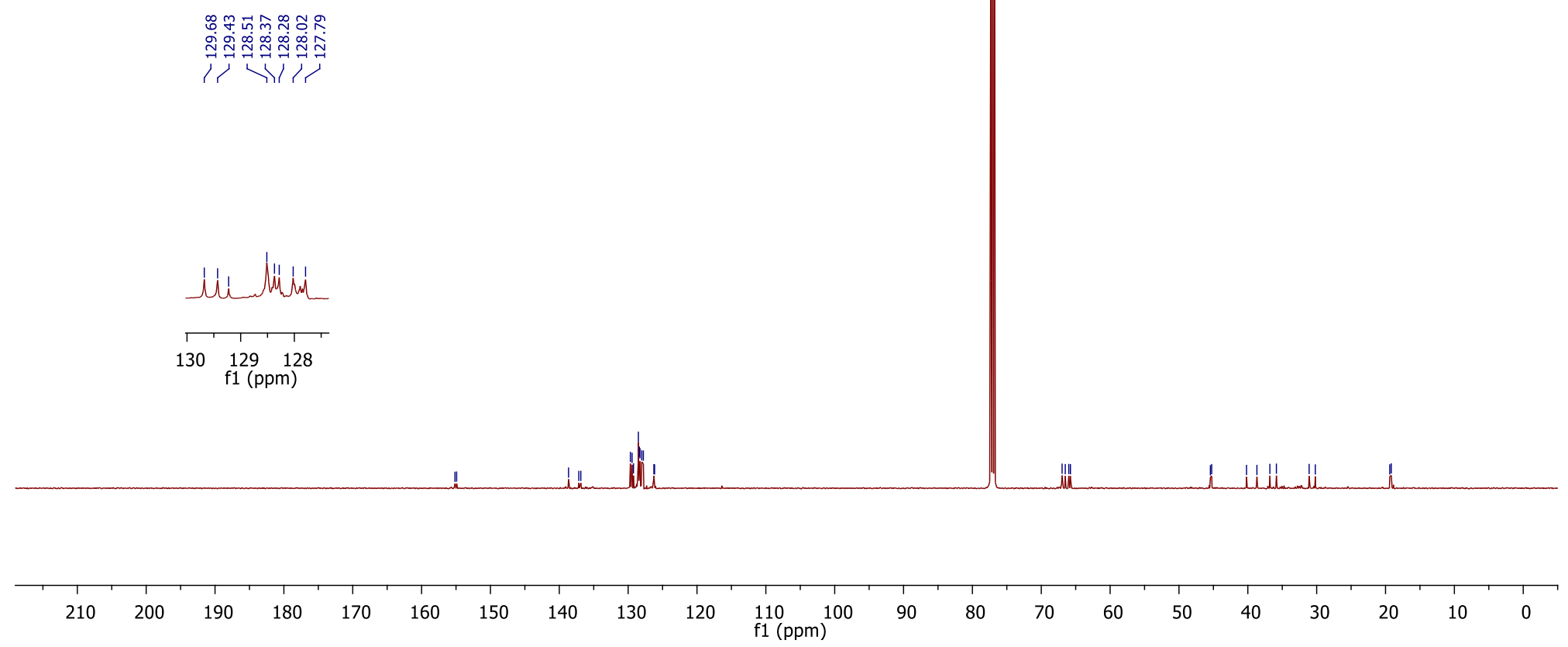


${ }^{1} \mathrm{H}-\mathrm{NMR}$ of compound $\mathbf{3 r}$
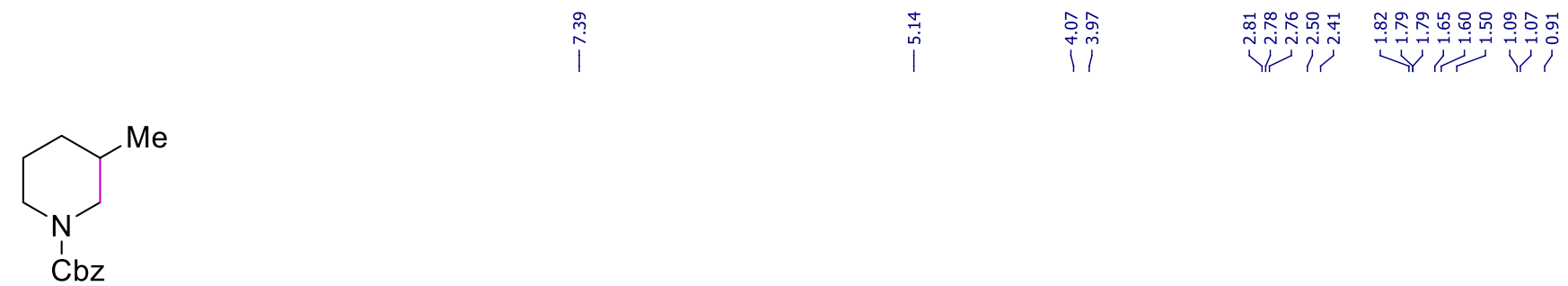

$3 \mathbf{r}$

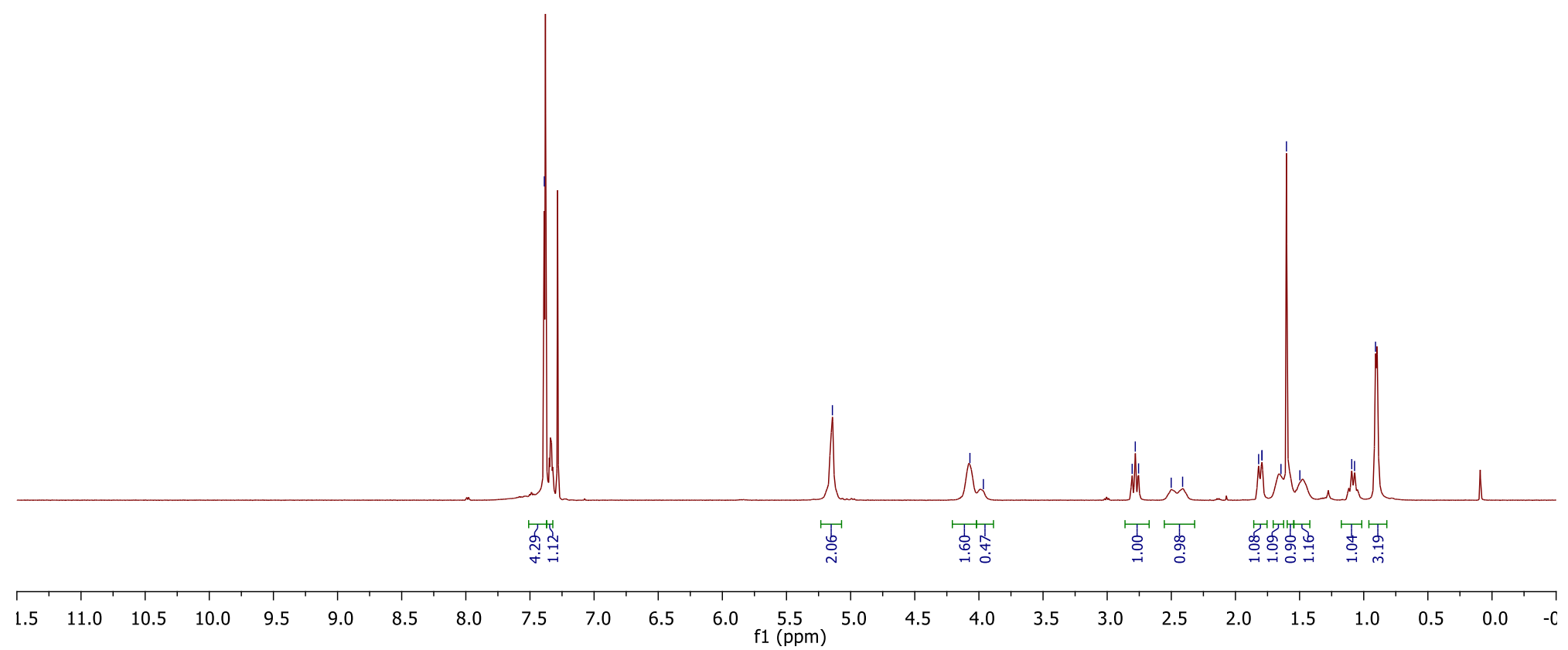


${ }^{13} \mathrm{C}$-NMR of compound $\mathbf{3 r}$

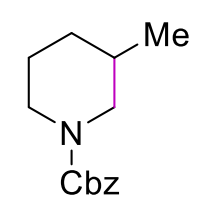

$3 r$

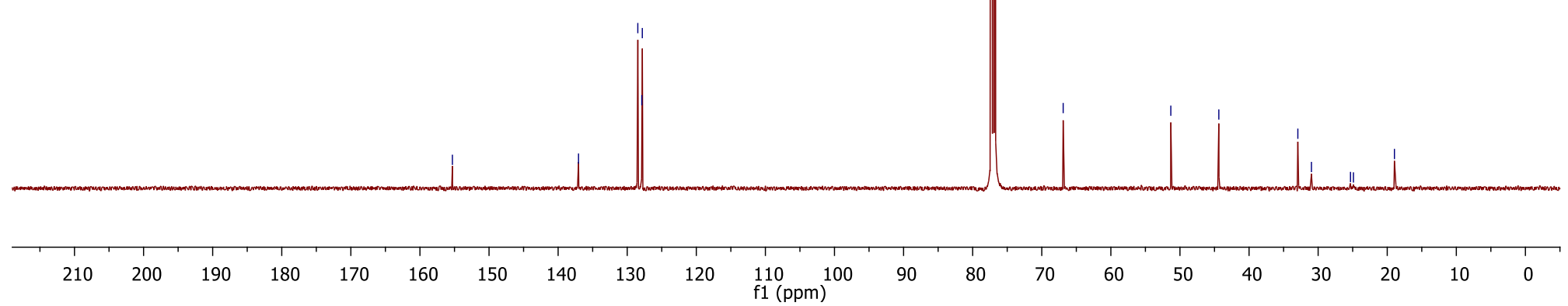


${ }^{1} \mathrm{H}-\mathrm{NMR}$ of compound $3 \mathrm{~s}$
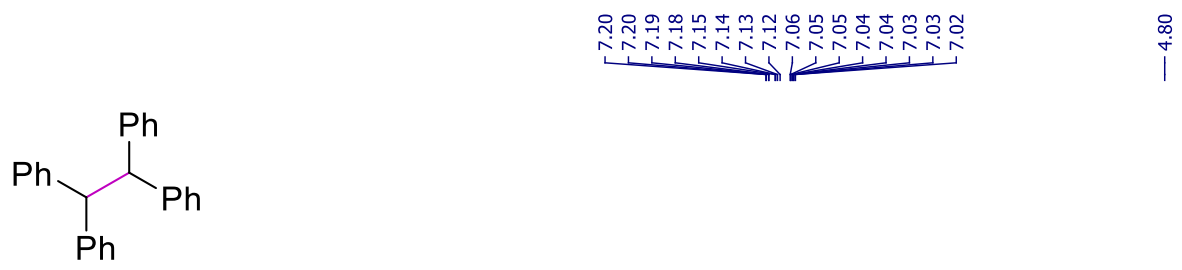

3s

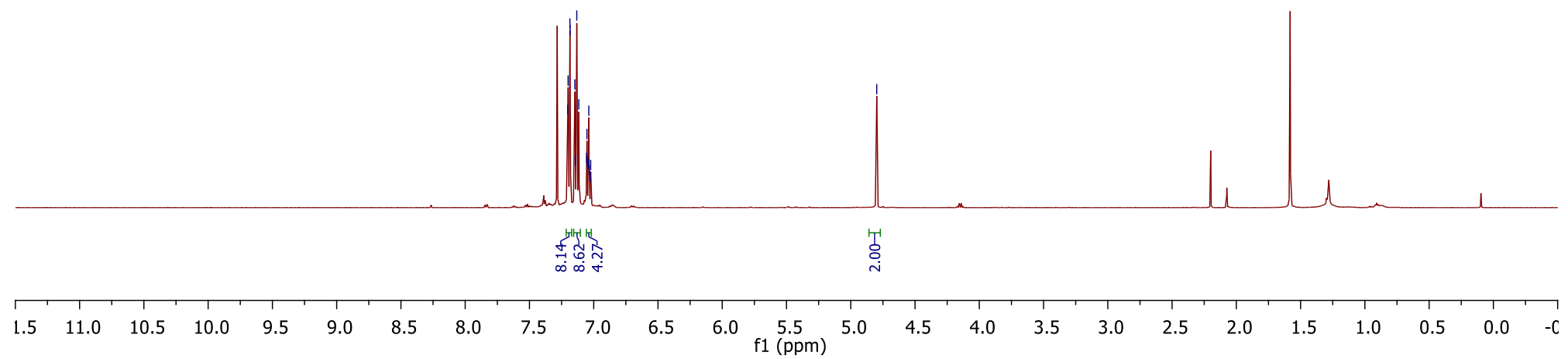


${ }^{13} \mathrm{C}-\mathrm{NMR}$ of compound 3s

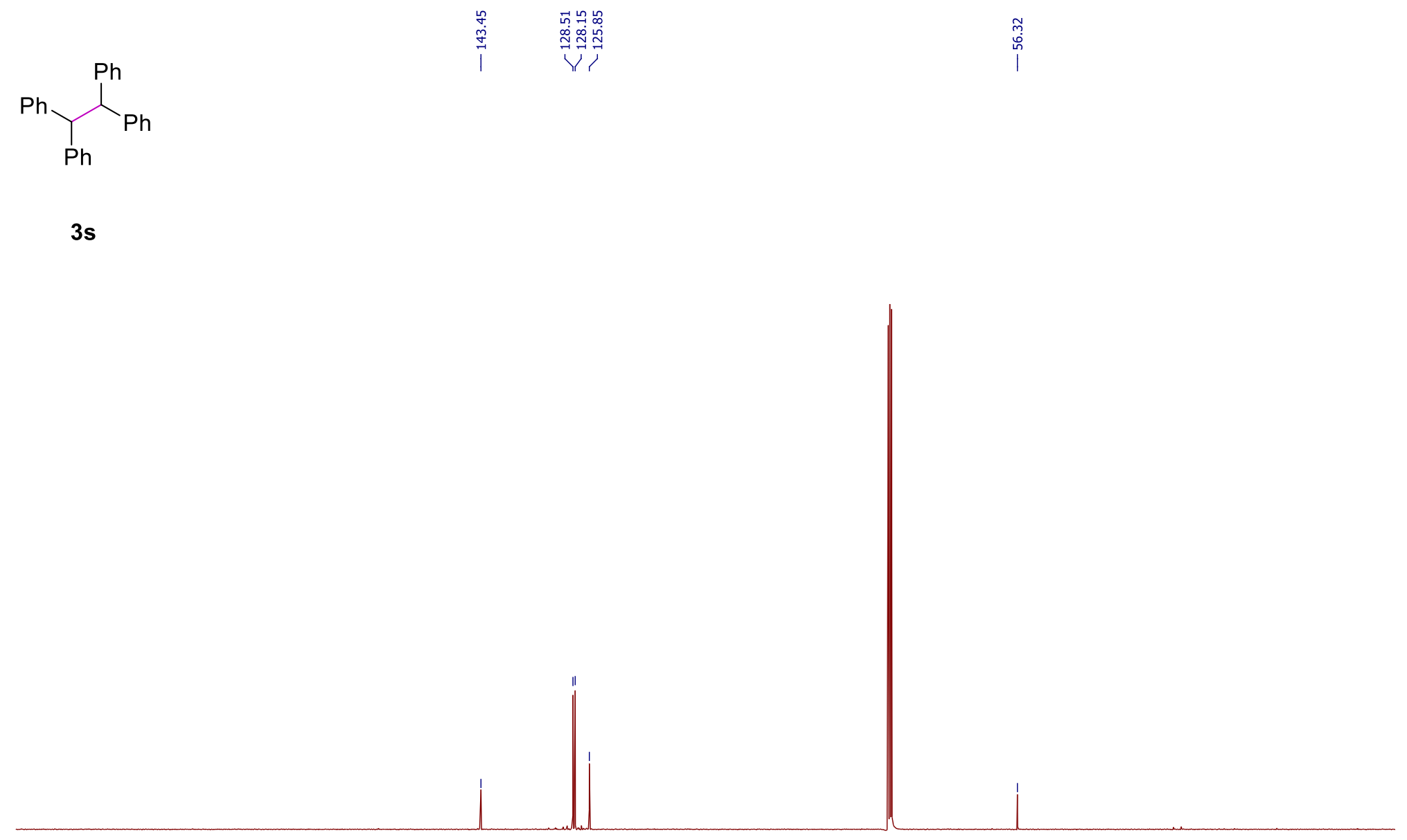

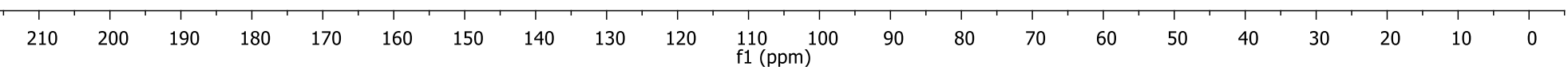


${ }^{1} \mathrm{H}$-NMR of compound $\mathbf{3 t}$

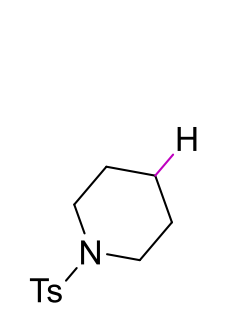

خ

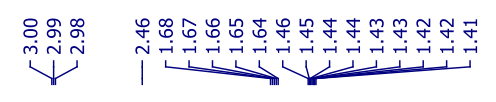

$3 t$

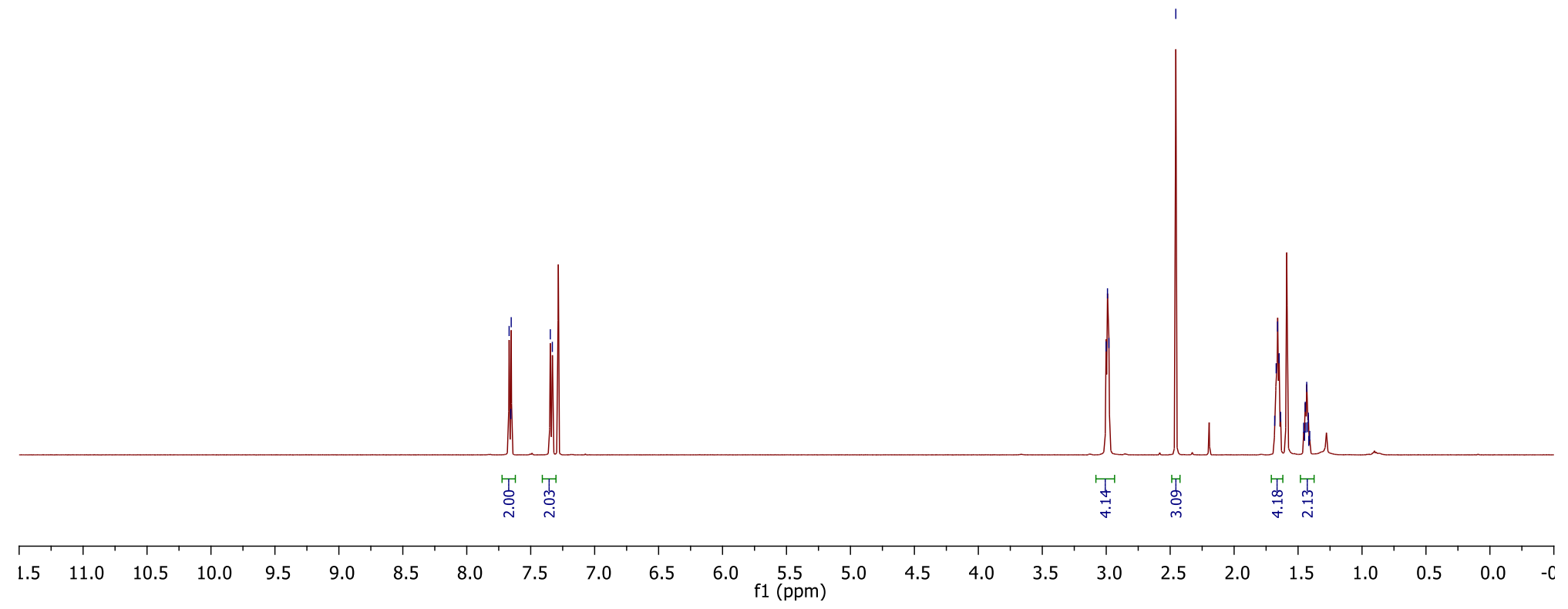


${ }^{13} \mathrm{C}-\mathrm{NMR}$ of compound $3 \mathrm{t}$

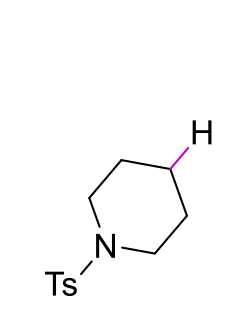

0
0
$m$

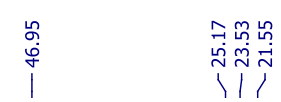

$3 t$

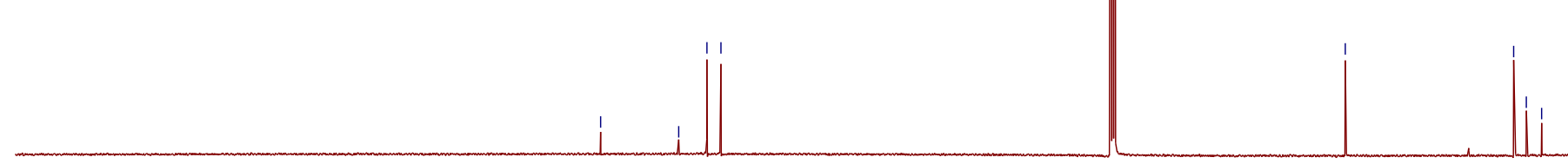

$210 \quad 200$

$190 \quad 180$

$\begin{array}{lllllll}170 & 170 & 160 & 150 & 140 & 130 & 120\end{array}$

$20 \quad \frac{110}{\mathrm{f} 1(\mathrm{ppm})} 100$

$90 \quad 80$

$\begin{array}{lll}70 & 60 & 50\end{array}$

40

20

$\begin{array}{ll}10 & 1 \\ 10 & 0\end{array}$ 
${ }^{1} \mathrm{H}-\mathrm{NMR}$ of compound $\mathbf{3 u}$

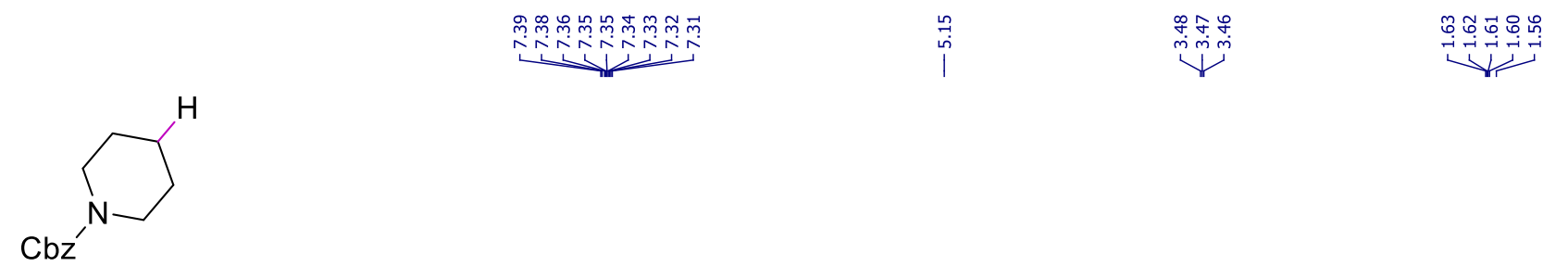

$3 u$

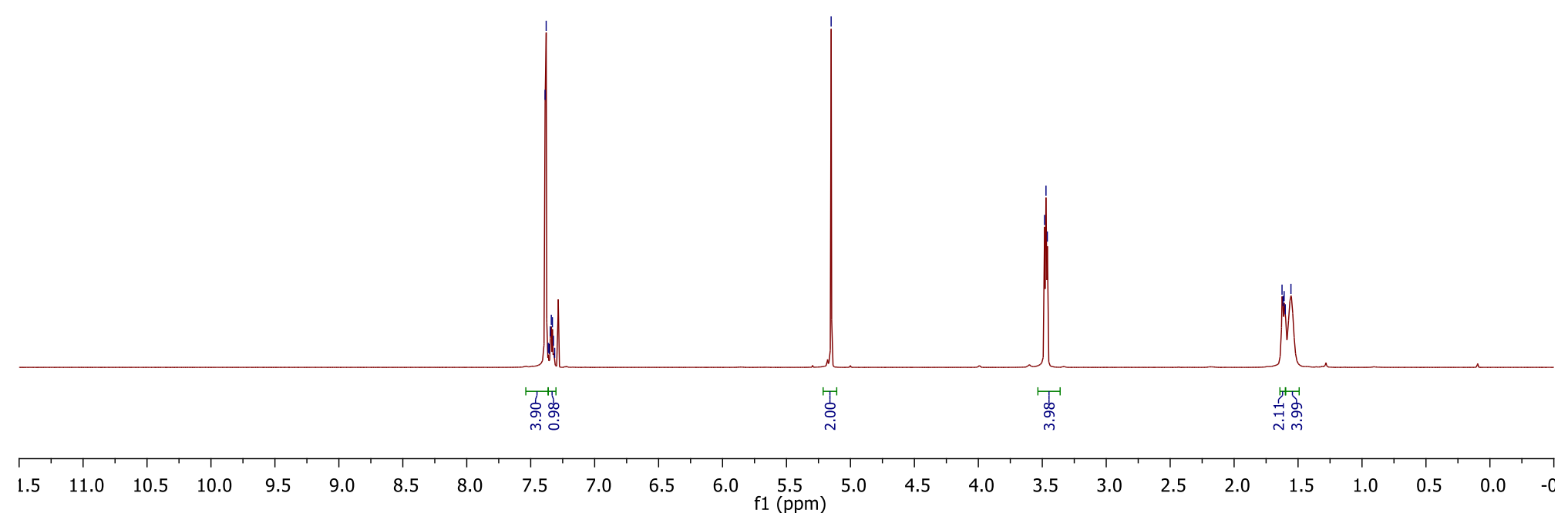


${ }^{13} \mathrm{C}-\mathrm{NMR}$ of compound $\mathbf{3 u}$

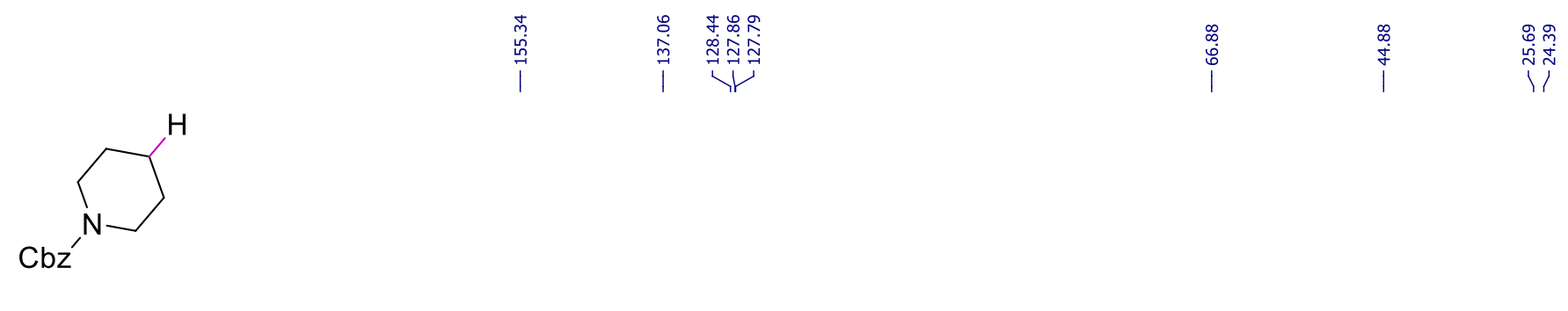

$3 u$

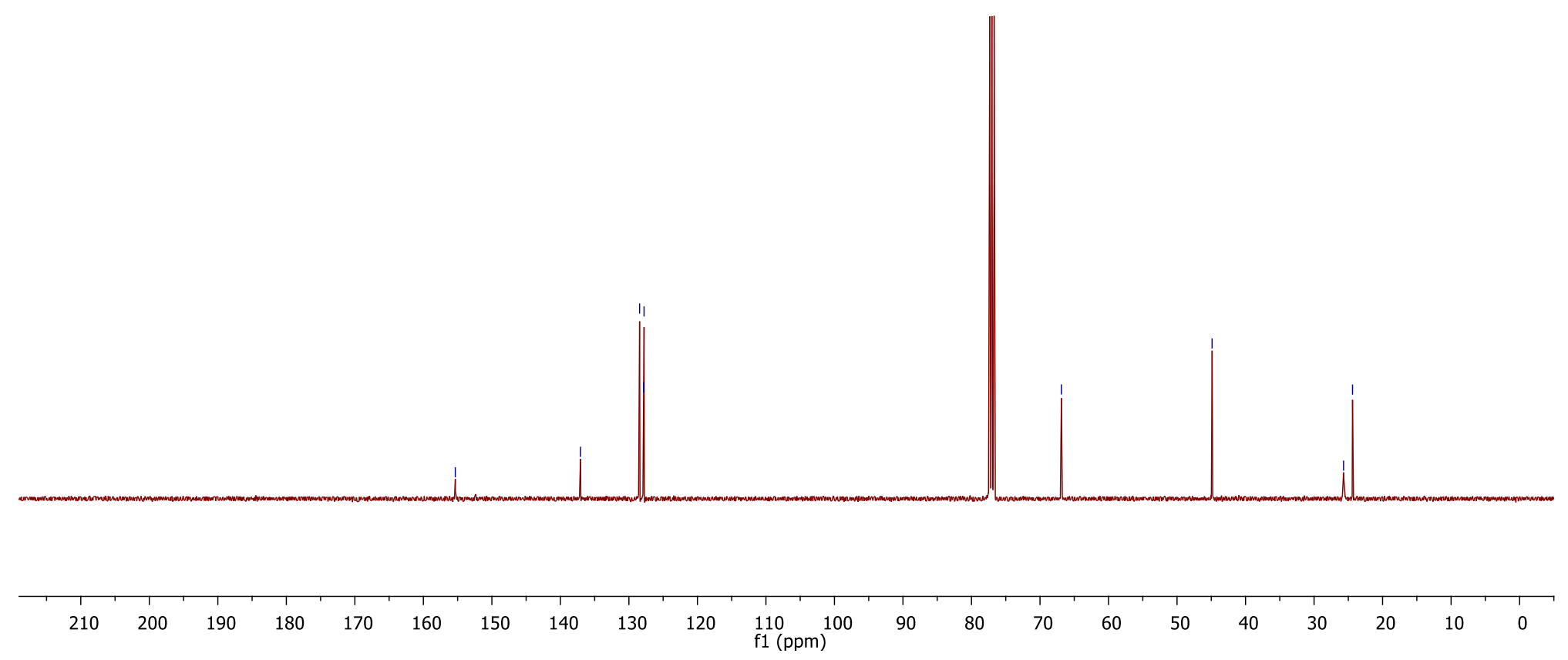


${ }^{1} \mathrm{H}-\mathrm{NMR}$ of compound $\mathbf{3 v}$

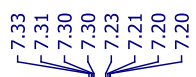

우수ํํํำ

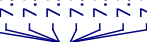

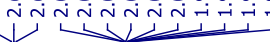

$\mathrm{Ph}_{\mathrm{Ph}} \longrightarrow \mathrm{H}$

3v

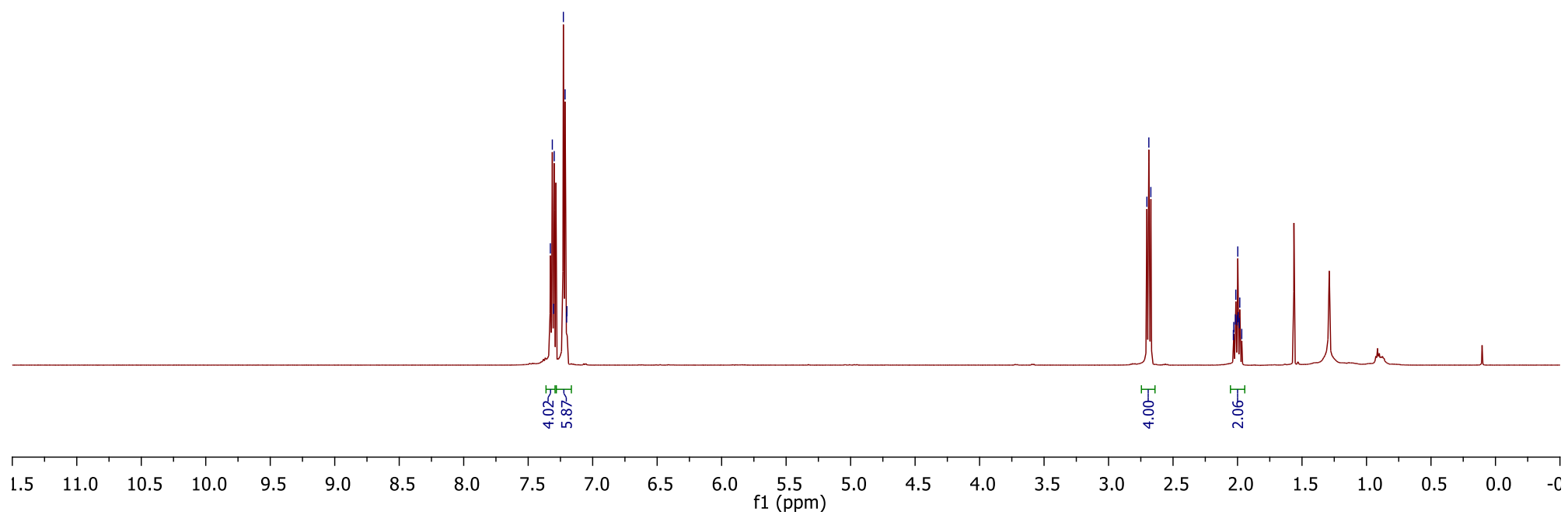


${ }^{13} \mathrm{C}-\mathrm{NMR}$ of compound $\mathbf{3 v}$

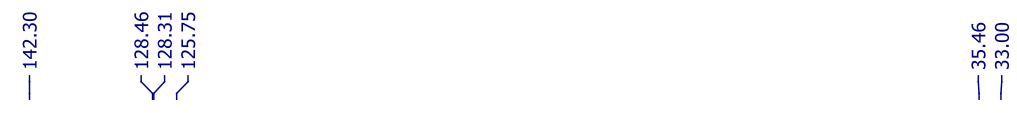

$$
\mathrm{Ph}_{\mathrm{Ph}} \longrightarrow \mathrm{H}
$$

$3 v$

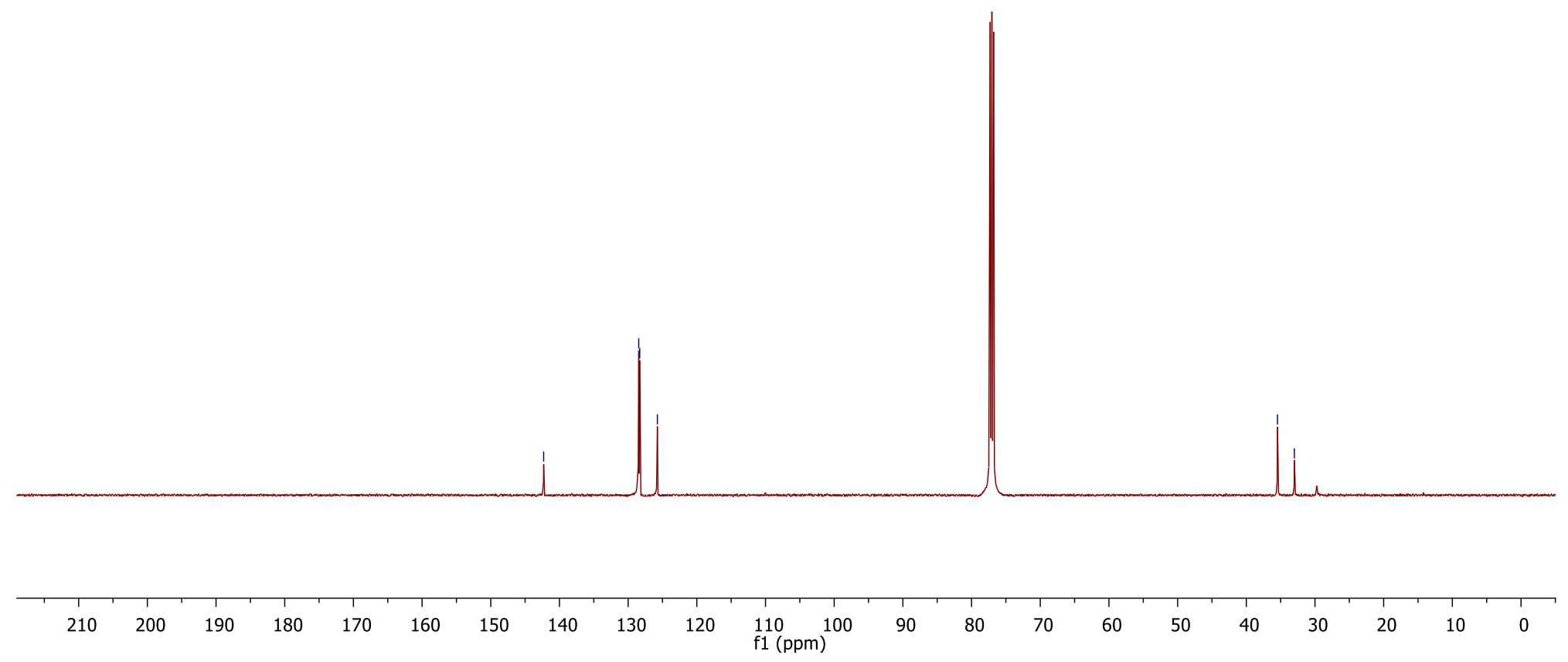


${ }^{1} \mathrm{H}$-NMR of compound $\mathbf{3 w}$

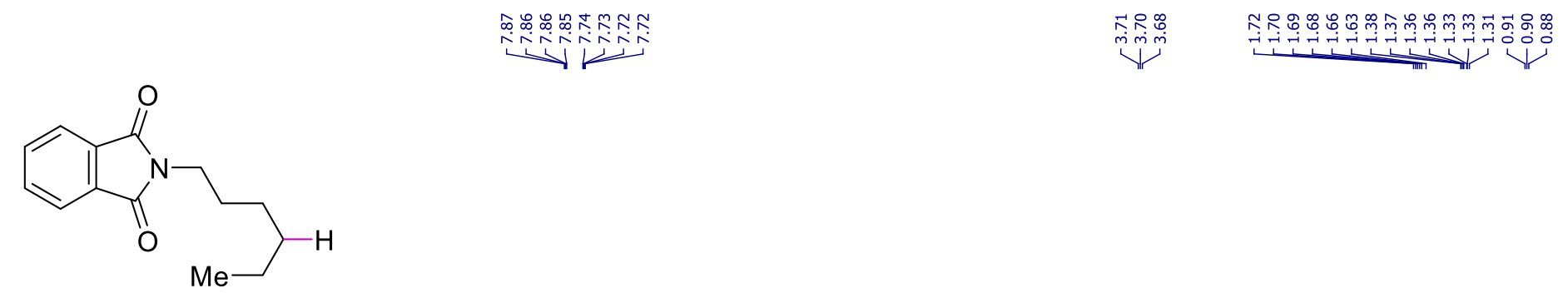

$3 w$

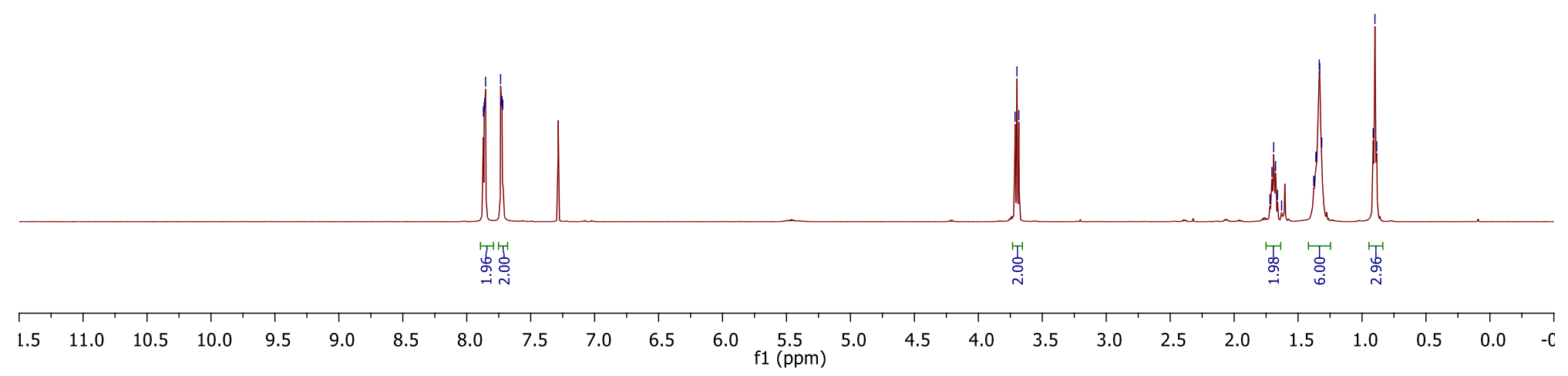


${ }^{13} \mathrm{C}-\mathrm{NMR}$ of compound $\mathbf{3 w}$

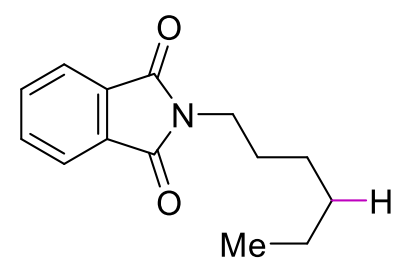

$3 w$

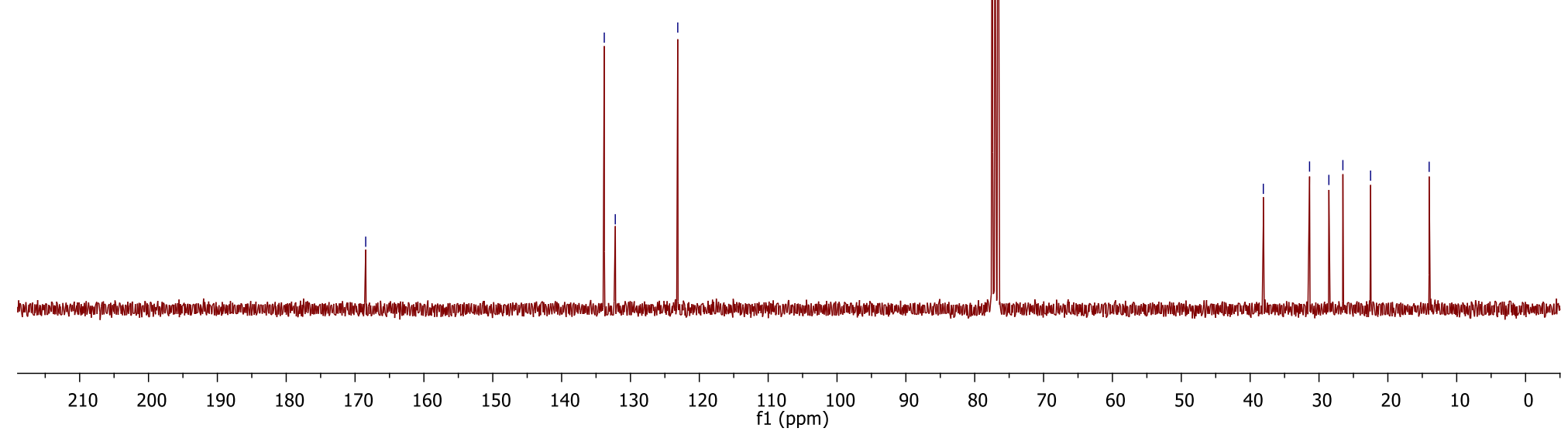


${ }^{1} \mathrm{H}-\mathrm{NMR}$ of compound $\mathbf{4 a}$

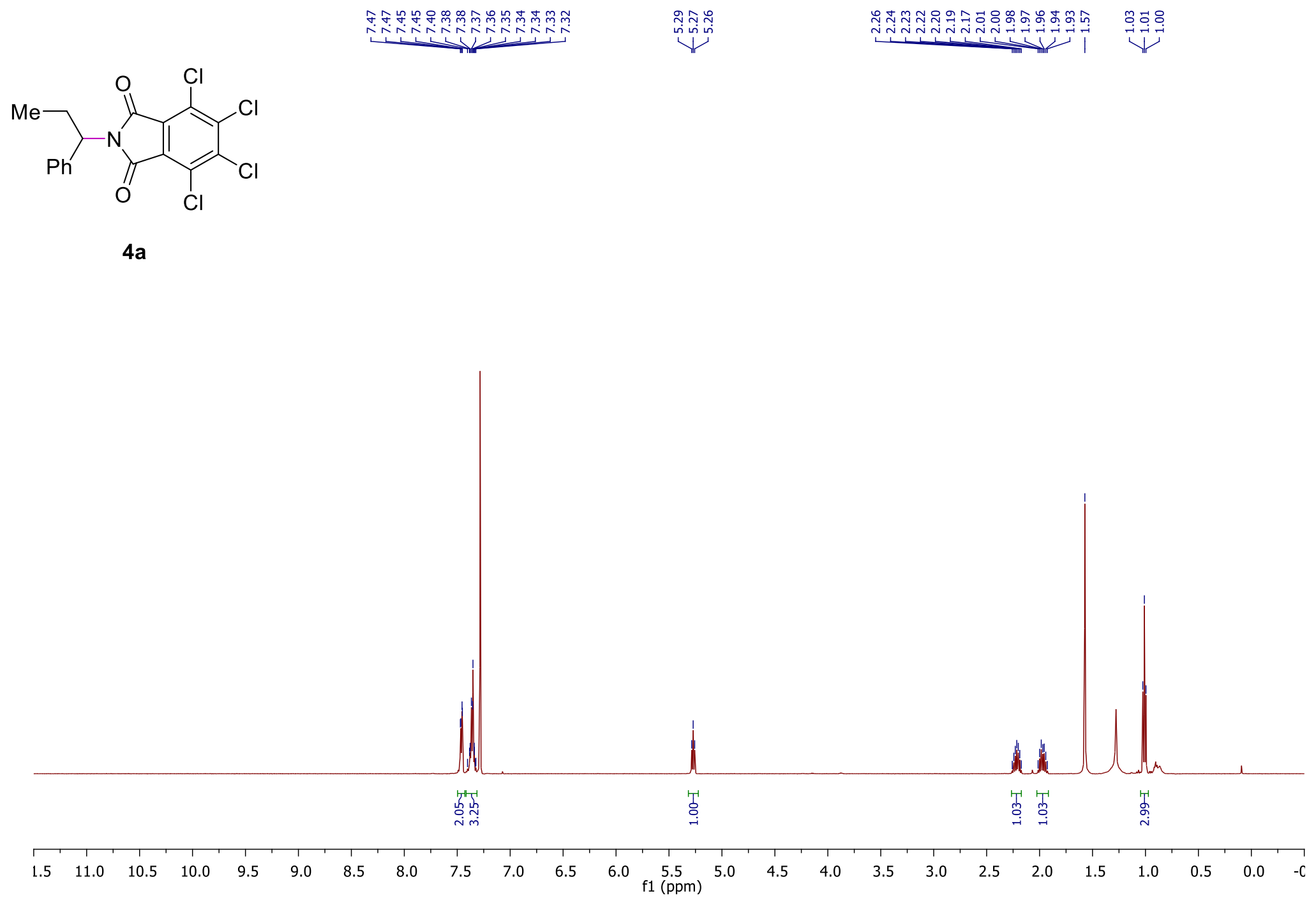


${ }^{13} \mathrm{C}-\mathrm{NMR}$ of compound $\mathbf{4 a}$

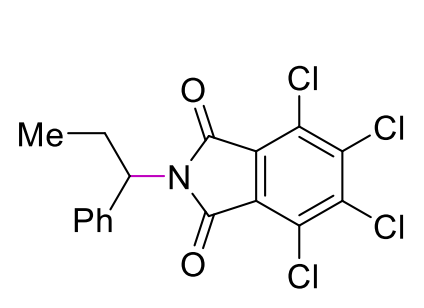

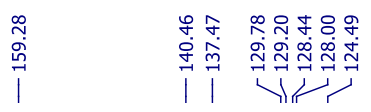

$\stackrel{\substack{\infty \\ i}}{\stackrel{?}{0}}$

$4 a$

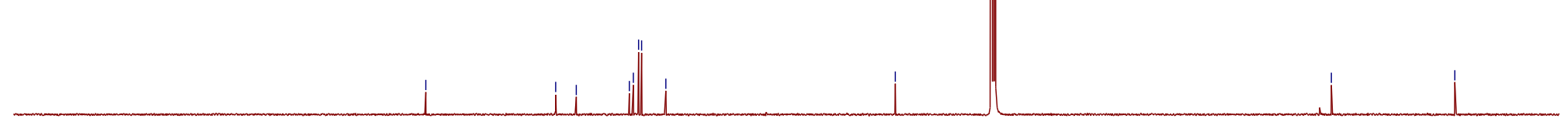

$210 \quad 200 \quad 190$

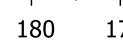

$160 \quad 150$ 
${ }^{1} \mathrm{H}$-NMR of compound $\mathbf{4 k}$

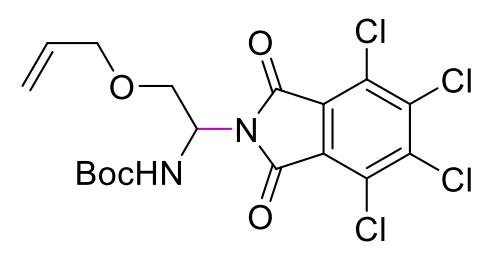

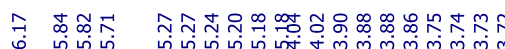

V!

$4 k$

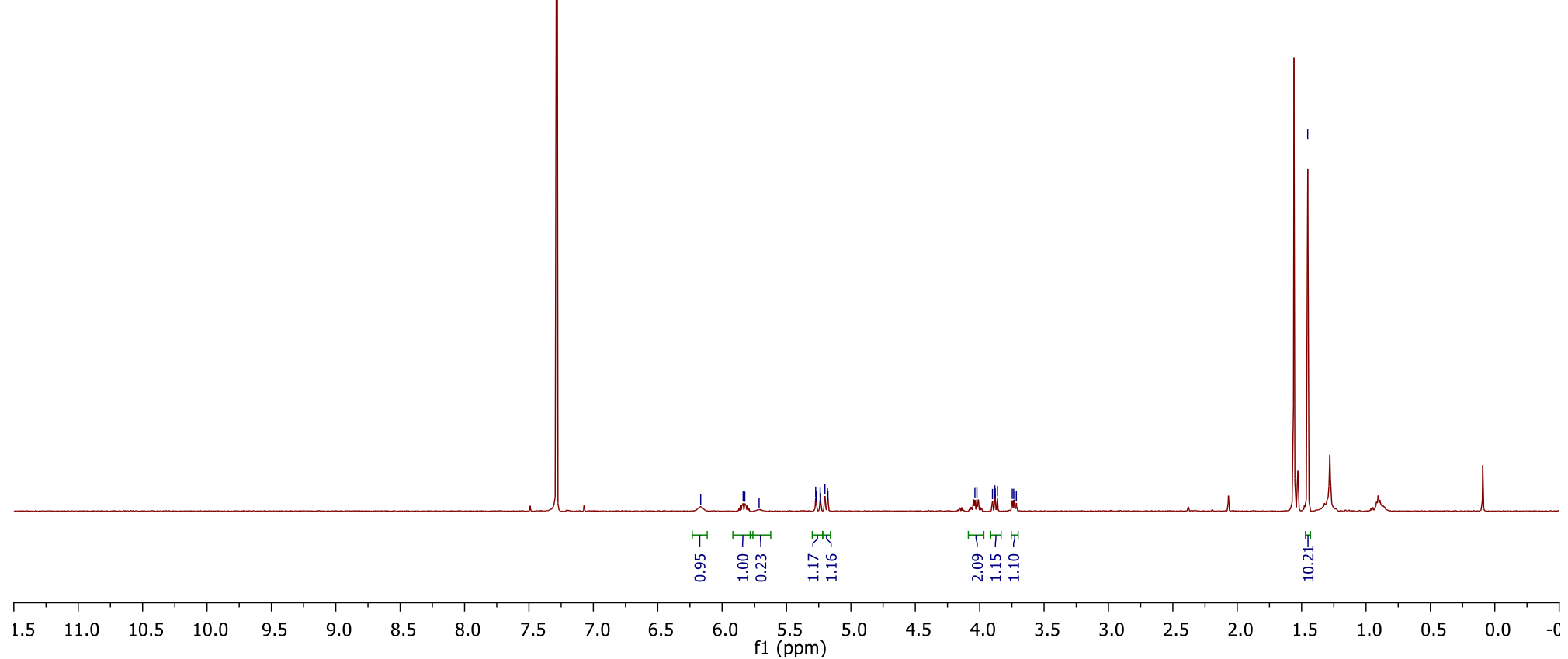


${ }^{13} \mathrm{C}-\mathrm{NMR}$ of compound $\mathbf{4 k}$

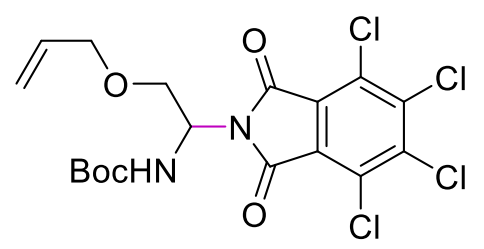

|

4k

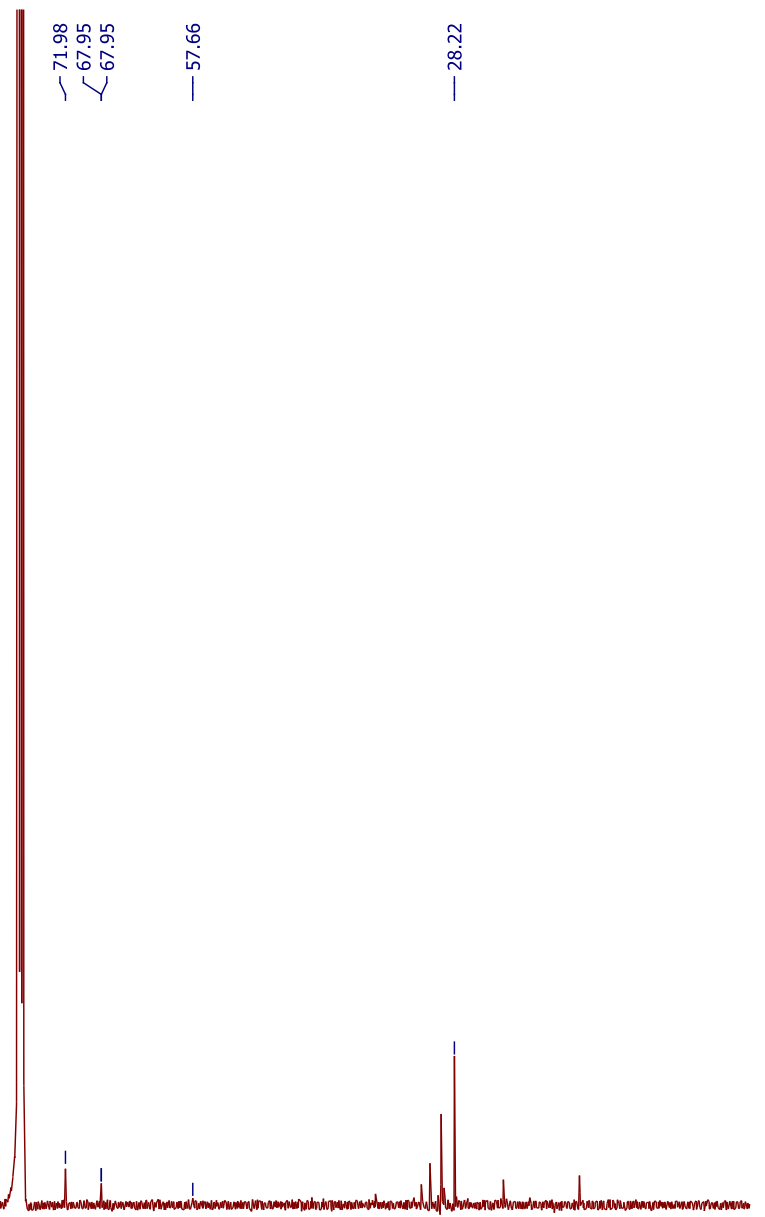

$210 \quad 200 \quad 190$

$180 \quad 17$

$160 \quad 15$

140

120

110
f1 (ppm)

90

80

$70 \quad 60$ 
${ }^{1} \mathrm{H}-\mathrm{NMR}$ of compound $4 \mathrm{o}$

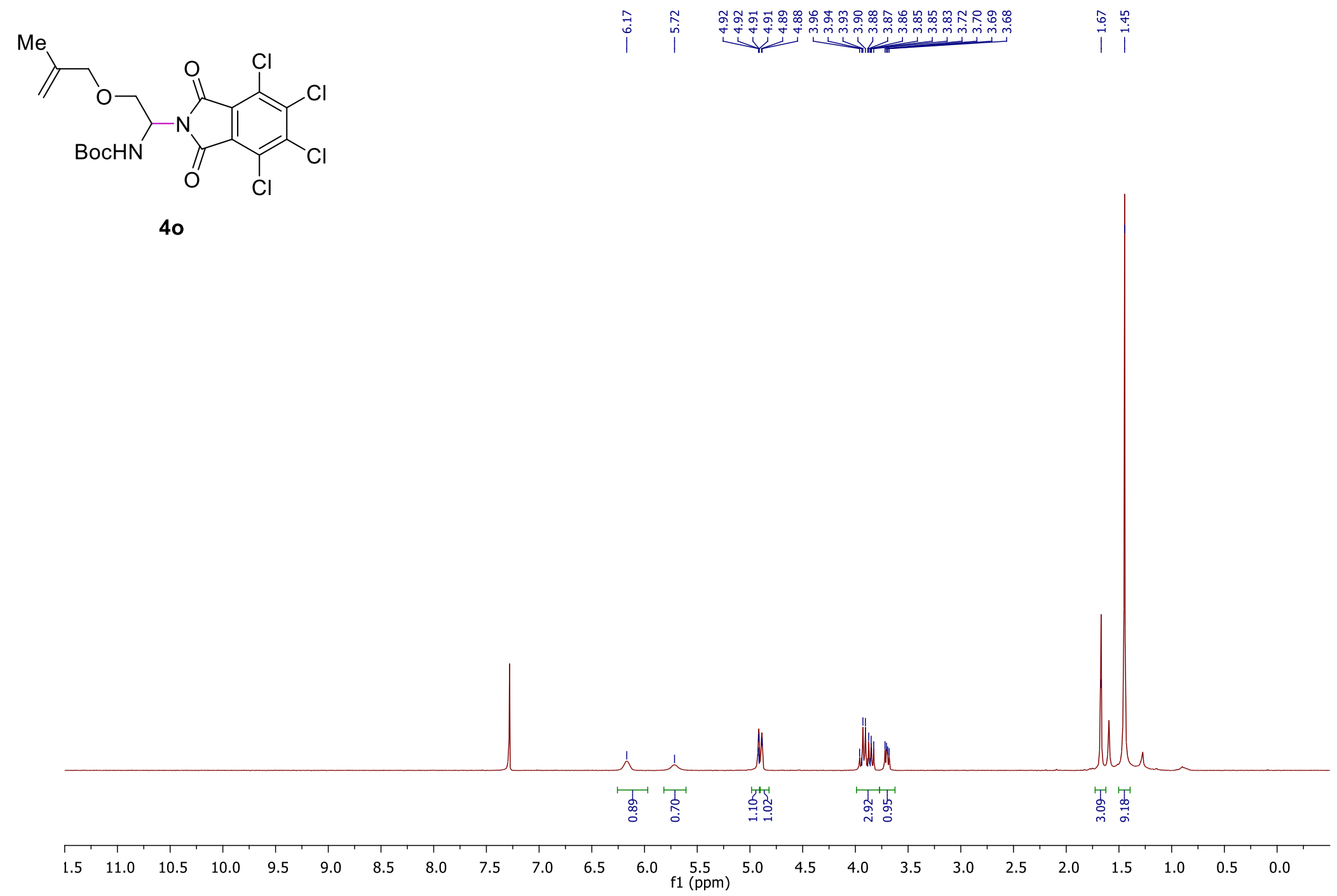


${ }^{13} \mathrm{C}-\mathrm{NMR}$ of compound $4 \mathrm{o}$

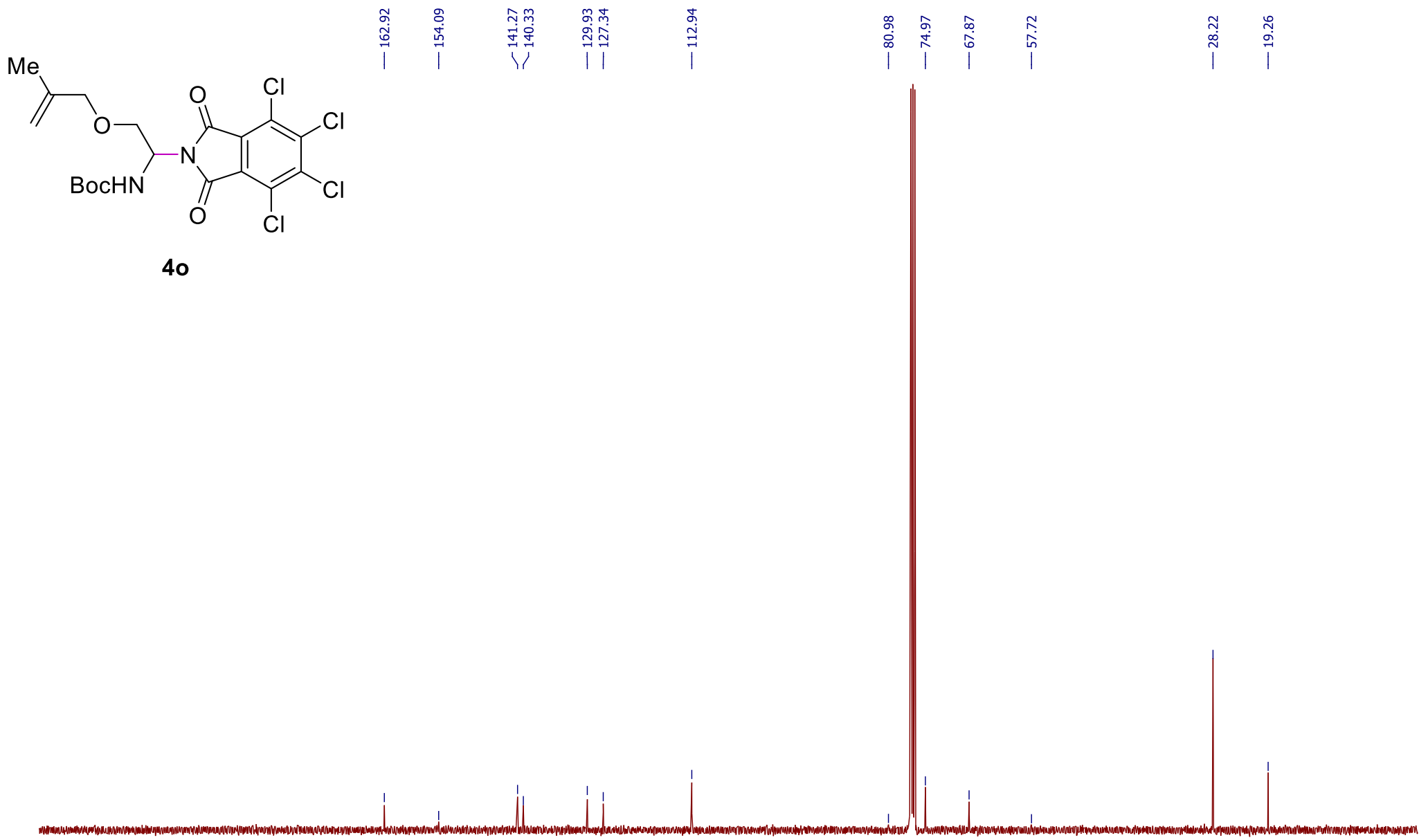

$\begin{array}{lllllllllllllllllllllll}210 & 200 & 190 & 180 & 170 & 160 & 150 & 140 & 130 & 120 & \underset{\mathrm{f} 1}{110}(\mathrm{ppm}) & 100 & 90 & 80 & 70 & 60 & 50 & 40 & 30 & 20 & 10 & 0\end{array}$ 
${ }^{1} \mathrm{H}$-NMR of compound $\mathbf{4 p}$
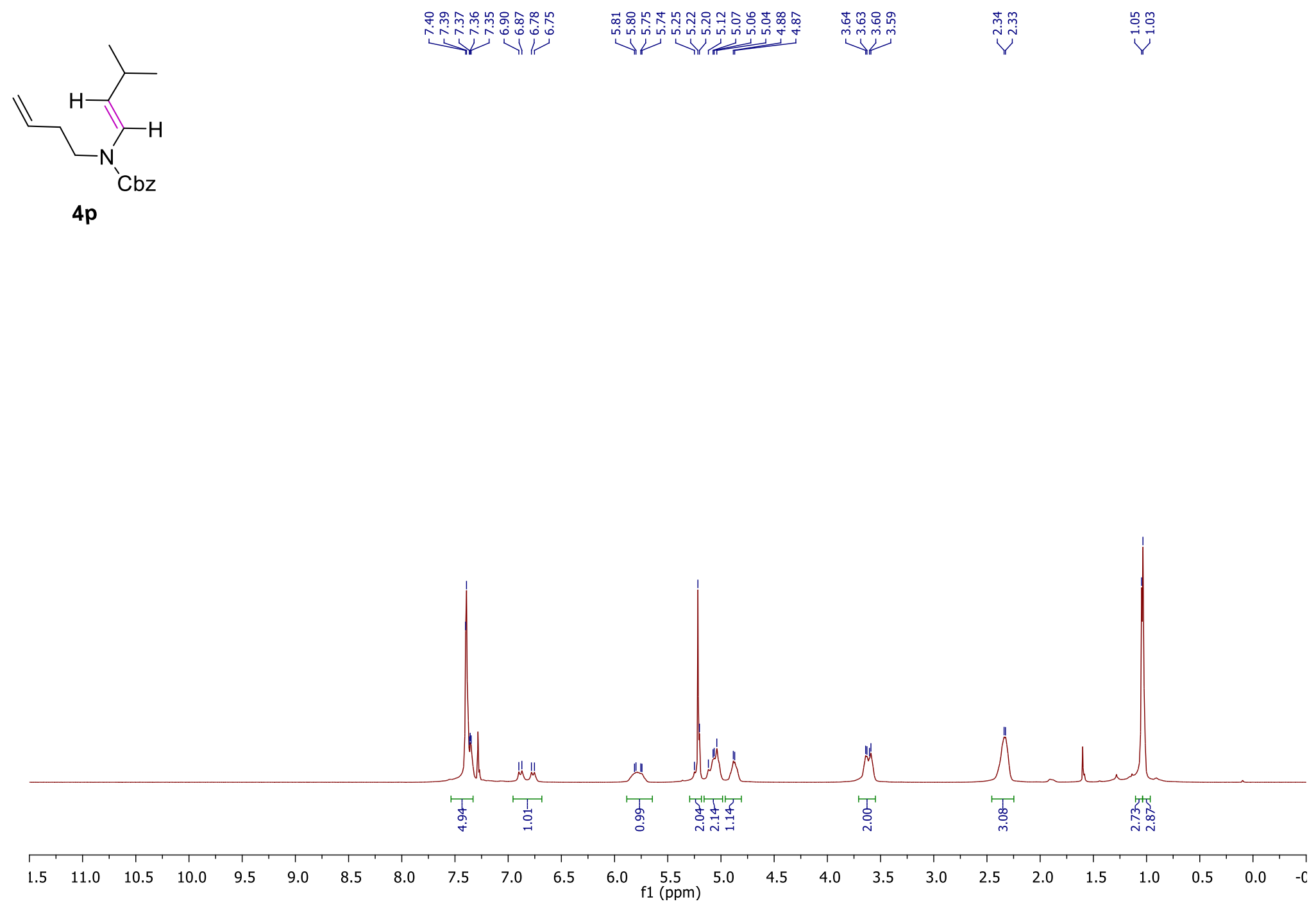
${ }^{13} \mathrm{C}$-NMR of compound $4 p$
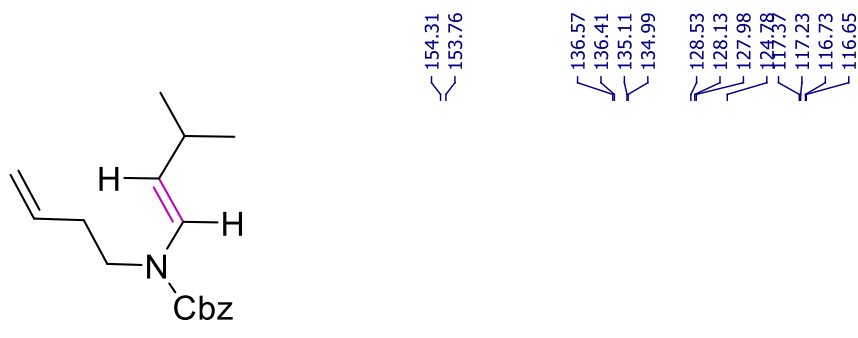

先

守

$4 p$

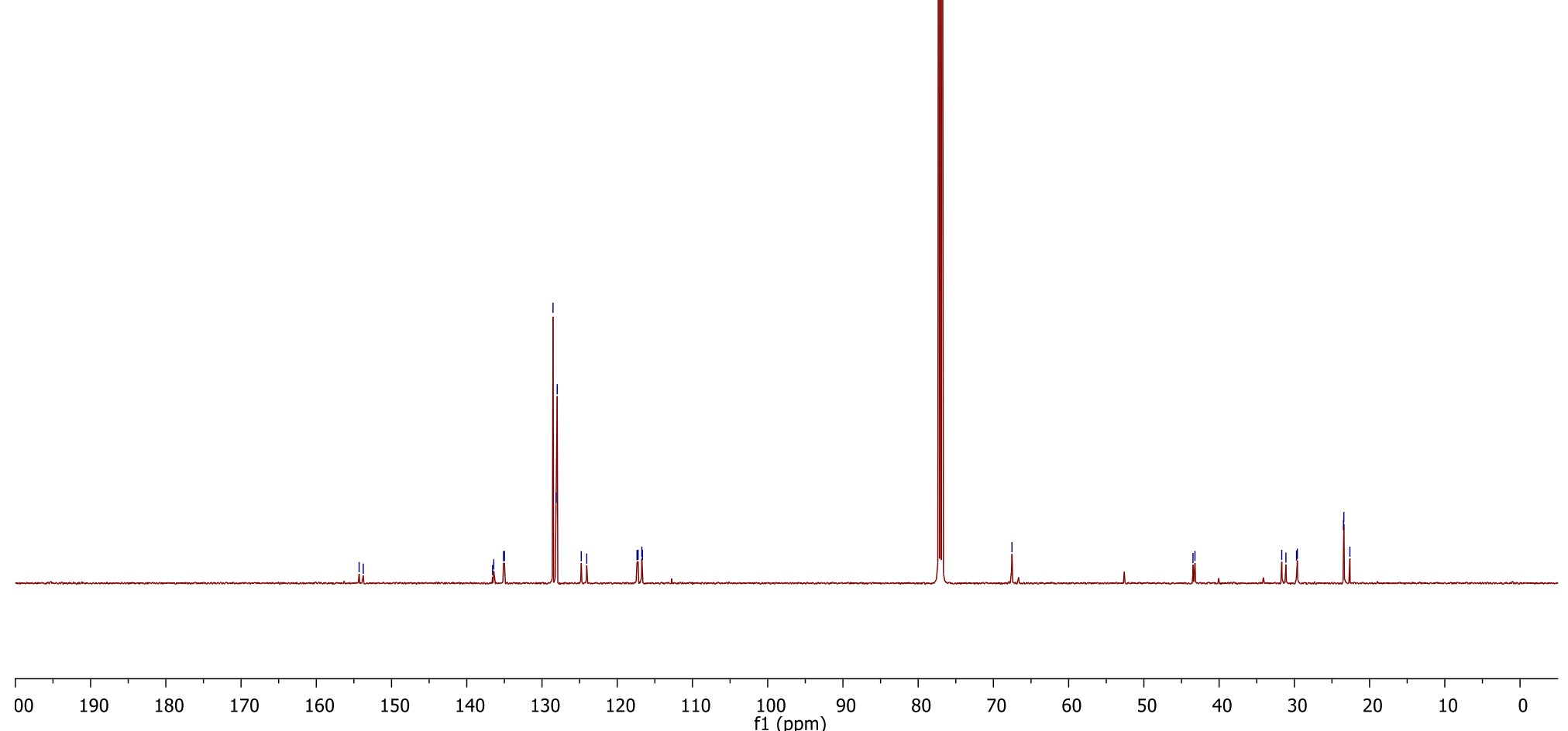


NOESY of compound $\mathbf{4 p}$

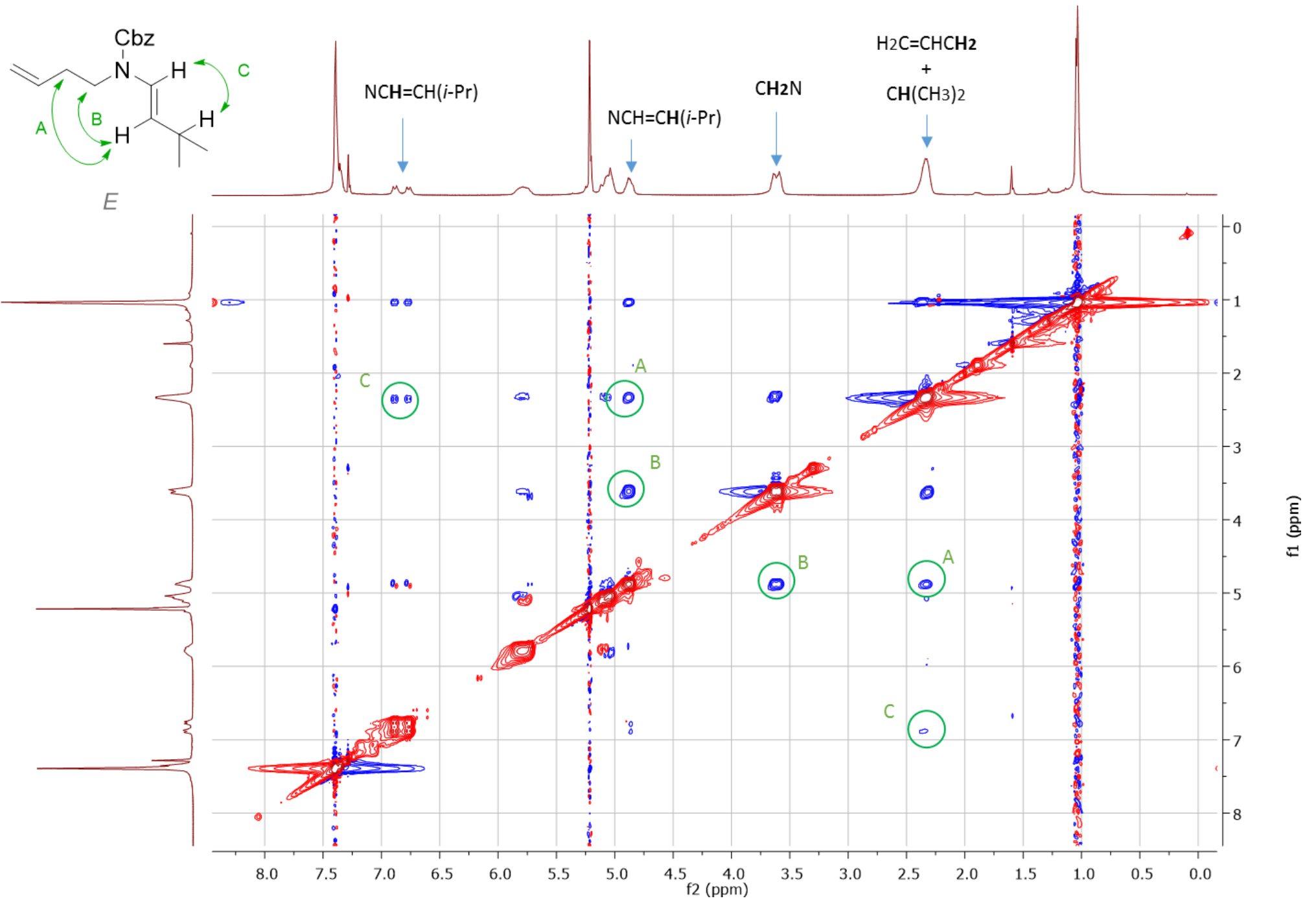


${ }^{1} \mathrm{H}$-NMR of compound $\mathbf{4 r}$
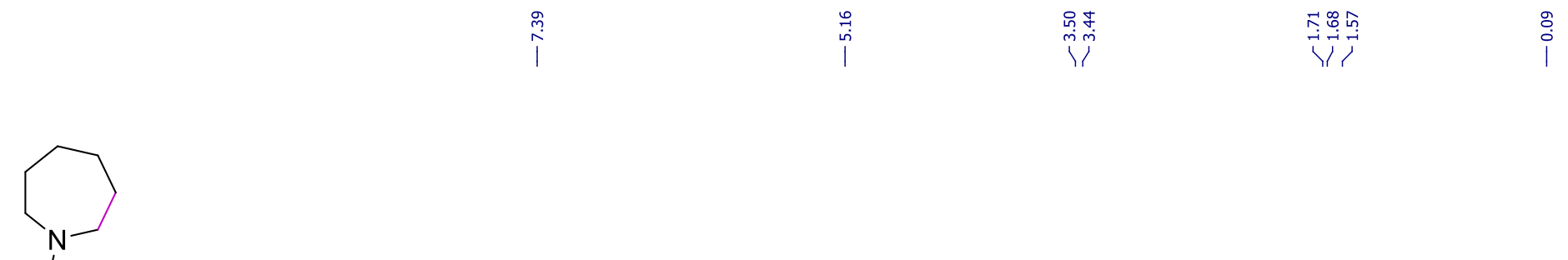

Cbz

$4 r$

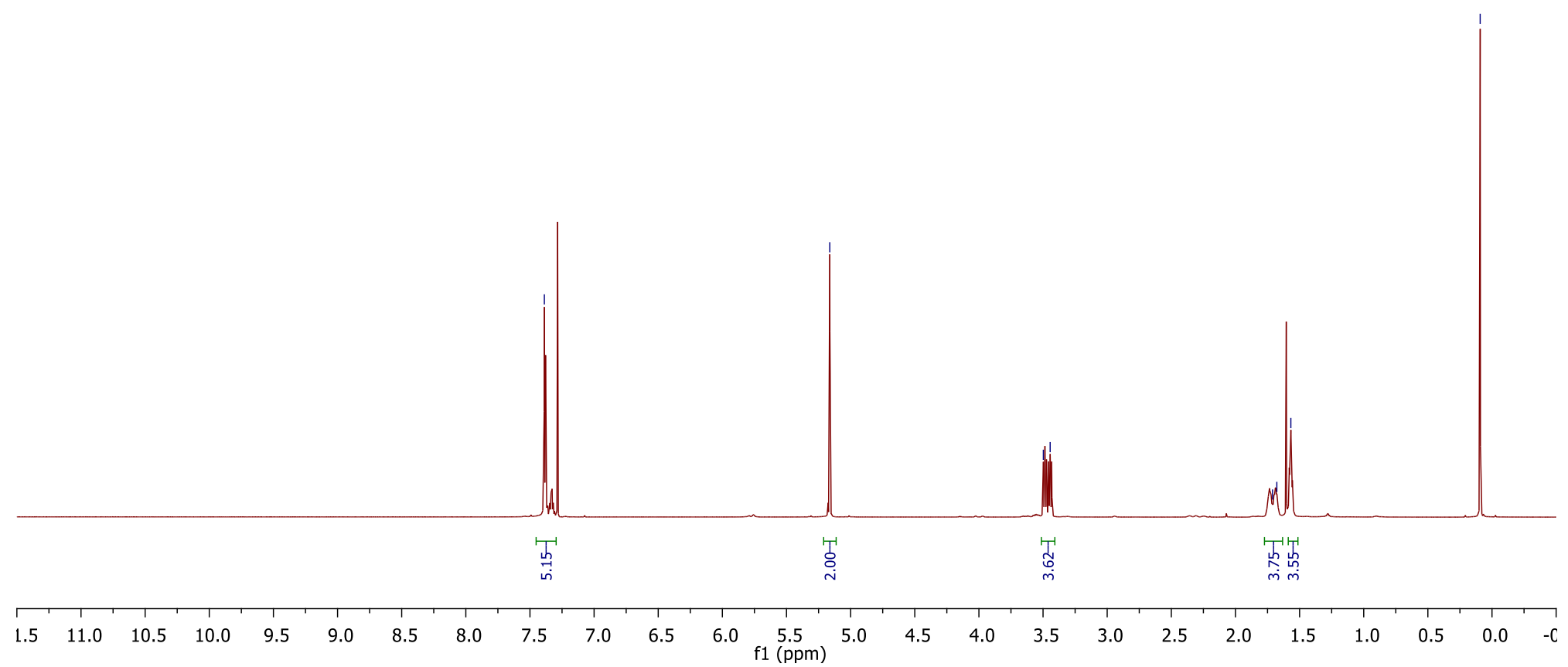


${ }^{13} \mathrm{C}-\mathrm{NMR}$ of compound $\mathbf{4 r}$
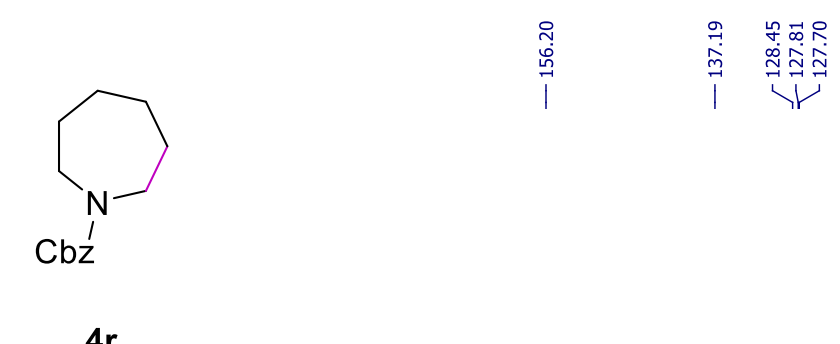

|

$4 r$

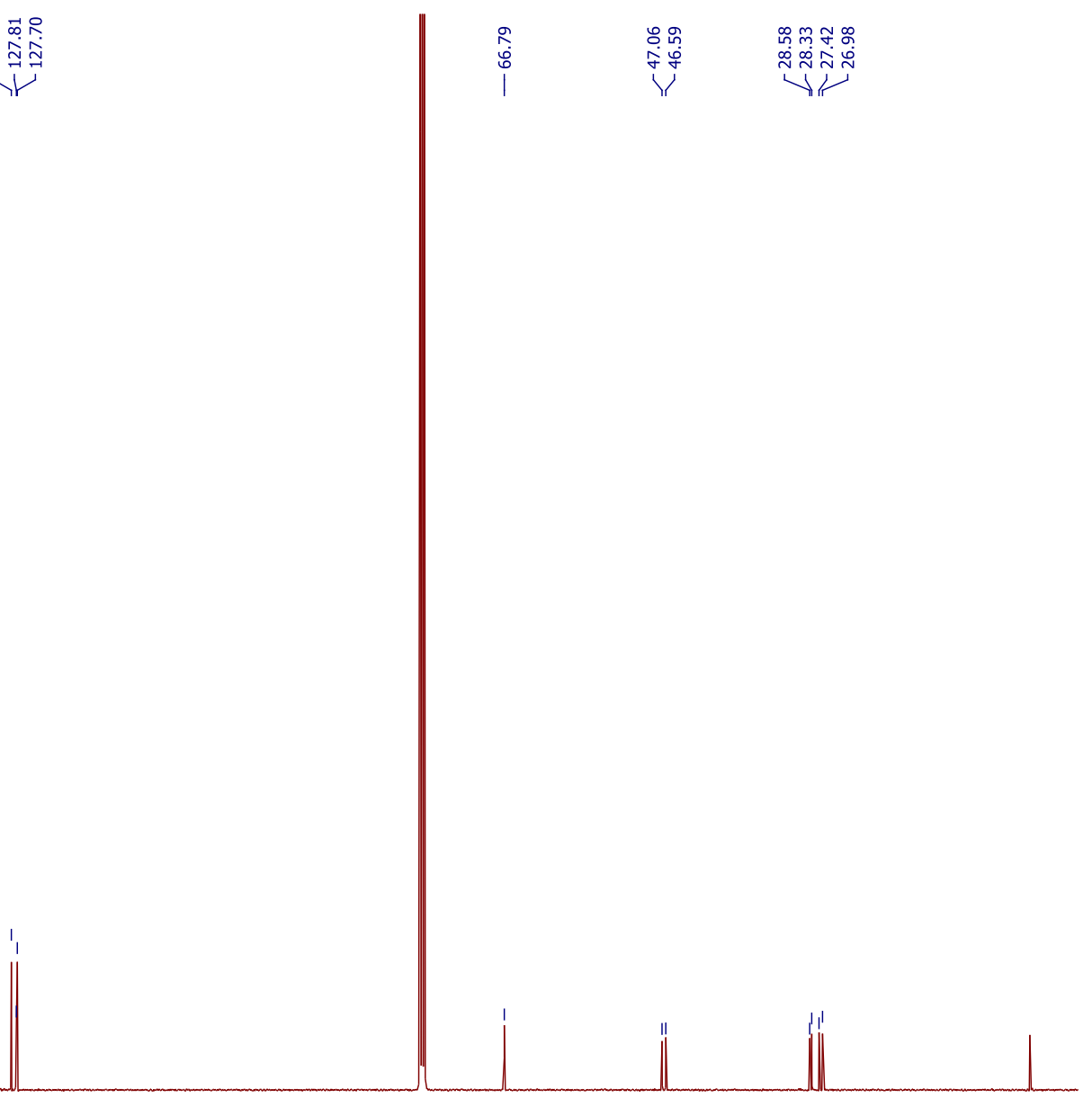

$210 \quad 200$

$90 \quad 180$

170

$160 \quad 150$

140

$130 \quad 120$

110100

$90 \quad 80$

$\begin{array}{lll}1 & 1 & 1 \\ 70 & 60 & 50\end{array}$ 
${ }^{1} \mathrm{H}-\mathrm{NMR}$ of compound $4 \mathrm{~s}$

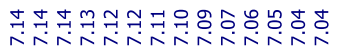

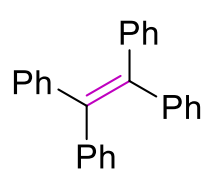

$4 s$

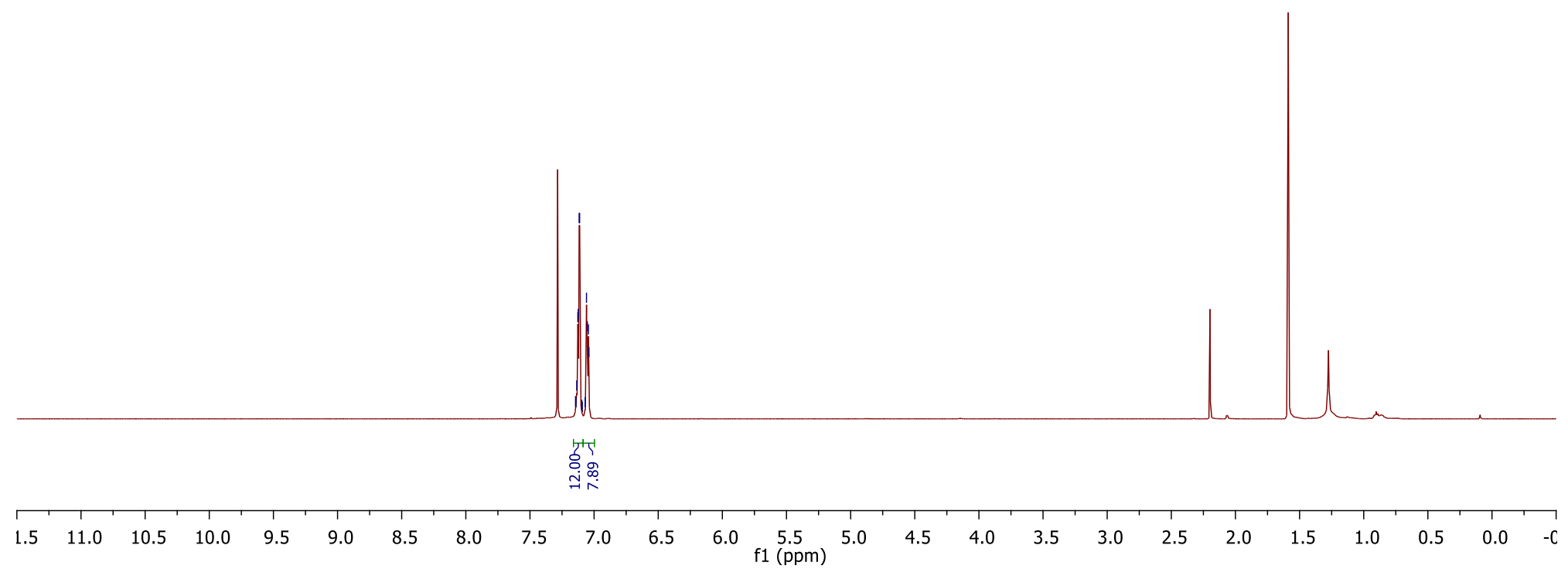


${ }^{13} \mathrm{C}-\mathrm{NMR}$ of compound $\mathbf{4 s}$

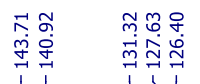

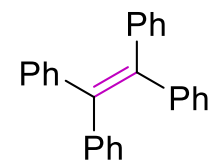

$4 s$

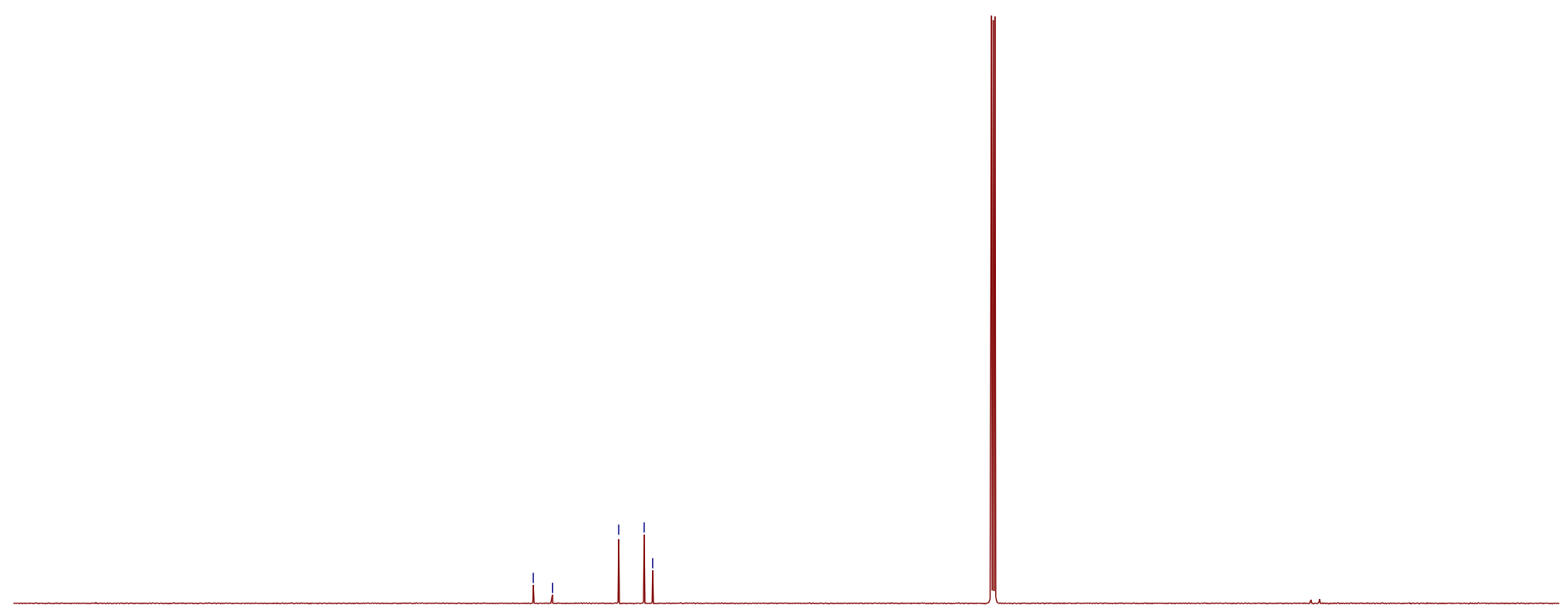

$210 \quad 200$

$90 \quad 180$

170

$160 \quad 150$

140

$30 \quad 120$

110100

$90 \quad 80$

$\begin{array}{lll}70 & 60 & 50\end{array}$ 
${ }^{1} \mathrm{H}-\mathrm{NMR}$ of compound $\mathbf{5 p}$
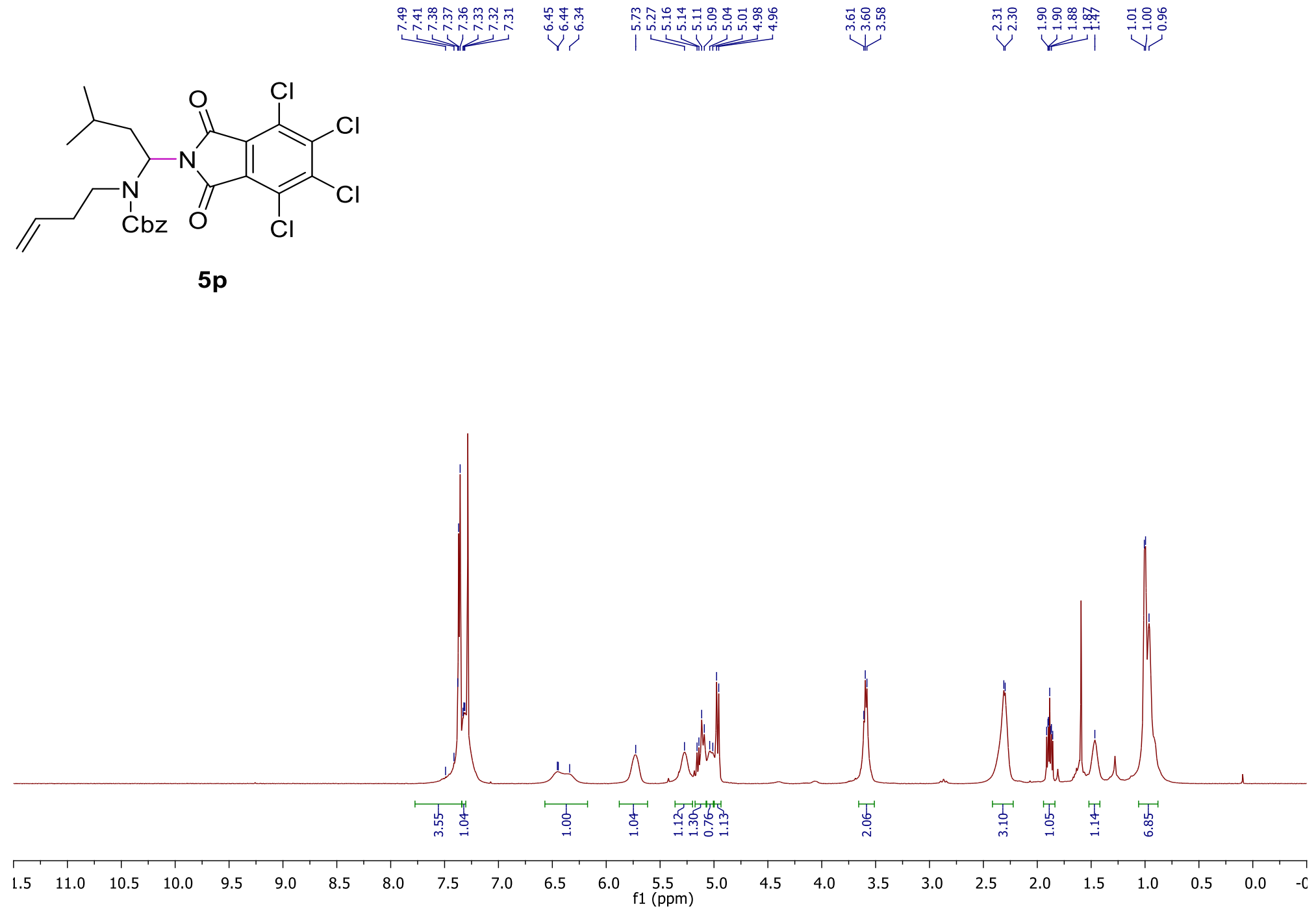
${ }^{13} \mathrm{C}-\mathrm{NMR}$ of compound $\mathbf{5 p}$

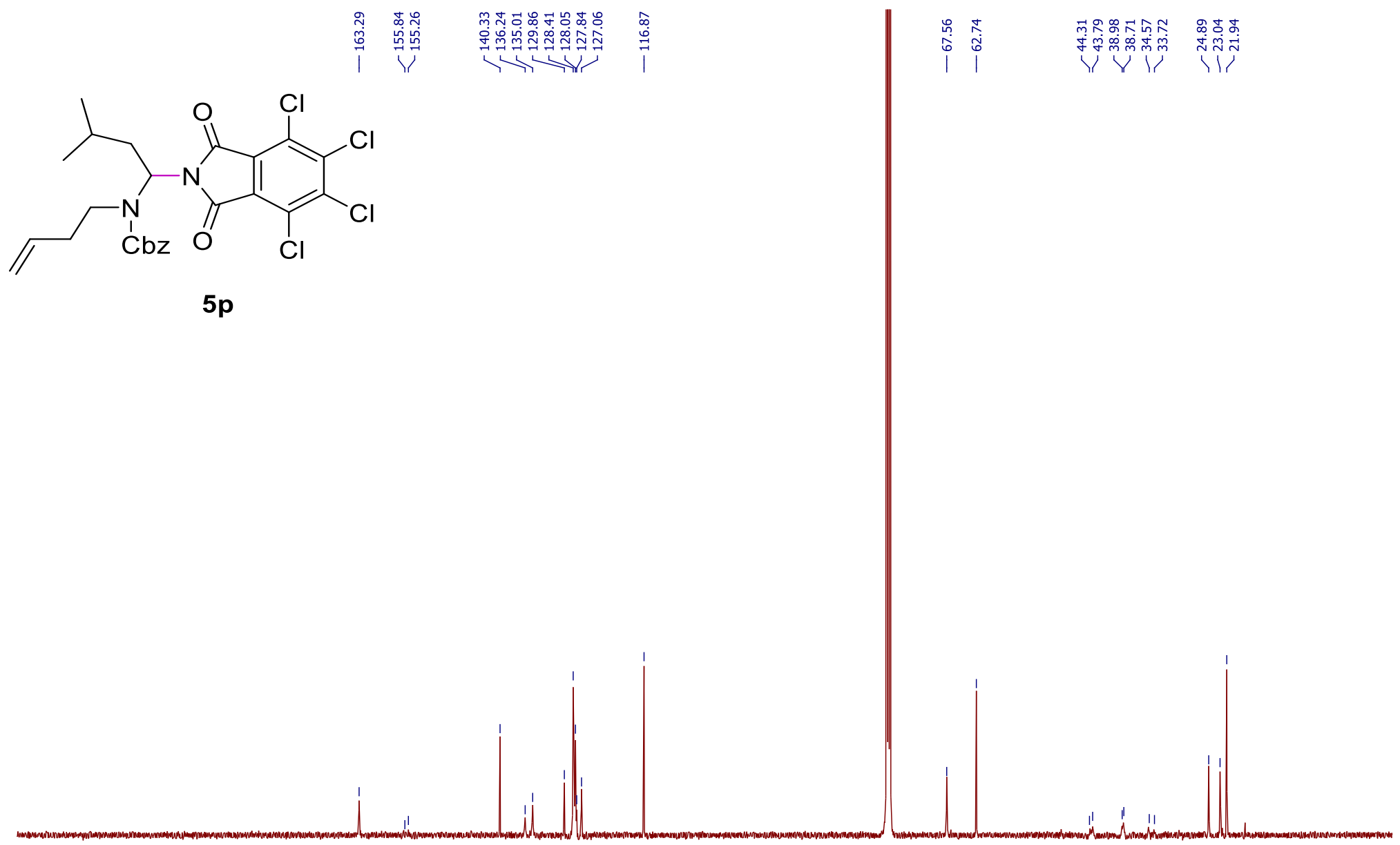

$\begin{array}{llllllllllllllllllllllllll}210 & 200 & 190 & 180 & 170 & 160 & 150 & 140 & 130 & 120 & 110 & 100 & 90 & 80 & 70 & 60 & 50 & 40 & 30 & 20 & 10 & 0\end{array}$ 
${ }^{1} \mathrm{H}-\mathrm{NMR}$ of compound S1e
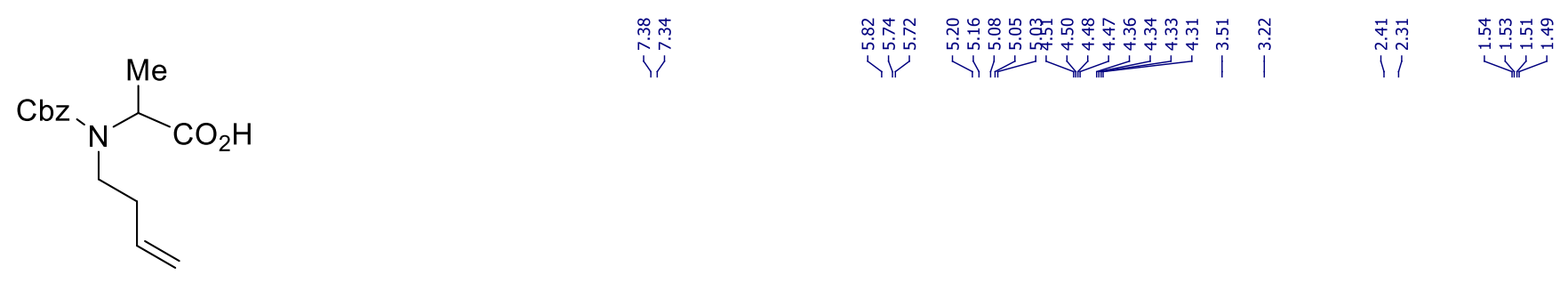

s1e

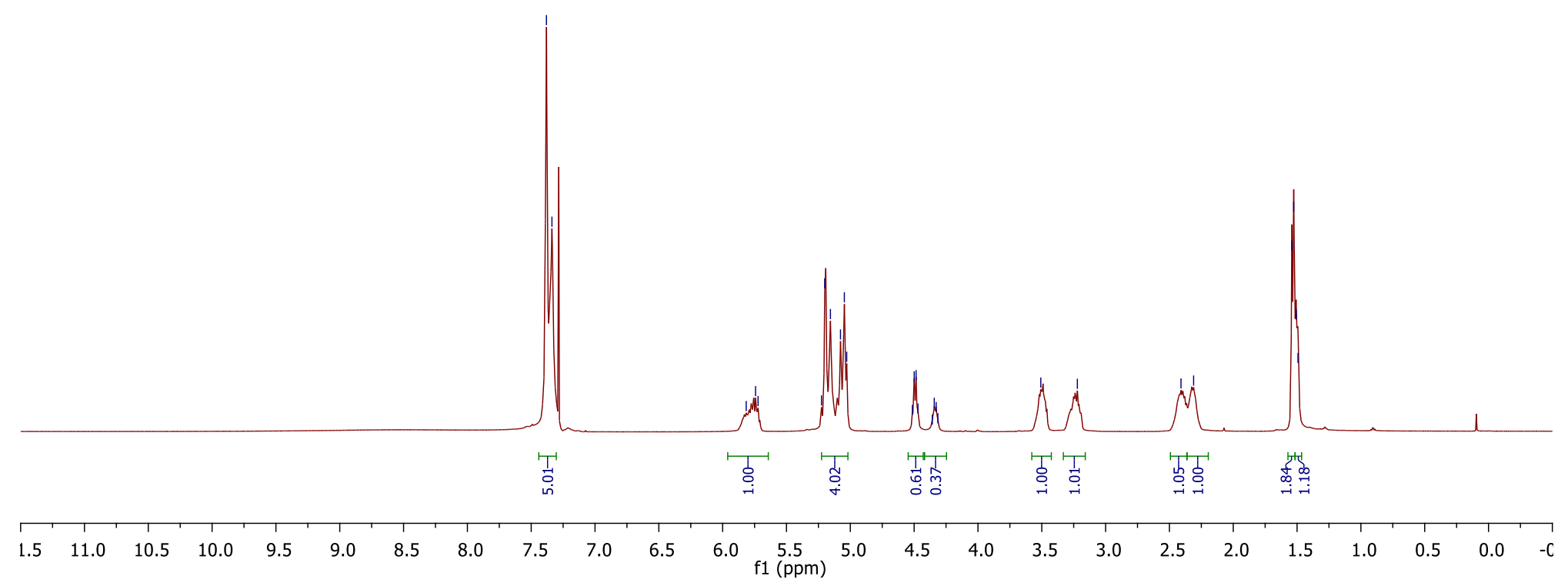


${ }^{13} \mathrm{C}-\mathrm{NMR}$ of compound S1e

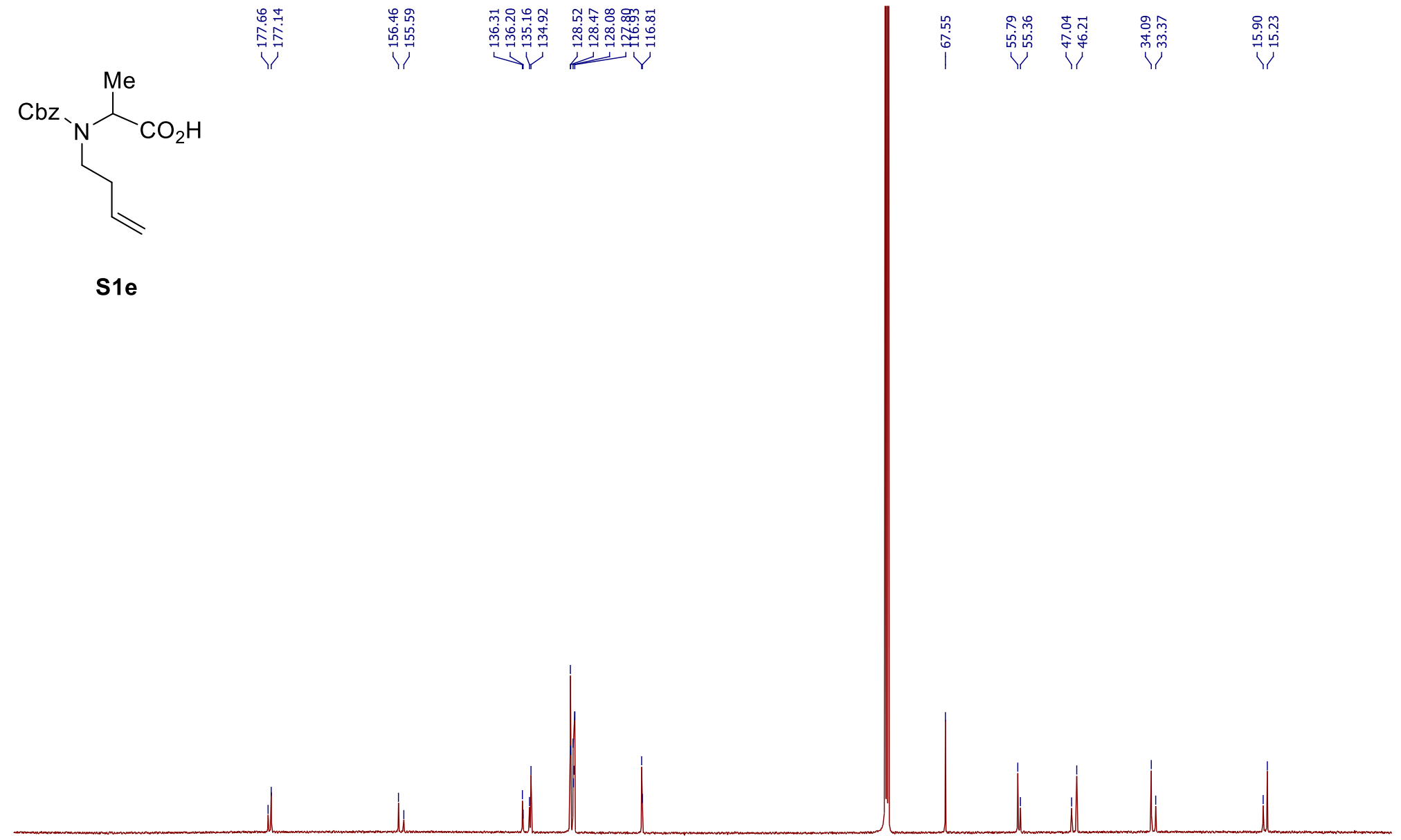

$\begin{array}{lllllllllllllllllllllllll}210 & 200 & 190 & 180 & 170 & 160 & 150 & 140 & 130 & 120 & 110 & 100 & 90 & 80 & 70 & 60 & 50 & 40 & 30 & 20 & 10 & 0\end{array}$ 
${ }^{1}$ H-NMR of compound S1h<smiles>C=CCCCOC(Cc1ccccc1)C(=O)O</smiles>

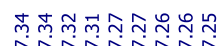

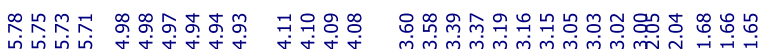

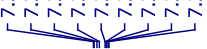

ivini

s1h

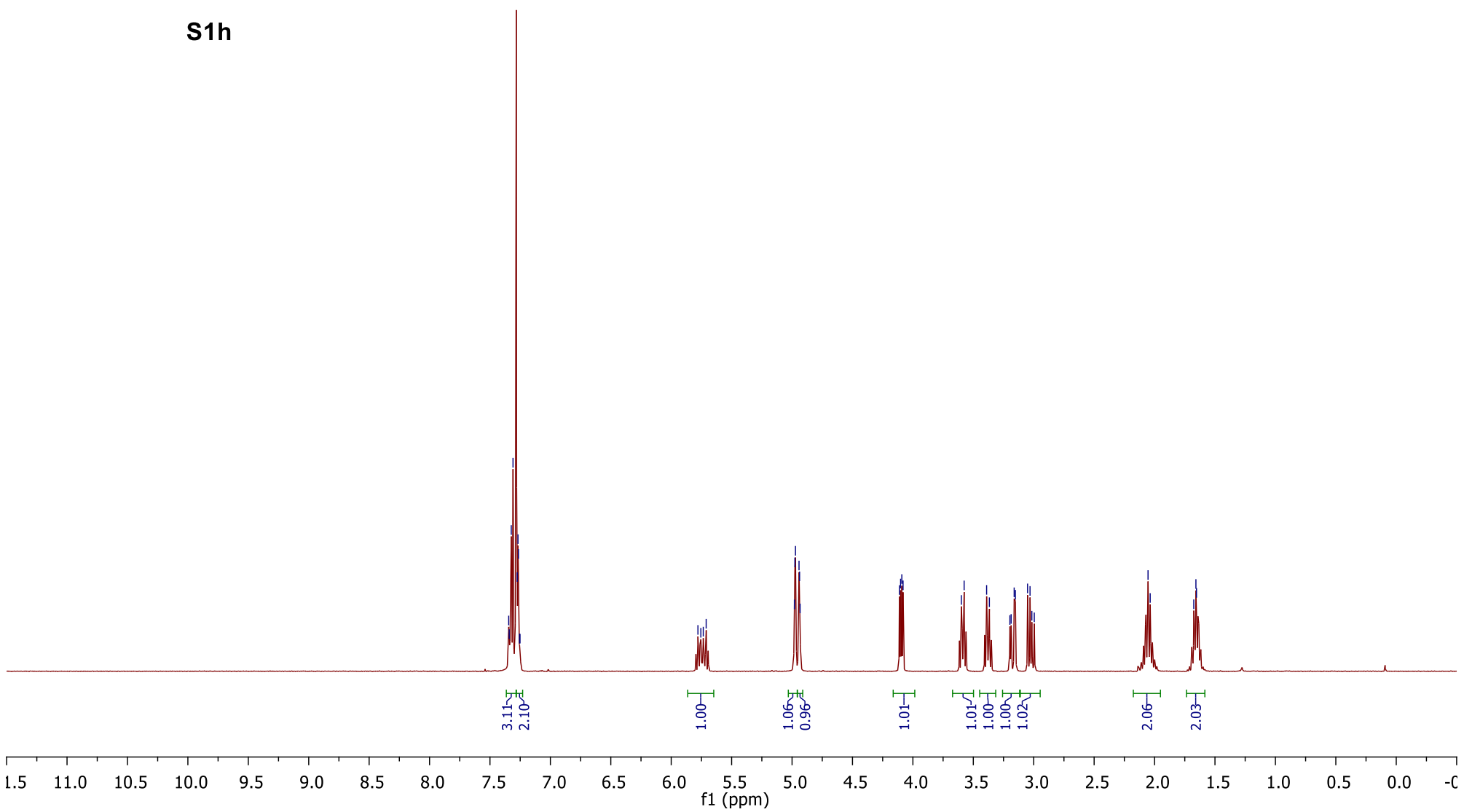


${ }^{13} \mathrm{C}$-NMR of compound S1h
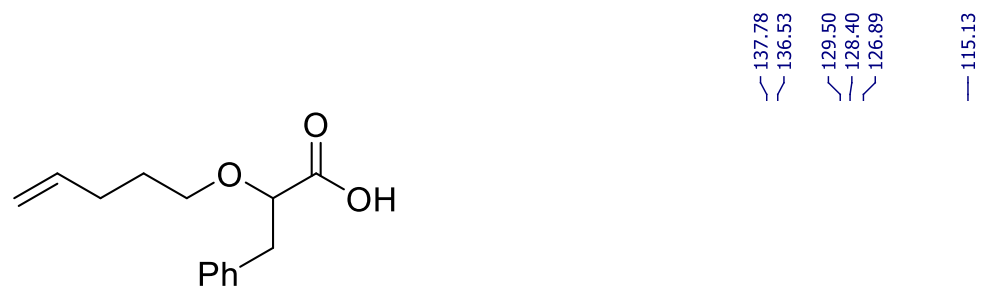

S1h

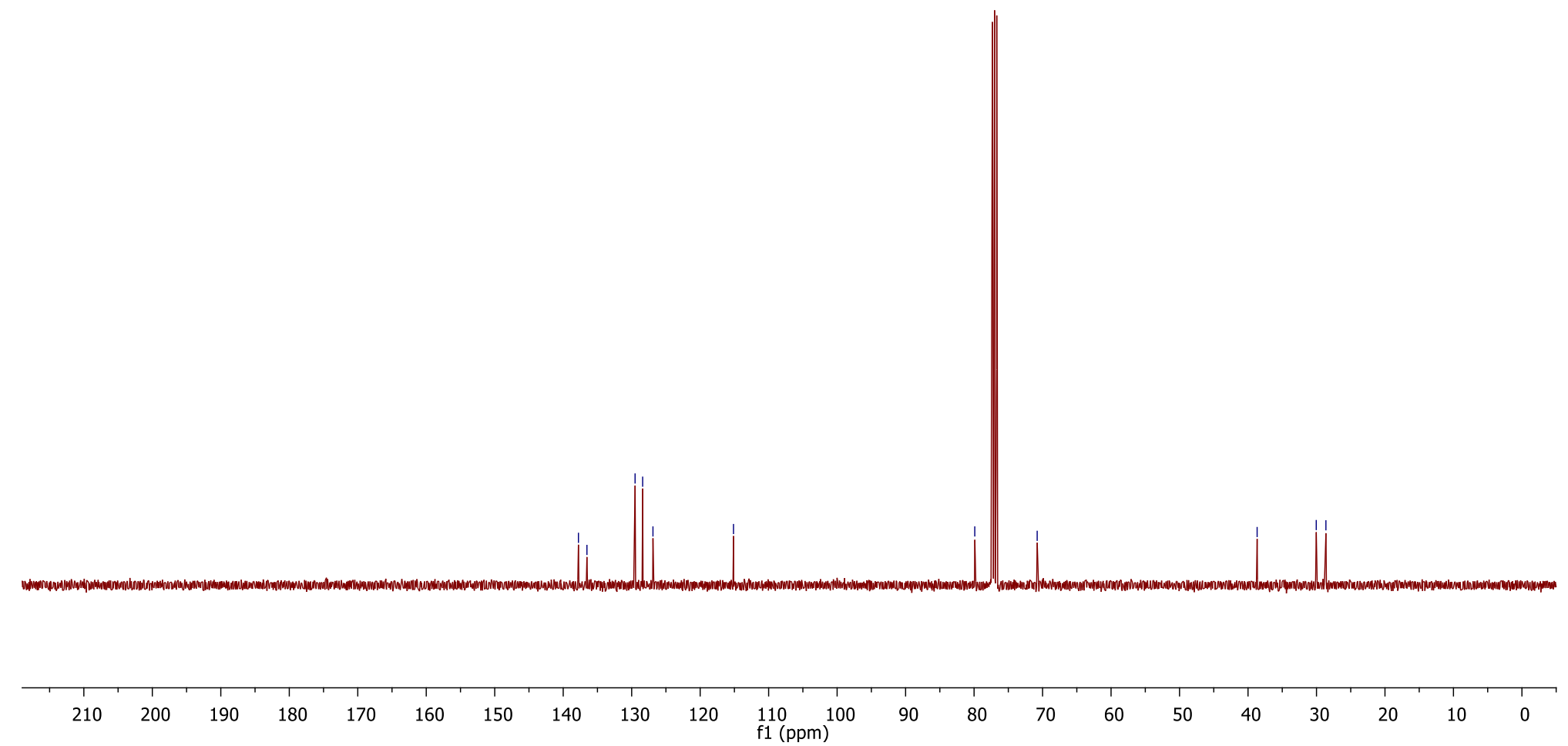


${ }^{1}$ H-NMR of compound S1p
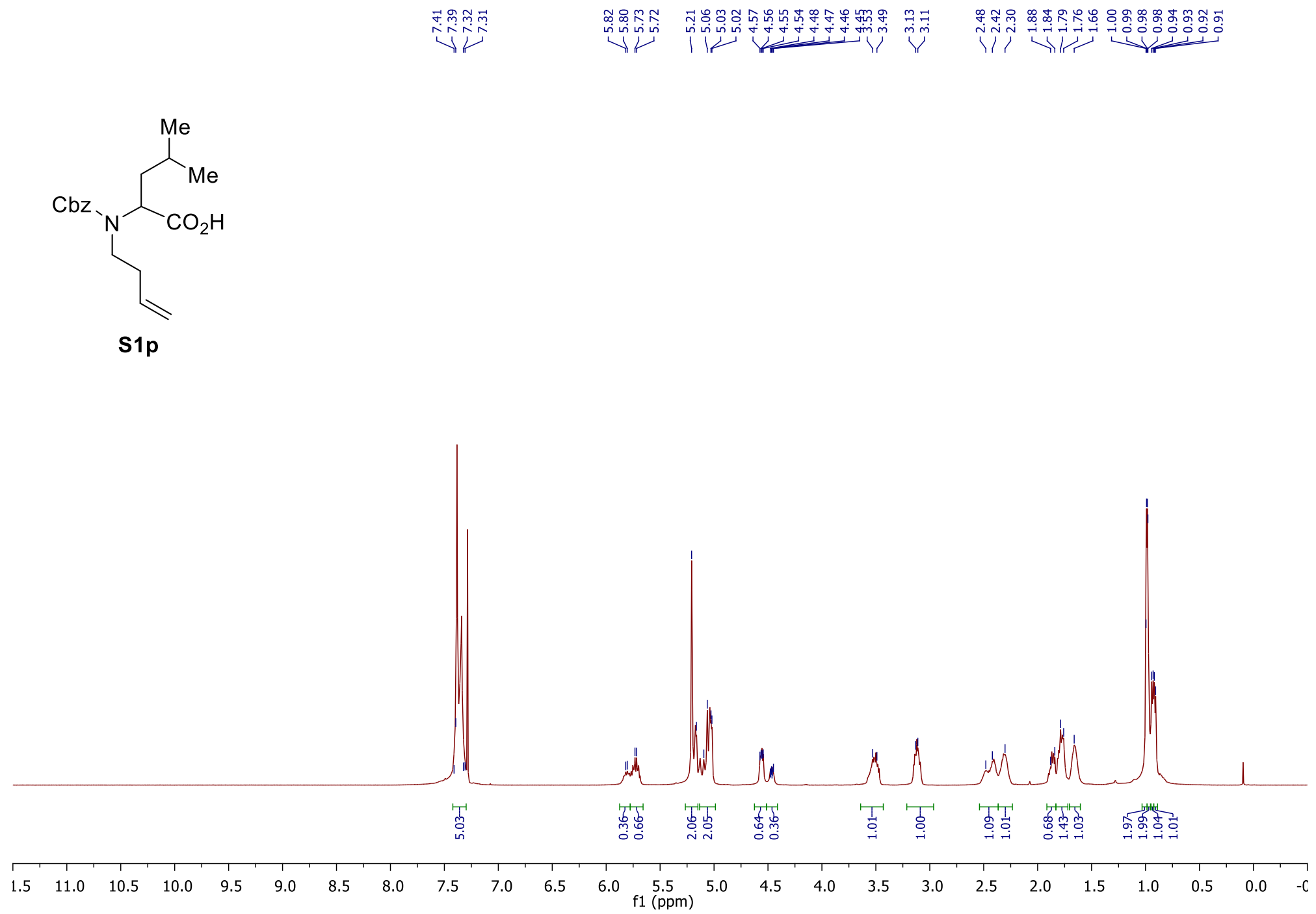
${ }^{13} \mathrm{C}$-NMR of compound S1p

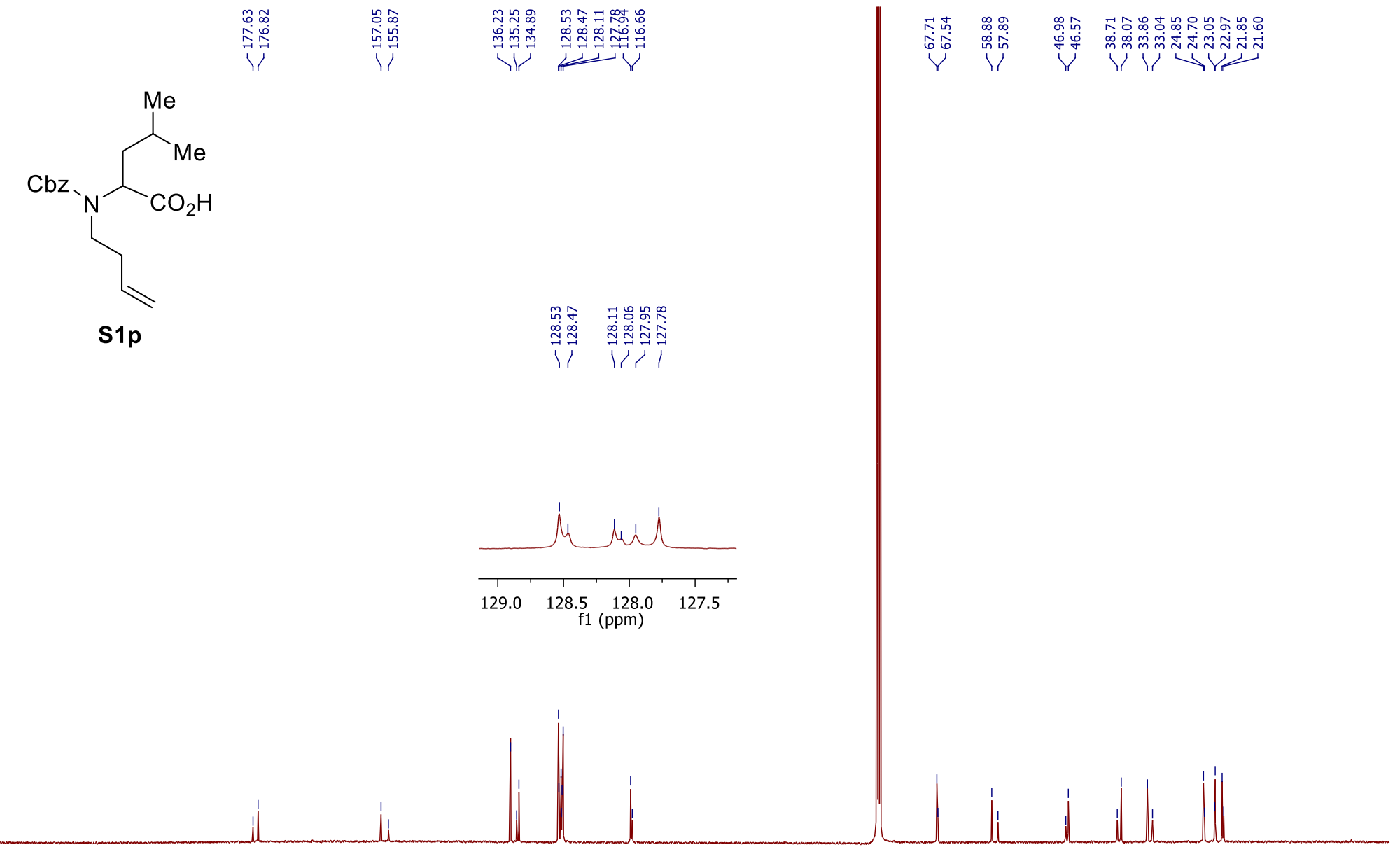

$\begin{array}{lllllllllllllllllllllllllll}210 & 200 & 190 & 180 & 170 & 160 & 150 & 140 & 130 & 120 & 110 & 100 & 90 & 80 & 70 & 60 & 50 & 40 & 30 & 20 & 10 & 0\end{array}$ 
${ }^{1}$ H-NMR of compound S1q

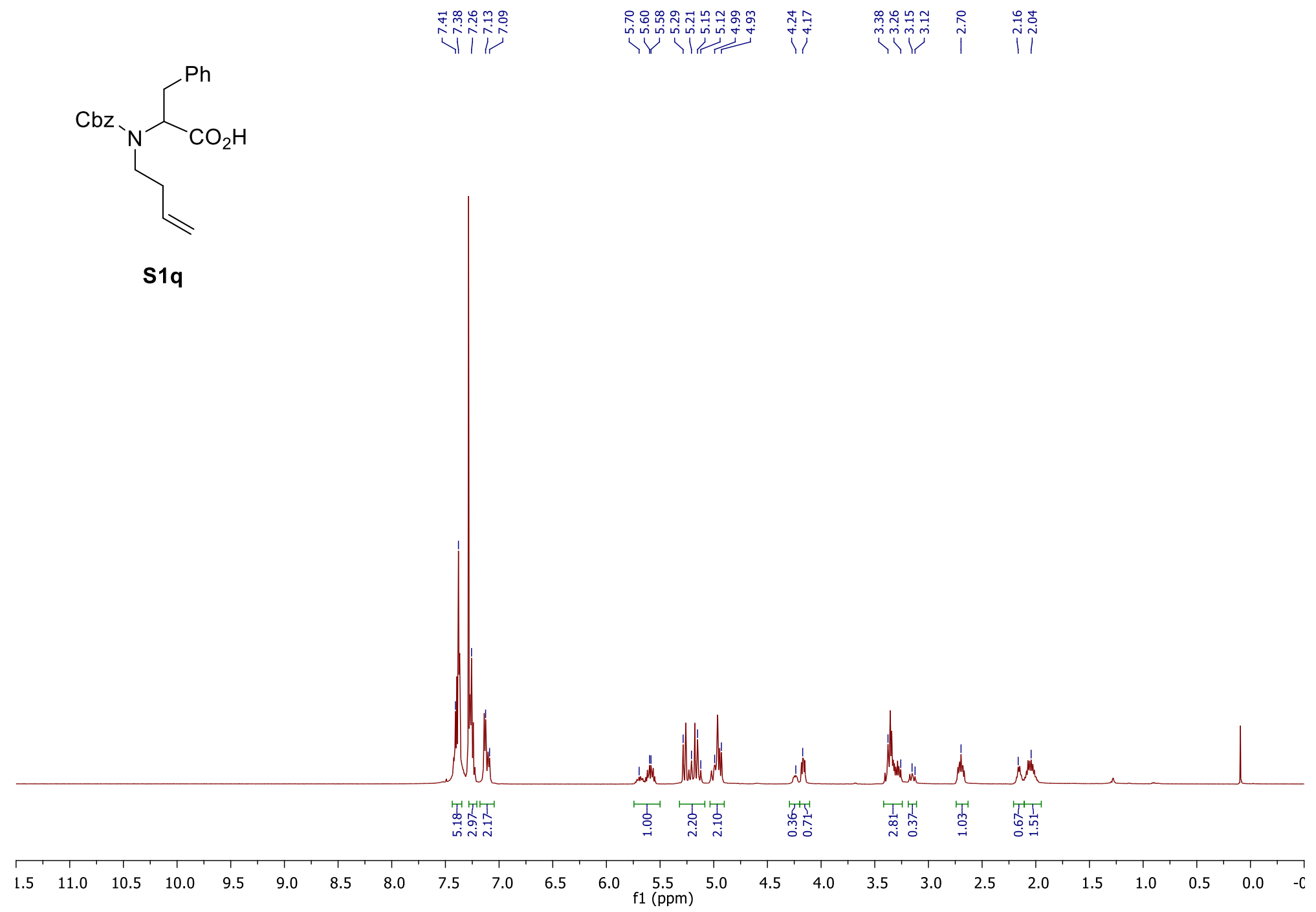


${ }^{13} \mathrm{C}-\mathrm{NMR}$ of compound S1q

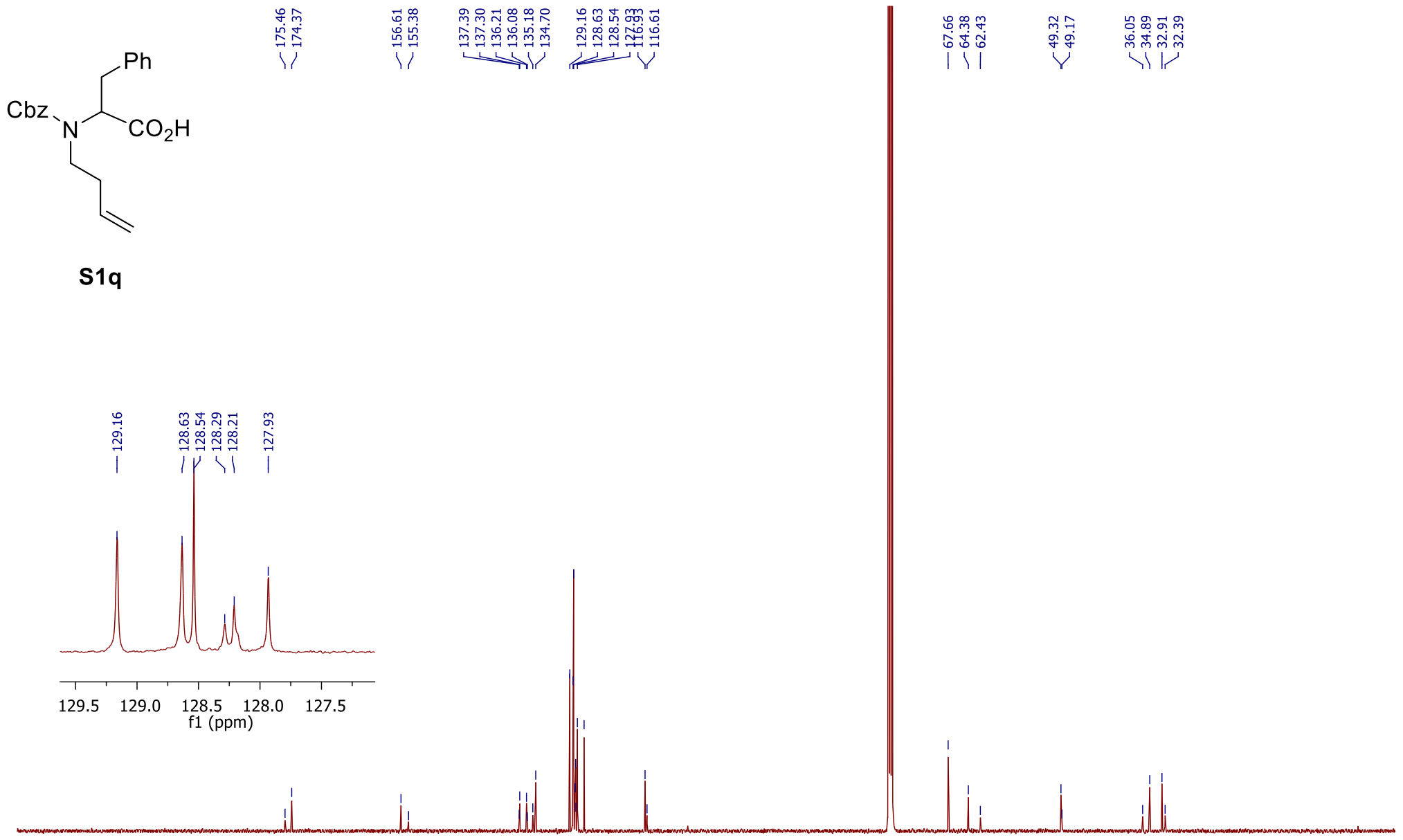


${ }^{1}$ H-NMR of compound $\mathbf{S 1 r}$

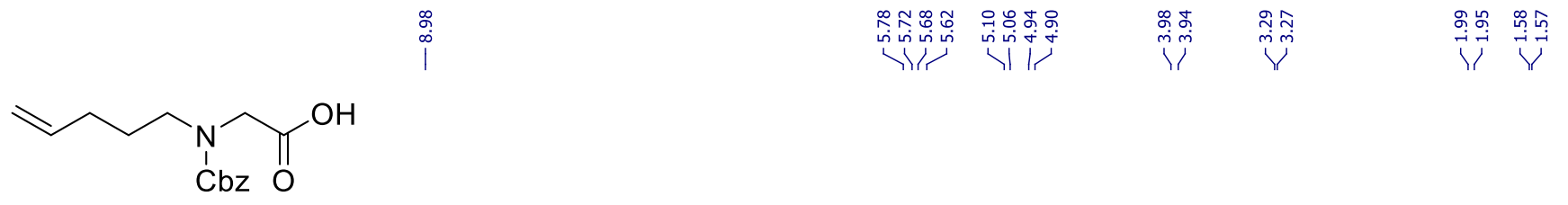

S1r

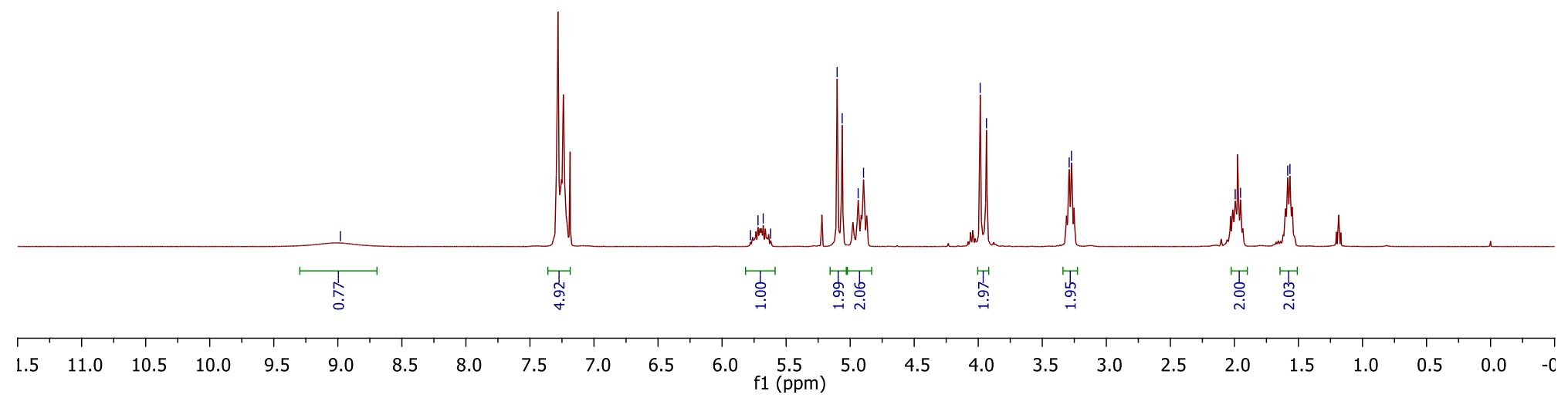


${ }^{13} \mathrm{C}-\mathrm{NMR}$ of compound $\mathbf{S 1 r}$

\begin{tabular}{|c|c|c|c|}
\hline 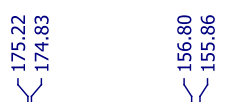 & 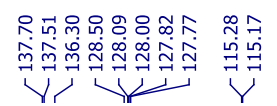 & 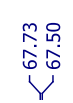 & 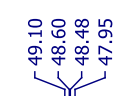 \\
\hline
\end{tabular}

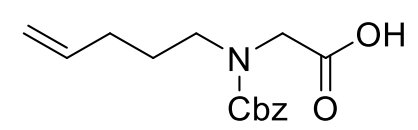

s1r

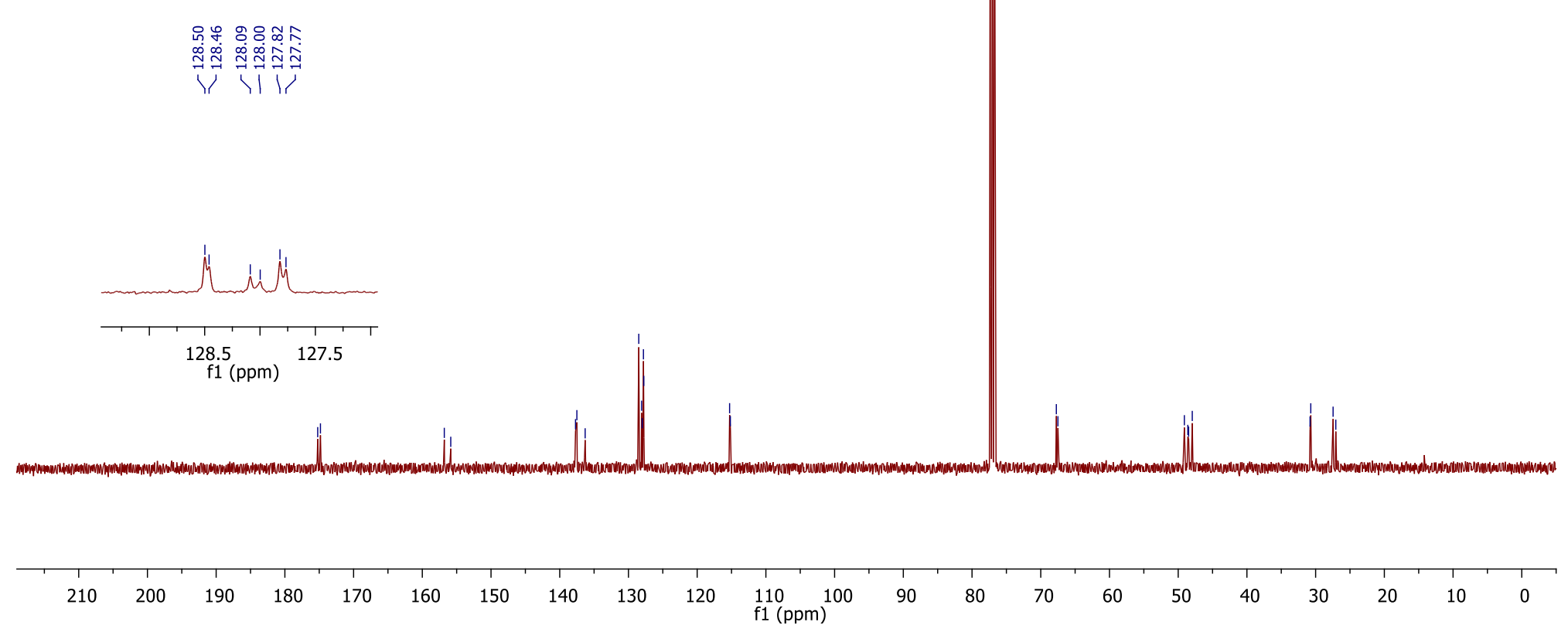


${ }^{1}$ H-NMR of compound S1t

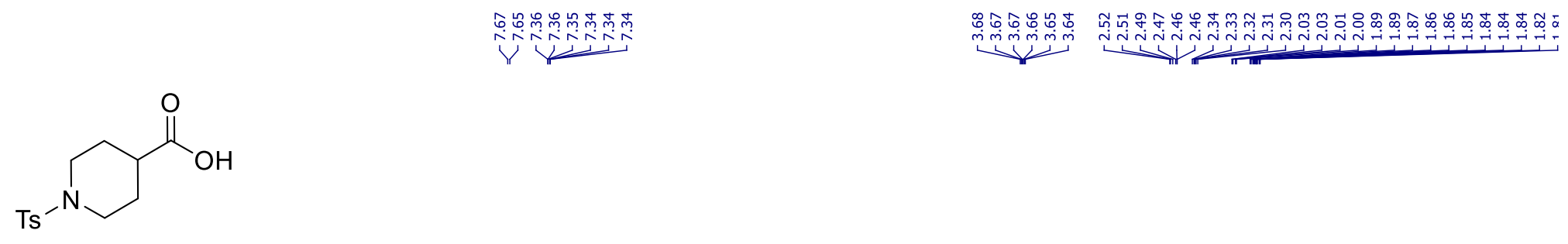

s1t

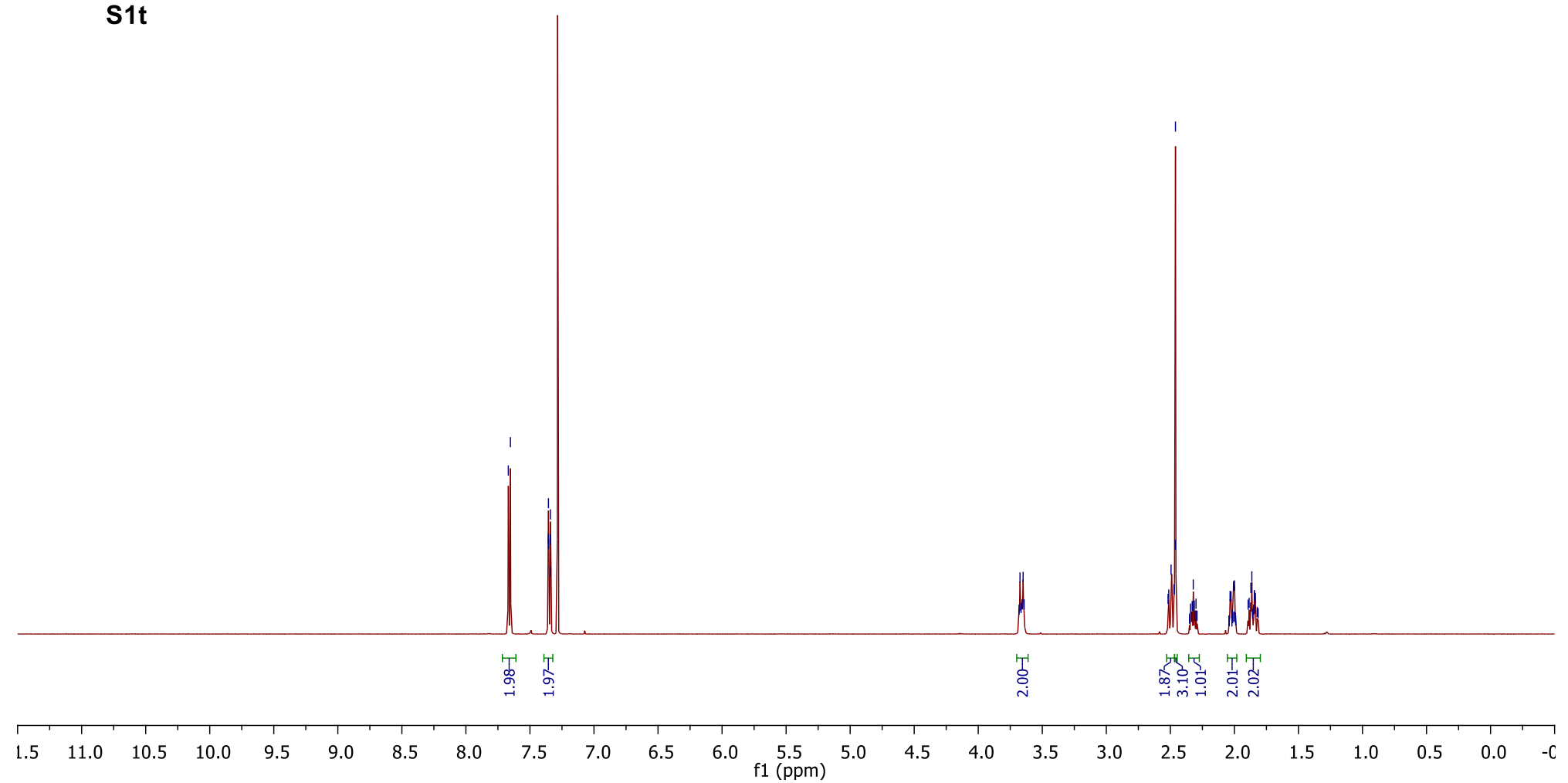


${ }^{13} \mathrm{C}-\mathrm{NMR}$ of compound S1t

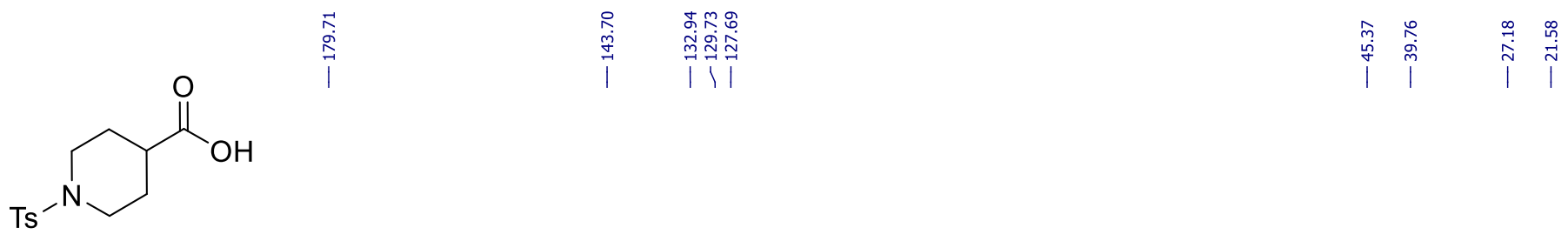

S1t

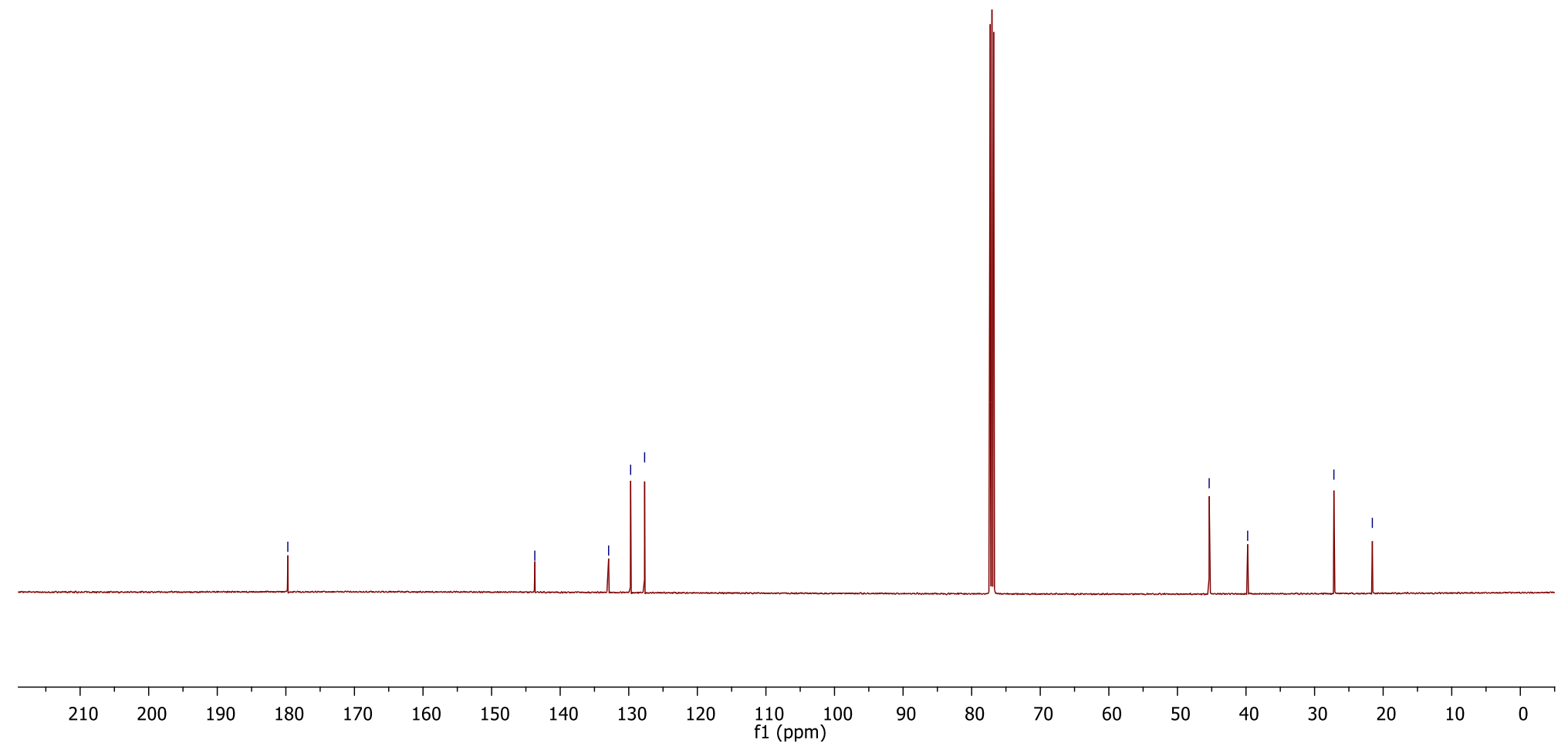


${ }^{1} \mathrm{H}$-NMR of compound S1u
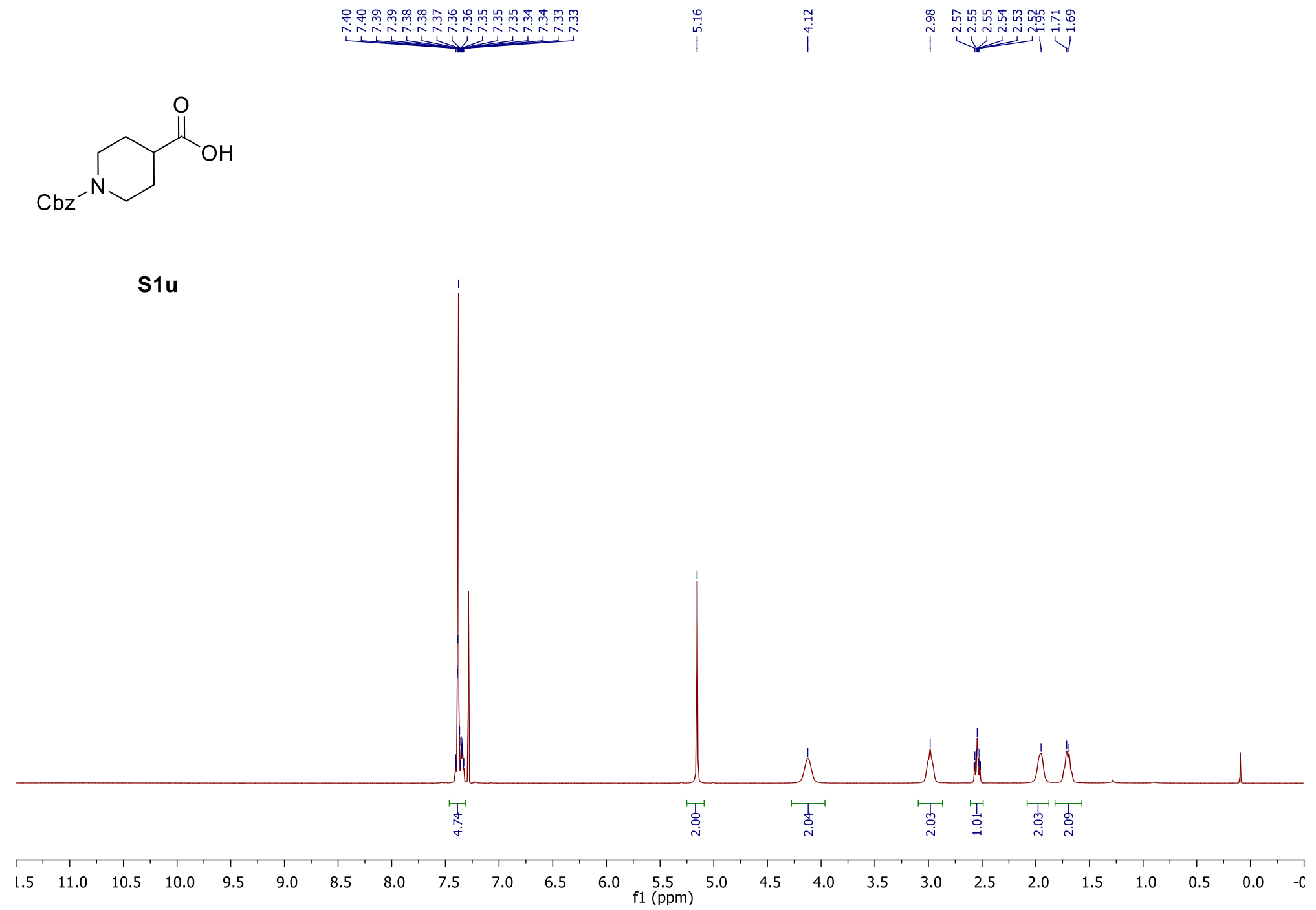
${ }^{13} \mathrm{C}-\mathrm{NMR}$ of compound S1u

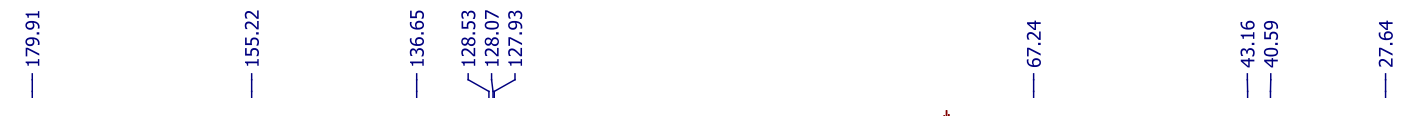<smiles>O=C(O)C1CCN(C(=O)OCc2ccccc2)CC1</smiles>

S1u

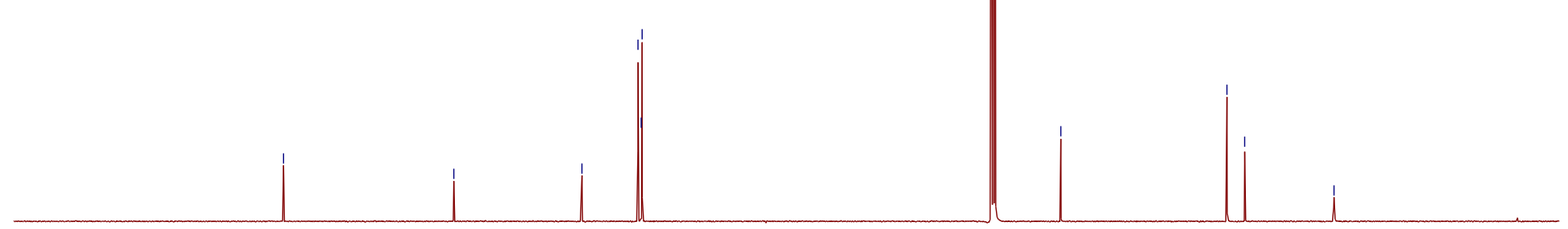


${ }^{1} \mathrm{H}-\mathrm{NMR}$ of compound $\mathbf{S 1 v}$

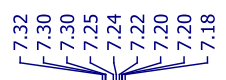

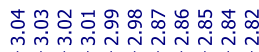

样

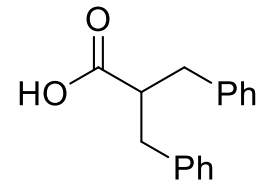

S1v

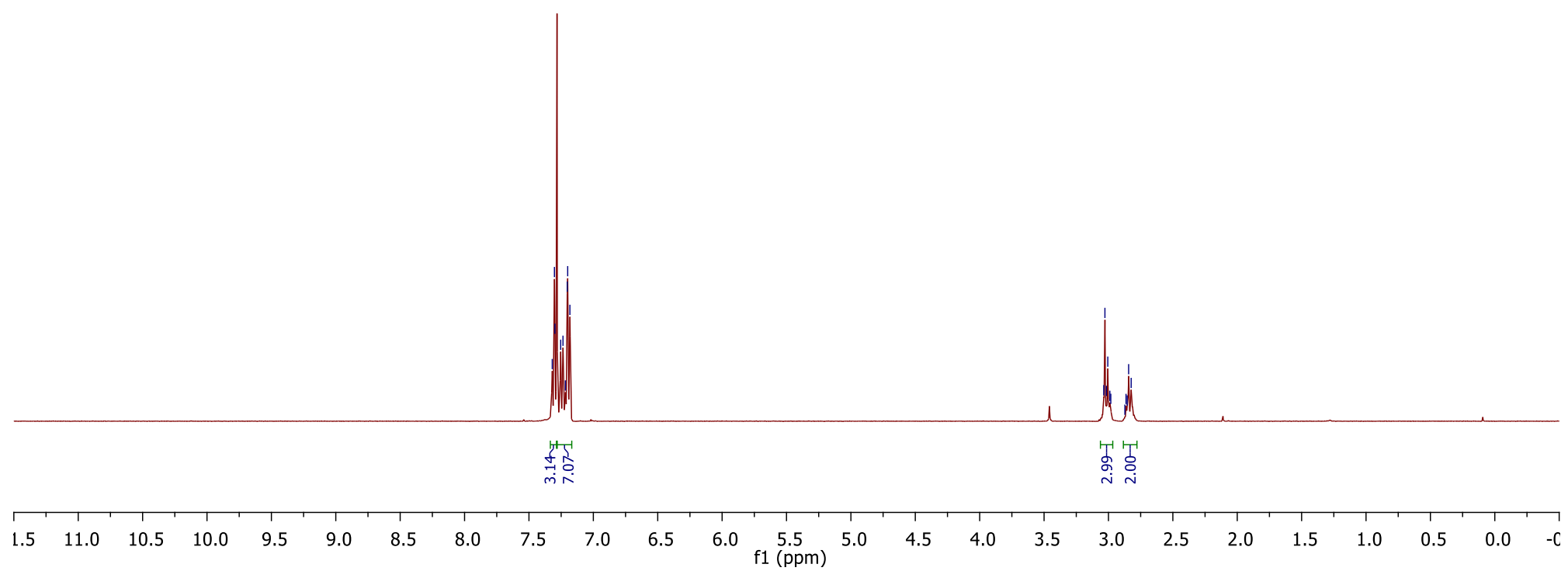


${ }^{13} \mathrm{C}$-NMR of compound S1v
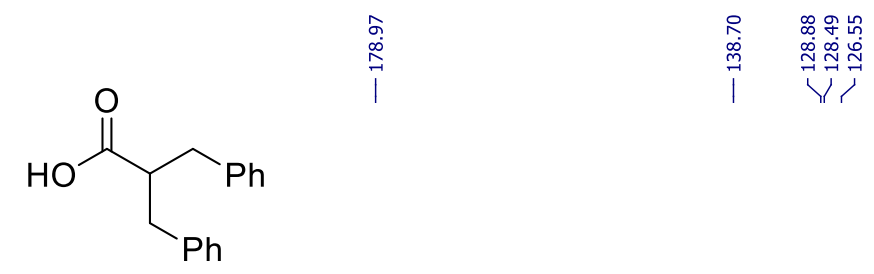

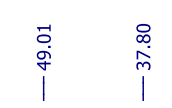

S1v

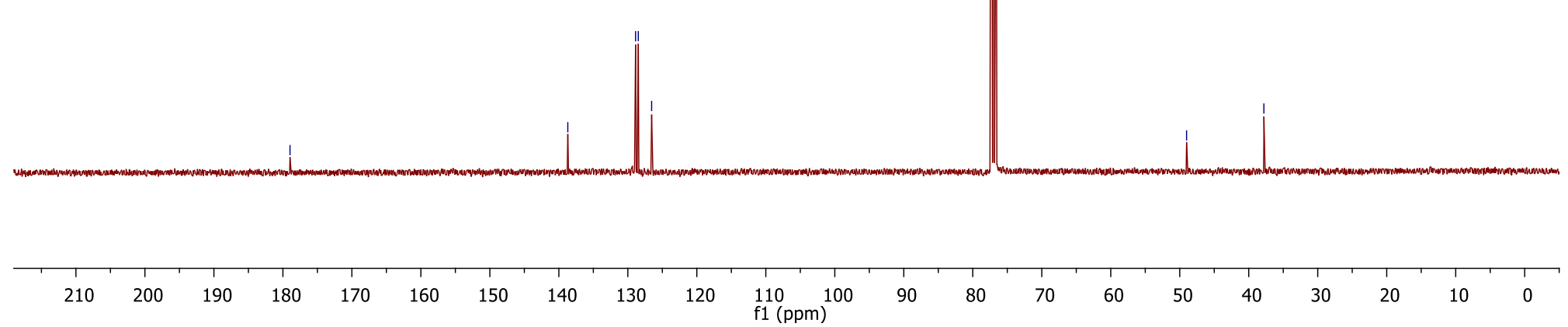


${ }^{1}$ H-NMR of compound $\mathbf{S 1 y}$
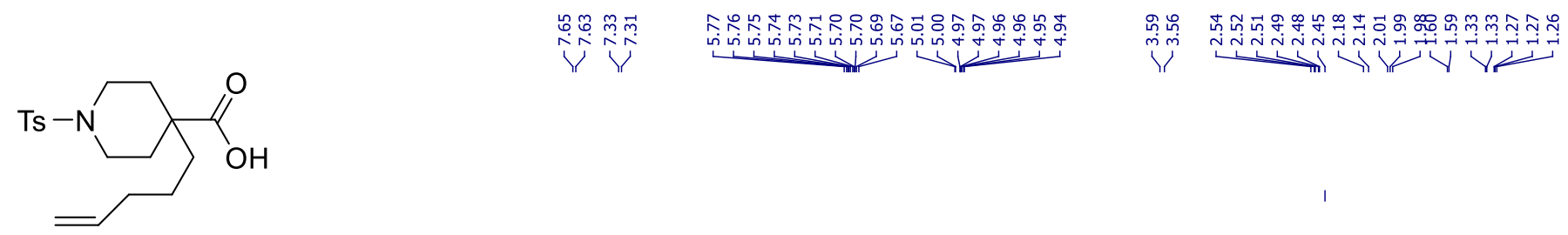

S1y

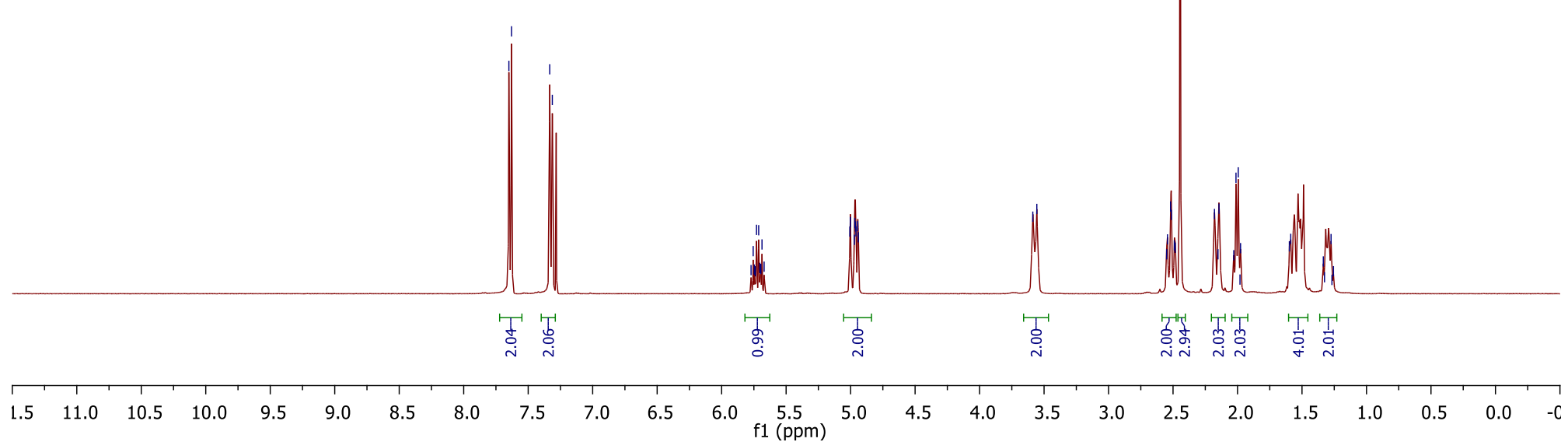


${ }^{13} \mathrm{C}-\mathrm{NMR}$ of compound $\mathbf{S 1 y}$

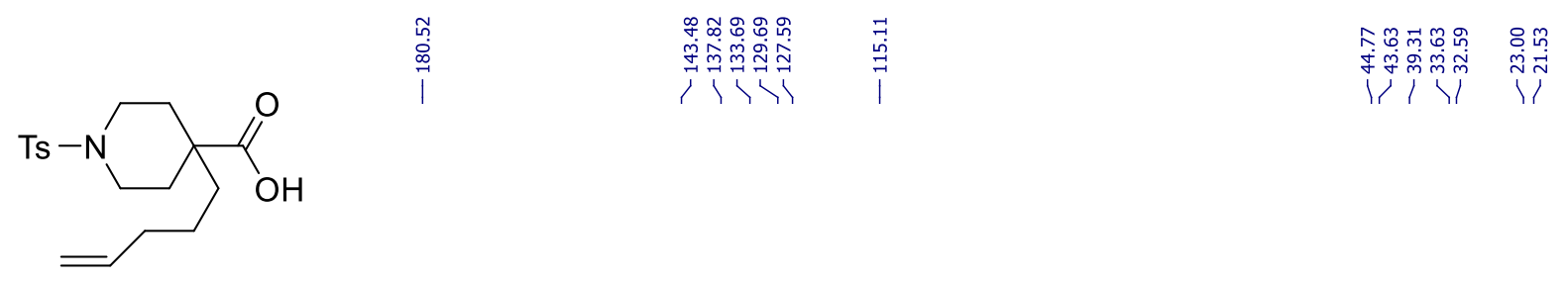

s1y

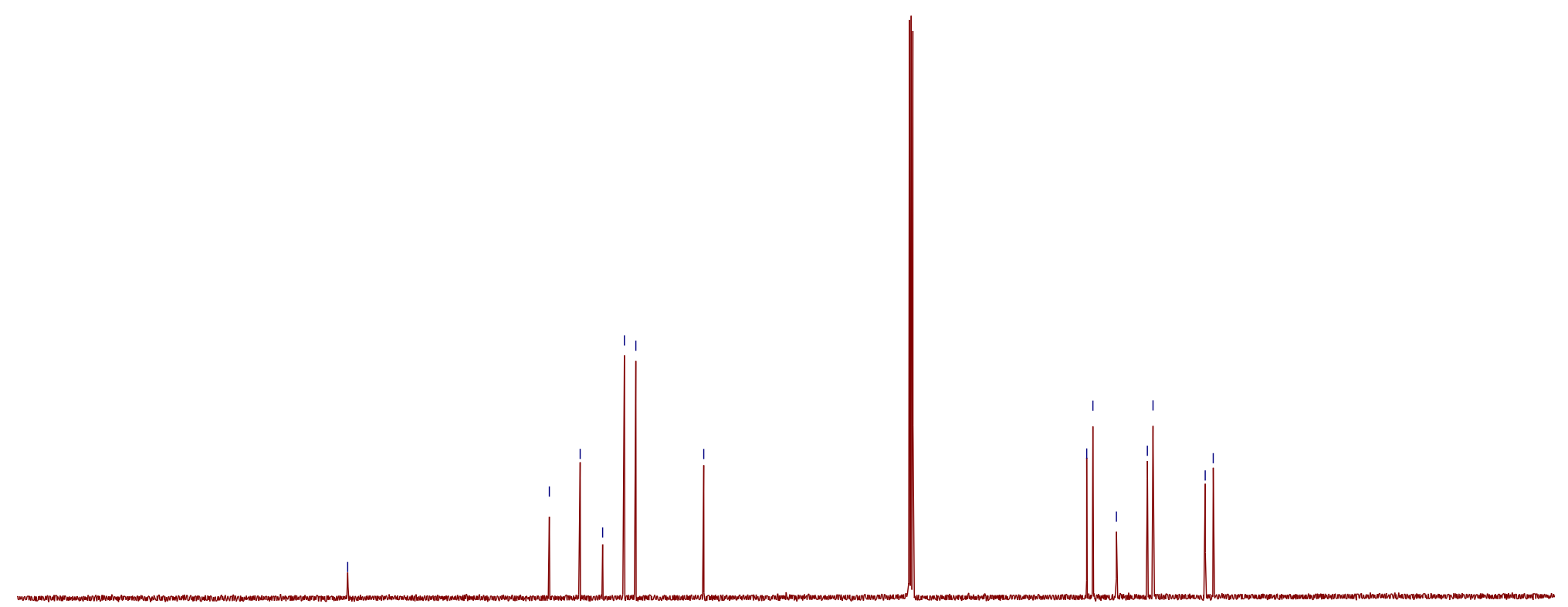

$\begin{array}{llllllllllllllllllllllllllllllllllllllllllllllllll}240 & 230 & 220 & 210 & 200 & 190 & 180 & 170 & 160 & 150 & 140 & 130 & 120 & 110 & 100 & 90 & 80 & 70 & 60 & 50 & 40 & 30 & 20 & 10 & 0 & -10 & -20 & -30 & -4\end{array}$ 
${ }^{1}$ H-NMR of compound S1z
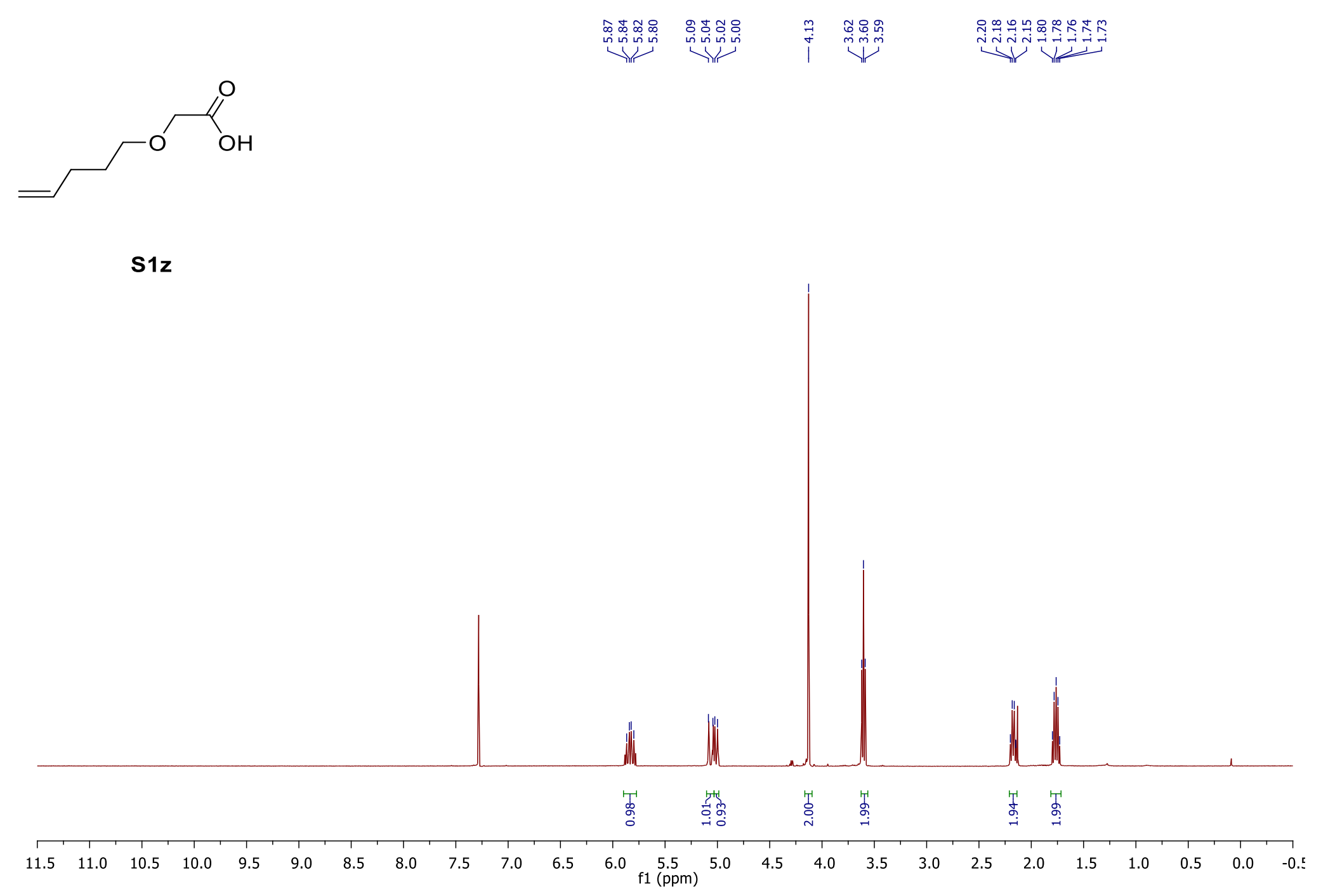
${ }^{13} \mathrm{C}$-NMR of compound $\mathbf{S 1 z}$

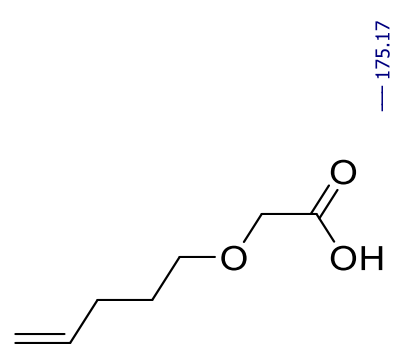

S1z

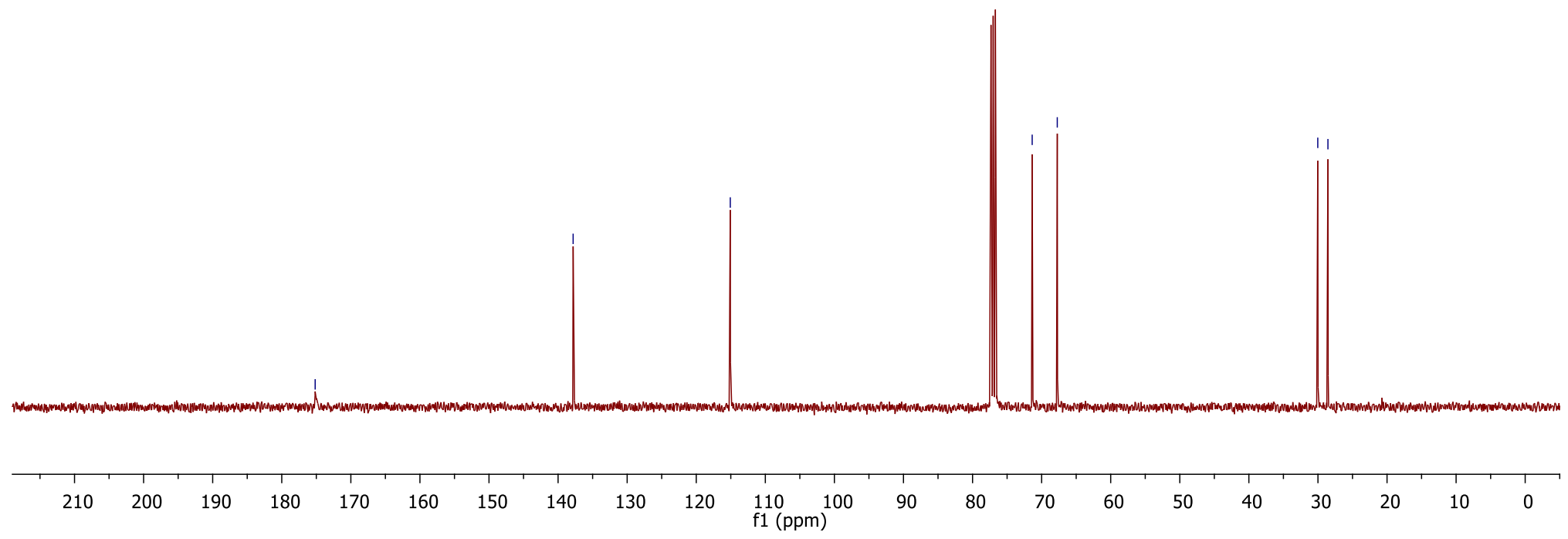


${ }^{1} \mathrm{H}-\mathrm{NMR}$ of compound S2e
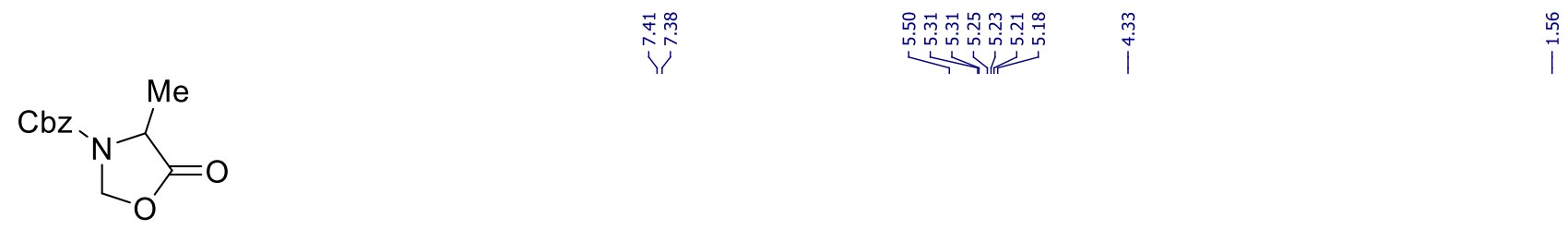

S2e

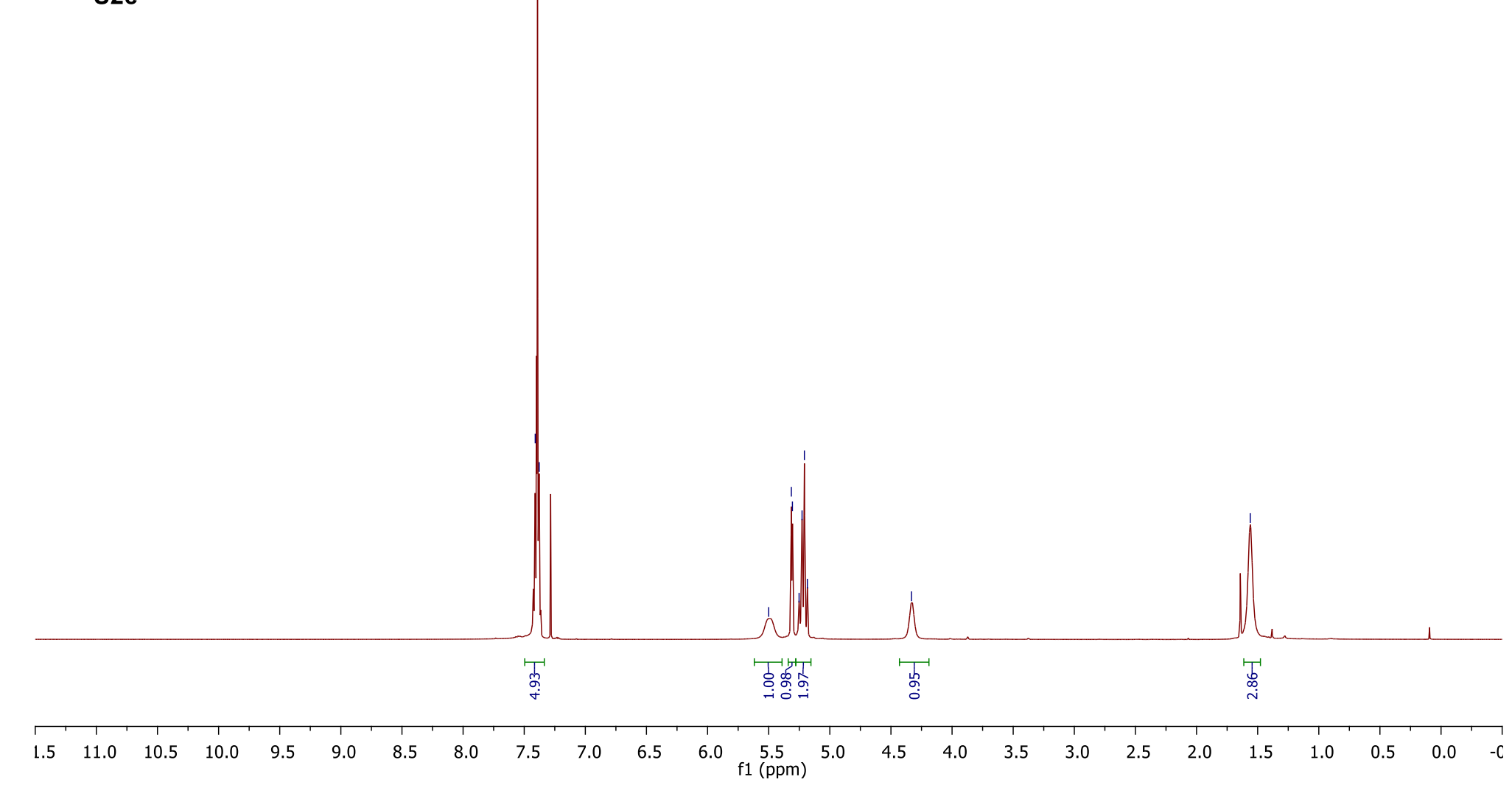


${ }^{13} \mathrm{C}$-NMR of compound S2e

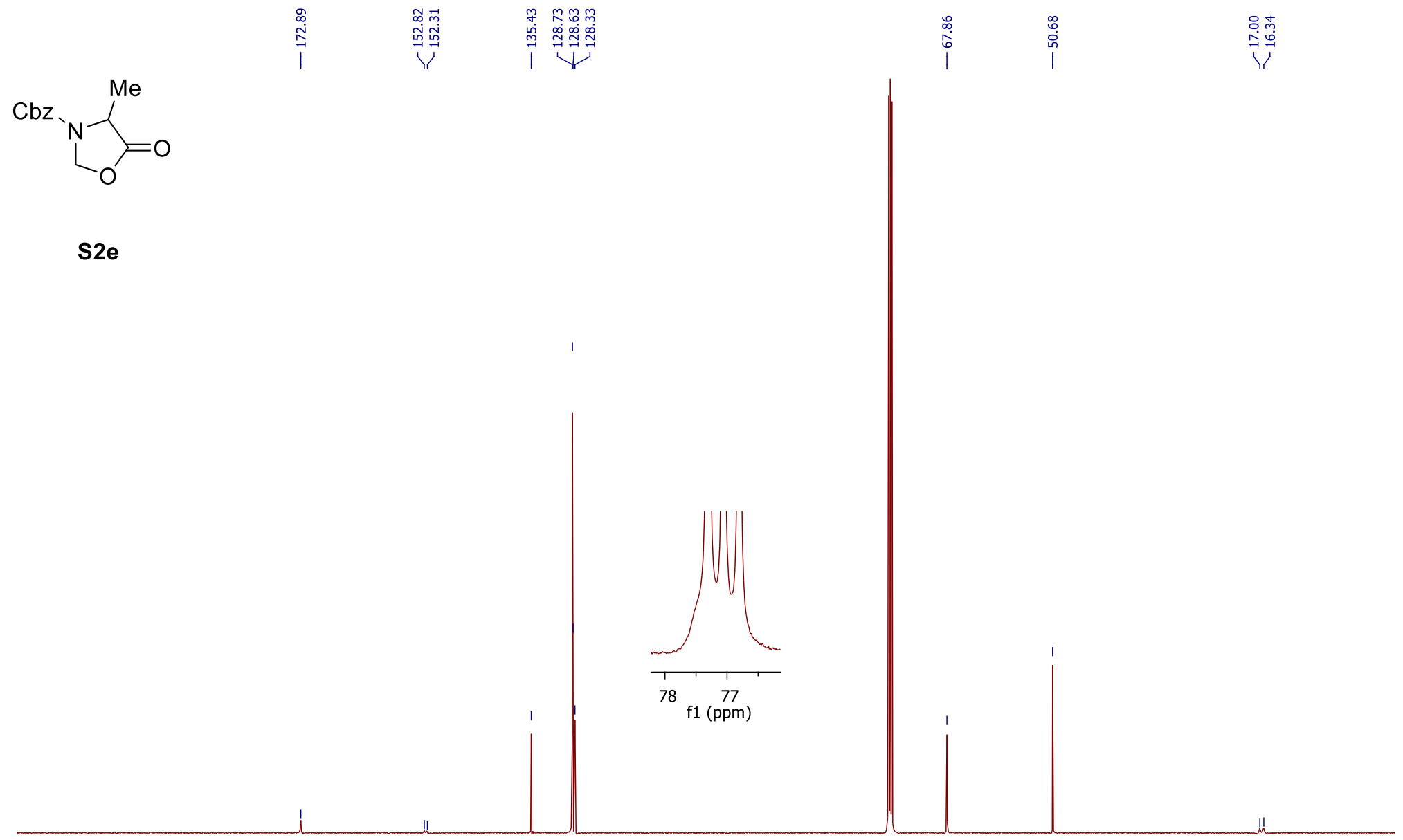

$\begin{array}{lllllllll}210 & 200 & 190 & 180 & 170 & 160 & 150 & 140 & 130\end{array}$

$120 \quad 110100$

$90 \quad 80$

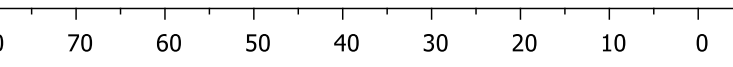


${ }^{1} \mathrm{H}-\mathrm{NMR}$ of compound $\mathbf{S 2 h}$

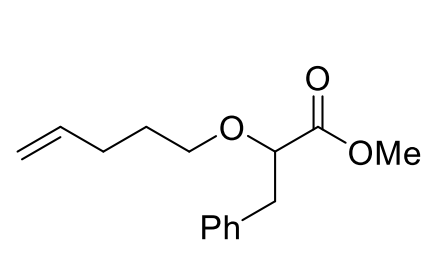

S2h

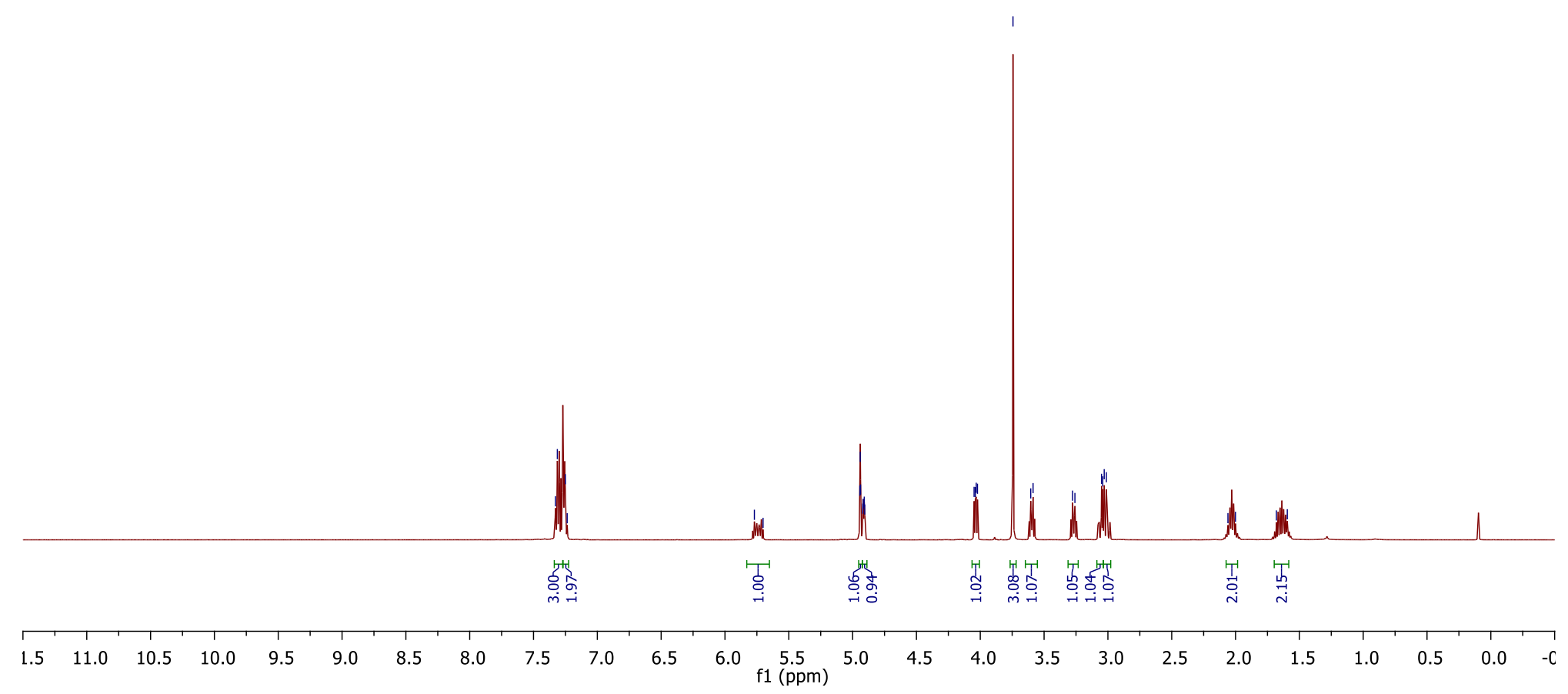


${ }^{13} \mathrm{C}-\mathrm{NMR}$ of compound $\mathbf{S 2 h}$

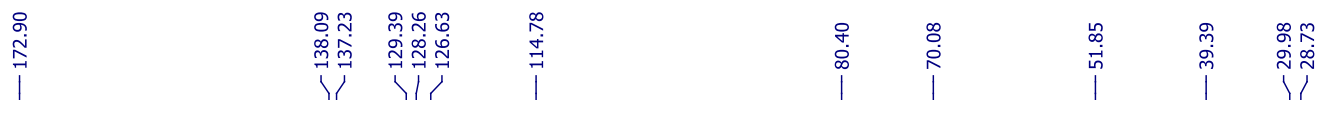<smiles>C=CCCCOC(Cc1ccccc1)C(=O)OC</smiles>

S2h

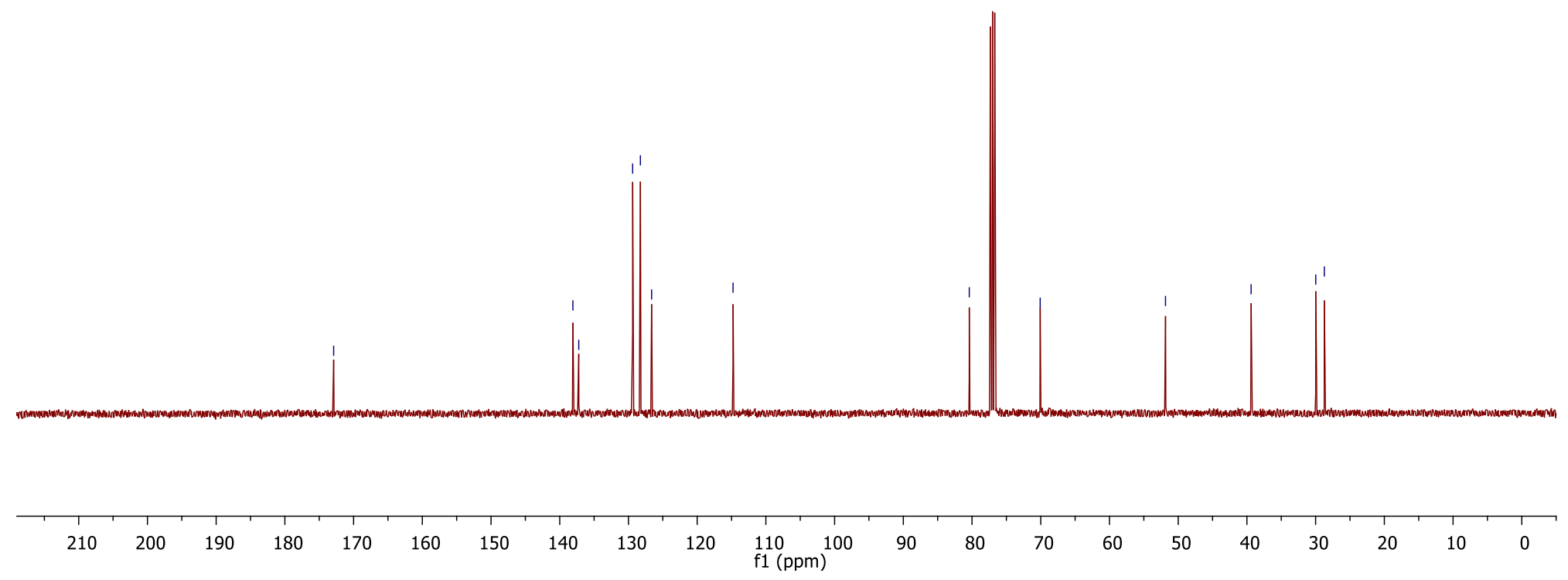


${ }^{1}$ H-NMR of compound S2p

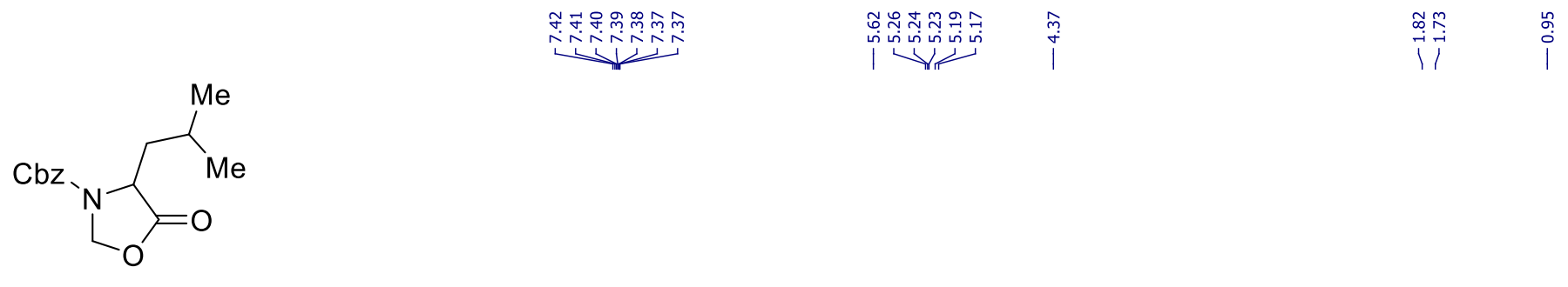

s2p

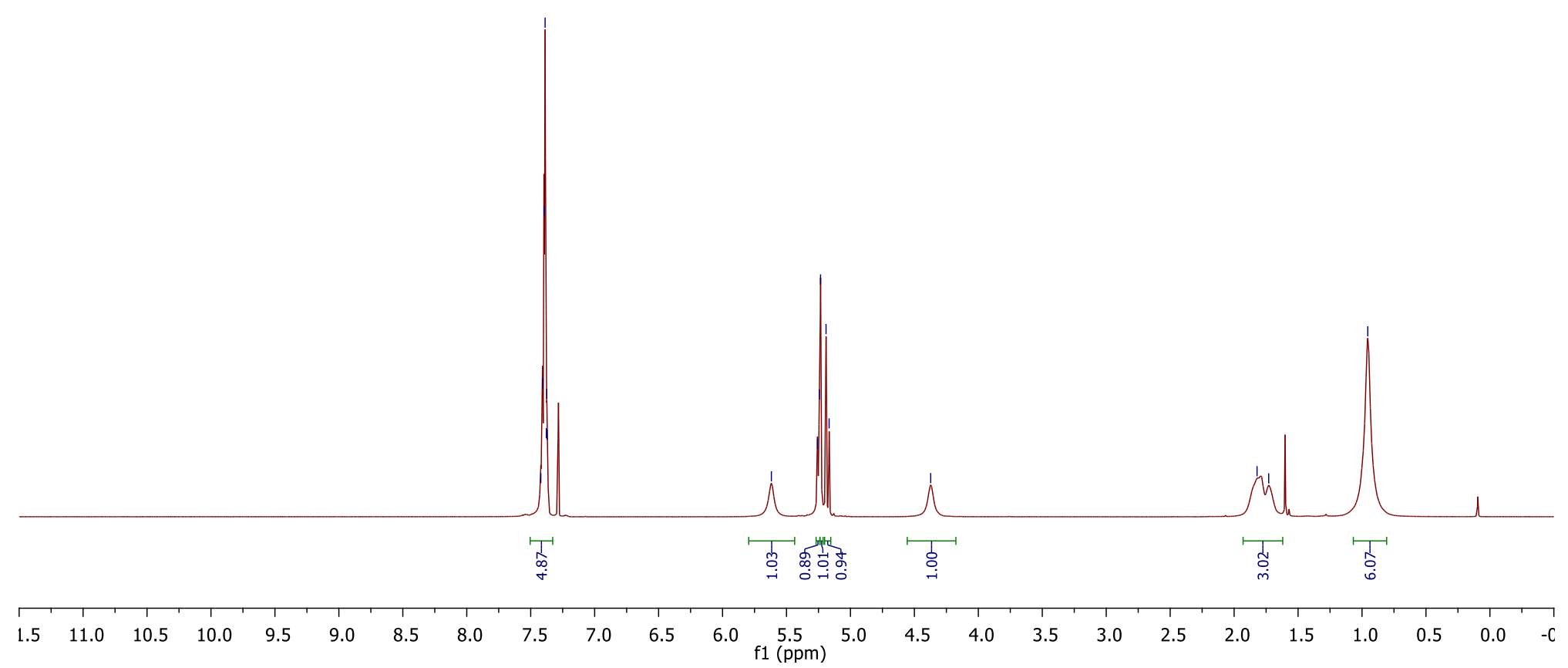


${ }^{13} \mathrm{C}$-NMR of compound S2p

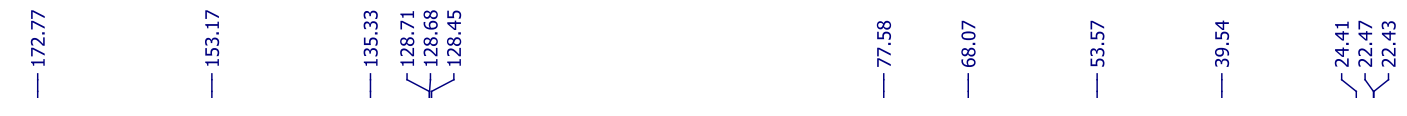

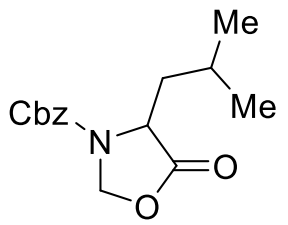

S2p

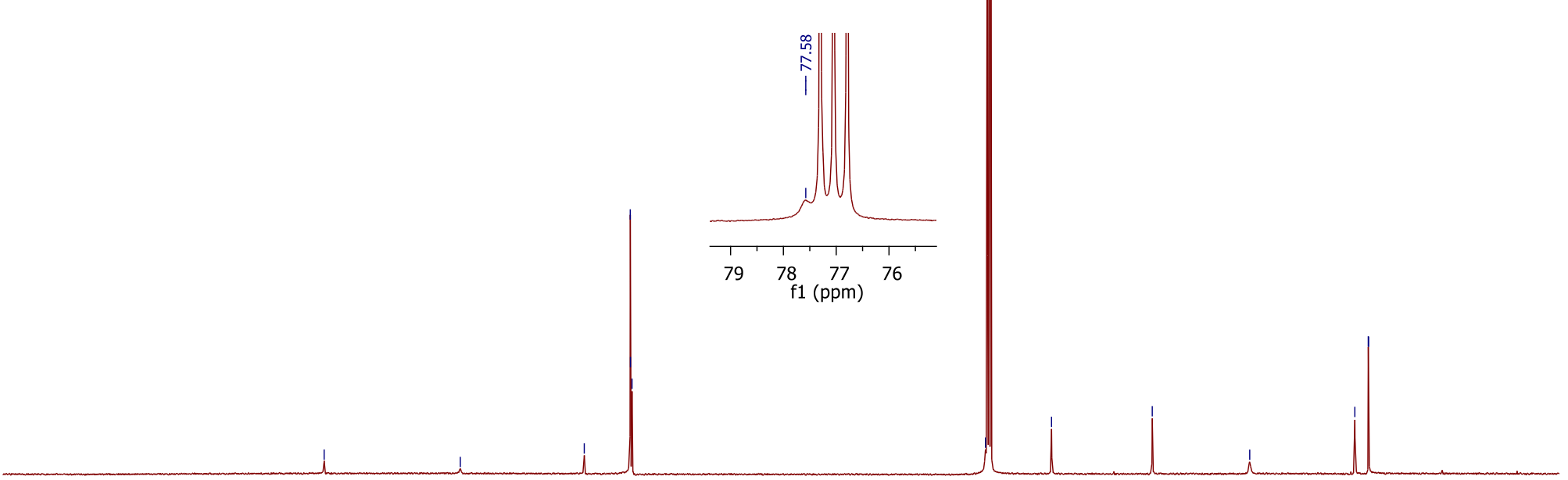


${ }^{1} \mathrm{H}-\mathrm{NMR}$ of compound $\mathbf{S 2 q}$

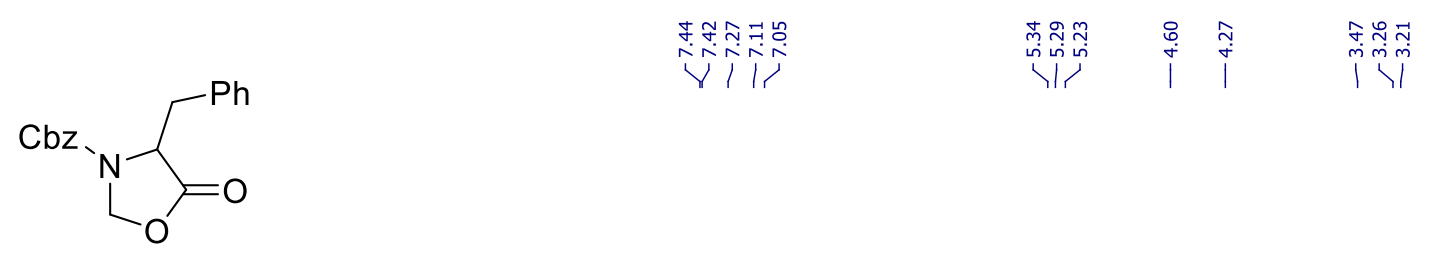

S2q

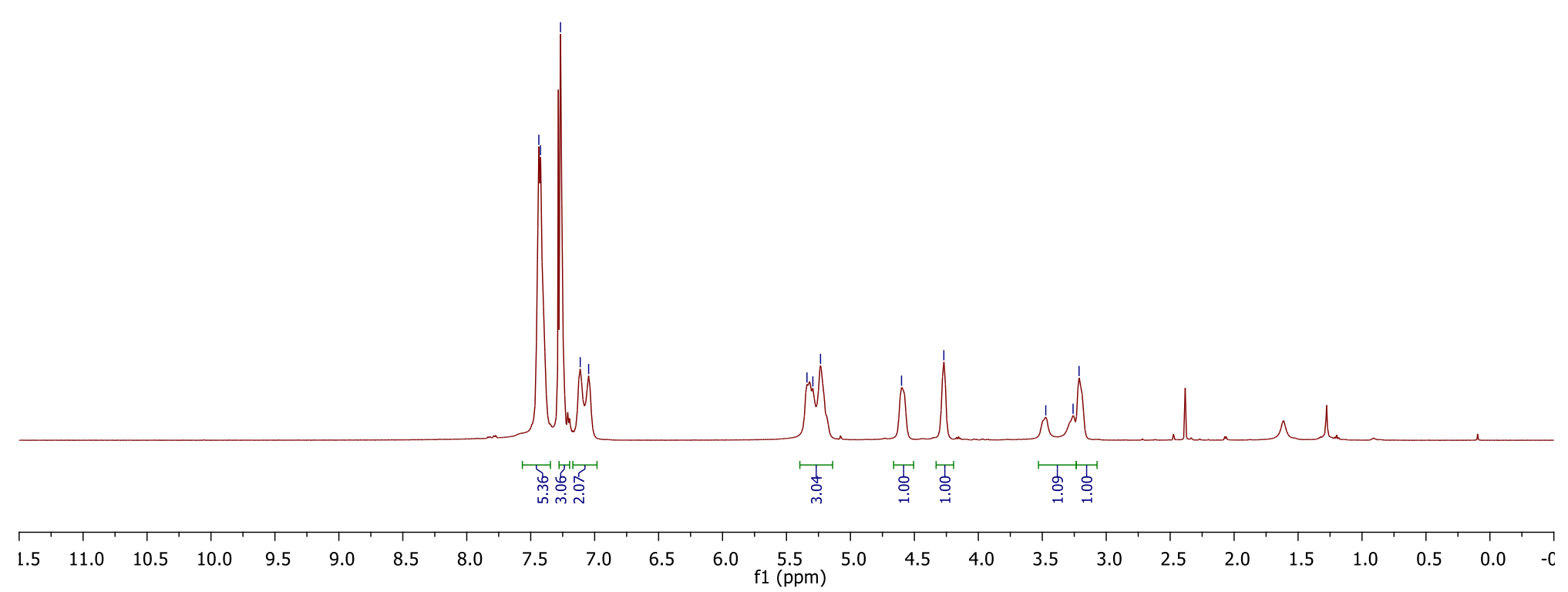


${ }^{13} \mathrm{C}$-NMR of compound S2q

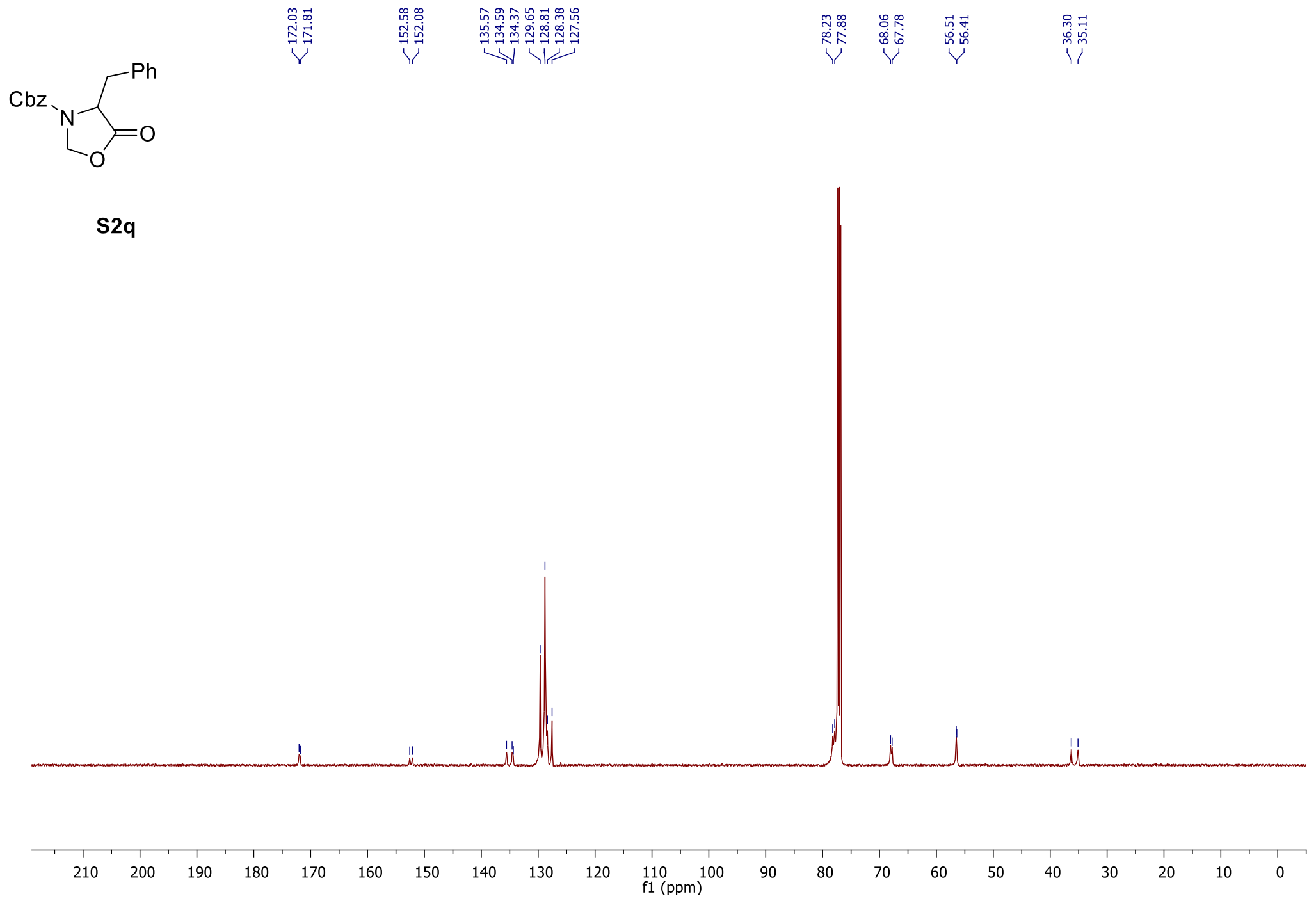


${ }^{1}$ H-NMR of compound S2r
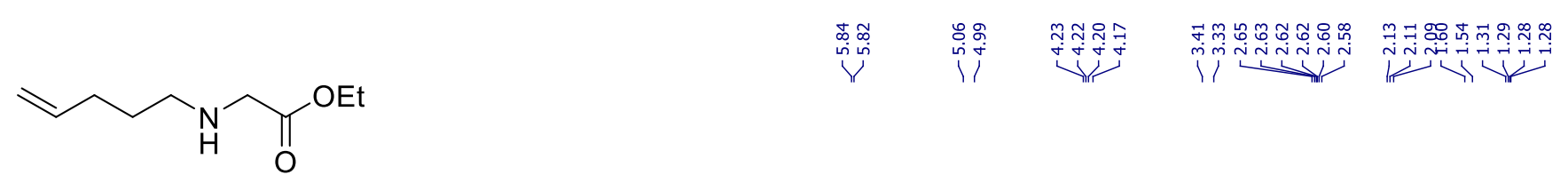

$\mathrm{s} 2 \mathrm{r}$

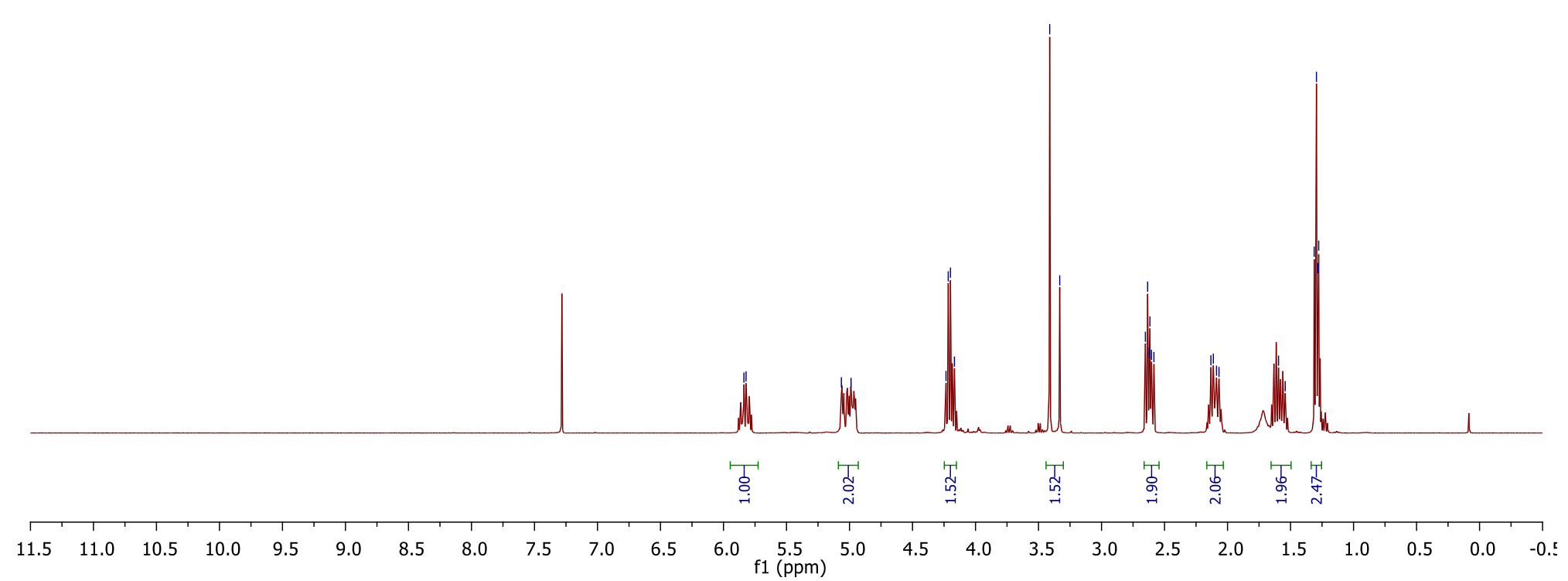


${ }^{13} \mathrm{C}$-NMR of compound S2r

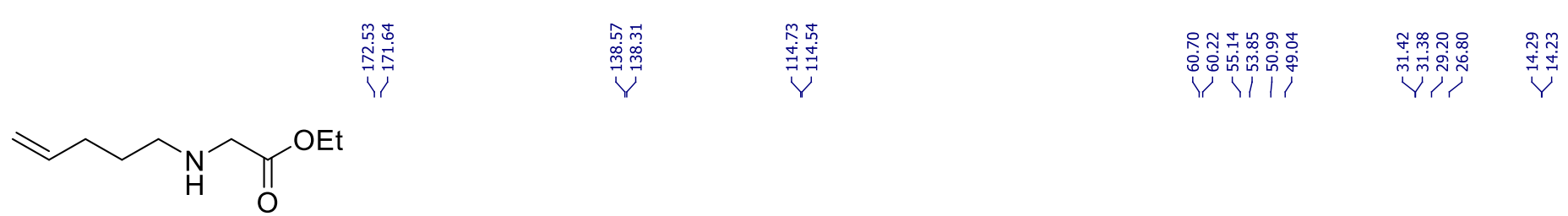

s2r

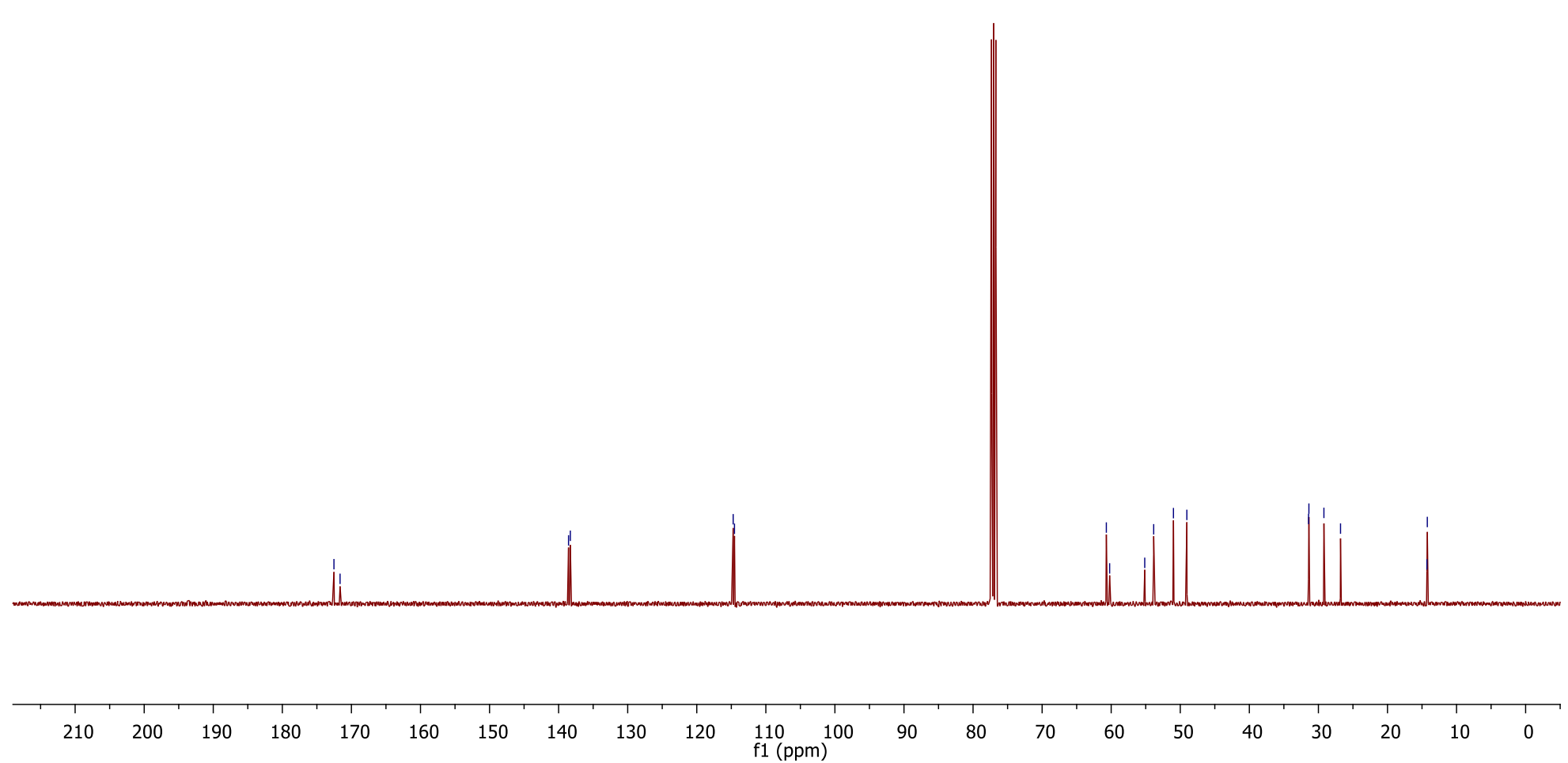


${ }^{1} \mathrm{H}-\mathrm{NMR}$ of compound $\mathbf{S 3 \mathbf { v }}$

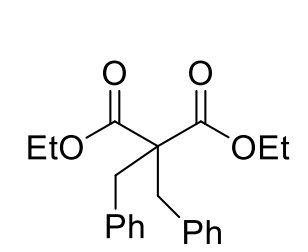

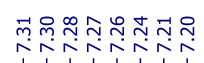

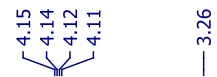

|ึ่

S3v

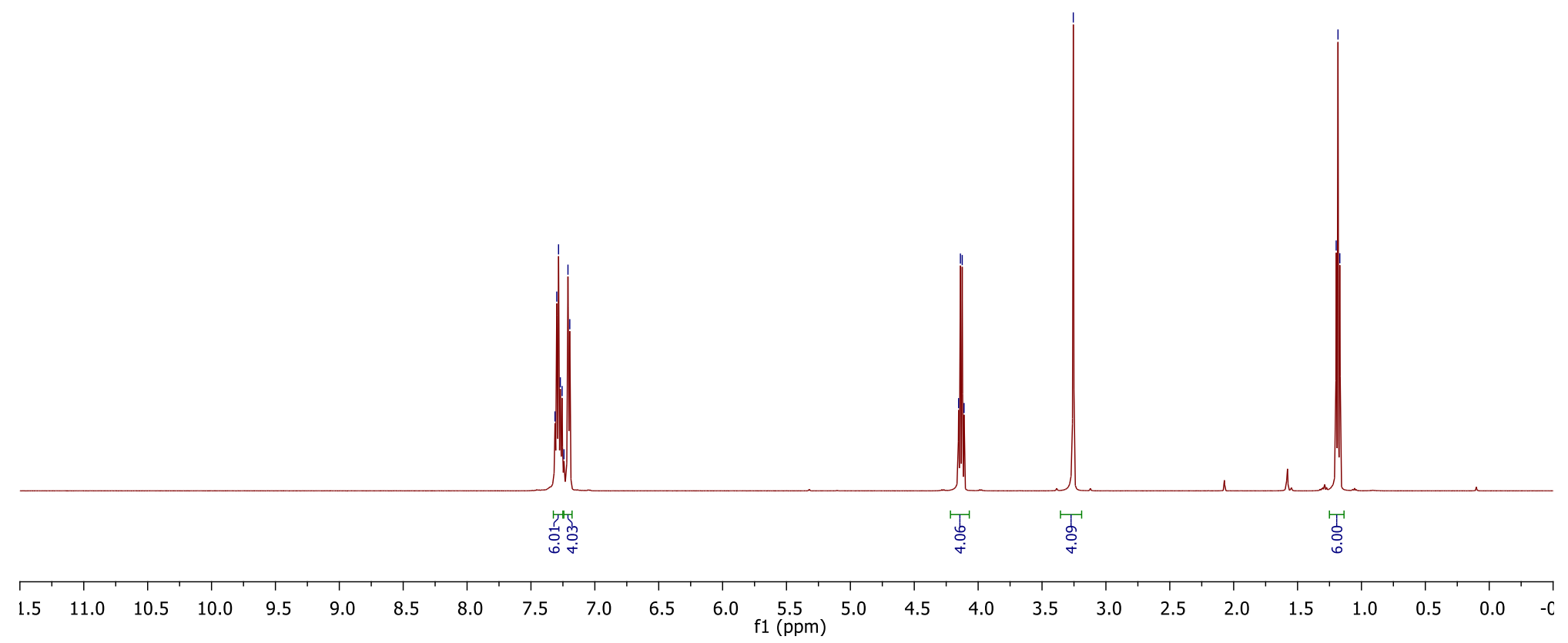


${ }^{13} \mathrm{C}$-NMR of compound $\mathbf{S 3 \mathbf { v }}$
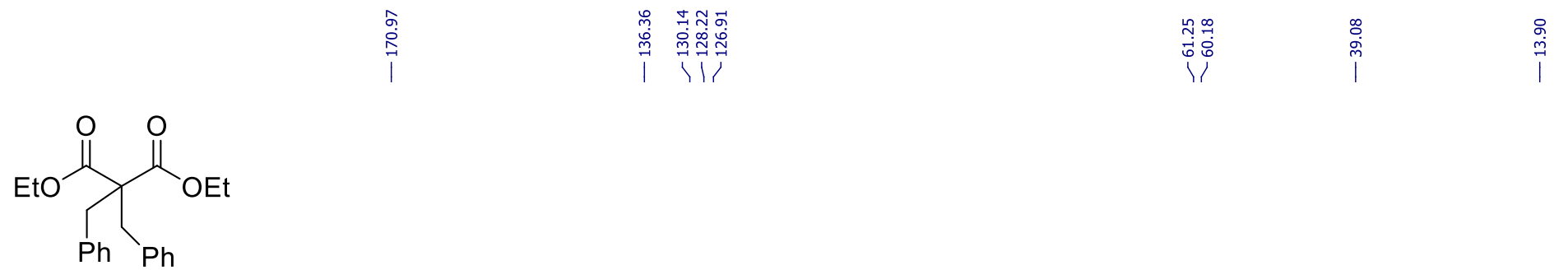

S3v

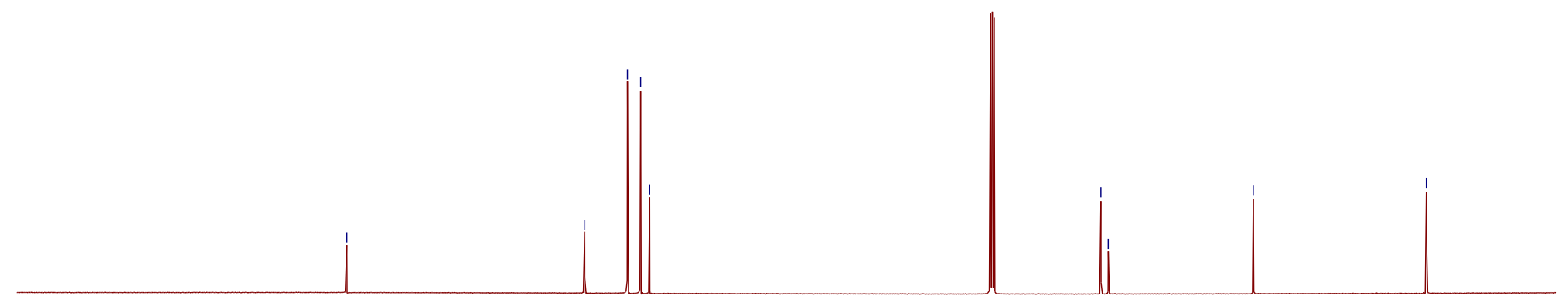


${ }^{1} \mathrm{H}$-NMR of compound S3w

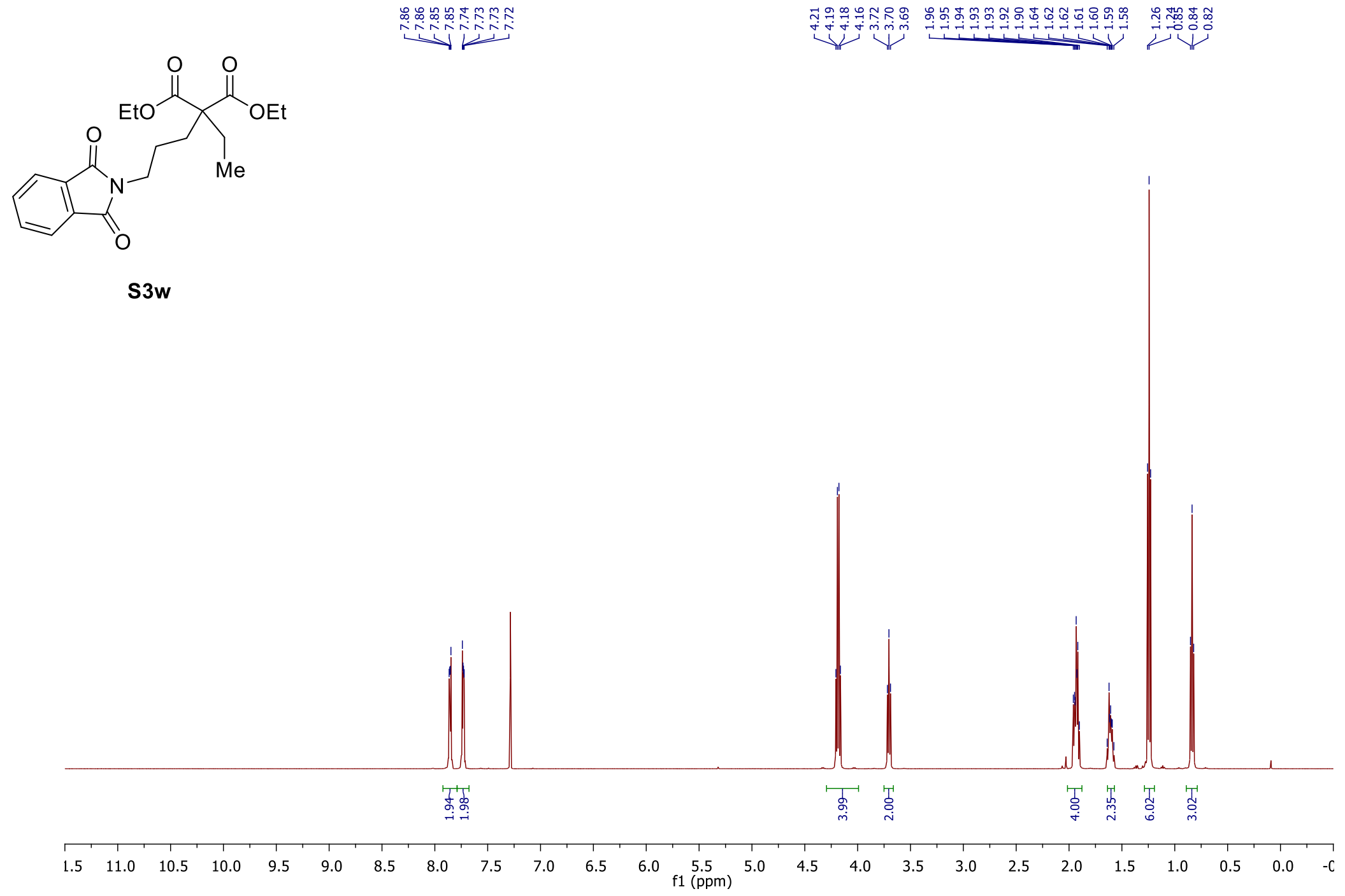


${ }^{13} \mathrm{C}-\mathrm{NMR}$ of compound $\mathbf{S 3 w}$

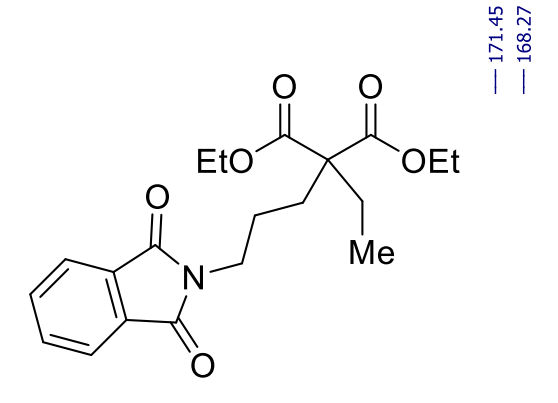

|

$\underset{1}{1}$

im

S3w

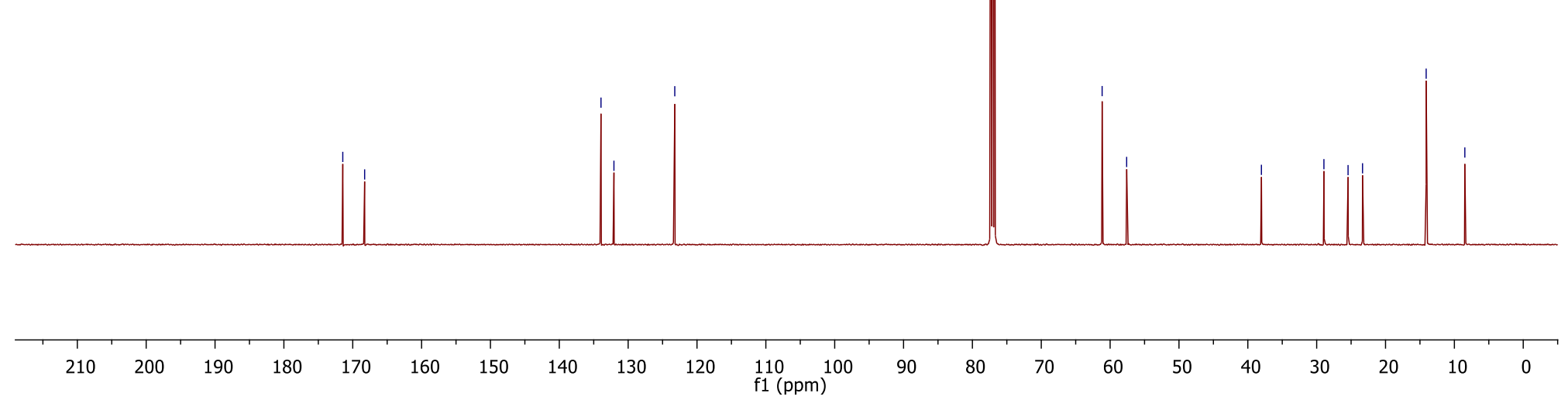


${ }^{1} \mathrm{H}-\mathrm{NMR}$ of compound $\mathbf{S 4} \mathbf{w}$

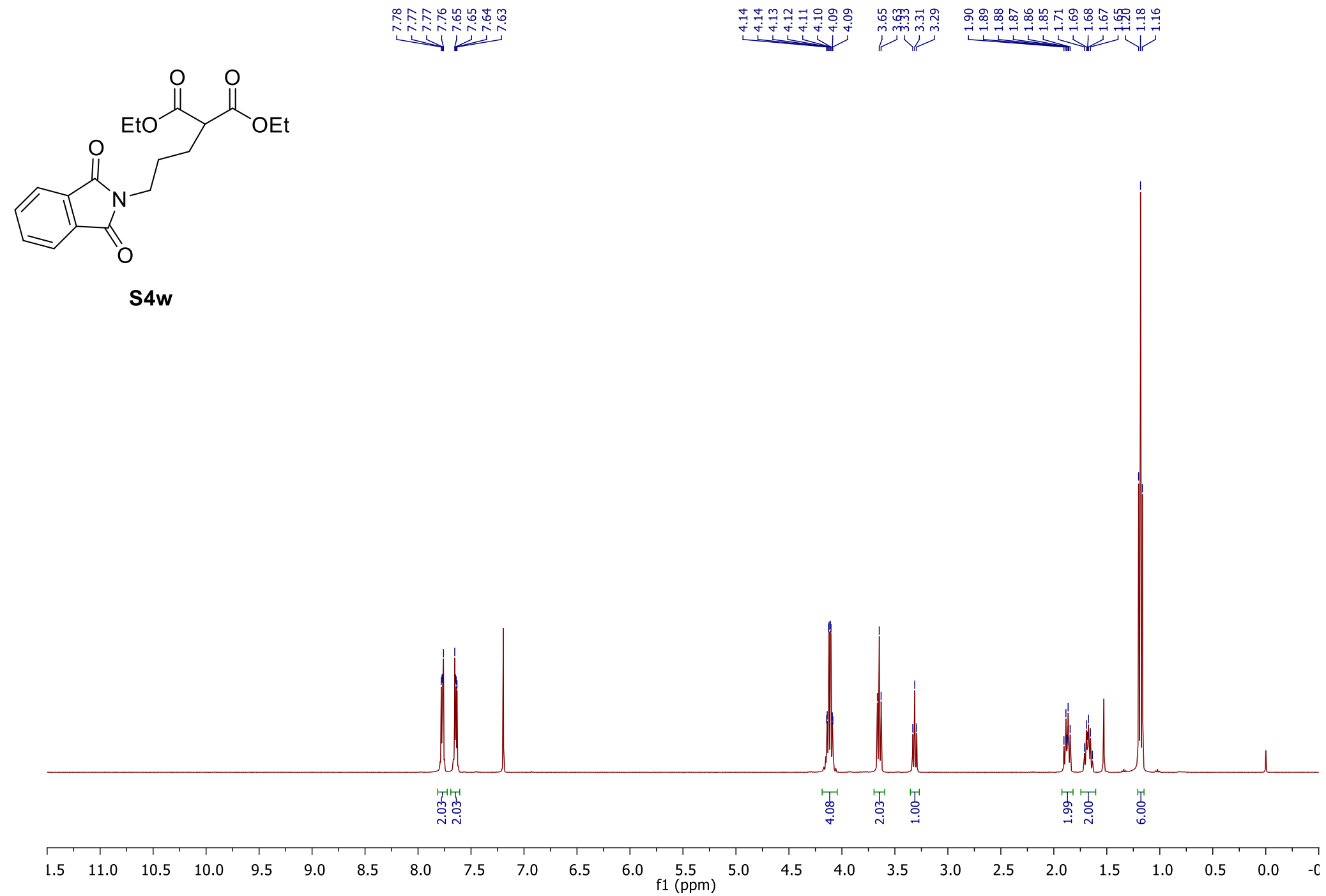


${ }^{13} \mathrm{C}-\mathrm{NMR}$ of compound $\mathbf{S 4 w}$

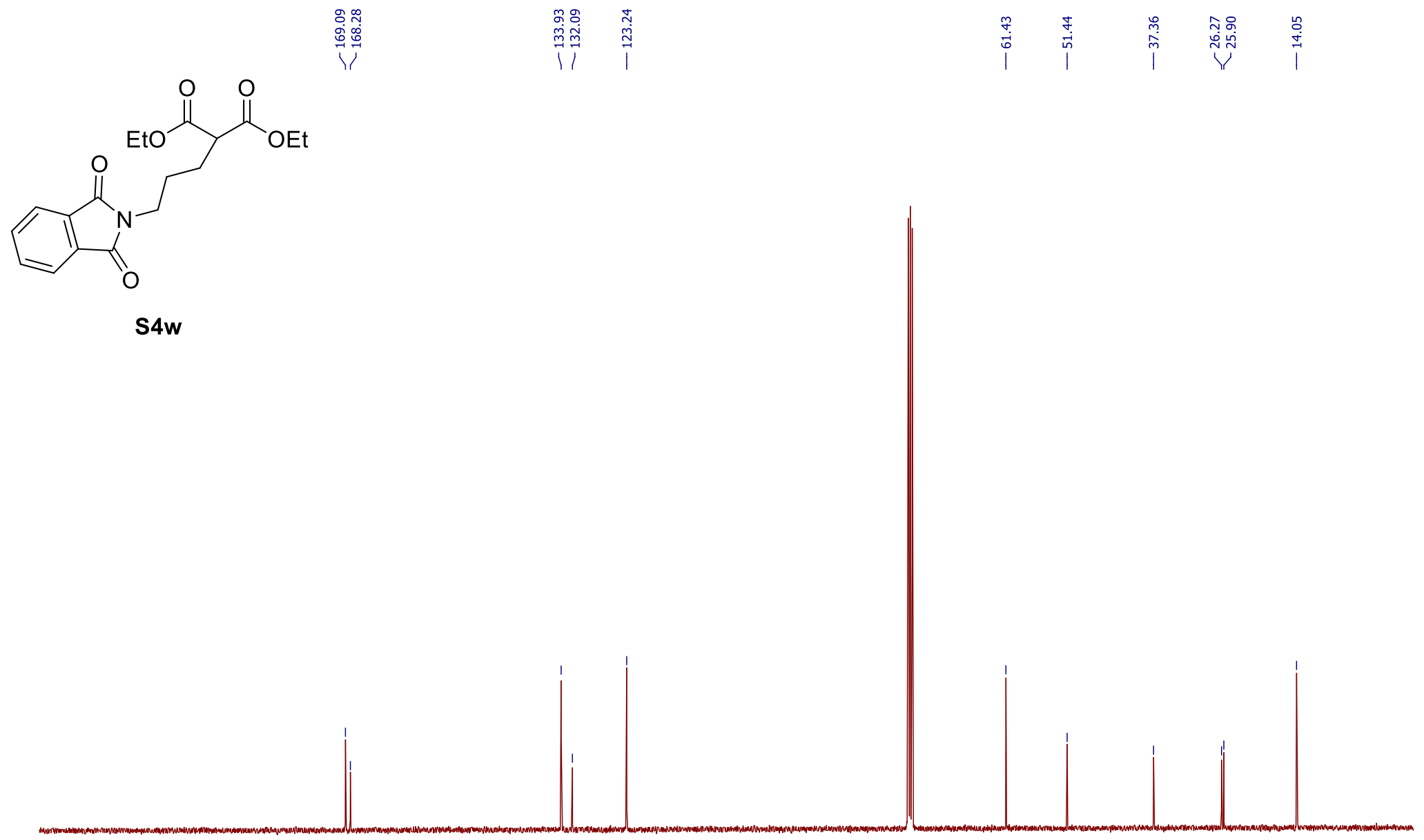

$\begin{array}{lllllllllllllllllllllllll}210 & 200 & 190 & 180 & 170 & 160 & 150 & 140 & 130 & 120 & 110 & 100 & 90 & 80 & 70 & 60 & 50 & 40 & 30 & 20 & 10 & 0\end{array}$ 
${ }^{1} \mathrm{H}-\mathrm{NMR}$ of compound S5w

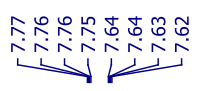

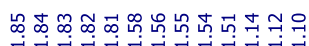

武诸

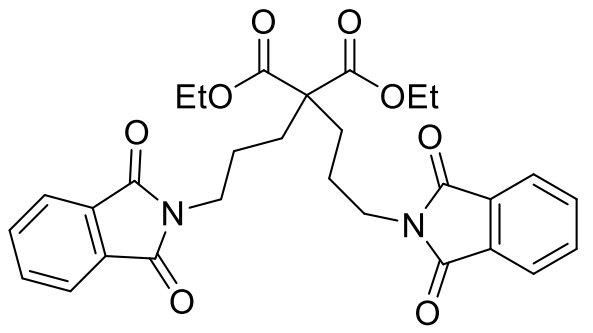

S5w

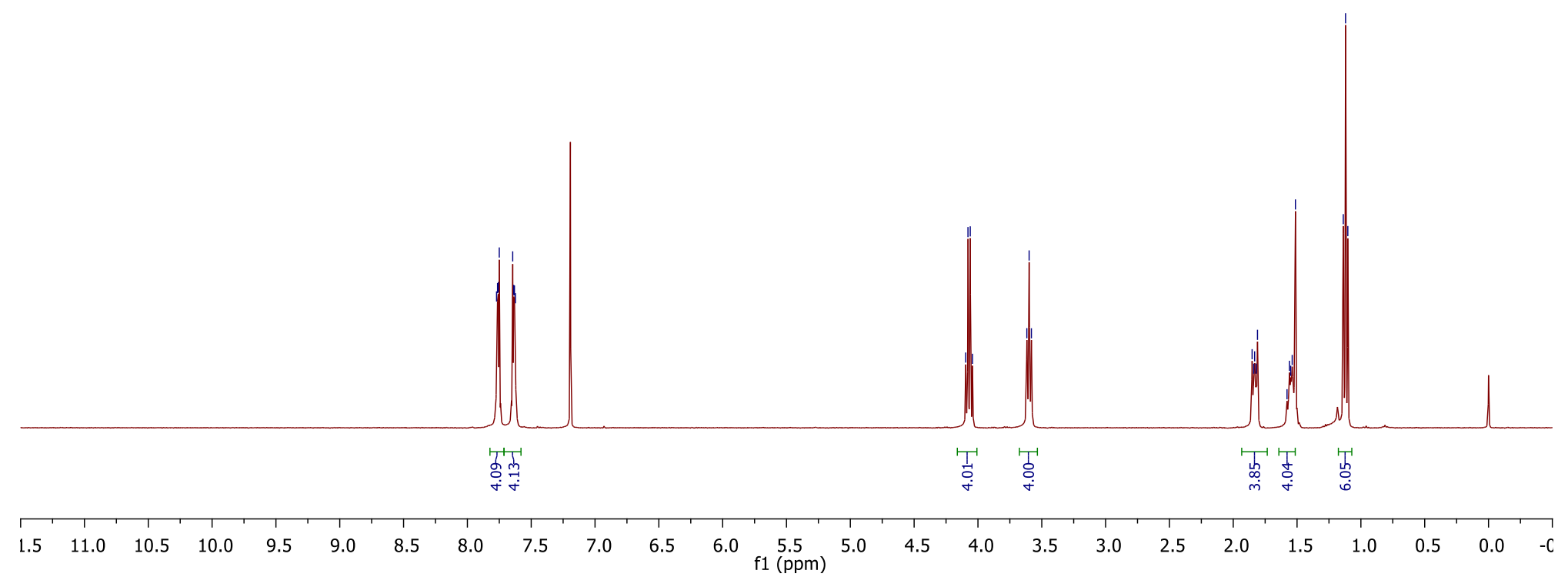


${ }^{13} \mathrm{C}-\mathrm{NMR}$ of compound $\mathbf{S 5} \mathbf{w}$

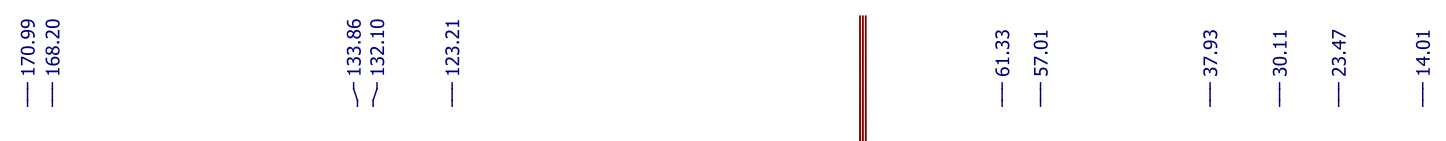

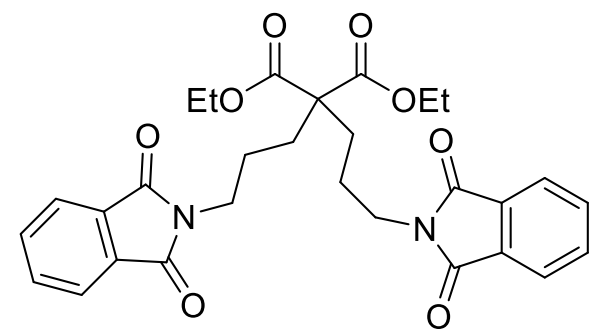

S5w

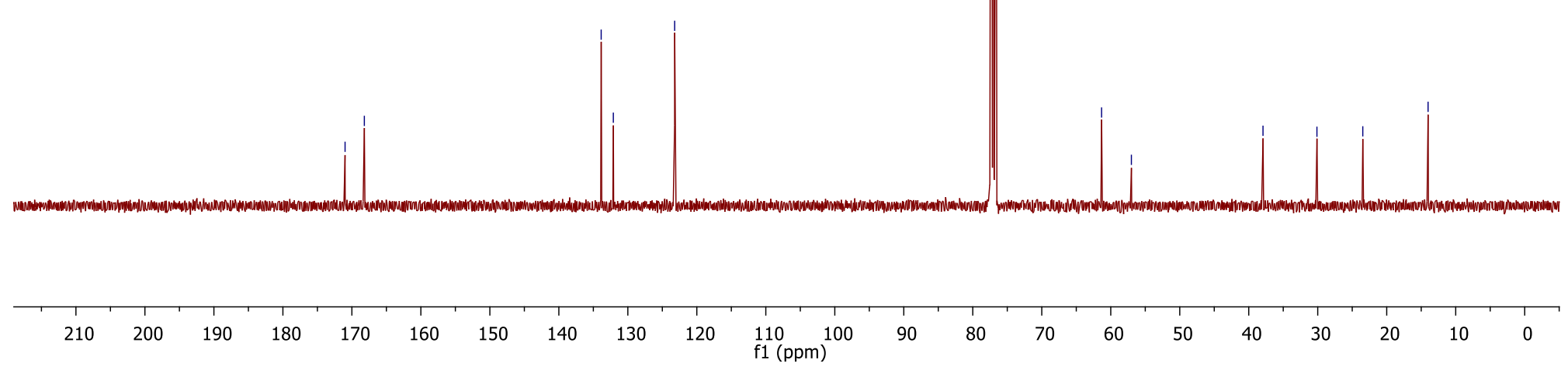




\section{References:}

[1] C. M. Tam, C. T. To, K. S. Chan, Organometallics 2016, 35, 2174-2177.

[2] J. H. Markgraf, R. Chang, J. R. Cort, J. L. Durant Jr, M. Finkelstein, A. W. Gross, M. H. Lavyne, C. M. Moore, R. C. Petersen, S. D. Ross, Tetrahedron 1997, 53, 10009-10018.

[3] F. W. Wassmundt, W. F. Kiesman, J. Org. Chem. 1995, 60, 1713-1719.

[4] M. Salamone, M. Milan, G. A. DiLabio, M. Bietti, J. Org. Chem. 2013, 78, 5909-5917.

[5] Y.-D. Wu, C.-L. Wong, K. W. K. Chan, G.-Z. Ji, X.-K. Jiang, J. Org. Chem. 1996, 61, 746-750.

[6] B. G. Gowenlock, P. P. Jones, J. R. Majer, Trans. Faraday Soc. 1961, 57, $23-27$.

[7] W.-Z. Liu, F. G. Bordwell, J. Org. Chem. 1996, 61, 4778-4783.

[8] M. Grübel, I. Bosque, P. J. Altmann, T. Bach, C. R. Hess, Chem. Sci. 2018, 9, 3313-3317.

[9] M. H. Shaw, V. W. Shurtleff, J. A. Terrett, J. D. Cuthbertson, D. W. C. MacMillan, Science (80-. ). 2016, 352, 1304-1308.

[10] D. Sikriwal, D. K. Dikshit, Tetrahedron 2011, 67, 210-215.

[11] S. Sugasawa, M. Murayama, Chem. Pharm. Bull. 1958, 6, 194-200. 